\title{
Deformações e isotopias de álgebras de Jordan
}

\author{
Maria Eugenia Martin
}

\author{
TESE APRESENTADA AO INSTITUTO DE \\ MATEMÁTICA E ESTATÍSTICA \\ DA UNIVERSIDADE DE SÃO PAULO \\ PARA OBTENÇÃO DO TÍTULO DE \\ DOUTOR EM MATEMÁTICA
}

Orientadora: Prof ${ }^{a}$. Dr ${ }^{\mathrm{a}}$. Iryna Kashuba

Durante o desenvolvimento deste trabalho o autor recebeu auxílio financeiro da CAPES e do CNPq.

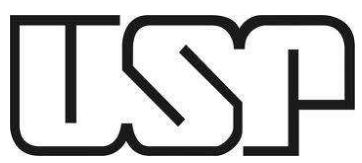

Universidade de São Paulo

São Paulo, 1o de outubro de 2013 



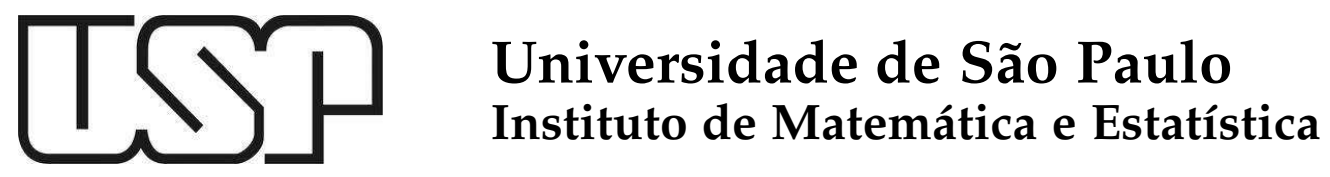

\title{
Deformações e isotopias de álgebras de Jordan
}

\author{
Maria Eugenia Martin
}

Orientadora: Prof ${ }^{\mathrm{a}}$. Dr ${ }^{\mathrm{a}}$. Iryna Kashuba

\begin{abstract}
Esta versão da tese contém as correções e alterações sugeridas pela Comissão Julgadora durante a defesa, realizada em 11/o9/2013. O original encontra-se disponível no Instituto de Matemática e Estatística da Universidade de São Paulo.
\end{abstract}

Comissão Julgadora:

- Prof ${ }^{\mathrm{a}}$. Dr ${ }^{\mathrm{a}}$. Iryna Kashuba (Orientadora) - IME - USP

口 Prof. Dr. Ivan Shestakov - IME - USP

- Prof $^{\mathrm{a}}$. Dr ${ }^{\mathrm{a}}$. Irina Sviridova - Universidade de Brasília

$\square$ Prof $^{\mathrm{a}}$. Dr ${ }^{\mathrm{a}}$. Maria Trushina - University State of Moscow

口 Prof. Dr. Roldão da Rocha - Universidade Federal do ABC

São Paulo, 10 de outubro de 2013 



\section{AGRADECIMENTOS}

Agradeço, primeiramente, à professora Iryna Kashuba por me introduzir nesta interessante área da matemática, pela sua orientação, amizade e dedicação.

Agradeço aos professores da banca pelas correções, comentários e sugestões. Aos professores I. Shestakov IME-USP e P. Koshlukov IMECC-UNICAMP pelos conselhos valiosos e por me introduzir ao conceito de isotopias.

Agradeço ao CNPq e a CAPES por terem financiado este projeto.

À minha família e amigos. Aos meus pais que sempre me incentivaram e estiveram comigo apesar da distância. Aos meus sogros por cuidarem da minha filha enquanto realizava este trabalho.

À minha filha Paloma pela paciência e compreensão dos momentos ausente.

E, por último, quero agradecer muito especialmente ao meu marido Daniel pela companhia, infinitos conselhos, muitas revisões, ajuda com a língua portuguesa, em fim por tudo o que ele fez para que seja possível a realização deste trabalho. 



\section{RESUMO}

Neste trabalho apresentamos a classificação algébrica e geométrica das álgebras de Jordan de dimensões pequenas sobre um corpo $\mathbf{k}$ algebricamente fechado de char $\mathbf{k} \neq 2$ e sobre o corpo dos números reais. A classificação algébrica foi realizada de duas maneiras: a menos de isomorfismos e a menos de isotopias. Enquanto que a classificação geométrica foi feita estudando as variedades de álgebras de Jordan Jor ${ }_{n}$ para $n \leqslant 4$ e $\mathrm{Jor}_{n}^{\mathbb{R}}$ para $n \leqslant 3$. Provamos que Jor 4 tem 73 órbitas sob a ação de GL(V) e que é a união dos fechos de Zariski das órbitas de 10 álgebras rígidas, cada um dos quais corresponde a uma componente irredutível. Analogamente, mostramos que $\mathrm{Jor}_{3}^{\mathbb{R}}$ tem 26 órbitas e é a união dos fechos de Zariski das órbitas de 8 álgebras rígidas. Também obtivemos que o número de componentes irredutíveis em Jor 5 é $\geqslant 26$. Construímos ainda três famílias de álgebras rígidas não associativas, não semisimples e indecomponíveis as quais correspondem a componentes irredutíveis de Jor $_{n}$ e $\operatorname{Jor}_{n}^{\mathbb{R}}$ para todo $n \geqslant 5$.

Palavras-chaves: Álgebras de Jordan, Deformação, Isotopia 



\section{ABSTRACT}

\section{Deformations and isotopies of Jordan algebras}

In this work we present the algebraic and geometric classification of Jordan algebras of small dimensions over an algebraically closed field $\mathbf{k}$ of char $\mathbf{k} \neq 2$ and over the field of real numbers. The algebraic classification was accomplished in two ways: up to isomorphism and up to isotopy. On the other hand, the geometric classification was obtained studying the varieties of Jordan algebras Jor ${ }_{n}$ for $n \leqslant 4$ and Jor $_{n}^{\mathbb{R}}$ for $n \leqslant 3$. We prove that Jor $_{4}$ has 73 orbits under the action of GL(V) and it is the union of Zariski closures of the orbits of 10 rigid algebras, each of which corresponds to one irreducible component. Analogously, we show that $\mathrm{Jor}_{3}^{\mathbb{R}}$ has 26 orbits and is the union of Zariski closures of the orbits of 8 rigid algebras. Also we obtain that the number of irreducible components in Jor $_{5}$ is $\geqslant 26$. We construct three families of indecomposable non-semisimple, non-associative rigid algebras which for any $n \geqslant 5$, correspond to irreducible components of Jor $_{n}$ and Jor $_{n}^{\mathbb{R}}$.

Keywords: Jordan Algebras, Deformation, Isotopy 



\section{SUMÁRIO}

Introdução $\quad 1$

$1 \quad$ Introdução às Álgebras de Jordan $\quad 7$

1.1 Definições e Exemplos 7

1.2 Resultados Principais 12

1.3 Decomposição de Peirce 15

1.4 Extensões e Cohomologia 17

2 Classificação Algébrica das Álgebras de Jordan de Dimensão 4 sobre um Corpo Algebricamente Fechado 21

2.1 Álgebras de Jordan de Dimensão menor que $4 \quad 22$

2.1.1 Álgebras de Jordan de Dimensão 122

2.1.2 Álgebras de Jordan de Dimensão 222

2.1.3 Álgebras de Jordan de Dimensão 322

2.2 Álgebras de Jordan de Dimensão $4 \quad 23$

2.2.1 Álgebras de Jordan Semissimples 24

2.2.2 Álgebras de Jordan com Radical de Dimensão $1 \quad 25$

2.2.3 Álgebras de Jordan com Radical de Dimensão $2 \quad 27$

2.2.4 Álgebras de Jordan com Radical de Dimensão 30

2.2.5 Álgebras de Jordan Nilpotentes 42

2.2.6 Observações 44

3 Classificação Algébrica das Álgebras de Jordan de Dimensão 3 sobre o Corpo dos Números Reais 45

3.1 Álgebras de Jordan Reais de Dimensão menor que $3 \quad 46$

3.1.1 Álgebras de Jordan Reais de Dimensão 146

3.1.2 Álgebras de Jordan Reais de Dimensão $2 \quad 46$

3.2 Álgebras de Jordan Reais de Dimensão 346

3.2.1 Álgebras de Jordan Reais Semissimples 47

3.2.2 Álgebras de Jordan Reais com Radical de dimensão $1 \quad 48$

3.2.3 Álgebras de Jordan Reais com Radical de dimensão $2 \quad 50$

3.2.4 Álgebras de Jordan Reais Nilpotentes $\quad 5^{1}$

3.2.5 Observações 53 
4 Deformações 55

4.1 Introdução à Geometria Algébrica 56

4.1.1 Variedades Algébricas 56

4.1.2 Dimensão 58

4.1.3 Ação de Grupos 59

4.2 Deformações Infinitesimais de Álgebras de Jordan 60

4.3 A Variedade Algébrica Jor ${ }_{n} \quad 64$

4.4 O Comportamento de uma Álgebra através de Deformação 74

4.5 Algumas Componentes Irredutíveis de Jor $_{m}$ para $m \geqslant 2 \quad 79$

5 Classificação Geométrica das Álgebras de Jordan de Dimensão menor ou igual a 4 sobre um Corpo Algebricamente Fechado $\quad 87$

5.1 A Variedade Algébrica Jor 187

5.2 A Variedade Algébrica Jor 288

5.3 A Variedade Algébrica Jor 390

5.4 A Variedade Algébrica Jor 496

5.5 Algumas Propriedades da Variedade Algébrica Jor $5 \quad 111$

6 Classificação Geométrica das Álgebras de Jordan de Dimensão menor ou igual a 3 sobre o Corpo dos Números Reais 115

6.1 A Variedade Algébrica Jor ${ }_{1}^{\mathbb{R}} \quad 115$

6.2 A Variedade Algébrica Jor ${ }_{2}^{\mathbb{R}} \quad 115$

6.3 A Variedade Algébrica Jor ${ }_{3}^{\mathbb{R}} \quad 117$

6.4 Comparação das Variedades $\operatorname{Jor}_{n}^{\mathbb{R}}$ e $\operatorname{Jor}_{n}^{\mathbb{C}} \quad 124$

7 Álgebras Isotópicas 127

7.1 Introdução às Isotopias 127

7.2 Classificação Algébrica a menos de Isotopia das álgebras de Jordan Reais de dimensão $3 \quad 130$

8 Conclusões e Sugestões para Pesquisas Futuras 135

A Programas em Mathematica 139

A.1 Define o Produto de Jordan na Base Canônica 139

A.2 Verifica se duas Álgebras são Isomorfas 141

A.3 Verifica se uma Álgebra é de Jordan, Flexível, Associativa, e/ou Alternativa. 142

A.4 Verifica se uma Álgebra é Unitária 144

A.5 Verifica se é Subálgebra. 145 
A.6 Calcula a Dimensão do Aniquilador de uma Álgebra 146

A.7 Calcula a Dimensão das Potências 146

A.8 Calcula a Dimensão do Grupo de Automorfismo 147

A.9 Calcula a dimensão de $\mathrm{H}^{2}$. 149

A.1o Calcula a dimensão do Centro Associador $\quad 151$

A.11 Realiza a Soma Direta de Álgebras de Jordan; 152

A.12 Decompõe uma Álgebra em Soma Direta 153

A.13 Exibe o Produto da Álgebra 154

A.14 Exibe Resumo das Propriedades da Álgebra 154

в Informações sobre as Álgebras de Jordan de Dimensão $4 \quad 157$

C Informações sobre as Álgebras de Jordan Reais de Dimensão 3209

Bibliografia 222 



\section{LISTA DE FIGURAS}

$\begin{array}{lll}\text { Fig. 4.1 Equivalências entre os diversos conceitos de rigidez } & 71\end{array}$

Fig. 5.1 Descrição completa das órbitas de Jor 289

Fig. 5.2 Descrição quase-completa das órbitas de Jor 393

Fig. 5.3 Descrição das órbitas de Jor 4 101

Fig. 5.4 Descrição mais completa das órbitas de Jor $4 \quad 102$

Fig. 6.1 Descrição completa das órbitas de Jor ${ }_{2}^{\mathbb{R}} \quad 116$

Fig. 6.2 Descrição quase-completa das órbitas de Jor $_{3}^{\mathbb{R}} \quad 123$ 



\section{LISTA DE TABELAS}

Tabela 2.1 $\quad$ k-álgebras de Jordan indecomponíveis de dimensão $2 \quad 22$

Tabela 2.2 k-álgebras de Jordan indecomponíveis de dimensão 322

Tabela 2.3 k-álgebras de Jordan semissimples de dimensão $4 \quad 25$

Tabela 2.4 k-álgebras de Jordan de dimensão 4 com radical unidimensional e parte semissimples $\mathbf{k}_{\mathbf{1}} \oplus \mathbf{k} \boldsymbol{e}_{2} \oplus \mathbf{k} \boldsymbol{e}_{3} \quad 26$

Tabela 2.5 k-álgebras de Jordan de dimensão 4 com radical unidimensional e parte semissimples $\mathcal{T}_{5} \quad 27$

Tabela $2.6 \quad k$-álgebras de Jordan de dimensão 4 com radical de tipo (2) 28

Tabela $2.7 \quad \mathbf{k}$-álgebras de Jordan de dimensão 4 com radical de tipo $(1,1) \quad 29$

Tabela $2.8 \quad \mathbf{k}$-álgebras de Jordan de dimensão 4 com radical de tipo (3) 31

Tabela $2.9 \quad$ k-álgebras de Jordan de dimensão 4 com radical de tipo $(1,1,1) \quad 32$

Tabela 2.10 k-álgebras de Jordan de dimensão 4 com radical de tipo $(2,1)$ e $\mathrm{N}=$ $\mathrm{N}_{0} \quad 34$

Tabela 2.11 k-álgebras de Jordan de dimensão 4 com radical de tipo $(2,1)$ e $\mathrm{N}=$ $\mathrm{N}_{1} \quad 35$

Tabela 2.12 k-álgebras de Jordan de dimensão 4 com radical de tipo $(2,1)$ e $\mathrm{N}=\mathrm{N}_{0} \oplus \mathrm{N}_{\frac{1}{2}}$ com $\operatorname{dim} \mathrm{N}_{\mathrm{O}}=2 \quad 35$

Tabela 2.13 k-álgebras de Jordan de dimensão 4 com radical de tipo $(2,1)$ e $\mathrm{N}=\mathrm{N}_{0} \oplus \mathrm{N}_{1}$ com $\operatorname{dim} \mathrm{N}_{0}=2 \quad 36$

Tabela 2.14 k-álgebras de Jordan de dimensão 4 com radical de tipo $(2,1)$ e $\mathrm{N}=\mathrm{N}_{0} \oplus \mathrm{N}_{\frac{1}{2}} \operatorname{com} \operatorname{dim} \mathrm{N}_{\frac{1}{2}}=2 \quad 37$

Tabela 2.15 k-álgebras de Jordan de dimensão 4 com radical de tipo $(2,1)$ e $\mathrm{N}=\mathrm{N}_{0} \oplus \mathrm{N}_{\frac{1}{2}} \oplus \mathrm{N}_{1}$

Tabela 2.16 k-álgebras de Jordan de dimensão 4 com radical de tipo $(2,1)$ e $\mathrm{N}=\mathrm{N}_{0} \oplus \mathrm{N}_{1}$ com $\operatorname{dim} \mathrm{N}_{1}=2 \quad 40$

Tabela 2.17 k-álgebras de Jordan de dimensão 4 com radical de tipo $(2,1)$ e $\mathrm{N}=\mathrm{N}_{\frac{1}{2}} \oplus \mathrm{N}_{1} \operatorname{com} \operatorname{dim} \mathrm{N}_{1}=2 \quad 4 \mathrm{O}$

Tabela 2.18 k-álgebras de Jordan de dimensão 4 com radical de tipo $(2,1)$ e $\mathrm{N}=\mathrm{N}_{\frac{1}{2}} \oplus \mathrm{N}_{1}$ com $\operatorname{dim} \mathrm{N}_{\frac{1}{2}}=2 \quad 41$

Tabela 2.19 k-álgebras de Jordan nilpotentes de dimensão $4 \quad 42$

Tabela 3.1 $\mathbb{R}$-álgebras de Jordan indecomponíveis de dimensão $2 \quad 46$

Tabela $3.2 \mathbb{R}$-álgebras de Jordan semissimples de dimensão $3 \quad 48$ 
Tabela 3.3 $\mathbb{R}$-álgebras de Jordan de dimensão 3 com radical unidimensional e parte semissimples $\mathbb{R e}_{1} \oplus \mathbb{R e}_{2} \quad 48$

Tabela 3.4 $\mathbb{R}$-álgebras de Jordan de dimensão 3 com radical unidimensional e parte semissimples $\mathcal{B}_{4}^{\prime} \quad 49$

Tabela 3.5 $\quad \mathbb{R}$-álgebras de Jordan de dimensão 3 com radical de tipo (2) 50

Tabela 3.6 R-álgebras de Jordan de dimensão 3 com radical de tipo $(1,1) \quad 51$

Tabela 3.7 $\quad \mathbb{R}$-álgebra de Jordan nilpotente de dimensão 3 de tipo (3) $\quad 51$

Tabela $3.8 \quad \mathbb{R}$-álgebra de Jordan nilpotente de dimensão 3 de tipo $(1,1,1) \quad 52$

Tabela $3.9 \mathbb{R}$-álgebras de Jordan nilpotentes de dimensão 3 de tipo $(2,1) \quad 52$

Tabela 5.1 Existência de deformações em Jor 290

Tabela 5.2 Existência de deformações em Jor $_{3} \quad 95$

Tabela 5.3 Existência de deformações em Jor $_{4} \quad 103$

Tabela 5.4 Existência de deformação em Jor 4105

Tabela 5.5 Existência de deformação em Jor $_{4} \quad 107$

Tabela 5.6 Existência de deformações em Jor $_{4} \quad 109$

Tabela 5.7 Algumas álgebras de Jordan rígidas de dimensão $5 \quad 112$

Tabela 6.1 Existência de deformações em $\operatorname{Jor}_{2}^{\mathbb{R}} \quad 117$

Tabela 6.2 Existência de deformações em Jor $_{3}^{\mathbb{R}} \quad 121$

Tabela 6.3 Existência de deformações em $\operatorname{Jor}_{3}^{\mathbb{R}} \quad 122$

Tabela 6.4 Existência de deformações em $\operatorname{Jor}_{3}^{\mathbb{R}} \quad 123$

Tabela 6.5 Comparação das variedades Jor $_{n}^{\mathrm{C}}$ e $\mathrm{Jor}_{\mathfrak{n}}^{\mathbb{R}} \quad 125$

Tabela 7.1 Comparação do número de álgebras de Jordan unitárias de dimensão 3133

Tabela 8.1 Número de órbitas e de componentes irredutíveis de $\operatorname{Jor}_{n}$ e Jor $_{n}^{\mathbb{R}} \quad 137$

Tabela B.1 k-álgebras de Jordan indecomponíveis de dimensão 2157

Tabela B.2 k-álgebras de Jordan indecomponíveis de dimensão $3 \quad 157$

Tabela C.1 $\mathbb{R}$-álgebras de Jordan indecomponíveis de dimensão 2209 


\section{INTRODUÇÃO}

Neste trabalho apresentamos a classificação algébrica e geométrica das álgebras de Jordan de dimensões pequenas sobre um corpo $\mathbf{k}$ algebricamente fechado de char $\mathbf{k} \neq 2 \mathrm{e}$ sobre o corpo dos números reais.

O problema de classificação algébrica foi abordado de duas maneiras: primeiramente determinamos todas as estruturas algébricas de uma certa dimensão $n$ a menos de isomorfismo e posteriormente utilizaremos esses resultados para refinar esta classificação ao determinar todas as estruturas algébricas a menos de isotopia.

Determinar as classes de isomorfismos de uma estrutura algébrica é um problema amplamente estudado. Apesar disso, a única classe de álgebras (associativas, Jordan e Lie) que foi completamente descrita é a classe das álgebras simples. Em geral, a lista de todas as álgebras é conhecida somente em dimensões pequenas. Em 1975, P. Gabriel apresentou em [13] a lista de todas as álgebras associativas unitárias sobre um corpo algebricamente fechado de dimensão menor ou igual a 4. Quatro anos mais tarde, G. Mazzola, em seu trabalho [31], estendeu a classificação para as álgebras de dimensão 5. Por outro lado, no contexto de álgebras de Lie a classificação é conhecida até dimensão 6, veja [26].

No que diz respeito às álgebras de Jordan, H. Wesseler em [48] descreveu as álgebras unitárias sobre um corpo algebricamente fechado até dimensão 6. Em, particular ele mostrou que essas álgebras são especiais. De modo mais geral, em 1989, H. Sherkulov em [43] classificou todas as álgebras de Jordan não associativas de dimensão menor ou igual a 4 também sobre um corpo algebricamente fechado e em 2011, em seu artigo [4], Ancochea Bermúdez e outros determinaram as leis das álgebras de Jordan nilpotentes de dimensão 3 e 4 sobre o corpo dos números complexos. Neste trabalho generalizaremos estes resultados e classificaremos a menos de isomorfismo todas as álgebras de Jordan (unitárias e não unitárias, associativas e não associativas) de dimensão $n$ para $n \leqslant 4$ sobre um corpo $\mathbf{k}$ algebricamente fechado de char $\mathbf{k} \neq 2$.

Quando consideramos um corpo arbitrário o problema da classificação algébrica é ainda mais complicado pois a classificação depende de maneira essencial do corpo que estivermos considerando e, em geral, não é possível obter uma classificação. Para corpos bases especiais, como por exemplo o corpo dos p-ádicos ou o corpo dos números reais o problema pode ser completamente resolvido mas mesmo com essas restrições a determinação de todas as classes de isomorfismos de álgebras de Jordan de dimen- 
são fixa sobre o corpo dos números reais $\mathbb{R}$ só é conhecida completamente no caso de álgebras de Jordan semissimples. Os resultados na literatura para álgebras de Jordan (e até mesmo Lie e associativas) sobre $\mathbb{R}$ são muito escassos, inclusive para dimensões pequenas. Em 2007, Ancochea Bermúdez e outros classificaram algebricamente as álgebras associativas de dimensão 2 sobre $\mathbb{R}$ no trabalho [5] e posteriormente, em [3], as álgebras de Jordan de dimensão 2. Neste trabalho apresentaremos a lista de todas as álgebras de Jordan de dimensão 3 sobre o corpo dos números reais.

Tendo obtido esta classificação temos metade do caminho percorrido em relação à questão de classificar álgebras de Jordan a menos de isotopia, pois se duas álgebras são isomorfas então elas são isotópicas. Uma álgebra homótopa de uma álgebra de Jordan é, resumidamente, uma nova álgebra de Jordan cujo produto é definido a partir de um elemento dado da álgebra original através do produto triplo de Jordan. Homotopia somente define uma relação de equivalência se a álgebra original é unitária e o elemento dado é invertível, em tal caso a álgebra homótopa é chamada de isótopa.

O conceito de isotopia foi introduzido em 1958 por N. Jacobson em [20] e em [19] ele determinou as identidades fundamentais nas quais a teoria de isotopias se baseia, i.e., identidades relativas ao produto triplo de Jordan. Já, M. Koecher, em seu trabalho [27], utilizou as propriedades funtoriais do conceito de homotopia (por ele denominado de "mutuação") para explicitar uma construção de espaços simétricos Hermitianos de tipo compacto como uma compactificação de uma álgebra de Jordan semissimples complexa de dimensão finita.

Neste trabalho determinamos todas as classes de equivalências dessa relação para as álgebras de Jordan unitárias de dimensão 3 sobre o corpo dos reais. A ideia de realizar a classificação neste trabalho surgiu como uma sugestão dos professores I. Shestakov IME-USP e P. Koshlukov IMECC-UNICAMP durante o exame de qualificação.

Ressaltamos que para álgebras associativas a noção de isotopia não desempenha papel importante pois os conceitos de álgebras isotópicas e isomorfas coincidem. Provaremos que isso não acontece com as álgebras de Jordan reais e, assim, que o conceito de isotopia fornece uma relação de equivalência mais ampla que o conceito de isomorfismo.

Nossa motivação para obter a classificação algébrica vem da intenção de entender a variedade das álgebras de Jordan, i.e., de descrever geometricamente tais álgebras. A saber, seja $\mathbf{k}$ um corpo algebricamente fechado de char $\mathbf{k} \neq 2$ ou o corpo dos números reais. Seja $V$ um espaço vetorial de dimensão finita $n$ sobre $\mathbf{k}$, com base $\left\{e_{1}, \ldots, e_{n}\right\}$ 
fixa, para introduzirmos uma estrutura de álgebra de Jordan em $V$ especificamos $n^{3}$ constantes estruturais $c_{i j}^{k} \in \mathbf{k}$, de modo que o produto na álgebra resultante é dado por

$$
e_{i} \cdot e_{j}=\sum_{k=1}^{n} c_{i j}^{k} e_{k}, \text { para todo } i, j \in\{1, \ldots, n\} .
$$

A escolha das constantes não é arbitrária e deve refletir os fatos que a álgebra é comutativa e que satisfaz a identidade de Jordan. Estas restrições se manifestam através de equações polinomiais nas constantes estruturais, e assim podemos interpretar o espaço das constantes estruturais como um subconjunto afim Zariski-fechado da variedade $\mathbf{k}^{\mathbf{n}^{3}}$ que chamaremos de variedade das álgebras de Jordan de dimensão $n$ e denotaremos por Jor $_{n}$. Um ponto qualquer $\left(c_{i j}^{k}\right) \in$ Jor $_{n}$ representa, na base fixa, uma k-álgebra de Jordan $\mathcal{J}$ de dimensão $n$.

O problema de determinar os pontos genéricos de $\mathrm{Jor}_{\mathrm{n}}$ ou, equivalentemente, as componentes irredutíveis da variedade algébrica pode ser formulado geometricamente como segue: o grupo geral linear $\mathrm{G}=\mathrm{GL}(\mathrm{V})$ age em Jor $\mathrm{r}_{\mathrm{n}}$ via "transporte de estrutura", decompondo a variedade Jor $\mathrm{n}_{\mathrm{n}}$ em G-órbitas as quais correspondem às classes de álgebras de Jordan isomorfas. Se uma álgebra $\mathcal{J}$ pertence ao fecho de Zariski da órbita de uma outra álgebra $\mathcal{J}^{\prime}$ da variedade então dizemos que $\mathcal{J}^{\prime}$ é uma deformação de $\mathcal{J}$. Uma álgebra $\mathcal{J}$ cuja órbita sob a ação de $G, \mathcal{J}^{G}$, é aberta na topologia de Zariski de $\mathcal{J o r}_{n}$ é chamada de rígida. Álgebras rígidas são de particular interesse pois o fecho da órbita de tais álgebras gera uma componente irredutível da variedade.

Note que analogamente podemos definir a variedade das álgebras associativas e de Lie. Denotaremos elas por Assoc $n$ e $\mathrm{Lie}_{n}$, respetivamente. A geometria de ambas é bastante complicada. Em 1890 E. Study em [45] considerou álgebras associativas complexas de dimensão 4 e mostrou que é impossível achar uma álgebra genérica, o equivalentemente, que $\mathrm{Assoc}_{4}$ tem mais de uma componente irredutível.

Em 1964, M. Gerstenhaber em seu trabalho [14] introduziu a noção formal de deformação infinitesimal entre álgebras associativas. Seja $\mathcal{A}$ uma álgebra associativa de dimensão $n$ e considere uma $n^{3}$-upla $g=\left\{g_{i j k}(t)\right\}$ de series de potências na variável $t$, tal que $\mathrm{g}(\mathrm{t})$ defina uma multiplicação associativa na álgebra $\mathcal{A}_{\mathrm{t}}=\mathrm{V} \otimes \mathbf{k}(\mathrm{t})$ e $\mathrm{g}_{\mathrm{ijk}}(0)$ coincida com as constantes estruturais de $\mathcal{A}$, então dizemos que $\mathcal{A}_{\mathrm{t}}$ é uma deformação de $\mathcal{A}$. É fácil ver que a definição analítica de deformação implica na geométrica, veja [11]. Em particular, uma álgebra de dimensão finita $\mathcal{A}$ é rígida se toda $\mathrm{g}_{i j k}(\mathrm{t})$ satisfazendo as condições anteriores define uma álgebra $\mathcal{A}_{\mathrm{t}}$ isomorfa a $\mathcal{A}$ para todo $\mathrm{t} \in \mathbf{k}$. Também foi provado por Gerstenhaber que se o segundo grupo de cohomologia de $\mathcal{A} \in$ Assoc $_{n}$ com coeficientes em $\mathcal{A}, \mathrm{H}^{2}(\mathcal{A}, \mathcal{A})$, é trivial então $\mathcal{A}$ é uma álgebra rígida. Quatro anos 
depois, a teoria de deformação analítica introduzida por Gerstenhaber para álgebras associativas foi estendida a álgebras de Lie por Nijenhuis e Richardson em [36].

Em 1968, F. Flanigan em [11] comparou a estrutura de uma álgebra deformada $\mathcal{A}_{\mathrm{t}}$ com a estrutura da álgebra original, tal comparação é análoga para álgebras de Jordan. Também em [12] ele mostrou que cada componente irredutível de Assoc $n$ ou é o fecho da órbita de uma álgebra rígida ou é o fecho da união de uma família infinita de órbitas de álgebras que chamou de semi-rígidas.

Em [13, 1975] P. Gabriel descreveu todas as álgebras genéricas da subvariedade fechada das álgebras associativas unitárias de Assoc $_{n}$ para $n \leqslant 4$. De fato, esta é uma boa referência para conferir as propriedades básicas que uma componente irredutível deve ter, tais como a dimensão do radical das álgebras, a dimensão do grupo de automorfismos, etc. Alem disso, em [31, 1979] G. Mazzola, aluno de Gabriel, presentou o diagrama de inclusão das órbitas das álgebras associativas unitárias de dimensão 5 e provou que existem dez componentes irredutíveis nesta subvariedade de Assoc 5 . Outra questão considerada por Mazzola é relacionada com a variedade das álgebras comutativas unitárias dentro de $\mathrm{Assoc}_{n}$, ele provou que esta variedade é irredutível para $n \leqslant 7$, veja $[32,1980]$ e suas referências. Em [26, 1987] A. Kirillov e Y. Neretin descreveram as componentes da variedade das álgebras de $\mathrm{Lie}_{n}$ para $n \leqslant 6$. Uma generalização recente desta teoria ocorre quando consideramos a variedade de representações de álgebras ao invés da variedade de álgebras.

Em relação à variedade Jor $_{n}$ as referencias são bastantes recentes. Em [25, 2005] I. Kashuba e I. Shestakov descreveram as componentes irredutíveis de Jor 3 e em [23, 2006] o primeiro autor fez um estudo análogo considerando a subvariedade fechada das álgebras de Jordan unitárias de Jor $n$ para $n \leqslant 5$. Nesse mesmo artigo, I. Kashuba estendeu para o caso de álgebras de Jordan algumas propriedades e fatos conhecidos para Assoc $n$ e Lie ${ }_{n}$. Finalmente, em [4, 2011] Ancochea Bermúdez e outros usaram deformações infinitesimais para estudar as álgebras de Jordan nilpotentes de dimensão 4 sobre o corpo dos complexos. O objetivo deste trabalho foi generalizar estes resultados para obter uma descrição completa das variedades Jor $_{n}$ para $n \leqslant 4$ sobre um corpo algebricamente fechado e para $n \leqslant 3$ sobre o corpo dos números reais.

Dividimos esta tese em 8 capítulos. O primeiro capítulo constitui uma introdução à teoria estrutural de álgebras de Jordan de dimensão finita, onde apresentaremos os principais conceitos e os resultados básicos.

No Capítulo 2 trabalhamos na classificação algébrica a menos de isomorfismo das álgebras de Jordan de dimensão $n$ para $n \leqslant 4$ sob a suposição que o corpo de definição $\mathbf{k}$ é algebricamente fechado e de char $\mathbf{k} \neq 2$. Estes resultados nos forneceram os conjuntos de classes de álgebras não isomorfas em cada dimensão menor ou igual que 4 o que 
nos permitiu determinar o número de órbitas sobre a ação de $G$ na variedade $J^{\circ} r_{n}$ que corresponde a: 2 órbitas em Jor 1,6 órbitas em Jor 2,20 órbitas em Jor 3 e finalmente 73 órbitas em Jor 4 . Os resultados deste capítulo foram publicados em International Journal of Mathematics, Game Theory and Algebra, veja [30, 2013].

No Capítulo 3, analogamente ao capítulo anterior trabalhamos na classificação algébrica das álgebras de Jordan a menos de isomorfismo, mas neste caso as álgebras foram consideradas sobre o corpo dos números reais e com dimensão menor ou igual a 3 . Denotamos a variedade de tais álgebras como sendo $\mathrm{Jor}_{n}^{\mathbb{R}}$. Obtivemos que o número de G-órbitas na variedade Jor $_{n}^{\mathbb{R}}$ é: 2 órbitas em $\operatorname{Jor}_{1}^{\mathbb{R}}, 7$ em Jor $_{2}^{\mathbb{R}}$ e, por último, temos 26 órbitas em Jor $_{3}^{\mathbb{R}}$.

O Capítulo 4 contém as principais ferramentas que serão necessárias para o objetivo da classificação geométrica. Começamos com uma rápida revisão de alguns conceitos e terminologia da geometria algébrica com ênfase especial nos grupos algébricos e sua ação em variedades. Definimos a variedade das álgebras de Jordan de dimensão $n$ e apresentamos os métodos usados nos capítulos seguintes para descrever as componentes irredutíveis assim como também as deformações entre as álgebras de Jordan, além de introduzir critérios que determinarão a não existência de deformação entre um par de álgebras dadas. Em particular, mostraremos como se comportam as dimensões do centro associador e do segundo grupo de cohomologia de uma álgebra através de uma deformação assim como também a dimensão de uma subálgebra associativa ou nula maximal. Provamos que rigidez é um conceito local e que não é preservada por adjunção formal de um elemento identidade. Mostraremos também que em toda variedade de álgebras de Jordan de dimensão $n$ maior ou igual a 5 existem pelo menos 3 componentes irredutíveis que provêm de álgebras rígidas que são indecomponíveis, não associativas e não semissimples. Faremos isso construindo as três famílias de tais álgebras rígidas. Para $n$ suficientemente grande esta família nos permite determinar no mínimo 12 novas álgebras rígidas.

No Capítulo 5 aplicamos os resultados do capítulo anterior para determinar e dar uma descrição completa das componentes irredutíveis de Jor ${ }_{n}$, para $n \leqslant 4$ e provamos que as variedades consideradas são conexas e têm dimensão $\mathrm{n}^{2}$. Concluímos que a variedade Jor 1 é irredutível, que a variedade Jor 2 tem 2 componentes irredutíveis e que a variedade $\mathrm{Jor}_{4}$ tem 10 componentes irredutíveis. Foi provado em [25] que a variedade Jor 3 tem 5 componentes irredutíveis, nos completamos esse trabalho dando uma descrição mais detalhada de tais componentes. Também mostramos que o número de componentes irredutíveis em Jor 5 é maior ou igual a 26 e que rigidez é preservada por soma direta em Jor $n$ para $n \leqslant 5$. Os resultados deste capítulo foram apresentados no artigo [24], aceito para publicação no Journal of Algebra. 
Um estudo análogo ao do Capítulo 5 foi feito no Capítulo 6 onde foram determinadas as componentes irredutíveis da variedade $\operatorname{Jor}_{n}^{\mathbb{R}}$ para $n \leqslant 3$, concluindo que a variedade $\mathrm{Jor}_{3}^{\mathbb{R}}$ tem 8 componentes irredutíveis. No final do capítulo foram comparados os resultados obtidos para Jor $_{n}$ e Jor $_{n}^{\mathbb{R}}$.

No Capítulo 7 foram dadas as definições e os resultados básicos relativos à teoria de álgebras isotópicas além da classificação algébrica das álgebras de Jordan reais de dimensão 3 a menos de isotopia. Provamos assim que o conceito de isotopia define uma relação de equivalência mais ampla que o conceito de isomorfismo pois encontramos álgebras isotópicas que não são isomorfas.

Por último, no Capítulo 8 foram apresentadas as conclusões e considerações finais deste trabalho assim como também sugestões e perspectivas para pesquisas futuras.

Este trabalho também conta com três apêndices: o Apêndice A reúne os códigos de vários programas de computação simbólica elaborados pela autora, os quais foram utilizados para calcular os invariantes geométricos das álgebras, i.e., os invariantes preservados por deformações, tais como: as dimensões dos grupos de automorfismos, as dimensões dos aniquiladores, as dimensões dos radicais nilpotentes, as dimensões das diferentes potências, subálgebras de dimensão 2 e 3, as identidades satisfeitas assim como também a dimensão do segundo grupo de cohomologia e do centro associador de cada álgebra. E nos Apêndices B e C apresentamos uma lista das propriedades das álgebras de Jordan de dimensão 4 e das álgebras de Jordan reais de dimensão 3, respetivamente, as quais foram obtidas utilizando os programas do Apêndice A. 


\section{INTRODUÇÃO ÀS ÁLGEBRAS DE JORDAN}

Neste capítulo apresentaremos, com a finalidade de fixar notação, os conceitos e os resultados básicos da teoria estrutural das álgebras de Jordan. Esta introdução será dividida em 4 seções, na primeira delas introduziremos os conceitos de álgebras de Jordan especiais e excepcionais e apresentaremos exemplos de tais álgebras. Lembraremos a definição de álgebra de Jordan nilpotente e definiremos o tipo de nilpotência destas álgebras, conceito que será de crucial para a classificação algébrica. Relacionados à definição de álgebras nilpotentes temos os conceitos de álgebras simples, semi-simples e de radical nilpotente.

Na segunda seção enunciaremos duas versões do teorema de Wedderburn para álgebras de Jordan de dimensão finita e os teoremas de classificação de álgebras de Jordan simples de dimensão finita, primeiramente sobre um corpo algebricamente fechado e logo após para um corpo arbitrário.

Outro resultado de suma importância para levar a cabo a classificação algébrica das álgebras de Jordan é a decomposição em subespaços de Peirce para álgebras que possuem um sistema de elementos idempotentes ortogonais um a um, este resultado será apresentado na seção 1.3.

Na quarta e última seção apresentaremos os conceitos de extensão nula e segundo grupo de cohomologia de uma álgebra de Jordan sobre um bimódulo e enunciaremos uma relação entre ambos no teorema 1.25 .

Salientamos que as álgebras consideradas neste capítulo serão definidas sobre um corpo arbitrário de característica diferente de 2 e reservamos o símbolo k para denotar tal corpo.

\subsection{DEFINIÇÕES E EXEMPLOS}

As álgebras de Jordan são uma classe importante de álgebras não associativas e foram introduzidas em 1932 pelo físico alemão Pascual Jordan em uma tentativa de formular o cenário da mecânica quântica, em termos do produto de Jordan em vez do produto associativo. A teoria estrutural das álgebras de Jordan iniciou-se com o trabalho [49] de 
P. Jordan, J. von Neumann e E. Wigner e foi desenvolvida por Albert em [2] onde foram introduzidos os conceitos que apresentaremos a seguir.

Definição 1.1. Um conjunto $A$ é chamado de uma álgebra sobre o corpo $\mathbf{k}$, se A possui uma estrutura de k-espaço vetorial e um produto · que satisfaz:

$$
\begin{aligned}
x \cdot(y+z) & =x \cdot y+x \cdot z \\
(x+y) \cdot z & =x \cdot z+y \cdot z \\
\alpha(x \cdot y) & =(\alpha x) \cdot y=x \cdot(\alpha y)
\end{aligned}
$$

para $x, y, z \in A$ e $\alpha \in \mathbf{k}$.

Chamaremos "álgebra sobre o corpo k" de k-álgebra ou, simplesmente, de álgebra e denotaremos o produto $x \cdot y$ simplesmente por $x y$ quando não houver ambiguidade.

Definição 1.2. Uma k-álgebra $\mathcal{J}$ é chamada de k-álgebra de Jordan (ou, simplesmente, álgebra de Jordan) se seu produto satisfaz a condição de comutatividade:

$$
x y=y x,
$$

para todo $x, y \in \mathcal{J}$ e uma versão debilitada da associatividade, chamada de identidade de Jordan:

$$
\left(x^{2} y\right) x=x^{2}(y x)
$$

para todo $x, y \in \mathcal{J}$.

Um subespaço vetorial $\mathcal{S} \subseteq \mathcal{J}$ que satisfaz $\mathcal{S} \mathcal{S} \subseteq \mathcal{S}$ é chamado de subálgebra de $\mathcal{J}$. Se um subespaço I $\subseteq \mathcal{J}$ satisfaz a condição mais forte $\mathcal{J I} \subseteq$ I então I é chamado de ideal da álgebra de Jordan $\mathcal{J}$.

Podemos reescrever a identidade de Jordan 1.2 em termos do associador $(x, y, z):=$ $(x y) z-x(y z)$, como sendo

$$
\left(x^{2}, y, x\right)=0
$$

Linearizando completamente esta última identidade e usando o fato que a álgebra é comutativa, obtemos:

$$
(x y, z, t)+(x t, z, y)+(y t, z, x)=0
$$

para todos $x, y, z, t \in \mathcal{J}$ (veja [50, eq.22, p.67]). 
Seja $\mathcal{A}$ uma k-álgebra associativa (i.e., $(x, y, z)=0$ para todo $x, y, z \in \mathcal{A})$. No espaço vetorial subjacente a $\mathcal{A}$ podemos definir uma nova operação de produto $\odot$ dada pela fórmula

$$
x \odot y=\frac{1}{2}(x y+y x)
$$

para todo $x, y \in \mathcal{A}$, onde $x y$ denota o produto em $\mathcal{A}$. Ao substituir o produto original de $\mathcal{A}$ por $\odot$, obtemos uma nova álgebra que será denotada por $\mathcal{A}^{(+)}$. É imediato ver que $\mathcal{A}^{(+)}$é uma álgebra de Jordan.

Definição 1.3. Uma álgebra de Jordan que é isomorfa a uma subálgebra de $\mathcal{A}^{(+)}$para alguma álgebra associativa $\mathcal{A}$ é chamada de especial.

As álgebras de Jordan que não são especiais são chamadas de excepcionais.

Observamos que a álgebra $\mathcal{A}^{(+)}$pode ser definida, analogamente, para uma k-álgebra $\mathcal{A}$ não necessariamente associativa, mas neste caso a álgebra resultante $\mathcal{A}^{(+)}$não necessariamente será de Jordan, veja o Exemplo 1.7.

Exemplo 1.4. Seja V um espaço vetorial sobre um corpo $\mathbf{k}$ que possui uma forma bilinear simétrica $f=f(x, y)$ definida em $V$. Consideramos a soma direta $\mathcal{J}(V, f)=\mathbf{k} \cdot 1 \oplus V$ do espaço vetorial $\mathrm{V}$ com o espaço vetorial unidimensional $\mathbf{k} \cdot 1=\{\alpha 1 \mid \alpha \in \mathbf{k}\}$ e definimos o produto em $\mathcal{J}(V, f)$ dado pela regra:

$$
(\alpha \cdot 1+x) \sqcup(\beta \cdot 1+y)=(\alpha \beta+f(x, y)) \cdot 1+(\beta x+\alpha y)
$$

onde $\alpha, \beta \in \mathbf{k}$ e $x, y \in V$. A álgebra $(\mathcal{J}(\mathrm{V}, \mathrm{f}), \square)$ é uma álgebra de Jordan e é uma subálgebra de $\mathcal{A}^{(+)}$onde $\mathcal{A}$ é a álgebra (associativa) de Clifford de $\mathrm{f}$ (veja [21, Cap.VII.1, Teo.1, p.261]). Logo $(\mathcal{J}(\mathrm{V}, \mathrm{f}), \square)$ é uma álgebra de Jordan especial, chamada de álgebra de Jordan da forma bilinear simétrica $f$. Se, além disso, a forma $f$ for não degenerada e a $\operatorname{dim}_{\mathbf{k}} \mathrm{V}>1$, a álgebra é também simples (veja [50, Ex. 2, pág. 57]).

Exemplo 1.5. Seja $\mathcal{U}$ uma álgebra e seja $i$ uma involução em $U$ (i.e., um endomorfismo

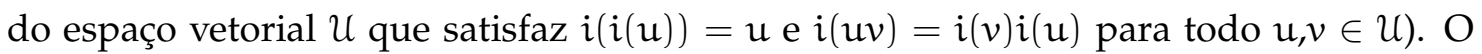
conjunto

$$
H(\mathcal{U}, \mathfrak{i})=\{\mathfrak{u} \in \mathcal{U} \mid \mathfrak{u}=\mathfrak{i}(\mathfrak{u})\}
$$

dos elementos de $\mathcal{U}$ fixos pela ação de $i$, é fechado em relação ao produto $\odot$ e é uma subálgebra da álgebra $\mathcal{U}^{(+)}$. Se $\mathcal{U}$ for associativa, então $\mathcal{U}^{(+)}$é de Jordan e $(H(\mathcal{U}, \mathfrak{i}), \odot)$ é uma álgebra de Jordan especial. 
Definição 1.6. Um elemento $e$ de uma álgebra $A$ é chamado de identidade ou unidade no caso que $e x=x e=x$ para todo $x \in A$. Se uma álgebra possui um elemento identidade então será chamada de álgebra unitária.

Uma k-álgebra unitária $\mathcal{D}$ é chamada de álgebra de composição se ela possui uma aplicação $n: \mathcal{D} \rightarrow \mathbf{k}$ que satisfaz:

1. $\mathfrak{n}(\alpha x)=\alpha^{2} \mathfrak{n}(x)$, onde $x \in \mathcal{D}$ e $\alpha \in \mathbf{k}$;

2. a função $f(x, y)=n(x+y)-n(x)-n(y)$ é uma forma bilinear simétrica não degenerada em $\mathcal{D}$; e

3. $n(x y)=n(x) n(y)$ para todo $x, y \in \mathcal{D}$.

Esta definição nos permite compreender o seguinte exemplo de uma álgebra de Jordan especial.

Exemplo 1.7. Seja $\mathcal{D}$ uma álgebra de composição com involução $i$ e seja $\mathcal{D}_{\mathrm{n}}=\operatorname{Mat}_{\mathrm{n}}(\mathcal{D})$ a álgebra das matrizes de ordem $n$ sobre $\mathcal{D}$. A aplicação $j: X \mapsto i(X)^{t}$ que leva uma matriz X em uma outra matriz obtida da anterior aplicando a involução $i$ a cada entrada de $\mathrm{X}$ e transpondo, é uma involução da álgebra $\mathcal{D}_{\mathrm{n}}$ chamada de involução padrão ${ }^{1}$ em $\mathcal{D}_{\mathrm{n}}$ associada a $i$. Como $\mathcal{D}_{\mathrm{n}}$ é associativa se $\mathcal{D}$ é associativa então, é claro que se $\mathcal{D}$ é associativa então $\left(H\left(\mathcal{D}_{n}, j\right), \odot\right)$ é uma álgebra de Jordan especial. Mas se $\mathcal{D}$ for a álgebra de Cayley-Dickson $\mathcal{C}$ (i.e., uma álgebra de tipo IV no Teorema de Classificação das Álgebras de Composição de [50, Teo.1, p.32]), a qual não é associativa mas é alternativa, então a álgebra $\left(H\left(\mathcal{C}_{n}, j\right), \odot\right)$ só é de Jordan para $n \leqslant 3$ (uma prova disto quando $n=3$ pode ser encontrada em [50, Teorema 1, p. 54$])$ e $\left(H\left(\mathcal{C}_{3}, j\right), \odot\right)$ é uma álgebra de Jordan excepcional (veja [1], [50, Teorema 2, p. 55]).

Se $\mathcal{J}$ é uma álgebra de Jordan sem unidade, então podemos considerar a soma direta

$$
\mathcal{J}^{\#}=\mathcal{J} \oplus \mathbf{k} \cdot 1,
$$

onde 1 é o elemento identidade de $\mathbf{k}$. O produto em $\partial^{\#}$ é definido por

$$
(x+\alpha \cdot 1)(y+\beta \cdot 1)=(x y+\alpha y+\beta x)+\alpha \beta \cdot 1,
$$

onde $\alpha, \beta \in \mathbf{k}$ e $x, y \in \mathcal{J}$. Chamaremos a álgebra $\mathcal{J}^{\#}$ de álgebra obtida pela adjunção formal de um elemento identidade à álgebra $\mathcal{\partial}$. É fácil ver que 1 é um elemento identidade para a álgebra $\partial^{\#}$ e $\mathcal{J}$ é uma subálgebra de $\partial^{\#}$. De [21, Teorema 6, p. 30] temos que $\partial^{\#}$ também é uma álgebra de Jordan.

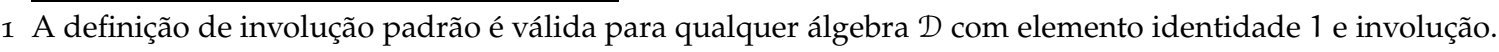


Definição 1.8. Sejam $\mathcal{J}$ e $\mathcal{J}^{\prime}$ duas álgebras de Jordan sobre o corpo k. O produto de Kronecker $\mathcal{J} \otimes_{\mathbf{k}} \mathcal{J}^{\prime}$ é o produto tensorial $\mathcal{J} \otimes_{\mathbf{k}} \mathcal{J}^{\prime}$ dos espaços vetoriais $\mathcal{J}, \mathcal{J}^{\prime}$ onde todos os elementos são somas $\sum x \otimes y$, com $x \in \mathcal{J}$ e $y \in \mathcal{J}^{\prime}$ e a multiplicação é definida por linearidade e

$$
\left(x_{1} \otimes y_{1}\right)\left(x_{2} \otimes y_{2}\right)=\left(x_{1} x_{2}\right) \otimes\left(y_{1} y_{2}\right), \quad \text { onde } x_{i} \in \mathcal{J}, y_{i} \in \mathcal{J}^{\prime}
$$

Se $\mathcal{J}$ e $\mathcal{J}^{\prime}$ têm dimensão finita sobre $\mathbf{k}$, então $\operatorname{dim}\left(\mathcal{J} \otimes_{\mathbf{k}} \mathcal{J}^{\prime}\right)=(\operatorname{dim} \mathcal{J})\left(\operatorname{dim} \mathcal{J}^{\prime}\right)$.

Em numerosas ocasiões depararemos-nos com o caso em que $J^{\prime}$ é um corpo, geralmente uma extensão $K$ de $\mathbf{k}$. Então $K$ contém $1, \log 0 \mathcal{J}_{K}=\mathcal{J} \otimes_{\mathbf{K}} K$ contém $\mathcal{J}$ como uma subálgebra sobre $\mathbf{k}$. Além disso, $\mathcal{J}_{K}$ é visto como uma álgebra sobre $K$ a qual é chamada de extensão escalar de $\mathcal{J}$ a uma álgebra sobre $K$. Pode-se verificar que a álgebra $\partial_{K}$ também é de Jordan.

Uma álgebra associativa é chamada de nilpotente se para algum número natural $n$ o produto de quaisquer $n$ elementos da álgebra é zero. Para álgebras não associativas, e em particular para álgebras de Jordan, deveríamos também indicar a ordem na qual os produtos são considerados, i.e., a distribuição dos parêntesis.

Definição 1.9. Uma álgebra de Jordan $\mathcal{J}$ é chamada de nilpotente se existe um número natural $\dot{n}$ tal que o produto de quaisquer $n$ elementos da álgebra, com quaisquer distribuição de parêntesis seja igual a zero.

O menor de tais números é denominado índice de nilpotência da álgebra $\mathcal{J}$.

Em $\mathcal{J}$ definimos indutivamente uma serie de subconjuntos

$$
\begin{aligned}
& \mathfrak{J}^{1}=\mathfrak{J}^{\langle 1\rangle}=\mathcal{J}, \\
& \mathfrak{g}^{n}=\mathfrak{J}^{n-1} \mathfrak{J}+\mathfrak{J}^{n-2} \mathfrak{J}^{2}+\cdots+\mathfrak{J}^{n-1}, \\
& \mathfrak{J}^{\langle n\rangle}=\mathfrak{J}^{\langle n-1\rangle} \mathcal{J} .
\end{aligned}
$$

O subconjunto $\mathcal{J}^{\mathfrak{n}}$ é chamado de $n$-ésima potência da álgebra $\mathcal{J}$. Segue de imediato da definição de potência que $\mathcal{J} \supseteq \mathcal{J}^{2} \supseteq \mathcal{J}^{3} \supseteq \cdots$ onde todos os membros desta cadeia são ideais da álgebra $\mathcal{J}$. Como $\mathcal{J}$ é comutativa a cadeia de subconjuntos $\mathcal{J}^{\langle 1\rangle} \supseteq \mathcal{J}^{\langle 2\rangle} \supseteq \cdots \supseteq$ $\mathcal{J}^{\langle\mathfrak{n}\rangle} \supseteq \cdots$ também consiste de ideais da álgebra $\mathcal{J}$ e é chamada de série central inferior de $\mathcal{J}$.

Se existir um $s \in \mathbb{N}$ tal que $\mathcal{J}^{\langle s\rangle}=0$ então, como $\mathcal{J}^{2^{s}} \subseteq \mathcal{J}^{\langle s\rangle}$ para qualquer $s \geqslant 1$ ([50, Prop. 1, p.82 ]), a álgebra $\mathcal{J}$ é nilpotente. O mínimo s para o qual $\mathcal{J}^{\langle s\rangle}=0$ será chamado de nil-índice de $\mathcal{J}$. 
Se s é o nil-índice de uma álgebra de Jordan nilpotente $\mathcal{J}$, definimos o tipo de nilpotência de $\mathcal{J}$ como sendo a sequência $\left(n_{1}, n_{2}, n_{3}, \cdots, n_{s-1}\right)$ onde $n_{i}=\operatorname{dim}\left(\mathcal{J}^{\langle i\rangle} / \mathcal{J}^{\langle i+1\rangle}\right)$.

Observamos que $n_{i}>0$ para todo $i$. De fato, suponha que existe um $i \in \mathbb{N}, 1 \leqslant$ $i \leqslant s-1$ tal que $n_{i}=0$ então $\operatorname{dim} \mathcal{g}^{\langle i\rangle}=\operatorname{dim} \mathcal{g}^{\langle i+1\rangle}$ e como $g^{\langle i+1\rangle} \subseteq g^{\langle i\rangle}$ temos $g^{\langle i\rangle}=g^{\langle i+1\rangle}$. Consequentemente $\mathcal{J}^{\langle i+2\rangle}=g^{\langle i\rangle} \cdot \mathcal{J}=g^{\langle i\rangle}$, por indução $\mathcal{J}^{\langle i\rangle}=g^{\langle k\rangle}$ para todo $k \in \mathbb{N}, k>i$. Em particular isto acontece para $k=s$, então $\mathcal{J}^{\langle i\rangle}=\mathcal{J}^{\langle s\rangle}=0$ o que impossível já que s é o nil-índice de $\mathcal{J}$.

Definição 1.10. Uma k-álgebra de Jordan de dimensão finita $\mathcal{J}$ é chamada de:

1. Simples se 0 e $\mathcal{J}$ são os únicos ideais de $\mathcal{J}$ e além disso $\mathcal{J}^{2} \neq 0$.

2. Semissimples se $\mathcal{J}$ é uma soma direta finita de álgebras simples.

3. Simples Central se $\mathcal{J}_{K}$ é simples para toda extensão $K$ de k.

4. Separável se $\mathcal{J}_{K}$ é semissimples para toda extensão $K$ de $\mathbf{k}$.

Observamos que toda álgebra $\mathcal{J}$ simples central é simples e toda álgebra $\mathcal{J}$ separável é semissimples.

\subsection{RESULTADOS PRINCIPAIS}

Nesta seção apresentaremos alguns resultados importantes da teoria estrutural das álgebras de Jordan que serão ferramentas necessárias na classificação algébrica. Omitiremos as provas de tales fatos que podem ser facilmente encontradas na literatura.

Começaremos enunciando o teorema que K. McCrimmon, em seu livro "A Taste of Jordan Algebras", chama de "Enlightenment Structure Theorem". Este teorema reúne as propriedades principais de uma álgebra de Jordan cuja dimensão sobre um corpo arbitrário (de char $\neq 2$ ) é finita.

Teorema 1.11. [33, Teo. 3.10, p. 79] Seja J uma álgebra de Jordan de dimensão finita sobre um corpo $\mathbf{k}$ de característica $\neq 2$.

1. Existe um único ideal nilpotente maximal de J, que chamaremos de radical (ou radical nilpotente) de J e será denotado por $\operatorname{Rad}(\mathcal{J})$.

2. O quociente $\mathrm{J} / \operatorname{Rad}(\mathcal{J})$ é uma álgebra de Jordan semissimples, à qual chamaremos de parte semissimples de $\mathcal{J}$ e será denotado por $\mathcal{J}_{\mathrm{ss}}$. 
3. Se J é semissimples então ela possui um elemento identidade e sua decomposição em álgebras simples é única.

A seguir enunciaremos um teorema conhecido como "Teorema Principal de Wedderburn" o qual tem sido provado para várias classes de álgebras. Uma prova da seguinte versão para álgebras de Jordan de dimensão finita pode ser encontrada em [46].

Teorema 1.12. [46] Se J é uma álgebra de Jordan de dimensão finita sobre um corpo $\mathbf{k}$ tal que $J_{\text {ss }}$ é separável então J contém uma subálgebra $\mathrm{C}$ tal que $\mathcal{J}=\mathrm{C}+\operatorname{Rad}(\mathcal{J})$ com $\mathrm{C} \cap \operatorname{Rad}(\mathcal{J})=0$ $e \mathrm{C} \simeq \mathrm{J}_{\mathrm{ss}}$.

Em [38] foi provado que quando o corpo tem char $\mathbf{k}=0$ o teorema de Wedderburn vale para toda álgebra de Jordan de dimensão finita, i.e., não é necessária a condição de $\partial_{\text {ss }}$ ser separável:

Teorema 1.13. [38] Seja J uma álgebra de Jordan de dimensão finita sobre um corpo $\mathbf{k}$ com char $\mathbf{k}=0$ então existe uma subálgebra $\mathrm{C}$ de $\mathcal{J}$ tal que $\mathcal{J}=\mathrm{C}+\operatorname{Rad}(\mathcal{J}) \operatorname{com} \mathrm{C} \cap \operatorname{Rad}(\mathcal{J})=0$ e $\mathrm{C} \simeq \mathfrak{J}_{\mathrm{ss}}$.

Observamos que se o corpo for algebricamente fechado então toda álgebra de Jordan de dimensão finita semissimples é separável [21, p.246], logo segue do Teorema 1.11 que $\mathcal{J}_{\text {ss }}$ é separável e portanto (Teorema 1.12) $\mathcal{J}$ admite decomposição $\mathcal{J}=\mathcal{J}_{\text {ss }} \oplus \operatorname{Rad}(\mathcal{J})$ como soma direta de subespaços.

Lembramos agora o teorema de Albert que fornece a classificação completa das álgebras de Jordan simples de dimensão finita sobre um corpo algebricamente fechado.

Teorema 1.14. [21, Corolário 2, pág. 204] Seja J uma álgebra de Jordan simples de dimensão finita sobre um corpo algebricamente fechado $\mathbf{k}$. Então temos as seguintes possibilidades para J:

1. $\mathcal{J}=\mathbf{k}$;

2. $\mathcal{J}=(\mathfrak{J}(\mathrm{V}, \mathrm{f}), \square)$ a álgebra de Jordan de uma forma bilinear simétrica não degenerada $\mathrm{f}$ sobre um espaço vetorial de dimensão finita $\mathrm{V}$ tal que $\operatorname{dim} \mathrm{V}>1$;

3. $\mathcal{J}=\left(\mathrm{H}\left(\mathcal{D}_{\mathrm{n}}, \mathrm{j}\right), \odot\right), \mathrm{n} \geqslant 3$, onde $(\mathcal{D}, \mathfrak{i})$ é uma álgebra de composição de dimensão 1,2 ou 4 se $\mathrm{n} \geqslant 4$ e de dimensão 1, 2, 4 ou 8 se $\mathrm{n}=3$ e j é a involução padrão de $\mathcal{D}_{\mathrm{n}}$ associada $a i$.

Seja $\mathcal{J}$ uma k-álgebra de Jordan, podemos associar a ela duas álgebras associativas de transformações lineares. Para isso considere a um elemento qualquer de $\mathcal{J}$. Definimos uma aplicação da álgebra $\mathcal{J}$ em si mesma:

$$
M_{a}: x \mapsto x a \quad \text { para todo } x \in \mathcal{J}
$$


Esta aplicação é um endomorfismo do espaço vetorial J e é chamada de operador de multiplicação pelo elemento a. A subálgebra da álgebra de endomorfismos do espaço vetorial $\mathcal{J}$ gerada por todos os possíveis operadores $M_{a}$ onde $a \in \mathcal{J}$, é chamada de álgebra de multiplicação da álgebra $\mathcal{J}$ e será denotada por $\mathfrak{M}(\mathcal{J})$. Os elementos $S \in$ $\mathfrak{M}(\mathcal{J})$ são da forma $S=\sum M_{a_{1}} M_{a_{2}} \cdots M_{a_{n}}$ com $a_{i} \in \mathcal{J}$.

A segunda álgebra que associaremos a $\mathcal{J}$ é o centroide $\mathfrak{C}(\mathcal{J})$ de $\mathcal{J}$ o qual é o centralizador de $\mathfrak{M}(\mathcal{J})$ em $\operatorname{Hom}_{\mathbf{k}}(\mathcal{J}, \mathcal{J})$, ou seja $\mathfrak{C}(\mathcal{J})$ é o conjunto de aplicações lineares $\mathrm{T}$ de $\mathcal{J}$ tais que $\mathrm{TS}=\mathrm{ST}$ para cada $\mathrm{S} \in \mathfrak{M}(\mathcal{J})$.

No caso em que $\mathfrak{J}$ tenha dimensão finita sobre $\mathbf{k}$ então o centroide $\mathfrak{C}(\mathcal{J})$ coincide com o centro de $\mathfrak{M}(\mathcal{J})([42$, Cap. II, p.13] ), i.e.,

$$
\mathfrak{C}(\mathcal{J})=\{\mathrm{S} \in \mathfrak{M}(\mathcal{J}) \mid \mathrm{TS}=\mathrm{ST} \text { para todo } \mathrm{T} \in \mathfrak{M}(\mathcal{J})\}
$$

Teorema 1.15. [42, Cap. II, p.13] Se J é uma k-álgebra de Jordan simples então o centroide $\mathfrak{C}(\mathcal{J})$ de J é um corpo (que contém $\mathbf{k}$ ). E quando consideramos J como uma álgebra sobre seu centroide é simples central.

Como consequência do Teorema 1.15 podemos reduzir o problema de classificar álgebras de Jordan simples de dimensão finita sobre um corpo arbitrário $\mathbf{k}$ ao problema de classificar álgebras de Jordan simples central (de dimensão finita sobre uma extensão do corpo arbitrário $\mathbf{k}$ ). Para isso, seja $\mathcal{J}$ uma tal álgebra e seja $\overline{\mathbf{k}}$ o fecho algébrico do corpo base $\mathbf{k}$, então $\mathcal{J}_{\overline{\mathbf{k}}}$ é uma álgebra de Jordan simples de dimensão finita sobre um corpo algebricamente fechado e logo $\mathcal{J}_{\overline{\mathbf{k}}}$ é uma das álgebras listadas no Teorema 1.14, usando essa informação para determinar $\mathcal{J}$ obtemos:

Teorema 1.16. [21, V.7][42, Cap.IV, p.36] Seja J uma álgebra de Jordan simples central de dimensão finita sobre um corpo $\mathbf{k}$ arbitrário. Então temos as seguintes possibilidades para $\mathrm{J}$ :

1. $\mathcal{J}=\mathbf{k}$,

2. $\mathcal{J}=(\mathcal{J}(\mathrm{V}, \mathrm{f}), \odot)$ a álgebra de Jordan de uma forma bilinear simétrica não degenerada $f$ num espaço vetorial $V$ de dimensão finita tal que $\operatorname{dim} V>1$,

3. $\mathcal{J}=(\mathrm{H}(\mathcal{A}, \mathrm{J}), \odot)$ onde $(\mathcal{A}, \mathrm{J})$ é uma álgebra associativa simples central de dimensão finita com involução $J$ de grau ${ }^{2} n \geqslant 3$, ou

4. J é uma álgebra tal que existe uma extensão finita $K$ do corpo base $\mathbf{k}$ tal que $\partial_{K} \simeq\left(H\left(\left(\mathcal{C}_{K}\right)_{3}, j\right), \odot\right)$ onde $\left(\mathcal{C}_{K}\right)_{3}$ é a álgebra das matrizes $3 \times 3$ com elementos

2 Chamamos de grau de $(\mathcal{A}, \mathrm{J})$ sobre $\mathbf{k}$ ao inteiro $\mathrm{n}$ tal que $\left(\mathcal{A}_{\overline{\mathbf{k}}^{\prime}} \mathrm{J}\right) \simeq\left(\mathcal{D}_{\mathrm{n}}, \mathrm{j}\right)$ onde $(\mathcal{D}, \mathfrak{i})$ é uma álgebra de composição associativa, veja [21, Cap.V.7, p.209]. 
numa álgebra de Cayley $\mathcal{C}$ sobre $\mathrm{K}$ e $\mathrm{j}$ é a involução padrão de $\left(\mathcal{C}_{\mathrm{K}}\right)_{3}$ associada com a involução de $\mathcal{C}$.

As únicas álgebras excepcionais na lista são as álgebras de Albert de dimensão 27 (de tipo 4) como prova Schafer em [42, Cap.IV, Teo.9, p.38]. Estas últimas foram determinadas em [41].

\subsection{DECOMPOSIÇÃ DE PEIRCE}

Nesta seção vamos desenvolver algumas das principais ferramentas para a teoria estrutural das álgebras de Jordan. Estamos falando da decomposição de uma álgebra de Jordan em subespaços de Peirce relativa a um conjunto finito de elementos idempotentes ortogonais dois a dois. Estes subespaços têm propriedades multiplicativas importantes que serão apresentadas nos Teoremas 1.18 e 1.19.

Definição 1.17. Um elemento e de uma álgebra de Jordan $\mathcal{J}$ é chamado de elemento idempotente se $e^{2}=e$. Um idempotente próprio é um elemento idempotente $e \neq 0,1$. Dois idempotentes $e, f$ são ditos ortogonais se $e f=0$.

Como em uma álgebra de Jordan $\mathcal{J}$ de dimensão finita (sobre um corpo $\mathbf{k}$ algebricamente fechado ou de char $\mathbf{k}=0$ ) temos decomposição $\mathcal{J}=\mathcal{J}_{\text {ss }} \oplus \operatorname{Rad}(\mathcal{J}) \operatorname{com} \mathcal{J}_{\text {ss }}$ uma álgebra de Jordan semissimples, pelo Teorema 1.11, existe um elemento identidade $\bar{e}$ em $\partial_{\text {ss }}$ que pode ser "levantado" a um idempotente e da álgebra $\mathcal{J}$. Então, sem perda de generalidade, podemos considerar que toda álgebra de Jordan a ser obtida nas classificações dos Capítulos 2 e 3, ou é nilpotente ou contém um elemento idempotente e.

Pela linearização da identidade de Jordan (1.3) temos que para qualquer elemento idempotente $e \in \mathcal{J}$ e qualquer elemento $x \in \mathcal{J}$

$$
\begin{aligned}
0 & =(x e, e, e)+(x e, e, e)+\left(e^{2}, e, x\right) \\
& =2(x e, e, e)+(e, e, x) \\
& =2((x e) e) e-3(x e) e+x e \\
& =\left[2 M_{e}^{3}-3 M_{e}^{2}+M_{e}\right](x),
\end{aligned}
$$

o que implica que $2 M_{e}^{3}-3 M_{e}^{2}+M_{e}=0$, isto é se $f(x)=(x-1)(2 x-1) x$ então $f\left(M_{e}\right)=$ 0 . Logo o polinômio minimal de $M_{e}$ divide $f(x)$ e as únicas possibilidades para as raízes características de $M_{e}$ são $0, \frac{1}{2}, 1$. Observe que 1 é com certeza uma raiz característica já que $M_{e}(e)=e^{2}=e \neq 0$. Também podemos deduzir que o polinômio minimal de $M_{e}$ 
tem raízes simples. Logo, pelo Teorema da Decomposição Primária [40, Teo.7.6,p.168], J é a soma direta de espaços vetoriais

$$
\mathcal{J}=\mathcal{P}_{1} \oplus \mathcal{P}_{\frac{1}{2}} \oplus \mathcal{P}_{0}, \quad \text { onde } \mathcal{P}_{\mathfrak{i}}=\{x \in \mathcal{J} \mid x e=i x\} \operatorname{com} i=0, \frac{1}{2}, 1 \text {. }
$$

Esta decomposição em soma de subespaços é chamada decomposição de Peirce da álgebra de Jordan $\mathcal{J}$ relativa ao idempotente $e$. Os subespaços $\mathcal{P}_{i}$ são chamados de componentes (ou subespaços) de Peirce da álgebra $\mathcal{J}$ e suas propriedades multiplicativas são enunciadas no seguinte teorema.

Teorema 1.18. [50, Teo. 4, p. 334] Seja J uma álgebra de Jordan com idempotente e. Então a tabela de multiplicação para a decomposição de Peirce de J é dada por:

$$
\begin{aligned}
\mathcal{P}_{1}^{2} \subseteq \mathcal{P}_{1}, \quad \mathcal{P}_{1} \mathcal{P}_{0}=(0), & \mathcal{P}_{0}^{2} \subseteq \mathcal{P}_{0} \\
\mathcal{P}_{0} \mathcal{P}_{\frac{1}{2}} \subseteq \mathcal{P}_{\frac{1}{2}}, & \mathcal{P}_{1} \mathcal{P}_{\frac{1}{2}} \subseteq \mathcal{P}_{\frac{1}{2}}, \quad \mathcal{P}_{\frac{1}{2}}^{2} \subseteq \mathcal{P}_{0}+\mathcal{P}_{1} .
\end{aligned}
$$

Observamos que a decomposição de Peirce é "herdada" por ideais ou por subálgebras de $\mathcal{J}$ contendo $e$, ou seja se I é um ideal de $\mathcal{J}$ ou uma subálgebra contendo e então $\mathrm{I}=\mathrm{I}_{0} \oplus \mathrm{I}_{\frac{1}{2}} \oplus \mathrm{I}_{1}$ onde $\mathrm{I}_{i}=\mathrm{I} \cap \mathcal{P}_{i}$, veja [33, Teo.8.1.2, p. 236].

Mais geralmente, no caso em que $\mathcal{J}$ seja uma álgebra de Jordan com unidade 1 a qual é soma de idempotentes $e_{i}$ dois a dois ortogonais, $1=\sum_{i=1}^{n} e_{i}$, então:

Teorema 1.19. [21, Lema 2, p. 120] J se decompõe em soma direta de subespaços

$$
\mathcal{J}=\bigoplus_{1 \leqslant i \leqslant j \leqslant n} \mathcal{P}_{i j}
$$

onde $\mathcal{P}_{i i}=\left\{x \in \mathcal{J} \mid x e_{i}=x\right\}, \mathcal{P}_{i j}=\left\{x \in \mathcal{J} \mid x e_{i}=x e_{j}=\frac{1}{2} x\right\}$ e as componentes $\mathcal{P}_{i j}$ satisfazem as seguintes relações:

$$
\begin{aligned}
\mathcal{P}_{i i}^{2} \subseteq \mathcal{P}_{i i}, \quad \mathcal{P}_{i j} \mathcal{P}_{i i} \subseteq \mathcal{P}_{i j}, \quad \mathcal{P}_{i j}^{2} \subseteq \mathcal{P}_{i i}+\mathcal{P}_{j j}, \\
\mathcal{P}_{i j} \mathcal{P}_{j k} \subseteq \mathcal{P}_{i k}, \quad \mathcal{P}_{i i} \mathcal{P}_{j j}=\mathcal{P}_{i i} \mathcal{P}_{j k}=\mathcal{P}_{i j} \mathcal{P}_{k l}=(0)
\end{aligned}
$$

onde os indices $i, j, k, l$ são todos distintos.

Neste caso a decomposição recebe o nome de decomposição de Peirce da álgebra de Jordan $\mathcal{J}$ relativa ao sistema de idempotentes $\left\{e_{1}, \ldots, e_{n}\right\}$ e também é herdada por ideais ou subálgebras de $\mathcal{J}$ contendo o sistema de idempotentes ortogonais ([33, Teo. 13.1.4, p.280]).

Segue do fato da soma ser direta que se $x \in \mathcal{P}_{i j}$ então $x e_{k}=0$ para todo $k \in$ $\{1, \cdots, n\} \backslash\{i, j\}$. 
Suponha agora que $\mathcal{J}$ não tenha unidade mas possua um número finito $e_{1}, \ldots, e_{n}$ de elementos idempotentes dois a dois ortogonais, então vamos considerar a álgebra $\mathcal{J}^{\#}=\mathcal{J} \oplus \mathbf{k} \cdot 1$ obtida pela adjunção formal de um elemento identidade à álgebra $\mathcal{J}$, para a qual temos garantida a decomposição de Peirce

$$
\partial^{\#}=\bigoplus_{0 \leqslant i \leqslant j \leqslant n} \mathcal{P}_{i j}
$$

relativa ao sistema de idempotentes $\left\{e_{0}, e_{1}, \ldots, e_{n}\right\}$ onde $e_{0}=1-e_{1}-\cdots-e_{n}$. Observamos que mesmo $\mathcal{J}$ sendo subálgebra de $\mathcal{J}^{\#}$ não podemos aplicar a herda da decomposição pois $e_{0} \notin \mathcal{J}$, mas a decomposição de Peirce de $\mathcal{J}^{\#}$ implica na seguinte decomposição de $\mathcal{J}$ em subespaços:

$$
\mathcal{J}=\bigoplus_{0 \leqslant i \leqslant j \leqslant n} \mathcal{P}_{i j}^{\prime}
$$

onde $\mathcal{P}_{i \mathrm{i}}^{\prime}=\left\{x \in \mathcal{J} \mid x e_{i}=x\right\}$ para $i \neq 0, \mathcal{P}_{00}^{\prime}=\left\{x \in \mathcal{J} \mid x e_{k}=0\right.$, para todo $\left.1 \leqslant k \leqslant n\right\}$, $\mathcal{P}_{i j}^{\prime}=\left\{x \in \mathcal{J} \mid x e_{i}=x e_{j}=\frac{1}{2} x\right\}$ para $0 \neq i<j$ e $\mathcal{P}_{{ }_{j}}^{\prime}=\left\{x \in \mathcal{J} \mid x e_{j}=\frac{1}{2} x\right\}$ para $j \neq 0$. É importante destacar que se $x$ é um elemento de $\mathcal{J}$ que pertence a $\mathcal{P}_{i j}^{\prime}$ então é conhecida a ação dos idempotentes $e_{1}, \ldots, e_{n}$ em $x$ e isto determina a ação de $e_{0}$ considerando $x$ como um elemento de $\mathcal{J}^{\#}, \log 0 x \in \mathcal{P}_{i j}$ quando considerado como um elemento da álgebra com unidade. É claro que a recíproca é também verdadeira. Como consequência disso temos que a tabela de multiplicação dos $\mathcal{P}_{i j}^{\prime}$ é análoga à tabela de multiplicação dos $\mathcal{P}_{i j}$ dada no Teorema 1.19.

Todo ideal $K$ de $\mathcal{J}$ é claramente um ideal de $\mathcal{J}^{\#}$, então $K$ herda a decomposição de Peirce $K=\bigoplus_{0 \leqslant i \leqslant j \leqslant n} K_{i j}$ onde $K_{i j}=K \cap \mathcal{P}_{i j}$ quando considerado como um ideal de $f^{\#}$ o que leva à decomposição $K=\bigoplus_{0 \leqslant i \leqslant j \leqslant n} K_{i j}^{\prime}$ onde $K^{\prime}{ }_{i j}=K \cap \mathcal{P}_{i j}^{\prime}$ quando considerado como ideal de $\mathcal{J}$. Novamente não podemos aplicar a herda a subálgebras de $\mathcal{J}$ já que estas ao igual que $\mathcal{J}$ não contém o idempotente $e_{0}$.

\subsection{EXTENSÕES E COHOMOLOGIA}

Nesta seção apresentaremos e relacionaremos os conceitos de extensões de uma álgebra de Jordan $\mathcal{J}$ por um bimódulo de Jordan $\mathcal{N}$, considerando $\mathcal{N}$ como uma álgebra de Jordan com multiplicação trivial e de segundo grupo de cohomologia de $\mathcal{J}$ com coeficientes em $\mathcal{M}$. Uma referência para esta seção é o livro de N. Jacobson [21, Cap. II, seção 5 e 8]. 
Começamos lembrando a definição de bimódulo de Jordan. Seja $\mathcal{J}$ uma álgebra de Jordan sobre um corpo $\mathbf{k}$, $\mathcal{M}$ um espaço vetorial sobre $\mathbf{k}$ e suponha que temos um par de aplicações bilineares $(a, m) \rightarrow a m$ e $(a, m) \rightarrow$ ma de $\mathcal{J} \otimes \mathcal{M}$ em $\mathcal{M}$, onde $a \in \mathcal{J}$ e $m \in \mathcal{M}$. Logo temos

$$
\begin{array}{ll}
\left(a_{1}+a_{2}\right) m=a_{1} m+a_{2} m, & a\left(m_{1}+m_{2}\right)=a m_{1}+a m_{2}, \\
\alpha(a m)=(\alpha a) m=a(\alpha m), & \alpha(m a)=(\alpha m) a=m(\alpha a)
\end{array}
$$

para $a, a_{1}, a_{2} \in \mathcal{J}, m, m_{1}, m_{2} \in \mathcal{M}$ e $\alpha \in \mathbf{k}$. Agora seja $\mathcal{F}=\mathcal{J} \oplus \mathcal{M}$ o espaço vetorial da soma direta de $\mathcal{J} \operatorname{com} \mathcal{M}$ e defina em $\mathcal{F}$ um produto dado por

$$
\left(a_{1}+m_{1}\right) \star\left(a_{2}+m_{2}\right)=a_{1} a_{2}+a_{1} m_{2}+m_{1} a_{2} .
$$

É imediato ver que este produto é bilinear, então $\mathcal{F}$ é uma álgebra. Mais ainda, J é uma subálgebra e $\mathcal{M}$ é um ideal de $\mathcal{F}$ tal que $\mathcal{M}^{2}=0$.

Definição 1.20. $\mathcal{M}$ e as duas aplicações bilineares constituem um bimódulo de Jordan para $\mathcal{J}$ se $(\mathcal{F}, \star)$ é uma álgebra de Jordan.

Definição 1.21. Seja $\mathcal{J}$ uma álgebra de Jordan e seja $\mathcal{M}$ um bimódulo de Jordan para $\mathcal{J}$. Uma aplicação bilinear $h: \mathcal{J} \times \mathcal{J} \rightarrow \mathcal{M}$ a qual, para todo $a, b \in \mathcal{J}$, satisfaz

$$
\begin{aligned}
h(a, b) & =h(b, a) \\
(h(a, a) b) a+h\left(a^{2}, b\right) a+h\left(a^{2} b, a\right) & =a^{2} h(b, a)+h(a, a)(b a)+h\left(a^{2}, b a\right)
\end{aligned}
$$

é chamada de 2-cociclo de $\mathcal{J}$ com coeficientes em $\mathcal{M}$. Denotamos o conjunto de todos os 2-cociclos de $\mathcal{J}$ com coeficientes em $\mathcal{M}$ por $Z^{2}(\mathcal{J}, \mathcal{M})$.

Definição 1.22. Dizemos que dois 2-cociclos $h$ e $h^{\prime}$ de $\mathcal{J}$ com coeficientes em $\mathcal{M}$ são equivalentes se existe uma aplicação linear $\mu: \mathcal{J} \rightarrow \mathcal{M}$ tal que para todo $a, b \in \mathcal{J}$ :

$$
h^{\prime}(a, b)=h(a, b)-\mu(a b)+a \mu(b)+\mu(a) b .
$$

Esta é uma relação de equivalência no conjunto de todos os 2-cociclos de $\mathcal{J}$ com coeficientes em $\mathcal{M}$. $\mathrm{O}$ conjunto das classes de equivalências determinado por esta relação é denotado por $\mathrm{H}^{2}(\mathcal{J}, \mathcal{M})$ e é denominado segundo grupo de cohomologia da álgebra $\mathcal{J}$ com coeficientes em $\mathcal{M}$. Chamaremos de 2 -cobordos de $\mathcal{J}$ com coeficientes em $\mathcal{M}$ aos 2-cociclos equivalentes a 0 . Denotaremos o conjunto de todos os 2-cobordos por $\mathrm{B}^{2}(\mathcal{J}, \mathcal{M})$.

Por outro lado vamos considerar as seguintes definições que veremos no final da seção como se relacionam com os conceitos de 2-cociclos e cohomologia. 
Definição 1.23. Sejam $\mathcal{J}$ e $\mathcal{M}$ duas álgebras de Jordan então definimos uma extensão de J por $\mathcal{M}$ como sendo uma sequência exata curta

$$
0 \rightarrow \mathcal{M} \stackrel{\alpha}{\rightarrow} \mathcal{F} \stackrel{\beta}{\rightarrow} \mathcal{J} \rightarrow 0
$$

tal que $\mathcal{F}$ é também uma álgebra de Jordan e $\alpha$ e $\beta$ são homomorfismos de álgebras.

Definição 1.24. Duas extensões são chamadas de equivalentes se existe um diagrama comutativo

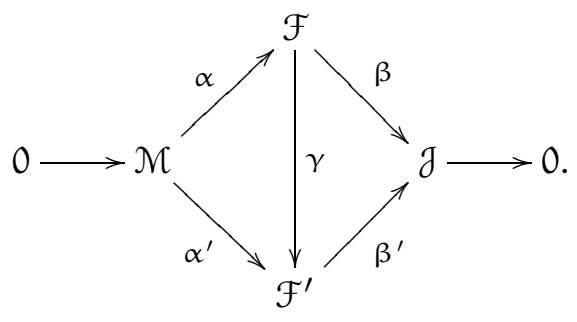

Segue da definição que $\gamma$ é um isomorfismo de $\mathcal{F}$ em $\mathcal{F}^{\prime}$.

Dizemos que a extensão (1.8) cinde se existe um homomorfismo $\delta: \mathcal{J} \rightarrow \mathcal{F}$ tal que $\beta \delta=\operatorname{Id}_{\mathcal{J}}$ seja a aplicação identidade em $\mathcal{J}$. Se este for o caso temos a decomposição em espaços vetoriais $\mathcal{F}=\delta(\mathcal{J}) \oplus \alpha(\mathcal{M})$ e $\delta(\mathcal{J})$ é uma subálgebra de $\mathcal{F}$ isomorfa a $\mathcal{J}$.

Se $\mathcal{M}$ é uma álgebra nula, no sentido que $\mathcal{M}^{2}=0$, então a extensão de $\mathcal{J}$ por $\mathcal{M}$ será chamada de extensão nula.

Consideremos este tipo de extensões. Notemos primeiro que (1.8) implica que $\alpha$ é injetora, logo podemos identificar $\mathcal{M} \operatorname{com} \alpha(\mathcal{M})$ e considerar $\alpha$ como sendo a inclusão de $\mathcal{M}$ em $\mathcal{F}$. Como $\mathcal{M}$ tem um subespaço vetorial complementar em $\mathcal{F}$, existe uma transformação linear $\delta: \mathcal{J} \rightarrow \mathcal{F}$ tal que $\beta \delta=I_{\mathcal{\jmath}}$. Assim temos a decomposição em espaços vetoriais $\mathcal{F}=\mathcal{M} \oplus \delta(\mathcal{J})$. Se $a, b \in \mathcal{J}$ então

$$
\beta(\delta(a) \delta(b)-\delta(a b))=a b-a b=0 .
$$

Como a sequência (1.8) é exata isto implica que $\delta(a) \delta(b)-\delta(a b) \in \mathcal{M}$. Logo temos

$$
\delta(a) \delta(b)=\delta(a b)+h(a, b)
$$

onde $(a, b) \rightarrow h(a, b)$ é uma aplicação bilinear de $\mathcal{J} \times \mathcal{J}$ em $\mathcal{M}$.

Observamos que $\mathcal{M}$ tem estrutura de bimódulo de Jordan para $\mathcal{J}$ relativa ao produto bilinear

$$
a m=m a=\delta(a) m
$$


onde $a \in \mathcal{J}, \mathrm{m} \in \mathcal{M}$ e o produto do lado direito é o produto de $\mathcal{F}$ (para uma prova deste fato veja [21, p. 92]) e destacamos que $h$ definida por $h(a, b)=\delta(a) \delta(b)-\delta(a b)$ é um 2-cociclo de $\mathcal{J}$ com coeficientes em $\mathcal{M}$ (considerando $\mathcal{M}$ como um bimódulo de Jordan para $\mathcal{J}$ via (1.9)) como consequência das condições impostas pela comutatividade e a identidade de Jordan (1.2) de $\mathcal{J}$.

Reciprocamente, seja $\mathcal{J}$ é uma álgebra de Jordan, $\mathcal{M}$ um bimódulo de Jordan para $\mathcal{J}$ e h um 2-cociclo de $\mathcal{J}$ com coeficientes em $\mathcal{M}$. Seja $\mathcal{F}$ um espaço vetorial contendo $\mathcal{M}$ tal que existe um isomorfismo linear $\delta: \mathcal{J} \rightarrow \mathcal{F}$ que satisfaz $\mathcal{F}=\delta(\mathcal{J}) \oplus \mathcal{M}$. Então $\mathcal{F}$ com a multiplicação definida por:

$$
(\delta(a)+m) \bullet(\delta(b)+n)=\delta(a b)+h(a, b)+a n+m b, \quad a, b \in \mathcal{J} \text { e } m, n \in \mathcal{M},
$$

é uma álgebra de Jordan e $\mathcal{M}$ é um ideal em $\mathcal{F}$ tal que $\mathcal{M}^{2}=0$ e a estrutura de bimódulo de $\mathcal{M}$ dada por (1.9) coincide com a original.

Estes fatos se resumem no seguinte teorema.

Teorema 1.25. [21, Teorema 12, p. 94] Seja J uma álgebra de Jordan e $\mathcal{M}$ um bimódulo de Jordan para $\mathcal{J}$, então existe uma bijeção de $\mathrm{H}^{2}(\mathcal{J}, \mathcal{M})$ no conjunto das classes de equivalências de extensões nulas de J por $\mathcal{M}$ tal que a estrutura de bimódulo em $\mathcal{M}$ dada por (1.9) coincide com a original. Nesta correspondência a classe de equivalência do $0 \mathrm{em} \mathrm{H}^{2}(\mathcal{J}, \mathcal{M})$ corresponde à classe de isomorfismos de extensões cindidas. 


\section{2 CLASSIFICAÇÃO ALGÉBRICA DAS ÁLGEBRAS DE JORDAN DE DIMENSÃO 4 SOBRE UM CORPO ALGEBRI- CAMENTE FECHADO}

Entendemos por "classificação algébrica" a determinação de todas as classes de isomorfismos de álgebras de Jordan de dimensão fixa. O primeiro passo na classificação algébrica de álgebras de Jordan foi dado por A. Albert quem determinou todas as álgebras de Jordan simples de dimensão finita sobre um corpo algebricamente fechado. Mas, exceto para dimensões baixas, uma classificação algébrica completa só é conhecida no caso de álgebras de Jordan semissimples.

Neste capítulo $\mathbf{k}$ denotará um corpo algebricamente fechado de char $\mathbf{k} \neq 2$. A seguir apresentaremos a lista de todas as álgebras de Jordan ${ }^{1}$ (unitárias e não unitárias, associativas e não associativas) de dimensão 4 sobre k. Faremos isto em duas seções, na primeira introduzimos as álgebras de Jordan indecomponíveis de dimensão menor que 4, com o fim de fixar e simplificar a notação. Na segunda descrevemos as álgebras de dimensão 4 não isomorfas de acordo com a dimensão do radical e dos possíveis valores do tipo de nilpotência.

Usaremos esta descrição para estudar deformações entre álgebras de Jordan e descrever a variedade Jor $_{4}$ no Capítulo 5 deste texto.

Como foi visto no capítulo anterior toda álgebra de Jordan de dimensão finita sobre um corpo algebricamente fechado pode-se decompor como soma direta de espaços vetoriais como $\mathcal{J}=\mathcal{J}_{s s} \oplus \operatorname{Rad}(\mathcal{J})$, onde $\mathcal{J}_{s s}=\mathcal{J} / \operatorname{Rad}(\mathcal{J})$ é semissimples ou 0 e $\operatorname{Rad}(\mathcal{J})$ é o radical de $\mathcal{J}$. Consequentemente denotaremos por $e_{i}$ os elementos em $\partial_{s s}$ e por $n_{i}$ aqueles elementos que pertencem a $\operatorname{Rad}(\mathcal{J})$.

1 A classificação foi realizada inspirada no trabalho feito por H. Sherkulov em [43] onde o autor somente apresentou a lista das álgebras de Jordan não associativas. 


\section{1 Álgebras De JORdAn DE Dimensão MENOR QUE 4}

\subsection{1 Álgebras de Jordan de Dimensão 1}

Existem somente duas k-álgebras de Jordan de dimensão 1 não isomorfas: a álgebra simples $\mathbf{k} e \operatorname{com} e^{2}=e$ e a álgebra nilpotente $\mathbf{k n} \operatorname{com} \mathrm{n}^{2}=0$.

\subsection{2 Álgebras de Jordan de Dimensão 2}

Do teorema de Albert temos que a única álgebra de Jordan semissimples de dimensão 2 é $\mathbf{k} e_{1} \oplus \mathbf{k} e_{2}$. Se $\operatorname{dim} \operatorname{Rad}(\mathcal{J})=1$ então $\mathcal{J}$ tem um elemento idempotente $e_{1}, \operatorname{Rad}(\mathcal{J})$ é gerado por $n_{1}$ e a ação de $e_{1}$ em $n_{1}$ é dada pelo Teorema 1.18, logo temos que $e_{1} n_{1}=i n_{1}$ para $i=0, \frac{1}{2}, 1$. Isto define 3 álgebras não isomorfas. Finalmente, existem duas álgebras nilpotentes: a álgebra "nula", isto é com multiplicação 0 e a álgebra gerada por $n_{1}$, com $n_{1}^{3}=0$. Portanto obtemos as seguintes três $\mathbf{k}$-álgebras de Jordan indecomponíveis de dimensão 2 , que denotaremos por $\mathcal{B}_{\mathfrak{i}}$ :

Tabela 2.1: k-álgebras de Jordan indecomponíveis de dimensão 2

\begin{tabular}{|c|ccc|c|}
\hline $\mathcal{B}$ & \multicolumn{3}{|c|}{ Tabela de Multiplicação } & Observação \\
\hline $\mathcal{B}_{1}$ & $e_{1}^{2}=e_{1}$ & $e_{1} n_{1}=n_{1}$ & $n_{1}^{2}=0$ & Associativa \\
\hline $\mathcal{B}_{2}$ & $e_{1}^{2}=e_{1}$ & $e_{1} n_{1}=\frac{1}{2} n_{1}$ & $n_{1}^{2}=0$ & Não Associativa \\
\hline $\mathcal{B}_{3}$ & $n_{1}^{2}=n_{2}$ & $n_{1} n_{2}=0$ & $n_{2}^{2}=0$ & Nilpotente, Associativa \\
\hline
\end{tabular}

\subsection{3 Álgebras de Jordan de Dimensão 3}

Em [25] foram descritas todas as álgebras de Jordan de dimensão 3 sobre um corpo algebricamente fechado. Dessa lista obtemos as seguintes 10 álgebras indecomponíveis de dimensão 3 , que denotaremos por $\mathcal{T}_{i}$ :

Tabela 2.2: k-álgebras de Jordan indecomponíveis de dimensão 3

\begin{tabular}{|c|cc|c|}
\hline $\mathcal{T}$ & Tabela de Multiplicação & Observação \\
\hline \multirow{2}{*}{$\mathcal{T}_{1}$} & $e_{1}^{2}=e_{1} \quad n_{1}^{2}=n_{2} \quad n_{2}^{2}=0$ & Unitária \\
& $e_{1} n_{1}=n_{1} \quad e_{1} n_{2}=n_{2} \quad n_{1} n_{2}=0$ & Associativa \\
\hline \multirow{2}{*}{$\mathcal{T}_{2}$} & $\begin{array}{c}e_{1}^{2}=e_{1} \quad n_{1}^{2}=0 \quad n_{2}^{2}=0 \\
e_{1} n_{1}=n_{1} \quad e_{1} n_{2}=n_{2} \quad n_{1} n_{2}=0\end{array}$ & Unitária \\
\end{tabular}




\begin{tabular}{|c|c|c|}
\hline $\mathcal{T}$ & Tabela de Multiplicação & Observação \\
\hline $\mathcal{T}_{3}$ & $\begin{array}{ccc}n_{1}^{2}=n_{2} & n_{2}^{2}=0 \quad n_{3}^{2}=0 \\
n_{1} n_{2}=n_{3} & n_{1} n_{3}=0 \quad n_{2} n_{3}=0\end{array}$ & $\begin{array}{l}\text { Associativa, } \\
\text { Nilpotente }\end{array}$ \\
\hline $\mathcal{T}_{4}$ & $\begin{array}{ccc}n_{1}^{2}=n_{2} & n_{2}^{2}=0 \quad n_{3}^{2}=0 \\
n_{1} n_{2}=0 \quad n_{1} n_{3}=n_{2} \quad n_{2} n_{3}=0\end{array}$ & $\begin{array}{l}\text { Associativa, } \\
\text { Nilpotente }\end{array}$ \\
\hline $\mathcal{T}_{5}$ & $\begin{aligned} e_{1}^{2} & =e_{1} \quad e_{2}^{2}=e_{2} \quad e_{3}^{2}=e_{1}+e_{2} \\
e_{1} e_{2} & =0 \quad e_{1} e_{3}=\frac{1}{2} e_{3} \quad e_{2} e_{3}=\frac{1}{2} e_{3}\end{aligned}$ & $\begin{array}{c}\text { Unitária, } \\
\text { Não Associativa, } \\
\text { Simples }\end{array}$ \\
\hline $\mathcal{T}_{6}$ & $\begin{aligned} & e_{1}^{2}=e_{1} n_{1}^{2}=0 \quad n_{2}^{2}=0 \\
& e_{1} n_{1}=\frac{1}{2} n_{1} \quad e_{1} n_{2}=n_{2} \quad n_{1} n_{2}=0\end{aligned}$ & Não Associativa \\
\hline $\mathcal{T}_{7}$ & $\begin{array}{c}e_{1}^{2}=e_{1} \quad n_{1}^{2}=0 \quad n_{2}^{2}=0 \\
e_{1} n_{1}=\frac{1}{2} n_{1} \quad e_{1} n_{2}=\frac{1}{2} n_{2} \quad n_{1} n_{2}=0\end{array}$ & Não Associativa \\
\hline $\mathcal{T}_{8}$ & $\begin{array}{rll}e_{1}^{2}=e_{1} & n_{1}^{2}=n_{2} & n_{2}^{2}=0 \\
e_{1} n_{1}=\frac{1}{2} n_{1} & e_{1} n_{2}=0 & n_{1} n_{2}=0\end{array}$ & Não Associativa \\
\hline $\mathcal{T}_{9}$ & $\begin{array}{rll}e_{1}^{2}=e_{1} & n_{1}^{2}=n_{2} \quad n_{2}^{2}=0 \\
e_{1} n_{1}=\frac{1}{2} n_{1} & e_{1} n_{2}=n_{2} \quad n_{1} n_{2}=0\end{array}$ & Não Associativa \\
\hline $\mathcal{T}_{10}$ & $\begin{array}{rll}e_{1}^{2}=e_{1} & e_{2}^{2}=e_{2} & n_{1}^{2}=0 \\
e_{1} e_{2}=0 & e_{1} n_{1}=\frac{1}{2} n_{1} & e_{2} n_{1}=\frac{1}{2} n_{1}\end{array}$ & $\begin{array}{c}\text { Unitária, } \\
\text { Não Associativa }\end{array}$ \\
\hline
\end{tabular}

\section{2 Álgebras DE JORdAn DE DIMENSÃO 4}

Nesta seção descreveremos todas as 73 álgebras de Jordan de dimensão 4 sobre um corpo $\mathbf{k}$ algebricamente fechado. A descrição será organizada de acordo com a dimensão do radical $\operatorname{Rad}(\mathcal{J})$ e subsequentemente com os possíveis valores de seu tipo de nilpotência. Durante a descrição será provado que qualquer outra álgebra de Jordan de dimensão 4 sobre um corpo algebricamente fechado é isomorfa a uma das 73 e por último no Teorema 2.2 veremos que as álgebras $\mathcal{J}_{1}$ a $\mathcal{J}_{73}$ são duas a duas não isomorfas.

Quando não houver ambiguidade em relação à álgebra $\mathcal{J}$ chamaremos de $\mathrm{N}$ ao radical nilpotente de $\mathcal{J}$ a fim de simplificar a notação. Também observamos que como $\mathfrak{d}^{i}=\mathcal{J}^{\langle i\rangle}$ para $i=1,2,3$ usaremos somente a notação de $i$-ésima potência nestes casos.

Para cada álgebra calcularemos a dimensão do seu grupo de automorfismos $\operatorname{Aut}(\mathcal{J})=$ $\{\mathrm{g} \in \mathrm{GL}(\mathcal{J}) \mid \mathrm{g}$ é um endomorfismo de $\mathcal{J}\}$, de seu aniquilador $\operatorname{Ann}(\mathcal{J})=\{\mathrm{a} \in \mathcal{J} \mid \mathrm{a} \mathcal{J}=0\}$ e de sua segunda potência $\mathcal{J}^{2}$. Para uma lista mais detalhada das propriedades de cada álgebra veja o Apêndice $B$. 


\subsection{1 Álgebras de Jordan Semissimples}

Para obter a classificação das álgebras semissimples é suficiente, por definição, conhecer as simples. Para um corpo algebricamente fechado e uma álgebra de dimensão finita estas últimas foram totalmente determinadas por A. Albert. Segue então do Teorema 1.14 que se $\mathcal{J}$ é uma álgebra de Jordan simples de dimensão menor ou igual a 4 sobre um corpo $\mathbf{k}$ algebricamente fechado então, ou $\mathcal{J}=\mathbf{k}$ ou $\mathcal{J}$ é a álgebra de Jordan de uma forma bilinear simétrica não degenerada. Observamos que desprezamos as álgebras de Jordan $\mathcal{J}=\left(H\left(\mathcal{D}_{n}, \mathfrak{j}\right), \odot\right)$ dos elementos de $\mathcal{D}_{n}$ para $n \geqslant 3$, onde $(\mathcal{D}, \mathfrak{i})$ é uma álgebra de composição, simétricos com respeito à involução padrão $j$ associada a $i$, pois no mínimo estas álgebras tem dimensão 6.

Assim, o problema de classificar as álgebras simples de dimensão menor ou igual a 4, fica reduzido ao problema de classificar formas bilineares simétricas não degeneradas sobre espaços vetoriais de dimensão finita.

Seja $V$ um espaço vetorial de dimensão finita $n \geqslant 2$ sobre $\mathbf{k}$, que possui uma forma bilinear simétrica não degenerada $f=f(x, y)$, de [40, Teo. 11.23, p.287] existe uma base $\left\{e_{1}, \ldots, e_{n}\right\}$ de $V$ tal que $f\left(e_{i}, e_{i}\right)=1$ e $f\left(e_{i}, e_{j}\right)=0$ para $i \neq j$. Então a álgebra de Jordan $(\mathcal{J}(V, f), \square)$ da forma bilinear $f$ tem base $\left\{1, e_{1}, \ldots, e_{n}\right\}$ e é definida pelos produtos

$$
1 \sqcup 1=1, \quad 1 \sqcup e_{i}=e_{i}, \quad e_{i} \sqcup e_{i}=1, \quad e_{i} \sqcup e_{j}=0 \text { para } i \neq j
$$

Logo existem somente 3 álgebras de Jordan simples não isomorfas de dimensão menor ou igual a 4:

1. $\mathbf{k}_{1}$ de dimensão 1 ,

2. $(\mathcal{J}(\mathrm{V}, \mathrm{f}), \bullet)$ onde $\operatorname{dim}_{\mathbf{k}} \mathrm{V}=2$, de dimensão 3 e

3. $(\mathcal{J}(V, f), \sqcup)$ onde $\operatorname{dim}_{\mathbf{k}} V=3$, de dimensão 4 .

Note que a álgebra do item 2 é isomorfa à álgebra tridimensional $\mathcal{T}_{5}$ via isomorfismo $\mathrm{T}:\left(\mathcal{T}_{5}, \cdot\right) \rightarrow(\mathcal{J}(\mathrm{V}, \mathrm{f}), \bullet)$ dado por

$$
\mathrm{T}\left(e_{1}\right)=\frac{1}{2} \cdot 1+\frac{1}{2} e_{1}, \quad \mathrm{~T}\left(e_{2}\right)=\frac{1}{2} \cdot 1-\frac{1}{2} e_{1}, \quad \mathrm{~T}\left(e_{3}\right)=e_{2} .
$$

Considerando somas diretas de tais álgebras obtemos todas as álgebras de Jordan semissimples de dimensão 4 sobre $\mathbf{k}$ : 
Tabela 2.3: k-álgebras de Jordan semissimples de dimensão 4

\begin{tabular}{|c|c|c|c|c|c|}
\hline $\mathcal{J}$ & Tabela de Multiplicação & $\begin{array}{c}\operatorname{dim} \\
\operatorname{Aut}(\mathcal{J})\end{array}$ & $\begin{array}{c}\operatorname{dim} \\
\operatorname{Ann}(\mathcal{J})\end{array}$ & $\begin{array}{c}\operatorname{dim} \\
\mathcal{J}^{2}\end{array}$ & Observação \\
\hline$\partial_{1}$ & $\mathcal{T}_{5} \oplus \mathbf{k} e_{4}$ & 1 & 0 & 4 & $\begin{array}{c}\text { Unitária, } \\
\text { Semissimples }\end{array}$ \\
\hline$\partial_{2}$ & $\begin{array}{c}e_{1}^{2}=e_{1} \quad e_{2}^{2}=e_{2}=\frac{1}{2} e_{3} \quad e_{1} e_{4}=\frac{1}{2} e_{4} \\
e_{2} e_{3}=\frac{1}{2} e_{3} \quad e_{2} e_{4}=\frac{1}{2} e_{4} \\
e_{3} e_{4}=\frac{1}{2}\left(e_{1}+e_{2}\right.\end{array}$ & 3 & 0 & 4 & $\begin{array}{c}\text { Unitária, } \\
\text { Simples }\end{array}$ \\
\hline$\partial_{3}$ & $\mathbf{k} e_{1} \oplus \mathbf{k} e_{2} \oplus \mathbf{k} e_{3} \oplus \mathbf{k} e_{4}$ & 0 & 0 & 4 & $\begin{array}{c}\text { Unitária, } \\
\text { Assissimples, } \\
\text { Associativa }\end{array}$ \\
\hline
\end{tabular}

Novamente, note que a álgebra do item 3 é isomorfa à álgebra $J_{2}$ via isomorfismo $\mathrm{T}:\left(\mathcal{J}_{2}, \cdot\right) \rightarrow(\mathcal{J}(\mathrm{V}, \mathrm{f}), \circlearrowright)$ dado por

$$
\mathrm{T}\left(e_{1}\right)=\frac{1}{2} \cdot 1-\frac{1}{2} e_{1}, \quad \mathrm{~T}\left(e_{2}\right)=\frac{1}{2} \cdot 1+\frac{1}{2} e_{1}, \quad \mathrm{~T}\left(e_{3}\right)=-i e_{2}+e_{3}, \quad \mathrm{~T}\left(e_{4}\right)=\frac{i}{4} e_{2}+\frac{1}{4} e_{3} .
$$

No que segue usaremos o produto dado por $\mathcal{I}_{2}$ já que estamos interessados em conhecer os elementos idempotentes ortogonais de cada álgebra que nos fornecem a correspondente decomposição de Peirce.

\subsection{2 Álgebras de Jordan com Radical de Dimensão 1}

Considere $\mathcal{J}$ uma k-álgebra de Jordan tal que $\operatorname{dim} N=1$, $\log$ a parte semissimples $\mathcal{J}_{\text {s }}$ tem dimensão 3 e pelo Teorema 1.14 temos as seguintes possibilidades:

$$
\text { ou } \mathcal{J}_{\mathrm{ss}}=\mathbf{k} e_{1} \oplus \mathbf{k} \mathbf{e}_{2} \oplus \mathbf{k} \mathrm{e}_{3} \text { ou } \mathcal{J}_{\mathrm{ss}}=\mathcal{T}_{5} \text {. }
$$

Quando não houver ambiguidade denotaremos as componentes de Peirce de $\mathcal{J}$ e $\mathrm{N}$, $\mathcal{P}_{i j}^{\prime}$ e $N_{i j}^{\prime}$, simplesmente por $\mathcal{P}_{i j}$ e $N_{i j}$ para simplificar a notação.

1) Suponha que $\mathfrak{J}_{s s}=\mathbf{k} e_{1} \oplus \mathbf{k} e_{2} \oplus \mathbf{k} e_{3}$. Então a álgebra $\mathcal{J}^{\#}=\mathcal{J} \oplus \mathbf{k} \cdot 1$ contém 4 idempotentes ortogonais $e_{1}, e_{2}, e_{3}$ e $e_{0}=1-e_{1}-e_{2}-e_{3}$, logo da decomposição de Peirce (1.5) temos:

$$
\mathcal{J}=\mathcal{P}_{00} \oplus \mathcal{P}_{01} \oplus \mathcal{P}_{02} \oplus \mathcal{P}_{03} \oplus \mathcal{P}_{11} \oplus \mathcal{P}_{12} \oplus \mathcal{P}_{13} \oplus \mathcal{P}_{22} \oplus \mathcal{P}_{23} \oplus \mathcal{P}_{33}
$$


e como o radical $\mathrm{N}$ é um ideal de $\mathcal{J}$ este herda a decomposição:

$$
\mathrm{N}=\mathrm{N}_{00} \oplus \mathrm{N}_{01} \oplus \mathrm{N}_{02} \oplus \mathrm{N}_{03} \oplus \mathrm{N}_{11} \oplus \mathrm{N}_{12} \oplus \mathrm{N}_{13} \oplus \mathrm{N}_{22} \oplus \mathrm{N}_{23} \oplus \mathrm{N}_{33},
$$

onde $N_{i j}=N \cap \mathcal{P}_{i j}$. Seja $n_{1}$ uma base de $N$, então $\mathcal{J}$ é completamente definida pelo subespaço de Peirce ao qual $n_{1}$ pertence. Assim obtemos as seguintes álgebras não isomorfas:

Tabela 2.4: k-álgebras de Jordan de dimensão 4 com radical unidimensional e parte semissimples $\mathbf{k} \mathbf{e}_{1} \oplus \mathbf{k} \mathbf{e}_{2} \oplus \mathbf{k} \mathbf{e}_{3}$

\begin{tabular}{|c|c|c|c|c|c|}
\hline $\mathcal{J}$ & Tabela de Multiplicação & $\begin{array}{c}\operatorname{dim} \\
\operatorname{Aut}(\mathcal{J})\end{array}$ & $\begin{array}{c}\operatorname{dim} \\
\operatorname{Ann}(\mathcal{J})\end{array}$ & $\begin{array}{c}\operatorname{dim} \\
\mathcal{J}^{2}\end{array}$ & Observação \\
\hline$\partial_{4}$ & 1 & 0 & 4 & $\begin{array}{c}\text { Unitária, } \\
\text { Associativa, } \\
\mathrm{n}_{1} \in \mathrm{N}_{11}\end{array}$ \\
\hline$\partial_{5}$ & $\mathbf{k} e_{1} \oplus \mathbf{k} e_{2} \oplus \mathbf{k} e_{2} \oplus \mathbf{k} e_{3} \oplus \mathbf{k} \mathrm{n}_{1}$ & 1 & 1 & 3 & $\begin{array}{c}\text { Associativa, } \\
\mathrm{n}_{1} \in \mathrm{N}_{00}\end{array}$ \\
\hline$\partial_{6}$ & $\mathcal{B}_{2} \oplus \mathbf{k} e_{2} \oplus \mathbf{k} e_{3}$ & 2 & 0 & 4 & $\mathrm{n}_{1} \in \mathrm{N}_{01}$ \\
\hline$J_{7}$ & $\mathcal{T}_{10} \oplus \mathbf{k} e_{3}$ & 2 & 0 & 4 & $\begin{array}{c}\text { Unitária, } \\
\mathrm{n}_{1} \in \mathrm{N}_{12}\end{array}$ \\
\hline
\end{tabular}

Observamos que no caso em que $n_{1}$ pertença aos subespaços $N_{i i}$ para $i=2,3$ temos um elemento idempotente na álgebra agindo como 1 em $n_{1}$, logo a álgebra obtida é isomorfa a $\partial_{4}$. Se $n_{1} \in N_{0 i}$ para $i=2,3$ temos um elemento idempotente na álgebra agindo como $\frac{1}{2}$ em $n_{1}, \operatorname{logo}$ a álgebra obtida é isomorfa a $\mathcal{J}_{6}$. Por último se $n_{1}$ pertence a algum dos subespaços $N_{i 3}$ temos dois elementos idempotentes na álgebra que agem como $\frac{1}{2}$ em $n_{1}$, portanto a álgebra correspondente é isomorfa a $\mathcal{J}_{7}$.

2) Suponha que $\mathcal{J}_{s s}=\mathcal{T}_{5}$. Então a álgebra $\mathfrak{J}^{\#}=\mathcal{J} \oplus \mathbf{k} \cdot 1$ contém 3 elementos idempotentes ortogonais $e_{1}, e_{2}, e_{0}=1-e_{1}-e_{2}$ o que implica na decomposição de Peirce do radical $\mathrm{N}$

$$
\mathrm{N}=\mathrm{N}_{00} \oplus \mathrm{N}_{01} \oplus \mathrm{N}_{02} \oplus \mathrm{N}_{11} \oplus \mathrm{N}_{12} \oplus \mathrm{N}_{22}
$$

Seja $n_{1}$ uma base de $N$ então a ação dos elementos idempotentes em $n_{1}$ fica definida pela componente $N_{i j}$ à qual $n_{1}$ pertence. Só resta estabelecer como age $e_{3}$ em $n_{1}$.

Da tabela de multiplicação de $\mathcal{T}_{5}$ (veja a Tabela 2.2) deduzimos que $e_{3} \in \mathcal{P}_{12}$. Suponha que $n_{1} \in N_{00}$, então do Teorema $1.19 e_{3} n_{1} \in \mathcal{P}_{12} \mathcal{P}_{00}=0$ é zero. Agora se $n_{1} \in N_{12}$, então $e_{3} n_{1} \in \mathcal{P}_{12}^{2} \subseteq \mathcal{P}_{11} \oplus \mathcal{P}_{22}$ e também $e_{3} n_{1} \in N=N_{12} \subseteq \mathcal{P}_{12} \operatorname{logo} e_{3} n_{1}=0$. 
Analogamente prova-se que se $n_{1} \in N_{0 i}$ ou $n_{1} \in N_{i i}$ para $i=1,2$ então $e_{3} n_{1}=0$ mas nestes casos existem elementos de $\mathcal{J}$ que não satisfazem a identidade (1.3)

$$
\begin{array}{ll}
\left(n_{1} e_{3}, e_{3}, e_{2}\right)+\left(n_{1} e_{2}, e_{3}, e_{3}\right)+\left(e_{3} e_{2}, e_{3}, n_{1}\right) \neq 0 & \text { se } n_{1} \in N_{01} \text { ou } n_{1} \in N_{11}, \\
\left(n_{1} e_{3}, e_{3}, e_{1}\right)+\left(n_{1} e_{1}, e_{3}, e_{3}\right)+\left(e_{3} e_{1}, e_{3}, n_{1}\right) \neq 0 & \text { se } n_{1} \in N_{02} \text { ou } n_{1} \in N_{22} .
\end{array}
$$

Logo as álgebras resultantes dos casos $n_{1} \in N_{0 i}$ ou $n_{1} \in N_{i i}$ para $i=1,2$ não são álgebras de Jordan, portanto só temos duas álgebras de Jordan não isomorfas:

Tabela 2.5: k-álgebras de Jordan de dimensão 4 com radical unidimensional e parte

\begin{tabular}{|c|c|c|c|c|c|}
\hline $\mathcal{J}$ & Tabela de Multiplicação & $\begin{array}{c}\operatorname{dim} \\
\operatorname{Aut}(\mathcal{J})\end{array}$ & $\begin{array}{c}\operatorname{dim} \\
\operatorname{Ann}(\mathcal{J})\end{array}$ & $\operatorname{dim}^{2}$ & Observação \\
\hline$\partial_{8}$ & $\mathcal{T}_{5} \oplus \mathbf{k} \mathbf{n}_{1}$ & 2 & 1 & 3 & $\mathrm{n}_{1} \in \mathrm{N}_{00}$ \\
\hline$\partial_{9}$ & $\begin{array}{c}e_{1}^{2}=e_{1} \quad e_{2}^{2}=e_{2} \\
e_{3}^{2}=e_{1}+e_{2} \\
e_{1} e_{3}=\frac{1}{2} e_{3} \quad e_{1} n_{1}=\frac{1}{2} n_{1} \\
e_{2} e_{3}=\frac{1}{2} e_{3} \quad e_{2} n_{1}=\frac{1}{2} n_{1}\end{array}$ & 4 & 0 & 4 & $\begin{array}{l}\text { Unitária, } \\
\mathrm{n}_{1} \in \mathrm{N}_{12}\end{array}$ \\
\hline
\end{tabular}
semissimples $\mathcal{T}_{5}$

\subsection{3 Álgebras de Jordan com Radical de Dimensão 2}

Seja $\mathcal{J}$ uma k-álgebra de Jordan com radical $\mathrm{N}$ de dimensão 2. Do Teorema de Albert temos que a única álgebra de Jordan semissimples de dimensão 2 é $\mathcal{J}_{s s}=\mathbf{k} e_{1} \oplus \mathbf{k} e_{2}$, logo a álgebra unitária $\mathfrak{f}^{\#}=\mathfrak{J} \oplus \mathbf{k} \cdot 1$ contém 3 elementos idempotentes ortogonais $e_{1}$, $e_{2}, e_{0}=1-e_{1}-e_{2}$ o que implica na seguinte decomposição de $\mathrm{N}$ em subespaços de Peirce:

$$
\mathrm{N}=\mathrm{N}_{00} \oplus \mathrm{N}_{01} \oplus \mathrm{N}_{02} \oplus \mathrm{N}_{11} \oplus \mathrm{N}_{12} \oplus \mathrm{N}_{22}
$$

O ideal nilpotente maximal $N$ pode ter dois tipos de nilpotência: $(2)$ ou $(1,1)$.

1) Suponha que $\mathrm{N}$ tem tipo de nilpotência (2). Logo, pela definição de tipo de nilpotência, $N^{2}=0$. Seja $\left\{n_{1}, n_{2}\right\}$ uma base de $N$ então, novamente, $\mathcal{J}$ fica completamente definida pelos subespaços de Peirce aos quais $n_{1}$ e $n_{2}$ pertencem. Assim obtemos 36 possíveis álgebras das quais as seguintes 13 são não isomorfas: 
Tabela 2.6: k-álgebras de Jordan de dimensão 4 com radical de tipo (2)

\begin{tabular}{|c|c|c|c|c|c|}
\hline $\mathcal{J}$ & Tabela de Multiplicação & $\begin{array}{c}\operatorname{dim} \\
\operatorname{Aut}(\mathcal{J})\end{array}$ & $\begin{array}{c}\operatorname{dim} \\
\operatorname{Ann}(\mathcal{J})\end{array}$ & $g^{2}$ & Observação \\
\hline$\partial_{10}$ & $\mathcal{B}_{2} \oplus \mathbf{k} \mathbf{e}_{2} \oplus \mathbf{k} n_{2}$ & 3 & 1 & 3 & $\mathrm{n}_{1} \in \mathrm{N}_{01}, \mathrm{n}_{2} \in \mathrm{N}_{00}$ \\
\hline$\partial_{11}$ & $\mathcal{T}_{10} \oplus \mathbf{k} n_{2}$ & 3 & 1 & 3 & $n_{1} \in N_{12}, n_{2} \in N_{00}$ \\
\hline$J_{12}$ & $\mathcal{T}_{7} \oplus \mathbf{k} e_{2}$ & 6 & 0 & 4 & $n_{1}, n_{2} \in N_{01}$ \\
\hline$\partial_{13}$ & $\mathcal{B}_{2} \oplus \mathcal{B}_{2}$ & 4 & 0 & 4 & $\mathrm{n}_{1} \in \mathrm{N}_{02}, \mathrm{n}_{2} \in \mathrm{N}_{01}$ \\
\hline$\partial_{14}$ & $\mathcal{T}_{6} \oplus \mathbf{k} e_{2}$ & 3 & 0 & 4 & $n_{1} \in N_{01}, n_{2} \in N_{11}$ \\
\hline$\partial_{15}$ & $\mathcal{B}_{2} \oplus \mathcal{B}_{1}$ & 3 & 0 & 4 & $n_{1} \in N_{11}, n_{2} \in N_{02}$ \\
\hline$\partial_{16}$ & $\begin{array}{c}e_{1}^{2}=e_{1} \quad e_{2}^{2}=e_{2} \\
e_{1} n_{1}=\frac{1}{2} n_{1} \\
e_{1} n_{2}=\frac{1}{2} n_{2} \quad e_{2} n_{1}=\frac{1}{2} n_{1}\end{array}$ & 4 & 0 & 4 & $\begin{array}{l}\mathrm{n}_{1} \in \mathrm{N}_{12} \\
\mathrm{n}_{2} \in \mathrm{N}_{01}\end{array}$ \\
\hline$\partial_{17}$ & $\begin{array}{c}e_{1}^{2}=e_{1} \quad e_{2}^{2}=e_{2} \\
e_{1} n_{1}=\frac{1}{2} n_{1} \\
e_{1} n_{2}=n_{2} \quad e_{2} n_{1}=\frac{1}{2} n_{1}\end{array}$ & 3 & 0 & 4 & $\begin{array}{c}\text { Unitária, } \\
\mathrm{n}_{1} \in \mathrm{N}_{12}, \mathrm{n}_{2} \in \mathrm{N}_{11}\end{array}$ \\
\hline$\partial_{18}$ & $\begin{aligned} & e_{1}^{2}=e_{1} e_{2}^{2}=e_{2} \\
& e_{1} n_{1}=\frac{1}{2} n_{1} e_{1} n_{2}=\frac{1}{2} n_{2} \\
& e_{2} n_{1}=\frac{1}{2} n_{1} e_{2} n_{2}=\frac{1}{2} n_{2} \\
&\end{aligned}$ & 6 & 0 & 4 & $\begin{array}{c}\text { Unitária, } \\
n_{1}, n_{2} \in N_{12}\end{array}$ \\
\hline$J_{19}$ & $\mathbf{k} e_{1} \oplus \mathbf{k} \mathbf{n}_{1} \oplus \mathbf{k} e_{2} \oplus \mathbf{k} \mathbf{n}_{2}$ & 4 & 2 & 2 & $\begin{array}{l}\text { Associativa } \\
\mathrm{n}_{1}, \mathrm{n}_{2} \in \mathrm{N}_{00}\end{array}$ \\
\hline$J_{20}$ & $\mathcal{B}_{1} \oplus \mathbf{k} e_{2} \oplus \mathbf{k} n_{2}$ & 2 & 1 & 3 & $\begin{array}{c}\text { Associativa } \\
\mathrm{n}_{1} \in \mathrm{N}_{11}, \mathrm{n}_{2} \in \mathrm{N}_{00}\end{array}$ \\
\hline$J_{21}$ & $\mathcal{T}_{2} \oplus \mathbf{k} e_{2}$ & 4 & 0 & 4 & $\begin{array}{c}\text { Unitária, } \\
\text { Associativa } \\
\mathrm{n}_{1}, \mathrm{n}_{2} \in \mathrm{N}_{11}\end{array}$ \\
\hline$\partial_{22}$ & $\mathcal{B}_{1} \oplus \mathcal{B}_{1}$ & 2 & 0 & 4 & $\begin{array}{c}\text { Unitária, } \\
\text { Associativa } \\
\mathrm{n}_{1} \in \mathrm{N}_{11}, \mathrm{n}_{2} \in \mathrm{N}_{22} \\
\end{array}$ \\
\hline
\end{tabular}

Qualquer outra das 26 possibilidades resulta em uma álgebra isomorfa a uma das álgebras $\mathcal{J}_{10}$ a $\mathcal{J}_{22}$, só basta aplicar o isomorfismo que troca $n_{1} \operatorname{com} n_{2}$ ou $e_{1} \operatorname{com} e_{2}$ ou que realiza ambas trocas.

2) Suponha que $N$ tem tipo de nilpotência $(1,1)$. Vamos provar que neste caso existe $n \in N$ tal que $N=\mathbf{k n}+\mathbf{k} n^{2} \operatorname{com} n^{3}=0$. Como $\operatorname{dim} N^{2}=1$ existe $m \in N$ tal que 
$\mathrm{N}^{2}=\mathbf{k m}$, completando a uma base de $\mathrm{N}$ então $\mathrm{N}=\mathbf{k} m+\mathbf{k n}$. Agora como $\mathrm{m} \in \mathrm{N}^{2}$ existem $n_{1}, n_{2} \in N$ tal que $m=n_{1} n_{2}, \operatorname{logo}$

$$
\begin{aligned}
0 \neq m & =n_{1} n_{2}=(\alpha m+\beta n)\left(\alpha^{\prime} m+\beta^{\prime} n\right) \\
& =\alpha \alpha^{\prime} m^{2}+\left(\alpha \beta^{\prime}+\alpha^{\prime} \beta\right) m n+\beta \beta^{\prime} n^{2}
\end{aligned}
$$

como $m^{2}, m n \in N^{3}=0$ temos que $m=\beta \beta^{\prime} n^{2} \log o N=\mathbf{k} n^{2}+\mathbf{k n}$.

Suponha primeiramente que $\mathrm{N}$ é dado por uma única componente de Peirce de dimensão 2, a saber $N=N_{i j}$ então do Teorema 1.19 $N_{i j}^{2} \subseteq N_{i i} \oplus N_{j j}$. Se $i \neq j$ então $N^{2}=0$ o que é uma contradição, isto implica que $i=j$ e $\log o N=N_{i i}, i=0,1,2$.

Suponha, agora, que $N=N_{i j} \oplus N_{k l} \operatorname{com}(i, j) \neq(k, l)$ e $\operatorname{dim} N_{i j}=\operatorname{dim} N_{k l}=1$. Analisaremos os seguintes casos no quais consideraremos os índices $i, j, k$ todos diferentes. Se $N=N_{i i} \oplus N_{i j}$, podemos escolher o $n$ da base como sendo um elemento de $N_{i j}$. De fato se $N_{i i}=\mathbf{k a}$ e $N_{i j}=\mathbf{k b}$, então temos $b^{2} \in N_{i i} \oplus N_{j j}$, mas $N_{j j}=0$, logo $b^{2}=\alpha a$ para algum $\alpha \in \mathbf{k}$. Note que $\alpha \neq 0$ pois pela nilpotência já temos que $a^{2}=a b=0$ e $N^{2}$ tem dimensão 1. Consequentemente $\mathrm{N}=\mathbf{k b}^{2}+\mathbf{k b}$.

Para qualquer outro par de índices $i, j$ e $k, l$ obtemos uma contradição ao fato de que o tipo de nilpotência de $\mathrm{N}$ é $(1,1)$.

i. Se $N=N_{i i} \oplus N_{j j}$, então pela nilpotência $N_{i i}^{2}=N_{j j}^{2}=0$ e da tabela de multiplicação das componentes de Peirce $N_{i i} N_{j j}=0$. Logo $N^{2}=0$ o que leva a uma contradição.

ii. Analogamente se $N=N_{i i} \oplus N_{j k}$, novamente pela nilpotência $N_{i i}^{2}=0$ e da tabela de multiplicação do Teorema $1.19 \mathrm{~N}_{j k}^{2} \subseteq \mathrm{N}_{j j} \oplus \mathrm{N}_{\mathrm{kk}}=0$ e $\mathrm{N}_{i i} \mathrm{~N}_{j k}=0$, o que é uma contradição.

iii. Finalmente se $N=N_{i j} \oplus N_{i k}$ então $N^{2} \subseteq N_{i i} \oplus N_{j j} \oplus N_{j k} \oplus N_{k k}=0$.

Assim temos que só existem 9 possíveis k-álgebras de Jordan de dimensão 4 com radical com tipo de nilpotência $(1,1)$ das quais as seguintes 5 álgebras são não isomorfas. Em todas elas $n_{1}^{2}=n_{2}$.

Tabela 2.7: k-álgebras de Jordan de dimensão 4 com radical de tipo $(1,1)$

\begin{tabular}{|c|c|c|c|c|c|}
\hline $\mathfrak{J}$ & Tabela de Multiplicação & $\begin{array}{c}\operatorname{dim} \\
\operatorname{Aut}(\mathcal{J})\end{array}$ & $\begin{array}{c}\operatorname{dim} \\
\operatorname{Ann}(\mathcal{J})\end{array}$ & $\begin{array}{c}\operatorname{dim} \\
\mathfrak{J}^{2}\end{array}$ & Observação \\
\hline $\mathfrak{J}_{23}$ & $\mathcal{T}_{8} \oplus \mathbf{k e}_{2}$ & 2 & 1 & 4 & $\begin{array}{c}\mathrm{n}_{1} \in \mathrm{N}_{01}, \\
\mathrm{n}_{2} \in \mathrm{N}_{00}\end{array}$ \\
\hline
\end{tabular}




\begin{tabular}{|c|c|c|c|c|c|}
\hline $\mathcal{J}$ & Tabela de Multiplicação & $\begin{array}{c}\operatorname{dim} \\
\operatorname{Aut}(\mathcal{J})\end{array}$ & $\begin{array}{c}\operatorname{dim} \\
\operatorname{Ann}(\mathcal{J})\end{array}$ & $\begin{array}{c}\operatorname{dim} \\
\mathcal{J}^{2}\end{array}$ & Observação \\
\hline$\partial_{24}$ & $\mathcal{T}_{9} \oplus \mathbf{k} e_{2}$ & 2 & 0 & 4 & $\begin{array}{c}\mathrm{n}_{1} \in \mathrm{N}_{01}, \\
\mathrm{n}_{2} \in \mathrm{N}_{11}\end{array}$ \\
\hline$\partial_{25}$ & $\begin{array}{c}e_{1}^{2}=e_{1} \quad e_{2}^{2}=e_{2} \\
e_{1} n_{2}=n_{2} \quad e_{1} n_{1}=\frac{1}{2} n_{1} \\
e_{2} n_{1}=\frac{1}{2} n_{1} \quad n_{1}^{2}=n_{2}\end{array}$ & 2 & 0 & 4 & $\begin{array}{c}\text { Unitária, } \\
n_{1} \in N_{12}, \\
n_{2} \in N_{11}\end{array}$ \\
\hline$\partial_{26}$ & $\mathcal{B}_{3} \oplus \mathbf{k} e_{1} \oplus \mathbf{k} e_{2}$ & 2 & 1 & 3 & $\begin{array}{c}\text { Associativa, } \\
n_{1}, n_{2} \in N_{00}\end{array}$ \\
\hline$J_{27}$ & $\mathcal{T}_{1} \oplus \mathbf{k} e_{2}$ & 2 & 0 & 4 & $\begin{array}{c}\text { Unitária, } \\
\text { Associativa, } \\
n_{1}, \mathrm{n}_{2} \in \mathrm{N}_{11}\end{array}$ \\
\hline
\end{tabular}

As outras 4 possibilidades: $n_{1} \in N_{02}$ e $n_{2} \in N_{00}, n_{1} \in N_{02}$ e $n_{2} \in N_{22}, n_{1}$ e $n_{2} \in N_{22}, n_{1} \in N_{12}$ e $n_{2} \in N_{22}$, resultam em uma álgebra isomorfa à álgebra $\mathcal{J}_{23}, J_{24}$, $\mathcal{J}_{27}$ e $\mathcal{J}_{25}$ respetivamente, com isomorfismo que permuta $e_{1}$ com $e_{2}$.

\subsection{4 Álgebras de Jordan com Radical de Dimensão 3}

No caso em que $\mathcal{J}$ seja uma álgebra de Jordan de dimensão 4 sobre $\mathbf{k}$, com radical nilpotente $\mathrm{N}$ de dimensão 3, então $J_{\text {ss }}$ é uma álgebra simples unidimensional, logo $\mathcal{J}_{s s}=\mathbf{k} e_{1}$ e $\mathcal{J}$ só tem um elemento idempotente $e_{1} \in \mathcal{J}$ que implica na decomposição de Peirce de N:

$$
\mathrm{N}=\mathrm{N}_{0} \oplus \mathrm{N}_{\frac{1}{2}} \oplus \mathrm{N}_{1}
$$

Primeiramente, provaremos o seguinte lema que será frequentemente usado no que resta da classificação.

Lema 2.1. Se $\operatorname{dim} \mathrm{N}_{i}=1$ então $\mathrm{N}_{i}^{2}=0$, para $i=0,1$. Se $\operatorname{dim} \mathrm{N}_{\frac{1}{2}}=1$ então $\mathrm{N}_{\frac{1}{2}} \mathrm{~N}_{i}=0$ para $i=0,1$.

Demonstração. Como para $i=1,2$ temos $N_{i}^{2} \subseteq N_{i}$, pela nilpotência esta inclusão é estrita $N_{i}^{2} \subsetneq N_{i}$. Pela hipóteses temos que $\operatorname{dim} N_{i}=1 \operatorname{logo} N_{i}^{2}=0$. Para a segunda parte note que $\mathrm{N}_{\frac{1}{2}} \mathrm{~N}_{i} \subseteq \mathrm{N}_{\frac{1}{2}}$. Suponha que acontece a igualdade, então

$$
0=N^{\langle 4\rangle} \supseteq\left(\left(N_{\frac{1}{2}} N_{i}\right) N_{i}\right) N_{i}=N_{\frac{1}{2}} \neq 0,
$$

logo devemos ter $N_{\frac{1}{2}} N_{i} \subsetneq N_{\frac{1}{2}}$. Finalmente, pela hipóteses temos que $\operatorname{dim} N_{\frac{1}{2}}=1$ segue que $\mathrm{N}_{\frac{1}{2}} \mathrm{~N}_{i}=0$. 
O radical $N$ pode ter os seguintes tipos de nilpotência: $(3),(1,1,1)$ ou $(2,1)$.

1) Suponha que $\mathrm{N}$ tem tipo de nilpotência (3). Logo, pela definição de tipo de nilpotência, $N^{2}=0$. Seja $\left\{n_{1}, n_{2}, n_{3}\right\}$ uma base de $N$ então, mais uma vez $\mathcal{J}$ fica completamente definida pelos subespaços de Peirce aos quais $n_{1}, n_{2}$ e $n_{3}$ pertencem. Assim obtemos 27 possíveis álgebras das quais as seguintes 10 são não isomorfas:

Tabela 2.8: k-álgebras de Jordan de dimensão 4 com radical de tipo (3)

\begin{tabular}{|c|c|c|c|c|c|}
\hline $\mathcal{J}$ & Tabela de Multiplicação & $\begin{array}{c}\operatorname{dim} \\
\operatorname{Aut}(\mathcal{J})\end{array}$ & $\begin{array}{c}\operatorname{dim} \\
\operatorname{Ann}(\mathcal{J})\end{array}$ & $\partial^{2}$ & Observação \\
\hline$\partial_{28}$ & $\mathcal{B}_{2} \oplus \mathbf{k} n_{2} \oplus \mathbf{k} n_{3}$ & 6 & 2 & 2 & $n_{1} \in N_{\frac{1}{2}}, n_{2}, n_{3} \in N_{0}$ \\
\hline$J_{29}$ & $\mathcal{T}_{6} \oplus \mathbf{k} n_{3}$ & 4 & 1 & 3 & $\begin{array}{c}\mathrm{n}_{1} \in \mathrm{N}_{\frac{1}{2}}, \\
\mathrm{n}_{2} \in \mathrm{N}_{1}, \mathrm{n}_{3} \in \mathrm{N}_{0}\end{array}$ \\
\hline$\partial_{30}$ & $\mathcal{T}_{7} \oplus \mathbf{k} n_{3}$ & 7 & 1 & 3 & $n_{1}, n_{2} \in N_{\frac{1}{2}}, n_{3} \in N_{0}$ \\
\hline$J_{31}$ & $\begin{array}{cl}e_{1}^{2}=e_{1} & e_{1} n_{1}=n_{1} \\
e_{1} n_{2}=n_{2} & e_{1} n_{3}=\frac{1}{2} n_{3}\end{array}$ & 6 & 0 & 4 & $\mathrm{n}_{1}, \mathrm{n}_{2} \in \mathrm{N}_{1}, \mathrm{n}_{3} \in \mathrm{N}_{\frac{1}{2}}$ \\
\hline$\partial_{32}$ & $\begin{array}{c}e_{1}^{2}=e_{1} \quad e_{1} n_{1}=\frac{1}{2} n_{1} \\
e_{1} n_{2}=\frac{1}{2} n_{2} \quad e_{1} n_{3}=n_{3}\end{array}$ & 7 & 0 & 4 & $\mathrm{n}_{1}, \mathrm{n}_{2} \in \mathrm{N}_{\frac{1}{2}}, \mathrm{n}_{3} \in \mathrm{N}_{1}$ \\
\hline$\partial_{33}$ & $\begin{aligned} e_{1}^{2} & =e_{1} \quad e_{1} n_{1}=\frac{1}{2} n_{1} \\
e_{1} n_{2} & =\frac{1}{2} n_{2} \quad e_{1} n_{3}=\frac{1}{2} n_{3}\end{aligned}$ & 12 & 0 & 4 & $n_{1}, n_{2}, n_{3} \in N_{\frac{1}{2}}$ \\
\hline$J_{34}$ & $\mathbf{k} e_{1} \oplus \mathbf{k} n_{1} \oplus \mathbf{k} n_{2} \oplus \mathbf{k} n_{3}$ & 9 & 3 & 1 & $\begin{array}{c}\text { Associativa, } \\
\mathrm{n}_{1}, \mathrm{n}_{2}, \mathrm{n}_{3} \in \mathrm{N}_{0}\end{array}$ \\
\hline$\partial_{35}$ & $\mathcal{B}_{1} \oplus \mathbf{k} n_{2} \oplus \mathbf{k} n_{3}$ & 5 & 2 & 2 & $\begin{array}{c}\text { Associativa, } \\
\mathrm{n}_{1} \in \mathrm{N}_{1}, \mathrm{n}_{2}, \mathrm{n}_{3} \in \mathrm{N}_{0}\end{array}$ \\
\hline$\partial_{36}$ & $\begin{array}{c}e_{1}^{2}=e_{1} \quad e_{1} n_{1}=n_{1} \\
e_{1} n_{2}=n_{2} \quad e_{1} n_{3}=n_{3}\end{array}$ & 9 & 0 & 4 & $\begin{array}{c}\text { Unitária, Associativa, } \\
n_{1}, n_{2}, n_{3} \in N_{1}\end{array}$ \\
\hline$\partial_{37}$ & $\mathcal{T}_{2} \oplus \mathbf{k} n_{3}$ & 5 & 1 & 3 & $\begin{array}{c}\text { Associativa, } \\
\mathrm{n}_{1}, \mathrm{n}_{2} \in \mathrm{N}_{1}, \mathrm{n}_{3} \in \mathrm{N}_{0}\end{array}$ \\
\hline
\end{tabular}

Qualquer outra das 17 possibilidades resulta em uma álgebra isomorfa a uma das álgebras $\mathcal{J}_{28}$ a $\mathcal{J}_{37}$, só basta aplicar o isomorfismo que troca $n_{1}, n_{2}$ e $n_{3}$.

2) Suponha que $N$ tem tipo de nilpotência $(1,1,1)$. Lembramos, pela definição de tipo de nilpotência, que isto significa que $\operatorname{dim} \mathrm{N}^{2}=2, \operatorname{dim} \mathrm{N}^{3}=1$ e $\mathrm{N}^{\langle 4\rangle}=0$.

Só existe uma (a menos de isomorfismo) álgebra de Jordan de dimensão 3 nilpotente com tipo de nilpotência $(1,1,1) \operatorname{logo} N=\mathcal{T}_{3}$ e somente temos duas álgebras de Jordan de dimensão 4 não isomorfas com esse radical: 
Tabela 2.9: k-álgebras de Jordan de dimensão 4 com radical de tipo $(1,1,1)$

\begin{tabular}{|c|c|c|c|c|c|}
\hline $\mathcal{J}$ & Tabela de Multiplicação & $\begin{array}{c}\operatorname{dim} \\
\operatorname{Aut}(\mathcal{J})\end{array}$ & $\begin{array}{c}\operatorname{dim} \\
\operatorname{Ann}(\mathcal{J})\end{array}$ & $\partial^{2}$ & Observação \\
\hline $\mathrm{J}_{38}$ & $\mathcal{T}_{3} \oplus \mathbf{k} e_{1}$ & 3 & 1 & 3 & $\begin{array}{c}\text { Associativa, } \\
\mathrm{N}=\mathrm{N}_{0}\end{array}$ \\
\hline$J_{39}$ & $\begin{aligned} e_{1}^{2}=e_{1} & n_{1}^{2}=n_{2} \\
e_{1} n_{1}=n_{1} & e_{1} n_{2}=n_{2} \\
e_{1} n_{3}=n_{3} & n_{1} n_{2}=n_{3}\end{aligned}$ & 3 & 0 & 4 & $\begin{array}{c}\text { Unitária, } \\
\text { Associativa, } \\
\mathrm{N}=\mathrm{N}_{1}\end{array}$ \\
\hline
\end{tabular}

Todos os outros casos levam a uma contradição, a saber:

i. Suponha que $N=N_{\frac{1}{2}}$ então, da tabela de multiplicação das componentes de Peirce do Teorema 1.18 temos $\mathrm{N}^{2} \subseteq \mathrm{N}_{0} \oplus \mathrm{N}_{1}=0$ o que contradiz o fato de $\mathrm{N}^{2}$ ter dimensão 2.

ii. Suponha agora que $N=N_{0} \oplus N_{1} \operatorname{com} \operatorname{dim} N_{0}=2$. Pelo Lema 2.1 $N_{1}^{2}=0$ e pela tabela de multiplicação do Teorema 1.18 temos que $\mathrm{N}_{0} \mathrm{~N}_{1}=0 \operatorname{logo} \mathrm{N}^{2} \subseteq \mathrm{N}_{0}$. Como $\operatorname{dim} \mathrm{N}^{2}=2=\operatorname{dim} \mathrm{N}_{0}$ eles coincidem, $\mathrm{N}^{2}=\mathrm{N}_{0}$. Como consequência, existe um elemento $n_{0} \in N_{0}$ que gera $N^{3}$, i.e., $N^{3}=\mathbf{k} n_{0}$, escolhendo algum $m_{0} \in N_{0}$ e $n_{1} \in N_{1}$ obtemos que $N^{2}=\mathbf{k n}_{0}+\mathbf{k m}_{0}$. Logo obtemos os seguintes produtos em $\mathrm{N}$ :

$$
\begin{aligned}
& \mathrm{N}^{\langle 4\rangle} \ni \mathrm{n}_{0}^{2}=\mathrm{n}_{0} \mathrm{~m}_{0}=0, \\
& \mathrm{n}_{1}^{2}=\mathrm{n}_{0} \mathrm{n}_{1}=\mathrm{m}_{0} \mathrm{n}_{1}=0,
\end{aligned}
$$

restando somente $\mathrm{m}_{0}^{2}$ não nulo, o que contradiz o fato de $\operatorname{dim} \mathrm{N}^{2}=2$. Analogamente chegamos a uma contradição se consideramos $N=N_{0} \oplus N_{1}$, com $\operatorname{dim} \mathrm{N}_{1}=2$.

iii. Suponha que $N=N_{0} \oplus N_{\frac{1}{2}}$ onde $\operatorname{dim} N_{0}=2$. Pelo Lema $2.1 N^{2} \subseteq N_{0}$ e como $\operatorname{dim} N^{2}=2=\operatorname{dim} N_{0}$, temos $N^{2}=N_{0}$. Consequentemente, existe $n_{0} \in N_{0}$ tal que $\mathrm{N}^{3}=\mathbf{k} n_{0}$, completando a uma base de $N_{0}$ temos que existe $m_{0} \in N_{0}$ tal que $\mathrm{N}^{2}=\mathbf{k} n_{0}+\mathbf{k} m_{0}$. Completando mais uma vez a uma base de $\mathrm{N}$ temos que existe $n_{\frac{1}{2}} \in N_{\frac{1}{2}}$ tal que temos os seguintes produtos em $N$ :

$$
\begin{aligned}
& N^{\langle 4\rangle} \ni n_{0}^{2}=n_{0} m_{0}=0, \quad N^{3} \ni m_{0}^{2}=\alpha n_{0} \\
& n_{0} n_{\frac{1}{2}}=m_{0} n_{\frac{1}{2}}=0, \quad N^{2} \ni n_{\frac{1}{2}}^{2}=\alpha^{\prime} n_{0}+\beta^{\prime} m_{0} .
\end{aligned}
$$


Obtemos uma álgebra de Jordan somente se $\alpha=0$ ou $\beta^{\prime}=0$, mas em ambos os casos isto contradiz o fato de $\operatorname{dim} \mathrm{N}^{2}=2$. Analogamente a prova leva a uma contradição se considerarmos $\mathrm{N}=\mathrm{N}_{1} \oplus \mathrm{N}_{\frac{1}{2}}, \operatorname{com} \operatorname{dim} \mathrm{N}_{1}=2$.

iv. Suponha que $N=N_{0} \oplus N_{\frac{1}{2}}$, onde $\operatorname{dim} N_{\frac{1}{2}}=2$. Primeiramente mostraremos que, neste caso,

$$
\left(N_{\frac{1}{2}} N_{0}\right) N_{0}=\left(N_{0} N_{\frac{1}{2}}\right) N_{\frac{1}{2}}=0
$$

Da tabela de multiplicação das componentes de Peirce do Teorema 1.18 temos que $\left(\mathrm{N}_{\frac{1}{2}} \mathrm{~N}_{0}\right) \mathrm{N}_{0} \subseteq \mathrm{N}_{\frac{1}{2}} \mathrm{~N}_{0} \subseteq \mathrm{N}_{\frac{1}{2}}$. Suponha que aconteça a igualdade $\mathrm{N}_{\frac{1}{2}} \mathrm{~N}_{0}=\mathrm{N}_{\frac{1}{2}}$ então

$$
0=N^{\langle 4\rangle} \supseteq\left(\left(N_{\frac{1}{2}} N_{0}\right) N_{0}\right) N_{0}=N_{\frac{1}{2}} \neq 0,
$$

logo a inclusão é estrita $N_{\frac{1}{2}} N_{0} \subsetneq N_{\frac{1}{2}}$. Considere agora a igualdade $\left(N_{\frac{1}{2}} N_{0}\right) N_{0}=$ $\mathrm{N}_{\frac{1}{2}} \mathrm{~N}_{0}$, neste caso

$$
0=N^{\langle 4\rangle} \supseteq\left(\left(N_{\frac{1}{2}} N_{0}\right) N_{0}\right) N_{0}=N_{\frac{1}{2}} N_{0}
$$

o que implica que $\mathrm{N}^{2}=\mathrm{N}_{0}^{2}+\mathrm{N}_{\frac{1}{2}}^{2} \subseteq \mathrm{N}_{0}$ mas $\operatorname{dim} \mathrm{N}^{2}=2$ enquanto que $\operatorname{dim} \mathrm{N}_{0}=1$. Portanto, ambas inclusões são estritas: $\left(\mathrm{N}_{\frac{1}{2}} \mathrm{~N}_{0}\right) \mathrm{N}_{0} \subsetneq \mathrm{N}_{\frac{1}{2}} \mathrm{~N}_{0} \subsetneq \mathrm{N}_{\frac{1}{2}}$ e, consequentemente,

$$
\operatorname{dim}\left[\left(\mathrm{N}_{\frac{1}{2}} \mathrm{~N}_{0}\right) \mathrm{N}_{0}\right]<\operatorname{dim}\left(\mathrm{N}_{\frac{1}{2}} \mathrm{~N}_{0}\right)<\operatorname{dim} \mathrm{N}_{\frac{1}{2}}=2
$$

$\operatorname{logo} \operatorname{dim}\left(\mathrm{N}_{\frac{1}{2}} \mathrm{~N}_{0}\right)=1$ e $\left(\mathrm{N}_{\frac{1}{2}} \mathrm{~N}_{0}\right) \mathrm{N}_{0}=0$ como queríamos provar.

Para provar a segunda igualdade de (2.1), procedemos de maneira análoga. Do Teorema $1.18\left(\mathrm{~N}_{0} \mathrm{~N}_{\frac{1}{2}}\right) \mathrm{N}_{\frac{1}{2}} \subseteq \mathrm{N}_{\frac{1}{2}}^{2} \subseteq \mathrm{N}_{0}$. Suponha que $\left(\mathrm{N}_{0} \mathrm{~N}_{\frac{1}{2}}\right) \mathrm{N}_{\frac{1}{2}}=\mathrm{N}_{0}$ então

$$
0=\mathrm{N}^{\langle 4\rangle} \supseteq\left(\left(\mathrm{N}_{0} \mathrm{~N}_{\frac{1}{2}}\right) \mathrm{N}_{\frac{1}{2}}\right) \mathrm{N}_{\frac{1}{2}}=\mathrm{N}_{0} \mathrm{~N}_{\frac{1}{2}} \neq 0,
$$

o que contradiz o fato de $\operatorname{dim}\left(\mathrm{N}_{0} \mathrm{~N}_{\frac{1}{2}}\right)=1$. Então, novamente a inclusão é estrita $\left(\mathrm{N}_{0} \mathrm{~N}_{\frac{1}{2}}\right) \mathrm{N}_{\frac{1}{2}} \subsetneq \mathrm{N}_{\mathrm{O}}$ e como por hipóteses $\operatorname{dim} \mathrm{N}_{\mathrm{O}}=1$, temos que $\left(\mathrm{N}_{0} \mathrm{~N}_{\frac{1}{2}}\right) \mathrm{N}_{\frac{1}{2}}=0$.

Agora, lembrando que $N=\mathcal{T}_{3}$, para o elemento $n_{1}$ da base dada na Tabela 2.2 temos $n_{1}=m_{0}+m_{\frac{1}{2}}$, onde $m_{i} \in N_{i}$ e $i=0, \frac{1}{2}$. Logo, usando que $N_{\frac{1}{2}}^{2} \subseteq N_{0}$, o Lema 2.1 e as identidades (2.1) obtemos que $n_{1}^{2}=m_{0}^{\prime} m_{\frac{1}{2}}+m_{\frac{1}{2}}^{2}$ e $n_{1}^{\frac{1}{2}}=m_{\frac{1}{2}}^{3}$. Portanto,

$$
\left(m_{\frac{1}{2}}^{2}, e_{1}, m_{\frac{1}{2}}\right)=\left(m_{\frac{1}{2}}^{2} e_{1}\right) m_{\frac{1}{2}}-m_{\frac{1}{2}}^{2}\left(e_{1} m_{\frac{1}{2}}\right)=-\frac{1}{2} n_{1}^{3} \neq 0
$$


e logo a álgebra $\mathcal{J}$ não é de Jordan.

Uma prova análoga mostra que não existe álgebra de Jordan de dimensão $4 \mathrm{com}$ radical de tipo $(1,1,1)$ e $\mathrm{N}=\mathrm{N}_{1} \oplus \mathrm{N}_{\frac{1}{2}}$, onde $\operatorname{dim} \mathrm{N}_{\frac{1}{2}}=2$.

v. Suponha que $N=N_{0} \oplus N_{\frac{1}{2}} \oplus N_{1}$ onde $\operatorname{dim} N_{i}=1$ para $i=0, \frac{1}{2}$, 1. Do Lema 2.1 e do Teorema 1.18 temos $\mathrm{N}^{2} \subseteq \mathrm{N}_{0} \oplus \mathrm{N}_{1}$ o que implica que $\mathrm{N}^{3} \subseteq\left(\mathrm{N}_{0} \oplus \mathrm{N}_{1}\right) \mathrm{N}=0$ o que é uma contradição ao fato de $\mathrm{N}^{3}$ ter dimensão 1 .

3) Suponha que $N$ tem tipo de nilpotência $(2,1)$. Lembramos que isto significa que $\operatorname{dim} \mathrm{N}^{2}=1$ e $\mathrm{N}^{3}=0$. Existem duas (a menos de isomorfismo) álgebras de Jordan de dimensão 3 nilpotente com tipo de nilpotência $(2,1) \log$ o ou $N=\mathcal{B}_{3} \oplus \mathbf{k} n_{3}$ ou $N=\mathcal{T}_{4}$.

Para provar que as únicas álgebras de Jordan neste caso são $\mathcal{J}_{40}$ a $\mathcal{J}_{60}$, consideraremos $(\mathcal{J}, \cdot)$ uma k-álgebra de Jordan com base $\left\{\mathrm{E}_{1}, \mathrm{~N}_{1}, \mathrm{~N}_{2}, \mathrm{~N}_{3}\right\}$ cujo radical tem tipo de nilpotência $(2,1)$ e cujo produto satisfaz as condições obtidas em cada caso. Concluiremos que $\mathcal{J}$ é isomorfa a uma das álgebras $\mathcal{J}_{40}$ a $\mathcal{J}_{60}$ exibindo um isomorfismo $(\mathcal{J}, \cdot) \rightarrow \mathcal{J}_{i}$ para algum $40 \leqslant i \leqslant 60$ que leva a base $\left\{E_{1}, N_{1}, N_{2}, N_{3}\right\}$ de $\mathcal{J}$ na base $\left\{e_{1}, n_{1}, n_{2}, n_{3}\right\}$ de $\partial_{i}$. Para simplificar a notação obviaremos os casos em que o isomorfismo leva $E_{1} \mapsto e_{1}$ e $N_{i} \mapsto n_{i}$, para $i=1,2,3$.

Analisaremos os seguintes casos dependendo da dimensão dos subespaços $N_{i}$.

i. Suponha que $N=N_{0}$. Isto implica que $e_{1} x=0$ para todo $x \in N$, $\log \mathcal{J}=\mathbf{k}_{1} \oplus N$ como soma direta de álgebras. Portanto obtemos duas álgebras não isomorfas:

Tabela 2.10: $\mathrm{k}$-álgebras de Jordan de dimensão 4 com radical de tipo $(2,1)$ e $\mathrm{N}=$

\begin{tabular}{|c|c|c|c|c|c|}
\hline $\mathcal{J}$ & Tabela de Multiplicação & $\begin{array}{c}\operatorname{dim} \\
\operatorname{Aut}(\mathcal{J})\end{array}$ & $\begin{array}{c}\operatorname{dim} \\
\operatorname{Ann}(\mathcal{J})\end{array}$ & $\begin{array}{c}\operatorname{dim} \\
\mathcal{J}^{2}\end{array}$ & Observação \\
\hline $\mathcal{J}_{40}$ & $\mathcal{B}_{3} \oplus \mathbf{k} e_{1} \oplus \mathbf{k n}_{3}$ & 5 & 2 & 2 & $\begin{array}{c}\text { Associativa, } \\
\mathrm{N}=\mathcal{B}_{3} \oplus \mathbf{k} n_{3}\end{array}$ \\
\hline $\mathcal{J}_{41}$ & $\mathcal{T}_{4} \oplus \mathbf{k} e_{1}$ & 4 & 1 & 2 & $\begin{array}{c}\text { Associativa, } \\
\mathrm{N}=\mathcal{T}_{4}\end{array}$ \\
\hline
\end{tabular}

ii. Suponha que $N=N_{1}$. Isto implica que $e_{1} x=x$ para todo $x \in N$, logo obtemos duas álgebras unitárias e associativas não isomorfas: 
Tabela 2.11: k-álgebras de Jordan de dimensão 4 com radical de tipo $(2,1)$ e $\mathrm{N}=$

\begin{tabular}{|c|c|c|c|c|c|}
\hline \multicolumn{6}{|c|}{$\mathrm{N}_{1}$} \\
\hline $\mathcal{J}$ & Tabela de Multiplicação & $\begin{array}{c}\operatorname{dim} \\
\operatorname{Aut}(\mathcal{J})\end{array}$ & $\begin{array}{c}\operatorname{dim} \\
\operatorname{Ann}(\mathcal{J})\end{array}$ & $\operatorname{dim}^{2}$ & Observação \\
\hline$J_{42}$ & $\begin{array}{c}\mathrm{e}_{1}^{2}=\mathrm{e}_{1} \quad \mathrm{n}_{1}^{2}=\mathrm{n}_{2} \\
\mathrm{e}_{1} \mathrm{n}_{1}=\mathrm{n}_{1} \quad \mathrm{e}_{1} \mathrm{n}_{2}=\mathrm{n}_{2} \\
\mathrm{e}_{1} \mathrm{n}_{3}=\mathrm{n}_{3}\end{array}$ & 5 & 0 & 4 & $\begin{array}{c}\text { Unitária, } \\
\text { Associativa, } \\
\mathrm{N}=\mathcal{B}_{3} \oplus \mathbf{k} n_{3}\end{array}$ \\
\hline $\mathcal{J}_{43}$ & $\begin{array}{rlrl}e_{1}^{2} & =e_{1} & n_{1}^{2}=n_{2} \\
e_{1} n_{1}=n_{1} & e_{1} n_{2}=n_{2} \\
e_{1} n_{3}=n_{3} & & n_{1} n_{3}=n_{2}\end{array}$ & 4 & 0 & 4 & $\begin{array}{c}\text { Unitária, } \\
\text { Associativa, } \\
\mathrm{N}=\mathcal{T}_{4}\end{array}$ \\
\hline
\end{tabular}

iii. Suponha que $N=N_{\frac{1}{2}}$. Então $N^{2} \subseteq N_{0} \oplus N_{1}=0$ o que contradiz o fato de $\operatorname{dim} N^{2}=1$ quando $N$ tem tipo de nilpotência $(2,1)$.

iv. Suponha que $N=N_{0} \oplus N_{\frac{1}{2}}, \operatorname{com} \operatorname{dim} N_{0}=2$. Pelo Lema 2.1 e pelo Teorema 1.18, $\mathrm{N}^{2} \subseteq \mathrm{N}_{0}$. Consequentemente, existe $n_{2} \in \mathrm{N}_{0}$ tal que $\mathrm{N}^{2}=\mathbf{k} n_{2}$, completando a uma base de $N$ temos que existem $n_{1} \in N_{\frac{1}{2}}$ e $n_{3} \in N_{0}$ tal que

$$
\begin{aligned}
& n_{2}^{2}, n_{2} n_{3} \in N^{3}=0, \quad n_{1} n_{2}=n_{1} n_{3}=0 \text { pelo Lema 2.1, } \\
& n_{3}^{2}=\alpha n_{2} \text { e } n_{1}^{2}=\beta n_{2} \text { pois ambos pertencem a } N^{2} .
\end{aligned}
$$

Para quaisquer valores de $\alpha$ e $\beta$ a álgebra obtida é de Jordan, mas se ambos coeficientes fossem nulos então $\mathrm{N}^{2}=0$, contradizendo o fato que $\mathrm{N}$ tem tipo de nilpotência $(2,1)$. Logo pelo menos um deles tem que ser não nulo. Assim obtemos as seguintes álgebras:

Tabela 2.12: k-álgebras de Jordan de dimensão 4 com radical de tipo $(2,1)$ e $\mathrm{N}=\mathrm{N}_{0} \oplus \mathrm{N}_{\frac{1}{2}}$ com $\operatorname{dim} \mathrm{N}_{0}=2$

\begin{tabular}{|c|c|c|c|c|c|}
\hline $\mathcal{J}$ & Tabela de Multiplicação & $\begin{array}{c}\operatorname{dim} \\
\operatorname{Aut}(\mathcal{J})\end{array}$ & $\begin{array}{c}\operatorname{dim} \\
\operatorname{Ann}(\mathcal{J})\end{array}$ & $\begin{array}{c}\operatorname{dim} \\
\mathfrak{J}^{2}\end{array}$ & Observação \\
\hline$\partial_{44}$ & $\mathcal{T}_{8} \oplus \mathbf{k} n_{3}$ & 4 & 2 & 3 & $\alpha=0, \beta \neq 0$ \\
\hline$\partial_{45}$ & $\begin{array}{c}e_{1}^{2}=e_{1} \quad n_{1}^{2}=n_{2} \\
n_{3}^{2}=n_{2} \quad e_{1} n_{1}=\frac{1}{2} n_{1}\end{array}$ & 3 & 1 & 3 & $\alpha \neq 0, \beta \neq 0$ \\
\hline$\partial_{46}$ & $\mathcal{B}_{2} \oplus \mathcal{B}_{3}$ & 4 & 1 & 3 & $\alpha \neq 0, \beta=0$ \\
\hline
\end{tabular}


Provaremos a seguir que para quaisquer outros valores de $\alpha$ e $\beta$ a álgebra obtida é isomorfa a uma das anteriores. Para isso suponha que o produto $\cdot$ de $\mathcal{J}$ satisfaz (2.2) para alguns $\alpha$ e $\beta$ tal que pelo menos um deles é não nulo.

Vamos supor primeiro que o coeficiente não nulo seja $\beta$. Então se $\alpha=0$ a álgebra $\mathcal{J}$ é isomorfa a $\mathcal{J}_{44}$ com isomorfismo definido por $N_{2} \mapsto \beta^{-1} n_{2}$. Mas se $\alpha$ for também não nulo então a álgebra $\mathcal{J}$ é isomorfa a $\mathcal{J}_{45}$ com isomorfismo dado por $N_{2} \mapsto \beta^{-1} n_{2}$ e $N_{3} \mapsto \frac{\sqrt{\alpha}}{\sqrt{\beta}} n_{3}$ cujo determinante $\frac{\sqrt{\alpha}}{(\sqrt{\beta})^{3}}$ é bem definido e não nulo para todo $\beta \neq 0$ e $\alpha \neq 0$. Suponha agora que o coeficiente não nulo seja $\alpha$. Se $\beta=0$ o isomorfismo definido por $N_{2} \mapsto \alpha^{-1} n_{2}$ mostra que $\mathcal{J} \simeq \mathcal{J}_{46}$.

v. Suponha que $N=N_{0} \oplus N_{1}$, com $\operatorname{dim} N_{0}=2$. Pelo Lema 2.1 e pelo Teorema 1.18, $N^{2} \subseteq N_{0}$. Consequentemente, existe $n_{2} \in N_{0}$ tal que $N^{2}=\mathbf{k n}_{2}$, completando a uma base de $N$ existem $n_{1} \in N_{0}$ e $n_{3} \in N_{1}$ tal que

$$
\begin{aligned}
& n_{2}^{2}, n_{2} n_{1}, n_{2} n_{3} \in N^{3}=0, n_{3}^{2}=n_{1} n_{3}=0, \\
& n_{1}^{2}=\alpha n_{2} \text { pois } n_{1}^{2} \in N^{2} .
\end{aligned}
$$

Para quaisquer valores de $\alpha$ a álgebra obtida é de Jordan, mas se $\alpha=0$ então $\mathrm{N}^{2}=0$ o que contradiz o fato de $\mathrm{N}$ ter tipo de nilpotência $(2,1)$. Logo $\alpha$ deve ser não nulo e obtemos uma única álgebra:

Tabela 2.13: k-álgebras de Jordan de dimensão 4 com radical de tipo $(2,1)$ e $\mathrm{N}=\mathrm{N}_{0} \oplus \mathrm{N}_{1} \operatorname{com} \operatorname{dim} \mathrm{N}_{0}=2$

\begin{tabular}{|c|c|c|c|c|c|}
\hline $\mathcal{J}$ & Tabela de Multiplicação & $\begin{array}{c}\operatorname{dim} \\
\operatorname{Aut}(\mathcal{J})\end{array}$ & $\begin{array}{c}\operatorname{dim} \\
\operatorname{Ann}(\mathcal{J})\end{array}$ & $\begin{array}{c}\operatorname{dim} \\
\mathfrak{J}^{2}\end{array}$ & Observação \\
\hline $\mathcal{J}_{47}$ & $\mathcal{B}_{1} \oplus \mathcal{B}_{3}$ & 3 & 1 & 3 & Associativa \\
\hline
\end{tabular}

Vejamos que para quaisquer outros valores de $\alpha$ a álgebra obtida é isomorfa a $\mathcal{J}_{47}$. Analogamente ao caso anterior suponha que o produto $\cdot$ de $\mathcal{J}$ satisfaz (2.3) para algum $\alpha \neq 0$ então o isomorfismo é definido por $\mathrm{N}_{2} \mapsto \alpha^{-1} n_{2}$.

vi. Suponha que $N=N_{0} \oplus N_{\frac{1}{2}}$, com $\operatorname{dim} N_{\frac{1}{2}}=2$. Considere $n_{1} \in N_{0}, n_{2}, n_{3} \in N_{\frac{1}{2}}$. Do Lema 2.1 segue que $n_{1}^{2}=0$. Por outro lado $n_{2}^{2}, n_{3}^{2}, n_{2} n_{3} \in N_{\frac{1}{2}}^{2} \subseteq N_{0} \operatorname{logo}$ $n_{2}^{2}=\alpha n_{1}, n_{3}^{2}=\beta n_{1}$ e $n_{2} n_{3}=\gamma n_{1}$. Agora, como $n_{1} n_{2}, n_{1} n_{3} \in N_{0} N_{\frac{1}{2}} \subseteq N_{\frac{1}{2}}$ temos $n_{1} n_{2}=\theta n_{2}+\delta n_{3}$ e $n_{1} n_{3}=\theta_{1} n_{2}+\delta_{1} n_{3}$.

Verificando que a base $\left\{e_{1}, n_{1}, n_{2}, n_{3}\right\}$ satisfaça a identidade de Jordan (1.3) obtemos condições para as constantes. Em todos os casos as álgebras que obtemos são isomorfas a uma das seguintes álgebras: 
Tabela 2.14: k-álgebras de Jordan de dimensão 4 com radical de tipo $(2,1)$ e $\mathrm{N}=\mathrm{N}_{0} \oplus \mathrm{N}_{\frac{1}{2}} \operatorname{com} \operatorname{dim} \mathrm{N}_{\frac{1}{2}}=2$

\begin{tabular}{|c|c|c|c|c|c|}
\hline $\mathcal{J}$ & Tabela de Multiplicação & $\begin{array}{c}\operatorname{dim} \\
\operatorname{Aut}(\mathcal{J})\end{array}$ & $\begin{array}{c}\operatorname{dim} \\
\operatorname{Ann}(\mathcal{J})\end{array}$ & $\operatorname{dim}^{2}$ & Observação \\
\hline$\partial_{48}$ & $\begin{aligned} e_{1}^{2}=e_{1} & n_{3}^{2}=n_{1} \\
e_{1} n_{2}=\frac{1}{2} n_{2} & e_{1} n_{3}=\frac{1}{2} n_{3}\end{aligned}$ & 5 & 1 & 4 & \\
\hline$J_{49}$ & $\begin{array}{c}e_{1}^{2}=e_{1} \quad n_{3}^{2}=n_{1} \\
e_{1} n_{2}=\frac{1}{2} n_{2} \quad e_{1} n_{3}=\frac{1}{2} n_{3} \\
n_{2} n_{3}=n_{1}\end{array}$ & 4 & 1 & 4 & \\
\hline$J_{50}$ & $\begin{array}{c}e_{1}^{2}=e_{1} \quad e_{1} n_{2}=\frac{1}{2} n_{2} \\
e_{1} n_{3}=\frac{1}{2} n_{3} \quad n_{1} n_{2}=n_{3}\end{array}$ & 5 & 0 & 3 & \\
\hline
\end{tabular}

Vejamos isso com mais detalhes, para isso considere que $(\mathcal{J}, \cdot)$ satisfaz as condições de nosso problema para certos coeficientes $\alpha, \beta, \gamma, \delta, \delta_{1}, \theta$ e $\theta_{1}$. Suponha, primeiramente, que char $\mathbf{k} \neq 3$, então obtemos ${ }^{2} 6$ condições que as constantes devem satisfazer para a álgebra ser de Jordan, a saber:

a) $\theta_{1}=\theta=\delta_{1}=\delta=\alpha=0$ e $\beta \neq 0$. Se $\gamma \neq 0$ o isomorfismo definido por $N_{1} \mapsto \beta^{-1} n_{1}$ e $N_{2} \mapsto-\gamma \beta^{-1} n_{2}+2 \gamma \beta^{-1} n_{3}$ cujo determinante $-\gamma \beta^{-2}$ é bem definido e não nulo, nos da $\mathcal{J} \simeq \mathcal{J}_{49}$. Mas se $\gamma=0$ então $\mathcal{J} \simeq \mathcal{J}_{48}$ com isomorfismo $N_{1} \mapsto \beta^{-1} n_{1}$.

b) $\theta_{1}=\theta=\delta_{1}=\gamma=\alpha=\beta=0$. Logo, necessariamente, $\delta \neq 0$ (caso contrário $\mathrm{N}^{2}=0$ contradizendo o tipo de nilpotência de $N$ ) e temos $\mathcal{J} \simeq \mathcal{J}_{50} \mathrm{com}$ isomorfismo $\mathrm{N}_{1} \mapsto \delta \mathrm{n}_{1}$.

c) $\theta_{1}=\theta=\delta_{1}=\delta=0$ e $\alpha \neq 0$. Se ambas constantes $\beta$ e $\gamma$ forem não nulas, temos duas possibilidades: ou $-\alpha \beta+\gamma^{2} \neq 0$ e neste caso $\mathcal{J} \simeq \mathcal{J}_{49}$ com isomorfismo $\mathrm{N}_{1} \mapsto \alpha^{-1} \mathrm{n}_{1}, \mathrm{~N}_{2} \mapsto \mathrm{n}_{3}$ e $\mathrm{N}_{3} \mapsto-\frac{\sqrt{-\alpha \beta+\gamma^{2}}}{\alpha} \mathrm{n}_{2}+\frac{\gamma+\sqrt{-\alpha \beta+\gamma^{2}}}{\alpha} \mathrm{n}_{3}$ de det $=\frac{\sqrt{-\alpha \beta+\gamma^{2}}}{\alpha^{2}}$, ou $-\alpha \beta+\gamma^{2}=0$ e então $\mathcal{J} \simeq \mathcal{J}_{48}$ com isomorfismo $N_{1} \mapsto \alpha^{-1} n_{1}, N_{2} \mapsto n_{2}+n_{3}$ e $N_{3} \mapsto \alpha^{-1} \gamma n_{3}$ de det $=\alpha^{-2} \gamma$. Agora, se $\beta=\gamma=0$ então $\mathcal{J} \simeq \mathcal{J}_{48}$ com isomorfismo dado por $N_{1} \mapsto \alpha^{-1} n_{1}$, $N_{2} \mapsto n_{3}$ e $N_{3} \mapsto n_{2}$ com det $=-\alpha^{-1}$. Se somente um deles for nulo então a álgebra obtida é $\mathcal{J}_{49}$ com isomorfismo $N_{1} \mapsto \alpha^{-1} n_{1}, N_{2} \mapsto n_{3}$ e $N_{3} \mapsto-\gamma \alpha^{-1} n_{2}+2 \gamma \alpha^{-1} n_{3}$ de det $=\gamma \alpha^{-2}$ se $\beta=0$, e com isomorfismo $N_{1} \mapsto-\beta^{-1} n_{1}, N_{2} \mapsto \frac{i \sqrt{\alpha}}{\sqrt{\beta}} n_{3}$ e $N_{3} \mapsto-n_{2}+n_{3} \operatorname{dedet}=\frac{-i \sqrt{\alpha}}{(\sqrt{\beta})^{3}}$ se $\gamma=0$.

\footnotetext{
2 Utilizando um programa de computação simbólica elaborado pela autora, veja Apêndice A.
} 
d) $\alpha=\beta=\gamma=0, \delta_{1}=-\theta, \delta=-\frac{\theta^{2}}{\theta_{1}} \operatorname{com} \theta_{1} \neq 0$ e $\theta \neq 0$. Neste caso $\mathcal{J} \simeq J_{50}$ com isomorfismo $N_{1} \mapsto-\frac{\theta^{2}}{\theta_{1}} n_{1}$ e $N_{3} \mapsto \frac{\theta_{1}}{\theta} n_{2}+n_{3}$ cujo determinante $-\frac{\theta^{2}}{\theta_{1}}$ é bem definido e não nulo.

e) $\theta=\delta_{1}=\delta=\alpha=\beta=\gamma=0$ e $\theta_{1} \neq 0$. Novamente $\mathcal{J} \simeq \mathcal{J}_{50}$ com isomorfismo $\mathrm{N}_{1} \mapsto \theta_{1} \mathrm{n}_{1}, \mathrm{~N}_{2} \mapsto \mathrm{n}_{3}$ e $\mathrm{N}_{3} \mapsto \mathrm{n}_{2}$ cujo determinante $-\theta_{1}$ é não nulo.

f) $\theta_{1}=\theta=\delta_{1}=\delta=\alpha=\beta=0$ e $\gamma \neq 0$. Neste caso $\mathcal{J} \simeq \mathcal{J}_{49}$ com isomorfismo $\mathrm{N}_{1} \mapsto \gamma^{-1} \mathrm{n}_{1}, \mathrm{~N}_{2} \mapsto-\frac{1}{2} \mathrm{n}_{2}+\mathrm{n}_{3}$ e $\mathrm{N}_{3} \mapsto \mathrm{n}_{2}$ cujo determinante $-\gamma^{-1}$ é bem definido e não nulo.

Suponha agora que $\mathbf{k}$ é um corpo algebricamente fechado de característica 3, então as condições dadas pela identidade (1.3) mudam. Assim obtemos as seguintes 7 condições sobre as constantes:

a) $\theta_{1}=\theta=\delta_{1}=\delta=0$ e $\alpha \beta+2 \gamma^{2} \neq 0$. Então considerando as diferentes possibilidades para $\alpha, \beta$ e $\gamma$ caímos nos casos a), c) e f) anteriores.

b) $\theta=\delta_{1}=\delta=\alpha=\gamma=0$ e $\theta_{1} \neq 0$. Então temos duas possibilidades para $\beta$ : ou $\beta=0$ e caímos no caso e) de char $\mathbf{k} \neq 3$ ou $\beta \neq 0$ mas neste caso $\left(N_{3}^{2}, E_{1}, N_{3}\right)=-\frac{1}{2} \beta \theta_{1} N_{2} \neq 0$ então $\mathcal{J}$ não é de Jordan.

c) $\theta_{1}=\theta=\delta_{1}=\beta=\gamma=0$ e $\delta \neq 0$. Então temos duas possibilidades para $\alpha$ : ou $\alpha=0$ e caímos no caso b) de char $\mathbf{k} \neq 3$ ou $\alpha \neq 0$ mas neste caso $\left(N_{2}^{2}, E_{1}, N_{2}\right)=-\frac{1}{2} \alpha \delta N_{3} \neq 0$ então $\mathcal{J}$ não é de Jordan.

d) $\theta_{1}=\theta=\delta_{1}=\delta=\beta=\gamma=0$. Então necessariamente $\alpha \neq 0$ (caso contrário $\mathrm{N}^{2}=0$ contradizendo o tipo de nilpotência de $\mathrm{N}$ ) e caímos no caso c) de char $\mathbf{k} \neq 3$.

e) $\alpha=\beta=\gamma=0, \delta_{1}=-\theta, \delta=-\frac{\theta^{2}}{\theta_{1}} \operatorname{com} \theta_{1} \neq 0$ e $\theta \neq 0$. Idem caso d) de char $\mathbf{k} \neq 3$.

f) $\theta_{1}=\theta=\delta_{1}=\delta=0, \alpha=\frac{-2 \gamma^{2}}{\beta}$ com $\beta \neq 0$ e $\gamma \neq 0$. Considerando que $-2=1$ temos que $\alpha=\frac{\gamma^{2}}{\beta}$ e $\log \mathcal{\partial} \simeq \mathcal{J}_{48}$ com isomorfismo $N_{1} \mapsto \alpha^{-1} n_{1}$, $\mathrm{N}_{2} \mapsto \mathrm{n}_{2}+\mathrm{n}_{3}$ e $\mathrm{N}_{3} \mapsto \alpha^{-1} \gamma \mathrm{n}_{3}$ cujo determinante $\alpha^{-2} \gamma$ é bem definido e não nulo. É interessante observar que se char $\mathbf{k}$ for diferente de 3 neste caso a álgebra seria $\mathcal{J}_{49}$.

g) $\theta_{1}=\theta=\delta_{1}=\delta=\alpha=\gamma=0$ com $\beta \neq 0$. Idem caso a) de char $\mathbf{k} \neq 3$ quando $\gamma=0$. 
vii. Suponha que $N=N_{0} \oplus N_{\frac{1}{2}} \oplus N_{1} \operatorname{com} \operatorname{dim} N_{i}=1$ para $i=0, \frac{1}{2}, 1$. Considere $n_{1} \in N_{\frac{1}{2}}, n_{2} \in N_{1}, n_{3} \in N_{0}$ então temos os seguintes produtos em $N$ :

$$
\begin{aligned}
& n_{3}^{2}=n_{2}^{2}=n_{1} n_{3}=n_{1} n_{2}=0 \text { pelo Lema 2.1, } n_{2} n_{3} \in N_{0} N_{1}=0, \\
& n_{1}^{2}=\alpha n_{3}+\beta n_{2} \text { pois } n_{1}^{2} \in N_{\frac{1}{2}}^{2} \subseteq N_{0} \oplus N_{1} .
\end{aligned}
$$

Para quaisquer valores de $\alpha$ e $\beta$ a álgebra obtida é de Jordan, mas se ambos coeficientes fossem nulos então $\mathrm{N}^{2}=0$, contradizendo o fato que $\mathrm{N}$ tem tipo de nilpotência $(2,1)$. Logo pelo menos um deles tem que ser não nulo. Assim obtemos as seguintes álgebras:

Tabela 2.15: k-álgebras de Jordan de dimensão 4 com radical de tipo $(2,1)$ e

$$
\mathrm{N}=\mathrm{N}_{0} \oplus \mathrm{N}_{\frac{1}{2}} \oplus \mathrm{N}_{1}
$$

\begin{tabular}{|c|c|c|c|c|c|}
\hline $\mathcal{J}$ & Tabela de Multiplicação & $\begin{array}{c}\operatorname{dim} \\
\operatorname{Aut}(\mathcal{J})\end{array}$ & $\begin{array}{c}\operatorname{dim} \\
\operatorname{Ann}(\mathcal{J})\end{array}$ & $\begin{array}{c}\operatorname{dim} \\
\mathcal{J}^{2}\end{array}$ & Observação \\
\hline$J_{51}$ & $\mathcal{T}_{9} \oplus \mathbf{k n}_{3}$ & 3 & 1 & 3 & $\begin{array}{l}\alpha=0 \\
\beta \neq 0\end{array}$ \\
\hline$J_{52}$ & $\begin{array}{c}e_{1}^{2}=e_{1} \quad n_{1}^{2}=n_{3} \\
e_{1} n_{2}=n_{2} \quad e_{1} n_{1}=\frac{1}{2} n_{1}\end{array}$ & 3 & 1 & 4 & $\begin{array}{l}\alpha \neq 0 \\
\beta=0\end{array}$ \\
\hline$J_{53}$ & $\begin{array}{c}e_{1}^{2}=e_{1} \quad n_{1}^{2}=n_{2}+n_{3} \\
e_{1} n_{2}=n_{2} \quad e_{1} n_{1}=\frac{1}{2} n_{1}\end{array}$ & 2 & 1 & 4 & $\begin{array}{l}\alpha \neq 0 \\
\beta \neq 0\end{array}$ \\
\hline
\end{tabular}

Para provar que não existe outra álgebra suponha que o produto $\cdot$ de $\mathcal{J}$ satisfaz (2.4) e que o coeficiente não nulo é $\beta$. Então se $\alpha=0$ o isomorfismo que leva $N_{2} \mapsto \beta^{-1} n_{2}$ nos dá $\mathcal{J} \simeq \mathcal{J}_{51}$, mas se $\alpha \neq 0$ então o isomorfismo dado por $N_{2} \mapsto \beta^{-1} n_{2}$ e $N_{3} \mapsto \alpha^{-1} n_{3}$ prova que $\mathcal{J} \simeq \mathcal{J}_{53}$. Caso o coeficiente não nulo seja $\alpha$ e $\beta=0$ então $\mathcal{J}$ é $\mathcal{J}_{52}$ com isomorfismo $N_{3} \mapsto \alpha^{-1} n_{3}$.

viii. Suponha que $N=N_{0} \oplus N_{1}$ com $\operatorname{dim} N_{1}=2$. Do Lema 2.1 e do Teorema 1.18 segue que $N^{2} \subseteq N_{1}$. Consequentemente, existe $n_{2} \in N_{1}$ tal que $N^{2}=\mathbf{k n}_{2}$, completando a uma base de $N$ existem $n_{1} \in N_{1}$ e $n_{3} \in N_{0}$ tal que

$$
\begin{aligned}
& n_{2}^{2}, n_{1} n_{2} \in N^{3}=0, \quad n_{3}^{2}=n_{2} n_{3}=n_{1} n_{3}=0, \\
& n_{1}^{2}=\alpha n_{2} \text { pois } n_{1}^{2} \in N^{2} .
\end{aligned}
$$

Para quaisquer valores de $\alpha$ a álgebra obtida é de Jordan, mas devido ao tipo de nilpotência que estamos considerando $\alpha$ deve ser não nulo. Consequentemente obtemos a seguinte álgebra: 
Tabela 2.16: k-álgebras de Jordan de dimensão 4 com radical de tipo $(2,1)$ e $\mathrm{N}=\mathrm{N}_{0} \oplus \mathrm{N}_{1}$ com $\operatorname{dim} \mathrm{N}_{1}=2$

\begin{tabular}{|c|c|c|c|c|c|}
\hline $\mathcal{J}$ & Tabela de Multiplicação & $\begin{array}{c}\operatorname{dim} \\
\operatorname{Aut}(\mathcal{J})\end{array}$ & $\begin{array}{c}\operatorname{dim} \\
\operatorname{Ann}(\mathcal{J})\end{array}$ & $\begin{array}{c}\operatorname{dim} \\
\mathcal{J}^{2}\end{array}$ & Observação \\
\hline $\mathcal{J}_{54}$ & $\mathcal{T}_{1} \oplus \mathbf{k n}_{3}$ & 3 & 1 & 3 & Associativa \\
\hline
\end{tabular}

Se $\mathcal{J}$ satisfaz (2.5) para algum $\alpha \neq 0$ então $\mathcal{J}$ é isomorfa a $\mathcal{J}_{54}$ com isomorfismo $\mathrm{N}_{2} \rightarrow \alpha^{-1} \mathrm{n}_{2}$.

ix. Suponha que $N=N_{\frac{1}{2}} \oplus N_{1}$ com $\operatorname{dim} N_{1}=2$. Pelo Lema 2.1 e pelo Teorema 1.18 segue que $N^{2} \subseteq N_{1}$. Consequentemente existe $n_{2} \in N_{1}$ tal que $N^{2}=\mathbf{k n}_{2}$, completando a uma base de $N$ temos que existem $n_{1} \in N_{1}$ e $n_{3} \in N_{\frac{1}{2}}$ tal que

$$
\begin{aligned}
& n_{2}^{2}, n_{2} n_{1} \in N^{3}=0, \quad n_{2} n_{3}=n_{1} n_{3}=0 \text { pelo Lema 2.1, } \\
& n_{1}^{2}=\alpha n_{2} \text { e } n_{3}^{2}=\beta n_{2} \text { pois ambos pertencem a } N^{2} .
\end{aligned}
$$

Para quaisquer valores de $\alpha$ e $\beta$ a álgebra obtida é de Jordan, mas devido ao tipo de nilpotência que estamos considerando não podem ser ambos nulos. Logo obtemos as seguintes álgebras:

Tabela 2.17: k-álgebras de Jordan de dimensão 4 com radical de tipo $(2,1)$ e

\begin{tabular}{|c|c|c|c|c|c|}
\hline $\mathcal{\partial}$ & Tabela de Multiplicação & $\begin{array}{c}\operatorname{dim} \\
\operatorname{Aut}(\mathcal{J})\end{array}$ & $\begin{array}{c}\operatorname{dim} \\
\operatorname{Ann}(\mathcal{J})\end{array}$ & $\begin{array}{c}\operatorname{dim} \\
\partial^{2}\end{array}$ & Observação \\
\hline$J_{55}$ & $\begin{array}{c}e_{1}^{2}=e_{1} \quad n_{3}^{2}=n_{2} \\
e_{1} n_{1}=n_{1} \\
e_{1} n_{2}=n_{2} \quad e_{1} n_{3}=\frac{1}{2} n_{3}\end{array}$ & 4 & 0 & 4 & $\begin{array}{l}\alpha=0 \\
\beta \neq 0\end{array}$ \\
\hline$J_{56}$ & $\begin{array}{c}e_{1}^{2}=e_{1} \quad n_{1}^{2}=n_{2} \\
e_{1} n_{1}=n_{1} \\
e_{1} n_{2}=n_{2} \quad e_{1} n_{3}=\frac{1}{2} n_{3}\end{array}$ & 4 & 0 & 4 & $\begin{array}{l}\alpha \neq 0 \\
\beta=0\end{array}$ \\
\hline$J_{57}$ & $\begin{array}{cc}e_{1}^{2}=e_{1} & n_{1}^{2}=n_{2} \\
n_{3}^{2}=n_{2} & e_{1} n_{1}=n_{1} \\
e_{1} n_{2}=n_{2} & e_{1} n_{3}=\frac{1}{2} n_{3}\end{array}$ & 3 & 0 & 4 & $\begin{array}{l}\alpha \neq 0 \\
\beta \neq 0\end{array}$ \\
\hline
\end{tabular}
$\mathrm{N}=\mathrm{N}_{\frac{1}{2}} \oplus \mathrm{N}_{1} \operatorname{com} \operatorname{dim} \mathrm{N}_{1}=2$

Suponha que $\mathcal{J}$ satisfaz (2.6) para alguns $\alpha$ e $\beta$, então se o coeficiente não nulo é $\beta$ e $\alpha=0$ a álgebra $\mathcal{J}$ é isomorfa a $\partial_{55}$ com isomorfismo que leva $N_{2} \mapsto \beta^{-1} n_{2}$. Mas se $\alpha$ for também não nulo então a álgebra $\mathcal{J}$ é isomorfa a $\mathcal{J}_{57}$ neste caso com 
isomorfismo $N_{1} \mapsto \frac{\sqrt{\alpha}}{\sqrt{\beta}} n_{1}$ e $N_{2} \mapsto \beta^{-1} n_{2}$ cujo determinante $\frac{\sqrt{\alpha}}{(\sqrt{\beta})^{3}}$ é bem definido e não nulo. Por último se o coeficiente não nulo é $\alpha$ e $\beta=0$ o isomorfismo $\mathrm{N}_{2} \mapsto \alpha^{-1} n_{2}$ prova que $\mathcal{J} \simeq \mathcal{J}_{56}$.

x. Suponha, por último, que $N=N_{\frac{1}{2}} \oplus N_{1} \operatorname{com} \operatorname{dim} N_{\frac{1}{2}}=2$. Considere $n_{1} \in N_{1}$, $n_{2}, n_{3} \in N_{\frac{1}{2}}$. Do Lema 2.1 segue que $n_{1}^{2}=0$. Por outro lado $n_{2}^{2}, n_{3}^{2}, n_{2} n_{3} \in$ $N_{\frac{1}{2}}^{2} \subseteq N_{1} \operatorname{logo} n_{2}^{2}=\alpha n_{1}, n_{3}^{2}=\beta n_{1}$ e $n_{2} n_{3}=\gamma n_{1}$. Agora, como $n_{1} n_{2}, n_{1} n_{3} \in$ $N_{1}^{2} N_{\frac{1}{2}} \subseteq N_{\frac{1}{2}}$, temos $n_{1} n_{2}=\theta n_{2}+\delta n_{3}$ e $n_{1} n_{3}=\theta_{1} n_{2}+\delta_{1} n_{3}$.

Verificando que a base $\left\{e_{1}, n_{1}, n_{2}, n_{3}\right\}$ satisfaça a identidade de Jordan (1.3) obtemos condições para as constantes. Em todos os casos as álgebras que obtemos são isomorfas a uma das seguintes álgebras:

Tabela 2.18: k-álgebras de Jordan de dimensão 4 com radical de tipo $(2,1)$ e $\mathrm{N}=\mathrm{N}_{\frac{1}{2}} \oplus \mathrm{N}_{1} \operatorname{com} \operatorname{dim} \mathrm{N}_{\frac{1}{2}}=2$

\begin{tabular}{|c|c|c|c|c|c|}
\hline$J$ & Tabela de Multiplicação & $\begin{array}{c}\operatorname{dim} \\
\operatorname{Aut}(\mathcal{J})\end{array}$ & $\begin{array}{c}\operatorname{dim} \\
\operatorname{Ann}(\mathcal{J})\end{array}$ & $\begin{array}{c}\operatorname{dim} \\
\mathcal{J}^{2}\end{array}$ & Observação \\
\hline$\partial_{58}$ & $\begin{array}{c}e_{1}^{2}=e_{1} \quad n_{3}^{2}=n_{1} \quad e_{1} n_{1}=n_{1} \\
e_{1} n_{2}=\frac{1}{2} n_{2} \quad e_{1} n_{3}=\frac{1}{2} n_{3}\end{array}$ & 5 & 0 & 4 & \\
\hline$\partial_{59}$ & $\begin{array}{c}e_{1}^{2}=e_{1} \quad n_{3}^{2}=n_{1} \\
e_{1} n_{1}=n_{1} \quad e_{1} n_{2}=\frac{1}{2} n_{2} \\
e_{1} n_{3}=\frac{1}{2} n_{3} \quad n_{2} n_{3}=n_{1}\end{array}$ & 4 & 0 & 4 & \\
\hline$\partial_{60}$ & $\begin{array}{c}e_{1}^{2}=e_{1} \quad e_{1} n_{1}=n_{1} \quad n_{1} n_{2}=n_{3} \\
e_{1} n_{2}=\frac{1}{2} n_{2} \quad e_{1} n_{3}=\frac{1}{2} n_{3}\end{array}$ & 5 & 0 & 4 & \\
\hline
\end{tabular}

Vejamos que só existem essas álgebras. Suponha, primeiramente, que char $\mathbf{k} \neq 3$, então obtemos ${ }^{3} 6$ condições que as constantes devem satisfazer para a álgebra ser de Jordan, que são as mesmas condições do caso vi. onde $N=N_{\frac{1}{2}} \oplus N_{0}$ com $\operatorname{dim} N_{\frac{1}{2}}=2$. Quando o isomorfismo não seja dado explicitamente se entenderá que é análogo ao isomorfismo correspondente do caso vi.

a) $\theta_{1}=\theta=\delta_{1}=\delta=\alpha=0$ e $\beta \neq 0$. Se $\gamma \neq 0$ então $\mathcal{\partial} \simeq \mathcal{J}_{59}$, mas se $\gamma=0$ então $\mathcal{J} \simeq \mathcal{J}_{58}$.

b) $\theta_{1}=\theta=\delta_{1}=\gamma=\alpha=\beta=0$. Logo, necessariamente, $\delta \neq 0$ (caso contrário $\mathrm{N}^{2}=0$ contradizendo o tipo de nilpotência de $N$ ) e temos $\mathcal{J} \simeq \mathcal{J}_{60}$ com isomorfismo que leva $N_{3} \mapsto \delta^{-1} n_{3}$.

c) $\theta_{1}=\theta=\delta_{1}=\delta=0$ e $\alpha \neq 0$. Se ambas constantes $\beta$ e $\gamma$ forem não nulas, temos duas possibilidades: ou $-\alpha \beta+\gamma^{2} \neq 0$ e neste caso $\mathcal{J} \simeq \mathcal{J}_{59}$, ou

\footnotetext{
3 Utilizando um programa de computação simbólica elaborado pela autora, veja Apêndice A.
} 
$-\alpha \beta+\gamma^{2}=0$ e então $\mathcal{J} \simeq \mathcal{J}_{58}$. Agora, se $\beta=\gamma=0$ então $\mathcal{J} \simeq \mathcal{J}_{58}$. Se somente um deles for nulo então a álgebra obtida é $\partial_{59}$.

d) $\alpha=\beta=\gamma=0, \delta_{1}=-\theta, \delta=-\frac{\theta^{2}}{\theta_{1}} \operatorname{com} \theta_{1} \neq 0$ e $\theta \neq 0$. Neste caso $\mathcal{J} \simeq \mathcal{J}_{60}$ com isomorfismo $N_{3} \mapsto \frac{\theta_{1}}{\theta} n_{2}-\frac{\theta_{1}}{\theta^{2}} n_{3}$ cujo determinante $-\frac{\theta_{1}}{\theta^{2}}$ é bem definido e não nulo.

e) $\theta=\delta_{1}=\delta=\alpha=\beta=\gamma=0$ e $\theta_{1} \neq 0$. Novamente $\mathcal{J} \simeq \mathcal{J}_{60}$ com isomorfismo $\mathrm{N}_{2} \mapsto \theta_{1}^{-1} \mathrm{n}_{3}$ e $\mathrm{N}_{3} \mapsto \mathrm{n}_{2}+\mathrm{n}_{3}$ cujo determinante $-\theta_{1}^{-1}$ é bem definido e não nulo.

f) $\theta_{1}=\theta=\delta_{1}=\delta=\alpha=\beta=0$ e $\gamma \neq 0$. Neste caso $\mathcal{J} \simeq \mathcal{J}_{59}$.

Suponha agora que $\mathbf{k}$ tem característica 3, então obtemos as mesmas 7 condições sobre as constantes que obtivemos no caso (vi). quando char $\mathbf{k}=3$, por isso só explicitaremos aquelas condições que não impliquem em algum dos casos anteriores.

a) $\theta=\delta_{1}=\delta=\alpha=\gamma=0$ e $\theta_{1} \neq 0$. Se $\beta \neq 0$ então $\left(N_{3}^{2}, E_{1}, N_{3}\right)=\frac{1}{2} \beta \theta_{1} N_{2} \neq 0$ logo $\mathcal{J}$ não é de Jordan.

b) $\theta_{1}=\theta=\delta_{1}=\beta=\gamma=0$ e $\delta \neq 0$. Se $\alpha \neq 0$ então $\left(N_{2}^{2}, E_{1}, N_{2}\right)=\frac{1}{2} \alpha \delta N_{3} \neq 0$ logo $\mathcal{J}$ não é de Jordan.

c) $\theta_{1}=\theta=\delta_{1}=\delta=0, \alpha=\frac{-2 \gamma^{2}}{\beta} \operatorname{com} \beta \neq 0$ e $\gamma \neq 0$. Considerando que $-2=1$ temos que $\alpha=\frac{\gamma^{2}}{\beta}$ e logo $\mathcal{J} \simeq \mathcal{J}_{58}$. É interessante observar que se char $\mathbf{k}$ for diferente de 3 neste caso a álgebra seria $\mathcal{J}_{59}$.

\subsection{5 Álgebras de Jordan Nilpotentes}

As álgebras de Jordan nilpotentes de dimensão 4 sobre o corpo dos números complexos foram descritas em [4]. Observamos que esta classificação é ainda válida para um corpo algebricamente fechado de char $\neq 2$, veja [17]. Temos as seguintes álgebras não isomorfas:

Tabela 2.19: k-álgebras de Jordan nilpotentes de dimensão 4

\begin{tabular}{|c|c|c|c|c|c|}
\hline $\mathcal{J}$ & Tabela de Multiplicação & $\begin{array}{c}\operatorname{dim} \\
\operatorname{Aut}(\mathcal{J})\end{array}$ & $\begin{array}{c}\operatorname{dim} \\
\operatorname{Ann}(\mathcal{J})\end{array}$ & $\operatorname{dim}_{\partial^{2}}$ & Observação \\
\hline $\mathcal{J}_{61}$ & $\begin{aligned} n_{1}^{2}=n_{2} & n_{2}^{2}=n_{4} \\
n_{1} n_{2}=n_{3} & n_{1} n_{3}=n_{4}\end{aligned}$ & 4 & 1 & 3 & $\begin{array}{c}\text { Associativa, } \\
\text { tipo de nilpotência } \\
(1,1,1,1)\end{array}$ \\
\hline
\end{tabular}




\begin{tabular}{|c|c|c|c|c|c|}
\hline $\mathcal{J}$ & Tabela de Multiplicação & $\begin{array}{c}\operatorname{dim} \\
\operatorname{Aut}(\mathcal{J})\end{array}$ & $\begin{array}{c}\operatorname{dim} \\
\operatorname{Ann}(\mathcal{J})\end{array}$ & $\begin{array}{c}\operatorname{dim} \\
g^{2} \\
\end{array}$ & Observação \\
\hline$\partial_{62}$ & $\begin{array}{c}\mathrm{n}_{1}^{2}=\mathrm{n}_{2} \quad \mathrm{n}_{4}^{2}=\mathrm{n}_{2} \\
\mathrm{n}_{1} \mathrm{n}_{2}=\mathrm{n}_{3}\end{array}$ & 3 & 1 & 2 & $\begin{array}{l}\text { tipo de nilpotência } \\
\qquad(2,1,1)\end{array}$ \\
\hline$\partial_{63}$ & $\begin{array}{c}\mathrm{n}_{1}^{2}=\mathrm{n}_{2} \quad \mathrm{n}_{4}^{2}=-\mathrm{n}_{2}-\mathrm{n}_{3} \\
\mathrm{n}_{1} \mathrm{n}_{2}=\mathrm{n}_{3} \quad \mathrm{n}_{2} \mathrm{n}_{4}=\mathrm{n}_{3}\end{array}$ & 4 & 1 & 2 & $\begin{array}{l}\text { tipo de nilpotência } \\
\qquad(2,1,1)\end{array}$ \\
\hline$\partial_{64}$ & $\begin{array}{cc}n_{1}^{2}=n_{2} & n_{4}^{2}=-n_{2} \\
n_{1} n_{2}=n_{3} & n_{2} n_{4}=n_{3}\end{array}$ & 5 & 1 & 2 & $\begin{array}{l}\text { tipo de nilpotência } \\
\qquad(2,1,1)\end{array}$ \\
\hline$\partial_{65}$ & $\begin{array}{c}n_{1}^{2}=n_{2} \quad n_{1} n_{2}=n_{3} \\
n_{2} n_{4}=n_{3}\end{array}$ & 4 & 1 & 2 & $\begin{array}{l}\text { tipo de nilpotência } \\
\qquad(2,1,1)\end{array}$ \\
\hline$\partial_{66}$ & $\begin{array}{c}n_{1}^{2}=n_{2} \quad n_{4}^{2}=n_{3} \\
n_{1} n_{2}=n_{3}\end{array}$ & 5 & 1 & 2 & $\begin{array}{c}\text { Associativa, } \\
\text { tipo de nilpotência } \\
(2,1,1)\end{array}$ \\
\hline$\partial_{67}$ & $\mathcal{T}_{3} \oplus \mathbf{k} n_{4}$ & 6 & 2 & 2 & $\begin{array}{c}\text { Associativa, } \\
\text { tipo de nilpotência } \\
(2,1,1)\end{array}$ \\
\hline$\partial_{68}$ & $\mathcal{B}_{3} \oplus \mathcal{B}_{3}$ & 6 & 2 & 2 & $\begin{array}{c}\text { Associativa, } \\
\text { tipo de nilpotência } \\
(2,2)\end{array}$ \\
\hline$\partial_{69}$ & $\begin{array}{c}n_{1}^{2}=n_{2} \\
n_{1} n_{3}=n_{4}\end{array}$ & 7 & 2 & 2 & $\begin{array}{c}\text { Associativa, } \\
\text { tipo de nilpotência } \\
(2,2) \\
\end{array}$ \\
\hline$\partial_{70}$ & $\begin{array}{c}n_{1}^{2}=n_{2} \\
n_{3} n_{4}=n_{2}\end{array}$ & 7 & 1 & 1 & $\begin{array}{c}\text { Associativa, } \\
\text { tipo de nilpotência } \\
(3,1)\end{array}$ \\
\hline$\partial_{71}$ & $\mathcal{T}_{4} \oplus \mathbf{k} n_{4}$ & 8 & 2 & 1 & $\begin{array}{c}\text { Associativa, } \\
\text { tipo de nilpotência } \\
(3,1)\end{array}$ \\
\hline$\partial_{72}$ & $\mathcal{B}_{3} \oplus \mathbf{k} n_{3} \oplus \mathbf{k} n_{4}$ & 10 & 3 & 1 & $\begin{array}{c}\text { Associativa, } \\
\text { tipo de nilpotência } \\
(3,1)\end{array}$ \\
\hline$\partial_{73}$ & $\mathbf{k} n_{1} \oplus \mathbf{k} n_{2} \oplus \mathbf{k} n_{3} \oplus \mathbf{k} n_{4}$ & 16 & 4 & 0 & $\begin{array}{c}\text { Associativa, } \\
\text { tipo de nilpotência } \\
\text { (4) }\end{array}$ \\
\hline
\end{tabular}




\subsubsection{Observações}

Vimos em cada caso que dada qualquer álgebra de Jordan $\mathcal{J}$ de dimensão 4 sobre um corpo algebricamente fechado $\mathbf{k}$ de char $\mathbf{k} \neq 2$ então $\mathcal{J}$ é uma das álgebras $\mathcal{J}_{1}$ a $\mathcal{J}_{73}$. Para finalizar a classificação algébrica só resta provar que todas elas são diferentes.

Teorema 2.2. As álgebras $\mathcal{J}_{1}$ a $\mathcal{J}_{73}$ são duas a duas não isomorfas.

Demonstração. Comparando os invariantes $\operatorname{dim} \operatorname{Rad}(\mathcal{J})$, tipo de nilpotência $\operatorname{de} \operatorname{Rad}(\mathcal{J})$, $\operatorname{dim} \operatorname{Ann}(\mathcal{J}), \operatorname{dim} \operatorname{Aut}(\mathcal{J})$ e $\operatorname{dim} \mathcal{J}^{2}$, junto com as propriedades de $\mathcal{J}$ ser indecomponível, associativa, não associativa e unitária, somente necessitamos provar que não existe isomorfismo entre $\mathcal{J}_{55}, \mathcal{J}_{56}, \mathcal{J}_{59}$ e entre $\mathcal{J}_{58}$ e $\mathcal{J}_{60}$.

Primeiro observe que a álgebra de Jordan não associativa $\mathcal{B}_{2}$ de dimensão 2 , é uma subálgebra de $J_{56}$ mas não é subálgebra de $J_{55}$ (veja Apêndice B), então $J_{55} \nsucceq \mathcal{J}_{56}$. Mais ainda, o segundo grupo de cohomologia $\mathrm{H}^{2}\left(\mathcal{J}_{59}, \mathcal{J}_{59}\right)=0$ enquanto que ambos $H^{2}\left(\mathcal{J}_{55}, \mathcal{J}_{55}\right)$ e $H^{2}\left(\mathcal{J}_{56}, \mathcal{J}_{56}\right)$ são não nulos, logo temos $\mathcal{J}_{59} \nsucceq \mathcal{J}_{55}$ e $\mathcal{J}_{59} \nsucceq \mathcal{J}_{56}$.

Por último veja que $\operatorname{Rad}\left(\mathcal{J}_{58}\right)=\mathcal{B}_{3} \oplus \mathbf{k} n_{2}$ enquanto que $\operatorname{Rad}\left(\mathcal{J}_{60}\right)=\mathcal{T}_{4}$, portanto $J_{58} \neq J_{60}$.

Como última observação temos o seguinte teorema.

Teorema 2.3. Todas as álgebras de este capítulo são especiais.

Demonstração. Em 1979, A. M. Slin'ko em [44] provou que álgebras de Jordan nilpotentes sobre um corpo arbitrário até dimensão 5 são especiais e em 1989, H. Sherkulov em [43] mostrou que as álgebras de Jordan não associativas e não nilpotentes sobre um corpo arbitrário até dimensão 4 são especiais. 


\section{3 CLASSIFICAÇÃO ALGÉBRICA DAS ÁLGEBRAS DE JORDAN DE DIMENSÃO 3 SOBRE O CORPO DOS NÚME- ROS REAIS}

O problema de classificar álgebras sobre um corpo arbitrário não é simples e a classificação depende de maneira essencial do corpo que estivermos considerando. Para corpos bases especiais, como por exemplo o corpo dos p-ádicos ou o corpo dos números reais, como é o nosso caso, o problema pode ser resolvido e podemos obter uma classificação completa das correspondentes álgebras de Jordan.

A determinação de todas as classes de isomorfismos de álgebras de Jordan de dimensão fixa sobre o corpo dos números reais $\mathbb{R}$ só é conhecida completamente no caso de álgebras de Jordan semissimples. Os resultados conhecidos para álgebras de Jordan sobre $\mathbb{R}$ são muito escassos, inclusive para dimensões pequenas.

Neste capítulo apresentaremos a lista de todas as álgebras de Jordan (unitárias e não unitárias, associativas e não associativas) de dimensão 3 sobre $\mathbb{R}$. Analogamente ao Capítulo 2, faremos isto em duas seções: na primeira introduzimos as álgebras de Jordan indecomponíveis de dimensão menor que 3 e na segunda descrevemos as álgebras de dimensão 3 não isomorfas de acordo com a dimensão do radical e dos possíveis valores do tipo de nilpotência.

Usaremos esta descrição para estudar deformações entre álgebras de Jordan e descrever a variedade $\mathrm{Jor}_{3}^{\mathbb{R}}$ das álgebras de Jordan de dimensão 3 sobre o corpo dos números reais no Capítulo 6 deste texto.

Como foi visto no Teorema 1.13 toda álgebra de Jordan de dimensão finita sobre um corpo de característica zero pode-se decompor em soma direta de espaços vetoriais como sendo $\mathcal{J}=\mathcal{J}_{s s} \oplus \operatorname{Rad}(\mathcal{J})$, logo denotaremos por $e_{i}$ os elementos em $\mathcal{J}_{s s}$ e por $n_{i}$ aqueles elementos que pertencem a $\operatorname{Rad}(\mathcal{J})$. 


\section{1 Álgebras DE JORdAN REAis DE Dimensão MENOR QUE 3}

\subsection{1 Álgebras de Jordan Reais de Dimensão 1}

Existem somente duas $\mathbb{R}$-álgebras de Jordan de dimensão 1: a álgebra simples $\mathbb{R e}$, onde $e^{2}=e$ e a álgebra nilpotente $\mathbb{R} n$, onde $n^{2}=0$.

\subsection{2 Álgebras de Jordan Reais de Dimensão 2}

Em [3] Ancochea Bermúdez e outros descreveram todas as álgebras de Jordan de dimensão 2 sobre $\mathbb{R}$. Dessa lista obtemos as seguintes 4 álgebras indecomponíveis, que denotaremos por $\mathcal{B}_{i}^{\prime}$ :

Tabela 3.1: $\mathbb{R}$-álgebras de Jordan indecomponíveis de dimensão 2

\begin{tabular}{|c|ccc|c|}
\hline $\mathcal{B}^{\prime}$ & \multicolumn{3}{|c|}{ Tabela de Multiplicação } & Observação \\
\hline $\mathcal{B}_{1}^{\prime}$ & $e_{1}^{2}=e_{1}$ & $e_{1} n_{1}=n_{1}$ & $n_{1}^{2}=0$ & Associativa \\
\hline $\mathcal{B}_{2}^{\prime}$ & $e_{1}^{2}=e_{1}$ & $e_{1} n_{1}=\frac{1}{2} n_{1}$ & $n_{1}^{2}=0$ & \\
\hline $\mathcal{B}_{3}^{\prime}$ & $n_{1}^{2}=n_{2}$ & $n_{1} n_{2}=0$ & $n_{2}^{2}=0$ & Associativa, Nilpotente \\
\hline $\mathcal{B}_{4}^{\prime}$ & $e_{1}^{2}=e_{1}$ & $e_{1} e_{2}=e_{2}$ & $e_{2}^{2}=-e_{1}$ & Associativa, Simples \\
\hline
\end{tabular}

Note que a álgebra $\mathcal{B}_{4}^{\prime}$ é na verdade a álgebra dos números complexos $\mathbb{C}$.

\section{2 Álgebras de JORdAn REAis DE DIMENSÃO 3}

Nesta seção descreveremos todas as 26 álgebras de Jordan de dimensão 3 sobre $\mathbb{R}$. A descrição será organizada de acordo com a dimensão do radical nilpotente $\mathrm{N}$ e subsequentemente com os possíveis valores de seu tipo de nilpotência. Analogamente ao capítulo anterior, provaremos durante a descrição que qualquer outra $\mathbb{R}$-álgebra de Jordan de dimensão 3 é isomorfa a uma das 26 e por último no Teorema 3.1 veremos que as álgebras $\mathcal{T}_{1}^{\prime}$ a $\mathcal{T}_{26}^{\prime}$ são duas a duas não isomorfas.

Para cada álgebra calcularemos a dimensão do seu grupo de automorfismos $\operatorname{Aut}(\mathcal{T})$, de seu aniquilador $\operatorname{Ann}(\mathcal{T})$ e de sua segunda potência $\mathcal{T}^{2}$. Para uma lista mais detalhada das propriedades de cada álgebra veja o Apêndice $C$. 


\subsection{1 Álgebras de Jordan Reais Semissimples}

Para obter a classificação das álgebras de Jordan semissimples sobre $\mathbb{R}$ é suficiente conhecer as álgebras simples. Como consequência do Teorema 1.15 do Capítulo 1 podemos reduzir o problema de classificar álgebras de Jordan simples de dimensão finita sobre $\mathbb{R}$ ao problema de classificar álgebras de Jordan simples centrais sobre uma extensão $K$ de $\mathbb{R}$. Segue, então, do Teorema 1.16 que se $\mathcal{T}$ é uma álgebra de Jordan simples de dimensão menor ou igual a 3 sobre $\mathbb{R}$ então ou $\mathcal{T}=\mathbb{R}$ ou $\mathcal{T}=\mathbb{C}$ ou $\mathcal{T}$ é a álgebra de Jordan de uma forma bilinear simétrica não degenerada. Observamos que desprezamos as álgebras de Jordan $(\mathrm{H}(\mathcal{A}, \mathrm{J}), \odot)$ dos elementos de uma álgebra $\mathcal{A}$ associativa simples central de grau $n \geqslant 3$, fixos pela ação da involução J de $\mathcal{A}$, pois no mínimo estas álgebras tem dimensão 6 . Por um argumento análogo podemos eliminar as formas bilineares definidas sobre espaços vetoriais sobre o corpo dos complexos.

Assim, o problema de classificar as álgebras simples de dimensão menor ou igual a 3, fica reduzido ao problema de classificar formas bilineares simétricas não degeneradas sobre espaços vetoriais de dimensão finita sobre $\mathbb{R}$.

Seja $V$ um espaço vetorial de dimensão finita $n \geqslant 2$ sobre $\mathbb{R}$, que possui uma forma bilinear simétrica não degenerada $f=f(x, y)$, pela Lei de inercia de Sylvester [40, Teo. 11.25, p.287] existe uma base $\left\{e_{1}, \ldots, e_{n}\right\}$ de $V$ tal que $f\left(e_{i}, e_{i}\right)=1$, para $1 \leqslant i \leqslant p$, $f\left(e_{i}, e_{i}\right)=-1$ para $p+1 \leqslant i \leqslant n$ e $f\left(e_{i}, e_{j}\right)=0$ para todo $i \neq j$. Então a álgebra de Jordan $(\mathcal{J}(V, f), \sqcup)$ da forma bilinear $f$ tem base $\left\{1, e_{1}, \ldots, e_{n}\right\}$ e é definida pelos produtos

$$
\begin{aligned}
& 1 \boxminus 1=1, \quad 1 \boxminus e_{i}=e_{i}, \quad e_{i} \sqcup e_{j}=0 \text { para } i \neq j \\
& e_{i} \sqcup e_{i}=1 \text { para } 1 \leqslant i \leqslant p, \quad e_{i} \sqcup e_{i}=-1 \text { para } p+1 \leqslant i \leqslant n
\end{aligned}
$$

Logo existem somente 5 álgebras de Jordan reais simples não isomorfas de dimensão menor ou igual a 3:

i. $\mathbb{R}$ de dimensão 1 ,

ii. C de dimensão 2,

iii. $(\mathcal{J}(V, f), \oslash)$ onde $\operatorname{dim}_{\mathbb{R}} V=2, f\left(e_{1}, e_{1}\right)=1$ e $f\left(e_{2}, e_{2}\right)=-1$ de dimensão 3 ,

iv. $(\mathcal{J}(V, f), \bullet)$ onde $\operatorname{dim}_{\mathbb{R}} V=2$ e $f\left(e_{i}, e_{i}\right)=-1$ para $i=1,2$, de dimensão 3 e

v. $(\mathcal{J}(V, f), \square)$ onde $\operatorname{dim}_{\mathbb{R}} V=2$ e $f\left(e_{i}, e_{i}\right)=1$ para $i=1,2$, de dimensão 3.

Considerando somas diretas de tais álgebras obtemos todas as álgebras de Jordan semissimples de dimensão 3 sobre $\mathbb{R}$ : 
Tabela 3.2: $\mathbb{R}$-álgebras de Jordan semissimples de dimensão 3

\begin{tabular}{|c|c|c|c|c|c|}
\hline $\mathcal{T}$ & Tabela de Multiplicação & $\begin{array}{c}\operatorname{dim} \\
\operatorname{Aut}(\mathcal{T})\end{array}$ & $\begin{array}{c}\operatorname{dim} \\
\operatorname{Ann}(\mathcal{T})\end{array}$ & $\begin{array}{c}\operatorname{dim} \\
\mathcal{T}^{2}\end{array}$ & Observação \\
\hline $\mathcal{T}_{1}^{\prime}$ & $\operatorname{Re}_{1} \oplus \mathbb{R} e_{2} \oplus \mathbb{R} e_{3}$ & 0 & 0 & 3 & $\begin{array}{c}\text { Associativa, } \\
\text { Unitária }\end{array}$ \\
\hline $\mathcal{T}_{2}^{\prime}$ & $\mathcal{B}_{4}^{\prime} \oplus \mathbb{R} e_{3}$ & 0 & 0 & 3 & $\begin{array}{c}\text { Associativa, } \\
\text { Unitária }\end{array}$ \\
\hline $\mathcal{T}_{3}^{\prime}$ & $e_{2}^{2}=e_{1} \quad e_{3}^{2}=-e_{1} \quad e_{1} e_{i}=e_{i} i=1,2,3$ & 1 & 0 & 3 & $\begin{array}{c}\text { Unitária, } \\
\text { item iii }\end{array}$ \\
\hline $\mathcal{T}_{4}^{\prime}$ & $e_{2}^{2}=e_{3}^{2}=-e_{1} \quad e_{1} e_{i}=e_{i} i=1,2,3$ & 1 & 0 & 3 & $\begin{array}{c}\text { Unitária, } \\
\text { item iv }\end{array}$ \\
\hline $\mathcal{T}_{5}^{\prime}$ & $e_{2}^{2}=e_{3}^{2}=e_{1} \quad e_{1} e_{i}=e_{i} i=1,2,3$ & 1 & 0 & 3 & $\begin{array}{c}\text { Unitária, } \\
\text { item } \mathrm{v}\end{array}$ \\
\hline
\end{tabular}

\subsection{2 Álgebras de Jordan Reais com Radical de dimensão 1}

Considere $\mathcal{T}$ uma álgebra de Jordan real tal que $\operatorname{dim} N=1$, logo a parte semissimples $\mathcal{T}_{\text {ss }}$ tem dimensão 2 e da seção anterior temos as seguintes possibilidades:

$$
\text { ou } \mathcal{T}_{\text {ss }}=\mathbb{R} e_{1} \oplus \mathbb{R e}_{2} \text { ou } \mathcal{T}_{\text {ss }}=\mathcal{B}_{4}^{\prime} \text {. }
$$

1) Suponha que $\mathcal{T}_{\text {ss }}=\mathbb{R} e_{1} \oplus \mathbb{R} e_{2}$. Então a álgebra $\mathcal{T}^{\#}=\mathcal{T} \oplus \mathbb{R} \cdot 1$ contem 3 idempotentes ortogonais $e_{1}, e_{2}$ e $e_{0}=1-e_{1}-e_{2}$ o que implica na decomposição de Peirce do radical $\mathrm{N}$ :

$$
\mathrm{N}=\mathrm{N}_{00} \oplus \mathrm{N}_{01} \oplus \mathrm{N}_{02} \oplus \mathrm{N}_{11} \oplus \mathrm{N}_{12} \oplus \mathrm{N}_{22}
$$

Seja $n_{1}$ uma base de $N$, então $\mathcal{T}$ fica completamente definida pelo subespaço de Peirce ao qual $n_{1}$ pertence. Das 6 possíveis álgebras obtemos as seguintes 4 não isomorfas:

Tabela 3.3: $\mathbb{R}$-álgebras de Jordan de dimensão 3 com radical unidimensional e parte

\begin{tabular}{|c|c|c|c|c|c|}
\hline $\mathcal{T}$ & Tabela de Multiplicação & $\begin{array}{c}\operatorname{dim} \\
\operatorname{Aut}(\mathcal{T})\end{array}$ & $\begin{array}{c}\operatorname{dim} \\
\operatorname{Ann}(\mathcal{T})\end{array}$ & $\underset{\mathcal{T}^{2}}{\operatorname{dim}}$ & Observação \\
\hline $\mathcal{T}_{6}^{\prime}$ & $\mathbb{R e}_{1} \oplus \mathbb{R} e_{2} \oplus \mathbb{R} n_{1}$ & 1 & 1 & 2 & $\begin{array}{c}\text { Associativa } \\
n_{1} \in N_{00}\end{array}$ \\
\hline $\mathcal{T}_{7}^{\prime}$ & $\mathcal{B}_{2}^{\prime} \oplus \mathbb{R} e_{2}$ & 2 & 0 & 3 & $\mathrm{n}_{1} \in \mathrm{N}_{01}$ \\
\hline
\end{tabular}
semissimples $\mathbb{R e}_{1} \oplus \mathbb{R} e_{2}$ 


\begin{tabular}{|c|c|c|c|c|c|}
\hline $\mathcal{T}$ & Tabela de Multiplicação & $\begin{array}{c}\operatorname{dim} \\
\operatorname{Aut}(\mathcal{T})\end{array}$ & $\begin{array}{c}\operatorname{dim} \\
\operatorname{Ann}(\mathcal{T})\end{array}$ & $\begin{array}{c}\operatorname{dim} \\
\mathcal{T}^{2}\end{array}$ & Observação \\
\hline $\mathcal{T}_{8}^{\prime}$ & $e_{i}^{2}=e_{i} \quad e_{i} n_{1}=\frac{1}{2} n_{1} i=1,2$ & 2 & 0 & 3 & $\begin{array}{c}\text { Unitária } \\
\mathrm{n}_{1} \in \mathrm{N}_{12}\end{array}$ \\
\hline $\mathcal{T}_{9}^{\prime}$ & $\mathcal{B}_{1}^{\prime} \oplus \mathbb{R} e_{2}$ & 1 & 0 & 3 & $\begin{array}{c}\text { Associativa, } \\
\text { Unitária } \\
n_{1} \in \mathrm{N}_{11}\end{array}$ \\
\hline
\end{tabular}

Observamos que no caso em que $n_{1}$ pertença ao subespaço $N_{22}$ temos um elemento idempotente na álgebra agindo como $1 \mathrm{em} n_{1}, \operatorname{logo}$ a álgebra obtida é isomorfa a $\mathcal{T}_{9}^{\prime}$. Se $n_{1} \in N_{02}$ temos somente um elemento idempotente na álgebra agindo como $\frac{1}{2}$ em $\mathrm{n}_{1}, \operatorname{logo}$ a álgebra obtida é isomorfa a $\mathcal{T}_{7}^{\prime}$.

2) Suponha que $\mathcal{T}_{s s}=\mathcal{B}_{4}^{\prime}$. Então, somente temos um elemento idempotente $e_{1}$ que determina a seguinte decomposição de $\mathrm{N}$ :

$$
N=N_{0} \oplus N_{1} \oplus N_{\frac{1}{2}}
$$

Seja $n_{1}$ uma base de $N$ então a ação de $e_{1}$ em $n_{1}$ fica definida pela componente $N_{i}$ à qual $n_{1}$ pertence. Só resta estabelecer como age $e_{2}$ em $n_{1}$. Da tabela de multiplicação de $\mathcal{B}_{4}^{\prime}$ (veja Tabela 3.1) deduzimos que $e_{2} \in \mathcal{P}_{1}$. Suponha que $n_{1} \in N_{0}$, então temos do Teorema 1.18 que $e_{2} n_{1} \in \mathcal{P}_{1} \mathcal{P}_{0}=0$. Agora se $n_{1} \in N_{1}$ como $e_{2} n_{1} \in N$ temos que $e_{2} n_{1}=\alpha n_{1}$ para algum $\alpha \in \mathbb{R}$. Verificando que a base $\left\{e_{1}, e_{2}, n_{1}\right\}$ satisfaça a identidade de Jordan (1.3) obtemos que a única condição real para a constante é $\alpha=0$. No último caso, quando $n_{1} \in N_{\frac{1}{2}}$, não obtemos uma álgebra de Jordan real já que as únicas soluções de $e_{2} n_{1}=\beta n_{1}$ são $\beta= \pm \frac{i}{2}$.

Logo obtemos as seguintes álgebras não isomorfas:

Tabela 3.4: $\mathbb{R}$-álgebras de Jordan de dimensão 3 com radical unidimensional e parte semissimples $\mathcal{B}_{4}^{\prime}$

\begin{tabular}{|c|c|c|c|c|c|}
\hline $\mathcal{T}$ & Tabela de Multiplicação & $\begin{array}{c}\operatorname{dim} \\
\operatorname{Aut}(\mathcal{T})\end{array}$ & $\begin{array}{c}\operatorname{dim} \\
\operatorname{Ann}(\mathcal{T})\end{array}$ & $\begin{array}{c}\operatorname{dim} \\
\mathcal{T}^{2}\end{array}$ & Observação \\
\hline $\mathcal{T}_{10}^{\prime}$ & $\mathcal{B}_{4}^{\prime} \oplus \mathbb{R} n_{1}$ & 1 & 1 & 2 & $\begin{array}{c}\text { Associativa } \\
n_{1} \in N_{0}\end{array}$ \\
\hline $\mathcal{T}_{11}^{\prime}$ & $\begin{array}{c}e_{2}^{2}=-e_{1} \quad e_{1} n_{1}=n_{1} \\
e_{1} e_{i}=e_{i} i=1,2\end{array}$ & 2 & 0 & 3 & $\begin{array}{c}\text { Unitária } \\
n_{1} \in N_{1}\end{array}$ \\
\hline
\end{tabular}




\subsection{3 Álgebras de Jordan Reais com Radical de dimensão 2}

Suponha agora que $\mathcal{T}$ seja uma $\mathbb{R}$-álgebra de Jordan com radical $\mathrm{N}$ de dimensão 2. A única álgebra de Jordan semissimples de dimensão 1 é $\mathcal{T}_{s \mathrm{~s}}=\mathbb{R e}_{1}$, o que implica na seguinte decomposição de Peirce de N:

$$
\mathrm{N}=\mathrm{N}_{0} \oplus \mathrm{N}_{\frac{1}{2}} \oplus \mathrm{N}_{1}
$$

O ideal $\mathrm{N}$ pode ter dois tipos de nilpotência: $(2)$ ou $(1,1)$.

1) Suponha que $N$ tem tipo de nilpotência (2). Logo $N^{2}=0$. Seja $\left\{n_{1}, n_{2}\right\}$ uma base de $N$, então $\mathcal{T}$ fica completamente definida pelos subespaços de Peirce aos quais $n_{1} e$ $n_{2}$ pertencem. Assim obtemos 9 possíveis álgebras das quais as seguintes 6 são não isomorfas:

Tabela 3.5: $\mathbb{R}$-álgebras de Jordan de dimensão 3 com radical de tipo (2)

\begin{tabular}{|c|c|c|c|c|c|}
\hline $\mathcal{T}$ & Tabela de Multiplicação & $\begin{array}{c}\operatorname{dim} \\
\operatorname{Aut}(\mathcal{T})\end{array}$ & $\begin{array}{c}\operatorname{dim} \\
\operatorname{Ann}(\mathcal{T})\end{array}$ & $\begin{array}{c}\operatorname{dim} \\
\mathcal{T}^{2}\end{array}$ & Observação \\
\hline $\mathcal{T}_{12}^{\prime}$ & $e_{1}^{2}=e_{1} \quad e_{1} n_{i}=\frac{1}{2} n_{i} i=1,2$ & 6 & 0 & 3 & $n_{1}, n_{2} \in N_{\frac{1}{2}}$ \\
\hline $\mathcal{T}_{13}^{\prime}$ & $e_{1}^{2}=e_{1} \quad e_{1} n_{i}=n_{i} \quad i=1,2$ & 4 & 0 & 3 & $\begin{array}{c}\text { Associativa, } \\
\text { Unitária, } \\
n_{1}, n_{2} \in N_{1}\end{array}$ \\
\hline $\mathcal{T}_{14}^{\prime}$ & $\mathcal{B}_{2}^{\prime} \oplus \mathbb{R} n_{2}$ & 3 & 1 & 2 & $n_{1} \in N_{\frac{1}{2}}, n_{2} \in N_{0}$ \\
\hline $\mathcal{T}_{15}^{\prime}$ & $\mathcal{B}_{1}^{\prime} \oplus \mathbb{R} n_{2}$ & 2 & 1 & 2 & $\begin{array}{c}\text { Associativa, } \\
n_{1} \in N_{1}, n_{2} \in N_{0}\end{array}$ \\
\hline $\mathcal{T}_{16}^{\prime}$ & $e_{1}^{2}=e_{1} \quad e_{1} n_{1}=\frac{1}{2} n_{1}$ & 3 & 0 & 3 & $\begin{array}{c}n_{1} \in N_{\frac{1}{2}}, n_{2} \in N_{1} \\
e_{1} n_{2}=n_{2}\end{array}$ \\
\hline $\mathcal{T}_{17}^{\prime}$ & $\mathbb{R} e_{1} \oplus \mathbb{R} n_{1} \oplus \mathbb{R} n_{2}$ & 4 & 2 & 1 & $\begin{array}{c}\text { Associativa, } \\
n_{1}, n_{2} \in N_{0}\end{array}$ \\
\hline
\end{tabular}

As outras 3 possibilidades: $n_{1} \in N_{0}$ e $n_{2} \in N_{\frac{1}{2}}, n_{1} \in N_{0}$ e $n_{2} \in N_{1}$ e $n_{1} \in N_{1}$ e $n_{2} \in N_{\frac{1}{2}}$ resultam em uma álgebra isomorfa à álgebra $\mathcal{T}_{14}^{\prime}, \mathcal{T}_{15}^{\prime}$ e $\mathcal{T}_{16}^{\prime}$ respetivamente, basta aplicar o isomorfismo que troca $n_{1} \operatorname{com} n_{2}$.

2) Suponha que $N$ tem tipo de nilpotência $(1,1)$. Analogamente ao caso sobre um corpo algebricamente fechado existe $n \in N$ tal que $N=\mathbb{R} n+\mathbb{R} n^{2}$ com $n^{3}=0$. Suponha primeiramente que $\mathrm{N}$ é dado por uma única componente de Peirce de dimensão 2, isto é $N=N_{i}$ então $N_{i}^{2} \subseteq N_{0} \oplus N_{1}$. Se $i=\frac{1}{2}$ então $N^{2}=0$ o que contradiz o fato de $N$ ter tipo de nilpotência $(1,1), \operatorname{logo} i=0$ ou $i=1$. Agora suponha que $N=N_{i} \oplus N_{\frac{1}{2}}$ com $\operatorname{dim} N_{i}=\operatorname{dim} N_{\frac{1}{2}}=1$ para $i=0,1$ então podemos escolher o $n$ da base como 
sendo um elemento de $N_{\frac{1}{2}}$. De fato se $N_{i}=\mathbb{R a}$ e $N_{\frac{1}{2}}=\mathbb{R} b$, então temos $b^{2} \in N_{i}$ $\operatorname{logo} b^{2}=\alpha a$ para algum $\alpha \in \mathbb{R}$. Note que $\alpha \neq 0$ pois pela nilpotência $a^{2}=a b=0$. Consequentemente $\mathrm{N}=\mathbb{R b} \oplus \mathbb{R} \mathrm{b}^{2}$.

Por último se $N=N_{0} \oplus N_{1}$, então da nilpotência de $N$ temos que $N_{0}^{2}=N_{1}^{2}=0$ e da tabela de multiplicação das componentes de Peirce $\mathrm{N}_{0} \mathrm{~N}_{1}=0 \operatorname{logo} \mathrm{N}^{2}=0$ o que é uma contradição.

Assim obtemos as seguintes álgebras não isomorfas, onde $n_{1}^{2}=n_{2}$.

Tabela 3.6: $\mathbb{R}$-álgebras de Jordan de dimensão 3 com radical de tipo $(1,1)$

\begin{tabular}{|c|c|c|c|c|c|}
\hline $\mathcal{T}$ & Tabela de Multiplicação & $\begin{array}{c}\operatorname{dim} \\
\operatorname{Aut}(\mathcal{T})\end{array}$ & $\begin{array}{c}\operatorname{dim} \\
\operatorname{Ann}(\mathcal{T})\end{array}$ & $\begin{array}{c}\operatorname{dim} \\
\mathcal{T}^{2}\end{array}$ & Observação \\
\hline $\mathcal{T}_{18}^{\prime}$ & $\begin{array}{c}e_{1}^{2}=e_{1} \quad n_{1}^{2}=n_{2} \\
e_{1} n_{i}=n_{i} i=1,2\end{array}$ & 2 & 0 & 3 & $\begin{array}{c}\text { Associativa, } \\
\text { Unitária, } \\
n_{1}, n_{2} \in N_{1}\end{array}$ \\
\hline $\mathcal{T}_{19}^{\prime}$ & $e_{1}^{2}=e_{1} \quad n_{1}^{2}=n_{2} \quad e_{1} n_{1}=\frac{1}{2} n_{1}$ & 2 & 1 & 3 & $\begin{array}{c}n_{1} \in N_{\frac{1}{2}} \\
n_{2} \in N_{0}\end{array}$ \\
\hline $\mathcal{T}_{20}^{\prime}$ & $e_{1}^{2}=e_{1} \quad n_{1}^{2}=e_{1} n_{2}=n_{2}$ & 2 & 0 & 3 & $\begin{array}{c}n_{1} \in N_{\frac{1}{2}} \\
n_{2} \in N_{1}\end{array}$ \\
\hline $\mathcal{T}_{21}^{\prime}$ & $e_{1} n_{1}=\frac{1}{2} n_{1}$ & 2 & 1 & 2 & $\begin{array}{c}\text { Associativa, } \\
n_{1}, n_{2} \in N_{0}\end{array}$ \\
\hline
\end{tabular}

\subsection{4 Álgebras de Jordan Reais Nilpotentes}

Seja $\mathcal{T}$ uma álgebra de Jordan nilpotente de dimensão 3 sobre $\mathbb{R}$, então $\mathcal{T}$ pode ter os seguintes tipos de nilpotência:

1) Suponha que $\mathcal{T}$ tem tipo de nilpotência (3). Isto significa que $\mathcal{T}^{2}=0$, logo só temos uma álgebra que satisfaz essa condição:

Tabela 3.7: $\mathbb{R}$-álgebra de Jordan nilpotente de dimensão 3 de tipo (3)

\begin{tabular}{|c|c|c|c|c|c|}
\hline $\mathcal{T}$ & $\begin{array}{c}\text { Tabela de } \\
\text { Multiplicação }\end{array}$ & $\begin{array}{c}\operatorname{dim} \\
\operatorname{Aut}(\mathcal{T})\end{array}$ & $\begin{array}{c}\operatorname{dim} \\
\operatorname{Ann}(\mathcal{T})\end{array}$ & $\begin{array}{c}\operatorname{dim} \\
\mathcal{T}^{2}\end{array}$ & Observação \\
\hline $\mathcal{T}_{22}^{\prime}$ & $\mathbb{R} n_{1} \oplus \mathbb{R} n_{2} \oplus \mathbb{R} n_{3}$ & 9 & 3 & 0 & Associativa \\
\hline
\end{tabular}

2) Suponha que $\mathcal{T}$ tem tipo de nilpotência $(1,1,1)$. Então por definição $\mathcal{T}^{\langle 4\rangle}=0$, $\operatorname{dim} \mathcal{T}^{3}=1$ e $\operatorname{dim} \mathcal{T}^{2}=2$. Seja $n_{1}$ uma base de $\mathcal{T}^{3}$, completando a uma base de $\mathcal{T}^{2}$ e de 
$\mathcal{T}$ temos que existem $n_{2} \in \mathcal{T}^{2}$ e $n_{3} \in \mathcal{T}$ tal que $\mathcal{T}^{2}=\mathbb{R} n_{1}+\mathbb{R} n_{2}$ e $\mathcal{T}=\mathbb{R} n_{1}+\mathbb{R} n_{2}+\mathbb{R} n_{3}$ com produtos

$$
\begin{aligned}
& n_{1}^{2}, n_{1} n_{2}, n_{1} n_{3} \in \mathcal{T}^{\langle 4\rangle}=0 \quad n_{3}^{2}=\alpha n_{1}+\beta n_{2} \text { pois } n_{3}^{2} \in \mathcal{T}^{2} \\
& n_{2} n_{3}=\gamma n_{1} \text { e } n_{2}^{2}=\delta n_{1} \text { pois } n_{2} n_{3}, n_{2}^{2} \in \mathcal{T}^{3} .
\end{aligned}
$$

Verificando que a base $\left\{n_{1}, n_{2}, n_{3}\right\}$ satisfaça a identidade de Jordan (1.3) obtemos ${ }^{1}$ condições para as constantes: ou $\beta=0$ ou $\delta=0$. Mas se $\beta=0$ então isto implicaria que $\operatorname{dim} \mathcal{T}^{2}=1$ o que é uma contradição, logo temos que $\beta \neq 0$ e, portanto, $\delta=0$. Pelo mesmo argumento concluímos que $\gamma \neq 0$. Assim neste caso somente existe uma álgebra:

Tabela 3.8: $\mathbb{R}$-álgebra de Jordan nilpotente de dimensão 3 de tipo $(1,1,1)$

\begin{tabular}{|c|c|c|c|c|c|}
\hline $\mathcal{T}$ & $\begin{array}{c}\text { Tabela de } \\
\text { Multiplicação }\end{array}$ & $\begin{array}{c}\operatorname{dim} \\
\operatorname{Aut}(\mathcal{T})\end{array}$ & $\begin{array}{c}\operatorname{dim} \\
\operatorname{Ann}(\mathcal{T})\end{array}$ & $\begin{array}{c}\operatorname{dim} \\
\mathcal{T}^{2}\end{array}$ & Observação \\
\hline $\mathcal{T}_{23}^{\prime}$ & $\mathrm{n}_{2} \mathrm{n}_{3}=\mathrm{n}_{1} \quad \mathrm{n}_{3}^{2}=\mathrm{n}_{2}$ & 3 & 1 & 2 & Associativa \\
\hline
\end{tabular}

Considere uma álgebra $(\mathcal{T}, \cdot)$ com base $\left\{N_{1}, N_{2}, N_{3}\right\}$ tal que o produto satisfaz (3.1) para certos coeficientes $\alpha, \beta \neq 0, \gamma \neq 0$ e $\delta=0$. Então $\mathcal{T}$ é isomorfa a $\mathcal{T}_{23}^{\prime}$ com isomorfismo dado por $N_{1} \mapsto \beta^{-1} \gamma^{-1} n_{1}$ e $N_{2} \mapsto-\frac{\alpha}{\beta^{2} \gamma} n_{1}+\beta^{-1} n_{2}$ cujo determinante $\frac{1}{\beta^{2} \gamma}$ é bem definido e não nulo.

3) Suponha que $\mathcal{T}$ tem tipo de nilpotência $(2,1)$. Então $\mathcal{T}^{3}=0$ e $\operatorname{dim} \mathcal{T}^{2}=1$. Seja $n_{3}$ uma base de $\mathcal{T}^{2}$, completando a uma base de $\mathcal{T}$ temos que existem $n_{1}, n_{2} \in \mathcal{T}$ tal que $\mathcal{T}=\mathbb{R} n_{1}+\mathbb{R} n_{2}+\mathbb{R} n_{3}$ com produtos

$$
\begin{aligned}
& n_{3}^{2}, n_{1} n_{3}, n_{2} n_{3} \in N^{3}=0 \\
& n_{1}^{2}=\alpha n_{3}, n_{2}^{2}=\beta n_{3}, n_{1} n_{2}=\gamma n_{3}
\end{aligned}
$$

Para quaisquer valores de $\alpha, \beta$ e $\gamma$ a álgebra obtida $\mathcal{T}$ é de Jordan, mas é claro que pelo menos um deles deve ser não nulo para $\mathcal{T}$ ter tipo de nilpotência $(2,1)$. Provaremos a seguir que se $(\mathcal{T}, \cdot)$ é uma álgebra com base $\left\{N_{1}, N_{2}, N_{3}\right\}$ tal que o produto $\cdot$ satisfaz (3.2) para certos coeficientes $\alpha, \beta, \gamma \in \mathbb{R}$ então $\mathcal{T}$ é isomorfa a uma das seguintes álgebras:

Tabela 3.9: $\mathbb{R}$-álgebras de Jordan nilpotentes de dimensão 3 de tipo $(2,1)$

\begin{tabular}{|c|c|c|c|c|c|}
\hline $\mathcal{T}$ & Tabela de & $\operatorname{dim}$ & $\operatorname{dim}$ & $\operatorname{dim}$ & Observação \\
& Multiplicação & $\operatorname{Aut}(\mathcal{T})$ & $\operatorname{Ann}(\mathcal{T})$ & $\mathcal{T}^{2}$ & \\
\hline
\end{tabular}

\footnotetext{
1 Utilizando um programa de computação simbólica elaborado pela autora, veja Apêndice A.
} 


\begin{tabular}{|c|c|c|c|c|c|}
\hline $\mathcal{T}$ & $\begin{array}{c}\text { Tabela de } \\
\text { Multiplicação }\end{array}$ & $\begin{array}{c}\operatorname{dim} \\
\operatorname{Aut}(\mathcal{T})\end{array}$ & $\begin{array}{c}\operatorname{dim} \\
\operatorname{Ann}(\mathcal{T})\end{array}$ & $\begin{array}{c}\operatorname{dim} \\
\mathcal{T}^{2}\end{array}$ & Observação \\
\hline $\mathcal{T}_{24}^{\prime}$ & $\mathrm{n}_{1}^{2}=\mathrm{n}_{2}^{2}=\mathrm{n}_{3}$ & 4 & 1 & 1 & Associativa \\
\hline $\mathcal{T}_{25}^{\prime}$ & $\mathcal{B}_{3}^{\prime} \oplus \mathbb{R} n_{3}$ & 5 & 2 & 1 & Associativa \\
\hline $\mathcal{T}_{26}^{\prime}$ & $\mathrm{n}_{1} \mathrm{n}_{2}=\mathrm{n}_{3}$ & 4 & 1 & 1 & Associativa \\
\hline
\end{tabular}

Suponha que $\beta=0$ então, se $\gamma \neq 0$ temos que $\mathcal{T} \simeq \mathcal{T}_{26}$ com isomorfismo $N_{1} \mapsto$ $n_{1}+\frac{\alpha}{2 \gamma} n_{2}$ e $N_{3} \mapsto \gamma^{-1} n_{3}$ de determinante $\gamma^{-1}$. Mas se $\gamma=0$, então necessariamente $\alpha \neq 0$ e neste caso $\mathcal{T}$ é isomorfa a $\mathcal{T}_{25}$ com isomorfismo definido por $\mathrm{N}_{2} \mapsto n_{3}$ e $\mathrm{N}_{3}=\alpha^{-1} \mathrm{n}_{2}$.

Por outro lado, se consideramos $\beta \neq 0$ temos três possibilidades. Vamos denotar $-\alpha \beta+\gamma^{2}$ por $\Delta$ para simplificar a notação, então:

i. Se $\Delta>0$, novamente $\mathcal{T}$ é $\mathcal{T}_{26}$ com isomorfismo dado por $N_{1} \mapsto\left(\frac{1}{2}+\frac{\gamma}{2 \sqrt{\Delta}}\right) n_{1}+$ $\left(-\frac{1}{2}+\frac{\gamma}{2 \sqrt{\Delta}}\right) n_{2}, N_{2} \mapsto \frac{\beta}{2 \sqrt{\Delta}} n_{1}+\frac{\beta}{2 \sqrt{\Delta}} n_{2}$ e $N_{3} \mapsto \frac{\beta}{2 \Delta} n_{3}$ cujo determinante $\frac{\beta^{2}}{4 \Delta^{\frac{3}{2}}}$ é bem definido a não nulo.

ii. Se $\Delta<0$, neste caso $\mathcal{T} \simeq \mathcal{T}_{24}$ com isomorfismo $N_{1} \mapsto n_{1}+\frac{\gamma}{\sqrt{-\Delta}} n_{2}, N_{2} \mapsto \frac{\beta}{\sqrt{-\Delta}} n_{2}$ e $N_{3} \mapsto-\frac{\beta}{\Delta} n_{3}$ de determinante $\frac{\beta^{2}}{(-\Delta)^{\frac{3}{2}}}$.

iii. Por último, se $\Delta=0$ então $\alpha=\frac{\gamma^{2}}{\beta}$ e $\mathcal{T} \simeq \mathcal{T}_{25}$. Se considerarmos que $\gamma=0$ (e $\operatorname{logo} \alpha=0$ ) então o isomorfismo é dado por $N_{1} \mapsto n_{3}, N_{2} \mapsto n_{1}$ e $N_{3} \mapsto \beta^{-1} n_{2}$, mas se supomos $\gamma \neq 0$ (e portanto $\alpha \neq 0$ ) então o isomorfismo é definido por $\mathrm{N}_{1} \mapsto \mathrm{n}_{1}+\mathrm{n}_{3}, \mathrm{~N}_{2} \mapsto \alpha^{-1} \gamma \mathrm{n}_{1}$ e $\mathrm{N}_{3} \mapsto \alpha^{-1} n_{2}$ cujo determinante $\frac{\gamma}{\alpha^{2}}$ é bem definido e não nulo.

\subsubsection{Observações}

Vimos em cada caso que dada qualquer álgebra de Jordan $\mathcal{T}$ de dimensão 3 sobre $\mathbb{R}$ então $\mathcal{T}$ é uma das álgebras $\mathcal{T}_{1}^{\prime}$ a $\mathcal{T}_{26}^{\prime}$. Para finalizar a classificação algébrica só resta provar que todas elas são diferentes.

Teorema 3.1. As álgebras $\mathcal{T}_{1}^{\prime}$ a $\mathcal{T}_{26}^{\prime}$ são duas a duas não isomorfas.

Demonstração. Comparando os invariantes $\operatorname{dim} \operatorname{Rad}(\mathcal{T})$, tipo de nilpotência $\operatorname{de} \operatorname{Rad}(\mathcal{T})$, $\operatorname{dim} \operatorname{Ann}(\mathcal{T}), \operatorname{dim} \operatorname{Aut}(\mathcal{T})$ e $\operatorname{dim} \mathcal{T}^{2}$, junto com as propriedades de $\mathcal{T}$ ser indecomponível, associativa, não associativa e unitária, somente necessitamos provar que não existe isomorfismo entre $\mathcal{T}_{3}^{\prime}, \mathcal{T}_{4}^{\prime}, \mathcal{T}_{5}^{\prime}$ e entre $\mathcal{T}_{24}^{\prime}$ e $\mathcal{T}_{26}^{\prime}$. 
Primeiro observamos que as álgebras $\mathcal{T}_{3}^{\prime}, \mathcal{T}_{4}^{\prime}$ e $\mathcal{T}_{5}^{\prime}$ proveem de formas bilineares simétricas não degeneradas não isomorfas as quais geram álgebras de Jordan não são isomorfas.

Por último veja que a álgebra nula $\mathbb{R} n_{1} \oplus \mathbb{R} n_{2}$ de dimensão 2 , é uma subálgebra de $\mathcal{T}_{26}^{\prime}$ mas não é subálgebra de $\mathcal{T}_{24}^{\prime}$ (veja Apêndice C), portanto $\mathcal{T}_{24}^{\prime} \nsucceq \mathcal{T}_{26}^{\prime}$.

Analogamente ao Teorema 2.3 temos o seguinte fato:

Teorema 3.2. Todas as álgebras de este capítulo são especiais. 
Nossa principal motivação para realizar a classificação algébrica das álgebras de Jordan nos capítulos anteriores vem da intenção de entender a variedade das álgebras de Jordan, i.e., de descrever geometricamente tais álgebras. A classificação geométrica é o problema de entender a geometria da variedade das álgebras de Jordan ,i.e., de determinar a lista completa das estruturas que denominaremos de rígidas (pois veremos que estas álgebras gerarão as componentes irredutíveis da variedade), de determinar o número de órbitas sob a ação do grupo geral linear $\mathrm{G}=\mathrm{GL}(\mathrm{V})$ e de encontrar todas as possíveis deformações entre tais álgebras.

Este capítulo foi dividido em 5 seções. Na primeira seção apresentaremos uma rápida revisão de alguns conceitos e terminologia da geometria algébrica que serão necessários para entender a geometria da variedade das álgebras de Jordan. Na segunda seção apresentaremos o conceito de deformação a um parâmetro introduzido por Gerstenhaber em [14] e os conceitos de rigidez analítica e infinitesimal. Provaremos que rigidez infinitesimal implica em rigidez analítica.

Na terceira seção introduziremos a definição de variedade das álgebras de Jordan de dimensão $n$, Jor ${ }_{n}$, assim como também as noções de deformação de uma álgebra e de rigidez geométrica. Reuniremos os resultados existentes na literatura que relacionam os três conceitos de rigidez. Provaremos que, sempre que a variedade tenha um número finito de G-órbitas, cada componente irredutível é determinada pelo fecho de Zariski da órbita de uma álgebra (geometricamente) rígida. Apresentaremos também certas "operações" que preservam algum dos tipos de rigidez e daremos contraexemplos de outras em que isso não acontece.

Na quarta seção introduziremos uma lista de critérios que determinarão a não existência de deformação entre um par de álgebras dadas, os quais nos permitirão determinar as álgebras rígidas e logo as componentes irredutíveis de uma variedade. Por último, na Seção 4.5 mostraremos que em toda variedade de álgebras de Jordan de dimensão maior ou igual a 5 existem pelo menos 3 componentes irredutíveis que provêm de álgebras rígidas que são indecomponíveis, não associativas e não semissimples. 


\subsection{IntRoduÇÃO À GEOMETRIA ALGÉBRICA}

Nesta seção daremos uma rápida revisão de alguns conceitos e terminologia da geometria algébrica com ênfase especial nos grupos algébricos e sua ação em variedades. No que se segue consideraremos que o corpo $\mathbf{k}$ de char $\mathbf{k} \neq 2$ é algebricamente fechado ou $\mathbb{R}$.

Como referências para os resultados que apresentaremos recomendamos os trabalhos [16], [8], [29] e [6].

\subsubsection{Variedades Algébricas}

Seja $P=\mathbf{k}\left[x_{1}, \ldots, x_{n}\right]$ o anel dos polinômios em $n$ variáveis sobre $\mathbf{k}$. Um polinômio $f \in P$ define uma função $f: \mathbf{k}^{n} \rightarrow \mathbf{k}$, o valor de $f$ em um ponto $\left(a_{1}, \ldots, a_{n}\right) \in \mathbf{k}^{n}$ é obtido substituindo os $x_{i}$ pelos $a_{i}$ em $f$, i.e., $f$ leva $\left(a_{1}, \ldots, a_{n}\right) \mapsto f\left(a_{1}, \ldots, a_{n}\right)$. A função definida por $f$ é chamada de função polinomial no espaço vetorial $\mathbf{k}^{n}$ de dimensão $n$ sobre $\mathbf{k}$, com valores em $\mathbf{k}$.

Diremos que $f$ se anula em $\left(a_{1}, \ldots, a_{n}\right)$ ou, equivalentemente, que $\left(a_{1}, \ldots, a_{n}\right)$ é um zero de $f$ se $f\left(a_{1}, \ldots, a_{n}\right)=0$.

Dado $S \subseteq$ P um subconjunto do anel dos polinômios definimos o anulador de $S$ como sendo

$$
V(S)=\left\{\left(a_{1}, \ldots, a_{n}\right) \in \mathbf{k}^{n} \mid f\left(a_{1}, \ldots, a_{n}\right)=0 \quad \forall f \in S\right\}
$$

Note que se I é o ideal de $\mathrm{P}$ gerado por $\mathrm{S}$ então $\mathrm{V}(\mathrm{I})=\mathrm{V}(\mathrm{S})$. Mais ainda, como P é um anel noetheriano, todo ideal I tem um número finito de geradores. Logo $\mathrm{V}(\mathrm{S})$ pode ser expressado como os zeros comuns de um conjunto finito de polinômios $f_{1}, \ldots, f_{r}$.

Definição 4.1. Um subconjunto de $\mathbf{k}^{n}$, é dito algébrico se ele é o anulador $\mathrm{V}(\mathrm{S})$ de algum conjunto de polinômios $\mathrm{S}$.

Como $\bigcap_{i} V\left(S_{i}\right)=V\left(\bigcup_{i} S_{i}\right)$, a interseção de qualquer coleção de conjuntos algébricos é um conjunto algébrico. Mais ainda, se definimos $S_{1} S_{2}$ como sendo o conjunto consistindo de todos os produtos de um elemento de $S_{1}$ por um elemento de $S_{2}$ então $\mathrm{V}\left(\mathrm{S}_{1}\right) \cup \mathrm{V}\left(\mathrm{S}_{2}\right)=\mathrm{V}\left(\mathrm{S}_{1} \mathrm{~S}_{2}\right)$ ou seja a união de dois conjuntos algébricos é de novo um conjunto algébrico. Por último $\emptyset=\mathrm{V}(1)$ e $\mathbf{k}^{n}=\mathrm{V}(0)$ são ambos conjuntos algébricos. Para a demostração destes fatos veja [16, Prop. 1.1 p.2].

As propriedades anteriores nos permitem definir uma topologia em $\mathbf{k}^{n}$ considerando os conjuntos fechados como sendo os conjuntos algébricos de $\mathbf{k}^{n}$. Esta topologia é chamada de topologia de Zariski. 
Definição 4.2. Um n-espaço afim $\mathbb{A}^{n}$ é um espaço topológico, o qual é $\mathbf{k}^{\mathfrak{n}}$ como conjunto dotado da topologia de Zariski.

O nosso objetivo é definir uma variedade algébrica, para isso precisaremos das seguintes duas definições:

Definição 4.3. Em um espaço topológico, um subconjunto é localmente fechado se é aberto no seu fecho ou, equivalentemente, se é a interseção de um conjunto aberto e um fechado.

Definição 4.4. Se $X \subseteq \mathbb{A}^{n}$ é localmente fechado, uma função $f: X \rightarrow \mathbf{k}$ é dita regular no ponto $p \in X$ se existe uma vizinhança aberta $U$ com $p \in U \subseteq X$ e polinômios $g, h \in P$ tal que $h$ nunca se anula em $U$ e $f=g / h$ em $U$. Denotamos o conjunto das funções regulares em $p$ por $\mathcal{O}_{p}$. Dizemos que $f$ é regular em $X$ se é regular em todo ponto de $X$. $O$ conjunto de aplicações regulares em $X$ é denotado por

$$
\mathcal{O}(X)=\{f: X \rightarrow \mathbf{k} \mid \text { f é regular em } X\} .
$$

Ou seja, funções regulares são funções que são localmente quocientes de polinômios.

Definição 4.5. Um subconjunto não vazio $X$ de um espaço topológico $Y$ é chamado de irredutível se não pode ser decomposto como a união de dois subconjuntos fechados próprios (onde a topologia de $X$ é a topologia induzida da topologia de $Y$ ). Ou seja, $X$ é irredutível se $\emptyset \neq X=X_{1} \cup X_{2} \operatorname{com} X_{1}$ e $X_{2}$ fechados, implica $X_{1}=X$ ou $X_{2}=X$.

Todo subconjunto aberto não vazio de um espaço irredutível é irredutível e denso. Além disso, se $X$ é um subconjunto irredutível de $Y$ então seu fecho $\bar{X}$ em $Y$ é também irredutível, [16, p. 3].

Definição 4.6. Uma variedade algébrica (ou simplesmente variedade) X é um subconjunto localmente fechado de $\mathbb{A}^{n}$ dotado de sua topologia e da coleção $\mathcal{O}(\mathrm{U})$ para todo $\mathrm{U}$ aberto em $\mathrm{X}$.

Claramente uma subvariedade de uma variedade algébrica $X$ é um subconjunto de $X$ que é uma variedade. Todo subconjunto fechado de uma variedade é uma subvariedade [29, p.12].

Definição 4.7. Um morfismo $\phi: X \rightarrow Y$ entre variedades é uma aplicação contínua tal que para todo $\mathrm{U} \subseteq \mathrm{Y}$ e toda aplicação regular $\theta: \mathrm{U} \rightarrow \mathbf{k}$ a composição

$$
\phi^{-1}(\mathrm{U}) \stackrel{\phi}{\longrightarrow} \mathrm{U} \stackrel{\theta}{\longrightarrow} \mathbf{k}
$$

é regular. 
Observamos que um isomorfismo neste caso é um morfismo com uma inversa, o que não é o mesmo que um morfismo bijetivo.

Conjuntos algébricos são exemplos de um tipo especial de variedade que definiremos a seguir.

Definição 4.8. Uma variedade afim é uma variedade que é isomorfa a um subconjunto fechado de $\mathbb{A}^{n}$ para algum $n$.

Definição 4.9. Seja $Y$ um subconjunto fechado de um espaço topológico $X$. Um ponto $x \in Y$ é chamado de ponto genérico de $Y$ se $Y=\overline{\{x\}}$, o fecho de $\{x\}$ em $X$.

Se $Y$ tem um ponto genérico então $Y$ é irredutível [29, p.25].

Toda variedade afim admite uma decomposição em um número finito de componentes irredutíveis, i.e., subconjuntos fechados irredutíveis maximais ([16, Prop.1.5, p.5], [29, Cor. 2.15, p.13]). Como as componentes irredutíveis são fechadas elas são subvariedades. Observamos que neste trabalho a definição de variedade algébrica não é a padrão. Geralmente, variedades são definidas como sendo irredutíveis, no entanto, de acordo com a literatura de classificação geométrica de estruturas algébricas, não impomos esta condição pois um dos objetivos do problema da classificação geométrica é determinar as componentes irredutíveis de uma variedade algébrica.

\subsubsection{Dimensão}

Nesta seção daremos um significado algébrico preciso à noção geométrica de dimensão.

Se $X$ é um espaço topológico, definimos a dimensão de $X$, que denotamos $\operatorname{dim} X$, como sendo o supremo dos comprimentos das cadeias $X_{0} \subset X_{1} \subset \cdots \subset X_{n}$ de subconjuntos fechados irredutíveis não vazios distintos de $X$. Em particular, definimos a dimensão de uma variedade algébrica como sendo sua dimensão como espaço topológico.

A dimensão de uma variedade algébrica possui as seguintes propriedades:

i. Se $X \subseteq Y$ então $\operatorname{dim} X \leqslant \operatorname{dim} Y$. A desigualdade é estrita se $Y$ é irredutível e $X$ é fechado.

ii. $\operatorname{dim} \mathbb{A}^{n}=n$.

iii. $\operatorname{dim} \emptyset=-\infty$.

iv. Se $U \neq \emptyset$ é aberto em uma variedade irredutível $X$ então $\operatorname{dim} U=\operatorname{dim} X$.

v. Se $X$ e $Y$ são variedades irredutíveis então $\operatorname{dim} X \times Y=\operatorname{dim} X+\operatorname{dim} Y$. 


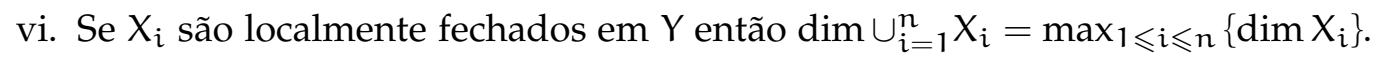

vii. $\operatorname{dim} X$ é independente da escolha do corpo de definição $\mathbf{k}$.

viii. Se $X$ é uma variedade algébrica afim então $\operatorname{dim} X$ é finita.

Para a demostração destes fatos consulte [29, Prop.II.3.11] e [8, Cap.3]

\subsubsection{Ação de Grupos}

Nesta seção apresentaremos alguns resultados sobre grupos algébricos, em especial sobre a ação desses grupos em variedades algébricas. Esses resultados serão importantes para a caracterização geométrica da variedade das álgebras de Jordan, Jor ${ }_{n}$, que apresentaremos na Seção 4.3.

Para maiores informações sobre os resultados aqui apresentados, veja $[7,18,9,10]$.

Definição 4.10. Um grupo algébrico é uma variedade $G$ junto com:

i. um elemento $e \in G$;

ii. um morfismo $\mu: G \times G \rightarrow G$ denotado por $(x, y) \mapsto x y$;

iii. um morfismo $i: G \times G \rightarrow G$ denotado por $x \mapsto x^{-1}$,

em relação aos quais (o conjunto) G é um grupo.

Um morfismo de grupos algébricos é um morfismo de variedades que é também um homomorfismo de grupos. Denominaremos isomorfismos os morfismos que possuam inversas e estas também sejam morfismos.

Exemplo 4.11. [18, Cap II.7.1 e II.7.3] O conjunto $\operatorname{Mat}_{\mathfrak{n}}(\mathbf{k})$ de todas as matrizes $\mathrm{n} \times \mathrm{n}$ com entradas em $\mathbf{k}$ pode ser identificado com $\mathbb{A}^{n^{2}}$ e o grupo geral linear $G L_{n}(\mathbf{k})$, i.e., o conjunto das matrizes invertíveis $\mathrm{n} \times \mathrm{n}$ com entradas em $\mathbf{k}$, com o subconjunto aberto definido pelo não-anulamento do polinômio det, visto assim como uma variedade afim, mais ainda $\mathrm{GL}_{n}(\mathbf{k})$ é uma variedade irredutível. As expressões para a multiplicação e inversão de matrizes deixam claro que $\mathrm{GL}_{n}(\mathbf{k})$ é um grupo algébrico.

Seja V um espaço vetorial sobre o corpo $\mathbf{k}$ dimensão finita $n$. O grupo geral linear de $\mathrm{V}$, i.e., o conjunto de todas as transformações lineares bijetivas de $\mathrm{V}$ em $\mathrm{V}$, denotado por $\mathrm{GL}(\mathrm{V})$, junto com a composição de funções como operação de grupo, é isomorfo a $\mathrm{GL}_{n}(\mathbf{k})$ e, portanto, é também um grupo algébrico.

Por simplicidade, doravante, vamos assumir que o grupo G é uma variedade irredutível pois nosso principal interesse está na ação do grupo algébrico GL(V) que é irredutível. 
Definição 4.12. Dizemos que um grupo algébrico $G$ age numa variedade algébrica $X$ se existe um morfismo $\varphi: G \times X \rightarrow X$ que é uma ação de grupo.

Seja $G$ um grupo algébrico agindo em uma variedade $X$. Para cada ponto $x$ em $X$ definimos a órbita de $x$ sobre a ação de $G$ como sendo o conjunto $x^{G}=\{g \cdot x \mid g \in G\}$ e o subgrupo estabilizador de $x$ como sendo o conjunto de todos os elementos em $G$ que fixam $x$, i.e., $\operatorname{Stab}_{\mathrm{G}}(x)=\{\mathrm{g} \in \mathrm{G} \mid \mathrm{g} \cdot \mathrm{x}=\mathrm{x}\}$.

As propriedades de grupo garantem que a relação definida por " $x \sim y$ se e somente se existe $g \in G$ tal que $g \cdot x=y$ " seja uma relação de equivalência. As órbitas são então as classes de equivalência sob essa relação. Logo dois elementos $x$ e y são equivalentes se e somente se suas órbitas são as mesmas, i.e., $x^{G}=y^{G}$.

Lema 4.13. [8, 18, Cap.8.2,8.3] Seja G um grupo algébrico agindo em uma variedade X, então:

i. Cada órbita $x^{G}$ é localmente fechada e irredutível.

ii. $\operatorname{dim} x^{G}=\operatorname{dim} G-\operatorname{dim} \operatorname{Stab}_{G}(x)$.

iii. $\overline{\chi^{G}} \backslash x^{G}$ é uma união de órbitas de dimensão estritamente menor que $\operatorname{dim} x^{G}$, onde $\overline{\chi^{\mathrm{G}}}$ representa o fecho algébrico da órbita de $x$ na topologia de Zariski. Em particular, órbitas de dimensão minimal são fechadas.

iv. O conjunto $\left\{x \in X \mid \operatorname{dim} x^{G} \leqslant s\right\}$ é fechado e o conjunto $\left\{x \in X \mid \operatorname{dim} x^{G}=s\right\}$ é localmente fechado.

v. Se $Y$ é uma componente irredutível de $X$ então $Y$ é invariante sob a ação de $G$, i.e., $Y=Y^{G}=\{g \cdot y \mid y \in Y$ e $g \in G\}$.

Segue do item iii. que se a variedade $X$ tem um número finito de órbitas então $\overline{\chi^{G}}=$ $\chi^{G} \cup\left(\bigcup_{i=1}^{n} x_{i}^{G}\right) \operatorname{com} \operatorname{dim} x_{i}^{G}<\operatorname{dim} x^{G}$ para todo $1 \leqslant i \leqslant n$. Então dos resultados da Seção 4.1.2 temos

$$
\operatorname{dim} \overline{x^{G}}=\max _{1 \leqslant i \leqslant n}\left\{\operatorname{dim} x^{G}, \operatorname{dim} x_{i}^{G}\right\}=\operatorname{dim} x^{G} .
$$

\subsection{DEFORMAÇÕES INFInitesimais DE ÁlgeBRAS DE JOR- $\mathrm{DAN}$}

Seja $\mathcal{J}$ uma álgebra de Jordan sobre um corpo $\mathbf{k}$ de char $\mathbf{k} \neq 2$ e $\mathrm{V}$ o espaço vetorial subjacente a $\mathcal{J}$. Denotamos por $R=\mathbf{k}[[t]]$ ao anel das séries de potências formais em uma 
variável $t$, por $K=\mathbf{k}((t))$ ao corpo de frações de $R$ e por $V_{K}$ ao espaço vetorial obtido de $\mathrm{V}$ estendendo o domínio de coeficientes de $\mathbf{k}$ a $\mathrm{K}$, i.e., $\mathrm{V}_{\mathrm{K}}=\mathrm{V} \otimes_{\mathbf{k}} \mathrm{K}$. Observamos que qualquer função bilinear $\mathrm{f}: \mathrm{V} \times \mathrm{V} \rightarrow \mathrm{V}$ (em particular o produto de $\mathcal{J}$ ) pode ser estendida a uma função bilinear sobre $K$ de $V_{K} \times V_{K}$ em $V_{K}$.

Suponha que é dada uma função bilinear $f_{t}: V_{K} \times V_{K} \rightarrow V_{K}$ expressa na forma

$$
f_{t}(a, b)=a b+t F_{1}(a, b)+t^{2} F_{2}(a, b)+\cdots,
$$

onde $F_{i}$ é uma função bilinear definida sobre $\mathbf{k}$ e $F_{0}(a, b)=a b$ é o produto em $J$. Suponha ainda que $f_{t}$ é um produto de Jordan, i.e.,

$$
\begin{aligned}
f_{t}(a, b) & =f_{t}(b, a), \\
f_{t}\left(f_{t}(a, a), f_{t}(b, a)\right) & =f_{t}\left(f_{t}\left(f_{t}(a, a), b\right), a\right),
\end{aligned}
$$

para todo $a, b \in V_{K}$ (ou equivalentemente, para todo $a, b \in V$ ). Então podemos considerar a álgebra de Jordan $\partial_{t}$ cujo espaço vetorial subjacente é $V_{K}$ e cujo produto é $f_{t}$.

Definição 4.14. Nas condições acima dizemos que $\mathcal{J}_{t}=\left(V_{K}, f_{t}\right)$ (ou, quando for necessário fazer referencia à multiplicação, diremos que $f_{t}$ ) é um elemento genérico de uma família de deformações a um parâmetro de $\mathcal{J}$.

Definição 4.15. Se $\mathcal{J}_{1}$ e $\mathcal{J}_{2}$ são duas k-álgebras de Jordan dizemos que $\mathcal{J}_{1}$ deformase em $\mathfrak{J}_{2}$ se existir um elemento genérico $\left(\mathcal{J}_{1}\right)_{t}$ de uma família de deformações a um parâmetro de $\mathcal{J}_{1}$ o qual é K-isomorfo a $\left(\mathcal{J}_{2}\right)_{K}=\mathcal{J}_{2} \otimes_{\mathbf{K}} \mathrm{K}$.

A condição (4.2) de que $f_{t}$ seja um produto de Jordan é equivalente às seguintes relações, para todo $a, b \in V$ e todo $v$ inteiro não negativo:

$$
\begin{aligned}
& F_{\nu}(a, b)=F_{\nu}(b, a), \\
& \sum_{\substack{\lambda+\mu+\gamma=v \\
\lambda, \mu, \gamma \geqslant 0}} F_{\gamma}\left(F_{\mu}(a, a), F_{\lambda}(b, a)\right)-F_{\gamma}\left(F_{\mu}\left(F_{\lambda}(a, a), b\right), a\right)=0 .
\end{aligned}
$$

Observe que para $v=0$ obtemos a comutatividade e a identidade de Jordan do produto original.

A primeira aplicação bilinear $F_{n}$ não nula logo após o produto de $\mathcal{J}$ na expressão (4.1) de $f_{t}$, considerada como uma aplicação de $V \times V$ em $V$, é chamada de diferencial da família. 
Para $v=n$, a relação (4.3) pode ser expressa da forma

$$
\begin{aligned}
& F_{n}(a, b)=F_{n}(b, a), \\
& F_{n}\left(a^{2}, b a\right)-F_{n}\left(a^{2} b, a\right)+a^{2} F_{n}(b, a)-F_{n}\left(a^{2}, b\right) a+F_{n}(a, b)(b a)-\left(F_{n}(a, a) b\right) a=0,
\end{aligned}
$$

ou seja $F_{n}$ é um elemento do grupo $Z^{2}(\mathcal{J}, \mathcal{J})$ de 2 -cociclos de $\mathcal{J}$ com coeficientes em $\mathcal{J}$ (veja Definição 1.21).

Observamos que um elemento arbitrário $h \in Z^{2}(\mathcal{J}, \mathcal{J})$ não necessariamente é o diferencial de uma família de deformações a um parâmetro de $\mathcal{J}$. Se esse for o caso, dizemos que $h$ é integrável.

Definição 4.16. Uma família de deformações a um parâmetro de uma álgebra de Jordan definida pelo produto $g_{t}$ é chamada de trivial se existe uma aplicação linear nãosingular $\Phi_{t}: V_{K} \rightarrow V_{K}$ (um automorfismo de $V_{K}$ ) da forma:

$$
\Phi_{t}(a)=a+t \phi_{1}(a)+t^{2} \phi_{2}(a)+\cdots,
$$

onde todos os $\phi_{i}: V_{K} \rightarrow V_{K}$ são aplicações lineais definidas sobre $\mathbf{k}$, tais que

$$
g_{t}(a, b)=\Phi_{t}^{-1}\left(\Phi_{t}(a) \cdot \Phi_{t}(b)\right)
$$

A álgebra então obtida $\partial_{t}$ é claramente isomorfa à álgebra $\partial_{K}=\mathcal{J} \otimes_{\mathbf{K}} K$, de fato o isomorfismo é dado pela aplicação linear $\Phi_{t}$ considerada como uma aplicação de $\mathcal{J}_{t}$ em $\partial_{K}$.

Definição 4.17. Duas famílias $f_{t}$ e $g_{t}$ de deformações a um parâmetro de uma álgebra de Jordan $\mathcal{J}$ serão chamadas de equivalentes se existir um automorfismo $\Phi_{t}: V_{K} \rightarrow V_{K}$ da forma (4.4) tal que

$$
g_{t}(a, b)=\Phi_{t}^{-1} f_{t}\left(\Phi_{t}(a), \Phi_{t}(b)\right) .
$$

Da última definição temos que a família $f_{t}$ é trivial se é equivalente à deformação identidade $g_{t}$ definida por $g_{t}(a, b)=a b$.

Definição 4.18. Uma álgebra de Jordan é chamada de analiticamente rígida se ela somente possui as deformações a um parâmetro triviais.

Em [14], Gerstenhaber denominou tais álgebras de "rígidas" mas, por precisão, nós utilizaremos o termo "analiticamente rígida" para diferenciar os três conceitos de rigidez, como feito em [15]. 
Diremos que uma álgebra de Jordan $\mathcal{J}$ é infinitesimalmente rígida se $\mathrm{H}^{2}(\mathcal{J}, \mathcal{J})=0$. Para álgebras de dimensão finita existe ainda um terceiro conceito de rigidez o qual será apresentado na Seção 4.3.

A seguinte proposição é um dos resultados fundamentais na teoria de deformações.

Proposição 4.19. [14] Seja $\mathrm{f}_{\mathrm{t}}$ uma família de deformações a um parâmetro de uma álgebra de Jordan J. Então $\mathrm{f}_{\mathrm{t}}$ é equivalente a uma família

$$
g_{t}(a, b)=a b+t^{n} F_{n}(a, b)+t^{n+1} F_{n+1}(a, b)+\cdots,
$$

onde o primeiro termo não nulo $\mathrm{F}_{\mathrm{n}}$ não é cohomólogo a zero.

Demonstração. Seja $f_{t}(a, b)=a b+t^{n} F_{n}(a, b)+\cdots$. Se $F_{n} \in B^{2}(\mathcal{J}, \mathcal{J})$ ou seja $F_{n}$ é um 2-cociclo equivalente a 0 , i.e., $F_{n}=-\delta \phi_{n}$ para alguma aplicação linear $\phi_{n}$, então escolhendo $\Phi_{t}(x)=x+t^{n} \phi_{n}(x)$ temos que

$$
\begin{aligned}
f_{t}\left(\Phi_{t}(a), \Phi_{t}(b)\right) & =f_{t}\left(a+t^{n} \phi_{n}(a), b+t^{n} \phi_{n}(b)\right) \\
& =\left(a+t^{n} \phi_{n}(a)\right)\left(b+t^{n} \phi_{n}(b)\right)+t^{n} F_{n}\left(a+t^{n} \phi_{n}(a), b+t^{n} \phi_{n}(b)\right)+\cdots \\
& =a b+t^{n}\left(b \phi_{n}(a)+a \phi_{n}(b)+F_{n}(a, b)\right)+t^{n+1}(\cdots)+\cdots
\end{aligned}
$$

e que

$$
\Phi_{t}\left(a b+t^{n+1} F_{n+1}(a, b)+\cdots\right)=a b+t^{n} \phi_{n}(a b)+t^{n+1}(\cdots)+\cdots,
$$

e portanto

$$
\Phi_{\mathrm{t}}^{-1} \mathrm{f}_{\mathrm{t}}\left(\Phi_{\mathrm{t}}(\mathrm{a}), \Phi_{\mathrm{t}}(\mathrm{b})\right)=\mathrm{ab}+\mathrm{t}^{\mathrm{n}+1} \mathrm{~F}_{\mathrm{n}+1}(\mathrm{a}, \mathrm{b})+\cdots
$$

e de novo $F_{n+1} \in Z^{2}($ J, J).

Agora, observe que se $H^{2}(\mathcal{J}, \mathcal{J})=0$ então $Z^{2}(\mathcal{J}, \mathcal{J})=B^{2}(\mathcal{J}, \mathcal{J})$ e se $f_{t}$ é uma família de deformações a um parâmetro de $\mathcal{J}$ então, procedendo indutivamente como na prova da proposição anterior, $f_{t}$ é equivalente à deformação trivial. Logo temos:

Proposição 4.20. Se uma álgebra de Jordan sobre um corpo $\mathbf{k}$ é infinitesimalmente rígida então ela é analiticamente rígida.

Este resultado foi originalmente obtido em [14, Cor, p.65] para álgebras associativas e de Lie.

Em particular segue de [47, Teo. 9.2.11 p. 310] que para qualquer álgebra separável de dimensão finita $A$ e qualquer bimódulo $M$ de $A, H^{n}(A, M)=0$ para $n>0$, isto implica que: 
Corolário 4.21. Toda álgebra de Jordan semissimples de dimensão finita sobre um corpo algebricamente fechado é analiticamente rígida.

Exemplo 4.22. Considere $\mathcal{B}_{2} \in$ Jor $_{2}$ (veja 2.1.2) e seja $h: \mathcal{B}_{2} \times \mathcal{B}_{2} \rightarrow \mathcal{B}_{2}$ uma aplicação bilinear satisfazendo (1.6) ou seja, h é um 2-cociclo de $\mathcal{B}_{2}$ com coeficientes em $\mathcal{B}_{2}$, então resolvendo as linearizações das equações (1.6) nos elementos da base de $\mathcal{B}_{2}$ obtemos:

$$
h\left(e_{1}, e_{1}\right)=\alpha e_{1}, \quad h\left(e_{1}, n_{1}\right)=\beta e_{1}+\frac{\alpha}{2} n_{1}, \quad h\left(n_{1}, n_{1}\right)=2 \beta n_{1}
$$

para quaisquer $\alpha, \beta \in \mathbf{k}$. Defina uma aplicação linear $\mu: \mathcal{B}_{2} \rightarrow \mathcal{B}_{2}$ como sendo $\mu\left(e_{1}\right)=$ $-\alpha e_{1}+n_{1}$ e $\mu\left(n_{1}\right)=-2 \beta e_{1}+n_{1}$, logo temos que

$$
0=h(a, b)-\mu(a b)+a \mu(b)+\mu(a) b,
$$

para todo $a, b \in \mathcal{B}_{2}$, i.e., $h$ é equivalente a 0 , portanto $H^{2}\left(\mathcal{B}_{2}, \mathcal{B}_{2}\right)=0$ o que implica que $\mathcal{B}_{2}$ é analiticamente rígida.

Vamos agora comparar a estrutura da álgebra deformada $\mathcal{J}_{t}$ com a de $\mathcal{J}$, mais precisamente com a de $\mathcal{J}_{K}$. Do Teorema 1.12 temos que qualquer álgebra de Jordan de dimensão finita (sobre um corpo algebricamente fechado ou $\mathbb{R}$ ) admite decomposição: $\mathcal{J}=\mathcal{J}_{s s} \oplus \operatorname{Rad}(\mathcal{J})$. Seja $\mathcal{J}_{t}$ um elemento genérico de uma família de deformações a um parâmetro de $\mathcal{J}$, com produto $f_{t}$. Então podemos construir uma deformação equivalente à dada tal que a nova deformação preserva tanto o produto original em $\left(\mathcal{J}_{K}\right)_{s s}$ como a ação de $\left(\mathcal{J}_{K}\right)_{s s}$ em $\operatorname{Rad}(\mathcal{J})$.

Teorema 4.23. [23, Teorema 2.3, p. 66] Seja $J_{\mathrm{t}}$ um elemento genérico de uma família a um parâmetro de deformações de J com multiplicação $f_{\mathrm{t}}$. Então existe $\mathcal{G}_{\mathrm{t}}$ um elemento genérico de uma família de deformações a um parâmetro de $\mathrm{J}$, com produto $\mathrm{g}_{\mathrm{t}}$ o qual é equivalente a $\mathrm{f}_{\mathrm{t}} e$ tal que o radical da álgebra $\mathcal{G}_{\mathrm{t}}$ é $\operatorname{Rad}\left(\mathcal{G}_{\mathrm{t}}\right)=\mathrm{R} \otimes_{\mathrm{K}} \mathrm{K}$, onde $\mathrm{R}$ é um ideal nilpotente de J. Mais ainda, para todo $\mathrm{x}, \mathrm{y} \in\left(\mathcal{J}_{\mathrm{K}}\right)_{\mathrm{ss}}, \mathrm{g}_{\mathrm{t}}(\mathrm{x}, \mathrm{y})=\mathrm{x} \cdot \mathrm{y}$ e para todo $\mathrm{x} \in\left(\mathcal{J}_{\mathrm{K}}\right)_{\mathrm{ss}} e z \in \operatorname{Rad}\left(\mathcal{J}_{\mathrm{K}}\right)$, $g_{\mathrm{t}}(x, z)=x \cdot z$.

\subsection{A VARIEDADE ALGÉBRICA Jor}

Nesta seção definiremos a variedade das álgebras de Jordan de dimensão $n$. O corpo de definição será denotado por $\mathbf{k}$ e representará um corpo algebricamente fechado de char $\mathbf{k} \neq 2$ ou $\mathbb{R}$. Denotaremos tal variedade por Jor $_{n}$ ou por Jor $_{n}^{\mathbb{R}}$ quando pretendamos enfatizar que o corpo base que estamos considerando é $\mathbb{R}$. 
Também explicaremos os métodos usados nos Capítulos 5 e 6 para descrever as componentes irredutíveis de Jor $n$ para $n \leqslant 4$ e de $\operatorname{Jor}_{n}^{\mathbb{R}}$ para $n \leqslant 3$, assim como também as deformações entre as álgebras de Jordan.

Seja $V$ um espaço vetorial de dimensão $n$ sobre um corpo $\mathbf{k}$ com base $\left\{e_{1}, \ldots, e_{n}\right\}$ fixa. O nosso objetivo é introduzir uma estrutura de álgebra de Jordan em $\mathrm{V}$, o que pode ser feito especificando $n^{3}$ constantes estruturais $c_{i j}^{k} \in \mathbf{k}$, de modo que o produto na álgebra resultante seja definido como:

$$
e_{i} \cdot e_{j}=\sum_{k=1}^{n} c_{i j}^{k} e_{k}, \text { para todo } i, j \in\{1, \ldots, n\} \text {. }
$$

A escolha das constantes estruturais não é arbitrária pois deve refletir os fatos que a álgebra é comutativa, isto é:

$$
c_{i j}^{k}=c_{j i}^{k}
$$

e que satisfaz a identidade de Jordan (1.2):

$$
\begin{aligned}
& \sum_{a=1}^{n} c_{i j}^{a} \sum_{b=1}^{n} c_{k l}^{b} c_{a b}^{p}-\sum_{a=1}^{n} c_{k l}^{a} \sum_{b=1}^{n} c_{j a}^{b} c_{i b}^{p}+\sum_{a=1}^{n} c_{l j}^{a} \sum_{b=1}^{n} c_{k i}^{b} c_{a b}^{p}- \\
& -\sum_{a=1}^{n} c_{k i}^{a} \sum_{b=1}^{n} c_{l a}^{b} c_{l b}^{p}+\sum_{a=1}^{n} c_{k j}^{a} \sum_{b=1}^{n} c_{i l}^{b} c_{a b}^{p}-\sum_{a=1}^{n} c_{i l}^{a} \sum_{b=1}^{n} c_{j a}^{b} c_{k b}^{p}=0,
\end{aligned}
$$

para todos $i, j, k, l, p \in\{1, \ldots, n\}$.

Estas restrições se manifestam através de equações polinomiais nas constantes estruturais, e assim podemos interpretar o espaço das constantes estruturais como uma variedade em $\mathbb{A}^{n^{3}}$ definida pelas equações (4.5) e (4.6) que chamaremos de variedade das álgebras de Jordan de dimensão $n$ e que denotaremos por Jor ${ }_{n}$. Um ponto qualquer $\left(c_{i j}^{k}\right) \in J^{\prime} r_{n}$ representa, na base fixa, uma k-álgebra de Jordan $\mathcal{J}$ de dimensão n.

As afirmações acima provam que:

Lema 4.24. A variedade Jor ${ }_{\mathrm{n}}$ é um conjunto algébrico e logo uma variedade afim.

O grupo $G=G L(V)$ age em Jor $n$ via "transporte de estrutura", i.e.,

$$
\begin{aligned}
& \mathrm{G} \times \mathcal{J O J}_{\mathrm{n}} \rightarrow \text { Jor }_{\mathrm{n}} \\
& (\mathrm{g},(\mathcal{J}, \cdot)) \mapsto(\mathcal{J}, \cdot g), \text { onde } x \cdot g \mathrm{y}=\mathrm{g}\left(\mathrm{g}^{-1} x \cdot \mathrm{g}^{-1} \mathrm{y}\right),
\end{aligned}
$$


para toda $\mathcal{J} \in \mathcal{J}^{\circ} r_{n}, g \in G$ e $x, y \in V$. As órbitas desta ação são dadas por

$$
\mathcal{J}^{\mathrm{G}}=\left\{(\mathcal{J}, \cdot \mathrm{g}) \in \mathcal{J O r}_{\mathrm{n}} \mid \mathrm{g} \in \mathrm{G}\right\}
$$

e o estabilizador de uma álgebra sob a ação de G,

$$
\operatorname{Stab}_{\mathrm{G}}(\mathcal{J})=\{\mathrm{g} \in \mathrm{G} \mid(\mathcal{J}, \cdot g)=(\mathcal{J}, \cdot)\}
$$

coincide com o grupo de automorfismos da álgebra

$$
\operatorname{Aut}(\mathcal{J})=\{\mathrm{g} \in \mathrm{G} \mid \mathrm{g} \text { é um endomorfismo (de álgebras) de } \mathcal{J}\} \text {. }
$$

De fato $g \in \operatorname{Stab}_{\mathrm{G}}(\mathcal{J})$ se e somente se a álgebra $\mathcal{J}$ com o produto ${ }_{g}$ coincide com a álgebra $\mathcal{J}$ com o produto original, i.e., $g^{-1}(x \cdot y)=g^{-1}(x) \cdot g^{-1}(y)$ se e somente se $g^{-1}$ é um endomorfismo se e somente se $g \in \operatorname{Aut}(\mathcal{J})$.

Dois conjuntos de constantes estruturais geram álgebras isomorfas se e somente se existe um elemento de G que leva uma álgebra na outra como em (4.7), assim temos o seguinte lema:

Lema 4.25. Sejam $(\mathcal{J}, \cdot) e\left(\mathcal{J}_{1}, \bullet\right) \in \mathcal{J} \mathrm{r}_{n}$. Então $\mathcal{J} \simeq \mathcal{J}_{1}$ se e somente se $\mathcal{J}^{\mathrm{G}}=\mathcal{J}_{1}$.

Como consequência deste lema temos que as órbitas de Jor ${ }_{n}$ sob a ação de G podem ser identificadas com a classe de isomorfismos de álgebras de Jordan de dimensão $n$.

Agora introduziremos um conceito muito importante no estudo da variedade Jor $_{n}$, a ideia de deformação, a qual nos permitirá determinar as componentes irredutíveis dessa variedade.

Definição 4.26. Dizemos que a álgebra de Jordan $\mathcal{J}_{1}$ de dimensão $n$, é uma deformação da álgebra de Jordan da mesma dimensão $\mathcal{J}_{2}$ ou, equivalentemente, que $\mathcal{J}_{1}$ domina $\mathcal{J}_{2}$ ou, ainda, que $\mathcal{J}_{2}$ deforma-se em $\mathcal{J}_{1}$, se a órbita $\mathcal{J}_{2}^{G}$ está contida no fecho de Zariski da órbita $\mathcal{J}_{1}^{G}$ e denotamos tal fato por $\mathcal{J}_{1} \rightarrow \mathcal{J}_{2}$.

Claramente sempre que $\mathcal{J}_{2} \in \mathcal{J}_{1}^{G}$ então $\mathcal{J}_{2}^{G}=\mathcal{J}_{1}^{G}$ ou seja $\mathcal{J}_{2} \simeq \mathcal{J}_{1}$ e também $\mathcal{J}_{2}^{G} \subseteq \overline{\mathcal{J}_{1}^{G}}$ $\operatorname{logo} \mathfrak{J}_{1} \rightarrow \mathfrak{J}_{2}$. Nos referiremos a uma deformação deste tipo como uma deformação trivial, caso contrário será denominada de deformação não trivial.

Proposição 4.27. A relação $\mathcal{J}_{1} \rightarrow \mathcal{J}_{2}$ é uma relação de ordem parcial em $\mathcal{J o r}_{n}$.

Demonstração. Para cada $\mathcal{J} \in \mathcal{J}^{\circ} r_{n}$ temos a deformação trivial $\mathcal{J} \rightarrow \mathcal{J}$. Por outro lado se $\mathcal{J} \rightarrow \mathcal{J}_{1}$ e $\mathcal{J}_{1} \rightarrow \mathcal{J}_{2}$ então $\mathcal{J}_{2}^{\mathrm{G}} \subseteq \overline{\mathcal{J}_{1}} \subseteq \overline{\mathcal{J}^{G}}$ o que implica que $\mathcal{J} \rightarrow \mathcal{J}_{2}$. Por último, se $\mathcal{J} \rightarrow \mathcal{J}_{1}$ e $\mathcal{J}_{1} \rightarrow \mathcal{J}$ então $\mathcal{J}_{1}^{\mathrm{G}} \subseteq \overline{\mathcal{J}^{\mathrm{G}}}$ e $\mathcal{J}^{\mathrm{G}} \subseteq \overline{\mathcal{J}_{1}^{\mathrm{G}}}$. Suponha que $\mathcal{J} \not \mathcal{J}_{1}$ então $\mathcal{J}^{\mathrm{G}} \neq \mathcal{J}_{1}^{\mathrm{G}}$ o que significa que $\mathcal{J}^{\mathrm{G}} \cap \mathcal{J}_{1}^{\mathrm{G}}=\emptyset$, logo $\mathcal{J}_{1}^{\mathrm{G}} \subseteq \overline{\mathcal{J}^{\mathrm{G}}} \backslash \mathcal{J}^{\mathrm{G}}$. Pelo Lema $4.13 \operatorname{dim} \mathcal{J}_{1}^{G}<\operatorname{dim} \mathcal{J}^{\mathrm{G}}$ e analogamente $\operatorname{dim} \mathcal{J}^{G}<\operatorname{dim} \mathcal{J}_{1}^{G}$ o que é uma contradição. Logo $\mathcal{J} \simeq \mathcal{J}_{1}$. 
O seguinte lema é uma ferramenta básica para a determinação da existência de uma deformação entre duas álgebras. A prova segue diretamente da definição de deformação.

Lema 4.28. [31, p.13] A existência de uma curva $\gamma$ em Jor $\mathrm{n}_{\mathrm{n}}$ que mora genericamente numa subvariedade $\mathcal{U}$ e a qual corta $\mathcal{J}^{\mathrm{G}}$ em um ponto especial implica que $\mathcal{J} \in \overline{\mathcal{U}}$ e reciprocamente.

Para ilustrar o lema vamos dar um exemplo considerando a variedade $J^{\circ} r_{4}$ :

Exemplo 4.29. Seja $\left\{e_{1}, e_{2}, e_{3}, e_{4}\right\}$ a base da álgebra de Jordan $\partial_{1}$ de dimensão 4 obtida na classificação algébrica da Seção 2.2. Considere a curva $A_{t}=e_{1}+e_{4}, B_{t}=e_{2}$, $C_{t}=t e_{3}$ e $D_{t}=t^{2} e_{1}$. Para qualquer $t \neq 0$ álgebra $J_{t}$ com base $\left\{A_{t}, B_{t}, C_{t}, D_{t}\right\}$ e produtos

$$
\begin{aligned}
& A_{t}^{2}=A_{t} \quad B_{t}^{2}=B_{t} \quad C_{t}^{2}=D_{t}+t^{2} B_{t} \quad D_{t}^{2}=t^{2} D_{t} \\
& A_{t} B_{t}=0 \quad A_{t} C_{t}=\frac{1}{2} C_{t} \quad A_{t} D_{t}=D_{t} \\
& B_{t} C_{t}=\frac{1}{2} C_{t} \quad B_{t} D_{t}=0 \quad C_{t} D_{t}=\frac{1}{2} t^{2} C_{t}
\end{aligned}
$$

é isomorfa a $\mathcal{J}_{1}$, i.e., $\mathcal{J}_{t} \in \mathcal{J}_{1}^{G}$ mas quando $t=0$ obtemos

$$
\begin{aligned}
& A_{t}^{2}=A_{t} \quad B_{t}^{2}=B_{t} \quad C_{t}^{2}=D_{t} \quad D_{t}^{2}=0 \\
& A_{t} B_{t}=0 \quad A_{t} C_{t}=\frac{1}{2} C_{t} \quad A_{t} D_{t}=D_{t} \\
& B_{t} C_{t}=\frac{1}{2} C_{t} \quad B_{t} D_{t}=0 \quad C_{t} D_{t}=0
\end{aligned}
$$

que é o produto na álgebra $\mathcal{J}_{25}$. Logo do lema anterior $\mathcal{J}_{1} \rightarrow \mathcal{J}_{25}$.

O seguinte lema relaciona as definições de deformações a um parâmetro (Definição 4.15) e de deformação (Definição 4.26) quando o corpo é algebricamente fechado.

Lema 4.30. [11, Lema $7 a, p .77$ ] Seja $\mathbf{k}$ um corpo algebricamente fechado. Se $\mathfrak{J}_{1}$ e $\mathfrak{J}_{2}$ são duas k-álgebras de Jordan tal que J1 é uma deformação de J2 no sentido da Definição 4.15, i.e., existe um elemento genérico $\left(\mathcal{J}_{2}\right)_{\mathrm{t}}$ de uma família de deformações a um parâmetro de $\mathcal{J}_{2}$ o qual é $\mathrm{K}$-isomorfo a $\left(\mathcal{J}_{1}\right)_{\mathrm{K}}$, onde $\mathrm{K}=\mathbf{k}((\mathrm{t}))$. Então a órbita $\mathrm{J}_{2}^{\mathrm{G}}$ está contida no fecho de Zariski da órbita $\partial_{1}^{\mathrm{G}}$.

Definição 4.31. Chamaremos de álgebras de Jordan geometricamente rígidas ou simplesmente de álgebras de Jordan rígidas, às álgebras para as quais sua órbita sob a ação de $\mathrm{G}$ é um subconjunto Zariski-aberto em Jor ${ }_{n}$.

Proposição 4.32. [15, p. 61] As seguintes condições são equivalentes: 


\section{i. Jé geometricamente rígida.}

ii. I tem uma vizinhança na qual todo ponto representa uma álgebra isomorfa a J sobre alguma extensão de $\mathbf{k}$.

iii. A componente de Jor $\mathrm{n}$ que contém J tem um ponto genérico o qual representa uma álgebra isomorfa a J sobre alguma extensão de $\mathbf{k}$ (a componente deve então ser única).

Uma das questões que nos perguntamos na hora da classificação geométrica sobre um corpo $\mathbf{k}$ algebricamente fechado e sobre $\mathbb{R}$ é se toda álgebra rígida permanece rígida sob uma extensão escalar, a resposta é consequência direta da proposição anterior:

Lema 4.33. [15, p. 61] J é geometricamente rígida se e somente se J $\otimes_{\mathbf{k}} \mathrm{L}$ é geometricamente rígida sempre que L é uma extensão de $\mathbf{k}$.

Uma das perguntas que surgem ao respeito de rigidez é como se relacionam os três conceitos de rigidez introduzidos neste capítulo. A resposta é dada nos Teoremas 4.34 e 4.35 a seguir:

Teorema 4.34. [15, Teo. 3.2,p.61] Para uma álgebra de Jordan de dimensão finita sobre um corpo $\mathbf{k}$ rigidez analítica implica rigidez geométrica.

Demonstração. Do lema anterior podemos assumir que k é algebricamente fechado. Seja $(c)=\left(c_{i j}^{k}\right)$ um ponto em $\mathcal{J o r}_{n}$ representando uma álgebra $\mathcal{J}$ analiticamente rígida e seja $(c(t))$ um ponto genérico com coeficientes em $\mathbf{k}[[t]]$ de uma componente irredutível de $\operatorname{Jor}_{n}$ contendo (c). Agora, o produto em $\mathcal{J}_{\mathbf{k}[[t]]}$ definido pelas estruturas constantes

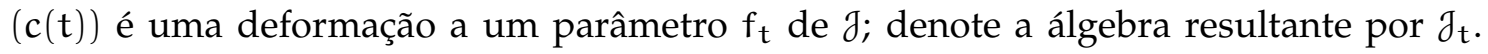
Pela hipótese, $f_{t}$ é equivalente ao produto original de $\mathcal{J}$. Mas isto nos diz que $J_{t}$ é isomorfa a $\mathcal{J}$ sobre $\mathbf{k}[[t]]$ e, logo, $\partial_{t}$ e $\mathcal{J}$ são isomorfas sobre o corpo $\mathbf{k}((t))$. Ou seja, é satisfeita a condição (iii) da Proposição (4.32).

Ou seja, quando consideramos álgebras de dimensão finita temos:

$$
\text { rigidez infinitesimal } \Rightarrow \text { rigidez analítica } \Rightarrow \text { rigidez geométrica }
$$

Estas implicâncias se aplicam igualmente bem a qualquer categoria de álgebras definidas por equações com as apropriadas modificações. Em característica positiva foi mostrado que todas as implicações inversas são falsas para álgebras associativas de dimensão finita. Em [15] M. Gerstenhaber e S. Schack construíram álgebras analiticamente rígidas associativas $\mathcal{A}$ de dimensão alta sobre um corpo de característica positiva tendo $\mathrm{H}^{2}(\mathcal{A}, \mathcal{A}) \neq 0$ e álgebras associativas geometricamente rígidas que não são analiticamente rígidas. 
O cenário muda quando assumimos que o corpo tem característica 0 , neste caso temos que os conceitos de rigidez geométrica e rigidez analítica coincidem:

Teorema 4.35. [15, Teo 7.1,p.73] Seja $\mathbf{k}$ um corpo de característica zero e seja J uma k-álgebra de Jordan de dimensão finita. Suponha que J é geometricamente rígida e seja $\mathrm{f}_{\mathrm{t}}(\mathrm{a}, \mathrm{b})=\mathrm{ab}+$ $\mathrm{tF}_{1}(\mathrm{a}, \mathrm{b})+\mathrm{t}^{2} \mathrm{~F}_{2}(\mathrm{a}, \mathrm{b})+\cdots$ uma deformação a um parâmetro de J. Então $\mathrm{f}_{\mathrm{t}}$ é trivial. Logo J é analiticamente rígida.

Surpreendentemente, a questão de quando existem álgebras associativas $\mathcal{A}$ analiti-

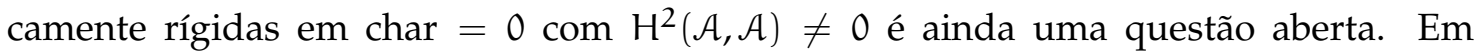
contrapartida, Richardson em [39] mostrou que existem álgebras de Lie complexas em toda dimensão par maior que 16 que são geometricamente rígidas mas não são infinitesimalmente rígidas.

Já para as álgebras associativas com unidade sobre um corpo algebricamente fechado P. Gabriel provou em [13, Corolário 2.5, p.142] que os conceitos de rigidez geométrica e rigidez infinitesimal coincidem. Uma outra prova desse fato pode ser encontrada em [37, Teo. 3, p.238].

Vimos na Seção 4.1 que toda variedade afim admite uma decomposição em um número finito de componentes irredutíveis, i.e., subconjuntos fechados irredutíveis maximais. É nessa hora, quando pretendemos decompor a variedade afim Jor $_{n}$ em componentes irredutíveis, que as álgebras rígidas demostram sua importância pois provaremos na seguinte proposição que cada álgebra rígida é um ponto genérico (veja Definição 4.9) de alguma componente irredutível de Jor $_{n}$.

Proposição 4.36. Se J $\in$ Jor $_{\mathrm{n}}$ é uma álgebra rígida então existe uma componente irredutível $\mathrm{T}$ tal que $\mathrm{T}=\overline{\jmath^{\mathrm{G}}}$.

Demonstração. Se J é uma álgebra rígida, então a órbita $\mathcal{J}^{\mathrm{G}}$ é um subconjunto Zariskiaberto (não vazio) de $\mathcal{J o r}_{n}$. Por outro lado

$$
\emptyset \neq \mathcal{J}^{\mathrm{G}} \subseteq \operatorname{Jor}_{\mathrm{n}}=\bigcup_{i=1}^{p} \mathrm{~T}_{\mathrm{i}}
$$

onde $T_{i}$ são as componentes irredutíveis de $\mathcal{J o r}_{n}$. Logo existe $T_{i}$ tal que $\mathcal{J} \in T_{i}$ e do Lema 4.13 segue que $T_{i}$ é invariante sob a ação de $G \log o \mathcal{J}^{G} \subseteq T_{i}$. Como $T_{i}$ é irredutível todo aberto é denso, assim o fecho de $\mathcal{J}^{G}$ em $T_{i}$ é $T_{i}$ o que implica que

$$
\mathrm{T}_{i}=\mathrm{T}_{i} \cap \overline{J^{\mathrm{G}}} \operatorname{logo} \mathrm{T}_{\mathrm{i}} \subseteq \overline{J^{\mathrm{G}}} .
$$

Agora, do Lema 4.13 cada órbita é irredutível, ou seja $\overline{\bar{J}^{\mathrm{G}}}$ é um subconjunto fechado e irredutível de Jor $_{n}$ que contém $T_{i} \operatorname{logo} T_{i}=\overline{\mathcal{J G}^{G}}$. 
A recíproca não é sempre verdadeira, i.e., não toda componente irredutível vem de uma álgebra rígida quando isto não acontece a componente é o fecho de uma família infinita de órbitas, como prova a seguinte proposição:

Proposição 4.37. Cada componente irredutível da variedade das álgebras de Jordan, Jor ${ }_{n}$ ou é o fecho de Zariski da órbita de uma álgebra rígida ou é o fecho de uma família infinita de órbitas.

Demonstração. Seja $T$ uma componente irredutível de Jor $_{n}$ e seja $\mathcal{J} \in T$. Segue do Lema 4.13 que $T$ é invariante sob a ação de $G \log o f^{G} \subseteq T$ e portanto $T=\cup_{\mathfrak{g} \in \mathrm{T}} \mathcal{J}^{\mathrm{G}}$.

Suponha que a família de órbitas seja finita, i.e. $T=\cup_{i=1}^{N} \mathcal{\partial}_{i}^{G}$, então

$$
m=\operatorname{dim}(T)=\operatorname{dim}\left(\bigcup_{i=1}^{N} \partial_{i}^{G}\right)=\max _{1 \leqslant i \leqslant N}\left(\operatorname{dim}\left(\mathcal{\partial}_{i}^{G}\right)\right)=\operatorname{dim} \partial_{i_{0}}^{G} .
$$

Em particular, $\mathcal{\partial}_{i_{0}} \subseteq \mathrm{T}$ e logo $\overline{\partial_{i_{0}}} \subseteq \mathrm{T}$, i.e., um conjunto fechado está contido num conjunto irredutível e segue da observação do Lema 4.13 que ambos tem a mesma dimensão, $\operatorname{logo} T=\overline{\mathcal{J}_{i_{0}}}$. Por outro lado, órbitas são localmente fechadas logo $\mathcal{J}_{i_{0}}^{G}$ é aberto em $\mathrm{T}$.

É importante destacar que as variedades que queremos descrever geometricamente tem um número finito de órbitas. Se o corpo for algebricamente fechado temos 2 órbitas em Jor 1,6 em Jor $_{2}, 20$ em Jor $_{3}$ e 73 em Jor $_{4}$. No caso de álgebras sobre $\mathbb{R}$ temos 2 órbitas em Jor 1,7 em Jor 2 e 26 em Jor 3 . Então o seguinte corolário será útil no objetivo deste trabalho.

Corolário 4.38. Se Jor $\mathrm{r}_{\mathrm{n}}$ se decompõe como um número finito de órbitas então cada componente irredutível da variedade é o fecho de Zariski da órbita de uma álgebra rígida.

Em [11], Flanigan mostra que a segunda alternativa da Proposição 4.37 de fato ocorre, exibindo uma componente de $\mathrm{Assoc}_{3}$ que consiste inteiramente das órbitas de uma família de álgebras nilpotentes. Outros exemplos para álgebras associativas com unidade sobre um corpo algebricamente fechado foram obtidos em [13] por Gabriel (a família infinita denominada por (18) dependendo continuamente do parâmetro $\lambda \neq 1$ ) em dimensão 4 e em [31] por Mazzola em dimensão 5.

Como consequência dos resultados anteriores temos:

Proposição 4.39. Seja $\mathcal{J} \in$ Jor $_{n}$ então:

i. Se J é rígida então qualquer deformação de J é isomorfa a J.

ii. Se Jor ${ }_{\mathrm{n}}$ se decompõe como um número finito de órbitas e toda deformação de J é isomorfa a J então J é rígida. 
iii. Se $\mathbf{k}$ é algebricamente fechado e toda deformação de J é isomorfa a J então J é rígida.

Demonstração. i. Seja $\mathcal{J}_{1}$ uma deformação de uma álgebra rígida $\mathcal{J}$, então por definição $\mathcal{J}^{G} \subseteq \overline{J_{1}^{G}}$. Seja $\mathcal{J}_{2} \in \mathcal{J}^{G}$ então como $\mathcal{J}_{2}$ pertence ao fecho de $\mathcal{J}_{1}^{G}$ para toda vizinhança $U$ de $\mathcal{J}_{2}$ temos que $U \cap \mathcal{J}_{1}^{\mathrm{G}} \neq \emptyset$. Em particular, $\mathcal{J}^{\mathrm{G}}$ é uma vizinhança de $\mathcal{J}_{2}, \log$ o $\mathcal{J}_{1}^{G} \cap \mathcal{J}^{G} \neq \emptyset$ o que implica que $\mathcal{J}^{G}=\mathcal{J}_{1}^{G}$ e do Lema 4.25 temos $\mathcal{J} \simeq \mathcal{J}_{1}$.

ii. Seja T uma componente irredutível de $\mathcal{J}_{\mathrm{n}} \mathrm{r}_{\mathrm{n}}$ que contém $\mathcal{J}$, como T é G-invariante $J^{\mathrm{G}} \subseteq \mathrm{T}$. Do Corolário 4.38 existe uma álgebra rígida $\mathcal{J}_{1}$ tal que $\mathrm{T}=\overline{\mathcal{J}_{1}}$, ou seja $\mathcal{J}^{\mathrm{G}} \subseteq \overline{\mathcal{J}_{1}^{\mathrm{G}}}$ o que implica por hipóteses que $\mathcal{J} \simeq \mathcal{J}_{1}$ e portanto $\mathcal{J}$ é rígida.

iii. Do Lema 4.30 J J somente tem as deformações a um parâmetro triviais, então é analiticamente rígida. Logo, do Teorema 4.34, segue que $\mathcal{J}$ é geometricamente rígida.

Resumimos os resultados até aqui obtidos no seguinte diagrama (Figura 4.1) que relaciona os três conceitos de rigidez e as noções de deformação e deformação a um parâmetro $^{1}$.

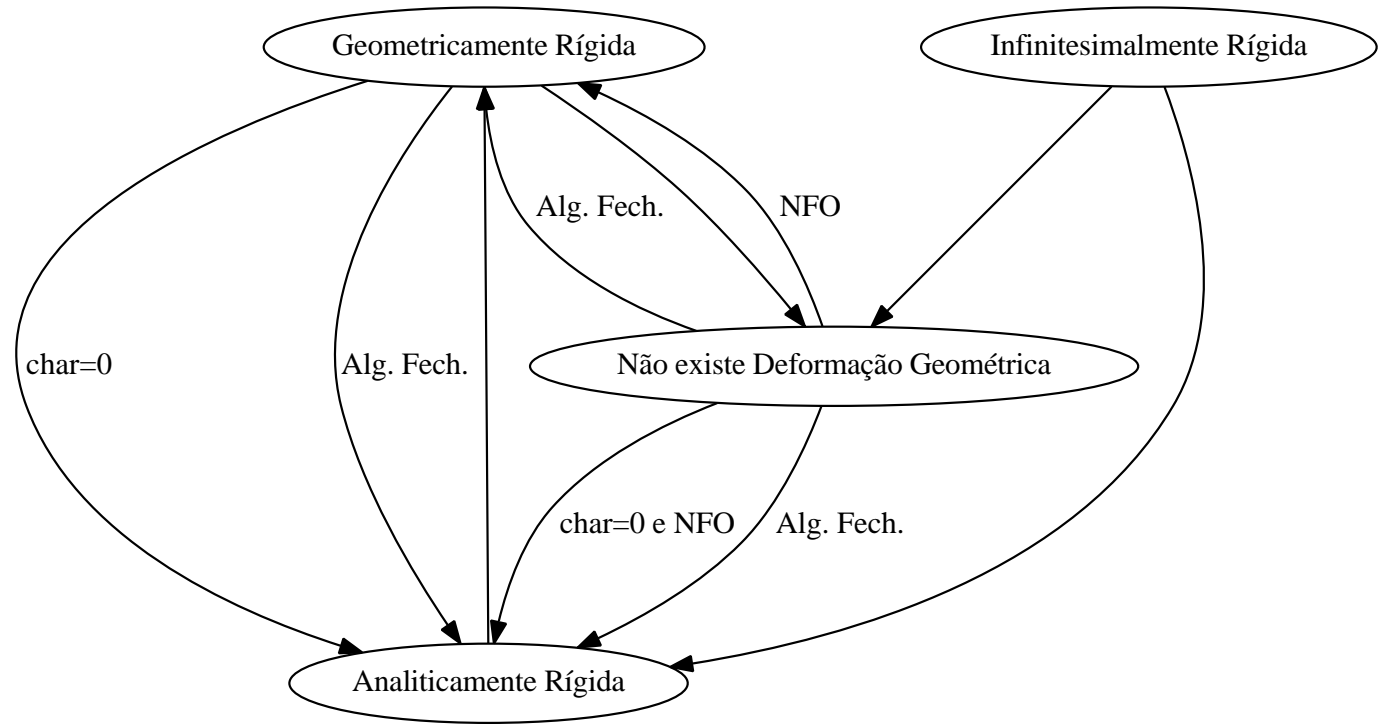

Fig. 4.1: Equivalências entre os diversos conceitos de rigidez

O seguinte lema é análogo à Proposição 2.2 de [13] enunciada no caso da variedade das álgebras associativas unitárias sobre um corpo algebricamente fechado. Mostrare-

1 No diagrama, a sigla NFO significa "Número Finito de Órbitas". 
mos que em Jor $_{n}$ existe uma única órbita fechada que consiste de álgebras isomorfas a $\oplus_{i=1}^{n} \mathbf{k} n_{i}$.

Lema 4.40. Seja $\mathcal{J} \in$ Jor $_{\mathrm{n}}$ uma k-álgebra de Jordan de dimensão $\mathrm{n}$. A órbita de J em Jor $\mathrm{n}$ é fechada sob a ação de $\mathrm{G}$ se e somente se $\mathcal{J} \simeq \oplus_{\mathfrak{i}=1}^{\mathrm{n}} \mathbf{k} \mathbf{n}_{\mathfrak{i}}$. Logo a variedade Jor ${ }_{\mathrm{n}}$ é conexa para todo n.

Demonstração. Mostraremos primeiramente que cada álgebra em Jor ${ }_{\mathrm{n}}$ é uma deformação da álgebra $\oplus_{i=1}^{n} \mathbf{k} n_{i}$. Para isso, seja $\mathcal{J} \in \mathcal{J o r}_{n}$ com base $\left\{e_{1}, \cdots, e_{n}\right\}$ tal que $e_{i} e_{j}=\sum_{k=1}^{n} c_{i j}^{k} e_{k}$ onde os coeficientes $c_{i j}^{k} \in \mathbf{k}$ são as constantes estruturais. Considere para $t \in \mathbf{k} \backslash 0$ a nova base de $J: f_{i}=t e_{i}$ para $i=1, \ldots, n$ em relação à qual as novas constantes estruturais são $d_{i j}^{k}=t c_{i j}^{k}$. Estas constantes estruturais definem uma álgebra $\partial_{t}$ a qual tende $a \oplus_{i=1}^{n} k n_{i}$ quando $t$ tende a 0 .

Isto implica que $\left(\oplus_{i=1}^{n} \mathbf{k} n_{i}\right)^{G} \subseteq \overline{J^{G}}$ para toda $\mathcal{J} \in$ Jor $_{\mathfrak{n}}$. Logo de Lema 4.13 iii temos que $\operatorname{dim}\left(\oplus_{i=1}^{n} \mathbf{k} n_{i}\right)^{G} \leqslant \operatorname{dim} \mathcal{J}^{G}$ para toda $\mathcal{J} \in \mathcal{J} \mathrm{Jr}_{\mathfrak{n}}$. Portanto a órbita de $\oplus_{i=1}^{n} \mathbf{k} n_{i}$ tem dimensão minimal logo é fechada. Reciprocamente, suponha agora que $\mathcal{J}$ tem órbita fechada, i.e., $\overline{J^{G}}=\mathcal{J}^{G} \operatorname{logo} \mathcal{J} \simeq \oplus_{i=1}^{n} \mathbf{k} n_{i}$.

A seguinte proposição, apesar de ser simples é muito útil e nos da uma condição suficiente para a existência de uma deformação entre álgebras decomponíveis.

Proposição 4.41. Sejam $\mathcal{J}_{i}, \mathcal{J}_{i}^{\prime} \in \mathcal{J} \mathrm{r}_{\mathfrak{n}_{i}}$ para $i=1$, 2. Se $\mathcal{J}_{i} \rightarrow \mathcal{J}_{\mathfrak{i}}^{\prime}$ então $\mathcal{J}_{1} \oplus \mathcal{J}_{2} \rightarrow \mathcal{J}_{1}^{\prime} \oplus \mathcal{J}_{2}^{\prime}$.

De fato, pelo Lema 4.28 temos que existem curvas $\gamma_{i}$ que moram genericamente em $\mathcal{J}_{i} G_{i}$ tais que estas curvas cortam $\mathcal{J}_{i}^{\prime G}$, então a curva $\gamma_{1} \times \gamma_{2}$ está genericamente contida em $\mathcal{J}_{1} \mathrm{G}_{1} \times \mathcal{J}_{2} \mathrm{G}_{2} \subseteq\left(\mathcal{J}_{1} \oplus \mathcal{J}_{2}\right)^{\mathrm{G}}$ e, claramente, essa curva corta $\mathcal{J}_{1}^{\prime} \oplus \mathcal{J}_{2}^{\prime}$.

Exemplo 4.42. Em Jor 2 , a deformação $\mathbf{k}_{1} \oplus \mathbf{k} e_{2} \rightarrow \mathcal{B}_{1}$ é dada pela mudança de base $A_{t}=e_{1}+e_{2}$ e $B_{t}=t e_{2}$ que para qualquer $t \neq 0$ tem det $=t \neq 0$ e logo a álgebra obtida pertence à órbita de $\mathbf{k} e_{1} \oplus \mathbf{k} e_{2}$ enquanto que uma conta simples mostra que para $t=0$ pertence à órbita de $\mathcal{B}_{1}$. Segue da Proposição 4.41 que $\mathcal{J}_{4} \rightarrow J_{22}$ pois $\partial_{4}=\mathcal{B}_{1} \oplus \mathbf{k} e_{2} \oplus \mathbf{k} e_{3}$ e $\mathcal{J}_{22}=\mathcal{B}_{1} \oplus \mathcal{B}_{1}$.

Em seu trabalho [32] G. Mazzola provou a recíproca da proposição anterior na variedade das álgebras associativas unitárias de dimensão $n$ sobre um corpo algebricamente fechado, Assoc ${ }_{n}^{1}$. A saber,

Teorema 4.43. [32, Teo. p.291] Sejam $A, A^{\prime} \in$ Assoc $_{n}^{1}$ tal que $A \simeq A_{1} \oplus A_{2} \operatorname{com} A_{i} \in$ Assoc $_{n_{i}}^{1}$ para $i=1$, 2. Se $A^{\prime} \rightarrow$ A então existem $A_{i}^{\prime} \in$ Assoc $_{n_{i}}^{1}$ satisfazendo $A_{i}^{\prime} \rightarrow A_{i}$ para $i=1,2$ e tal que $A^{\prime} \simeq A_{1}^{\prime} \oplus A_{2}^{\prime}$.

Observamos que o teorema anterior não é válido na variedade das álgebras de Jordan: 
Exemplo 4.44. Considere as álgebras $\mathcal{T}_{5}$ e $\mathcal{T}_{8}$ da variedade $\mathcal{J o r}_{3}$, segue de [25] que $\mathcal{T}_{5} \rightarrow$ $\mathcal{T}_{8}$. Somando diretamente a álgebra unidimensional $\mathbf{k n}$ obtemos então da Proposição 4.41 que $\mathcal{T}_{5} \oplus \mathbf{k n} \rightarrow \mathcal{T}_{8} \oplus \mathbf{k n}$, isto é $\mathcal{J}_{8} \rightarrow \mathcal{J}_{44}$ na variedade $\mathcal{J o r}_{4}$. Mas existe uma álgebra indecomponível, $\mathfrak{J}_{45} \in \mathrm{J}_{4} \mathrm{r}_{4}$, tal que

$$
\mathcal{J}_{8} \simeq \mathcal{T}_{5} \oplus \mathbf{k n} \rightarrow \mathcal{J}_{45} \rightarrow \mathcal{T}_{8} \oplus \mathbf{k n} \simeq \mathcal{J}_{44},
$$

veja a Seção 5.4 para uma descrição de tais deformações.

Mais ainda, a condição da existência da unidade é essencial no teorema de Mazzola, pois existem álgebras decomponíveis na variedade das álgebras associativas Assoc $_{n}$ (sem unidade) tal que uma é deformação da outra mas os somandos da mesma dimensão não o são, veja o seguinte exemplo onde as álgebras envolvidas, $\mathcal{J}_{37}$ e $\mathcal{J}_{67}$, são álgebras de Jordan associativas:

Exemplo 4.45. Considere para $t \neq 0$ a mudança de base de $\mathfrak{J}_{37}=\mathcal{T}_{2} \oplus \mathbf{k} n_{3}$ dada por

$$
\begin{aligned}
\mathrm{A}_{\mathrm{t}} & =\mathrm{t} e_{1}+\mathrm{n}_{2}+\mathrm{n}_{3} \\
\mathrm{~B}_{\mathrm{t}} & =\mathrm{t}^{2} \mathrm{e}_{1}+2 \mathrm{tn} \mathrm{n}_{2} \\
\mathrm{C}_{\mathrm{t}} & =\mathrm{t}^{3} \mathrm{e}_{1}+3 \mathrm{t}^{2} \mathrm{n}_{2} \\
\mathrm{D}_{\mathrm{t}} & =\mathrm{n}_{1} .
\end{aligned}
$$

Calculado os produtos e fazendo $t=0$ obtemos a estrutura da álgebra $\mathcal{J}_{67}=\mathcal{T}_{3} \oplus \mathbf{k n}_{4}$, $\operatorname{logo} \mathcal{T}_{2} \oplus \mathbf{k} n_{3} \rightarrow \mathcal{T}_{3} \oplus \mathbf{k} n_{4}$. Mas $\mathcal{T}_{2} \not \rightarrow \mathcal{T}_{3}$ pois

$$
\operatorname{dim} \operatorname{Aut}\left(\mathcal{T}_{2}\right)=4 \geqslant 3=\operatorname{dim} \operatorname{Aut}\left(\mathcal{T}_{3}\right)
$$

e isto representa uma violação à Proposição $4 \cdot 51$ da próxima seção.

Uma das perguntas que surge quando consideramos soma direta de álgebras é o que acontece se as álgebras somadas são rígidas, a rigidez é preservada? Para dar respostas a essas questões enunciaremos o seguinte teorema cuja prova para o caso geral, i.e., o caso quando k é um anel comutativo, $\mathcal{J}$ e $\mathcal{J}^{\prime}$ são k-álgebras arbitrárias e $\mathcal{M}$ e $\mathcal{M}^{\prime}$ são $\mathcal{J}$ e $f^{\prime}$-bimódulos respetivamente, pode ser encontrada em [47].

Teorema 4.46. [47, Teorema 9.1.8, p.305] Sejam J e J' duas k-álgebras de Jordan, seja $\mathcal{M}$ um bimódulo de Jordan para J e respetivamente $\mathcal{M}^{\prime}$ um bimódulo de Jordan para J'. Então $\mathrm{H}^{\mathrm{n}}\left(\mathcal{J} \oplus \mathcal{J}^{\prime}, \mathcal{M} \oplus \mathcal{M}^{\prime}\right) \simeq \mathrm{H}^{\mathrm{n}}(\mathcal{J}, \mathcal{M}) \oplus \mathrm{H}^{\mathrm{n}}\left(\mathcal{J}^{\prime}, \mathcal{M}^{\prime}\right)$.

Como corolário imediato do teorema anterior temos que: 
Corolário 4.47. Soma direta de álgebras infinitesimalmente rígidas é infinitesimalmente rígida.

E, como consequência, soma direta de álgebras de dimensão finita infinitesimalmente rígidas é analiticamente rígida e geometricamente rígida. Provaremos no Teorema $5 \cdot 3$ da Seção 5.5 que soma direta de álgebras geometricamente rígidas é geometricamente rígida em Jor ${ }_{n}$ para $n \leqslant 5$.

No contexto das álgebras de Lie, Yu. Neretin em seu trabalho [35] mostrou que as álgebras de Lie $(\operatorname{sl}(2) \oplus \mathrm{C}) \wedge \mathrm{V}_{2}$ e $\operatorname{sl}(2) \wedge \mathrm{V}_{2}$ são analiticamente rígidas e indecomponíveis mas sua soma direta não é analiticamente rígida. Onde $\measuredangle$ designa o produto semi-direto, $\mathrm{V}_{\alpha}$ é o sl(2)-módulo irredutível de dimensão $\alpha$ e $\mathrm{C}$ é uma álgebra abeliana.

Por último provaremos a seguinte proposição:

Proposição 4.48. Seja Jor $\mathrm{n}_{\mathrm{n}}$ uma variedade com um número finito de órbitas sob a ação de $\mathrm{G}$ ou uma variedade sobre um corpo algebricamente fechado. Seja $\mathcal{J} \in \mathcal{J} \mathrm{r}_{\mathrm{n}}$ uma álgebra rígida tal que $\mathcal{J}=\mathcal{J}_{1} \oplus \mathcal{J}_{2}$ com $\mathcal{J}_{i} \in \mathcal{J o r}_{n_{i}}$ para $i=1$, 2 . Então $\mathcal{J}_{1}$ e $\mathcal{J}_{2}$ devem ser álgebras rígidas.

Demonstração. Suponha que uma delas $\mathcal{J}_{1}$ não é rígida, então da Proposição 4.39 segue que existe uma deformação não trivial, i.e., existe $\mathcal{J}_{1}^{\prime} \in \mathcal{J o r}_{n_{1}}, \mathcal{J}_{1}^{\prime} \neq \mathcal{J}_{1}$ e tal que $\mathcal{J}_{1}^{\prime} \rightarrow \mathcal{J}_{1}$. Logo da Proposição 4.41 $\mathcal{J}_{1}^{\prime} \oplus \mathcal{J}_{2} \rightarrow \mathcal{J}_{1} \oplus \mathcal{J}_{2}$ e $\mathcal{J}_{1}^{\prime} \oplus \mathcal{J}_{2} \not \mathcal{J}_{1} \oplus \mathcal{J}_{2}$ contradizendo a rigidez de $\mathcal{J}$.

Finalizaremos esta seção com um exemplo que mostra que rigidez é um conceito local, i.e., que depende da variedade que estivermos considerando. Mostraremos que o fato de uma álgebra ser rígida na interseção de duas variedades não implica que seja rígida em cada uma das variedades independentemente.

Exemplo 4.49. Foi provado em [4] que as álgebras $J_{61}$ e $J_{62}$ são álgebras rígidas na variedade das álgebras de Jordan nilpotentes de dimensão 4. Mas, como veremos no Capítulo 5 , a álgebra $\mathcal{J}_{47}$ é uma deformação de $\mathcal{J}_{61}$ o que implica que $J_{61}$ não é uma álgebra rígida na variedade das álgebras associativas de dimensão 4. Por outro lado, a álgebra $\mathcal{J}_{53}$ é uma deformação de $\mathcal{J}_{62} \log \mathcal{J}_{62}$ não é uma álgebra rígida na variedade das álgebras de Jordan de dimensão 4.

\subsection{O COMportamento de uma Álgebra ATRAVÉS DE DE- FORMAÇ $\tilde{A} O$}

A não existência de deformação entre um par de álgebras dadas, $\mathcal{J}_{1} \nrightarrow \mathcal{J}$, pode ser obtida da violação de uma das condições que apresentamos a seguir. De modo paradigmático, essas condições são definidas de tal modo que as álgebras que as satisfaçam 
formem subconjuntos fechados invariantes de $\mathcal{J o r}_{n}$ e assim qualquer deformação de $\mathcal{J}$ deve satisfazer a mesma condição.

Proposição 4.50. [23] Se $\mathcal{J} \rightarrow \mathcal{J}_{1}$ é uma deformação não trivial então $\operatorname{dim} \mathcal{J}^{\mathrm{G}}>\operatorname{dim} \mathcal{J}_{1}^{\mathrm{G}}$.

Demonstração. Da definição de deformação não trivial temos que $\jmath_{1}^{G} \subseteq \overline{J^{G}} \backslash J^{G}$. Do Lema 4.13 iii segue que $\operatorname{dim}\left(\overline{\jmath^{G}} \backslash \jmath^{G}\right)<\operatorname{dim} \jmath^{G}$.

Segue do Lema 4.13 ii. que $\operatorname{dim} \mathcal{J}^{G}=\operatorname{dim} G-\operatorname{dim} \operatorname{Stab}_{G}(\mathcal{J})$ e como provamos no início da Seção 4.3, quando $G=G L(V)$, $\operatorname{Stab}_{G}(\mathcal{J})$ coincide com o grupo de automorfismo de $\mathcal{J}$, logo como consequência da proposição anterior segue que:

Proposição 4.51. [23] Se $\mathcal{J} \rightarrow \mathcal{J}_{1}$ é uma deformação não trivial então $\operatorname{dim} \operatorname{Aut}(\mathcal{J})<\operatorname{dim} \operatorname{Aut}\left(\mathcal{J}_{1}\right)$.

A seguinte proposição relaciona as dimensões dos radicais nilpotentes de uma álgebra e de sua deformação e mostra que tal dimensão não aumenta através de uma deformação.

Proposição 4.52. [23] Se $\mathcal{J} \rightarrow \mathcal{J}_{1}$, então $\operatorname{dim} \operatorname{Rad}(\mathcal{J}) \leqslant \operatorname{dim} \operatorname{Rad}\left(\mathcal{J}_{1}\right)$.

Demonstração. É suficiente provar que os conjuntos $\left\{\mathcal{J} \in \mathcal{J o r}_{\mathfrak{n}} \mid \operatorname{dim} \operatorname{Rad}(\mathcal{J}) \geqslant s\right\}$ são fechados na topologia de Zariski em Jor ${ }_{n}$ para todo $s \in \mathbb{N}$. Isto implicará que se $\mathcal{J}_{1} \rightarrow \mathcal{J}_{2}$ e $\operatorname{dim} \operatorname{Rad}\left(\mathcal{J}_{1}\right)=s$ então $\mathcal{J}_{2}^{G} \subseteq \overline{\mathcal{J}_{1}^{G}} \subseteq\left\{\mathcal{J} \in \mathcal{J o r}_{n} \mid \operatorname{dim} \operatorname{Rad}(\mathcal{J}) \geqslant s\right\} \operatorname{logo} \operatorname{dim} \operatorname{Rad}\left(\mathcal{J}_{2}\right) \geqslant$ $\operatorname{dim} \operatorname{Rad}\left(\mathcal{J}_{1}\right)$.

Considere para qualquer número natural $m \leqslant n$ a Grassmaniana $\operatorname{Gr}(m, n)$, i.e., o conjunto dos subespaços de dimensão $m$ de um $\mathbf{k}$-espaço vetorial $\mathrm{V} \simeq \mathbf{k}^{\mathrm{n}}$. Considere também os pares $(\mathcal{J}, I)$ no produto de variedades $\mathcal{J} r_{n} \times \operatorname{Gr}(m, n)$, onde I é um ideal nilpotente de dimensão $m$ de $\mathcal{J}$. Tais pares formam um conjunto fechado $\Omega_{m}$ em $\mathrm{Jor}_{n} \times \operatorname{Gr}(m, n)$. Como $\operatorname{Gr}(m, n)$ é completa como uma variedade algébrica (i.e., para qualquer variedade $Y$ o morfismo projeção $\operatorname{Gr}(m, n) \times Y \rightarrow Y$ é uma aplicação fechada) a projeção $F_{m}$ de $\Omega_{m}$ em Jor $n$ é fechada na topologia de Zariski. Então segue de

$$
\left\{\mathcal{J} \in \operatorname{Jor}_{n} \mid \operatorname{dim} \operatorname{Rad}(\mathcal{J}) \geqslant s\right\}=\bigcup_{s \leqslant m \leqslant n} F_{m}
$$

que o conjunto $\left\{\mathcal{J} \in \mathcal{J} r_{\mathfrak{n}} \mid \operatorname{dim} \operatorname{Rad}(\mathcal{J}) \geqslant s\right\}$ é Zariski-fechado.

Observamos que a desigualdade não é estrita no caso da dimensão do radical nilpotente como é no caso da dimensão do grupo de automorfismos ou da dimensão da órbita, pois de fato em [4] foram obtidas deformações entre álgebras nilpotentes não isomorfas.

Analogamente ao que acontece com $\operatorname{Rad}(\mathcal{J})$ temos o seguinte resultado que relaciona as dimensões dos aniquiladores das álgebras. Lembrando que $\operatorname{Ann}(\mathcal{J})=\{a \in \mathcal{J} \mid \mathfrak{a} \mathcal{J}=0\}$. 
Proposição 4.53. [22] Se $\mathcal{J} \rightarrow \mathcal{J}_{1}$, então $\operatorname{dim} \operatorname{Ann}(\mathcal{J}) \leqslant \operatorname{dim} \operatorname{Ann}\left(\mathcal{J}_{1}\right)$.

Demonstração. Procederemos de maneira análoga à prova da Proposição 4.52, mostrando que o conjunto $\left\{\mathcal{J} \in \mathcal{J o r}_{n} \mid \operatorname{dim} \operatorname{Ann}(\mathcal{J}) \geqslant s\right\}$ é fechado. Vamos descrever o que é $\operatorname{dim} \operatorname{Ann}(\mathcal{J})$. Seja $\left\{e_{1}, \cdots, e_{n}\right\}$ uma base da álgebra $\mathcal{J} \in \mathcal{J o r}_{n}$ e seja $a=\sum_{i=1}^{n} \alpha_{i} e_{i}$ um elemento arbitrário de $\operatorname{Ann}(\mathcal{J}), \operatorname{logo} a \mathcal{J}=0$ ou seja $a e_{j}=0$ para todo $j=1, \cdots, n$. As equações $a e_{j}=0$ se escrevem como $a e_{j}=\sum_{k=1}^{n} \beta_{k}^{j} e_{k}=0$ onde cada $\beta_{k}^{j}$ é uma combinação linear dos $\alpha_{i}$ e é um polinômio nas constantes estruturais. Ou seja cada uma das $n$ equações $a e_{j}=0$ resulta em $n$ novas equações $\beta_{k}^{j}=0$ para $k=1, \cdots, n$ com os $\alpha_{i}$ como incógnitas. Seja então $P_{n^{2}, n}$ a matriz deste sistema de $n^{2}$ equações com $n$ incógnitas. Então $\operatorname{dim} \operatorname{Ann}(\mathcal{J})=n-\operatorname{rank}\left(P_{n^{2}, n}\right), \operatorname{logo} \operatorname{dim} \operatorname{Ann}(\mathcal{J}) \geqslant s$ é equivalente a $n-s+1>\operatorname{rank}\left(P_{n^{2}, n}\right)$ o que a sua vez é equivalente ao fato de que todos os menores de ordem $n-s+1$ de $P_{n^{2}, n}$ sejam nulos. Ou seja o conjunto $\left\{\mathcal{J} \in \operatorname{Jor}_{n} \mid \operatorname{dim} \operatorname{Ann}(\mathcal{J}) \geqslant s\right\}$ fica definido por um número finito de identidades polinomiais logo é Zariski-fechado.

Em contrapartida do que acontece com $\operatorname{Rad}(\mathcal{J})$ e $\operatorname{Ann}(\mathcal{J})$ temos que a dimensão de qualquer potência de $\mathcal{J}$ não diminui através de uma deformação.

Proposição 4.54. [23] Se $\mathcal{J} \rightarrow \mathcal{J}_{1}$ então $\operatorname{dim} \mathcal{J}^{r} \geqslant \operatorname{dim} \mathcal{J}_{1}^{r}$, para qualquer inteiro positivo $\mathrm{r}$.

Demonstração. Seja $\left\{e_{1}, \cdots, e_{n}\right\}$ uma base da álgebra $\mathcal{J} \in \operatorname{Jor}_{n}$ e considere $\Omega_{n, r}$ o conjunto de todas as palavras não associativas de comprimento $r$ nas variáveis $e_{1}, \ldots, e_{n}$. Denotemos por $l(r)=\left|\Omega_{n, r}\right|$ a cardinalidade de $\Omega_{n, r}$. Qualquer tal palavra pode ser escrita como $f_{1}^{l} e_{1}+\cdots+f_{n}^{l} e_{n}$ onde cada $f_{i}^{l}$ é um polinômio nas constantes estruturais $c_{i j}^{k}$ de $\mathcal{J}$. Considere a matriz $\mathrm{P}_{l(\mathrm{r}), n}$, onde cada linha consiste das $n$ coordenadas da palavra correspondente. Então $\operatorname{dim} \mathcal{J}^{r}=\operatorname{rank}\left(\mathrm{P}_{l(\mathrm{r}), \mathrm{n}}\right)$ e o fato de $\operatorname{dim} \mathcal{J}^{r} \leqslant s$ é equivalente ao fato que todos os menores de ordem $s+1$ sejam nulos e portanto $\left\{\mathcal{J} \in \mathcal{J o r}_{n} \mid \operatorname{dim} \mathcal{J}^{r} \leqslant s\right\}$ é fechado.

Como corolário da proposição anterior temos:

Corolário 4.55. Se J e J $\mathcal{J}_{1}$ são álgebras de Jordan nilpotentes tal que $\mathcal{J} \rightarrow J_{1}$ então o índice de nilpotência de J deve ser maior ou igual ao indice de nilpotência de $\mathfrak{J}_{1}$.

Provaremos a seguir que as identidades polinomiais de $\mathcal{J}$ são preservadas quando $\mathcal{J}$ domina uma outra álgebra $\mathcal{J}_{1}$ :

Proposição 4.56. [25] Se $\mathcal{J} \rightarrow \mathcal{J}_{1}$ então toda identidade polinomial de $\mathcal{J}$ é válida em $\mathcal{J}_{1}$. Em particular, qualquer deformação de uma álgebra não associativa é uma álgebra não associativa. 
Demonstração. Seja $S$ um conjunto de identidades polinomiais satisfeitas por $\mathcal{J}$. Então $\mathrm{V}(\mathrm{S})$ é um conjunto algébrico e logo é fechado na topologia de Zariski de $\mathbb{A}^{\mathrm{n}^{3}}$ o que implica que $\mathrm{V}(\mathrm{S}) \cap \mathrm{Jor}_{\mathrm{n}}$ é um subconjunto fechado de $\mathcal{J o r}_{n}$, G-invariante e tal que $\mathcal{J} \in$ $\mathrm{V}(\mathrm{S}) \cap \mathcal{J o r}_{n}, \operatorname{logo} \mathcal{J}_{1}^{\mathrm{G}} \subseteq \overline{\mathcal{J}^{G}} \subseteq \mathrm{V}(\mathrm{S}) \bigcap \mathcal{J o r}_{n}$ e portanto $\mathcal{J}_{1}$ também satisfaz as identidades polinomiais $S$.

Seja I um conjunto finito de identidades polinomiais. Denote por

$$
M_{I}(\mathcal{J})=\max \{\operatorname{dim} S \mid S \text { é uma subálgebra de } \mathcal{J} \text { que satisfaz } \mathrm{I}\} .
$$

Então usando um argumento análogo à prova da Proposição 4.52 temos o seguinte resultado:

Proposição 4.57. Se $\mathcal{J} \rightarrow \mathcal{J}_{1}$ então $M_{\mathrm{I}}(\mathcal{J}) \leqslant M_{\mathrm{I}}\left(\mathcal{J}_{1}\right)$.

Demonstração. Considere, para qualquer número natural $m \leqslant n$, a Grassmaniana $\operatorname{Gr}(m, n)$. Considere também os pares $(\mathcal{J}, S)$ no produto de variedades $\operatorname{Jor}_{n} \times \operatorname{Gr}(m, n)$, onde $S$ é uma subálgebra de dimensão $m$ de $\mathcal{J}$ satisfazendo I. Tais pares formam um conjunto fechado que denotaremos por $\Omega_{m}$ em $\operatorname{Jor}_{n} \times \operatorname{Gr}(m, n)$. Como $\operatorname{Gr}(m, n)$ é completa a projeção $F_{m}$ de $\Omega_{m}$ em Jor $_{n}$ é fechada na topologia de Zariski. Então segue de

$$
\left\{\mathcal{J} \in \operatorname{Jor}_{\mathrm{n}} \mid M_{\mathrm{I}}(\mathcal{J}) \geqslant s\right\}=\bigcup_{s \leqslant m \leqslant n} F_{m}
$$

que o conjunto $\left\{\mathcal{J} \in \mathcal{J o r}_{n} \mid M_{I}(\mathcal{J}) \geqslant s\right\}$ é Zariski-fechado.

Em particular, o resultado vale para subálgebras associativas, nilpotentes e nulas, i.e., subálgebras que satisfazem a identidade $x y=0$ para todo $x$ e $y$. Denotaremos $M_{I}(\mathcal{\partial})$ nesses casos particulares como sendo $M_{A}(\mathcal{J}), M_{N}(\mathcal{J})$ e $M_{N u l}(\mathcal{J})$ respetivamente.

Considere a subálgebra dos elementos de $\mathcal{J}$ que "associam" com todos os outros, i.e., o conjunto definido por

$$
\mathrm{Z}(\mathcal{J})=\{a \in \mathcal{J} \mid(a, \mathcal{J}, \mathcal{J})=(\mathcal{J}, a, \mathcal{J})=(\mathcal{J}, \mathcal{J}, a)=0\}
$$

que chamaremos de centro associativo de $\mathcal{J}$. Então temos:

Proposição 4.58. Se $\mathcal{J} \rightarrow \mathcal{J}_{1}$ então $\operatorname{dim} Z(\mathcal{J}) \leqslant \operatorname{dim} Z\left(\mathcal{J}_{1}\right)$.

Demonstração. Seja $\left\{e_{1}, \cdots, e_{n}\right\}$ uma base de $\mathcal{J} \in \mathcal{J O r}_{n}$ e $a=\sum_{i=1}^{n} \alpha_{i} e_{i}$ um elemento de $Z(\mathcal{J})$, logo cada uma das $3 n^{2}$ equações $\left(a, e_{i}, e_{j}\right)=0,\left(e_{i}, a, e_{j}\right)=0,\left(e_{i}, e_{j}, a\right)=0$ para $i, j=1, \cdots, n$ resulta em $n$ novas equações tendo os $\alpha_{i}$ como incógnitas. Seja então $P_{3 n^{3}, n}$ a matriz deste sistema de $3 n^{3}$ equações com $n$ incógnitas. Então $\operatorname{dim} Z(\mathcal{J})=$ 
$n-\operatorname{rank}\left(P_{3 n^{3}, n}\right), \operatorname{logo} \operatorname{dim} Z(\mathcal{J}) \geqslant s$ é equivalente a $n-s+1>\operatorname{rank}\left(P_{3 n^{3}, n}\right)$ o que a sua vez é equivalente ao fato de que todos os menores de ordem $n-s+1$ de $P_{3 n^{3}, n}$ sejam nulos. Ou seja o conjunto $\left\{\mathcal{J} \in \mathcal{J}^{\circ} r_{\mathfrak{n}} \mid \operatorname{dim} Z(\mathcal{J}) \geqslant s\right\}$ fica definido por um número finito de identidades polinomiais logo é Zariski-fechado.

Usaremos frequentemente o fato que deformação preserva tanto o produto original em $\mathcal{J}_{s s}$ como a ação de $\mathcal{J}_{s s}$ em $\operatorname{Rad}(\mathcal{J})$. Este resultado é análogo ao Teorema 4.23 enunciado para família de deformações a um parâmetro. A saber,

Proposição 4.59. [25, Prop.2.2] Para uma álgebra de Jordan não nilpotente J considere o par $\left(\mathcal{J}_{s \mathrm{~s}}, \Gamma(\mathcal{J})\right)$, onde $\Gamma(\mathcal{J})$ é J como espaço vetorial e tem estrutura de $\mathcal{J}_{\mathrm{ss}}$-bimódulo. Então para qualquer componente irredutível $\mathrm{T}$ de $\mathrm{Jor}_{\mathrm{n}},\left(\mathcal{J}_{\mathrm{ss}}, \Gamma(\mathcal{J})\right)$ é constante em um subconjunto aberto de $\mathrm{T}$. Se $\mathcal{J}_{1} \in \mathrm{T}$ então $\left(\mathcal{J}_{1}\right)_{\mathrm{ss}}$ é uma subálgebra de $\mathcal{J}_{\mathrm{ss}}$ e $\Gamma\left(\mathcal{J}_{1}\right)$ é a restrição de $\Gamma(\mathcal{J})$ em $\left(\mathcal{J}_{1}\right)_{s s}$.

Como consequências diretas da proposição anterior temos os seguintes dois resultados:

Proposição 4.60. Se $\mathcal{J} \rightarrow \mathcal{J}_{1}$ e $\mathcal{J}_{1}$ admite decomposição de Peirce em relação a um sistema de $\mathrm{r}$ idempotentes ortogonais então em J existem $\mathrm{r}$ idempotentes ortogonais tal que a decomposição de Peirce de J em relação a esse sistema coincide com a de J.

Proposição 4.61. Se J $\rightarrow \mathcal{J}_{1}$ e J $\mathcal{J}_{1}$ é uma álgebra unitária, então J deve ser também uma álgebra unitária.

Provaremos a seguir que a dimensão do conjunto dos 2-cociclos de $\mathcal{I}$ com coeficientes em $\mathcal{J}$ não aumenta através de uma deformação.

Proposição 4.62. Se $\mathcal{J} \rightarrow \mathcal{J}_{1}$ então $\operatorname{dim} Z^{2}(\mathcal{J}, \mathcal{J}) \leqslant \operatorname{dim} Z^{2}\left(\mathcal{J}_{1}, \mathcal{J}_{1}\right)$.

Demonstração. Seja $\mathrm{h}: \mathcal{J} \times \mathcal{J} \rightarrow \mathcal{J}$ um 2-cociclo de $\mathcal{J}$ com coeficientes em $\mathcal{J}$. Então $h$ fica definida pelas $\mathrm{n}^{3}$ constantes $\alpha_{i j}^{k} \in \mathbf{k}$ dadas por $h\left(e_{i}, e_{j}\right)=\sum_{k=1}^{n} \alpha_{i j}^{k} e_{k}$. Linearizando completamente as identidades de 2-cociclo (1.6) obtemos

$$
\begin{aligned}
& (h(x, y) w) z+(h(x, z) w) y+(h(y, z) w) x+h((x y) w, z)+h((x z) w, y)+h((y z) w, x)+ \\
& +h(x y, w) z+h(x z, w) y+h(y z, w) x=(x y) h(w, z)+(x z) h(w, y)+(y z) h(w, x)+ \\
& +h(x, y)(w z)+h(x, z)(w y)+h(y, z)(w x)+h(x y, w z)+h(x z, w y)+h(y z, w x) .
\end{aligned}
$$

Calcular essas equações e a condição de comutatividade de $h$ nos elementos da base resulta em $l(n)=\left(n^{4}+\frac{n(n-1)}{2}\right) n$ equações tendo os $\alpha_{i j}^{k}$ como incógnitas. Então $\operatorname{dim} Z^{2}(\mathcal{J}, \mathcal{J})=n^{3}-\operatorname{rank}\left(P_{l(n), n^{3}}\right)$, onde como antes $P_{l(n), n^{3}}$ representa a matriz do sistema de equações, assim $\operatorname{dim} Z^{2}(\mathcal{J}, \mathcal{J}) \geqslant s$ é equivalente ao fato de que todos os menores de ordem $n-s+1$ de $P_{l(n), n^{3}}$ sejam nulos. Logo o conjunto $\left\{\mathcal{J} \in \operatorname{Jor}_{n} \mid \operatorname{dim} Z^{2}(\mathcal{J}, \mathcal{J}) \geqslant s\right\}$ é Zariski-fechado. 
E analogamente,

Proposição 4.63. Se $\mathcal{J} \rightarrow \mathcal{J}_{1}$ então $\operatorname{dim} B^{2}(\mathcal{J}, \mathcal{J}) \geqslant \operatorname{dim} B^{2}\left(\mathcal{J}_{1}, \mathcal{J}_{1}\right)$.

Demonstração. Seja $f \in B^{2}(\mathcal{J}, \mathcal{J})$ um 2-cobordo de $\mathcal{J}$ com coeficientes em $\mathcal{J}$ então existe uma aplicação linear $\Phi: \mathcal{J} \rightarrow \mathcal{J}$ tal que $f=\delta \Phi$. Assim $f$ fica definida por $f\left(e_{i}, e_{j}\right)=$ $\Phi\left(e_{i} e_{j}\right)-e_{i} \Phi\left(e_{j}\right)-\Phi\left(e_{i}\right) e_{j}$ dependendo das $n^{2}$ constantes $\alpha_{i j} \in \mathbf{k}$ que definem $\Phi\left(e_{i}\right)=$ $\sum_{j=1}^{n} \alpha_{i j} e_{j}$.

Calcular essas condições nos elementos da base resulta em $n^{3}$ equações tendo os $\alpha_{i j}$ como incógnitas. Então dim $B^{2}(\mathcal{J}, \mathcal{J})=\operatorname{rank}\left(P_{n^{3}, n^{2}}\right)$, onde $P_{n^{3}, n^{2}}$ representa a matriz do sistema de equações, assim $\operatorname{dim} B^{2}(\mathcal{J}, \mathcal{J}) \leqslant s$ é equivalente ao fato de que todos os menores de ordem $s+1$ de $P_{n^{3}, n^{2}}$ sejam nulos. Logo o conjunto $\left\{\mathcal{J} \in \operatorname{Jor}_{n} \mid \operatorname{dim} B^{2}(\mathcal{J}, \mathcal{J}) \leqslant s\right\}$ é Zariski-fechado.

Combinando as Proposições 4.62 e 4.63 obtemos o seguinte resultado:

Proposição 4.64. Se $\mathcal{J} \rightarrow \mathcal{J}_{1}$ então $\operatorname{dim} H^{2}(\mathcal{J}, \mathcal{J}) \leqslant \operatorname{dim} H^{2}\left(\mathcal{J}_{1}, \mathcal{J}_{1}\right)$.

\subsection{Algumas COMPOnEntes irRedutíveis DE Jor mara $_{m}$ PA $m \geqslant 2$}

Provamos na Proposição 4.36 que toda álgebra rígida gera uma componente irredutível. Os seguintes teoremas mostram que em toda variedade de álgebras de Jordan de dimensão maior ou igual a 5 temos pelo menos 3 componentes irredutíveis que provem de álgebras infinitesimalmente rígidas que são indecomponíveis, não associativas e não semissimples.

Teorema 4.65. Seja $\mathrm{m} \in \mathbb{N}$ finito e $\mathrm{m} \geqslant 2$. A álgebra de Jordan $\mathrm{A}_{\mathrm{m}} \in \mathrm{Jor}_{\mathrm{m}}$ sobre um corpo $\mathbf{k}$ com char $\mathbf{k} \neq 2$, que possui base $\left\{e_{1}, n_{1}, \cdots, n_{m-1}\right\}$ tal que $e_{i}$ é idempotente e $e_{1} n_{i}=n_{i} e_{1}=$ $\frac{1}{2} n_{i}$ e $n_{i} n_{j}=0$ para todo $i, j=1, \cdots, m-1$ é uma álgebra rígida e a componente irredutível por ela determinada tem dimensão $\mathrm{m}$.

Demonstração. Primeiramente provaremos que de fato a álgebra $A_{m}$ assim definida é de Jordan, i.e., satisfaz a identidade (1.2) para todo $x, y \in A_{m}$ pois já é comutativa 
por definição. Sejam então $x=\alpha e_{1}+\sum_{i=1}^{m-1} \beta_{i} n_{i}$ e $y=\alpha^{\prime} e_{1}+\sum_{i=1}^{m-1} \beta_{i}^{\prime} n_{i}$ para $\alpha, \alpha^{\prime}, \beta_{i}, \beta_{i}^{\prime} \in \mathbf{k}, \operatorname{logo}$ temos

$$
\begin{aligned}
\left(x^{2}, y, x\right) & =\left(\alpha^{2} e_{1}+\sum_{i=1}^{m-1} \alpha \beta_{i} n_{i}, \alpha^{\prime} e_{1}+\sum_{i=1}^{m-1} \beta_{i}^{\prime} n_{i}, \alpha e_{1}+\sum_{i=1}^{m-1} \beta_{i} n_{i}\right) \\
& =\left(\alpha^{2} e_{1}, \alpha^{\prime} e_{1}, \alpha e_{1}\right)+\left(\alpha^{2} e_{1}, \alpha^{\prime} e_{1}, \sum_{i=1}^{m-1} \beta_{i} n_{i}\right)+ \\
& +\left(\alpha^{2} e_{1}, \sum_{i=1}^{m-1} \beta_{i}^{\prime} n_{i}, \alpha e_{1}\right)+\left(\alpha^{2} e_{1}, \sum_{i=1}^{m-1} \beta_{i}^{\prime} n_{i}, \sum_{i=1}^{m-1} \beta_{i} n_{i}\right)+ \\
& +\left(\sum_{i=1}^{m-1} \alpha \beta_{i} n_{i}, \alpha^{\prime} e_{1}, \alpha e_{1}\right)+\left(\sum_{i=1}^{m-1} \alpha \beta_{i} n_{i}, \alpha^{\prime} e_{1}+\sum_{i=1}^{m-1} \beta_{i} n_{i}\right)+ \\
& +\left(\sum_{i=1}^{m-1} \alpha \beta_{i} n_{i}, \sum_{i=1}^{m-1} \beta_{i}^{\prime} n_{i}, \alpha e_{1}\right)+\left(\sum_{i=1}^{m-1} \alpha \beta_{i} n_{i}, \sum_{i=1}^{m-1} \beta_{i}^{\prime} n_{i}, \sum_{i=1}^{m-1} \beta_{i} n_{i}\right) \\
& =\sum_{i=1}^{m-1} \frac{1}{4} \alpha^{2} \alpha^{\prime} \beta_{i} n_{i}-\sum_{i=1}^{m-1} \frac{1}{4} \alpha^{2} \alpha^{\prime} \beta_{i} n_{i}=0
\end{aligned}
$$

Agora, seja $T \in \operatorname{Aut}\left(A_{m}\right)$ então $T$ é definido por $T\left(e_{1}\right)=e_{1}+\sum_{i=1}^{m-1} a_{0 i} n_{i}$ com $a_{0 i} \in \mathbf{k}$ e $T\left(n_{i}\right)=\sum_{j=1}^{m-1} a_{i j} n_{j}$ para $i=1, \cdots, m-1$ e com $a_{i j} \in \mathbf{k}$. Ou seja dos $m^{2}$ coeficientes que definem $T$ temos $(m-1)+(m-1)^{2}$ livres. Isto é $\operatorname{dim} \operatorname{Aut}\left(A_{m}\right)=$ $m^{2}-m \log 0$

$$
\operatorname{dim} \overline{A_{m}^{G}}=m^{2}-\operatorname{dim} \operatorname{Aut}\left(A_{m}\right)=m
$$

Considere $h \in Z^{2}\left(A_{m}, A_{m}\right)$ um 2-cociclo de $A_{m}$ com coeficientes em $A_{m}$, então $h$ : $A_{m} \times A_{m} \rightarrow A_{m}$ é uma aplicação bilinear satisfazendo (1.6). Resolvendo as equações de 2-cociclos linearizadas (veja Proposição 4.62) nos elementos da base de $A_{m}$ obtemos:

$$
h\left(e_{1}, e_{1}\right)=\alpha e_{1} \quad h\left(e_{1}, n_{i}\right)=\beta_{i} e_{1}+\frac{\alpha}{2} n_{i} \quad h\left(n_{i}, n_{j}\right)=\beta_{j} n_{i}+\beta_{i} n_{j}
$$

para quaisquer $\alpha, \beta_{i} \in \mathbf{k}$ e $1 \leqslant i, j \leqslant m-1$. Ou seja temos $1+(m-1)$ coeficientes livres definindo um 2-cociclo arbitrário, $\operatorname{logo} \operatorname{dim} Z^{2}\left(A_{m}, A_{m}\right)=m$. Seja, agora $f \in$ $B^{2}\left(A_{m}, A_{m}\right)$ um 2-cobordo de $A_{m}$ com coeficientes em $A_{m}$ então existe uma aplicação linear $\Phi: A_{m} \rightarrow A_{m}$ tal que $f=\delta \Phi$. Então $f$ deve ser definido por:

$$
f\left(e_{1}, e_{1}\right)=-\alpha^{\prime} e_{1} \quad f\left(e_{1}, n_{i}\right)=-\frac{1}{2} \beta_{i}^{\prime} e_{1}-\frac{1}{2} \alpha^{\prime} n_{i} \quad f\left(n_{i}, n_{j}\right)=-\frac{1}{2} \beta_{j}^{\prime} n_{i}-\frac{1}{2} \beta_{i}^{\prime} n_{j}
$$


para quaisquer $\alpha^{\prime}, \beta_{i}^{\prime} \in \mathbf{k}$ e $1 \leqslant i, j \leqslant m-1$. Logo $f$ depende de $1+(m-1)$ parâmetros e portanto $\operatorname{dim} B^{2}\left(A_{m}, A_{m}\right)=m$ o que implica que $H^{2}\left(A_{m}, A_{m}\right)=0$.

Uma das questões que surgiu durante a realização deste trabalho foi o que acontece com uma álgebra rígida à qual adjuntamos formalmente um elemento identidade, a resposta foi que nem sempre ela permanece rígida:

Lema 4.66. Rigidez não é preservada por adjunção formal de um elemento identidade.

Demonstração. Seja $n \geqslant 3$ e seja $A_{n-1} \in$ Jor $_{n-1}$ a álgebra rígida do Teorema 4.65. Então a álgebra $A_{n-1}^{\#} \in J^{\circ} r_{n}$ é uma álgebra de Jordan unitária que resulta de adjuntar formalmente o elemento identidade de $\mathbf{k}$ a $A_{n-1}$ e tem produto definido por (1.4). Seja $\mathcal{J}(\mathrm{V}, \mathrm{f}) \in \mathcal{J o r}_{\mathrm{n}}$ a álgebra de Jordan da forma bilinear simétrica não degenerada $\mathrm{f}: \mathrm{V} \times \mathrm{V} \rightarrow \mathbf{k}$, com $\mathrm{V}$ um $\mathbf{k}$-espaço vetorial de $\operatorname{dim}_{\mathbf{k}} \mathrm{V}=\mathrm{n}-1$ e base $\left\{e_{2}, \cdots, e_{\mathrm{n}}\right\}$, dada por $f\left(e_{i}, e_{i}\right)=1$ e $f\left(e_{i}, e_{j}\right)=0$ para $i \neq j$ e $2 \leqslant i, j \leqslant n$. Claramente $\mathcal{J}(V, f) \not A_{n-1}^{\#}$ pois $\mathcal{J}(\mathrm{V}, \mathrm{f})$ é uma álgebra simples enquanto que $\operatorname{dim} \operatorname{Rad}\left(A_{n-1}^{\#}\right)=n-2 \geqslant 1$. Logo $A_{n-1}^{\#}$ não é uma álgebra rígida pois $\mathcal{J}(V, f)$ é uma deformação (não trivial) de $A_{n-1}^{\#}$ dada pela curva

$$
\begin{aligned}
& E_{1}^{t}=e_{1} \\
& E_{2}^{t}=\frac{1}{2} e_{1}+\frac{1}{2} e_{2} \\
& E_{i}^{t}=t e_{i} \quad i=3, \cdots, n .
\end{aligned}
$$

Uma outra família de álgebras rígidas é descrita no seguinte teorema:

Teorema 4.67. Seja $\mathrm{m} \in \mathbb{N}$ finito e $\mathrm{m} \geqslant 4$. A álgebra de Jordan $\mathrm{B}_{\mathrm{m}} \in$ Jor $_{\mathrm{m}}$ sobre um corpo $\mathbf{k}$ com char $\mathbf{k} \neq 2$, que possui base $\left\{\mathrm{e}_{1}, \mathrm{e}_{2}, \mathrm{n}_{1}, \cdots, \mathrm{n}_{\mathrm{m}-2}\right\}$ onde $\mathrm{e}_{1}$ e e $\mathrm{e}_{2}$ são idempotentes ortogonais e o produto comutativo é definido por

$$
\begin{array}{ll}
\mathrm{e}_{1} \mathrm{n}_{\mathrm{j}}=\frac{1}{2} \mathrm{n}_{j} & \mathrm{n}_{\mathrm{i}} \mathrm{n}_{\mathrm{j}}=0 \text { para } \mathrm{i}, \mathrm{j}=1, \cdots, \mathrm{m}-2 \\
\mathrm{e}_{2} \mathrm{n}_{1}=\frac{1}{2} \mathrm{n}_{1} & \mathrm{e}_{2} \mathrm{n}_{\mathrm{k}}=0 \text { para } \mathrm{k}=2, \cdots, \mathrm{m}-2
\end{array}
$$

é uma álgebra rígida. 
Demonstração. Primeiramente provaremos que de fato a álgebra $B_{m}$ assim definida é uma álgebra de Jordan. Sejam $x=\sum_{i=1}^{2} \alpha_{i} e_{i}+\sum_{i=1}^{m-2} \beta_{i} n_{i}$ e $y=\sum_{i=1}^{2} \alpha_{i}^{\prime} e_{i}+$ $\sum_{i=1}^{m-2} \beta_{i}^{\prime} n_{i}$ para $\alpha_{i}, \alpha_{i}^{\prime}, \beta_{i}, \beta_{i}^{\prime} \in \mathbf{k}$, logo temos

$$
\begin{aligned}
\left(x^{2}, y, x\right) & =\left(\sum_{i=1}^{2} \alpha_{i}^{2} e_{i}+\sum_{i=1}^{m-2} \alpha_{1} \beta_{i} n_{i}+\alpha_{2} \beta_{1} n_{1}, y, x\right) \\
& =\left(\sum_{i=1}^{2} \alpha_{i}^{2} e_{i}, y, x\right)+\left(\sum_{i=1}^{m-2} \alpha_{1} \beta_{i} n_{i}, y, x\right)+\left(\alpha_{2} \beta_{1} n_{1}, y, x\right) \\
& =\frac{1}{4}\left(\left(-\alpha_{2}^{2} \alpha_{1}^{\prime}+\alpha_{2}^{2} \alpha_{2}^{\prime}-\alpha_{1}^{2} \alpha_{2}^{\prime}\right) \beta_{1} n_{1}+\sum_{i=1}^{m-2} \alpha_{1}^{2} \alpha_{1}^{\prime} \beta_{i} n_{i}\right)- \\
& -\frac{1}{4}\left(\left(-\alpha_{2} \alpha_{1} \alpha_{1}^{\prime}+\alpha_{2} \alpha_{1} \alpha_{2}^{\prime}-\alpha_{1}^{2} \alpha_{2}^{\prime}\right) \beta_{1} n_{1}+\sum_{i=1}^{m-2} \alpha_{1}^{2} \alpha_{1}^{\prime} \beta_{i} n_{i}\right)+ \\
& +\frac{1}{4}\left(-\alpha_{2} \alpha_{1} \alpha_{1}^{\prime}+\alpha_{2} \alpha_{1} \alpha_{2}^{\prime}+\alpha_{2}^{2} \alpha_{1}^{\prime}-\alpha_{2}^{2} \alpha_{2}^{\prime}\right) \beta_{1} n_{1} \\
& =0
\end{aligned}
$$

Seja, agora $f \in B^{2}\left(B_{m}, B_{m}\right)$ um 2-cobordo de $B_{m}$ com coeficientes em $B_{m}$ então existe uma aplicação linear $\Phi: B_{m} \rightarrow B_{m}$ tal que $f=\delta \Phi$. Então $f$ deve ser definido por:

$$
\begin{aligned}
& f\left(e_{1}, e_{1}\right)=-u_{11} e_{1}+u_{12} e_{2} \\
& f\left(e_{2}, e_{2}\right)=u_{21} e_{1}-u_{22} e_{2}+\sum_{i=2}^{m-2} u_{2,(i+2)} n_{i} \\
& f\left(n_{1}, n_{1}\right)=-\left(u_{31}+u_{32}\right) n_{1} \\
& f\left(n_{i}, n_{i}\right)=-u_{(i+2), 1} n_{i} \\
& f\left(e_{1}, e_{2}\right)=-u_{21} e_{1}-u_{12} e_{2}-\frac{1}{2}\left(u_{13}+u_{23}\right) n_{1}-\frac{1}{2} \sum_{i=2}^{m-2} u_{2,(i+2)} n_{i} \\
& f\left(e_{1}, n_{1}\right)=-\frac{1}{2} u_{31} e_{1}+\frac{1}{2} u_{32} e_{2}-\frac{1}{2}\left(u_{11}+u_{12}\right) n_{1} \\
& f\left(e_{2}, n_{1}\right)=\frac{1}{2} u_{31} e_{1}-\frac{1}{2} u_{32} e_{2}-\frac{1}{2}\left(u_{21}+u_{22}\right) n_{1}+\frac{1}{2} \sum_{i=2}^{m-2} u_{3,(i+2)} n_{i} \\
& f\left(e_{1}, n_{i}\right)=-\frac{1}{2} u_{(i+2), 1} e_{1}+\frac{1}{2} u_{(i+2), 2} e_{2}-\frac{1}{2} u_{11} n_{i} \\
& f\left(e_{2}, n_{i}\right)=-u_{(i+2) 2} e_{2}-\frac{1}{2} u_{(i+2), 3} n_{1}-\frac{1}{2} u_{21} n_{i} \\
& f\left(n_{1}, n_{i}\right)=-\frac{1}{2}\left(u_{(i+2), 1}+u_{(i+2), 2}\right) n_{1}-\frac{1}{2} u_{31} n_{i}
\end{aligned}
$$




$$
f\left(n_{i}, n_{j}\right)=-\frac{1}{2} u_{(j+2), 1} n_{i}-\frac{1}{2} u_{(i+2), 1} n_{j}
$$

onde $i \neq j$ e $i, j=2, \cdots, m-2$. Logo $f$ depende de $1+3 \cdot 2+5(m-3)$ parâmetros $u_{i j} \in \mathbf{k}$ e portanto $\operatorname{dim} B^{2}\left(B_{m}, B_{m}\right)=7+5(m-3)$.

Considere $h \in Z^{2}\left(B_{m}, B_{m}\right)$, resolvendo as equações de 2-cociclos linearizadas nos elementos da base de $B_{m}$ obtemos:

$$
\begin{aligned}
& h\left(e_{1}, e_{1}\right)=a_{11}^{1} e_{1}+a_{11}^{2} e_{2} \\
& h\left(e_{2}, e_{2}\right)=-a_{12}^{1} e_{1}+2 a_{22}^{2} e_{2}-2 \sum_{i=2}^{m-2} a_{12}^{(i+2)} n_{i} \\
& h\left(n_{1}, n_{1}\right)=2\left(a_{13}^{1}-a_{13}^{2}\right) n_{1} \\
& h\left(n_{i}, n_{i}\right)=2 a_{1,(i+2), 1}^{1} n_{i} \\
& h\left(e_{1}, e_{2}\right)=a_{12}^{1} e_{1}-a_{11}^{2} e_{2}+a_{12}^{3} n_{1}+\sum_{i=2}^{m-2} a_{12}^{(i+2)} n_{i} \\
& h\left(e_{1}, n_{1}\right)=a_{13}^{1} e_{1}+a_{13}^{2} e_{2}+\frac{1}{2}\left(a_{11}^{1}-a_{11}^{2}\right) n_{1} \\
& h\left(e_{2}, n_{1}\right)=-a_{13}^{1} e_{1}-a_{13}^{2} e_{2}+\frac{1}{2}\left(a_{12}^{1}+a_{22}^{2}\right) n_{1}+\sum_{i=2}^{m-2} a_{23}^{(i+2)} n_{i} \\
& h\left(e_{1}, n_{i}\right)=a_{1,(i+2)}^{1} e_{1}+a_{1,(i+2)}^{2} e_{2}+\frac{1}{2} a_{11}^{1} n_{i} \\
& h\left(e_{2}, n_{i}\right)=-2 a_{1,(i+2)}^{2} e_{2}+a_{2,(i+2)}^{3} n_{1}+\frac{1}{2} a_{12}^{1} n_{i} \\
& h\left(n_{1}, n_{i}\right)=\left(a_{1,(i+2)}^{1}-a_{1,(i+2)}^{2}\right) n_{1}+a_{13}^{1} n_{i} \\
& h\left(n_{i}, n_{j}\right)=a_{1,(j+2)}^{1} n_{i}+a_{1(i+2)}^{1} n_{j}
\end{aligned}
$$

onde $i \neq j$ e $i, j=2, \cdots, m-2$. Logo $h$ depende de $1+3 \cdot 2+5(m-3)$ parâmetros $a_{i j}^{k} \in \mathbf{k}$ e portanto $\operatorname{dim} Z^{2}\left(B_{m}, B_{m}\right)=7+5(m-3)$, segue que $H^{2}\left(B_{m}, B_{m}\right)=0$.

Teorema 4.68. Seja $\mathrm{m} \in \mathbb{N}$ finito e $\mathrm{m} \geqslant 4$. A álgebra de Jordan $\mathrm{C}_{\mathrm{m}} \in$ Jor $_{\mathrm{m}}$ sobre um corpo $\mathbf{k}$ com char $\mathbf{k} \neq 2$, que possui base $\left\{\mathrm{e}_{1}, \mathrm{e}_{2}, \mathrm{n}_{1}, \cdots, \mathrm{n}_{\mathrm{m}-2}\right\}$ onde $\mathrm{e}_{1} e \mathrm{e}_{2}$ são idempotentes ortogonais e o produto comutativo é definido por

$$
\begin{aligned}
& e_{1} n_{j}=\frac{1}{2} n_{j} \quad n_{i} n_{j}=0 \text { para } i, j=1, \ldots, m-2 \\
& e_{2} n_{1}=0 \quad e_{2} n_{k}=\frac{1}{2} n_{k} \text { para } k=2, \cdots, m-2
\end{aligned}
$$


é uma álgebra rígida.

Demonstração. Uma conta longa, análoga à do teorema anterior mostra que a álgebra $C_{m}$ assim definida é uma álgebra de Jordan. Suponha agora que $f \in B^{2}\left(C_{m}, C_{m}\right)$. Então $f$ deve ser definido por:

$$
\begin{aligned}
& f\left(e_{1}, e_{1}\right)=-u_{11} e_{1}+u_{12} e_{2} \\
& f\left(e_{2}, e_{2}\right)=u_{21} e_{1}-u_{22} e_{2}+u_{23} n_{1} \\
& f\left(n_{1}, n_{1}\right)=-u_{31} n_{1} \\
& f\left(n_{i}, n_{i}\right)=-\left(u_{(i+2), 1}+u_{(i+2), 2}\right) n_{i} \\
& f\left(e_{1}, e_{2}\right)=-u_{21} e_{1}-u_{12} e_{2}-\frac{1}{2} u_{23} n_{1}-\frac{1}{2} \sum_{i=2}^{m-2}\left(u_{1,(i+2)}+u_{2,(i+2)}\right) n_{i} \\
& f\left(e_{1}, n_{1}\right)=-\frac{1}{2} u_{31} e_{1}+\frac{1}{2} u_{32} e_{2}-\frac{1}{2} u_{11} n_{1} \\
& f\left(e_{2}, n_{1}\right)=-u_{32} e_{2}-\frac{1}{2} u_{21} n_{1}-\frac{1}{2} \sum_{i=2}^{m-2} u_{3,(i+2)} n_{i} \\
& f\left(e_{1}, n_{i}\right)=-\frac{1}{2} u_{(i+2), 1} e_{1}+\frac{1}{2} u_{(i+2), 2} e_{2}-\frac{1}{2}\left(u_{11}+u_{12}\right) n_{i} \\
& f\left(e_{2}, n_{i}\right)=\frac{1}{2} u_{(i+2), 1} e_{1}-\frac{1}{2} u_{(i+2) 2} e_{2}+\frac{1}{2} u_{(i+2), 3} n_{1}-\frac{1}{2}\left(u_{21}+u_{22}\right) n_{i} \\
& f\left(n_{1}, n_{i}\right)=-\frac{1}{2} u_{(i+2), 1} n_{1}-\frac{1}{2}\left(u_{31}+u_{32}\right) n_{i} \\
& f\left(n_{i}, n_{j}\right)=-\frac{1}{2}\left(u_{(j+2), 1}+u_{(j+2), 2} n_{i}-\frac{1}{2}\left(u_{(i+2), 1}+u_{(i+2), 2}\right) n_{j}\right.
\end{aligned}
$$

onde $i \neq j$ e $i, j=2, \cdots, m-2$. Logo $f$ depende de $1+3 \cdot 2+5(m-3)$ parâmetros $u_{i j} \in \mathbf{k}$ e portanto $\operatorname{dim} B^{2}\left(C_{m}, C_{m}\right)=7+5(m-3)$. Considere agora $h \in Z^{2}\left(C_{m}, C_{m}\right)$ então:

$$
\begin{aligned}
& h\left(e_{1}, e_{1}\right)=a_{11}^{1} e_{1}+a_{11}^{2} e_{2} \\
& h\left(e_{2}, e_{2}\right)=-a_{12}^{1} e_{1}+a_{22}^{2} e_{2}-2 a_{12}^{3} n_{1} \\
& h\left(n_{1}, n_{1}\right)=2 a_{13}^{1} n_{1} \\
& h\left(n_{i}, n_{i}\right)=2\left(a_{1,(i+2), 1}^{1}-a_{1,(i+2)}^{2}\right) n_{i} \\
& h\left(e_{1}, e_{2}\right)=a_{12}^{1} e_{1}-a_{11}^{2} e_{2}+a_{12}^{3} n_{1}+\sum_{i=2}^{m-2} a_{12}^{(i+2)} n_{i} \\
& h\left(e_{1}, n_{1}\right)=a_{13}^{1} e_{1}+a_{13}^{2} e_{2}+\frac{1}{2} a_{11}^{1} n_{1}
\end{aligned}
$$




$$
\begin{aligned}
& h\left(e_{2}, n_{1}\right)=-2 a_{13}^{2} e_{2}+\frac{1}{2} a_{12}^{1} n_{1}+\sum_{i=2}^{m-2} a_{23}^{(i+2)} n_{i} \\
& h\left(e_{1}, n_{i}\right)=a_{1,(i+2)}^{1} e_{1}+a_{1,(i+2)}^{2} e_{2}+\frac{1}{2}\left(a_{11}^{1}-a_{11}^{2}\right) n_{i} \\
& h\left(e_{2}, n_{i}\right)=-a_{1,(i+2)}^{1} e_{1}-a_{1,(i+2)}^{2} e_{2}+a_{2,(i+2)}^{3} n_{1}+\frac{1}{2}\left(a_{12}^{1}+a_{22}^{2}\right) n_{i} \\
& h\left(n_{1}, n_{i}\right)=a_{1,(i+2)}^{1} n_{1}+\left(a_{13}^{1}-a_{13}^{2}\right) n_{i} \\
& h\left(n_{i}, n_{j}\right)=\left(a_{1,(j+2)}^{1}-a_{1,(j+2)}^{2}\right) n_{i}+\left(a_{1(i+2)}^{1}-a_{1,(i+2)}^{2}\right) n_{j}
\end{aligned}
$$

onde $i \neq j$ e $i, j=2, \cdots, m-2$. Logo $\operatorname{dim} Z^{2}\left(C_{m}, C_{m}\right)=7+5(m-3)$ portanto segue que $\mathrm{H}^{2}\left(\mathrm{C}_{\mathrm{m}}, \mathrm{C}_{\mathrm{m}}\right)=0$.

Para finalizar observamos que se $m=4$ a álgebra $B_{4}$ coincide com a álgebra $C_{4}$ mas, para cada $m \geqslant 5$, a álgebra de Jordan $J_{12}$ é uma subálgebra de dimensão 4 de $B_{m}$ mas não é subálgebra de $C_{m}$, logo elas não são isomorfas. Assim, sempre que $m$ for maior ou igual a 5 os teoremas anteriores nos fornecem 3 álgebras rígidas não isomorfas, não semissimples, não associativas e indecomponíveis que determinam 3 componentes irredutíveis em Jor $\mathrm{m}_{\mathrm{m}}$. Agora, como estas álgebras são infinitesimalmente rígidas, para $m$ suficientemente grande, podemos considerar somas diretas em diferentes dimensões e obter, no mínimo, 12 novas álgebras rígidas.

De modo análogo, uma estimativa, a grosso modo, do número de componentes irredutíveis de Jor $_{m}$ pode ser obtida do fato que em cada dimensão temos pelo menos uma álgebra infinitesimalmente rígida e que somas diretas de tais álgebras também são rígidas. Assim temos pelo menos tantas componentes irredutíveis quanto o número de partições $p(m)$ do inteiro positivo $m$, o qual satisfaz (veja [34, Cap. 15]) a fórmula assintótica:

$$
p(m) \sim \frac{e^{\pi \sqrt{\frac{2}{3} m}}}{4 \sqrt{3} m} .
$$





\section{5 \\ CLASSIFICAÇÃO GEOMÉTRICA DAS ÁLGEBRAS DE JORDAN DE DIMENSÃO MENOR OU IGUAL A 4 SO- BRE UM CORPO ALGEBRICAMENTE FECHADO}

Neste capítulo classificaremos geometricamente as álgebras de Jordan de dimensão 4. Consideraremos tais álgebras definidas sobre um corpo $\mathbf{k}$ algebricamente fechado de char $\mathbf{k} \neq 2$. O objetivo principal deste capítulo é determinar as componentes irredutíveis da variedade $\mathrm{Jor}_{4}$. Para isso primeiramente descreveremos as álgebras rígidas das variedades Jor $_{n}$ para $n \leqslant 3$ e, sempre que possível, daremos uma descrição completa das órbitas e componentes irredutíveis em cada caso.

A lista completa das 73 órbitas de $\mathrm{Jor}_{4}$ sobre a ação de GL(V), i.e., as 73 álgebras de Jordan de dimensão 4 não isomorfas, foi obtida no Capítulo 2. Para cada uma destas álgebras foram calculados os invariantes: grupo de automorfismos, dimensão do grupo de automorfismos, dimensão do aniquilador, dimensão do radical nilpotente, dimensão da segunda, terceira e quarta potência, dimensão do grupo de 2-cociclos, dimensão do grupo de 2-cobordos, dimensão do segundo grupo de cohomologia, subálgebras de dimensões 2 e 3 e as dimensões das subálgebras associativas, nilpotentes e nulas maximais. Para levar a cabo tais contas foram programados diferentes algoritmos usando o aplicativo Mathematica, os respectivos códigos se encontram no Apêndice A. Toda a informação obtida para cada uma das 73 álgebras se encontra disponível no Apêndice B e segundo, os resultados da Seção 4.4, nos fornecerá as ferramentas necessárias para determinar a não existência de deformação entre um par de álgebras dadas e logo nos permitirá descrever as álgebras rígidas.

\subsection{A VARIEDADE ALGÉBRICA Jor ${ }_{1}$}

Em Jor 1 a única álgebra rígida é a álgebra simples $\mathbf{k} e$ e é claro que $\mathbf{k} e \rightarrow \mathbf{k n}$. Logo só temos uma componente irredutível em Jor ${ }_{1}$ dada pelo fecho de Zariski da órbita da álgebra ke, portanto Jor 1 é uma variedade afim conexa é irredutível de dimensão 1 com 2 órbitas sob a ação de GL(V). 


\subsection{A VARIEDADE ALGÉBRICA Jor 2}

Existem seis k-álgebras de Jordan de dimensão 2 não isomorfas, a saber: $\mathcal{B}_{1}, \mathcal{B}_{2}, \mathcal{B}_{3}$ indecomponíveis, descritas na Tabela 2.1, e as álgebras decomponíveis $\mathbf{k e}_{1} \oplus \mathbf{k} \mathbf{e}_{2}, \mathbf{k} \boldsymbol{e}_{1} \oplus$ $\mathbf{k} n_{1}$ e $\mathbf{k} n_{1} \oplus \mathbf{k} n_{2}$.

Teorema 5.1. A variedade algébrica Jor 2 é uma variedade afim conexa de dimensão 4 com 6 órbitas sob a ação de $\mathrm{GL}(\mathrm{V})$ e 2 componentes irredutíveis dadas pelos fechos de Zariski das órbitas das álgebras $\mathbf{k e}_{1} \oplus \mathbf{k}_{2}$ e $\mathcal{B}_{2}$. A primeira determina uma componente de dimensão 4 e a segunda uma componente de dimensão 2.

Demonstração. A álgebra $\mathbf{k} e_{1} \oplus \mathbf{k} e_{2}$ é rígida por ser uma álgebra semissimples e a álgebra $\mathcal{B}_{2}$ é rígida pois foi provado no Exemplo 4.22 que $\mathrm{H}^{2}\left(\mathcal{B}_{2}, \mathcal{B}_{2}\right)=0$.

Todas as álgebras associativas de dimensão 2 podem ser deformadas em $\mathbf{k} \mathbf{e}_{1} \oplus \mathbf{k} \mathbf{e}_{2}$, de fato: $\mathbf{k} e_{1} \oplus \mathbf{k} e_{2} \rightarrow \mathbf{k} e_{1} \oplus \mathbf{k} n_{1}$ pela Proposição 4.41 e do Exemplo 4.42 segue que $\mathbf{k e}_{1} \oplus \mathbf{k} e_{2} \rightarrow \mathcal{B}_{1}$. Para ver que $\mathcal{B}_{1} \rightarrow \mathcal{B}_{3}$, para $t \neq 0$ escolha a base $A_{t}=t e_{1}+n_{1} \mathrm{e}$ $B_{t}=-t^{2} e_{1}$ de $\mathcal{B}_{1}$, então obtemos a álgebra $\mathcal{B}_{3}$ quando $t=0$. A mudança de base $A_{t}=t e_{1}+n_{1}$ e $B_{t}=t^{2} e_{1}$ de $\mathbf{k} e_{1} \oplus \mathbf{k} n_{1}$ mostra que $\mathbf{k}_{1} \oplus \mathbf{k} n_{1} \rightarrow \mathcal{B}_{3}$. Lembramos do Lema 4.40 que a órbita de $\mathbf{k} n_{1} \oplus \mathbf{k} n_{2}$ é fechada e logo toda $\mathcal{B} \in$ Jor $_{2}$ é uma deformação de $\mathbf{k} n_{1} \oplus \mathbf{k} n_{2}$ o que implica que Jor 2 é uma variedade afim conexa.

Como Jor 2 é uma união finita de órbitas que são localmente fechadas então a dimensão da variedade é o máximo das dimensões de suas órbitas, assim temos que

$$
\operatorname{dim} \text { Dor }_{2}=\operatorname{dim}\left(\mathbf{k} e_{1} \oplus \mathbf{k} e_{2}\right)^{G}=2^{2}-\operatorname{dim} \operatorname{Aut}\left(\mathbf{k} e_{1} \oplus \mathbf{k} e_{2}\right)=4 .
$$

Por último, a dimensão da componente é determinada pela dimensão da órbita da álgebra rígida que a gera, logo as componentes irredutíveis de $\mathrm{Jor}_{2}$ têm dimensão:

$$
\operatorname{dim}\left(\mathbf{k} e_{1} \oplus \mathbf{k} e_{2}\right)^{G}=4 \quad \operatorname{dim} \mathcal{B}_{2}^{G}=4-\operatorname{dim} \operatorname{Aut}\left(\mathcal{B}_{2}\right)=2 .
$$

Finalmente, para completar a descrição geométrica de Jor 2 falta mostrar que essas são as únicas deformações não triviais que existem. Para isso observe que $\operatorname{dim} \operatorname{Aut}\left(\mathbf{k}_{1} \oplus\right.$ $\left.\mathbf{k n}_{1}\right)=\operatorname{dim} \operatorname{Aut}\left(\mathcal{B}_{1}\right)=1$ logo, pela Proposição 4.51 , temos $\mathbf{k}_{1} \oplus \mathbf{k} n_{1} \not \rightarrow \mathcal{B}_{1}$ e $\mathcal{B}_{1} \not \rightarrow$ $\mathbf{k e}_{1} \oplus \mathbf{k} \mathbf{n}_{1}$. Analogamente $\operatorname{dim} \operatorname{Aut}\left(\mathcal{B}_{2}\right)=\operatorname{dim} \operatorname{Aut}\left(\mathcal{B}_{3}\right)=2 \operatorname{logo} \mathcal{B}_{2} \not \rightarrow \mathcal{B}_{3}$ e, também pela Proposição $4.51 \mathcal{B}_{2} \not \rightarrow \mathbf{k} e_{1} \oplus \mathbf{k} n_{1}$ nem $\mathcal{B}_{2} \not \rightarrow \mathcal{B}_{1}$. O restante das existências ou não existências de deformações segue das propriedades de transitividade e antissimetria da relação de ordem parcial " $\rightarrow$ ". 
Uma completa descrição das órbitas de Jor 2 é dada na Tabela 5.1 e o diagrama das álgebras de Jordan de dimensão 2 é dado na Figura 5.1.
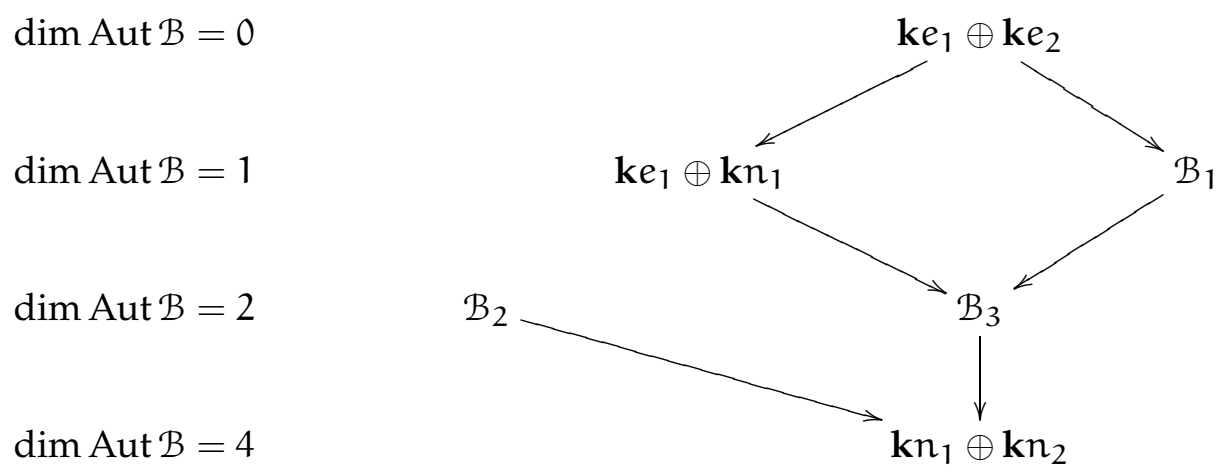

Fig. 5.1: Descrição completa das órbitas de Jor $_{2}$

Um resumo da existência de deformações é dado na seguinte tabela, onde $\rightarrow$ representa que a álgebra da linha é uma deformação da álgebra na coluna e ł que a álgebra da linha não é uma deformação da álgebra na coluna. Os símbolos estão acompanhados de siglas que justificam tales fatos, i.e.:

i. $\rightarrow{ }_{\mathrm{DT}}$ : existe deformação trivial;

ii. $\rightarrow_{\mathrm{T}}$ : existe deformação pela propriedade transitiva da relação de ordem parcial $\rightarrow$;

iii. $\rightarrow$ : existe deformação e foi exibida anteriormente;

iv. $\nrightarrow_{\mathrm{H}^{2}}$ : não existe deformação, pois a álgebra na coluna tem segundo grupo de cohomologia nulo;

v. ł ss: não existe deformação, pois a álgebra na coluna é semissimples;

vi. $\nrightarrow_{A S}$ : não existe deformação pela propriedade antissimétrica da relação de ordem parcial $\rightarrow$;

vii. $\nrightarrow_{\text {Aut: }}$ não existe deformação pela Proposição 4.51 que relaciona a dimensão dos grupos de automorfismos das álgebras envolvidas. 
Tabela 5.1: Existência de deformações em Jor $_{2}$

\begin{tabular}{|c|c|c|c|c|c|c|}
\hline$\rightarrow$ & $\mathbf{k} \mathbf{e}_{1} \oplus \mathbf{k} \mathbf{e}_{2}$ & $\mathbf{k} \mathbf{e}_{1} \oplus \mathbf{k} \mathbf{n}_{1}$ & $\mathcal{B}_{1}$ & $\mathcal{B}_{2}$ & $\mathcal{B}_{3}$ & $\mathbf{k} n_{1} \oplus \mathbf{k} n_{2}$ \\
\hline $\mathbf{k} \mathbf{e}_{1} \oplus \mathbf{k} \mathbf{e}_{2}$ & $\rightarrow_{\mathrm{DT}}$ & $\rightarrow$ & $\rightarrow$ & $t_{\mathrm{H}^{2}}$ & $\rightarrow_{\mathrm{T}}$ & $\rightarrow_{\mathrm{T}}$ \\
\hline $\mathbf{k e}_{1} \oplus \mathbf{k} \mathbf{n}_{1}$ & $t_{\mathrm{ss}}$ & $\rightarrow$ DT & $t_{\text {Aut }}$ & $t_{\mathrm{H}^{2}}$ & $\rightarrow$ & $\rightarrow_{\mathrm{T}}$ \\
\hline $\mathcal{B}_{1}$ & $\not \rightarrow s s$ & $\not \rightarrow$ Aut & $\rightarrow \rightarrow_{\mathrm{DT}}$ & $t_{\mathrm{H}^{2}}$ & $\rightarrow$ & $\rightarrow_{\mathrm{T}}$ \\
\hline $\mathcal{B}_{2}$ & $\not \rightarrow s s$ & $\not \rightarrow$ Aut & $t_{\text {Aut }}$ & $\rightarrow \rightarrow_{\mathrm{DT}}$ & $t_{\text {Aut }}$ & $\rightarrow$ \\
\hline $\mathcal{B}_{3}$ & $\not \rightarrow s s$ & $t_{A S}$ & $t_{A S}$ & $t_{\mathrm{H}^{2}}$ & $\rightarrow \rightarrow_{\mathrm{DT}}$ & $\rightarrow$ \\
\hline $\mathbf{k} \mathbf{n}_{1} \oplus \mathbf{k} \mathbf{n}_{2}$ & t $\rightarrow \mathrm{ss}$ & $t_{A S}$ & $t_{A S}$ & $t_{\mathrm{H}^{2}}$ & $t_{A S}$ & $\rightarrow \mathrm{DT}$ \\
\hline
\end{tabular}

\subsection{A VARIEDADE ALGÉBRICA Jor 3}

Em [25] I. Kashuba e I. Shestakov determinaram que $\mathrm{Jor}_{3}$ é uma variedade afim conexa de dimensão 9 com 5 componentes irredutíveis dadas pelos fechos de Zariski das órbitas das álgebras $\mathbf{k}_{1} \oplus \mathbf{k} e_{2} \oplus \mathbf{k} e_{3}, \mathcal{T}_{5}, \mathcal{T}_{7}, \mathcal{T}_{9}$ e $\mathcal{B}_{2} \oplus \mathbf{k} e_{2}$. Cada álgebra gera uma componente irredutível de dimensão 9, 8, 3, 7 e 7, respetivamente.

Daremos aqui uma descrição quase-completa das órbitas de Jor $_{3}$. Com quase-completa queremos dizer que conseguimos determinar em um $98,5 \%$ das vezes se uma álgebra de Jordan de dimensão 3 pertence ou não ao fecho de Zariski da órbita de uma outra álgebra.

Sabemos que em $\mathrm{Jor}_{3}$ existem 20 órbitas representadas pelas 10 álgebras indecomponíveis $\mathcal{T}_{i}$ para $1 \leqslant i \leqslant 10$ dadas na Tabela 2.2 e as 10 álgebras que são soma direta de álgebras de dimensões menores: $\mathcal{T}_{11}=\mathbf{k} \boldsymbol{e}_{1} \oplus \mathbf{k} \boldsymbol{e}_{2} \oplus \mathbf{k} e_{3}, \mathcal{T}_{12}=\mathcal{B}_{1} \oplus \mathbf{k} e_{2}$, $\mathcal{T}_{13}=\mathbf{k} e_{1} \oplus \mathbf{k} e_{2} \oplus \mathbf{k} n_{1}, \mathcal{T}_{14}=\mathcal{B}_{1} \oplus \mathbf{k} n_{2}, \mathcal{T}_{15}=\mathcal{B}_{3} \oplus \mathbf{k} e_{1}, \mathcal{T}_{16}=\mathbf{k} e_{1} \oplus \mathbf{k} n_{1} \oplus \mathbf{k} n_{2}$, $\mathcal{T}_{17}=\mathcal{B}_{3} \oplus \mathbf{k} n_{3}, \mathcal{T}_{18}=\mathbf{k n}_{1} \oplus \mathbf{k} n_{2} \oplus \mathbf{k} n_{3}, \mathcal{T}_{19}=\mathcal{B}_{2} \oplus \mathbf{k} e_{2}$ e $\mathcal{T}_{20}=\mathcal{B}_{2} \oplus \mathbf{k} n_{2}$

Por ser soma direta de alguma álgebra de Jor 2 com ke ou kn, segue da Proposição 4.41 e da classificação geométrica da seção anterior que:

$$
\begin{aligned}
& \mathcal{T}_{11} \rightarrow \mathcal{T}_{12} ; \quad \mathcal{T}_{11} \rightarrow \mathcal{T}_{13} ; \quad \mathcal{T}_{13} \rightarrow \mathcal{T}_{15} ; \quad \mathcal{T}_{15} \rightarrow \mathcal{T}_{16} ; \\
& \mathcal{T}_{12} \rightarrow \mathcal{T}_{14} \quad \mathcal{T}_{12} \rightarrow \mathcal{T}_{15} ; \quad \mathcal{T}_{13} \rightarrow \mathcal{T}_{14} ; \quad \mathcal{T}_{16} \rightarrow \mathcal{T}_{17} ; \\
& \mathcal{T}_{17} \rightarrow \mathcal{T}_{18} ; \quad \mathcal{T}_{19} \rightarrow \mathcal{T}_{16} ; \quad \mathcal{T}_{19} \rightarrow \mathcal{T}_{20} .
\end{aligned}
$$

Segue de [25] e [22] que existem as seguintes deformações:

$$
\begin{aligned}
& \mathcal{T}_{12} \rightarrow \mathcal{T}_{1} ; \quad \mathcal{T}_{1} \rightarrow \mathcal{T}_{2} ; \quad \mathcal{T}_{14} \rightarrow \mathcal{T}_{3} ; \quad \mathcal{T}_{5} \rightarrow \mathcal{T}_{10} ; \\
& \mathcal{T}_{3} \rightarrow \mathcal{T}_{4} ; \quad \mathcal{T}_{4} \rightarrow \mathcal{T}_{17} ; \quad \mathcal{T}_{5} \rightarrow \mathcal{T}_{8} ; \quad \mathcal{T}_{9} \rightarrow \mathcal{T}_{6} ; \quad \mathcal{T}_{10} \rightarrow \mathcal{T}_{20} .
\end{aligned}
$$


E das seguintes transformações obtemos as respectivas deformações:

i. Considere, para $t \neq 0$, a mudança de base $A_{t}=e_{1}, B_{t}=n_{2}$ e $C_{t}=t n_{1}$ de $\mathcal{T}_{8}$. Fazendo $t$ tender a zero obtemos a estrutura da álgebra $\mathcal{T}_{20}$. Logo $\mathcal{T}_{8} \rightarrow \mathcal{T}_{20}$.

ii. Analogamente se consideramos a base $A_{t}=t e_{1}+t n_{1}, B_{t}=t^{2} n_{1}$ e $C_{t}=t^{2} e_{1}+$ $t^{3} n_{1}+n_{2}$ de $\mathcal{T}_{2}$ obtemos a álgebra $\mathcal{T}_{17}$ quando $t$ tende a zero. Portanto, $\mathcal{T}_{2} \rightarrow \mathcal{T}_{17}$.

iii. A mudança de base $A_{t}=e_{1}+e_{2}, B_{t}=t e_{2}$ e $C_{t}=n_{1}$ de $\mathcal{T}_{10}$, nos da $\mathcal{T}_{10} \rightarrow \mathcal{T}_{2}$.

iv. A mudança de base $A_{t}=e_{1}+e_{2}, B_{t}=n_{1}$ e $C_{t}=t e_{2}$ de $\mathcal{T}_{19}$ tende a $\mathcal{T}_{6}$ quando $\mathrm{t} \rightarrow 0$ o que implica que $\mathcal{T}_{19} \rightarrow \mathcal{T}_{6}$.

v. Para o caso da deformação $\mathcal{T}_{1} \rightarrow \mathcal{T}_{3}$ considere a família de automorfismo $A_{t}=$ te $e_{1}+n_{1}, B_{t}=-t^{2} e_{1}+n_{2}$ e $C_{t}=t^{3} e_{1}$ de $\mathcal{T}_{1}$, fazendo $t$ tender a 0 obtemos a álgebra $\mathcal{T}_{3}$.

vi. A família de automorfismos $A_{t}=t e_{1}+n_{1}, B_{t}=t^{2} e_{1}+t^{3} n_{1}+n_{2}$ e $C_{t}=t^{3} e_{1}$ de $\mathcal{T}_{15}$ nos da $\mathcal{T}_{15} \rightarrow \mathcal{T}_{3}$.

vii. Se considerarmos a mudança de base $A_{t}=t e_{1}+n_{2}, B_{t}=t^{2} e_{1}+2 t n_{2}$ e $C_{t}=$ $n_{1}+2 n_{2}$ de $\mathcal{T}_{6}$, fazendo $t=0$ temos a álgebra $\mathcal{T}_{4}$ e portanto $\mathcal{T}_{6} \rightarrow \mathcal{T}_{4}$.

viii. Por último, a mudança de base $A_{t}=t e_{1}+n_{1}, B_{t}=t^{2} e_{1}$ e $C_{t}=2 n_{1}+n_{2}$ de $\mathcal{T}_{20}$ nos da $\mathcal{T}_{20} \rightarrow \mathcal{T}_{4}$.

Para completar a descrição geométrica de Jor $_{3}$ provaremos que não existem outras deformações.

Da Proposição 4.56 toda deformação de uma álgebra não associativa é uma álgebra não associativa, $\log \mathcal{T}_{i} \not \rightarrow \mathcal{T}_{j}$ para $i=1,2,3,11,12,13,14,15,16$ e $j=6,8,10,20$. Segue da Proposição 4.59 que a ação da parte semissimples $\mathcal{T}_{S S}$ é preservada através de uma deformação, logo:

$$
\begin{array}{ll}
\mathcal{T}_{1} \nrightarrow \mathcal{T}_{16} ; & \mathcal{T}_{5} \nrightarrow \rightarrow \mathcal{T}_{i} \text { para } i=6,14,15,16 ; \quad \mathcal{T}_{6} \not \rightarrow \mathcal{T}_{i} \text { para } i=2,16 \\
\mathcal{T}_{8} \not \rightarrow \mathcal{T}_{16} ; & \mathcal{T}_{9} \not \rightarrow \mathcal{T}_{i} \text { para } i=2,16,20 ; \quad \mathcal{T}_{10} \not \rightarrow \mathcal{T}_{i} \text { para } i=6,16 \\
\mathcal{T}_{14} \not \rightarrow \mathcal{T}_{16} ; & \mathcal{T}_{19} \nrightarrow \rightarrow \mathcal{T}_{2} ; \quad \mathcal{T}_{20} \not \rightarrow \mathcal{T}_{16} .
\end{array}
$$

As álgebras $\mathcal{T}_{3}$ e $\mathcal{T}_{4}$ são nilpotentes logo elas não podem ser deformações de álgebras não nilpotentes, assim temos $\mathcal{T}_{3}, \mathcal{T}_{4} \not \rightarrow \mathcal{T}_{2}, \mathcal{T}_{16}$ e como $\operatorname{dim} \operatorname{Rad}\left(\mathcal{T}_{8}\right)=\operatorname{dim} \operatorname{Rad}\left(\mathcal{T}_{9}\right)=2$ e $\operatorname{dim} \operatorname{Rad}\left(\mathcal{T}_{10}\right)=1$ da Proposição 4.52 segue que $\mathcal{T}_{8}, \mathcal{T}_{9} \not \rightarrow \mathcal{T}_{10}$. 
Da Proposição 4.53 segue que a dimensão do aniquilador de uma álgebra não aumenta sob deformação, portanto temos:

$$
\begin{aligned}
& \mathcal{T}_{8} \not \rightarrow \mathcal{T}_{1}, \mathcal{T}_{2} \mathcal{T}_{6} ; \quad \mathcal{T}_{13} \not \rightarrow \mathcal{T}_{1}, \mathcal{T}_{12} ; \quad \mathcal{T}_{14}, \mathcal{T}_{15} \not \rightarrow \mathcal{T}_{1}, \mathcal{T}_{2} ; \\
& \mathcal{T}_{16} \not \rightarrow \mathcal{T}_{2}, \mathcal{T}_{4} ; \quad \mathcal{T}_{20} \not \rightarrow \mathcal{T}_{2}, \mathcal{T}_{6}
\end{aligned}
$$

pois $\operatorname{dim} \operatorname{Ann}\left(\mathcal{T}_{\mathfrak{i}}\right)=0$ para $i=1,2,6,12, \operatorname{dim} \operatorname{Ann}\left(\mathcal{T}_{\mathfrak{i}}\right)=1$ para $i=4,8,13,14,15,20$ e $\operatorname{dim} \operatorname{Ann}\left(\mathcal{T}_{16}\right)=2$.

Segue da Proposição 4.51 que a dimensão do grupo de automorfismos diminui através de uma deformação não trivial, logo dado que

$$
\operatorname{dim} \operatorname{Aut}\left(\mathcal{T}_{i}\right)= \begin{cases}1, & i=5,12,13 \\ 2, & i=1,8,9,10,14,15,19 \\ 3, & i=3,6,20 \\ 4, & i=2,4,16 \\ 5, & i=17 \\ 6, & i=6\end{cases}
$$

temos:

$$
\begin{aligned}
& \mathcal{T}_{1} \not \rightarrow \mathcal{T}_{i} \text { para } i=13,14,15 ; \quad \mathcal{T}_{2} \not \rightarrow \mathcal{T}_{i} \text { para } i=3,4,14,15,16 ; \\
& \mathcal{T}_{5} \not \rightarrow \mathcal{T}_{i} \text { para } i=12,13 ; \quad \mathcal{T}_{6} \not \rightarrow \mathcal{T}_{i} \text { para } i=1,3,8,10,12,13,14,15,20 ; \\
& \mathcal{T}_{7} \not \rightarrow \mathcal{T}_{i} \text { para } i=1,2,3,4,6,8,10,12,13,14,15,16,17,20 ; \\
& \mathcal{T}_{8} \not \rightarrow \mathcal{T}_{i} \text { para } i=12,13,14,15 ; \quad \mathcal{T}_{9} \not \rightarrow \mathcal{T}_{i} \text { para } i=1,8,12,13,14,15 ; \\
& \mathcal{T}_{10} \not \rightarrow \mathcal{T}_{i} \text { para } i=1,8,12,13,14,15 ; \quad \mathcal{T}_{12} \not \rightarrow \mathcal{T}_{13} ; \quad \mathcal{T}_{14} \not \rightarrow \mathcal{T}_{15} ; \\
& \mathcal{T}_{15} \nrightarrow \rightarrow \mathcal{T}_{14} ; \quad \mathcal{T}_{16} \nrightarrow \rightarrow \mathcal{T}_{i} \text { para } i=1,3,14 ; \\
& \mathcal{T}_{19} \not \rightarrow \mathcal{T}_{i} \text { para } i=1,8,10,12,13,14,15 ; \quad \mathcal{T}_{20} \nrightarrow \rightarrow \mathcal{T}_{i} \text { para } i=1,3,12,13,14,15 ;
\end{aligned}
$$

Por último não pôde ser determinado se existem ou não as seguintes deformações: $\mathcal{T}_{5} \stackrel{?}{\rightarrow} \mathcal{T}_{1}$ e $\mathcal{T}_{5}, \mathcal{T}_{8}, \mathcal{T}_{9}, \mathcal{T}_{10}, \mathcal{T}_{19} \stackrel{?}{\rightarrow} \mathcal{T}_{3}$. O restante das existências ou não existências de deformações segue das propriedades de transitividade e antissimetria da relação de ordem parcial " $\rightarrow$ ".

O diagrama completo das álgebras de Jordan de dimensão 3, a menos de 6 deformações, é dado na Figura 5.2. Os super-índices A, S, U e N nos nomes das álgebras representam que $\mathcal{T}_{i}$ é Associativa, Semissimples, Unitária ou Nilpotente, respetivamente. Isto nos permitirá enxergar como se comportam estas características sob deformações. 
$\operatorname{dim} \operatorname{Aut}(\mathcal{T})$

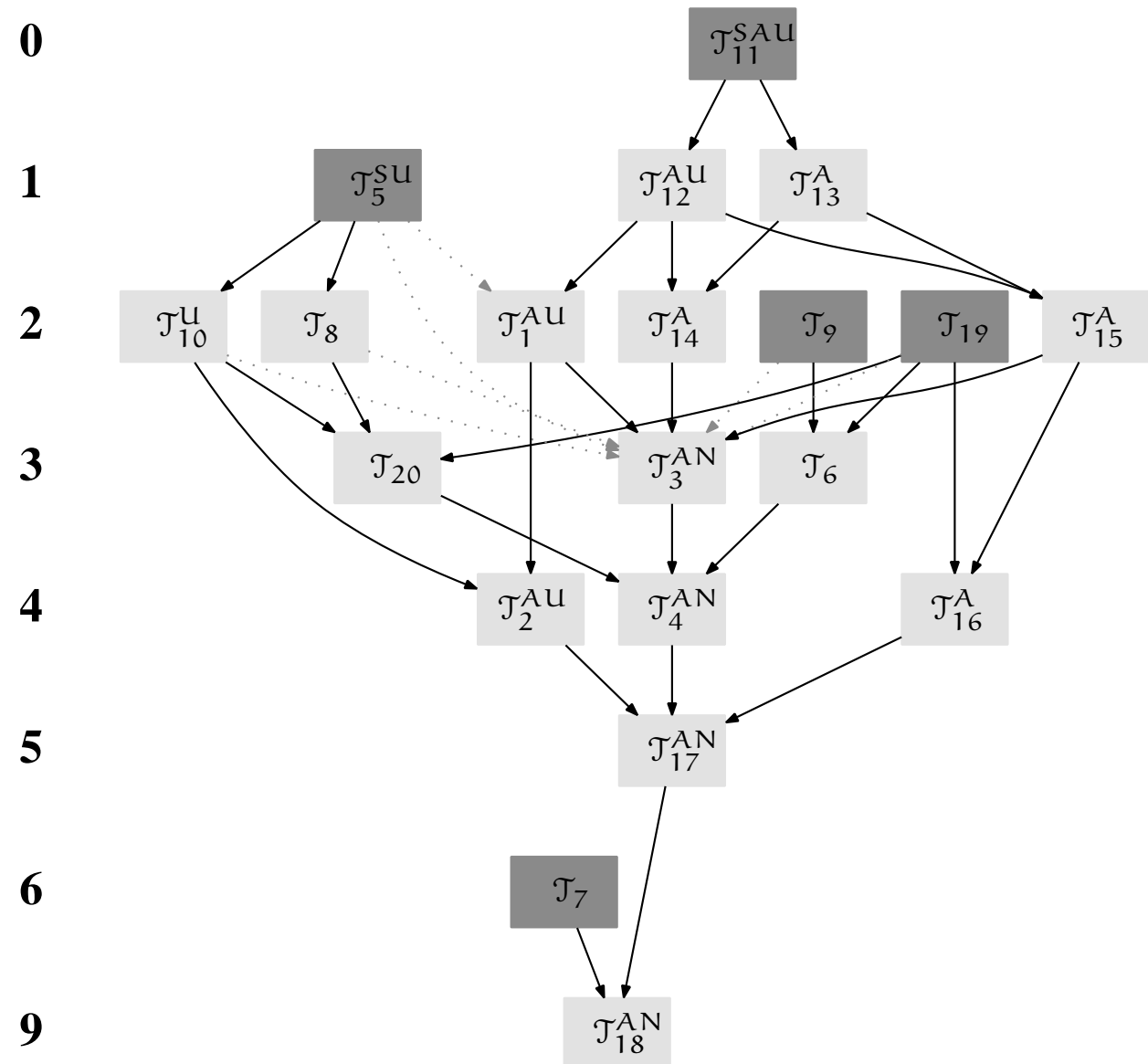

Fig. 5.2: Descrição quase-completa das órbitas de Jor $_{3}$

Um resumo da existência de deformações é dado na seguinte tabela. Observamos que não foram colocadas as colunas das álgebras rígidas $\mathcal{T}_{5}, \mathcal{T}_{7}, \mathcal{T}_{9}, \mathcal{T}_{11}$ e $\mathcal{T}_{19}$ com o propósito de ganhar espaço, já que a tabela ficou muito grande. Nessas colunas só teria o símbolo " $\rightarrow_{\mathrm{R}}$ " o qual representa que "não existe deformação, pois foi provado em [25] que a álgebra da coluna é rígida", além de obviamente " $\rightarrow \mathrm{DT}$ " a existência da deformação trivial.

Os símbolos $\rightarrow$ e $\nrightarrow$ estão acompanhados de siglas que justificam tales fatos de acordo com a seção anterior e adicionamos os seguintes:

i. $\nrightarrow_{\text {Ass }}$ : não existe deformação pela Proposição 4.56, pois a álgebra da linha é associativa e a álgebra da coluna não o é. 
ii. $\not_{\text {npa }}$ : não existe deformação pois não é preservada a ação da parte semissimples, Proposição 4.59.

iii. $\dashv_{\text {Rad }}$ : não existe deformação pela Proposição 4.52 que relaciona as dimensões dos radicais nilpotentes das álgebras envolvidas.

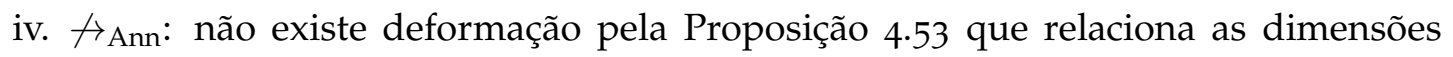
dos aniquiladores das álgebras envolvidas.

v. $\stackrel{?}{-\rightarrow} \rightarrow$ não foi possível determinar a existência ou não existência de deformação. 
Tabela 5.2: Existência de deformações em $\mathrm{Jor}_{3}$

\begin{tabular}{|c|c|c|c|c|c|c|c|c|c|c|c|c|c|c|c|}
\hline$\rightarrow$ & $\mathcal{T}_{1}$ & $\mathcal{T}_{2}$ & $\mathcal{T}_{3}$ & $\mathcal{T}_{4}$ & $\mathcal{T}_{6}$ & $\mathcal{T}_{8}$ & $\mathcal{T}_{10}$ & $\mathcal{T}_{12}$ & $\mathcal{T}_{13}$ & $\mathcal{T}_{14}$ & $\mathcal{T}_{15}$ & $\mathcal{T}_{16}$ & $\mathcal{T}_{17}$ & $\mathcal{T}_{18}$ & $\mathcal{T}_{20}$ \\
\hline $\mathcal{T}_{1}$ & $\rightarrow_{\mathrm{DT}}$ & $\rightarrow$ & $\rightarrow$ & $\rightarrow_{\mathrm{T}}$ & $\not \rightarrow$ Ass & $\not A_{\text {Ass }}$ & $\not A_{\text {Ass }}$ & $\not A A S$ & $A_{\text {Aut }}$ & $A_{\text {Aut }}$ & $A_{\text {Aut }}$ & $A_{\text {npa }}$ & $\rightarrow_{\mathrm{T}}$ & $\rightarrow_{\mathrm{T}}$ & $\not_{\text {Ass }}$ \\
\hline $\mathcal{T}_{2}$ & $A_{A S}$ & $\rightarrow{ }_{\mathrm{DT}}$ & $A_{\text {Aut }}$ & $A_{\text {Aut }}$ & $A_{\text {Ass }}$ & $A_{\text {Ass }}$ & $\not A A S$ & $\not A$ AS & $A_{A S}$ & $A_{\text {Aut }}$ & $A_{\text {Aut }}$ & $A_{\text {Aut }}$ & $\rightarrow$ & $\rightarrow_{\mathrm{T}}$ & $\not A_{\text {Ass }}$ \\
\hline $\mathcal{T}_{3}$ & $\not A_{A S}$ & $t_{\mathrm{Rad}}$ & $\rightarrow \mathrm{DT}$ & $\rightarrow$ & $A_{\text {Ass }}$ & $\not \rightarrow$ Ass & $\not A_{\text {Ass }}$ & $\not \rightarrow A S$ & $\not A_{A S}$ & $\not A A S$ & $\not \rightarrow A S$ & $t_{\mathrm{Rad}}$ & $\rightarrow_{\mathrm{T}}$ & $\rightarrow_{\mathrm{T}}$ & $\not \rightarrow_{\text {Ass }}$ \\
\hline $\mathcal{T}_{4}$ & $A_{A S}$ & $A_{\mathrm{Rad}}$ & $A_{A S}$ & $\rightarrow$ DT & $A_{A S}$ & $\not A A S$ & $A_{A S}$ & $\not A A S$ & $\not A A S$ & $A_{A S}$ & $\not A A S$ & $A_{\text {Rad }}$ & $\rightarrow$ & $\rightarrow_{\mathrm{T}}$ & $\not A A S$ \\
\hline $\mathcal{T}_{5}$ & $\stackrel{?}{-\rightarrow}$ & $\rightarrow_{\mathrm{T}}$ & $\stackrel{?}{?}+\rightarrow$ & $\rightarrow_{\mathrm{T}}$ & $\not_{\text {npa }}$ & $\rightarrow$ & $\rightarrow$ & $A_{\text {Aut }}$ & $f_{\text {Aut }}$ & $A_{\text {npa }}$ & $A_{\text {npa }}$ & $f_{\text {npa }}$ & $\rightarrow_{\mathrm{T}}$ & $\rightarrow_{\mathrm{T}}$ & $\rightarrow_{\mathrm{T}}$ \\
\hline $\mathcal{T}_{6}$ & $A_{\text {Aut }}$ & $A_{\text {npa }}$ & $A_{\text {Aut }}$ & $\rightarrow$ & $\rightarrow \mathrm{DT}$ & $A_{\text {Aut }}$ & $A_{\text {Aut }}$ & $A_{\text {Aut }}$ & $A_{\text {Aut }}$ & $A_{\text {Aut }}$ & $A_{\text {Aut }}$ & $A_{\text {npa }}$ & $\rightarrow_{\mathrm{T}}$ & $\rightarrow \rightarrow_{\mathrm{T}}$ & $A_{\text {Aut }}$ \\
\hline $\mathcal{T}_{7}$ & $A_{\text {Aut }}$ & $A_{\text {Aut }}$ & $A_{\text {Aut }}$ & $A_{\text {Aut }}$ & $\not \rightarrow_{\text {Aut }}$ & $A_{\text {Aut }}$ & $A_{\text {Aut }}$ & $A_{\text {Aut }}$ & $A_{\text {Aut }}$ & $A_{\text {Aut }}$ & $A_{\text {Aut }}$ & $A_{\text {Aut }}$ & $\nrightarrow_{\text {Aut }}$ & $\rightarrow$ & $\nrightarrow_{\text {Aut }}$ \\
\hline $\mathcal{T}_{8}$ & $t_{\text {Ann }}$ & $t_{\text {Ann }}$ & $\stackrel{?}{-\rightarrow}$ & $\rightarrow_{\mathrm{T}}$ & $t_{\text {Ann }}$ & $\rightarrow \mathrm{DT}$ & $t_{\mathrm{Rad}}$ & $A_{\text {Aut }}$ & $f_{\text {Aut }}$ & $\nrightarrow_{\text {Aut }}$ & $A_{\text {Aut }}$ & $f_{\text {npa }}$ & $\rightarrow_{\mathrm{T}}$ & $\rightarrow_{\mathrm{T}}$ & $\rightarrow$ \\
\hline $\mathcal{T}_{9}$ & $A_{\text {Aut }}$ & $f_{\text {npa }}$ & $\stackrel{?}{-\rightarrow}$ & $\rightarrow_{\mathrm{T}}$ & $\rightarrow$ & $A_{\text {Aut }}$ & $A_{\text {Rad }}$ & $A_{\text {Aut }}$ & $\phi_{\text {Aut }}$ & $\nrightarrow_{\text {Aut }}$ & $\nrightarrow_{\text {Aut }}$ & $\not_{\text {npa }}$ & $\rightarrow_{\mathrm{T}}$ & $\rightarrow_{\mathrm{T}}$ & $\not_{\text {npa }}$ \\
\hline $\mathcal{T}_{10}$ & $\nrightarrow_{\text {Aut }}$ & $\rightarrow$ & $\stackrel{?}{-\rightarrow}$ & $\rightarrow_{\mathrm{T}}$ & $A_{\text {npa }}$ & $\not \rightarrow_{\text {Aut }}$ & $\rightarrow$ DT & $\nrightarrow_{\text {Aut }}$ & $\not \rightarrow_{\text {Aut }}$ & $\nrightarrow_{\text {Aut }}$ & $\not_{\text {Aut }}$ & $\not_{\text {npa }}$ & $\rightarrow_{\mathrm{T}}$ & $\rightarrow_{\mathrm{T}}$ & $\rightarrow$ \\
\hline $\mathcal{T}_{11}$ & $\rightarrow_{\mathrm{T}}$ & $\rightarrow_{\mathrm{T}}$ & $\rightarrow_{\mathrm{T}}$ & $\rightarrow_{\mathrm{T}}$ & $A_{\text {Ass }}$ & $A_{\text {Ass }}$ & $A_{\text {Ass }}$ & $\rightarrow$ & $\rightarrow$ & $\rightarrow_{\mathrm{T}}$ & $\rightarrow_{\mathrm{T}}$ & $\rightarrow_{\mathrm{T}}$ & $\rightarrow_{\mathrm{T}}$ & $\rightarrow_{\mathrm{T}}$ & $f_{\text {Ass }}$ \\
\hline $\mathcal{T}_{12}$ & $\rightarrow$ & $\rightarrow_{\mathrm{T}}$ & $\rightarrow_{\mathrm{T}}$ & $\rightarrow_{\mathrm{T}}$ & $A_{\text {Ass }}$ & $\not A_{\text {Ass }}$ & $\not A_{\text {Ass }}$ & $\rightarrow \mathrm{DT}$ & $A_{\text {Aut }}$ & $\rightarrow$ & $\rightarrow$ & $\rightarrow_{\mathrm{T}}$ & $\rightarrow \mathrm{T}$ & $\rightarrow_{\mathrm{T}}$ & $A_{\text {Ass }}$ \\
\hline $\mathcal{T}_{13}$ & $\not_{\text {Ann }}$ & $\rightarrow$ & $\rightarrow_{\mathrm{T}}$ & $\rightarrow_{\mathrm{T}}$ & $\not A_{\text {Ass }}$ & $\not \rightarrow_{\text {Ass }}$ & $\not \rightarrow_{\text {Ass }}$ & $A_{\text {Ann }}$ & $\rightarrow \mathrm{DT}$ & $\rightarrow$ & $\rightarrow$ & $\rightarrow_{\mathrm{T}}$ & $\rightarrow \mathrm{T}$ & $\rightarrow_{\mathrm{T}}$ & $A_{\text {Ass }}$ \\
\hline $\mathcal{T}_{14}$ & $A_{\text {Ann }}$ & $A_{\text {Ann }}$ & $\rightarrow$ & $\rightarrow_{\mathrm{T}}$ & $\not A_{\text {Ass }}$ & $A_{\text {Ass }}$ & $A_{\text {Ass }}$ & $A_{\mathrm{AS}}$ & $A_{A S}$ & $\rightarrow_{\mathrm{DT}}$ & $A_{\text {Aut }}$ & $A_{\text {npa }}$ & $\rightarrow_{\mathrm{T}}$ & $\rightarrow_{\mathrm{T}}$ & $A_{\text {Ass }}$ \\
\hline $\mathcal{T}_{15}$ & $t_{\text {Ann }}$ & $t_{\text {Ann }}$ & $\rightarrow$ & $\rightarrow_{\mathrm{T}}$ & $\not A_{\text {Ass }}$ & $\not \rightarrow_{\text {Ass }}$ & $\not A_{\text {Ass }}$ & $\not A A S$ & $\not A_{A S}$ & $A_{\text {Aut }}$ & $\rightarrow \mathrm{DT}$ & $\rightarrow$ & $\rightarrow_{\mathrm{T}}$ & $\rightarrow_{\mathrm{T}}$ & $\not A_{\text {Ass }}$ \\
\hline $\mathcal{T}_{16}$ & $A_{\text {Aut }}$ & $t_{\text {Ann }}$ & $A_{\text {Aut }}$ & $t_{\text {Ann }}$ & $A_{\text {Ass }}$ & $\not A_{\text {Ass }}$ & $\nrightarrow_{\text {Ass }}$ & $A_{A S}$ & $A_{A S}$ & $A_{\text {Aut }}$ & $A_{A S}$ & $\rightarrow \mathrm{DT}$ & $\rightarrow$ & $\rightarrow_{\mathrm{T}}$ & $\not A_{\text {Ass }}$ \\
\hline $\mathcal{T}_{17}$ & $A_{A S}$ & $A_{A S}$ & $A_{A S}$ & $\not A A S$ & $A_{A S}$ & $\not A A S$ & $\not A A S$ & $A_{\mathrm{AS}}$ & $A_{A S}$ & $A_{A S}$ & $\not \rightarrow A S$ & $A_{A S}$ & $\rightarrow_{\mathrm{DT}}$ & $\not \rightarrow A S$ & $A_{\mathrm{AS}}$ \\
\hline $\mathcal{T}_{18}$ & $A_{A S}$ & $A_{A S}$ & $A_{A S}$ & $\not \rightarrow A S$ & $\not \rightarrow A S$ & $A_{A S}$ & $\not \rightarrow A S$ & $A_{A S}$ & $\not \rightarrow A S$ & $\not \rightarrow A S$ & $\not \rightarrow A S$ & $A_{A S}$ & $\not A_{A S}$ & $\rightarrow \mathrm{DT}$ & $A_{A S}$ \\
\hline $\mathcal{T}_{19}$ & $\nrightarrow_{\text {Aut }}$ & $\not A_{\text {npa }}$ & $\stackrel{?}{-} \rightarrow$ & $\rightarrow_{\mathrm{T}}$ & $\rightarrow$ & $t_{\text {Aut }}$ & $A_{\text {Aut }}$ & $\nrightarrow_{\text {Aut }}$ & $\nrightarrow_{\text {Aut }}$ & $\nrightarrow_{\text {Aut }}$ & $\nrightarrow_{\text {Aut }}$ & $\rightarrow$ & $\rightarrow_{\mathrm{T}}$ & $\rightarrow_{\mathrm{T}}$ & $\rightarrow$ \\
\hline $\mathcal{T}_{20}$ & $A_{\text {Aut }}$ & $A_{\text {Ann }}$ & $A_{\text {Aut }}$ & $\rightarrow$ & $A_{\text {Ann }}$ & $\not \rightarrow A S$ & $\not A A S$ & $A_{\text {Aut }}$ & $A_{\text {Aut }}$ & $A_{\text {Aut }}$ & $A_{\text {Aut }}$ & $\not_{\text {npa }}$ & $\rightarrow_{\mathrm{T}}$ & $\rightarrow_{\mathrm{T}}$ & $\rightarrow \mathrm{DT}$ \\
\hline
\end{tabular}




\subsection{A VARIEDADE ALGÉBRICA Jor 4}

Agora estamos prontos para enunciar o principal teorema deste capítulo.

Teorema 5.2. A variedade algébrica Jor 4 é uma variedade afim conexa de dimensão 16 com 73 órbitas sob a ação de GL(V) e 10 componentes irredutíveis dadas pelos fechos de Zariski das órbitas das álgebras

$$
\Omega=\left\{\mathcal{J}_{1}, J_{2}, J_{3}, J_{6}, J_{12}, J_{13}, J_{16}, J_{24}, J_{33}, J_{59}\right\},
$$

as quais determinam componentes de dimensões: 15, 11, 16, 14, 10,12, 12, 14, 4 e 12, respetivamente.

Demonstração. Vamos dividir a prova em duas partes: primeiramente, mostraremos que as álgebras em $\Omega$ são rígidas e depois provaremos que elas são as únicas rígidas, i.e., provaremos que toda outra álgebra de $\mathcal{J}_{1}$ a $\mathcal{J}_{73}$ da classificação algébrica do Capítulo 2 pode ser deformada em uma das álgebras anteriores.

Existem três álgebras semissimples $\mathcal{J}_{1}, \mathcal{J}_{2}$ e $\mathcal{J}_{3}$ logo pelo Corolário 4.21 elas são rígidas. Note que as álgebras $\mathcal{J}_{16}$ e $\mathcal{J}_{33}$ pertencem às famílias de álgebras dos Teoremas $4.67 \mathrm{e}$ 4.65 ( $B_{4}$ e $A_{4}$ respetivamente). Portanto $\mathcal{J}_{16}$ e $\mathcal{J}_{33}$ são rígidas.

Nenhuma álgebra em $J r_{4}$ domina $\partial_{6}$ : Da Proposição 4.52 as únicas possíveis candidatas a serem deformação de $\mathcal{J}_{6}$ são $\mathcal{J}_{1}$ a $\mathcal{J}_{9}$, pela Proposição $4.5^{1}$ podemos excluir $\mathcal{J}_{2}$, $\mathcal{J}_{7}$ e $\mathcal{J}_{9}$ da lista, enquanto que as álgebras $\mathcal{J}_{5}$ e $\mathcal{J}_{8}$ não dominam $\mathcal{J}_{6}$ devido à Proposição 4.53. Uma álgebra não associativa não pode ser deformada em uma álgebra associativa, portanto $\partial_{3} \nrightarrow \partial_{6}$ e $\partial_{4} \nrightarrow J_{6}$. Finalmente, a dimensão do grupo de 2-cociclos de $\partial_{1}$ é 15 enquanto que para $\mathcal{J}_{6}$ é $14, \operatorname{logo} \mathcal{J}_{1}$ não domina $\mathcal{J}_{6}$ pela Proposição 4.62. Isto prova que $J_{6}$ é rígida.

Considere $\mathcal{J} \in \mathcal{J O r}_{4}$, tal que $\mathcal{J} \rightarrow \mathcal{J}_{12}$, então pela Proposição 4.59 em uma base adequada $\left(\mathcal{J}_{12}\right)_{s s}$ é uma subálgebra de $\mathcal{J}_{s s}$ tal que a ação da parte semissimples é preservada. Escolhemos a base $\{a, b, c, d\}$ de $\mathcal{J}$ onde os produtos são $a^{2}=a, b^{2}=b, a c=\frac{1}{2} c$, $a d=\frac{1}{2} d$ e $a b=b c=b d=0$ logo, usando a tabela de multiplicação para a decomposição de Peirce (veja o Teorema 1.19) temos $c^{2}=\alpha a, d^{2}=\beta a$ e $c d=\gamma a$ para certos $\alpha, \beta, \gamma \in \mathbf{k}$. Como a base deve satisfazer a versão linearizada da identidade de Jordan (1.3) obtemos que $\alpha=\beta=\gamma=0$. $\log \mathcal{\partial} \simeq \mathcal{J}_{12}$ e $\mathcal{J}_{12}$ é rígida.

Verificaremos que $\mathcal{J}_{13}$ é rígida. Como $\operatorname{dim} \operatorname{Rad}\left(\mathcal{J}_{13}\right)=2$ temos que se $\mathcal{J}_{i} \rightarrow \mathcal{J}_{13}$ então $1 \leqslant i \leqslant 27$. Mais ainda, $\partial_{i}$ tem que ser não associativa $\operatorname{logo} i \neq 3,4,22$ e 27 , enquanto que usando o argumento da dimensão do grupo de automorfismo e da dimensão do aniquilador, deduzimos que $J_{13}$ não se deforma em $\partial_{9}, J_{12}, J_{16}, J_{18}, J_{19}$ e $J_{21}$, respetivamente em $\mathcal{J}_{5}, \partial_{8}, \mathcal{J}_{10}, \mathcal{J}_{11}, \mathcal{J}_{20}, \mathcal{J}_{23}$ e $\mathcal{J}_{26}$. As álgebras $\mathcal{J}_{14}, \mathcal{J}_{15}$ e $\mathcal{J}_{17}$ não são 
deformações de $\mathcal{J}_{13}$ pela Proposição 4.59. A álgebra $\mathcal{J}_{2}$ não domina $\mathcal{J}_{13}$ pela Proposição 4.58 pois a dimensão do centro associador $Z\left(\mathcal{J}_{2}\right)$ é 1 enquanto que $\operatorname{dim} Z\left(\mathcal{J}_{13}\right)=0$. Finalmente, note que $\mathcal{J}_{13}$ não tem nenhuma subálgebra associativa de dimensão 3 , logo nenhuma das álgebras restantes poderia dominar $\mathcal{J}_{13}$ pela Proposição 4.57.

Observe que não existe álgebra em $\mathcal{J}_{4} r_{4}$ que deforme-se em $\mathcal{J}_{24}$ : $\mathcal{J}_{24}$ é não associativa e ambas dimensões do aniquilador e do radical coincidem com as dimensões de $\mathcal{J}_{13}, \mathrm{o}$ que reduz a lista de possíveis deformações a

$$
\Delta=\left\{\mathcal{J}_{1}, \mathcal{J}_{2} \mathcal{J}_{6}, \mathcal{J}_{7}, \mathcal{J}_{9}, \mathcal{J}_{12}, \mathcal{J}_{13}, \mathcal{J}_{14}, \mathcal{J}_{15}, \mathcal{J}_{16}, \mathcal{J}_{17}, \mathcal{J}_{18}, \mathcal{J}_{19}, \mathcal{J}_{25}\right\}
$$

A $\operatorname{dim}$ Aut $\mathcal{J}_{24}=2$ portanto as possíveis álgebras $\mathcal{J}$ que podem dominar $\mathcal{J}_{24}$ são aquelas que pertencem a $\Delta$ e que $\operatorname{dim} \operatorname{Aut} \mathcal{J}<2$, o que leva somente a $\mathcal{J}_{1}$. Mas $\operatorname{dim} Z^{2}\left(\mathcal{J}_{1}, \mathcal{J}_{1}\right)=$ 15 enquanto que $\operatorname{dim} Z^{2}\left(\mathfrak{d}_{24}, \mathfrak{J}_{24}\right)=14 \operatorname{logo}$, Proposição $4.62, \mathfrak{J}_{1} \nrightarrow \mathfrak{J}_{24}$ e $\mathfrak{J}_{24}$ é rígida.

Finalmente, suponha que $\mathcal{J} \in \mathcal{J}^{2} r_{4}$ e $\mathcal{J} \rightarrow J_{59}$ então pela Proposição 4.59 podemos escolher uma base tal que $\left(\mathcal{J}_{59}\right)_{s s} \subseteq \mathcal{J}_{s s}$ e a ação de $\left(\mathcal{J}_{59}\right)_{\text {ss }}$ deveria ser preservada, então $\mathcal{J}$ somente poderia ser $\mathcal{J}_{12}, \mathcal{J}_{13}, \mathcal{J}_{32}, \mathcal{J}_{58}$ ou $\mathcal{J}_{60}$ mas então $\operatorname{dim} \operatorname{Aut}(\mathcal{J}) \geqslant 4$, portanto $\mathcal{J}_{59}$ é rígida.

Resta mostrar que para qualquer álgebra $\mathcal{J} \in \mathcal{J o r}_{4}$ existe uma álgebra $\mathcal{J}^{\prime} \in \Omega$ tal que $\mathcal{J}^{\prime}$ é uma deformação de $\mathcal{J}$. No que segue todas as transformações serão dadas usando as bases do Capítulo 2 na ordem $\left\{e_{i}, n_{j}\right\}$ onde $1 \leqslant i, j \leqslant 4$.

Mostraremos que $\mathcal{J}_{1}$ domina $J_{7}, \partial_{8}, J_{10}, J_{11}, J_{17}, J_{23}, J_{25}, J_{28}, J_{44}, J_{45}, J_{46}, J_{52}$, $\mathcal{J}_{53}, \mathcal{J}_{62}, \mathcal{J}_{63}, \mathcal{J}_{64}$ e $\mathcal{J}_{65}$. Do Exemplo 4.29 temos $\mathcal{J}_{1} \rightarrow \mathcal{J}_{25}$. Além disso $\mathcal{J}_{25} \rightarrow \mathcal{J}_{17}$, de fato para $t \neq 0$ escolhemos a base $A_{t}=e_{1}, B_{t}=e_{2}, C_{t}=t n_{1}$ e $D_{t}=n_{2}$ de $\partial_{25}$, em particular $C_{t}^{2}=t^{2} D_{t}$ e $\operatorname{logo} \mathcal{J}_{25} \rightarrow \mathcal{J}_{17}$ quando $t \rightarrow 0$. A deformação $\mathcal{J}_{1} \rightarrow \mathcal{J}_{53}$ é dada pela curva $A_{t}=e_{1}+e_{4}, B_{t}=t e_{3}, C_{t}=t^{2} e_{1}$ e $D_{t}=t^{2} e_{2}$, e a deformação $J_{53} \rightarrow J_{62}$ é dada por $A_{t}=t e_{1}+t n_{2}-t n_{3}, B_{t}=t^{2} n_{2}+t^{2} n_{3}, C_{t}=-t^{3} n_{3}$ e $D_{t}=t n_{1}$.

Em [4] foram descritas as "contrações" entre as álgebras de Jordan nilpotentes, mas se $\mathcal{J}$ é uma contração de $\mathcal{J}^{\prime}$ então $\mathcal{J}$ corresponde a um ponto no fecho da órbita de $\mathcal{J}^{\prime}$, ou seja $\mathcal{J}^{\prime}$ é uma deformação de $\mathcal{J}$. Logo de [4] temos as seguintes deformações entre álgebras nilpotentes: $\mathcal{J}_{62} \rightarrow \mathcal{J}_{65}, \mathcal{J}_{62} \rightarrow \mathcal{J}_{63}$ e $\mathcal{J}_{63} \rightarrow \mathcal{J}_{64}$

Por outro lado, combinando os resultados para álgebras de Jordan de dimensão 3 da Seção 5.3 com a Proposição 4.41 obtemos $\partial_{1} \rightarrow \partial_{23}, \partial_{1} \rightarrow \partial_{7}, \partial_{7} \rightarrow \partial_{10}, \partial_{1} \rightarrow \partial_{8}$ e $\mathfrak{\partial}_{8} \rightarrow \mathfrak{J}_{11}$. Para mostrar que $\mathfrak{J}_{11} \rightarrow \partial_{46}$, considere a mudança de base $A_{t}=e_{1}, B_{t}=n_{1}$, $\mathrm{C}_{\mathrm{t}}=\mathrm{te}_{2}-\mathrm{n}_{2}$ e $\mathrm{D}_{\mathrm{t}}=\mathrm{tn}_{2}$, para $\mathrm{t} \neq 0$, de $\mathcal{J}_{11}$. Como $\mathrm{B}_{\mathrm{t}} \mathrm{C}_{\mathrm{t}}=\mathrm{t} \frac{\mathrm{B}_{\mathrm{t}}}{2} \mathrm{e} \mathrm{C}_{\mathrm{t}}^{2}=\mathrm{D}_{\mathrm{t}}+\mathrm{t} \mathrm{C}_{\mathrm{t}}$ obtemos a estrutura de $\mathcal{J}_{46}$ quando $t$ tende a zero.

Para a deformação $\partial_{8} \rightarrow \partial_{45}$, considere a base $A_{t}=e_{1}, B_{t}=t e_{3}, C_{t}=t^{2} e_{2} e$ $D_{t}=t e_{2}+n_{1}$ de $\mathcal{J}_{8}$, então quando $t=0$ obtemos $\mathcal{J}_{45}$. A mudança de base $A_{t}=e_{1}$, 
$B_{t}=n_{1}, C_{t}=n_{2}$ e $D_{t}=t_{3}$ de $\mathcal{J}_{45}$ funciona para $\partial_{45} \rightarrow J_{44}$, enquanto $\mathcal{J}_{44} \rightarrow \partial_{28}$ tomando $A_{t}=e_{1}, B_{t}=t_{1}, C_{t}=n_{2}$ e $D_{t}=n_{3}$ em $\partial_{44}$.

Finalmente, para ver que $J_{23} \rightarrow J_{52}$, considere a mudança de base $A_{t}=e_{1}+e_{2}$, $\mathrm{B}_{\mathrm{t}}=\mathrm{n}_{1}, \mathrm{C}_{\mathrm{t}}=\mathrm{te}_{2}$ e $\mathrm{D}_{\mathrm{t}}=\mathrm{n}_{2}$ de $\mathcal{J}_{23}$.

Agora mostraremos que $\mathcal{J}_{2}$ domina $\mathcal{J}_{9}, \mathcal{J}_{18}, \mathcal{J}_{48}$ e $\mathcal{J}_{49}$. Para ver que $\mathcal{J}_{2} \rightarrow \mathcal{J}_{9}$ considere, para $t \neq 0$, a base $A_{t}=e_{1}, B_{t}=e_{2}, C_{t}=e_{3}+e_{4}$ e $D_{t}=t e_{4}$ de $J_{2}$. Como $C_{t}^{2}=A_{t}+B_{t}$, $C_{t} D_{t}=t\left(\frac{A_{t}+B_{t}}{2}\right)$ e $D_{t}^{2}=0$ obtemos a álgebra $\partial_{q}$ quando $t=0$. Para a deformação $\partial_{9} \rightarrow \partial_{18}$, é suficiente considerar a base $A_{t}=e_{1}, B_{t}=e_{2}, C_{t}=t e_{3}$ e $D_{t}=n_{1}$ de $\partial_{9}$. Então, como $C_{t}^{2}=t^{2}\left(A_{t}+B_{t}\right)$, fazendo $t$ tender a zero obtemos $J_{18}$. Analogamente $\mathcal{J}_{2} \rightarrow \mathcal{J}_{49}$ com base $A_{t}=e_{1}, B_{t}=t e_{2}, C_{t}=2 t e_{3}$ e $D_{t}=t e_{3}+e_{4}$ e $\mathcal{J}_{49} \rightarrow J_{48}$ com base $A_{t}=e_{1}, B_{t}=n_{1}, C_{t}=t_{2}$ e $D_{t}=n_{3}$.

Todas as álgebras associativas, ou seja $\mathcal{J}_{4}, \mathcal{J}_{5}, \mathcal{J}_{19}, \mathcal{J}_{20}, \mathcal{J}_{21}, \mathcal{J}_{22}, \mathcal{J}_{26}, \mathcal{J}_{27}, \mathcal{J}_{34}, \mathcal{J}_{35}, \mathcal{J}_{36}$, $\mathcal{J}_{37}, \mathcal{J}_{38}, \mathcal{J}_{39}, \mathcal{J}_{40}, \mathcal{J}_{41}, \mathcal{J}_{42}, \mathcal{J}_{43}, \mathcal{J}_{47}, \mathcal{J}_{54}, \mathcal{J}_{61}, J_{66}, \mathcal{J}_{67}, \mathcal{J}_{68}, \mathcal{J}_{69}, \mathcal{J}_{70}, \mathcal{J}_{71}, \mathcal{J}_{72}$ e $\mathcal{J}_{73}$ são dominadas por $\mathcal{J}_{3}$.

Combinando a Proposição 4.41 com a descrição geométrica de Jor 2 dada na Seção 5.2 obtemos $\mathcal{J}_{3} \rightarrow \mathcal{J}_{4}, J_{3} \rightarrow \mathcal{J}_{5}, J_{5} \rightarrow \mathcal{J}_{20}, J_{5} \rightarrow J_{26}, J_{26} \rightarrow J_{19}, J_{19} \rightarrow J_{35}, J_{40} \rightarrow J_{34}$, $J_{72} \rightarrow J_{73}, J_{4} \rightarrow J_{22}$ e $J_{26} \rightarrow J_{47}$. Novamente, usando a Proposição 4.41 e a descrição geométrica de $\mathrm{J} \mathrm{r}_{3}$ dada na seção anterior temos $\mathcal{J}_{4} \rightarrow J_{27}, J_{27} \rightarrow J_{21}, J_{21} \rightarrow J_{37}$, $\mathfrak{J}_{20} \rightarrow \mathcal{J}_{54}, \mathcal{J}_{38} \rightarrow \mathcal{J}_{41}, \mathcal{J}_{41} \rightarrow \mathcal{J}_{40}$ e $\mathcal{J}_{71} \rightarrow \mathcal{J}_{72}$. Finalmente, de [4] temos as seguintes deformações entre álgebras nilpotentes $\mathcal{J}_{61} \rightarrow \mathcal{J}_{67}, \mathcal{J}_{66} \rightarrow \mathcal{J}_{68}, \mathcal{J}_{66} \rightarrow \mathcal{J}_{70}, \mathcal{J}_{68} \rightarrow \mathcal{J}_{69}$ e $\partial_{69} \rightarrow \partial_{71}$.

Para ver que $J_{20} \rightarrow J_{38}$, para $t \neq 0$ consideramos a mudança de base $A_{t}=e_{2}$, $B_{t}=t e_{1}+n_{1}+n_{2}, C_{t}=t n_{1}-t_{2}$ e $D_{t}=t^{2} n_{1}$ de $\mathcal{J}_{20}$. Analogamente a dominância $J_{38} \rightarrow J_{66}$ é dada pela base $A_{t}=t n_{1}, B_{t}=t^{2} n_{2}, C_{t}=t^{3} n_{3}$ e $D_{t}=t e_{1}-t^{2} n_{3}$ de $\partial_{38}$. Para a deformação $\partial_{47} \rightarrow \partial_{61}$ tome $t \neq 0$ e considere a base $A_{t}=t e_{1}+n_{1}+n_{2}$, $B_{t}=t n_{1}-t_{2}+n_{3}, C_{t}=t^{2} n_{1}-t_{3}$ e $D_{t}=t^{2} n_{3}$ de $J_{47}$, então quando $t=0$ obtemos $J_{61}$.

Para mostrar que $\mathcal{J}_{22} \rightarrow \mathcal{J}_{39}$, para $t \neq 0$ usamos a base $A_{t}=e_{1}+e_{2}, B_{t}=t e_{2}+n_{1}+$ $n_{2}, C_{t}=-t_{1}+t_{2}$ e $D_{t}=t^{2} n_{2}$ de $\partial_{22}$. Como $B_{t}^{2}=C_{t}+t B_{t}$ e $B_{t} D_{t}=t D_{t}$ obtemos a estrutura da álgebra $\mathcal{J}_{39}$ quando $t$ tende a zero. No caso de $\mathcal{J}_{39} \rightarrow \mathcal{J}_{43}$ consideramos a base $A_{t}=e_{1}, B_{t}=-t n_{1}, C_{t}=-t^{2} n_{3}$ e $D_{t}=t n_{2}+t n_{3}$ de $J_{39}$. Analogamente, para mostrar que $\mathcal{J}_{43} \rightarrow \mathcal{J}_{42}$ é suficiente considerar a mudança de base $A_{t}=e_{1}, B_{t}=n_{1}$, $\mathrm{C}_{\mathrm{t}}=\mathrm{n}_{2}$ e $\mathrm{D}_{\mathrm{t}}=\mathrm{tn}_{1}-\mathrm{tn}_{3}$ de $\mathcal{J}_{43}$.

Por último, para completar a dominância de $\mathcal{J}_{3}$ só resta provar que $\mathcal{J}_{42} \rightarrow \mathcal{J}_{36}$. Para $t \neq 0$ considere a mudança de base $A_{t}=e_{1}, B_{t}=t n_{1}, C_{t}=n_{2}$ e $D_{t}=n_{3}$ de $\mathcal{J}_{42}$.

A dominância das álgebras rígidas $\mathcal{J}_{6}$ sobre $\mathcal{J}_{15}$ e $\mathcal{J}_{12}$ sobre $\mathcal{J}_{30}$ segue da Proposição 4.41. Mostraremos a seguir que $J_{12} \rightarrow J_{32}$, para isso considere a base $A_{t}=e_{1}+e_{2}$, 
$B_{t}=n_{1}, C_{t}=n_{2}$ e $D_{t}=t e_{2}$, para $t \neq 0$, de $\mathcal{J}_{12}$. Como $D_{t}^{2}=t D_{t}$ obtemos a estrutura da álgebra $\partial_{32}$ quando $t=0$.

Agora, vejamos que $\mathfrak{J}_{13}$ domina $\mathfrak{J}_{60}$. Para $t \neq 0$, é suficiente considerar a base $A_{t}=e_{1}+e_{2}, B_{t}=-2 t e_{1}, C_{t}=n_{1}+n_{2}$ e $D_{t}=t n_{2}$ de $J_{13}$ e então quando fazemos $t$ tender a zero obtemos $J_{60}$. Analogamente $\mathcal{J}_{16}$ domina $\mathcal{J}_{50}$, usando a base $A_{t}=e_{1}$, $B_{t}=t e_{2}, C_{t}=2 n_{1}+n_{2}$ e $D_{t}=t n_{1}$ de $\mathcal{J}_{16}$.

As álgebras $\mathcal{J}_{14}, J_{29}, \partial_{31}, J_{51}, J_{55}, J_{56}$ e $\mathcal{J}_{57}$ são dominadas por $\mathcal{J}_{24}$. Uma vez mais combinamos a Proposição 4.41 e as deformações obtidas na Seção 5.3 e obtemos que $\mathfrak{J}_{24} \rightarrow J_{51}, J_{51} \rightarrow J_{29}$ e $\mathcal{J}_{24} \rightarrow \mathcal{J}_{14}$. No caso de $\mathfrak{J}_{14} \rightarrow \mathcal{J}_{56}$ e $\mathfrak{J}_{24} \rightarrow J_{57}$ usamos, para ambas álgebras, a base $A_{t}=e_{1}+e_{2}, B_{t}=t^{2} e_{1}+n_{2}, C_{t}=t^{2} n_{2}$ e $D_{t}=t n_{1}$, então quando $t=0$ temos $J_{56}$ e $J_{57}$ respetivamente. Para provar que $J_{56} \rightarrow J_{31}$ e $J_{57} \rightarrow J_{55}$ considere a base $A_{t}=e_{1}, B_{t}=t n_{1}, C_{t}=n_{2}$ e $D_{t}=n_{3}, t \neq 0$ de $J_{56}$ e $J_{57}$, como em ambos casos $B_{t}^{2}=t^{2} C_{t}$, fazendo $t=0$ obtemos $\mathcal{J}_{31}$ e $\mathcal{J}_{55}$, respetivamente.

Para completar a descrição das componentes irredutíveis da variedade $\mathrm{Jor}_{4}$ só resta mostrar que $J_{59}$ domina $J_{58}$. Para $t \neq 0$, é suficiente considerar a base: $A_{t}=e_{1}$, $B_{t}=n_{1}, C_{t}=t n_{2}$ e $D_{t}=n_{3}$ de $J_{59}$. Como $C_{t} D_{t}=t B_{t}$ é claro que as contantes estruturais desta base tendem a aquelas de $\mathcal{J}_{58}$ quando fazemos $t=0$.

A órbita de $\mathcal{J}_{73}$ é fechada (Lema 4.40) e logo toda $\mathcal{J} \in \mathcal{J} r_{4}$ é uma deformação de $\mathcal{J}_{73}$ o que implica que $\mathrm{Jor}_{4}$ é uma variedade afim conexa.

Como $\mathrm{Jor}_{4}$ é uma união finita de órbitas que são localmente fechadas então a dimensão da variedade é o máximo das dimensões de suas órbitas, assim temos que

$$
\operatorname{dim} \partial_{0 r_{4}}=\max _{1 \leqslant i \leqslant 73}\left\{\operatorname{dim} \mathcal{J}_{i}^{G}\right\}=\operatorname{dim} \mathcal{J}_{3}^{G}=4^{2}-\operatorname{dim} \operatorname{Aut}\left(\mathcal{J}_{3}\right)=16
$$

Por último, a dimensão de cada componente é determinada pela dimensão da órbita da álgebra rígida que a gera, segue então das informações do Apêndice B que as dimensões das componentes irredutíveis de Jor 4 são: 15, 11, 16, 10, 4, duas componentes de dimensão 14 e três componentes de dimensão 12.

Um diagrama das álgebras de Jordan de dimensão 4 no qual é possível compreender a prova do Teorema 5.2 é dado na Figura 5.3. Uma descrição quase-completa (e assim menos compreensível) das órbitas e, portanto, das componentes irredutíveis de Jor 4 é dado na Figura 5.4. Com quase-completa queremos dizer que conseguimos determinar em um 96,53\% das vezes se uma álgebra de Jordan de dimensão 4 pertence ou não ao fecho de Zariski da órbita de uma outra álgebra. Novamente, usamos os super-índices A, $S$, U e N nos nomes das álgebras para fazer referência a que $\partial_{i}$ é Associativa, Semissimples, Unitária e Nilpotente respetivamente, com o fim de poder enxergar melhor o comportamento dessas características sob deformações. 
Resumimos a existência ou não existência de deformações em várias tabelas. A simbologia utilizada respeita o padrão das tabelas das seções anteriores e adicionamos os seguintes:

i. $\nrightarrow_{1}$ : não existe deformação pela Proposição 4.61, pois a álgebra da linha não é unitária e a álgebra da coluna sim o é.

ii. $t_{\mathfrak{g} m}$ : não existe deformação pela Proposição 4.54 que relaciona as dimensões das potencias das álgebras envolvidas.

iii. $\dashv_{Z^{2}}$ : não existe deformação pela Proposição 4.62 que relaciona as dimensões dos grupos de 2-cociclos das álgebras envolvidas.

iv. $\nrightarrow_{M_{A}}$ : não existe deformação pela Proposição 4.57 que relaciona as dimensões das subálgebras associativas maximais das álgebras envolvidas.

v. $\not_{\rightarrow} M_{\mathrm{Nul}}$ : não existe deformação pela Proposição 4.57 que relaciona as dimensões das subálgebras nulas maximais das álgebras envolvidas.

vi. $\rightarrow_{\oplus}$ : existe deformação pela Proposição 4.41, pois as álgebras envolvidas são soma direta de álgebras de dimensões menores, tal que uma é uma deformação da outra.

vii. $\rightarrow$ Anc: existe deformação e a contração foi dada em [4].

Observamos que propositalmente as álgebras não aparecem na ordem lexicográfica. Colocamos separadamente álgebras associativas de não associativas e álgebras nilpotentes de não nilpotentes com o fim de poupar um pouco de espaço. Desse modo não foi colocada uma tabela de 1020 células onde todas elas só teriam o símbolo " $f_{\text {Ass }}$ " o qual representa que "Associativa $\nrightarrow$ † Não-Associativa". 
$\operatorname{dim} \operatorname{Aut}(\mathcal{J})$

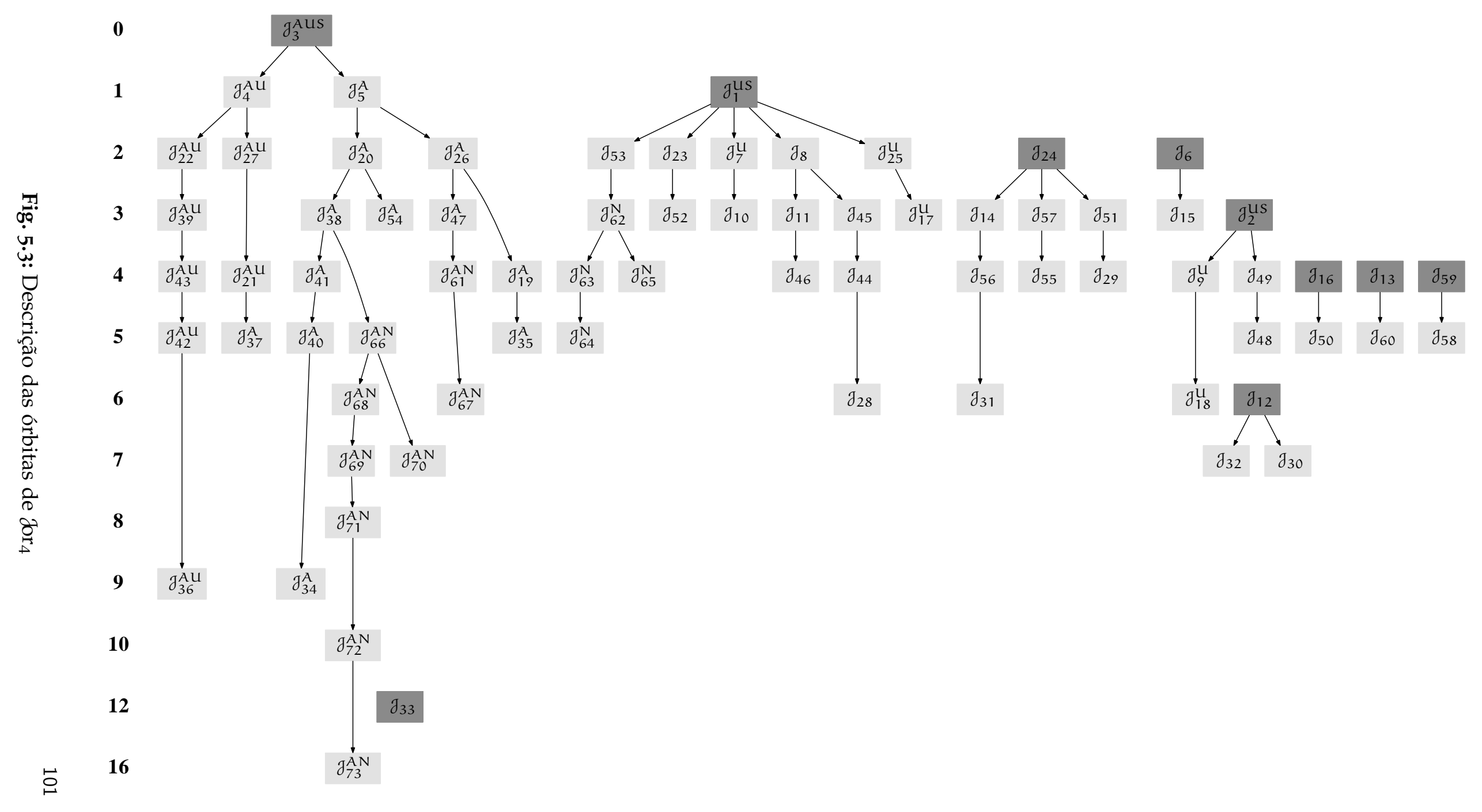


$\operatorname{dim} \operatorname{Aut}(\mathcal{J})$

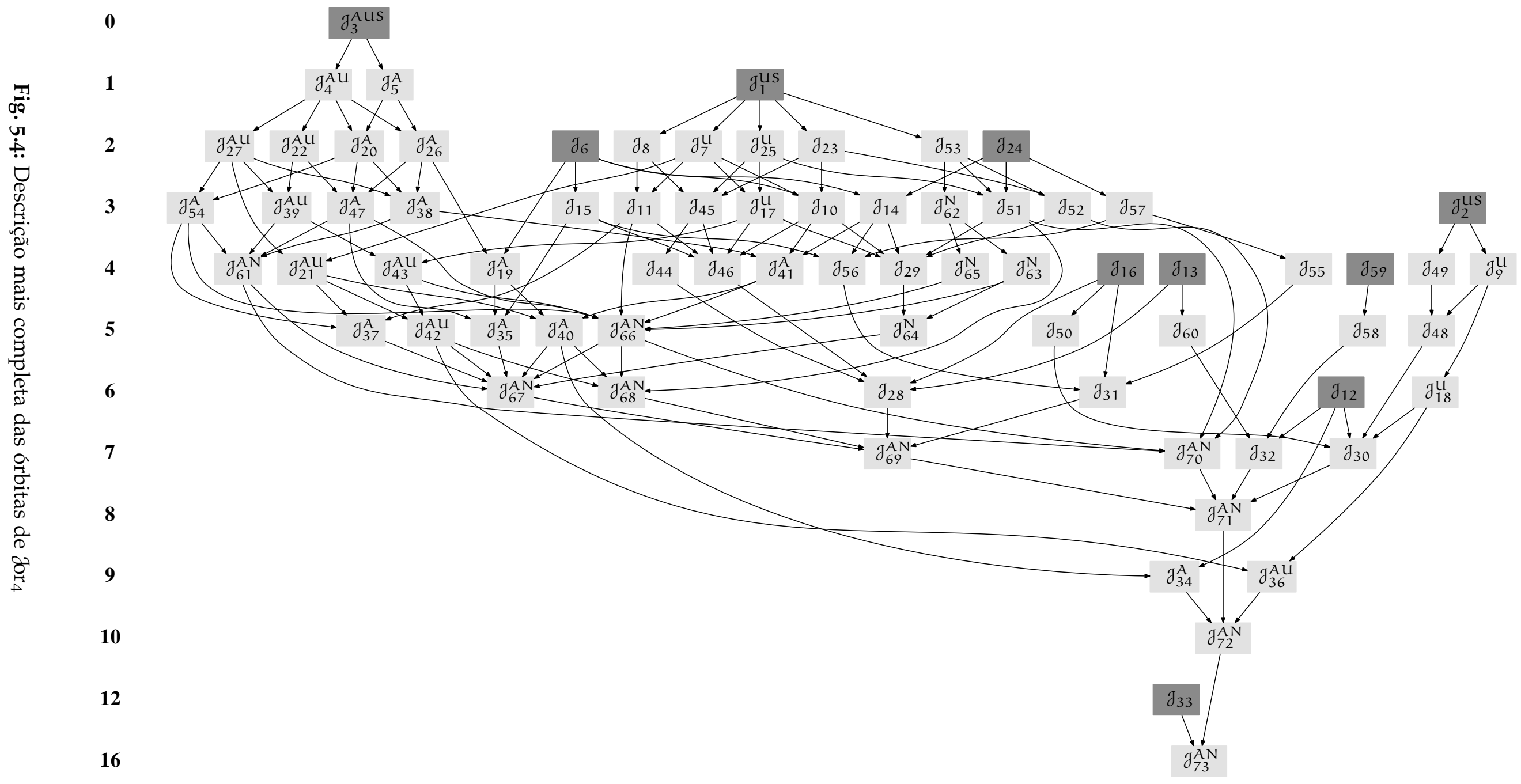


Tabela 5.3: Existência de deformações em $\mathrm{Jor}_{4}$

\begin{tabular}{|c|c|c|c|c|c|c|c|c|c|c|c|c|c|c|c|c|c|c|}
\hline & $\partial_{61}$ & $\partial_{66}$ & $\partial_{67}$ & $J_{68}$ & $\partial_{69}$ & $\partial_{70}$ & $J_{71}$ & $\partial_{72}$ & $\partial_{73}$ & $\partial_{3}$ & $\mathrm{~d}_{4}$ & $\partial_{5}$ & $J_{39}$ & $J_{38}$ & $\partial_{41}$ & $\partial_{43}$ & $\partial_{40}$ & $\partial_{47}$ \\
\hline$\partial_{61}$ & $\rightarrow_{\mathrm{DT}}$ & $f_{\text {Anc }}$ & $\rightarrow_{\mathrm{Anc}}$ & $f_{A n c}$ & $\rightarrow_{\mathrm{T}}$ & $\rightarrow_{\mathrm{Anc}}$ & $\rightarrow_{\mathrm{T}}$ & $\rightarrow_{\mathrm{T}}$ & $\rightarrow_{\mathrm{T}}$ & $t_{\mathrm{Rad}}$ & $f_{\mathrm{Rad}}$ & $f_{\mathrm{Rad}}$ & $f_{\mathrm{Rad}}$ & $f_{\mathrm{Rad}}$ & $f_{\text {Rad }}$ & $f_{\mathrm{Rad}}$ & $f_{\mathrm{Rad}}$ & $A_{\mathrm{Rac}}$ \\
\hline$\partial_{66}$ & $A_{\text {Aut }}$ & $\rightarrow \mathrm{DT}$ & $\rightarrow$ Anc & $\rightarrow$ Anc & $\rightarrow_{\mathrm{T}}$ & $\rightarrow$ Anc & $\rightarrow_{\mathrm{T}}$ & $\rightarrow_{\mathrm{T}}$ & $\rightarrow_{\mathrm{T}}$ & $t_{\mathrm{Rad}}$ & $t_{\mathrm{Rad}}$ & $t_{\mathrm{Rad}}$ & $t_{\mathrm{Rad}}$ & $t_{\text {Rad }}$ & $\not t_{\text {Rad }}$ & $t_{\mathrm{Rad}}$ & $t_{\mathrm{Rad}}$ & $t_{\mathrm{Rac}}$ \\
\hline$J_{67}$ & $f_{\text {Aut }}$ & $f_{\text {Aut }}$ & $\rightarrow_{\mathrm{DT}}$ & $t_{\text {Aut }}$ & $\rightarrow_{\mathrm{Anc}}$ & $f_{\text {Ann }}$ & $\rightarrow_{\mathrm{T}}$ & $\rightarrow_{\mathrm{T}}$ & $\rightarrow_{\mathrm{T}}$ & $f_{\mathrm{Rad}}$ & $t_{\mathrm{Rad}}$ & $f_{\mathrm{Rad}}$ & $f_{\mathrm{Rad}}$ & $t_{\mathrm{Rad}}$ & $f_{\mathrm{Rad}}$ & $f_{\mathrm{Rad}}$ & $f_{\mathrm{Rad}}$ & $t_{\mathrm{Rac}}$ \\
\hline$\partial_{68}$ & $A_{\text {Aut }}$ & $t_{\text {Aut }}$ & $t_{\text {Aut }}$ & $\rightarrow{ }_{\mathrm{DT}}$ & $\rightarrow_{\text {Anc }}$ & $t_{A n n}$ & $\rightarrow_{\mathrm{T}}$ & $\rightarrow_{\mathrm{T}}$ & $\rightarrow_{\mathrm{T}}$ & $t_{\mathrm{Rad}}$ & $t_{\mathrm{Rad}}$ & $t_{\mathrm{Rad}}$ & $t_{\mathrm{Rad}}$ & $\not t_{\text {Rad }}$ & $\not t_{\text {Rad }}$ & $t_{\mathrm{Rad}}$ & $t_{\mathrm{Rad}}$ & $t_{\mathrm{Rac}}$ \\
\hline$J_{69}$ & $A_{\text {Aut }}$ & $\not_{\text {Aut }}$ & $A_{\text {Aut }}$ & $t_{\text {Aut }}$ & $\rightarrow_{\mathrm{DT}}$ & $\not_{\text {Aut }}$ & $\rightarrow$ Anc & $\rightarrow_{\mathrm{T}}$ & $\rightarrow_{\mathrm{T}}$ & $t_{\mathrm{Rad}}$ & $A_{\mathrm{Rad}}$ & $t_{\mathrm{Rad}}$ & $t_{\mathrm{Rad}}$ & $t_{\mathrm{Rad}}$ & $t_{\text {Rad }}$ & $t_{\mathrm{Rad}}$ & $t_{\mathrm{Rad}}$ & $t_{\mathrm{Rac}}$ \\
\hline $\mathcal{J}_{70}$ & $f_{\text {Aut }}$ & $A_{\text {Aut }}$ & $f_{\text {Aut }}$ & $A_{\text {Aut }}$ & $\not_{A u t}$ & $\rightarrow_{\mathrm{DT}}$ & $\rightarrow_{\mathrm{Anc}}$ & $\rightarrow_{\mathrm{T}}$ & $\rightarrow_{\mathrm{T}}$ & $t_{\mathrm{Rad}}$ & $A_{\mathrm{Rad}}$ & $t_{\mathrm{Rad}}$ & $t_{\mathrm{Rad}}$ & $t_{\mathrm{Rad}}$ & $t_{\text {Rad }}$ & $t_{\mathrm{Rad}}$ & $t_{\mathrm{Rad}}$ & $t_{\mathrm{Rac}}$ \\
\hline$\partial_{71}$ & $f_{\text {Aut }}$ & $A_{\text {Aut }}$ & $A_{\text {Aut }}$ & $t_{\text {Aut }}$ & $\not_{A u t}$ & $\not_{\text {Aut }}$ & $\rightarrow$ DT & $\rightarrow_{\oplus}$ & $\rightarrow_{\mathrm{T}}$ & $t_{\mathrm{Rad}}$ & $t_{\mathrm{Rad}}$ & $t_{\mathrm{Rad}}$ & $t_{\mathrm{Rad}}$ & $t_{\text {Rad }}$ & $t_{\mathrm{Rad}}$ & $t_{\mathrm{Rad}}$ & $t_{\mathrm{Rad}}$ & $\not_{\mathrm{Rac}}$ \\
\hline$\partial_{72}$ & $f_{\text {Aut }}$ & $t_{\text {Aut }}$ & $t_{\text {Aut }}$ & $f_{\text {Aut }}$ & $t_{\text {Aut }}$ & $f_{\text {Aut }}$ & $f_{\text {Aut }}$ & $\rightarrow_{\mathrm{DT}}$ & $\rightarrow_{\oplus}$ & $f_{\mathrm{Rad}}$ & $t_{\mathrm{Rad}}$ & $f_{\mathrm{Rad}}$ & $f_{\mathrm{Rad}}$ & $t_{\mathrm{Rad}}$ & $f_{\mathrm{Rad}}$ & $f_{\mathrm{Rad}}$ & $f_{\mathrm{Rad}}$ & $t_{\mathrm{Rac}}$ \\
\hline$\partial_{73}$ & $f_{\text {Aut }}$ & $t_{\text {Aut }}$ & $t_{\text {Aut }}$ & $t_{\mathrm{Aut}}$ & $t_{\text {Aut }}$ & $t_{\text {Aut }}$ & $t_{\text {Aut }}$ & $t_{\text {Aut }}$ & $\rightarrow_{\mathrm{DT}}$ & $t_{\mathrm{Rad}}$ & $t_{\mathrm{Rad}}$ & $t_{\mathrm{Rad}}$ & $t_{\mathrm{Rad}}$ & $t_{\mathrm{Rad}}$ & $t_{\mathrm{Rad}}$ & $t_{\mathrm{Rad}}$ & $t_{\mathrm{Rad}}$ & $t_{\text {Rac }}$ \\
\hline$J_{3}$ & $\rightarrow_{\mathrm{T}}$ & $\rightarrow_{\mathrm{T}}$ & $\rightarrow_{\mathrm{T}}$ & $\rightarrow_{\mathrm{T}}$ & $\rightarrow_{\mathrm{T}}$ & $\rightarrow_{\mathrm{T}}$ & $\rightarrow_{\mathrm{T}}$ & $\rightarrow_{\mathrm{T}}$ & $\rightarrow_{\mathrm{T}}$ & $\rightarrow_{\mathrm{DT}}$ & $\rightarrow$ & $\rightarrow$ & $\rightarrow_{\mathrm{T}}$ & $\rightarrow_{\mathrm{T}}$ & $\rightarrow_{\mathrm{T}}$ & $\rightarrow_{\mathrm{T}}$ & $\rightarrow_{\mathrm{T}}$ & $\rightarrow_{\mathrm{T}}$ \\
\hline$\partial_{4}$ & $\rightarrow_{\mathrm{T}}$ & $\rightarrow_{\mathrm{T}}$ & $\rightarrow_{\mathrm{T}}$ & $\rightarrow_{\mathrm{T}}$ & $\rightarrow_{\mathrm{T}}$ & $\rightarrow_{\mathrm{T}}$ & $\rightarrow_{\mathrm{T}}$ & $\rightarrow_{\mathrm{T}}$ & $\rightarrow_{\mathrm{T}}$ & $t_{\text {Aut }}$ & $\rightarrow_{\mathrm{DT}}$ & $\not_{\text {Aut }}$ & $\rightarrow_{\mathrm{T}}$ & $\rightarrow_{\mathrm{T}}$ & $\rightarrow_{\mathrm{T}}$ & $\rightarrow_{\mathrm{T}}$ & $\rightarrow_{\mathrm{T}}$ & $\rightarrow_{\mathrm{T}}$ \\
\hline$J_{5}$ & $\rightarrow \mathrm{T}$ & $\rightarrow_{\mathrm{T}}$ & $\rightarrow \mathrm{T}$ & $\rightarrow_{\mathrm{T}}$ & $\rightarrow_{\mathrm{T}}$ & $\rightarrow_{\mathrm{T}}$ & $\rightarrow_{\mathrm{T}}$ & $\rightarrow_{\mathrm{T}}$ & $\rightarrow_{\mathrm{T}}$ & $\nrightarrow_{\text {Aut }}$ & $t_{\text {Aut }}$ & $\rightarrow \mathrm{DT}$ & $f_{A n n}$ & $\rightarrow_{\mathrm{T}}$ & $\rightarrow_{\mathrm{T}}$ & $\not_{\mathrm{Ann}}$ & $\rightarrow_{\mathrm{T}}$ & $\rightarrow_{\mathrm{T}}$ \\
\hline$J_{39}$ & $\rightarrow$ & $\rightarrow_{\mathrm{T}}$ & $\rightarrow_{\mathrm{T}}$ & $\rightarrow_{\mathrm{T}}$ & $\rightarrow_{\mathrm{T}}$ & $\rightarrow_{\mathrm{T}}$ & $\rightarrow_{\mathrm{T}}$ & $\rightarrow_{\mathrm{T}}$ & $\rightarrow_{\mathrm{T}}$ & $t_{\text {Aut }}$ & $t_{\text {Aut }}$ & $t_{\text {Aut }}$ & $\rightarrow \rightarrow_{\mathrm{DT}}$ & $t_{\text {Aut }}$ & $A_{\text {npa }}$ & $\rightarrow$ & $\not_{\text {npa }}$ & $t_{\mathrm{Au}}$ \\
\hline$J_{38}$ & $\rightarrow$ & $\rightarrow_{\mathrm{T}}$ & $\rightarrow_{\mathrm{T}}$ & $\rightarrow_{\mathrm{T}}$ & $\rightarrow_{\mathrm{T}}$ & $\rightarrow_{\mathrm{T}}$ & $\rightarrow_{\mathrm{T}}$ & $\rightarrow_{\mathrm{T}}$ & $\rightarrow_{\mathrm{T}}$ & $A_{\text {Aut }}$ & $A_{\text {Aut }}$ & $f_{\text {Aut }}$ & $t_{\text {Aut }}$ & $\rightarrow_{\mathrm{DT}}$ & $\rightarrow_{\oplus}$ & $f_{A n n}$ & $\rightarrow_{\mathrm{T}}$ & $A_{\mathrm{Au}}$ \\
\hline$J_{41}$ & $t_{\text {Aut }}$ & $\rightarrow$ & $\rightarrow_{\mathrm{T}}$ & $\rightarrow_{\mathrm{T}}$ & $\rightarrow_{\mathrm{T}}$ & $\rightarrow_{\mathrm{T}}$ & $\rightarrow_{\mathrm{T}}$ & $\rightarrow_{\mathrm{T}}$ & $\rightarrow_{\mathrm{T}}$ & $\nrightarrow_{\text {Aut }}$ & $f_{\text {Aut }}$ & $t_{\text {Aut }}$ & $f_{\text {Aut }}$ & $\nrightarrow_{\text {Aut }}$ & $\rightarrow_{\mathrm{DT}}$ & $t_{\text {Aut }}$ & $\rightarrow_{\oplus}$ & $t_{\mathrm{Au}}$ \\
\hline$J_{43}$ & $A_{\text {Aut }}$ & $\rightarrow$ & $\rightarrow_{\mathrm{T}}$ & $\rightarrow_{\mathrm{T}}$ & $\rightarrow_{\mathrm{T}}$ & $\rightarrow_{\mathrm{T}}$ & $\rightarrow_{\mathrm{T}}$ & $\rightarrow_{\mathrm{T}}$ & $\rightarrow_{\mathrm{T}}$ & $t_{\mathrm{Aut}}$ & $t_{\text {Aut }}$ & $A_{\text {Aut }}$ & $t_{\text {Aut }}$ & $t_{\text {Aut }}$ & $t_{\text {Aut }}$ & $\rightarrow \mathrm{DT}$ & $A_{\text {npa }}$ & $\not A_{\mathrm{Au}}$ \\
\hline$J_{40}$ & $f_{\text {Aut }}$ & $t_{\text {Aut }}$ & $\rightarrow_{\oplus}$ & $\rightarrow_{\oplus}$ & $\rightarrow_{\mathrm{T}}$ & $f_{\text {Ann }}$ & $\rightarrow_{\mathrm{T}}$ & $\rightarrow_{\mathrm{T}}$ & $\rightarrow_{\mathrm{T}}$ & $f_{\text {Aut }}$ & $f_{\text {Aut }}$ & $A_{\text {Aut }}$ & $f_{\text {Aut }}$ & $t_{\text {Aut }}$ & $t_{\text {Aut }}$ & $f_{\text {Aut }}$ & $\rightarrow_{\mathrm{DT}}$ & $t_{\mathrm{Au}}$ \\
\hline$\partial_{47}$ & $\rightarrow$ & $\rightarrow$ & $\rightarrow_{\mathrm{T}}$ & $\rightarrow_{\mathrm{T}}$ & $\rightarrow_{\mathrm{T}}$ & $\rightarrow_{\mathrm{T}}$ & $\rightarrow_{\mathrm{T}}$ & $\rightarrow_{\mathrm{T}}$ & $\rightarrow_{\mathrm{T}}$ & $t_{\text {Aut }}$ & $t_{\text {Aut }}$ & $t_{\text {Aut }}$ & $t_{\text {Aut }}$ & $t_{\text {Aut }}$ & $t_{\text {npa }}$ & $t_{A n n}$ & $A_{\text {npa }}$ & $\rightarrow{ }_{\mathrm{DT}}$ \\
\hline$J_{54}$ & $\rightarrow$ & $\rightarrow$ & $\rightarrow_{\mathrm{T}}$ & $\rightarrow_{\mathrm{T}}$ & $\rightarrow_{\mathrm{T}}$ & $\rightarrow_{\mathrm{T}}$ & $\rightarrow_{\mathrm{T}}$ & $\rightarrow_{\mathrm{T}}$ & $\rightarrow_{\mathrm{T}}$ & $t_{\text {Aut }}$ & $f_{\text {Aut }}$ & $t_{\text {Aut }}$ & $f_{\text {Aut }}$ & $t_{\text {Aut }}$ & $t_{\text {npa }}$ & $f_{A n n}$ & $t_{\text {npa }}$ & $A_{\mathrm{Au}}$ \\
\hline$\partial_{42}$ & $A_{\text {Aut }}$ & $t_{\text {Aut }}$ & $\rightarrow$ & $\rightarrow$ & $\rightarrow_{\mathrm{T}}$ & $\stackrel{?}{\rightarrow}$ & $\rightarrow_{\mathrm{T}}$ & $\rightarrow_{\mathrm{T}}$ & $\rightarrow_{\mathrm{T}}$ & $t_{\text {Aut }}$ & $\not \rightarrow_{\text {Aut }}$ & $A_{\text {Aut }}$ & $\not t_{\text {Aut }}$ & $t_{\text {Aut }}$ & $t_{\text {Aut }}$ & $A_{\text {Aut }}$ & $A_{\text {Aut }}$ & $t_{\mathrm{Au}}$ \\
\hline$J_{34}$ & $f_{\text {Aut }}$ & $t_{\text {Aut }}$ & $f_{\text {Aut }}$ & $t_{\text {Aut }}$ & $t_{\text {Aut }}$ & $t_{\text {Aut }}$ & $t_{\text {Aut }}$ & $\rightarrow_{\oplus}$ & $\rightarrow_{\mathrm{T}}$ & $t_{\text {Aut }}$ & $f_{\text {Aut }}$ & $A_{\text {Aut }}$ & $f_{\text {Aut }}$ & $t_{\text {Aut }}$ & $t_{\text {Aut }}$ & $A_{\text {Aut }}$ & $A_{\text {Aut }}$ & $\not_{\mathrm{Au}}$ \\
\hline$J_{35}$ & $t_{\text {Aut }}$ & $f_{\text {Aut }}$ & $\rightarrow_{\oplus}$ & $f_{M_{N u l}}$ & $\rightarrow_{\mathrm{T}}$ & $A_{A}$ nn & $\rightarrow_{\mathrm{T}}$ & $\rightarrow_{\mathrm{T}}$ & $\rightarrow_{\mathrm{T}}$ & $t_{\text {Aut }}$ & $A_{\text {Aut }}$ & $t_{\text {Aut }}$ & $t_{\text {Aut }}$ & $t_{\text {Aut }}$ & $\nrightarrow_{\text {Aut }}$ & $t_{\text {Aut }}$ & $t_{\text {Aut }}$ & $\not A_{\mathrm{Au}}$ \\
\hline$J_{36}$ & $A_{\text {Aut }}$ & $\not_{\text {Aut }}$ & $A_{\text {Aut }}$ & $\phi_{\text {Aut }}$ & $t_{\text {Aut }}$ & $A_{\text {Aut }}$ & $t_{\text {Aut }}$ & $\rightarrow$ & $\rightarrow_{\mathrm{T}}$ & $t_{\text {Aut }}$ & $t_{\text {Aut }}$ & $t_{\text {Aut }}$ & $t_{\text {Aut }}$ & $t_{\text {Aut }}$ & $\nrightarrow_{\text {Aut }}$ & $A_{\text {Aut }}$ & $A_{\text {Aut }}$ & $A_{\mathrm{Au}}$ \\
\hline$\partial_{37}$ & $t_{\text {Aut }}$ & $f_{\text {Aut }}$ & $\rightarrow$ & $f_{M_{N u l}}$ & $\rightarrow_{\mathrm{T}}$ & $f_{M_{N u l}}$ & $\rightarrow_{\mathrm{T}}$ & $\rightarrow_{\mathrm{T}}$ & $\rightarrow_{\mathrm{T}}$ & $t_{\text {Aut }}$ & $\nrightarrow_{A u t}$ & $t_{A u t}$ & $t_{\text {Aut }}$ & $\nrightarrow_{\text {Aut }}$ & $\nrightarrow_{\text {Aut }}$ & $t_{\text {Aut }}$ & $A_{\text {Aut }}$ & $A_{\mathrm{Au}}$ \\
\hline$J_{19}$ & $A_{\text {Aut }}$ & $t_{A n n}$ & $\rightarrow_{\mathrm{T}}$ & $\rightarrow_{\mathrm{T}}$ & $\rightarrow_{\mathrm{T}}$ & $t_{A n n}$ & $\rightarrow_{\mathrm{T}}$ & $\rightarrow_{\mathrm{T}}$ & $\rightarrow_{\mathrm{T}}$ & $\nrightarrow_{\text {Aut }}$ & $\nrightarrow_{\text {Aut }}$ & $A_{\text {Aut }}$ & $\nrightarrow_{\text {Aut }}$ & $\nrightarrow_{\text {Aut }}$ & $\nrightarrow_{\text {Aut }}$ & $\nrightarrow_{\text {Aut }}$ & $\rightarrow_{\oplus}$ & $A_{A u}$ \\
\hline$\partial_{20}$ & $\rightarrow_{\mathrm{T}}$ & $\rightarrow_{\mathrm{T}}$ & $\rightarrow_{\mathrm{T}}$ & $\rightarrow_{\mathrm{T}}$ & $\rightarrow \mathrm{T}$ & $\rightarrow_{\mathrm{T}}$ & $\rightarrow_{\mathrm{T}}$ & $\rightarrow_{\mathrm{T}}$ & $\rightarrow_{\mathrm{T}}$ & $t_{\mathrm{Aut}}$ & $t_{\text {Aut }}$ & $t_{\text {Aut }}$ & $t_{A n n}$ & $\rightarrow_{\oplus}$ & $\rightarrow \mathrm{T}$ & $t_{\mathrm{Ann}}$ & $\rightarrow_{\mathrm{T}}$ & $\rightarrow_{\oplus}$ \\
\hline$J_{21}$ & $A_{\text {Aut }}$ & $\stackrel{?}{\rightarrow}$ & $\rightarrow_{\mathrm{T}}$ & $\rightarrow_{\mathrm{T}}$ & $\rightarrow_{\mathrm{T}}$ & $\stackrel{?}{\rightarrow}$ & $\rightarrow_{\mathrm{T}}$ & $\rightarrow_{\mathrm{T}}$ & $\rightarrow_{\mathrm{T}}$ & $\nrightarrow_{\text {Aut }}$ & $\not_{\text {Aut }}$ & $A_{\text {Aut }}$ & $\nrightarrow_{\text {Aut }}$ & $t_{\text {Aut }}$ & $\nrightarrow_{\text {Aut }}$ & $\nrightarrow_{\text {Aut }}$ & $\rightarrow_{\oplus}$ & $A_{\mathrm{Au}}$ \\
\hline$f_{22}$ & $\rightarrow_{\mathrm{T}}$ & $\rightarrow_{\mathrm{T}}$ & $\rightarrow_{\mathrm{T}}$ & $\rightarrow_{\mathrm{T}}$ & $\rightarrow_{\mathrm{T}}$ & $\rightarrow_{\mathrm{T}}$ & $\rightarrow_{\mathrm{T}}$ & $\rightarrow_{\mathrm{T}}$ & $\rightarrow_{\mathrm{T}}$ & $f_{\text {Aut }}$ & $f_{\text {Aut }}$ & $A_{\text {Aut }}$ & $\rightarrow$ & $f_{\text {npa }}$ & A $_{\text {npa }}$ & $\rightarrow_{\mathrm{T}}$ & $t_{\text {npa }}$ & $\rightarrow_{\oplus}$ \\
\hline$\partial_{26}$ & $\rightarrow_{\mathrm{T}}$ & $\rightarrow_{\mathrm{T}}$ & $\rightarrow_{\mathrm{T}}$ & $\rightarrow_{\mathrm{T}}$ & $\rightarrow_{\mathrm{T}}$ & $\rightarrow_{\mathrm{T}}$ & $\rightarrow_{\mathrm{T}}$ & $\rightarrow_{\mathrm{T}}$ & $\rightarrow_{\mathrm{T}}$ & $t_{\text {Aut }}$ & $t_{\text {Aut }}$ & $t_{\text {Aut }}$ & $t_{A n n}$ & $\rightarrow_{\oplus}$ & $\rightarrow_{\mathrm{T}}$ & $t_{A n n}$ & $\rightarrow_{\mathrm{T}}$ & $\rightarrow_{\oplus}$ \\
\hline$\partial_{27}$ & $\rightarrow_{\mathrm{T}}$ & $\rightarrow_{\mathrm{T}}$ & $\rightarrow_{\mathrm{T}}$ & $\rightarrow_{\mathrm{T}}$ & $\rightarrow_{\mathrm{T}}$ & $\rightarrow_{\mathrm{T}}$ & $\rightarrow_{\mathrm{T}}$ & $\rightarrow_{\mathrm{T}}$ & $\rightarrow_{\mathrm{T}}$ & $t_{\text {Aut }}$ & $f_{\text {Aut }}$ & $f_{\text {Aut }}$ & $\rightarrow$ & $\rightarrow_{\oplus}$ & $\rightarrow_{\mathrm{T}}$ & $\rightarrow_{\mathrm{T}}$ & $\rightarrow_{\mathrm{T}}$ & $t_{n p a}$ \\
\hline$\partial_{10}$ & $\stackrel{?}{\rightarrow}$ & $\rightarrow_{\mathrm{T}}$ & $\rightarrow_{\mathrm{T}}$ & $\rightarrow_{\mathrm{T}}$ & $\rightarrow_{\mathrm{T}}$ & $\rightarrow_{\mathrm{T}}$ & $\rightarrow_{\mathrm{T}}$ & $\rightarrow_{\mathrm{T}}$ & $\rightarrow_{\mathrm{T}}$ & $t_{\text {Aut }}$ & $t_{\text {Aut }}$ & $t_{\text {Aut }}$ & $t_{\text {Aut }}$ & $t_{\text {Aut }}$ & $\rightarrow_{\oplus}$ & $t_{\mathrm{Ann}}$ & $\rightarrow_{\mathrm{T}}$ & $\nrightarrow_{\mathrm{Au}}$ \\
\hline$\partial_{11}$ & $\stackrel{?}{\rightarrow}$ & $\rightarrow$ & $\rightarrow_{\mathrm{T}}$ & $\rightarrow_{\mathrm{T}}$ & $\rightarrow_{\mathrm{T}}$ & $\rightarrow_{\mathrm{T}}$ & $\rightarrow_{\mathrm{T}}$ & $\rightarrow_{\mathrm{T}}$ & $\rightarrow_{\mathrm{T}}$ & $t_{\text {Aut }}$ & $t_{\text {Aut }}$ & $\not_{\text {Aut }}$ & $\nrightarrow_{\text {Aut }}$ & $\nrightarrow_{\text {Aut }}$ & $t_{\text {npa }}$ & $t_{\mathrm{Ann}}$ & $t_{\text {npa }}$ & $t_{\mathrm{Au}}$ \\
\hline
\end{tabular}




\begin{tabular}{|c|c|c|c|c|c|c|c|c|c|c|c|c|c|c|c|c|c|c|}
\hline & $J_{61}$ & $J_{66}$ & $J_{67}$ & $J_{68}$ & $\mathrm{~J}_{69}$ & $\partial_{70}$ & $J_{71}$ & $\mathcal{J}_{72}$ & $\mathcal{J}_{73}$ & $f_{3}$ & $J_{4}$ & $J_{5}$ & $f_{39}$ & $J_{38}$ & $f_{41}$ & $\partial_{43}$ & $J_{40}$ & $f_{47}$ \\
\hline$J_{12}$ & $\nrightarrow_{A \text { Aut }}$ & $t_{\text {Aut }}$ & $\not_{\text {Aut }}$ & $\nexists_{\text {Aut }}$ & $\stackrel{?}{\rightarrow}$ & $\stackrel{?}{\rightarrow}$ & $\rightarrow_{\mathrm{T}}$ & $\rightarrow_{\mathrm{T}}$ & $\rightarrow_{\mathrm{T}}$ & $\nrightarrow_{\text {Aut }}$ & $\nexists_{\text {Aut }}$ & $\Rightarrow_{\text {Aut }}$ & $\not_{\text {Aut }}$ & $\nrightarrow_{\text {Aut }}$ & $\nrightarrow_{\text {Aut }}$ & $\nrightarrow_{\text {Aut }}$ & $\nexists_{\text {Aut }}$ & $f_{\text {Aut }}$ \\
\hline$J_{13}$ & $f_{\text {Aut }}$ & $\stackrel{?}{\rightarrow}$ & $\stackrel{?}{\rightarrow}$ & $\stackrel{?}{\rightarrow}$ & $\rightarrow_{\mathrm{T}}$ & $\stackrel{?}{\rightarrow}$ & $\rightarrow_{\mathrm{T}}$ & $\rightarrow_{\mathrm{T}}$ & $\rightarrow_{\mathrm{T}}$ & $\not_{\text {Aut }}$ & $t_{\text {Aut }}$ & $\not_{\text {Aut }}$ & $f_{\text {Aut }}$ & $\neg_{\text {Aut }}$ & $\phi_{\text {Aut }}$ & $\not_{\text {Aut }}$ & $t_{\text {npa }}$ & $f_{\text {Aut }}$ \\
\hline$\partial_{14}$ & $\stackrel{?}{\rightarrow}$ & $\rightarrow_{\mathrm{T}}$ & $\rightarrow_{\mathrm{T}}$ & $\rightarrow_{\mathrm{T}}$ & $\rightarrow_{\mathrm{T}}$ & $\rightarrow_{\mathrm{T}}$ & $\rightarrow_{\mathrm{T}}$ & $\rightarrow_{\mathrm{T}}$ & $\rightarrow_{\mathrm{T}}$ & $\nrightarrow_{\text {Aut }}$ & $f_{\text {Aut }}$ & $t_{A \mathrm{Aut}}$ & $t_{\text {Aut }}$ & $t_{A_{\text {Aut }}}$ & $\rightarrow_{\oplus}$ & $t_{1}$ & $\rightarrow_{\mathrm{T}}$ & $f_{\text {Aut }}$ \\
\hline$J_{15}$ & $\stackrel{?}{\rightarrow}$ & $\stackrel{?}{\rightarrow}$ & $\rightarrow_{\mathrm{T}}$ & $\stackrel{?}{\rightarrow}$ & $\rightarrow_{\mathrm{T}}$ & $\stackrel{?}{\rightarrow}$ & $\rightarrow_{\mathrm{T}}$ & $\rightarrow_{\mathrm{T}}$ & $\rightarrow_{\mathrm{T}}$ & $f_{\text {Aut }}$ & $\nrightarrow_{\text {Aut }}$ & $\not_{\text {Aut }}$ & $\nrightarrow_{\text {Aut }}$ & $A_{\text {Aut }}$ & $f_{\text {npa }}$ & $\not_{1}$ & $f_{\text {npa }}$ & $f_{\text {Aut }}$ \\
\hline$J_{16}$ & $f_{\text {Aut }}$ & $\stackrel{?}{\rightarrow}$ & $\stackrel{?}{\rightarrow}$ & $\stackrel{?}{\rightarrow}$ & $\rightarrow_{\mathrm{T}}$ & $\stackrel{?}{\rightarrow}$ & $\rightarrow_{\mathrm{T}}$ & $\rightarrow_{\mathrm{T}}$ & $\rightarrow_{\mathrm{T}}$ & $f_{\mathrm{Aut}}$ & $f_{\mathrm{Aut}}$ & $\Rightarrow_{\mathrm{Aut}}$ & $t_{\mathrm{Aut}}$ & $f_{\mathrm{Aut}}$ & $t_{\text {Aut }}$ & $f_{\text {Aut }}$ & $t_{\text {npa }}$ & $f_{\text {Aut }}$ \\
\hline$\partial_{17}$ & $\stackrel{?}{\rightarrow}$ & $\rightarrow_{\mathrm{T}}$ & $\rightarrow_{\mathrm{T}}$ & $\rightarrow_{\mathrm{T}}$ & $\rightarrow_{\mathrm{T}}$ & $\rightarrow_{\mathrm{T}}$ & $\rightarrow_{\mathrm{T}}$ & $\rightarrow_{\mathrm{T}}$ & $\rightarrow_{\mathrm{T}}$ & $f_{\text {Aut }}$ & $t_{\text {Aut }}$ & $\not_{\mathrm{Aut}}$ & $\nrightarrow_{\text {Aut }}$ & $f_{\text {Aut }}$ & $f_{\text {npa }}$ & $\rightarrow$ & $t_{\text {npa }}$ & $f_{\text {Aut }}$ \\
\hline$\partial_{18}$ & $t_{\mathrm{Aut}}$ & $t_{\text {Aut }}$ & $t_{\text {Aut }}$ & $t_{\text {Aut }}$ & $\stackrel{?}{\rightarrow}$ & $\stackrel{?}{\rightarrow}$ & $\rightarrow_{\mathrm{T}}$ & $\rightarrow_{\mathrm{T}}$ & $\rightarrow_{\mathrm{T}}$ & $f_{\text {Aut }}$ & $t_{\text {Aut }}$ & $\not \not_{\mathrm{Aut}}$ & $f_{\text {Aut }}$ & $\nrightarrow_{\text {Aut }}$ & $f_{\text {Aut }}$ & $\nrightarrow_{\text {Aut }}$ & $\not_{\text {Aut }}$ & $f_{\text {Aut }}$ \\
\hline$\partial_{23}$ & $\stackrel{?}{\rightarrow}$ & $\rightarrow_{\mathrm{T}}$ & $\rightarrow_{\mathrm{T}}$ & $\rightarrow_{\mathrm{T}}$ & $\rightarrow_{\mathrm{T}}$ & $\rightarrow_{\mathrm{T}}$ & $\rightarrow_{\mathrm{T}}$ & $\rightarrow_{\mathrm{T}}$ & $\rightarrow_{\mathrm{T}}$ & $t_{\text {Aut }}$ & $t_{\text {Aut }}$ & $t_{A \mathrm{Aut}}$ & $t_{\text {Ann }}$ & $\stackrel{?}{\rightarrow}$ & $\rightarrow_{\mathrm{T}}$ & $t_{\mathrm{Ann}}$ & $\rightarrow_{\mathrm{T}}$ & $f_{\text {npa }}$ \\
\hline$J_{24}$ & $\stackrel{?}{\rightarrow}$ & $\rightarrow_{\mathrm{T}}$ & $\rightarrow_{\mathrm{T}}$ & $\rightarrow_{\mathrm{T}}$ & $\rightarrow_{\mathrm{T}}$ & $\rightarrow_{\mathrm{T}}$ & $\rightarrow_{\mathrm{T}}$ & $\rightarrow_{\mathrm{T}}$ & $\rightarrow_{\mathrm{T}}$ & $\nexists_{\text {Aut }}$ & $\nrightarrow_{\text {Aut }}$ & $\not \Rightarrow_{\text {Aut }}$ & $t_{1}$ & $\stackrel{?}{\rightarrow}$ & $\rightarrow_{\mathrm{T}}$ & $t_{1}$ & $\rightarrow_{\mathrm{T}}$ & $f_{\text {npa }}$ \\
\hline$J_{25}$ & $\stackrel{?}{\rightarrow}$ & $\rightarrow_{\mathrm{T}}$ & $\rightarrow_{\mathrm{T}}$ & $\rightarrow_{\mathrm{T}}$ & $\rightarrow_{\mathrm{T}}$ & $\rightarrow_{\mathrm{T}}$ & $\rightarrow_{\mathrm{T}}$ & $\rightarrow_{\mathrm{T}}$ & $\rightarrow_{\mathrm{T}}$ & $\nrightarrow_{\text {Aut }}$ & $\nrightarrow_{\text {Aut }}$ & $\nrightarrow_{\text {Aut }}$ & $\stackrel{?}{\rightarrow}$ & $A_{\text {npa }}$ & $A_{\text {npa }}$ & $\rightarrow_{\mathrm{T}}$ & $A_{\text {npa }}$ & $t_{\text {npa }}$ \\
\hline$J_{28}$ & $f_{\text {Aut }}$ & $f_{\text {Aut }}$ & $\not_{\text {Aut }}$ & $f_{\text {Aut }}$ & $\rightarrow$ & $f_{A n n}$ & $\rightarrow_{\mathrm{T}}$ & $\rightarrow_{\mathrm{T}}$ & $\rightarrow_{\mathrm{T}}$ & $f_{\text {Aut }}$ & $f_{\text {Aut }}$ & $f_{\text {Aut }}$ & $f_{\text {Aut }}$ & $f_{\text {Aut }}$ & $f_{\text {Aut }}$ & $f_{\text {Aut }}$ & $f_{A \text { Aut }}$ & $\not \Rightarrow_{\text {Aut }}$ \\
\hline$J_{29}$ & $f_{\text {Aut }}$ & $f_{M_{N}}$ & $\rightarrow_{\mathrm{T}}$ & $A_{M_{\mathrm{Nul}}}$ & $\rightarrow_{\mathrm{T}}$ & $\not \rightarrow_{M_{\mathrm{Nul}}}$ & $\rightarrow_{\mathrm{T}}$ & $\rightarrow_{\mathrm{T}}$ & $\rightarrow_{\mathrm{T}}$ & $f_{\text {Aut }}$ & $f_{\text {Aut }}$ & $\not \Rightarrow_{\text {Aut }}$ & $\not f_{\text {Aut }}$ & $\Rightarrow_{\text {Aut }}$ & $\Rightarrow_{\text {Aut }}$ & $\Rightarrow_{\text {Aut }}$ & $\not A_{M_{N W}}$ & $\not_{\text {Aut }}$ \\
\hline$J_{30}$ & $f_{\text {Aut }}$ & $f_{\text {Aut }}$ & $f_{\text {Aut }}$ & $f_{A}$ Aut & $f_{\text {Aut }}$ & $f_{\mathrm{Aut}}$ & $\rightarrow$ & $\rightarrow_{\mathrm{T}}$ & $\rightarrow_{\mathrm{T}}$ & $f_{\text {Aut }}$ & $f_{\text {Aut }}$ & $f_{\text {Aut }}$ & $f_{\text {Aut }}$ & $f_{\text {Aut }}$ & $f_{\text {Aut }}$ & $f_{\text {Aut }}$ & $f_{\text {Aut }}$ & $\not A_{\text {Aut }}$ \\
\hline$J_{31}$ & $f_{\text {Aut }}$ & $f_{\text {Aut }}$ & $f_{\text {Aut }}$ & $f_{\text {Aut }}$ & $\rightarrow$ & $f_{M_{N u l}}$ & $\rightarrow_{\mathrm{T}}$ & $\rightarrow_{\mathrm{T}}$ & $\rightarrow_{\mathrm{T}}$ & $f_{\text {Aut }}$ & $f_{\text {Aut }}$ & $f_{A}$ Aut & $f_{\text {Aut }}$ & $f_{\text {Aut }}$ & $f_{\text {Aut }}$ & $f_{\text {Aut }}$ & $f_{A \text { Aut }}$ & $f_{\text {Aut }}$ \\
\hline$\partial_{32}$ & $f_{\text {Aut }}$ & $f_{\text {Aut }}$ & $f_{\text {Aut }}$ & $f_{\text {Aut }}$ & $f_{\text {Aut }}$ & $f_{\mathrm{Aut}}$ & $\rightarrow$ & $\rightarrow_{\mathrm{T}}$ & $\rightarrow_{\mathrm{T}}$ & $f_{\text {Aut }}$ & $f_{\text {Aut }}$ & $\not_{\text {Aut }}$ & $f_{\text {Aut }}$ & $f_{\text {Aut }}$ & $f_{\text {Aut }}$ & $f_{\text {Aut }}$ & $\not_{\text {Aut }}$ & $\not \nexists_{\text {Aut }}$ \\
\hline$J_{33}$ & $f_{\text {Aut }}$ & $f_{\text {Aut }}$ & $\not_{\text {Aut }}$ & $f_{\text {Aut }}$ & $f_{\text {Aut }}$ & $f_{\text {Aut }}$ & $f_{\text {Aut }}$ & $f_{\text {Aut }}$ & $\rightarrow$ & $f_{\text {Aut }}$ & $f_{\text {Aut }}$ & $f_{A \text { Aut }}$ & $f_{\text {Aut }}$ & $f_{\text {Aut }}$ & $f_{\text {Aut }}$ & $f_{\text {Aut }}$ & $f_{\text {Aut }}$ & $f_{\text {Aut }}$ \\
\hline$\partial_{51}$ & $\stackrel{?}{\rightarrow}$ & $\stackrel{?}{\rightarrow}$ & $\rightarrow_{\mathrm{T}}$ & $\rightarrow$ & $\rightarrow_{\mathrm{T}}$ & $\rightarrow$ & $\rightarrow_{\mathrm{T}}$ & $\rightarrow_{\mathrm{T}}$ & $\rightarrow_{\mathrm{T}}$ & $A_{\text {Aut }}$ & $\nrightarrow_{\text {Aut }}$ & $\not_{\text {Aut }}$ & $\phi_{\text {Aut }}$ & $\nrightarrow_{\text {Aut }}$ & $A_{\text {npa }}$ & $\not_{A n n}$ & $A_{\text {npa }}$ & $f_{\text {Aut }}$ \\
\hline$\partial_{52}$ & $\stackrel{?}{\rightarrow}$ & $\stackrel{?}{\rightarrow-}$ & $\rightarrow_{\mathrm{T}}$ & $\stackrel{?}{\rightarrow-}$ & $\rightarrow_{\mathrm{T}}$ & $\rightarrow$ & $\rightarrow_{\mathrm{T}}$ & $\rightarrow_{\mathrm{T}}$ & $\rightarrow_{\mathrm{T}}$ & $f_{\text {Aut }}$ & $\nrightarrow_{\text {Aut }}$ & $\not \not_{\text {Aut }}$ & $\not_{\text {Aut }}$ & $\not_{\mathrm{Aut}}$ & $A_{\text {npa }}$ & $f_{\mathrm{Ann}}$ & $t_{\text {npa }}$ & $f_{\text {Aut }}$ \\
\hline$\partial_{53}$ & $\stackrel{?}{\rightarrow-}$ & $\rightarrow_{\mathrm{T}}$ & $\rightarrow_{\mathrm{T}}$ & $\rightarrow_{\mathrm{T}}$ & $\rightarrow_{\mathrm{T}}$ & $\rightarrow_{\mathrm{T}}$ & $\rightarrow_{\mathrm{T}}$ & $\rightarrow_{\mathrm{T}}$ & $\rightarrow_{\mathrm{T}}$ & $f_{\mathrm{Aut}}$ & $f_{\mathrm{Aut}}$ & $\Rightarrow_{\mathrm{Aut}}$ & $t_{\mathrm{Ann}}$ & $f_{\text {npa }}$ & $f_{\text {npa }}$ & $f_{\mathrm{Ann}}$ & $A_{\text {npa }}$ & $f_{\text {npa }}$ \\
\hline$\partial_{44}$ & $t_{\text {Aut }}$ & $f_{\mathrm{Ann}}$ & $\stackrel{?}{\rightarrow}$ & $\stackrel{?}{\rightarrow}$ & $\rightarrow_{\mathrm{T}}$ & $f_{\text {Ann }}$ & $\rightarrow_{\mathrm{T}}$ & $\rightarrow_{\mathrm{T}}$ & $\rightarrow_{\mathrm{T}}$ & $f_{\text {Aut }}$ & $f_{\text {Aut }}$ & $\not_{\mathrm{Aut}}$ & $f_{\text {Aut }}$ & $f_{\text {Aut }}$ & $f_{\text {Aut }}$ & $f_{\text {Aut }}$ & $t_{\text {npa }}$ & $f_{\text {Aut }}$ \\
\hline$\partial_{45}$ & $\stackrel{?}{\rightarrow}$ & $\stackrel{?}{\rightarrow}$ & $\stackrel{?}{\rightarrow}$ & $\stackrel{?}{\rightarrow}$ & $\rightarrow_{\mathrm{T}}$ & $\stackrel{?}{\rightarrow}$ & $\rightarrow_{\mathrm{T}}$ & $\rightarrow_{\mathrm{T}}$ & $\rightarrow_{\mathrm{T}}$ & $\Rightarrow_{\text {Aut }}$ & $\Rightarrow_{\text {Aut }}$ & $t_{\mathrm{Aut}}$ & $\Rightarrow_{\text {Aut }}$ & $t_{\text {Aut }}$ & $A_{\text {npa }}$ & $t_{\mathrm{Ann}}$ & $t_{\text {npa }}$ & $f_{\text {Aut }}$ \\
\hline$\partial_{46}$ & $f_{\text {Aut }}$ & $\stackrel{?}{\rightarrow}$ & $\stackrel{?}{\rightarrow}$ & $\stackrel{?}{\rightarrow-}$ & $\rightarrow_{\mathrm{T}}$ & $\stackrel{?}{\rightarrow}$ & $\rightarrow_{\mathrm{T}}$ & $\rightarrow_{\mathrm{T}}$ & $\rightarrow_{\mathrm{T}}$ & $\nrightarrow_{\text {Aut }}$ & $f_{\text {Aut }}$ & $\not_{\text {Aut }}$ & $\rightarrow_{\text {Aut }}$ & $\not_{\text {Aut }}$ & $\nrightarrow_{\text {Aut }}$ & $\Rightarrow_{\text {Aut }}$ & $t_{\text {npa }}$ & $f_{\text {Aut }}$ \\
\hline$\partial_{55}$ & $f_{\text {Aut }}$ & $\stackrel{?}{\rightarrow}$ & $\stackrel{?}{\rightarrow}$ & $\stackrel{?}{\rightarrow}$ & $\rightarrow_{\mathrm{T}}$ & $\stackrel{?}{\rightarrow}$ & $\rightarrow_{\mathrm{T}}$ & $\rightarrow_{\mathrm{T}}$ & $\rightarrow_{\mathrm{T}}$ & $\Rightarrow_{\text {Aut }}$ & $f_{\text {Aut }}$ & $\not \Rightarrow_{\mathrm{Aut}}$ & $\not_{\text {Aut }}$ & $\not_{\text {Aut }}$ & $\nexists_{\text {Aut }}$ & $\Rightarrow_{\text {Aut }}$ & $t_{\text {npa }}$ & $A_{\text {Aut }}$ \\
\hline$\partial_{56}$ & $t_{\text {Aut }}$ & $\stackrel{?}{\rightarrow}$ & $\stackrel{?}{\rightarrow}$ & $\stackrel{?}{\rightarrow}$ & $\rightarrow_{\mathrm{T}}$ & $\stackrel{?}{\rightarrow}$ & $\rightarrow_{\mathrm{T}}$ & $\rightarrow_{\mathrm{T}}$ & $\rightarrow_{\mathrm{T}}$ & $t_{\text {Aut }}$ & $t_{\text {Aut }}$ & $t_{A \text { Aut }}$ & $\nrightarrow_{\text {Aut }}$ & $A_{\text {Aut }}$ & $\nrightarrow_{\text {Aut }}$ & $\nrightarrow_{\text {Aut }}$ & $t_{\text {npa }}$ & $A_{\text {Aut }}$ \\
\hline$\partial_{57}$ & $\stackrel{?}{\rightarrow}$ & $\stackrel{?}{\rightarrow}$ & $\stackrel{?}{\rightarrow}$ & $\stackrel{?}{\rightarrow}$ & $\rightarrow_{\mathrm{T}}$ & $\stackrel{?}{\rightarrow}$ & $\rightarrow_{\mathrm{T}}$ & $\rightarrow_{\mathrm{T}}$ & $\rightarrow_{\mathrm{T}}$ & $f_{\text {Aut }}$ & $f_{\text {Aut }}$ & $f_{\mathrm{Aut}}$ & $f_{\text {Aut }}$ & $f_{\text {Aut }}$ & $f_{\text {npa }}$ & $t_{1}$ & $t_{\text {npa }}$ & $f_{\text {Aut }}$ \\
\hline$\partial_{48}$ & $A_{\text {Aut }}$ & $\nrightarrow_{\text {Aut }}$ & $\stackrel{?}{\rightarrow}$ & $\stackrel{?}{\rightarrow}$ & $\stackrel{?}{\rightarrow}$ & $\stackrel{?}{\rightarrow}$ & $\rightarrow_{\mathrm{T}}$ & $\rightarrow_{\mathrm{T}}$ & $\rightarrow_{\mathrm{T}}$ & $\nrightarrow_{\text {Aut }}$ & $t_{\text {Aut }}$ & $t_{\mathrm{Aut}}$ & $\nrightarrow_{\text {Aut }}$ & $t_{\text {Aut }}$ & $\nrightarrow_{\text {Aut }}$ & $\nrightarrow_{\text {Aut }}$ & $t_{\text {Aut }}$ & $t_{\mathrm{Aut}}$ \\
\hline$\partial_{49}$ & $f_{\text {Aut }}$ & $\stackrel{?}{\rightarrow}$ & $\stackrel{?}{\rightarrow}$ & $\stackrel{?}{\rightarrow \rightarrow}$ & $\stackrel{?}{\rightarrow}$ & $\stackrel{?}{\rightarrow}$ & $\rightarrow_{\mathrm{T}}$ & $\rightarrow_{\mathrm{T}}$ & $\rightarrow_{\mathrm{T}}$ & $\Rightarrow_{\text {Aut }}$ & $f_{\text {Aut }}$ & $\not \Rightarrow_{\mathrm{Aut}}$ & $\Rightarrow_{\text {Aut }}$ & $\not \Rightarrow_{\text {Aut }}$ & $\nrightarrow_{\text {Aut }}$ & $\nrightarrow_{\text {Aut }}$ & $t_{\text {npa }}$ & $A_{\mathrm{Aut}}$ \\
\hline$\partial_{50}$ & $f_{\text {Aut }}$ & $\Rightarrow_{\text {Aut }}$ & $\stackrel{?}{\rightarrow-}$ & $\stackrel{?}{\rightarrow}$ & $\stackrel{?}{\rightarrow}$ & $\stackrel{?}{\rightarrow}$ & $\rightarrow_{\mathrm{T}}$ & $\rightarrow_{\mathrm{T}}$ & $\rightarrow_{\mathrm{T}}$ & $\Rightarrow_{\text {Aut }}$ & $f_{\text {Aut }}$ & $\not \Rightarrow_{\mathrm{Aut}}$ & $\nexists_{\text {Aut }}$ & $\not_{\text {Aut }}$ & $\nexists_{\text {Aut }}$ & $\Rightarrow_{\text {Aut }}$ & $\not_{\text {Aut }}$ & $A_{\mathrm{Aut}}$ \\
\hline$\partial_{58}$ & $\not_{\text {Aut }}$ & $t_{\text {Aut }}$ & $\stackrel{?}{\rightarrow}$ & $\stackrel{?}{\rightarrow}$ & $\stackrel{?}{\rightarrow}$ & $\stackrel{?}{\rightarrow}$ & $\rightarrow_{\mathrm{T}}$ & $\rightarrow_{\mathrm{T}}$ & $\rightarrow_{\mathrm{T}}$ & $A_{\text {Aut }}$ & $t_{\text {Aut }}$ & $t_{\mathrm{Aut}}$ & $\nrightarrow_{\text {Aut }}$ & $f_{\text {Aut }}$ & $\nrightarrow_{\text {Aut }}$ & $t_{\mathrm{Aut}}$ & $t_{\text {Aut }}$ & $A_{\text {Aut }}$ \\
\hline
\end{tabular}




\begin{tabular}{|c|c|c|c|c|c|c|c|c|c|c|c|c|c|c|c|c|c|c|}
\hline & $\partial_{61}$ & $\partial_{66}$ & $\partial_{67}$ & $\partial_{68}$ & $\partial_{69}$ & $\partial_{70}$ & $\partial_{71}$ & $\partial_{72}$ & $\partial_{73}$ & $\partial_{3}$ & $\partial_{4}$ & $\partial_{5}$ & $J_{39}$ & $\partial_{38}$ & $\partial_{41}$ & $\partial_{43}$ & $\partial_{40}$ & $\partial_{47}$ \\
\hline$J_{59}$ & $\not_{\text {Aut }}$ & $\stackrel{?}{\rightarrow}$ & $\stackrel{?}{\rightarrow}$ & $\stackrel{?}{\rightarrow}$ & $\stackrel{?}{\rightarrow}$ & $\stackrel{?}{\rightarrow}$ & $\rightarrow_{\mathrm{T}}$ & $\rightarrow_{\mathrm{T}}$ & $\rightarrow_{\mathrm{T}}$ & $\not A_{\text {Aut }}$ & $\not_{\text {Aut }}$ & $\not_{\text {Aut }}$ & $A_{\text {Aut }}$ & $\nrightarrow_{\text {Aut }}$ & $\not_{\text {Aut }}$ & $\nexists_{\text {Aut }}$ & $A_{\text {npa }}$ & $t_{\text {Aut }}$ \\
\hline$\partial_{60}$ & $t_{\text {Aut }}$ & $f_{\text {Aut }}$ & $\stackrel{?}{\rightarrow}$ & $\stackrel{?}{\rightarrow}$ & $\stackrel{?}{\rightarrow}$ & $\stackrel{?}{\rightarrow}$ & $\rightarrow_{\mathrm{T}}$ & $\rightarrow_{\mathrm{T}}$ & $\rightarrow_{\mathrm{T}}$ & $A_{\text {Aut }}$ & $\nrightarrow_{\text {Aut }}$ & $t_{\text {Aut }}$ & $\nrightarrow_{\text {Aut }}$ & $\rightarrow_{\text {Aut }}$ & $t_{\text {Aut }}$ & $\nrightarrow_{\text {Aut }}$ & $\not A_{\text {Aut }}$ & $f_{\text {Aut }}$ \\
\hline$\partial_{6}$ & $\stackrel{?}{\rightarrow} \rightarrow$ & $\rightarrow_{\mathrm{T}}$ & $\rightarrow \mathrm{T}$ & $\rightarrow \mathrm{T}$ & $\rightarrow_{\mathrm{T}}$ & $\rightarrow_{\mathrm{T}}$ & $\rightarrow_{\mathrm{T}}$ & $\rightarrow_{\mathrm{T}}$ & $\rightarrow_{\mathrm{T}}$ & $\not \rightarrow_{\text {Aut }}$ & $\not_{\text {Aut }}$ & $f_{\text {Aut }}$ & $t_{1}$ & $\stackrel{?}{\rightarrow}$ & $\rightarrow_{\mathrm{T}}$ & $t_{1}$ & $\rightarrow_{\mathrm{T}}$ & $\stackrel{?}{\rightarrow}$ \\
\hline$\partial_{7}$ & $\stackrel{?}{-\rightarrow}$ & $\rightarrow_{\mathrm{T}}$ & $\rightarrow_{\mathrm{T}}$ & $\rightarrow_{\mathrm{T}}$ & $\rightarrow_{\mathrm{T}}$ & $\rightarrow_{\mathrm{T}}$ & $\rightarrow_{\mathrm{T}}$ & $\rightarrow_{\mathrm{T}}$ & $\rightarrow_{\mathrm{T}}$ & $A_{\text {Aut }}$ & $\not A_{\text {Aut }}$ & $f_{\text {Aut }}$ & $\stackrel{?}{\rightarrow}$ & $\stackrel{?}{\rightarrow}$ & $\rightarrow_{\mathrm{T}}$ & $\rightarrow_{\mathrm{T}}$ & $\rightarrow_{\mathrm{T}}$ & $t_{\text {npa }}$ \\
\hline$\partial_{8}$ & $\stackrel{?}{\rightarrow}$ & $\rightarrow_{\mathrm{T}}$ & $\rightarrow_{\mathrm{T}}$ & $\rightarrow_{\mathrm{T}}$ & $\rightarrow_{\mathrm{T}}$ & $\rightarrow_{\mathrm{T}}$ & $\rightarrow_{\mathrm{T}}$ & $\rightarrow_{\mathrm{T}}$ & $\rightarrow_{\mathrm{T}}$ & $A_{\text {Aut }}$ & $t_{\text {Aut }}$ & $t_{\text {Aut }}$ & $t_{\text {Ann }}$ & $t_{\text {npa }}$ & $t_{\text {npa }}$ & $\not_{\text {Ann }}$ & $A_{\text {npa }}$ & $A_{\text {npa }}$ \\
\hline$\partial_{9}$ & $t_{\text {Aut }}$ & $\stackrel{?}{\rightarrow}$ & $\stackrel{?}{\rightarrow}$ & $\stackrel{?}{\rightarrow}$ & $\stackrel{?}{\rightarrow}$ & $\stackrel{?}{\rightarrow}$ & $\rightarrow_{\mathrm{T}}$ & $\rightarrow_{\mathrm{T}}$ & $\rightarrow_{\mathrm{T}}$ & $A_{\text {Aut }}$ & $t_{\text {Aut }}$ & $t_{\text {Aut }}$ & $t_{\text {Aut }}$ & $t_{\text {Aut }}$ & $t_{\text {Aut }}$ & $\not_{\text {Aut }}$ & $A_{\text {npa }}$ & $A_{\text {Aut }}$ \\
\hline$\partial_{1}$ & $\stackrel{?}{\rightarrow}$ & $\rightarrow_{\mathrm{T}}$ & $\rightarrow_{\mathrm{T}}$ & $\rightarrow_{\mathrm{T}}$ & $\rightarrow_{\mathrm{T}}$ & $\rightarrow_{\mathrm{T}}$ & $\rightarrow_{\mathrm{T}}$ & $\rightarrow_{\mathrm{T}}$ & $\rightarrow_{\mathrm{T}}$ & $\nrightarrow_{\text {Aut }}$ & $\nrightarrow_{\text {Aut }}$ & $f_{\text {Aut }}$ & $\stackrel{?}{\rightarrow}$ & $\stackrel{?}{-\rightarrow}$ & $\rightarrow_{\mathrm{T}}$ & $\rightarrow_{\mathrm{T}}$ & $\rightarrow_{\mathrm{T}}$ & $A_{\text {npa }}$ \\
\hline$\partial_{2}$ & $\stackrel{?}{\rightarrow}$ & $\stackrel{?}{\rightarrow}$ & $\stackrel{?}{\rightarrow}$ & $\stackrel{?}{\rightarrow} \rightarrow$ & $\stackrel{?}{\rightarrow}$ & $\stackrel{?}{\rightarrow}$ & $\rightarrow_{\mathrm{T}}$ & $\rightarrow_{\mathrm{T}}$ & $\rightarrow_{\mathrm{T}}$ & $t_{\text {Aut }}$ & $\not_{\text {Aut }}$ & $f_{\text {Aut }}$ & $t_{\text {Aut }}$ & $t_{\text {Aut }}$ & $t_{\text {npa }}$ & $\stackrel{?}{\rightarrow} \rightarrow$ & $f_{\text {npa }}$ & $f_{\text {Aut }}$ \\
\hline$\partial_{62}$ & $t_{g^{2}}$ & $\rightarrow_{\mathrm{T}}$ & $\rightarrow_{\mathrm{T}}$ & $\rightarrow_{\mathrm{T}}$ & $\rightarrow_{\mathrm{T}}$ & $\rightarrow_{\mathrm{T}}$ & $\rightarrow_{\mathrm{T}}$ & $\rightarrow_{\mathrm{T}}$ & $\rightarrow_{\mathrm{T}}$ & $f_{\mathrm{Rad}}$ & $f_{\mathrm{Rad}}$ & $f_{\mathrm{Rad}}$ & $t_{\mathrm{Rad}}$ & $f_{\mathrm{Rad}}$ & $f_{\mathrm{Rad}}$ & $f_{\mathrm{Rad}}$ & $t_{\mathrm{Rad}}$ & $t_{\mathrm{Rad}}$ \\
\hline$\partial_{63}$ & $f_{\text {Aut }}$ & $\rightarrow_{\mathrm{Anc}}$ & $\rightarrow_{\mathrm{T}}$ & $\rightarrow_{\mathrm{T}}$ & $\rightarrow_{\mathrm{T}}$ & $\rightarrow_{\mathrm{T}}$ & $\rightarrow_{\mathrm{T}}$ & $\rightarrow_{\mathrm{T}}$ & $\rightarrow_{\mathrm{T}}$ & $f_{\text {Rad }}$ & $A_{\mathrm{Rad}}$ & $f_{\mathrm{Rad}}$ & $t_{\mathrm{Rad}}$ & $t_{\mathrm{Rad}}$ & $t_{\mathrm{Rad}}$ & $t_{\mathrm{Rad}}$ & $f_{\mathrm{Rad}}$ & $t_{\mathrm{Rad}}$ \\
\hline$\partial_{64}$ & $f_{\text {Aut }}$ & $f_{\text {Aut }}$ & $\rightarrow_{\mathrm{Anc}}$ & $f_{M_{N}}$ & $\rightarrow_{\mathrm{T}}$ & $t_{M_{N u 1}}$ & $\rightarrow \mathrm{T}$ & $\rightarrow_{\mathrm{T}}$ & $\rightarrow_{\mathrm{T}}$ & $t_{\mathrm{Rad}}$ & $A_{\mathrm{Rad}}$ & $f_{\mathrm{Rad}}$ & $t_{\mathrm{Rad}}$ & $t_{\mathrm{Rad}}$ & $f_{\mathrm{Rad}}$ & $f_{\mathrm{Rad}}$ & $f_{\mathrm{Rad}}$ & $t_{\mathrm{Rad}}$ \\
\hline$J_{65}$ & $t_{\text {Aut }}$ & $\rightarrow_{\mathrm{Anc}}$ & $\rightarrow_{\mathrm{T}}$ & $\rightarrow_{\mathrm{T}}$ & $\rightarrow_{\mathrm{T}}$ & $\rightarrow_{\mathrm{T}}$ & $\rightarrow_{\mathrm{T}}$ & $\rightarrow_{\mathrm{T}}$ & $\rightarrow_{\mathrm{T}}$ & $t_{\mathrm{Rad}}$ & $t_{\mathrm{Rad}}$ & $f_{\mathrm{Rad}}$ & $t_{\mathrm{Rad}}$ & $f_{\mathrm{Rad}}$ & $\not f_{\mathrm{Rad}}$ & $f_{\mathrm{Rad}}$ & $t_{\mathrm{Rad}}$ & $t_{\mathrm{Rad}}$ \\
\hline
\end{tabular}

Tabela 5.4: Existência de deformação em $\mathrm{Jor}_{4}$

\begin{tabular}{|c|c|c|c|c|c|c|c|c|c|c|c|c|c|c|c|c|c|c|}
\hline & $J_{54}$ & $J_{42}$ & $\partial_{34}$ & $J_{35}$ & $J_{36}$ & $J_{37}$ & $J_{19}$ & $f_{20}$ & $\partial_{21}$ & $J_{22}$ & $f_{26}$ & $J_{27}$ & $J_{10}$ & $\partial_{11}$ & $f_{12}$ & $\mathcal{J}_{13}$ & $\partial_{14}$ & $J_{15}$ \\
\hline$\partial_{61}$ & $f_{\text {Rad }}$ & $f_{\mathrm{Rad}}$ & $f_{\text {Rad }}$ & $f_{\mathrm{Rad}}$ & $f_{\mathrm{Rad}}$ & $f_{\mathrm{Rad}}$ & $f_{\text {Rad }}$ & $f_{\mathrm{Rad}}$ & $f_{\mathrm{Rad}}$ & $f_{\mathrm{Rad}}$ & $A_{\mathrm{Rad}}$ & $f_{\mathrm{Rad}}$ & $\not A_{\text {Ass }}$ & $\not_{\text {Ass }}$ & $t_{\text {Ass }}$ & $f_{\text {Ass }}$ & $t_{A}$ Ass & $\not \rightarrow_{\text {Ass }}$ \\
\hline$\partial_{66}$ & $f_{\text {Rad }}$ & $A_{\mathrm{Rad}}$ & $\rightarrow \mathrm{Rad}$ & $A_{\mathrm{Rad}}$ & $f_{\mathrm{Rad}}$ & $A_{\mathrm{Rad}}$ & $A_{\mathrm{Rad}}$ & $f_{\mathrm{Rad}}$ & $f_{\text {Rad }}$ & $A_{\mathrm{Rad}}$ & $A_{\mathrm{Rad}}$ & $f_{\mathrm{Rad}}$ & $f_{\text {Ass }}$ & $f_{\text {Ass }}$ & $A_{\text {Ass }}$ & $A_{\text {Ass }}$ & $f_{\text {Ass }}$ & $\not A_{\text {Ass }}$ \\
\hline$\partial_{67}$ & $f_{\mathrm{Rad}}$ & $f_{\mathrm{Rad}}$ & $f_{\text {Rad }}$ & $f_{\mathrm{Rad}}$ & $f_{\mathrm{Rad}}$ & $f_{\mathrm{Rad}}$ & $f_{\mathrm{Rad}}$ & $f_{\mathrm{Rad}}$ & $f_{\mathrm{Rad}}$ & $f_{\mathrm{Rad}}$ & $A_{\mathrm{Rad}}$ & $f_{\mathrm{Rad}}$ & $\not A_{\text {Ass }}$ & $f_{\text {Ass }}$ & $f_{\text {Ass }}$ & $A_{\text {Ass }}$ & $f_{\text {Ass }}$ & $\not A_{\text {Ass }}$ \\
\hline$\partial_{68}$ & $A_{\mathrm{Rad}}$ & $f_{\mathrm{Rad}}$ & $A_{\mathrm{Rad}}$ & $A_{\mathrm{Rad}}$ & $f_{\mathrm{Rad}}$ & $t_{\mathrm{Rad}}$ & $f_{\mathrm{Rad}}$ & $f_{\mathrm{Rad}}$ & $f_{\mathrm{Rad}}$ & $f_{\mathrm{Rad}}$ & $A_{\mathrm{Rad}}$ & $f_{\mathrm{Rad}}$ & $\not_{\text {Ass }}$ & $A_{\text {Ass }}$ & $f_{\text {Ass }}$ & $A_{\text {Ass }}$ & $A_{\text {Ass }}$ & $\not f_{\text {Ass }}$ \\
\hline$J_{69}$ & $f_{\mathrm{Rad}}$ & $f_{\text {Rad }}$ & $f_{\mathrm{Rad}}$ & $A_{\mathrm{Rad}}$ & $f_{\mathrm{Rad}}$ & $f_{\mathrm{Rad}}$ & $f_{\mathrm{Rad}}$ & $f_{\mathrm{Rad}}$ & $f_{\mathrm{Rad}}$ & $f_{\mathrm{Rad}}$ & $A_{\mathrm{Rad}}$ & $f_{\mathrm{Rad}}$ & $f_{\text {Ass }}$ & $f_{\text {Ass }}$ & $\not A_{\text {Ass }}$ & $A_{\text {Ass }}$ & $\not_{\text {Ass }}$ & $A_{\text {Ass }}$ \\
\hline$\partial_{70}$ & $A_{\mathrm{Rad}}$ & $A_{\mathrm{Rad}}$ & $f_{\mathrm{Rad}}$ & $A_{\mathrm{Rad}}$ & $t_{\mathrm{Rad}}$ & $t_{\mathrm{Rad}}$ & $f_{\mathrm{Rad}}$ & $t_{\mathrm{Rad}}$ & $f_{\mathrm{Rad}}$ & $f_{\mathrm{Rad}}$ & $t_{\mathrm{Rad}}$ & $f_{\mathrm{Rad}}$ & $t_{\text {Ass }}$ & $t_{\text {Ass }}$ & $t_{\mathrm{Ass}}$ & $t_{\text {Ass }}$ & $f_{\text {Ass }}$ & $A_{\text {Ass }}$ \\
\hline$\partial_{71}$ & $f_{\mathrm{Rad}}$ & $A_{\mathrm{Rad}}$ & $A_{\mathrm{Rad}}$ & $A_{\mathrm{Rad}}$ & $t_{\mathrm{Rad}}$ & $f_{\mathrm{Rad}}$ & $f_{\mathrm{Rad}}$ & $f_{\mathrm{Rad}}$ & $f_{\mathrm{Rad}}$ & $f_{\mathrm{Rad}}$ & $A_{\mathrm{Rad}}$ & $f_{\mathrm{Rad}}$ & $f_{\text {Ass }}$ & $f_{\text {Ass }}$ & $f_{\text {Ass }}$ & $f_{\text {Ass }}$ & $\not_{\text {Ass }}$ & $\not_{\text {Ass }}$ \\
\hline$\partial_{72}$ & $f_{\text {Rad }}$ & $f_{\mathrm{Rad}}$ & $f_{\text {Rad }}$ & $f_{\mathrm{Rad}}$ & $t_{\mathrm{Rad}}$ & $t_{\mathrm{Rad}}$ & $f_{\mathrm{Rad}}$ & $t_{\mathrm{Rad}}$ & $t_{\mathrm{Rad}}$ & $f_{\mathrm{Rad}}$ & $A_{\mathrm{Rad}}$ & $f_{\mathrm{Rad}}$ & $f_{\text {Ass }}$ & $t_{\text {Ass }}$ & $t_{\text {Ass }}$ & $f_{\text {Ass }}$ & $f_{\text {Ass }}$ & $f_{\text {Ass }}$ \\
\hline$\partial_{73}$ & $f_{\text {Rad }}$ & $f_{\mathrm{Rad}}$ & $A_{\mathrm{Rad}}$ & $f_{\mathrm{Rad}}$ & $f_{\mathrm{Rad}}$ & $A_{\mathrm{Rad}}$ & $f_{\mathrm{Rad}}$ & $f_{\mathrm{Rad}}$ & $f_{\mathrm{Rad}}$ & $f_{\mathrm{Rad}}$ & $A_{\mathrm{Rad}}$ & $f_{\mathrm{Rad}}$ & $t_{\text {Ass }}$ & $t_{\text {Ass }}$ & $A_{\text {Ass }}$ & $f_{\text {Ass }}$ & $f_{\text {Ass }}$ & $f_{\text {Ass }}$ \\
\hline$J_{3}$ & $\rightarrow_{\mathrm{T}}$ & $\rightarrow_{\mathrm{T}}$ & $\rightarrow_{\mathrm{T}}$ & $\rightarrow_{\mathrm{T}}$ & $\rightarrow_{\mathrm{T}}$ & $\rightarrow_{\mathrm{T}}$ & $\rightarrow_{\mathrm{T}}$ & $\rightarrow_{\mathrm{T}}$ & $\rightarrow_{\mathrm{T}}$ & $\rightarrow_{\mathrm{T}}$ & $\rightarrow_{\mathrm{T}}$ & $\rightarrow_{\mathrm{T}}$ & $f_{\text {Ass }}$ & $f_{\text {Ass }}$ & $t_{\text {Ass }}$ & $f_{\text {Ass }}$ & $A_{\text {Ass }}$ & $f_{\text {Ass }}$ \\
\hline$\partial_{4}$ & $\rightarrow_{\mathrm{T}}$ & $\rightarrow_{\mathrm{T}}$ & $\rightarrow_{\mathrm{T}}$ & $\rightarrow_{\mathrm{T}}$ & $\rightarrow_{\mathrm{T}}$ & $\rightarrow_{\mathrm{T}}$ & $\rightarrow_{\mathrm{T}}$ & $\rightarrow_{\oplus}$ & $\rightarrow_{\mathrm{T}}$ & $\rightarrow_{\oplus}$ & $\rightarrow_{\oplus}$ & $\rightarrow_{\oplus}$ & $t_{\text {Ass }}$ & $A_{\text {Ass }}$ & $t_{\mathrm{Ass}}$ & $f_{\text {Ass }}$ & $f_{\text {Ass }}$ & $t_{\text {Ass }}$ \\
\hline $\mathcal{J}_{5}$ & $\rightarrow_{\mathrm{T}}$ & $A_{\text {Ann }}$ & $\rightarrow_{\mathrm{T}}$ & $\rightarrow_{\mathrm{T}}$ & $f_{\text {Ann }}$ & $\rightarrow_{\mathrm{T}}$ & $\rightarrow_{\mathrm{T}}$ & $\rightarrow_{\oplus}$ & $t_{1}$ & $t_{1}$ & $\rightarrow_{\oplus}$ & $A_{1}$ & $f_{\text {Ass }}$ & $A_{\text {Ass }}$ & $f_{\text {Ass }}$ & $A_{\text {Ass }}$ & $A_{\text {Ass }}$ & $f_{\text {Ass }}$ \\
\hline$J_{39}$ & $f_{\text {Aut }}$ & $\rightarrow_{\mathrm{T}}$ & $A_{\text {npa }}$ & $t_{\text {npa }}$ & $\rightarrow_{\mathrm{T}}$ & $f_{\text {npa }}$ & $t_{\mathrm{Rad}}$ & $t_{\text {Aut }}$ & $f_{\mathrm{Rad}}$ & $\not_{\text {Aut }}$ & $f_{\text {Aut }}$ & $f_{\text {Aut }}$ & $A_{\text {Ass }}$ & $t_{\text {Ass }}$ & $t_{A}$ Ass & $t_{\text {Ass }}$ & $t_{\text {Ass }}$ & $t_{\text {Ass }}$ \\
\hline$J_{38}$ & $f_{\text {Aut }}$ & $A_{1}$ & $\rightarrow_{\mathrm{T}}$ & $A_{g^{4}}$ & $f_{\text {Ann }}$ & $A_{\text {npa }}$ & $A_{\mathrm{Rad}}$ & $A_{\text {Aut }}$ & $A_{\mathrm{Rad}}$ & $A_{\text {Aut }}$ & $A_{\text {Aut }}$ & $A_{\text {Aut }}$ & $f_{\text {Ass }}$ & $f_{\text {Ass }}$ & $\not \rightarrow$ Ass & $f_{\text {Ass }}$ & $A_{\text {Ass }}$ & $\not A_{\text {Ass }}$ \\
\hline$\partial_{41}$ & $A_{\text {Aut }}$ & $\not_{1}$ & $\rightarrow_{\mathrm{T}}$ & $A_{z^{2}}$ & $f_{\text {Ann }}$ & $A_{y^{2}}$ & $f_{\text {Aut }}$ & $\not_{\text {Aut }}$ & $A_{\text {Aut }}$ & $A_{\text {Aut }}$ & $A_{\text {Aut }}$ & $A_{\text {Aut }}$ & $f_{\text {Ass }}$ & $f_{\text {Ass }}$ & $f_{\text {Ass }}$ & $f_{\text {Ass }}$ & $A_{\text {Ass }}$ & $f_{\text {Ass }}$ \\
\hline
\end{tabular}

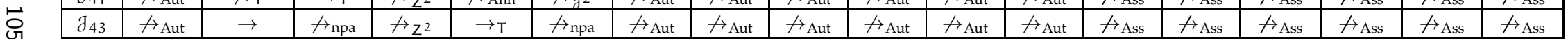




\begin{tabular}{|c|c|c|c|c|c|c|c|c|c|c|c|c|c|c|c|c|c|c|}
\hline & $J_{54}$ & $J_{42}$ & $J_{34}$ & $J_{35}$ & $J_{36}$ & $J_{37}$ & $J_{19}$ & $\mathrm{~J}_{20}$ & $\mathrm{~J}_{21}$ & $J_{22}$ & $\mathrm{~J}_{26}$ & $J_{27}$ & $\partial_{10}$ & $\partial_{11}$ & $J_{12}$ & $\partial_{13}$ & $J_{14}$ & $J_{15}$ \\
\hline$\partial_{40}$ & $\nexists_{\text {Aut }}$ & $\nexists_{\mathrm{Aut}}$ & $\rightarrow \oplus$ & $f_{A \mathrm{Aut}}$ & $f_{\text {Ann }}$ & $f_{A \mathrm{Aut}}$ & $f_{\mathrm{Aut}}$ & $f_{\mathrm{Aut}}$ & $f_{\mathrm{Aut}}$ & $f_{\mathrm{Aut}}$ & $\nexists_{\text {Aut }}$ & $f_{A}$ Aut & $f_{\text {Ass }}$ & $f_{\text {Ass }}$ & $\not \neg_{\text {Ass }}$ & $f_{\text {Ass }}$ & $\neg_{\text {Ass }}$ & $f_{\text {Ass }}$ \\
\hline$J_{47}$ & $f_{\text {Aut }}$ & $t_{1}$ & $f_{\text {npa }}$ & $\rightarrow_{\oplus}$ & $\Delta_{\mathrm{Ann}}$ & $f_{\text {npa }}$ & $t_{\mathrm{Rad}}$ & $\nexists_{\text {Aut }}$ & $t_{\mathrm{Rad}}$ & $f_{\text {Aut }}$ & $f_{\mathrm{Aut}}$ & $\not_{p_{\text {Aut }}}$ & $f_{\text {Ass }}$ & $f_{\mathrm{Ass}}$ & $f_{\text {Ass }}$ & $\not_{A \text { Ass }}$ & $\not_{\text {Ass }}$ & $f_{\text {Ass }}$ \\
\hline$\partial_{54}$ & $\rightarrow \rightarrow_{\mathrm{DT}}$ & $t_{1}$ & $t_{\text {npa }}$ & $A_{\text {npa }}$ & $t_{\mathrm{Ann}}$ & $\rightarrow_{\oplus}$ & $f_{\mathrm{Rad}}$ & $f_{\mathrm{Aut}}$ & $A_{\mathrm{Rad}}$ & $\not \Rightarrow_{\mathrm{Aut}}$ & $\nexists_{\mathrm{Aut}}$ & $f_{\text {Aut }}$ & $f_{\text {Ass }}$ & $f_{\text {Ass }}$ & $f_{\text {Ass }}$ & $f_{\text {Ass }}$ & $f_{\text {Ass }}$ & $f_{\text {Ass }}$ \\
\hline$J_{42}$ & $f_{\text {Aut }}$ & $\rightarrow_{\mathrm{DT}}$ & $A_{\text {nppa }}$ & $f_{\text {Aut }}$ & $\rightarrow$ & $f_{\text {Aut }}$ & $f_{\text {Aut }}$ & $f_{\text {Aut }}$ & $f_{\text {Aut }}$ & $f_{\text {Aut }}$ & $f_{A_{\text {Aut }}}$ & $f_{\text {Aut }}$ & $f_{A_{\text {Ass }}}$ & $f_{\text {Ass }}$ & $f_{\text {Ass }}$ & $f_{\text {Ass }}$ & $f_{\text {Ass }}$ & $f_{\text {Ass }}$ \\
\hline$\partial_{34}$ & $A_{\text {Aut }}$ & $A_{\text {Aut }}$ & $\rightarrow \rightarrow_{\mathrm{DT}}$ & $f_{A \mathrm{Aut}}$ & $A_{\text {Aut }}$ & $f_{A u t}$ & $A_{\mathrm{Aut}}$ & $f_{A u t}$ & $f_{A}$ Aut & $f_{\mathrm{Aut}}$ & $A_{\mathrm{Aut}}$ & $f_{\text {Aut }}$ & $f_{\text {Ass }}$ & $f_{\text {Ass }}$ & $f_{\text {Ass }}$ & $f_{\text {Ass }}$ & $f_{\text {Ass }}$ & $f_{\text {Ass }}$ \\
\hline$J_{35}$ & $f_{\text {Aut }}$ & $f_{\text {Aut }}$ & $f_{\text {npa }}$ & $\rightarrow \rightarrow_{\mathrm{DT}}$ & $\not_{A n n}$ & $f_{\text {Aut }}$ & $f_{\text {Aut }}$ & $f_{\text {Aut }}$ & $f_{\text {Aut }}$ & $f_{\mathrm{Aut}}$ & $f_{\text {Aut }}$ & $f_{\text {Aut }}$ & $f_{\text {Ass }}$ & $f_{\text {Ass }}$ & $f_{\text {Ass }}$ & $f_{\text {Ass }}$ & $\not f_{\text {Ass }}$ & $f_{\text {Ass }}$ \\
\hline$J_{36}$ & $f_{\text {Aut }}$ & $f_{\text {Aut }}$ & $f_{\text {Aut }}$ & $f_{\text {Aut }}$ & $\rightarrow \rightarrow_{\mathrm{DT}}$ & $f_{\text {Aut }}$ & $f_{\text {Aut }}$ & $f_{\text {Aut }}$ & $f_{\mathrm{Aut}}$ & $f_{\text {Aut }}$ & $f_{\mathrm{Aut}}$ & $f_{\text {Aut }}$ & $f_{\text {Ass }}$ & $f_{\text {Ass }}$ & $f_{\text {Ass }}$ & $f_{\text {Ass }}$ & $f_{\text {Ass }}$ & $f_{\text {Ass }}$ \\
\hline$J_{37}$ & $\nexists_{\text {Aut }}$ & $A_{\mathrm{Aut}}$ & $A_{\text {npa }}$ & $f_{\text {Aut }}$ & $A_{\mathrm{Ann}}$ & $\rightarrow \rightarrow_{\mathrm{DT}}$ & $f_{\mathrm{Aut}}$ & $A_{\text {Aut }}$ & $f_{\text {Aut }}$ & $f_{\mathrm{Aut}}$ & $\nexists_{\mathrm{Aut}}$ & $f_{\text {Aut }}$ & $f_{\text {Ass }}$ & $f_{\text {Ass }}$ & $f_{\text {Ass }}$ & $f_{\text {Ass }}$ & $f_{\text {Ass }}$ & $f_{\text {Ass }}$ \\
\hline$J_{19}$ & $f_{\text {Aut }}$ & $t_{1}$ & $\rightarrow_{\mathrm{T}}$ & $\rightarrow_{\oplus}$ & $f_{\text {Ann }}$ & $f_{\text {Ann }}$ & $\rightarrow_{\mathrm{DT}}$ & $f_{\text {Aut }}$ & $f_{\mathrm{Aut}}$ & $f_{\text {Aut }}$ & $f_{\mathrm{Aut}}$ & $f_{\text {Aut }}$ & $f_{\text {Ass }}$ & $f_{\text {Ass }}$ & $f_{\text {Ass }}$ & $f_{\text {Ass }}$ & $f_{A_{\text {Ass }}}$ & $f_{\text {Ass }}$ \\
\hline$J_{20}$ & $\rightarrow_{\oplus}$ & $A_{1}$ & $\rightarrow \mathrm{T}$ & $\rightarrow \rightarrow_{\mathrm{T}}$ & $A_{\text {Ann }}$ & $\rightarrow_{\mathrm{T}}$ & $f_{\text {npa }}$ & $\rightarrow \rightarrow_{\mathrm{DT}}$ & $A_{1}$ & $f_{\mathrm{Aut}}$ & $t_{\mathrm{Aut}}$ & $f_{\text {Aut }}$ & $f_{\text {Ass }}$ & $f_{\text {Ass }}$ & $f_{\text {Ass }}$ & $f_{\text {Ass }}$ & $f_{\text {Ass }}$ & $f_{\text {Ass }}$ \\
\hline$J_{21}$ & $f_{\text {Aut }}$ & $\rightarrow$ & $\rightarrow_{\mathrm{T}}$ & $f_{Z_{Z^{2}}}$ & $\rightarrow_{\mathrm{T}}$ & $\rightarrow_{\oplus}$ & $f_{\text {Aut }}$ & $f_{\text {Aut }}$ & $\rightarrow_{\mathrm{DT}}$ & $f_{\text {Aut }}$ & $f_{\mathrm{Aut}}$ & $f_{\text {Aut }}$ & $f_{\text {Ass }}$ & $f_{\text {Ass }}$ & $f_{\text {Ass }}$ & $f_{\text {Ass }}$ & $f_{\text {Ass }}$ & $f_{\text {Ass }}$ \\
\hline$J_{22}$ & $A_{\text {npa }}$ & $\rightarrow_{\mathrm{T}}$ & $f_{\text {npa }}$ & $\rightarrow_{\mathrm{T}}$ & $\rightarrow \mathrm{T}$ & $A_{\text {npa }}$ & $A_{\text {npa }}$ & $f_{\text {Aut }}$ & $f_{\text {npa }}$ & $\rightarrow_{\mathrm{DT}}$ & $f_{\text {Aut }}$ & $f_{\text {Aut }}$ & $f_{\text {Ass }}$ & $f_{\text {Ass }}$ & $f_{\text {Ass }}$ & $f_{\text {Ass }}$ & $f_{\text {Ass }}$ & $f_{\text {Ass }}$ \\
\hline$\partial_{26}$ & $A_{\text {npa }}$ & $t_{1}$ & $\rightarrow \mathrm{T}$ & $\rightarrow \mathrm{T}$ & $f_{A}$ Ann & $f_{\text {npa }}$ & $\rightarrow_{\oplus}$ & $f_{A \text { Aut }}$ & $t_{1} 1$ & $f_{\mathrm{Aut}}$ & $\rightarrow$ DT & $f_{A \text { Aut }}$ & $f_{\text {Ass }}$ & $f_{\text {Ass }}$ & $f_{\text {Ass }}$ & $f_{\text {Ass }}$ & $\lambda_{A}$ Ass & $f_{\text {Ass }}$ \\
\hline$J_{27}$ & $\rightarrow_{\oplus}$ & $\rightarrow_{\mathrm{T}}$ & $\rightarrow_{\mathrm{T}}$ & $A_{\text {npa }}$ & $\rightarrow_{\mathrm{T}}$ & $\rightarrow_{\mathrm{T}}$ & $A_{\text {npa }}$ & $f_{\text {Aut }}$ & $\rightarrow_{\oplus}$ & $f_{\mathrm{Aut}}$ & $f_{\text {Aut }}$ & $\rightarrow_{\mathrm{DT}}$ & $f_{A_{\text {Ass }}}$ & $f_{\text {Ass }}$ & $f_{\text {Ass }}$ & $f_{\text {Ass }}$ & $f_{\text {Ass }}$ & $f_{\text {Ass }}$ \\
\hline$\partial_{10}$ & $\nexists_{\text {Aut }}$ & $t_{1}$ & $\rightarrow \rightarrow_{\mathrm{T}}$ & $A_{\text {npa }}$ & $t_{\mathrm{Ann}}$ & $A_{\text {npa }}$ & $A_{\text {npa }}$ & $f_{\mathrm{Aut}}$ & $t_{1}$ & $f_{\mathrm{Aut}}$ & $\nexists_{\mathrm{Aut}}$ & $t_{\text {Aut }}$ & $\rightarrow \rightarrow_{\mathrm{DT}}$ & $t_{\text {Aut }}$ & $\phi_{A n n}$ & $A_{\text {Ann }}$ & $f_{\text {Aut }}$ & $f_{\text {Aut }}$ \\
\hline$J_{11}$ & $f_{\text {Aut }}$ & $t_{1}$ & $A_{\text {npa }}$ & $A_{\text {npa }}$ & $f_{\mathrm{Ann}}$ & $\rightarrow_{\oplus}$ & $f_{\text {npa }}$ & $f_{\text {Aut }}$ & $f_{1}$ & $f_{\text {Aut }}$ & $f_{\mathrm{Aut}}$ & $f_{\text {Aut }}$ & $f_{\text {Aut }}$ & $\rightarrow \rightarrow_{\mathrm{DT}}$ & $f_{A}$ Ann & $f_{A}$ An & $f_{\text {Aut }}$ & $f_{\text {Aut }}$ \\
\hline$J_{12}$ & $A_{\text {Aut }}$ & $A_{\text {Aut }}$ & $\rightarrow_{\oplus}$ & $\nexists_{\mathrm{Aut}}$ & $t_{1}$ & $f_{\mathrm{Aut}}$ & $f_{\text {Aut }}$ & $\not \rightarrow_{\text {Aut }}$ & $A_{\mathrm{Aut}}$ & $\not \Rightarrow_{\mathrm{Aut}}$ & $\nexists_{\mathrm{Aut}}$ & $f_{\text {Aut }}$ & $f_{\mathrm{Aut}}$ & $t_{\text {Aut }}$ & $\rightarrow \rightarrow_{\mathrm{DT}}$ & $A_{\text {Aut }}$ & $f_{\mathrm{Aut}}$ & $f_{\mathrm{Aut}}$ \\
\hline$J_{13}$ & $f_{\text {Aut }}$ & $t_{1}$ & $f_{\text {nppa }}$ & $f_{\text {npa }}$ & $f_{1}$ & $f_{\text {npa }}$ & $f_{\text {Aut }}$ & $f_{\text {Aut }}$ & $f_{\mathrm{Aut}}$ & $f_{\text {Aut }}$ & $f_{\mathrm{Aut}}$ & $f_{\mathrm{Aut}}$ & $f_{\text {Aut }}$ & $f_{\text {Aut }}$ & $f_{z^{2}}$ & $\rightarrow_{\mathrm{DT}}$ & $f_{\text {Aut }}$ & $f_{\text {Aut }}$ \\
\hline$J_{14}$ & $A_{\text {Aut }}$ & $A_{1}$ & $\rightarrow_{\mathrm{T}}$ & $f_{\text {npa }}$ & $t_{1}$ & $f_{\text {npa }}$ & $f_{\text {npa }}$ & $f_{A}$ Aut & $t_{1}$ & $f_{\mathrm{Aut}}$ & $A_{\text {Aut }}$ & $f_{\mathrm{Aut}}$ & $f_{\text {Aut }}$ & $f_{\text {Aut }}$ & $t_{\mathrm{z} z}$ & $t_{\mathrm{z} z}$ & $\rightarrow \rightarrow_{\mathrm{DT}}$ & $f_{A}$ Aut \\
\hline$J_{15}$ & $t_{\text {Aut }}$ & $t_{1}$ & $A_{\text {npa }}$ & $\rightarrow_{\oplus}$ & $t_{1}$ & $f_{\text {npa }}$ & $A_{\text {npa }}$ & $A_{\text {Aut }}$ & $t_{1}$ & $A_{\text {Aut }}$ & $\nexists_{\mathrm{Aut}}$ & $A_{\text {Aut }}$ & $t_{\text {Aut }}$ & $\lambda_{\text {Aut }}$ & $\Rightarrow z z$ & $A_{z}$ & $f_{\text {Aut }}$ & $\rightarrow \rightarrow_{\mathrm{DT}}$ \\
\hline$J_{16}$ & $f_{\text {Aut }}$ & $f_{1}$ & $f_{\text {npa }}$ & $A_{\text {npa }}$ & $f_{1}$ & $A_{\text {npa }}$ & $f_{\text {Aut }}$ & $f_{A}$ Aut & $f_{\text {Aut }}$ & $f_{\mathrm{Aut}}$ & $f_{\mathrm{Aut}}$ & $f_{\text {Aut }}$ & $f_{\text {Aut }}$ & $f_{A \text { Aut }}$ & $f_{z^{2}}$ & $f_{A}$ Aut & $f_{A}$ Aut & $f_{A}$ Aut \\
\hline$J_{17}$ & $A_{\text {Aut }}$ & $\rightarrow \mathrm{T}$ & $A_{\text {npa }}$ & $A_{\text {npa }}$ & $\rightarrow \mathrm{T}$ & $f_{\text {npa }}$ & $A_{\text {npa }}$ & $f_{\text {Aut }}$ & $f_{\text {npa }}$ & $f_{\mathrm{Aut}}$ & $t_{\mathrm{Aut}}$ & $f_{\mathrm{Aut}}$ & $A_{\text {Aut }}$ & $A_{\text {Aut }}$ & $A_{z} z$ & $A_{A z}$ & $f_{\text {Aut }}$ & $f_{A u t}$ \\
\hline$J_{18}$ & $f_{\text {Aut }}$ & $f_{\text {Aut }}$ & $A_{\text {npa }}$ & $f_{\mathrm{Aut}}$ & $\rightarrow$ & $f_{\text {Aut }}$ & $f_{\mathrm{Aut}}$ & $f_{\text {Aut }}$ & $f_{\mathrm{Aut}}$ & $f_{\mathrm{Aut}}$ & $f_{\mathrm{Aut}}$ & $A_{\mathrm{Aut}}$ & $f_{\text {Aut }}$ & $f_{\text {Aut }}$ & $f_{\text {Aut }}$ & $f_{\text {Aut }}$ & $f_{\text {Aut }}$ & $f_{\mathrm{Aut}}$ \\
\hline$J_{23}$ & $A_{\text {npa }}$ & $t_{1}$ & $\rightarrow \mathrm{T}$ & $A_{\text {npa }}$ & $f_{\text {Ann }}$ & $A_{\text {npa }}$ & $f_{\text {npa }}$ & $f_{\text {Aut }}$ & $t_{1}$ & $f_{\mathrm{Aut}}$ & $A_{\mathrm{Aut}}$ & $f_{\text {Aut }}$ & $\rightarrow_{\oplus}$ & $A_{\text {npa }}$ & $f_{\text {Ann }}$ & $\not f_{\text {Ann }}$ & $f_{\text {Ann }}$ & $f_{A n n}$ \\
\hline$J_{24}$ & $A_{\text {npa }}$ & $f_{1}$ & $\rightarrow_{\mathrm{T}}$ & $f_{\text {npa }}$ & $A_{1}$ & $f_{\text {npa }}$ & $f_{\text {npa }}$ & $f_{A \mathrm{Aut}}$ & $f_{1}$ & $f_{\text {Aut }}$ & $f_{\mathrm{Aut}}$ & $f_{\mathrm{Aut}}$ & $A_{\text {npa }}$ & $A_{\text {npa }}$ & $f_{z}$ & $A_{z}$ & $\rightarrow_{\oplus}$ & $f_{\text {npa }}$ \\
\hline$J_{25}$ & $A_{\text {npa }}$ & $\rightarrow_{\mathrm{T}}$ & $A_{\text {npa }}$ & $A_{\text {npa }}$ & $\rightarrow_{\mathrm{T}}$ & $f_{\text {npa }}$ & $A_{\text {npa }}$ & $f_{\text {Aut }}$ & $A_{\text {npa }}$ & $f_{A \mathrm{Aut}}$ & $f_{\text {Aut }}$ & $f_{\text {Aut }}$ & $A_{\text {npa }}$ & $A_{\text {npa }}$ & $f_{z} z$ & $f_{\mathrm{z}}$ & $f_{\text {npa }}$ & $A_{Z^{2}}$ \\
\hline$J_{28}$ & $f_{\text {Aut }}$ & $f_{\text {Aut }}$ & $A_{\text {npa }}$ & $f_{\text {Aut }}$ & $f_{\text {Ann }}$ & $f_{\text {Aut }}$ & $f_{\mathrm{Aut}}$ & $f_{A \text { Aut }}$ & $f_{\text {Aut }}$ & $\not_{\mathrm{Aut}}$ & $f_{\text {Aut }}$ & $f_{\text {Aut }}$ & $f_{\text {Aut }}$ & $f_{\text {Aut }}$ & $f_{\text {Aut }}$ & $f_{\text {Aut }}$ & $f_{A \text { Aut }}$ & $f_{A \text { Aut }}$ \\
\hline$J_{29}$ & $f_{\text {Aut }}$ & $f_{M_{N u}}$ & $A_{\text {npa }}$ & $f_{\text {npa }}$ & $f_{A n n}$ & $f_{\text {npa }}$ & $f_{\text {Aut }}$ & $f_{\text {Aut }}$ & $f_{\text {Aut }}$ & $f_{\text {Aut }}$ & $f_{\mathrm{Aut}}$ & $f_{\text {Aut }}$ & $f_{\text {Aut }}$ & $f_{\text {Aut }}$ & $f_{\mathrm{Rad}}$ & $f_{\text {Aut }}$ & $f_{\text {Aut }}$ & $f_{\mathrm{Aut}}$ \\
\hline$J_{30}$ & $f_{\text {Aut }}$ & $f_{\text {Aut }}$ & $f_{\text {npa }}$ & $f_{A \text { Aut }}$ & $f_{A n n}$ & $f_{A \text { Aut }}$ & $f_{A \text { Aut }}$ & $f_{A \text { Aut }}$ & $\not_{A_{\text {Aut }}}$ & $f_{A}$ Aut & $f_{\text {Aut }}$ & $f_{\text {Aut }}$ & $f_{\text {Aut }}$ & $f_{\text {Aut }}$ & $f_{\text {Aut }}$ & $f_{\text {Aut }}$ & $f_{A \text { Aut }}$ & $f_{A \text { Aut }}$ \\
\hline$J_{31}$ & $f_{\text {Aut }}$ & $f_{\text {Aut }}$ & $A_{\text {nppa }}$ & $f_{\mathrm{Aut}}$ & $f_{1}$ & $f_{\mathrm{Aut}}$ & $f_{\text {Aut }}$ & $f_{\text {Aut }}$ & $f_{\text {Aut }}$ & $f_{\text {Aut }}$ & $f_{\mathrm{Aut}}$ & $f_{\text {Aut }}$ & $f_{\text {Aut }}$ & $f_{\text {Aut }}$ & $f_{\text {Aut }}$ & $f_{\text {Aut }}$ & $f_{\mathrm{Aut}}$ & $f_{\mathrm{Aut}}$ \\
\hline$J_{32}$ & $A_{\text {Aut }}$ & $A_{\mathrm{Aut}}$ & $A_{\text {npa }}$ & $A_{\mathrm{Aut}}$ & $t_{1}$ & $t_{\mathrm{Aut}}$ & $f_{\mathrm{Aut}}$ & $f_{A}$ Aut & $f_{\mathrm{Aut}}$ & $f_{\mathrm{Aut}}$ & $A_{\mathrm{Aut}}$ & $f_{\mathrm{Aut}}$ & $f_{\text {Aut }}$ & $A_{\text {Aut }}$ & $f_{\mathrm{Aut}}$ & $f_{\text {Aut }}$ & $f_{A}$ Aut & $f_{A}$ Aut \\
\hline$J_{33}$ & $f_{\text {Aut }}$ & $f_{\text {Aut }}$ & $f_{\text {Aut }}$ & $f_{\text {Aut }}$ & $f_{\text {Aut }}$ & $f_{\text {Aut }}$ & $f_{\text {Aut }}$ & $f_{\text {Aut }}$ & $\not_{\text {Aut }}$ & $\not_{\text {Aut }}$ & $f_{\text {Aut }}$ & $f_{\text {Aut }}$ & $f_{\text {Aut }}$ & $f_{\text {Aut }}$ & $f_{\text {Aut }}$ & $f_{\text {Aut }}$ & $f_{\text {Aut }}$ & $f_{\text {Aut }}$ \\
\hline$\partial_{51}$ & $f_{\text {Aut }}$ & $t_{1}$ & $A_{\text {npa }}$ & $f_{\text {npa }}$ & $f_{\text {Ann }}$ & $f_{\text {npa }}$ & $f_{\mathrm{R}} \mathrm{ad}$ & $f_{\text {Aut }}$ & $f_{\mathrm{R}} \mathrm{dad}$ & $f_{\text {Aut }}$ & $f_{\text {Aut }}$ & $f_{\text {Aut }}$ & $f_{\text {Aut }}$ & $f_{\text {Aut }}$ & $\not A_{\mathrm{Rad}}$ & $f_{\mathrm{Rad}}$ & $f_{\text {Aut }}$ & $f_{A}$ Aut \\
\hline & $\phi_{A \text { Aut }}$ & $f_{1}$ & $A_{\text {npa }}$ & $A_{\text {npp }}$ & $f_{\text {Ann }}$ & $A_{\text {nppa }}$ & $f_{\mathrm{Rad}}$ & $f_{\text {Aut }}$ & $\phi_{\mathrm{R} \text { Rad }}$ & $f_{\text {Aut }}$ & $\phi_{f_{\mathrm{Aut}}}$ & $\phi_{\text {Aut }}$ & $\phi_{\text {Aut }}$ & $\phi_{\text {Aut }}$ & $\not A_{\mathrm{Rad}}$ & $t_{\mathrm{Rad}}$ & $f_{\text {Aut }}$ & $A_{\mathrm{Aut}}$ \\
\hline
\end{tabular}




\begin{tabular}{|c|c|c|c|c|c|c|c|c|c|c|c|c|c|c|c|c|c|c|}
\hline & $J_{54}$ & $\mathrm{~J}_{42}$ & $J_{34}$ & $J_{35}$ & $J_{36}$ & $J_{37}$ & $\partial_{19}$ & $J_{20}$ & $J_{21}$ & $J_{22}$ & $\mathcal{J}_{26}$ & $J_{27}$ & $J_{10}$ & $J_{11}$ & $J_{12}$ & $J_{13}$ & $\partial_{14}$ & $\partial_{15}$ \\
\hline$\partial_{53}$ & $A_{\text {npa }}$ & $A_{1}$ & $f_{\text {npa }}$ & $f_{\text {npa }}$ & $\not A_{\text {Ann }}$ & $f_{\text {npa }}$ & $f_{\mathrm{Rad}}$ & $f_{\text {Aut }}$ & $f_{\mathrm{Rad}}$ & $f_{\text {Aut }}$ & $\not_{\text {Aut }}$ & $f_{\text {Aut }}$ & $f_{\mathrm{Rad}}$ & $f_{\mathrm{Rad}}$ & $f_{\mathrm{Rad}}$ & $f_{\text {Rad }}$ & $A_{\mathrm{Rad}}$ & $\not A_{\mathrm{Rad}}$ \\
\hline$\partial_{44}$ & $A_{\text {Aut }}$ & $\not t_{1}$ & npa & npa & $f_{\mathrm{Ann}}$ & $f_{\text {Ann }}$ & $A_{\text {Aut }}$ & $f_{\text {Aut }}$ & $A_{\text {Aut }}$ & $f_{\text {Aut }}$ & $f_{\text {Aut }}$ & $f_{\text {Aut }}$ & $f_{\text {Aut }}$ & $f_{\text {Aut }}$ & $f_{\text {Rad }}$ & $A_{\text {Aut }}$ & $f_{\text {Aut }}$ & $f_{\text {Aut }}$ \\
\hline$\partial_{45}$ & Aut & $t_{1}$ & $t_{\text {npa }}$ & $7_{\text {npa }}$ & $A_{\text {Ann }}$ & $A_{\text {npa }}$ & $4_{\mathrm{Rad}}$ & $A_{\text {Aut }}$ & $f_{\mathrm{Rad}}$ & $t_{\text {Aut }}$ & $f_{\text {Aut }}$ & $f_{\text {Aut }}$ & $t_{A_{\text {Aut }}}$ & $f_{\text {Aut }}$ & $A_{\mathrm{Rad}}$ & $f_{\mathrm{Rad}}$ & $f_{\text {Aut }}$ & $A_{\text {Aut }}$ \\
\hline$\partial_{46}$ & $\nrightarrow_{\text {Aut }}$ & $\not_{1}$ & $f_{\text {npa }}$ & $f_{\text {npa }}$ & $f_{\text {Ann }}$ & $A_{\text {npa }}$ & $\nrightarrow_{\text {Aut }}$ & $\nrightarrow_{\text {Aut }}$ & $\not_{\text {Aut }}$ & $\nrightarrow_{\text {Aut }}$ & $f_{\text {Aut }}$ & $f_{\text {Aut }}$ & $\nrightarrow_{\text {Aut }}$ & $\nrightarrow_{\text {Aut }}$ & $A_{\mathrm{Rad}}$ & $\not_{\text {Aut }}$ & $\nrightarrow_{\text {Aut }}$ & $f_{\text {Aut }}$ \\
\hline$\partial_{55}$ & $A_{\text {Aut }}$ & $\not \rightarrow_{1}$ & $A_{\text {npa }}$ & $f_{\text {npa }}$ & $t_{1}$ & $f_{\text {npa }}$ & $t_{\text {Aut }}$ & $f_{\text {Aut }}$ & $f_{\text {Aut }}$ & $A_{\text {Aut }}$ & $t_{\text {Aut }}$ & $f_{\text {Aut }}$ & $t_{\text {Aut }}$ & $f_{\text {Aut }}$ & $t_{\mathrm{Rad}}$ & $f_{\text {Aut }}$ & $f_{\text {Aut }}$ & $A_{\text {Aut }}$ \\
\hline$J_{56}$ & $A_{\text {Aut }}$ & $\not \lambda_{1}$ & $A_{\text {npa }}$ & $f_{\text {npa }}$ & $t_{1}$ & $A_{\text {npa }}$ & $t_{\text {Aut }}$ & $t_{\text {Aut }}$ & $\not \rightarrow_{\text {Aut }}$ & $A_{\text {Aut }}$ & $f_{\text {Aut }}$ & $f_{\text {Aut }}$ & $t_{\text {Aut }}$ & $f_{\text {Aut }}$ & $t_{\mathrm{Rad}}$ & $t_{\text {Aut }}$ & $t_{\text {Aut }}$ & $\not \rightarrow_{\text {Aut }}$ \\
\hline$\partial_{57}$ & $A_{\text {Aut }}$ & $t_{1}$ & $f_{\text {npa }}$ & $f_{\text {npa }}$ & $t_{1}$ & $f_{\text {npa }}$ & $f_{\mathrm{Rad}}$ & $\not A_{\text {Aut }}$ & $f_{\mathrm{Rad}}$ & $A_{\text {Aut }}$ & $\not \rightarrow_{\text {Aut }}$ & $f_{\text {Aut }}$ & $t_{\text {Aut }}$ & $t_{\text {Aut }}$ & $A_{\mathrm{Rad}}$ & $f_{\mathrm{Rad}}$ & $f_{\text {Aut }}$ & $\not \rightarrow_{\text {Aut }}$ \\
\hline$\partial_{48}$ & $A_{\text {Aut }}$ & $A_{\text {Aut }}$ & $f_{\text {npa }}$ & $f_{\text {Aut }}$ & $f_{\text {Ann }}$ & $A_{\text {Aut }}$ & $f_{\text {Aut }}$ & $\not_{\text {Aut }}$ & $\not_{\text {Aut }}$ & $A_{\text {Aut }}$ & $f_{\text {Aut }}$ & $f_{\text {Aut }}$ & $t_{\text {Aut }}$ & $f_{\text {Aut }}$ & $f_{\text {Rad }}$ & $t_{\text {Aut }}$ & $f_{\text {Aut }}$ & $\not \rightarrow_{\text {Aut }}$ \\
\hline$\partial_{49}$ & $A_{\text {Aut }}$ & $\not A_{1}$ & $A_{\text {npa }}$ & $f_{\text {npa }}$ & $f_{\mathrm{Ann}}$ & $A_{\text {npa }}$ & $f_{\text {Aut }}$ & $t_{\text {Aut }}$ & $f_{\text {Aut }}$ & $A_{\text {Aut }}$ & $f_{\text {Aut }}$ & $f_{\text {Aut }}$ & $t_{\text {Aut }}$ & $f_{\text {Aut }}$ & $f_{\mathrm{Rad}}$ & $t_{\text {Aut }}$ & $f_{\text {Aut }}$ & $A_{\text {Aut }}$ \\
\hline$\partial_{50}$ & $A_{\text {Aut }}$ & $A_{\text {Aut }}$ & $A_{\text {npa }}$ & $f_{\text {Aut }}$ & $t_{y^{2}}$ & $A_{\text {Aut }}$ & $t_{\text {Aut }}$ & $t_{\text {Aut }}$ & $f_{\text {Aut }}$ & $t_{\text {Aut }}$ & $f_{\text {Aut }}$ & $f_{\text {Aut }}$ & $t_{\text {Aut }}$ & $t_{\text {Aut }}$ & $t_{\mathrm{Rad}}$ & $t_{\text {Aut }}$ & $f_{\text {Aut }}$ & $t_{\text {Aut }}$ \\
\hline$\partial_{58}$ & $A_{\text {Aut }}$ & $\nrightarrow_{\text {Aut }}$ & $f_{\text {npa }}$ & $f_{\text {Aut }}$ & $t_{1}$ & $A_{\text {Aut }}$ & $A_{\text {Aut }}$ & $A_{\text {Aut }}$ & $A_{\text {Aut }}$ & $A_{\text {Aut }}$ & $f_{\text {Aut }}$ & $f_{\text {Aut }}$ & $f_{\text {Aut }}$ & $A_{\text {Aut }}$ & $A_{\mathrm{Rad}}$ & $f_{\text {Aut }}$ & $f_{\text {Aut }}$ & $f_{A u t}$ \\
\hline$\partial_{59}$ & $A_{\text {Aut }}$ & $\not \rightarrow_{1}$ & $f_{\text {npa }}$ & $f_{\text {npa }}$ & $t_{1}$ & $A_{\text {npa }}$ & $t_{\text {Aut }}$ & $t_{\text {Aut }}$ & $t_{\text {Aut }}$ & $A_{\text {Aut }}$ & $f_{\text {Aut }}$ & $t_{\text {Aut }}$ & $t_{\text {Aut }}$ & $t_{\text {Aut }}$ & $t_{\mathrm{Rad}}$ & $t_{\text {Aut }}$ & $f_{\text {Aut }}$ & $\not \rightarrow_{\text {Aut }}$ \\
\hline$\partial_{60}$ & $A_{\text {Aut }}$ & $t_{A_{\text {Aut }}}$ & $A_{\text {npa }}$ & $A_{\text {Aut }}$ & $t_{1}$ & $A_{\text {Aut }}$ & $f_{\text {Aut }}$ & $t_{\text {Aut }}$ & $t_{\text {Aut }}$ & $A_{\text {Aut }}$ & $f_{\text {Aut }}$ & $f_{\text {Aut }}$ & $f_{\text {Aut }}$ & $f_{\text {Aut }}$ & $f_{\text {Rad }}$ & $f_{\text {Aut }}$ & $f_{\text {Aut }}$ & $t_{\mathrm{Aut}}$ \\
\hline$J_{6}$ & $A_{\text {npa }}$ & $t_{1}$ & $\rightarrow_{\mathrm{T}}$ & $\rightarrow_{\mathrm{T}}$ & $t_{1}$ & $f_{\text {npa }}$ & $\rightarrow_{\oplus}$ & $t_{\text {Aut }}$ & $t_{1}$ & $f_{\text {Aut }}$ & $f_{\text {Aut }}$ & $f_{\text {Aut }}$ & $\rightarrow_{\oplus}$ & $t_{\text {npa }}$ & $t_{z}$ & $t_{z}$ & $\rightarrow_{\oplus}$ & $\rightarrow_{\oplus}$ \\
\hline$\partial_{7}$ & $\stackrel{?}{\rightarrow} \rightarrow$ & $\rightarrow_{\mathrm{T}}$ & $\rightarrow_{\mathrm{T}}$ & $t_{\text {npa }}$ & $\rightarrow_{\mathrm{T}}$ & $\rightarrow_{\mathrm{T}}$ & $t_{\text {npa }}$ & $t_{\text {Aut }}$ & $\rightarrow_{\oplus}$ & $\rightarrow_{\text {Aut }}$ & $A_{\text {Aut }}$ & $t_{\text {Aut }}$ & $\rightarrow_{\oplus}$ & $\rightarrow_{\oplus}$ & $t_{\mathrm{z}}$ & $t_{z} z$ & $A_{\text {npa }}$ & $t_{Z^{2}}$ \\
\hline$\partial_{8}$ & $\stackrel{?}{\rightarrow} \rightarrow$ & $t_{1}$ & $A_{\text {npa }}$ & $t_{\text {npa }}$ & $A_{\mathrm{Ann}}$ & $\rightarrow_{\mathrm{T}}$ & $t_{\text {npa }}$ & $t_{\text {Aut }}$ & $t \rightarrow_{1}$ & $\rightarrow_{\text {Aut }}$ & $\not_{\text {Aut }}$ & $f_{\text {Aut }}$ & $\not_{\text {npa }}$ & $\rightarrow_{\oplus}$ & $A_{\text {Ann }}$ & $t_{\text {Ann }}$ & $\nexists_{\text {Ann }}$ & $t_{\mathrm{Ann}}$ \\
\hline$\partial_{9}$ & $t_{\text {Aut }}$ & $\stackrel{?}{\rightarrow}$ & $A_{\text {npa }}$ & $t_{\text {npa }}$ & $\rightarrow_{\mathrm{T}}$ & $A_{\text {npa }}$ & $t_{\text {Aut }}$ & $t_{\text {Aut }}$ & $t_{\text {Aut }}$ & $\rightarrow_{\text {Aut }}$ & $A_{\text {Aut }}$ & $t_{\text {Aut }}$ & $t_{\text {Aut }}$ & $t_{\text {Aut }}$ & $t_{z^{2}}$ & $t_{\text {Aut }}$ & $t_{\text {Aut }}$ & $t_{\text {Aut }}$ \\
\hline$\partial_{1}$ & $\stackrel{?}{\rightarrow}$ & $\rightarrow_{\mathrm{T}}$ & $\rightarrow_{\mathrm{T}}$ & $t_{\text {npa }}$ & $\rightarrow_{\mathrm{T}}$ & $\rightarrow_{\mathrm{T}}$ & $t_{\text {npa }}$ & $f_{\text {npa }}$ & $\rightarrow_{\mathrm{T}}$ & $t_{\text {npa }}$ & $f_{\text {npa }}$ & $\stackrel{?}{\rightarrow}$ & $\rightarrow_{\mathrm{T}}$ & $\rightarrow_{\mathrm{T}}$ & $t_{\mathrm{z}}$ & $t_{z} z$ & $A_{\text {npa }}$ & $t_{z^{2}}$ \\
\hline$\partial_{2}$ & $A_{\text {Aut }}$ & $\stackrel{?}{\rightarrow} \rightarrow$ & $f_{\text {npa }}$ & $f_{\text {npa }}$ & $\rightarrow_{\mathrm{T}}$ & $t_{\text {npa }}$ & $f_{\text {npa }}$ & $t_{\text {Aut }}$ & $t_{\text {npa }}$ & $t_{\text {Aut }}$ & $t_{\text {Aut }}$ & $f_{\text {Aut }}$ & $t_{\text {Aut }}$ & $t_{\text {Aut }}$ & $t_{z^{2}}$ & $t_{z}$ & $\not_{\text {Aut }}$ & $t_{\mathrm{Aut}}$ \\
\hline$\partial_{62}$ & $A_{\mathrm{Rad}}$ & $A_{\mathrm{Rad}}$ & $A_{\mathrm{Rad}}$ & $f_{\mathrm{Rad}}$ & $t_{\mathrm{Rad}}$ & $f_{\mathrm{Rad}}$ & $f_{\mathrm{Rad}}$ & $t_{\mathrm{Rad}}$ & $t_{\mathrm{Rad}}$ & $f_{\mathrm{Rad}}$ & $f_{\mathrm{Rad}}$ & $f_{\mathrm{Rad}}$ & $t_{\mathrm{Rad}}$ & $f_{\mathrm{Rad}}$ & $A_{\mathrm{Rad}}$ & $t_{\mathrm{Rad}}$ & $A_{\mathrm{Rad}}$ & $f_{\mathrm{Rad}}$ \\
\hline$\partial_{63}$ & $f_{\mathrm{Rad}}$ & $f_{\mathrm{Rad}}$ & $t_{\mathrm{Rad}}$ & $f_{\mathrm{Rad}}$ & $t_{\mathrm{Rad}}$ & $t_{\mathrm{Rad}}$ & $t_{\mathrm{Rad}}$ & $t_{\mathrm{Rad}}$ & $f_{\mathrm{Rad}}$ & $t_{\mathrm{Rad}}$ & $t_{\mathrm{Rad}}$ & $t_{\mathrm{Rad}}$ & $t_{\mathrm{Rad}}$ & $t_{\mathrm{Rad}}$ & $f_{\mathrm{Rad}}$ & $t_{\mathrm{Rad}}$ & $f_{\mathrm{Rad}}$ & $t_{\mathrm{Rad}}$ \\
\hline$\partial_{64}$ & $A_{\mathrm{Rad}}$ & $A_{\mathrm{Rad}}$ & $A_{\mathrm{Rad}}$ & $A_{\mathrm{Rad}}$ & $f_{\mathrm{Rad}}$ & $A_{\mathrm{Rad}}$ & $f_{\mathrm{Rad}}$ & $f_{\mathrm{Rad}}$ & $f_{\mathrm{Rad}}$ & $A_{\mathrm{Rad}}$ & $A_{\mathrm{Rad}}$ & $A_{\mathrm{Rad}}$ & $t_{\mathrm{Rad}}$ & $f_{\mathrm{Rad}}$ & $A_{\mathrm{Rad}}$ & $t_{\mathrm{Rad}}$ & $A_{\mathrm{Rad}}$ & $f_{\mathrm{Rad}}$ \\
\hline$J_{65}$ & $A_{\text {Rad }}$ & $f_{\mathrm{Rad}}$ & $A_{\mathrm{Rad}}$ & $A_{\text {Rad }}$ & $A_{\text {Rad }}$ & $A_{\mathrm{Rad}}$ & $f_{\mathrm{Rad}}$ & $f_{\mathrm{Rad}}$ & $f_{\mathrm{Rad}}$ & $f_{\mathrm{Rad}}$ & $A_{\mathrm{Rad}}$ & $f_{\mathrm{Rad}}$ & $t_{\mathrm{Rad}}$ & $f_{\mathrm{Rad}}$ & $A_{\mathrm{Rad}}$ & $t_{\mathrm{Rad}}$ & $A_{\mathrm{Rad}}$ & $f_{\mathrm{Rad}}$ \\
\hline
\end{tabular}

Tabela 5.5: Existência de deformação em Jor $_{4}$

\begin{tabular}{|c|c|c|c|c|c|c|c|c|c|c|c|c|c|c|c|c|c|c|}
\hline & $\partial_{16}$ & $\partial_{17}$ & $\partial_{18}$ & $\partial_{23}$ & $\partial_{24}$ & $J_{25}$ & $\partial_{28}$ & $J_{29}$ & $\partial_{30}$ & $\partial_{31}$ & $\partial_{32}$ & $J_{33}$ & $\partial_{51}$ & $\partial_{52}$ & $\partial_{53}$ & $\partial_{44}$ & $\partial_{45}$ & $\partial_{46}$ \\
\hline$\partial_{10}$ & $f_{\text {Ann }}$ & $\not_{\text {Aut }}$ & $\not_{\mathrm{Ann}}$ & $t_{\text {Aut }}$ & $\nrightarrow_{\mathrm{Aut}}$ & $\not_{A \mathrm{Aut}}$ & $\rightarrow_{\mathrm{T}}$ & $\rightarrow_{\oplus}$ & $A_{z}$ & $\not_{\mathrm{Ann}}$ & $t_{A n n}$ & $\not_{\mathrm{Ann}}$ & $\not_{\mathrm{Aut}}$ & $\not_{\text {Aut }}$ & $\nrightarrow_{\mathrm{Aut}}$ & $\stackrel{?}{\rightarrow}$ & $t_{\mathrm{Aut}}$ & $\rightarrow_{\oplus}$ \\
\hline$\partial_{11}$ & $\not_{\text {Ann }}$ & $t_{\mathrm{Aut}}$ & $t_{A n n}$ & $A_{\text {Aut }}$ & $t_{\mathrm{Aut}}$ & $A_{\mathrm{Aut}}$ & $\rightarrow_{\mathrm{T}}$ & $t_{\mathrm{npa}}$ & $A_{z}$ & $t_{\text {Ann }}$ & $t_{A n n}$ & $t_{A n n}$ & $t_{A u t}$ & $t_{\mathrm{Aut}}$ & $t_{\mathrm{Aut}}$ & $\stackrel{?}{\rightarrow}$ & $t_{\text {Aut }}$ & $\rightarrow$ \\
\hline$\partial_{12}$ & $t_{\text {Aut }}$ & $t_{\text {Aut }}$ & $t_{\text {Aut }}$ & $t_{\text {Aut }}$ & $t_{\text {Aut }}$ & $\not_{\text {Aut }}$ & $t_{\text {Aut }}$ & $t_{\mathrm{Aut}}$ & $\rightarrow_{\oplus}$ & $t_{\text {Aut }}$ & $\rightarrow$ & $t_{z}$ & $t_{\text {Aut }}$ & $t_{\text {Aut }}$ & $t_{\text {Aut }}$ & $t_{\text {Aut }}$ & $t_{\text {Aut }}$ & $t_{\text {Aut }}$ \\
\hline$\partial_{13}$ & $A_{\text {Aut }}$ & $t_{\text {Aut }}$ & $t_{1}$ & $t_{\text {Aut }}$ & $t_{\text {Aut }}$ & $\not_{\text {Aut }}$ & $\rightarrow_{\oplus}$ & $t_{A u t}$ & $t_{\text {npa }}$ & $f_{\text {npa }}$ & $\rightarrow_{\mathrm{T}}$ & $\not A_{z^{2}}$ & $\not_{\text {Aut }}$ & $\nrightarrow_{\text {Aut }}$ & $\not_{\text {Aut }}$ & $\not_{\text {Aut }}$ & $\nexists_{\text {Aut }}$ & $\not_{\text {Aut }}$ \\
\hline$\partial_{14}$ & $A_{z}$ & $f_{\text {Aut }}$ & $A_{z}$ & $t_{\text {Aut }}$ & $t_{\text {Aut }}$ & $f_{\text {Aut }}$ & $f_{\text {npa }}$ & $\rightarrow_{\oplus}$ & $t_{z}$ & $\rightarrow_{\mathrm{T}}$ & $t_{z}$ & $A_{z} z$ & $t_{\text {Aut }}$ & $f_{\text {Aut }}$ & $t_{\text {Aut }}$ & $f_{\text {npa }}$ & $t_{\text {Aut }}$ & $f_{\text {npa }}$ \\
\hline
\end{tabular}




\begin{tabular}{|c|c|c|c|c|c|c|c|c|c|c|c|c|c|c|c|c|c|c|}
\hline & 16 & $\partial_{17}$ & 18 & $J_{23}$ & 24 & $y_{25}$ & $J_{28}$ & $f_{29}$ & $\mathrm{~J}_{30}$ & $J_{31}$ & $f_{32}$ & $f_{33}$ & $\mathcal{J}_{51}$ & $J_{52}$ & $J_{53}$ & $J_{44}$ & $\partial_{45}$ & $\partial_{46}$ \\
\hline$\partial_{15}$ & $\rightarrow z$ & $\not_{A_{\text {Aut }}}$ & $\Delta z$ & $f_{\mathrm{Aut}}$ & Aut & $7_{\text {Aut }}$ & $\rightarrow_{\mathrm{T}}$ & $\not_{\text {npa }}$ & $A_{z}$ & $\rightarrow_{\mathrm{T}}$ & $A_{z}$ & $t_{z}$ & $A_{\text {Aut }}$ & $f_{\text {Aut }}$ & $f_{A_{\text {Aut }}}$ & $\stackrel{?}{\rightarrow}$ & $f_{\text {Aut }}$ & $\rightarrow_{\oplus}$ \\
\hline$J_{16}$ & $\mathrm{DT}$ & Aut & $t_{1}$ & & Aut & \rangle$_{\text {Aut }}$ & $\rightarrow$ & Aut & $\rightarrow_{\mathrm{T}}$ & $\rightarrow$ & $f_{\text {npa }}$ & $f_{\mathrm{Z}^{2}}$ & Aut & $7_{\text {Aut }}$ & & $f_{\text {Aut }}$ & $4_{\mathrm{Aut}}$ & $\mathrm{As}_{\Delta}$ \\
\hline$f_{17}$ & $t_{z}$ & DT & $\Rightarrow z$ & Aut & Aut & ${ }_{\text {Aut }}$ & $\rightarrow_{\mathrm{T}}$ & $\rightarrow$ & $A_{z}$ & $\rightarrow_{\text {npa }}$ & $f_{z}$ & $A_{\mathrm{z}}$ & Aut & $\mathrm{Au}$ & $\gg_{\text {Aut }}$ & $\stackrel{?}{\rightarrow}$ & $7_{\text {Aut }}$ & $\rightarrow$ \\
\hline $\mathcal{J}_{18}$ & Aut & Aut & $\mathrm{DT}$ & $\mathrm{Aut}_{\mathrm{A}}$ & Aut & $4_{\text {Aut }}$ & $4_{\text {Aut }}$ & $\triangle_{\mathrm{Aut}}$ & $\rightarrow$ & $t_{\text {Aut }}$ & $t_{\text {npa }}$ & $\not \neg z$ & $\$_{\text {Aut }}$ & $\not \phi_{\text {Aut }}$ & $\nabla_{\text {Aut }}$ & $\Rightarrow_{\text {Aut }}$ & $\phi_{\mathrm{Aut}}$ & $\bar{\Delta}_{\mathrm{Aut}}$ \\
\hline$J_{23}$ & $\mathrm{Ann}$ & $f_{\text {Ann }}$ & $\mathrm{A}_{\mathrm{Ann}}$ & $\mathrm{DT}$ & $f_{\text {Aut }}$ & \rangle$_{\text {Aut }}$ & $\rightarrow_{\mathrm{T}}$ & $\rightarrow_{\mathrm{T}}$ & $f_{A z}$ & $f_{A}$ Ann & $f_{\text {Ann }}$ & $f_{A}$ Ann & $f_{\text {npa }}$ & $\rightarrow$ & $f_{\text {Aut }}$ & $\rightarrow \rightarrow_{\mathrm{T}}$ & $\rightarrow$ & $\rightarrow_{\mathrm{T}}$ \\
\hline$J_{24}$ & $A z$ & $t_{1}$ & $\not \rightarrow z$ & $t_{\text {Aut }}$ & $\rightarrow \rightarrow_{\mathrm{DT}}$ & $t_{A_{\mathrm{Aut}}}$ & $t_{\text {npa }}$ & $\rightarrow_{\mathrm{T}}$ & $f_{\rightarrow z}$ & $\rightarrow_{\mathrm{T}}$ & $t_{\mathrm{Az}}$ & $A_{\mathrm{z}}$ & $\rightarrow_{\oplus}$ & $\stackrel{?}{\rightarrow}$ & $\Rightarrow_{\mathrm{Aut}}$ & $f_{\text {npa }}$ & $f_{\text {npa }}$ & $\not_{\text {npa }}$ \\
\hline $\mathcal{J}_{25}$ & $4 z$ & $\rightarrow$ & $\Rightarrow \mathrm{z}$ & $\not_{A \mathrm{Aut}}$ & $4_{\text {Aut }}$ & $\rightarrow \mathrm{DT}$ & $\rightarrow_{\mathrm{T}}$ & $\rightarrow_{\mathrm{T}}$ & $A_{z}$ & $\not_{\text {npa }}$ & $t_{\mathrm{z}}$ & $A_{\mathrm{z}}$ & $\rightarrow$ & $\stackrel{?}{\rightarrow}$ & $4_{\text {Aut }}$ & $\rightarrow_{\mathrm{T}}$ & $\rightarrow$ & $\rightarrow_{\mathrm{T}}$ \\
\hline 28 & Aut & $f_{\text {Aut }}$ & Aut & $4_{\text {Aut }}$ & $f_{A \text { Aut }}$ & $\overrightarrow{4}_{\mathrm{Aut}}$ & $\mathrm{DT}$ & $7_{\text {Aut }}$ & $t_{\mathrm{Ann}}$ & $\rightarrow$ Aut & $t_{\text {Ann }}$ & $p_{\text {Ann }}$ & $f_{A}$ Aut & $A_{\text {Aut }}$ & $\Rightarrow_{\text {Aut }}$ & $\Rightarrow_{\text {Aut }}$ & $p_{\text {Aut }}$ & $\Rightarrow_{\text {Aut }}$ \\
\hline 29 & Aut & Aut & $\mathrm{Rad}$ & Aut & Aut & \rangle$_{\text {Aut }}$ & npa & $\overrightarrow{D D T}_{\mathrm{DT}}$ & $A_{z}$ & $\mathrm{~A}_{\mathrm{Ann}}$ & $\mathrm{Ann}$ & $4_{\mathrm{Ann}}$ & $\rightarrow_{\text {Aut }}$ & $\not_{\mathrm{Aut}}$ & 4 Aut & $4_{\text {Aut }}$ & $A_{\text {Aut }}$ & $\phi_{\mathrm{Aut}}$ \\
\hline$J_{30}$ & Aut & Aut & $\overline{\text { Aut }}$ & Aut & Aut & $\succ_{\text {Aut }}$ & Aut & $\mathrm{Au}$ & $\rightarrow_{\mathrm{DT}}$ & $A_{\text {Aut }}$ & $\not f_{\text {Aut }}$ & $\not_{A_{A n n}}$ & $\phi_{\text {Aut }}$ & $f_{\text {Aut }}$ & $f_{\text {Aut }}$ & $\not f_{\text {Aut }}$ & Aut & $\bar{A}_{\mathrm{Aut}}$ \\
\hline 31 & Aut & Aut & Aut & 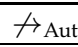 & Aut & $7_{\text {Aut }}$ & $\overline{\text { Aut }}$ & Aut & $t_{\mathrm{z} z}$ & $\rightarrow_{\mathrm{DT}}$ & $A_{z}$ & $t_{z}$ & $p_{\mathrm{Aut}}$ & $f_{f_{\text {Aut }}}$ & $f_{\text {Aut }}$ & $f_{f_{\text {Aut }}}$ & Aut & $\bar{f}_{\mathrm{Aut}}$ \\
\hline$J_{32}$ & Aut & & $A_{1}$ & 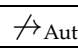 & Aut & \rangle$_{\text {Aut }}$ & $\mathrm{Au}$ & $\$_{\text {Aut }}$ & $f_{\text {Aut }}$ & $\Rightarrow_{\text {Aut }}$ & $\rightarrow_{\mathrm{DT}}$ & $\Rightarrow$ & $\$_{\text {Aut }}$ & $f_{\text {Aut }}$ & Aut & $\Rightarrow_{\text {Aut }}$ & $\not \Rightarrow_{\text {Aut }}$ & $\partial_{\mathrm{Aut}}$ \\
\hline$J_{33}$ & Aut & & Aut & & Aut & Aut & & $f_{\text {Aut }}$ & $f_{\text {Aut }}$ & $A_{A}$ & $f_{A \text { Aut }}$ & & $4_{\text {Aut }}$ & $f_{\mathrm{Aut}_{\mathrm{At}}}$ & Aut & $4_{\text {Aut }}$ & $f_{\text {Aut }}$ & $\overrightarrow{4}_{\mathrm{Al}_{1}}$ \\
\hline 51 & $\mathrm{Rad}$ & & & & Aut & $4_{\mathrm{Aut}}$ & npa & $\rightarrow_{\oplus}$ & $t \rightarrow z$ & $4 \mathrm{~A}$ & $f_{\text {Ann }}$ & $P_{\mathrm{Ann}}$ & $\rightarrow \mathrm{DT}$ & $f_{\mathrm{Aut}}$ & $f_{\text {Aut }}$ & $f_{\text {npa }}$ & $\not_{A_{\text {Aut }}}$ & $\bar{A}_{\mathrm{npa}}$ \\
\hline $\mathcal{J}_{52}$ & $\mathrm{Rad}$ & Aut & & & Aut & Aut & np & $\rightarrow$ & $f_{z}$ & $\nabla_{A n n}$ & $f_{\text {Ann }}$ & $\nabla_{A n n}$ & $f_{\mathrm{Aut}}$ & $\rightarrow_{\mathrm{DT}}$ & $f_{\text {Aut }}$ & $f_{\text {npa }}$ & $f_{\text {Aut }}$ & $\bar{A}_{\mathrm{npa}}$ \\
\hline$f_{53}$ & $\mathrm{Rad}$ & $f_{\mathrm{Rad}}$ & $\overline{\mathrm{Rad}}$ & 7 & $\mathrm{Aut}_{\mathrm{Aut}}$ & $f_{\text {Aut }}$ & $f_{\text {npa }}$ & $\rightarrow_{\mathrm{T}}$ & $t_{z}$ & $\Rightarrow_{A_{A}}$ & $f_{A n n}$ & $f_{A}$ Ann & $\rightarrow$ & $\rightarrow$ & & $f_{\text {npa }}$ & & $\bar{A}_{\mathrm{npa}}$ \\
\hline 44 & Aut & & & & Aut & $f_{\text {Aut }}$ & $\rightarrow_{\oplus}$ & Aut & $f_{\text {Ann }}$ & ${\nexists A_{A}}_{A}$ & $f_{\text {Ann }}$ & $f_{\text {Ann }}$ & $\phi_{\mathrm{Aut}}$ & $\Rightarrow_{A \text { Aut }}$ & $\nabla_{\text {Aut }}$ & $\rightarrow \rightarrow_{\mathrm{DT}}$ & $\not \nexists_{\text {Aut }}$ & $\Rightarrow_{A_{\mathrm{Aut}}}$ \\
\hline$f_{45}$ & Rad & & & & Aut & $7_{\text {Aut }}$ & & npa & $A_{z}$ & $A_{t}$ & $f_{\text {Ann }}$ & $7_{A n n}$ & $4_{\mathrm{Aut}}$ & $f_{\text {Aut }}$ & $4_{\text {Aut }}$ & $\rightarrow$ & & $\rightarrow$ \\
\hline$f_{46}$ & Aut & $\Rightarrow s$ & & & $f_{\text {Aut }}$ & Aut & $\rightarrow$ & $\mathrm{Aut}_{\mathrm{Aut}}$ & $f_{\mathrm{z}}$ & $f_{\text {Ann }}$ & $f_{\text {Ann }}$ & $f_{\text {Ann }}$ & $f_{\text {Aut }}$ & $f_{\text {Aut }}$ & $\mathrm{Aut}_{\mathrm{Aut}}$ & $f_{\text {Aut }}$ & tut & $\rightarrow_{\mathrm{DT}}$ \\
\hline & & & & & Aut & Aut & & & $f_{z z}$ & $\rightarrow$ & $f_{z}$ & & $\mathrm{~A}_{\mathrm{Aut}}$ & $\Rightarrow_{A \text { Aut }}$ & & $f_{A}$ Aut & $\not A$ & $\Rightarrow_{\mathrm{Aut}}$ \\
\hline$f_{56}$ & $7_{\text {Aut }}$ & $\not_{\mathrm{Au}}$ & $\mathrm{Rad}$ & $A_{A}$ & $7_{\text {Aut }}$ & $f_{\text {Aut }}$ & $\mathrm{np}$ & \rangle$_{\text {Aut }}$ & $A_{z z}$ & - & $A_{z}$ & & $\phi_{\mathrm{Aut}}$ & $f_{\mathrm{Aut}}$ & 4 Aut & $\not \neg_{\text {Aut }}$ & $\phi_{\text {Aut }}$ & $\overline{\phi_{\mathrm{Au}}}$ \\
\hline$J_{57}$ & $\mathrm{Rad}$ & $\not t_{A u}$ & & & $7_{\text {Aut }}$ & $f_{\text {Aut }}$ & npa & npa & $A_{\mathrm{Az}}$ & $\rightarrow$ & $A_{z}$ & $\Rightarrow \rightarrow z$ & $f_{f_{\text {Aut }}}$ & $f_{A}$ Aut & 4 Aut & $f_{\text {npa }}$ & $\not f_{\text {Aut }}$ & $f_{\text {npa }}$ \\
\hline & & & & & & & & & $\rightarrow$ & & & $\triangle_{\mathrm{Ann}}$ & $\not \rightarrow_{\text {Aut }}$ & & & $f_{A}$ Aut & & $f_{\mathrm{Aut}}$ \\
\hline$f_{49}$ & Aut & $f_{\mathrm{Au}}$ & & & Aut & $4_{\text {Aut }}$ & & Aut & $\rightarrow_{\mathrm{T}}$ & $A_{A}$ & $f_{A}$ Ann & $f_{\mathrm{Ann}}$ & $f_{A}$ Aut & $f_{A \text { Aut }}$ & $f_{\mathrm{Aut}}$ & $f_{A \text { Aut }}$ & $f_{A}$ Aut & $\not_{\mathrm{Al}_{1}}$ \\
\hline $\mathcal{J}_{50}$ & $7_{\text {Aut }}$ & $f_{A \text { Aut }}$ & & & $f_{A \text { Aut }}$ & $f_{\text {Aut }}$ & & Aut & $\rightarrow$ & $t \rightarrow$ & $f_{g^{2}}$ & $f_{f^{\prime}}$ & $f_{\mathrm{Aut}}$ & $f_{A}$ Aut & 4 Aut & $f_{A}$ Aut & $f_{A}$ Aut & $f_{\mathrm{Aut}}$ \\
\hline$J_{58}$ & & & & & & & & & & & & & $f_{\mathrm{Aut}}$ & & & $\not \rightarrow$ Aut & & $\bar{\partial}_{\mathrm{Aut}}$ \\
\hline$J_{59}$ & Aut & $f_{A}$ Aut & & & & Aut & & & npa & & -1 & & $f_{\text {Aut }}$ & $f_{\text {Aut }}$ & Aut & $f_{A}$ Aut & $f_{\text {Aut }}$ & $\phi_{\mathrm{Au}}$ \\
\hline $\mathcal{J}_{60}$ & $\not \rightarrow_{\text {Aut }}$ & $\not \Rightarrow_{\text {Aut }}$ & $t_{\mathrm{R}}$ & $f_{\text {Aut }}$ & $\not \Rightarrow_{\text {Aut }}$ & $\not_{\text {Aut }}$ & $f_{\text {npa }}$ & $\not A_{\text {Aut }}$ & $A_{\text {npa }}$ & $f_{\text {npa }}$ & $\rightarrow$ & $\not t_{z^{2}}$ & $f_{\text {Aut }}$ & $\not \neg_{\text {Aut }}$ & $f_{\text {Aut }}$ & $\not \rightarrow_{\text {Aut }}$ & $\not \Rightarrow_{\text {Aut }}$ & $\not \neg_{\mathrm{Aut}}$ \\
\hline$\partial_{6}$ & $t_{z}$ & $t_{1}$ & $t_{z}$ & $t_{\mathrm{Aut}}$ & $\not_{\mathrm{Aut}}$ & $\not_{\mathrm{Aut}}$ & $\rightarrow_{\mathrm{T}}$ & $\rightarrow_{\mathrm{T}}$ & $\nrightarrow_{z}$ & $\rightarrow_{\mathrm{T}}$ & $t_{z}$ & $t_{z}$ & $\stackrel{?}{\rightarrow}$ & $\stackrel{?}{\rightarrow}$ & $t_{\text {Aut }}$ & $\stackrel{?}{\rightarrow}$ & $\stackrel{?}{\rightarrow}$ & $\rightarrow_{\mathrm{T}}$ \\
\hline$\partial_{7}$ & $\not \rightarrow z$ & $\rightarrow$ & $\not \Rightarrow z$ & 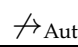 & $t_{\mathrm{Aut}}$ & $\Rightarrow_{A}$ Aut & $\rightarrow_{\mathrm{T}}$ & $\rightarrow_{\mathrm{T}}$ & $t_{\mathrm{z}}$ & & $t_{\mathrm{z}}$ & f & $\stackrel{?}{\rightarrow}$ & $\stackrel{?}{\rightarrow}$ & Aut & $\stackrel{?}{\rightarrow}$ & $\stackrel{?}{\rightarrow}$ & $\rightarrow_{\mathrm{T}}$ \\
\hline$\partial_{8}$ & $\mathrm{Ann}$ & $f_{A_{A n}}$ & $f_{A n n}$ & $f_{\mathrm{Au}}$ & $f_{\text {Aut }}$ & $f_{\text {Aut }}$ & $\rightarrow_{1}$ & $f_{\text {npa }}$ & $f_{z}$ & $A_{A}$ & $f_{A}$ Ann & $f_{\text {Ann }}$ & $f_{\text {npa }}$ & $t_{g_{g}}$ & $f_{\text {Aut }}$ & $\rightarrow_{\mathrm{T}}$ & $\rightarrow$ & $\rightarrow_{\mathrm{T}}$ \\
\hline$\partial_{9}$ & $\not_{A}$ Aut & $f_{\text {Aut }}$ & $\rightarrow$ & $f_{A \text { Aut }}$ & $t_{A \text { Aut }}$ & $f_{A \text { Aut }}$ & 7 inpa & $t_{\text {Aut }}$ & $\rightarrow \mathrm{T}$ & & $A_{\text {npa }}$ & $\not \rightarrow z$ & $\not A_{\text {Aut }}$ & $\not A_{\text {Aut }}$ & $t_{A \text { Aut }}$ & $\not_{A}$ Aut & $f_{A \text { Aut }}$ & $f_{\mathrm{Au}}$ \\
\hline$J_{1}$ & $f_{z}$ & $\rightarrow_{\mathrm{T}}$ & $A_{z}$ & $\rightarrow_{\oplus}$ & $t_{z_{z^{2}}}$ & $\rightarrow$ & $\rightarrow_{\mathrm{T}}$ & $\rightarrow \mathrm{T}$ & $A_{z}$ & $f_{\text {npa }}$ & $A_{\mathrm{z}}$ & $A_{z}$ & $\rightarrow_{\mathrm{T}}$ & $\rightarrow \mathrm{T}$ & $\rightarrow$ & $\rightarrow_{\mathrm{T}}$ & $\rightarrow_{\mathrm{T}}$ & \\
\hline
\end{tabular}




\begin{tabular}{|c|c|c|c|c|c|c|c|c|c|c|c|c|c|c|c|c|c|c|}
\hline & $\partial_{16}$ & $J_{17}$ & $\partial_{18}$ & $\partial_{23}$ & $\partial_{24}$ & $\partial_{25}$ & $J_{28}$ & $J_{29}$ & $\partial_{30}$ & $\partial_{31}$ & $J_{32}$ & $\partial_{33}$ & $\partial_{51}$ & $J_{52}$ & $\partial_{53}$ & $\partial_{44}$ & $\partial_{45}$ & $\partial_{46}$ \\
\hline$f_{2}$ & $A_{z}$ & $t_{A}$ Aut & $\rightarrow_{\mathrm{T}}$ & $t_{\text {Aut }}$ & $t_{\text {Aut }}$ & $\not A_{A u t}$ & $f_{\text {npa }}$ & $t_{\text {npa }}$ & $\rightarrow_{\mathrm{T}}$ & $t_{\text {npa }}$ & $t_{\text {npa }}$ & $A_{z}$ & $t_{\text {Aut }}$ & $\nrightarrow_{\text {Aut }}$ & $t_{\text {Aut }}$ & $t_{\text {npa }}$ & $t_{A}$ Aut & $\not_{\text {npa }}$ \\
\hline$J_{62}$ & $t_{\mathrm{Rad}}$ & $t_{\mathrm{Rad}}$ & $t_{\mathrm{Rad}}$ & $\not_{\mathrm{Rad}}$ & $f_{\mathrm{Rad}}$ & $A_{\mathrm{Rad}}$ & $t_{\mathrm{Rad}}$ & $t_{\mathrm{Rad}}$ & $t_{\mathrm{Rad}}$ & $A_{\mathrm{Rad}}$ & $t_{\mathrm{Rad}}$ & $t_{\mathrm{Rad}}$ & $f_{\mathrm{Rad}}$ & $A_{\mathrm{Rad}}$ & $A_{\mathrm{Rad}}$ & $f_{\mathrm{Rad}}$ & $t_{\mathrm{Rad}}$ & $\not A_{\mathrm{Rad}}$ \\
\hline$J_{63}$ & $t_{\mathrm{Rad}}$ & $t_{\mathrm{Rad}}$ & $t_{\mathrm{Rad}}$ & $t_{\mathrm{Rad}}$ & $t_{\mathrm{Rad}}$ & $t_{\mathrm{Rad}}$ & $t_{\mathrm{Rad}}$ & $f_{\mathrm{Rad}}$ & $f_{\mathrm{Rad}}$ & $t_{\mathrm{Rad}}$ & $t_{\mathrm{Rad}}$ & $f_{\mathrm{Rad}}$ & $f_{\mathrm{Rad}}$ & $t_{\mathrm{Rad}}$ & $t_{\mathrm{Rad}}$ & $t_{\mathrm{Rad}}$ & $t_{\mathrm{Rad}}$ & $t_{\mathrm{Rad}}$ \\
\hline$J_{64}$ & $f_{\mathrm{Rad}}$ & $t_{\mathrm{Rad}}$ & $f_{\mathrm{Rad}}$ & $t_{\mathrm{Rad}}$ & $t_{\mathrm{Rad}}$ & $t_{\mathrm{Rad}}$ & $f_{\mathrm{Rad}}$ & $f_{\mathrm{Rad}}$ & $f_{\mathrm{Rad}}$ & $t_{\mathrm{Rad}}$ & $t_{\mathrm{Rad}}$ & $f_{\mathrm{Rad}}$ & $f_{\mathrm{Rad}}$ & $t_{\mathrm{Rad}}$ & $A_{\mathrm{Rad}}$ & $t_{\mathrm{Rad}}$ & $t_{\mathrm{Rad}}$ & $A_{\mathrm{Rad}}$ \\
\hline$J_{65}$ & $f_{\mathrm{Rad}}$ & $t_{\mathrm{Rad}}$ & $t_{\mathrm{Rad}}$ & $t_{\mathrm{Rad}}$ & $f_{\mathrm{Rad}}$ & $f_{\mathrm{Rad}}$ & $t_{\mathrm{Rad}}$ & $f_{\mathrm{Rad}}$ & $f_{\mathrm{Rad}}$ & $t_{\mathrm{Rad}}$ & $t_{\mathrm{Rad}}$ & $f_{\mathrm{Rad}}$ & $f_{\mathrm{Rad}}$ & $t_{\mathrm{Rad}}$ & $t_{\mathrm{Rad}}$ & $f_{\mathrm{Rad}}$ & $t_{\mathrm{Rad}}$ & $t_{\text {Rad }}$ \\
\hline
\end{tabular}

Tabela 5.6: Existência de deformações em $\mathrm{Jor}_{4}$

\begin{tabular}{|c|c|c|c|c|c|c|c|c|c|c|c|c|c|c|c|c|c|c|c|}
\hline & $\partial_{55}$ & $\partial_{56}$ & $\partial_{57}$ & $\partial_{48}$ & $\partial_{49}$ & $\partial_{50}$ & $J_{58}$ & $J_{59}$ & $\partial_{60}$ & $\partial_{6}$ & $\partial_{7}$ & $\partial_{8}$ & $\partial_{9}$ & $\partial_{1}$ & $\partial_{2}$ & $\partial_{62}$ & $\partial_{63}$ & $\partial_{64}$ & $\partial_{65}$ \\
\hline$\partial_{10}$ & $\not_{\text {Ann }}$ & $f_{\text {Ann }}$ & $\nrightarrow_{\text {Aut }}$ & $f_{\mathrm{z}}$ & $A_{\mathrm{z}}$ & $\not_{\mathrm{Ann}}$ & $\not_{\mathrm{Ann}}$ & $f_{\text {Ann }}$ & $\not_{\mathrm{Ann}}$ & $\nrightarrow_{\text {Aut }}$ & $\nrightarrow_{\text {Aut }}$ & $\nrightarrow_{A}$ Aut & $t_{\mathrm{Rad}}$ & $\nrightarrow_{A \text { Aut }}$ & $\nrightarrow_{\text {Aut }}$ & $f_{\text {Aut }}$ & $\stackrel{?}{\rightarrow}$ & $\rightarrow_{\mathrm{T}}$ & $\stackrel{?}{\rightarrow}$ \\
\hline$\partial_{11}$ & $A_{\text {Ann }}$ & $A_{\text {Ann }}$ & $A_{\text {Aut }}$ & $t_{z} z$ & $A_{z}$ & $A_{\text {Ann }}$ & $t_{\text {Ann }}$ & $A_{\text {Ann }}$ & $A_{\mathrm{Ann}}$ & $t_{\text {Aut }}$ & $t_{\text {Aut }}$ & $\Rightarrow_{\text {Aut }}$ & $t_{\mathrm{Rad}}$ & $A_{\text {Aut }}$ & $A_{\text {Aut }}$ & $t_{A}$ Aut & $\stackrel{?}{-\stackrel{?}{\rightarrow}}$ & $\stackrel{?}{-\rightarrow}$ & $\stackrel{?}{\rightarrow} \rightarrow$ \\
\hline$\partial_{12}$ & $t_{\text {Aut }}$ & $f_{\text {Aut }}$ & $f_{\text {Aut }}$ & $f_{\text {Aut }}$ & $f_{\text {Aut }}$ & $t_{A_{\text {Aut }}}$ & $f_{\text {Aut }}$ & $f_{\text {Aut }}$ & $f_{\text {Aut }}$ & $f_{\text {Aut }}$ & $f_{\text {Aut }}$ & $f_{\text {Aut }}$ & $f_{\text {Aut }}$ & $f_{\text {Aut }}$ & $t_{\text {Aut }}$ & $t_{\text {Aut }}$ & $f_{\text {Aut }}$ & $t_{\text {Aut }}$ & $f_{\text {Aut }}$ \\
\hline$\partial_{13}$ & $\not_{\text {Aut }}$ & $f_{\text {Aut }}$ & $\not_{\text {Aut }}$ & $f_{\text {npa }}$ & $\not_{\text {Aut }}$ & $f_{\text {npa }}$ & $\stackrel{?}{\rightarrow}$ & $\not_{\text {Aut }}$ & $\rightarrow$ & $\not_{\text {Aut }}$ & $\nrightarrow_{\text {Aut }}$ & $f_{\text {Aut }}$ & $\not_{\text {Aut }}$ & $\not_{\text {Aut }}$ & $f_{\text {Aut }}$ & $f_{\text {Aut }}$ & $f_{\text {Aut }}$ & $\stackrel{?}{\rightarrow}$ & $\nrightarrow_{\text {Aut }}$ \\
\hline$\partial_{14}$ & $A_{z^{2}}$ & $\rightarrow$ & $\nrightarrow_{\text {Aut }}$ & $A_{z}$ & $t_{z}$ & $A_{z}$ & $A_{z}$ & $t_{z}$ & $A_{z}$ & $t_{\text {Aut }}$ & $\nrightarrow_{\text {Aut }}$ & $\nrightarrow_{\text {Aut }}$ & $t_{\mathrm{Rad}}$ & $\nrightarrow_{A \text { Aut }}$ & $\nrightarrow_{A \text { Aut }}$ & $\nrightarrow_{\text {Aut }}$ & $\stackrel{?}{\rightarrow}$ & $\rightarrow_{\mathrm{T}}$ & $\stackrel{?}{-\rightarrow}$ \\
\hline$\partial_{15}$ & $\stackrel{?}{-\rightarrow}$ & $\rightarrow$ & $\not_{\text {Aut }}$ & $f_{z}$ & $A_{\mathrm{z}}$ & $A_{z}$ & $t_{z} z$ & $A_{z}$ & $t_{z}$ & $\not_{\mathrm{Aut}}$ & $\nrightarrow_{\text {Aut }}$ & $\not_{\text {Aut }}$ & $t_{\mathrm{Rad}}$ & $\not_{\text {Aut }}$ & $\nrightarrow_{A \mathrm{Aut}}$ & $\nrightarrow_{\text {Aut }}$ & $\stackrel{?}{\rightarrow} \rightarrow$ & $\stackrel{?}{\rightarrow}$ & $\stackrel{?}{\rightarrow}$ \\
\hline$\partial_{16}$ & $\not_{\text {Aut }}$ & $t_{\text {Aut }}$ & $\nrightarrow_{\text {Aut }}$ & $\stackrel{?}{\rightarrow}$ & $\not_{\text {Aut }}$ & $\rightarrow$ & $A_{\text {npa }}$ & $f_{\text {Aut }}$ & $t_{\text {npa }}$ & $t_{\text {Aut }}$ & $\nrightarrow_{\text {Aut }}$ & $\not_{\text {Aut }}$ & $t_{\text {Aut }}$ & $\nrightarrow_{\text {Aut }}$ & $\not_{\mathrm{Aut}}$ & $t_{\mathrm{Aut}}$ & $\not_{\text {Aut }}$ & $\stackrel{?}{\rightarrow}$ & $\nrightarrow_{\text {Aut }}$ \\
\hline$\partial_{17}$ & $A_{z^{2}}$ & $t_{\text {npa }}$ & $t_{\text {Aut }}$ & $A_{z}$ & $t_{z}$ & $A_{z}$ & $t_{z}$ & $A_{z}$ & $A_{z}$ & $t_{\text {Aut }}$ & $\nrightarrow_{\mathrm{Aut}}$ & $\not_{\text {Aut }}$ & $t_{\mathrm{Rad}}$ & $f_{\text {Aut }}$ & $t_{\mathrm{Aut}}$ & $\nrightarrow_{\mathrm{Aut}}$ & $\stackrel{?}{\rightarrow}$ & $\rightarrow_{\mathrm{T}}$ & $\stackrel{?}{\rightarrow}$ \\
\hline$\partial_{18}$ & $A_{\text {Aut }}$ & $t_{\text {Aut }}$ & $f_{\text {Aut }}$ & $t_{\text {Aut }}$ & $f_{\text {Aut }}$ & $t_{\text {Aut }}$ & $t_{\text {Aut }}$ & $t_{\text {Aut }}$ & $t_{\text {Aut }}$ & $t_{\text {Aut }}$ & $f_{\text {Aut }}$ & $t_{\text {Aut }}$ & $t_{\text {Aut }}$ & $t_{\text {Aut }}$ & $t_{\mathrm{Aut}}$ & $t_{\text {Aut }}$ & $t_{\text {Aut }}$ & $f_{\text {Aut }}$ & $f_{\text {Aut }}$ \\
\hline$\partial_{23}$ & $\not_{\text {Ann }}$ & $f_{\text {Ann }}$ & $\not_{\mathrm{Ann}}$ & $A_{z}$ & $t_{z}$ & $\not_{\text {Ann }}$ & $t_{\text {Ann }}$ & $\not_{\text {Ann }}$ & $t_{\mathrm{Ann}}$ & $t_{\text {Aut }}$ & $t_{\mathrm{Aut}}$ & $\not_{\text {Aut }}$ & $t_{\mathrm{Rad}}$ & $f_{\text {Aut }}$ & $t_{\mathrm{Rad}}$ & $\stackrel{?}{\rightarrow}$ & $\stackrel{?}{\rightarrow}$ & $\rightarrow_{\mathrm{T}}$ & $\stackrel{?}{\rightarrow}$ \\
\hline$\partial_{24}$ & $\rightarrow_{\mathrm{T}}$ & $\rightarrow_{\mathrm{T}}$ & $\rightarrow$ & $A_{z}$ & $t_{z}$ & $t_{z}$ & $A_{z}$ & $t_{z}$ & $A_{z}$ & $t_{\text {Aut }}$ & $t_{\mathrm{Aut}}$ & $f_{\text {Aut }}$ & $t_{\mathrm{Rad}}$ & $\nrightarrow_{\text {Aut }}$ & $t_{\mathrm{Rad}}$ & $\stackrel{?}{\rightarrow}$ & $\stackrel{?}{\rightarrow}$ & $\rightarrow_{\mathrm{T}}$ & $\stackrel{?}{\rightarrow}$ \\
\hline$\partial_{25}$ & $A_{z^{2}}$ & $t_{\text {npa }}$ & $A_{z^{2}}$ & $A_{z}$ & $A_{z}$ & $A_{\mathrm{z}}$ & $A_{z}$ & $A_{z}$ & $A_{z}$ & $A_{\text {Aut }}$ & $f_{\text {Aut }}$ & $A_{\text {Aut }}$ & $f_{\mathrm{Rad}}$ & $A_{A \mathrm{Aut}}$ & $f_{\mathrm{Rad}}$ & $\stackrel{?}{\rightarrow}$ & $\stackrel{?}{\rightarrow}$ & $\rightarrow_{\mathrm{T}}$ & $\stackrel{?}{\rightarrow}$ \\
\hline$\partial_{28}$ & $f_{\text {Aut }}$ & $f_{\text {Aut }}$ & $f_{\text {Aut }}$ & $f_{\text {Aut }}$ & $\nrightarrow_{\text {Aut }}$ & $\not_{\text {Aut }}$ & $f_{\text {Aut }}$ & $\not_{\text {Aut }}$ & $f_{\text {Aut }}$ & $f_{\text {Aut }}$ & $f_{\text {Aut }}$ & $\not_{\text {Aut }}$ & $\nrightarrow_{\text {Aut }}$ & $A_{A u t}$ & $f_{\text {Aut }}$ & $\not_{A u t}$ & $f_{A u t}$ & $f_{\text {Aut }}$ & $f_{\text {Aut }}$ \\
\hline$\partial_{29}$ & $\nexists_{\text {Aut }}$ & $t_{\text {Aut }}$ & $A_{\text {Aut }}$ & $A_{z}$ & $\not_{\text {Aut }}$ & $\not A_{\text {Ann }}$ & $f_{\text {Ann }}$ & $\not A_{\text {Aut }}$ & $f_{\text {Ann }}$ & $f_{\text {Aut }}$ & $f_{\text {Aut }}$ & $f_{\text {Aut }}$ & $f_{\text {Aut }}$ & $f_{\text {Aut }}$ & $f_{\text {Aut }}$ & $f_{\text {Aut }}$ & $f_{\text {Aut }}$ & $\rightarrow$ & $f_{\text {Aut }}$ \\
\hline$J_{30}$ & $f_{\text {Aut }}$ & $f_{\text {Aut }}$ & $\nrightarrow_{\text {Aut }}$ & $f_{\text {Aut }}$ & $f_{\text {Aut }}$ & $\not_{\mathrm{Aut}}$ & $t_{A u t}$ & $f_{\text {Aut }}$ & $\not A_{\text {Aut }}$ & $f_{\text {Aut }}$ & $\nrightarrow_{\text {Aut }}$ & $f_{\text {Aut }}$ & $f_{\text {Aut }}$ & $\not_{A}$ Aut & $A_{\text {Aut }}$ & $\nrightarrow_{A \mathrm{Aut}}$ & $A_{A u t}$ & $\not_{\text {Aut }}$ & $A_{\text {Aut }}$ \\
\hline$J_{31}$ & $f_{\text {Aut }}$ & $t_{\text {Aut }}$ & $f_{\text {Aut }}$ & $t_{\text {Aut }}$ & $t_{\text {Aut }}$ & $f_{\text {Aut }}$ & $A_{\text {Aut }}$ & $t_{\text {Aut }}$ & $\not A_{\text {Aut }}$ & $t_{\text {Aut }}$ & $f_{\text {Aut }}$ & $t_{\text {Aut }}$ & $t_{\text {Aut }}$ & $t_{\text {Aut }}$ & $A_{\text {Aut }}$ & $A_{\text {Aut }}$ & $t_{\text {Aut }}$ & $A_{\text {Aut }}$ & $t_{\text {Aut }}$ \\
\hline$J_{32}$ & $t_{\text {Aut }}$ & $A_{\text {Aut }}$ & $A_{\text {Aut }}$ & $t_{\text {Aut }}$ & $t_{\text {Aut }}$ & $A_{\text {Aut }}$ & $A_{\text {Aut }}$ & $A_{\text {Aut }}$ & $t_{\text {Aut }}$ & $t_{\text {Aut }}$ & $A_{\text {Aut }}$ & $t_{\text {Aut }}$ & $t_{\text {Aut }}$ & $t_{\text {Aut }}$ & $t_{\text {Aut }}$ & $t_{\text {Aut }}$ & $t_{\text {Aut }}$ & $t_{\text {Aut }}$ & $t_{\text {Aut }}$ \\
\hline$\partial_{33}$ & $t_{\text {Aut }}$ & $f_{\text {Aut }}$ & $f_{\text {Aut }}$ & $f_{\text {Aut }}$ & $t_{\text {Aut }}$ & $t_{\text {Aut }}$ & $f_{\text {Aut }}$ & $\not A_{\text {Aut }}$ & $t_{\text {Aut }}$ & $t_{\text {Aut }}$ & $t_{\text {Aut }}$ & $t_{\text {Aut }}$ & $f_{\text {Aut }}$ & $t_{\text {Aut }}$ & $f_{\text {Aut }}$ & $t_{\text {Aut }}$ & $t_{\text {Aut }}$ & $t_{\text {Aut }}$ & $f_{\text {Aut }}$ \\
\hline$\partial_{51}$ & $\not_{\text {Ann }}$ & $t_{\text {Ann }}$ & $t_{\text {Aut }}$ & $A_{z}$ & $t_{z}$ & $\nrightarrow_{\text {Ann }}$ & $t_{\text {Ann }}$ & $t_{\text {Ann }}$ & $\not_{\text {Ann }}$ & $t_{\text {Aut }}$ & $t_{\text {Aut }}$ & $t_{\text {Aut }}$ & $t_{\mathrm{Rad}}$ & $\not_{\text {Aut }}$ & $t_{\text {Aut }}$ & $t_{\text {Aut }}$ & $\stackrel{?}{\rightarrow}$ & $\rightarrow_{\mathrm{T}}$ & $\stackrel{?}{\rightarrow}$ \\
\hline$\partial_{52}$ & $\nrightarrow_{\mathrm{Ann}}$ & $t_{\mathrm{Ann}}$ & $\nrightarrow_{\text {Aut }}$ & $f_{z}$ & $A_{z}$ & $\nrightarrow_{\mathrm{Ann}}$ & $\nrightarrow_{\mathrm{Ann}}$ & $t_{\text {Ann }}$ & $f_{\text {Ann }}$ & $f_{\text {Aut }}$ & $f_{\text {Aut }}$ & $f_{\text {Aut }}$ & $t_{\mathrm{Rad}}$ & $\nrightarrow_{\text {Aut }}$ & $A_{\text {Aut }}$ & $\nrightarrow_{A \text { Aut }}$ & $\stackrel{?}{\rightarrow}$ & $\rightarrow_{\mathrm{T}}$ & $\stackrel{?}{\rightarrow}$ \\
\hline$\partial_{53}$ & $f_{\text {Ann }}$ & $\not_{\text {Ann }}$ & $f_{A n n}$ & $A_{z}$ & $t_{z}$ & $A_{\text {Ann }}$ & $f_{\text {Ann }}$ & $f_{\text {Ann }}$ & $f_{\text {Ann }}$ & $t_{\text {Aut }}$ & $t_{\text {Aut }}$ & $t_{\text {Aut }}$ & $t_{\mathrm{Rad}}$ & $\not_{\text {Aut }}$ & $f_{\mathrm{Rad}}$ & $\rightarrow$ & $\rightarrow_{\mathrm{T}}$ & $\rightarrow_{\mathrm{T}}$ & $\rightarrow_{\mathrm{T}}$ \\
\hline$\partial_{44}$ & $\nrightarrow_{\text {Aut }}$ & $f_{\text {Aut }}$ & $\nrightarrow_{\text {Aut }}$ & $\not_{\text {Ann }}$ & $f_{\text {Aut }}$ & $A_{\text {Ann }}$ & $f_{A n n}$ & $\nrightarrow_{\text {Aut }}$ & $f_{\text {Ann }}$ & $A_{\text {Aut }}$ & $\nrightarrow_{\text {Aut }}$ & $f_{\text {Aut }}$ & $f_{\text {Aut }}$ & $\nrightarrow_{\text {Aut }}$ & $A_{\text {Aut }}$ & $\nrightarrow_{\text {Aut }}$ & $\nrightarrow_{\text {Aut }}$ & $A_{\text {Ann }}$ & $\nrightarrow_{\text {Aut }}$ \\
\hline
\end{tabular}




\begin{tabular}{|c|c|c|c|c|c|c|c|c|c|c|c|c|c|c|c|c|c|c|c|}
\hline & $\partial_{55}$ & $\partial_{56}$ & $\partial_{57}$ & $\partial_{48}$ & $\partial_{49}$ & $\partial_{50}$ & $\partial_{58}$ & $J_{59}$ & $\partial_{60}$ & $\partial_{6}$ & $\partial_{7}$ & $\partial_{8}$ & $\partial_{9}$ & $\partial_{1}$ & $\partial_{2}$ & $\partial_{62}$ & $\partial_{63}$ & $\partial_{64}$ & $\partial_{65}$ \\
\hline$\partial_{45}$ & $\not A_{\text {Ann }}$ & $\not A_{\text {Ann }}$ & $\not \rightarrow_{\text {Aut }}$ & $f_{z}$ & $t_{z}$ & $\not_{\text {Ann }}$ & $\not A_{\mathrm{Ann}}$ & $\not A_{\text {Ann }}$ & $\not_{\mathrm{Ann}}$ & $A_{\text {Aut }}$ & $\not \rightarrow_{\text {Aut }}$ & $\not \nrightarrow_{\text {Aut }}$ & $A_{\text {Rad }}$ & $\not_{\text {Aut }}$ & $\not_{\text {Aut }}$ & $\not \rightarrow_{\text {Aut }}$ & $\stackrel{?}{\rightarrow}$ & $\stackrel{?}{\rightarrow}$ & $\stackrel{?}{\rightarrow}$ \\
\hline$J_{46}$ & $\not \rightarrow_{\text {Aut }}$ & $\not_{\text {Aut }}$ & $\not \rightarrow_{\text {Aut }}$ & $A_{z}$ & $\not \rightarrow_{\text {Aut }}$ & $\not A_{\text {Ann }}$ & $A_{\text {Ann }}$ & $\not \rightarrow_{\text {Aut }}$ & $t_{\text {Ann }}$ & $\rightarrow_{\text {Aut }}$ & $\not \rightarrow_{\text {Aut }}$ & $\not \rightarrow_{\text {Aut }}$ & $\not \rightarrow_{\text {Aut }}$ & $A_{\text {Aut }}$ & $\not \rightarrow_{\text {Aut }}$ & $\not \rightarrow_{\text {Aut }}$ & $A_{\text {Aut }}$ & $\stackrel{?}{\rightarrow}$ & $\not \rightarrow_{\text {Aut }}$ \\
\hline$\partial_{55}$ & $\rightarrow_{\mathrm{DT}}$ & $\not_{\text {Aut }}$ & $\not \rightarrow_{\text {Aut }}$ & $A_{z}$ & $\not_{\text {Aut }}$ & $t_{z}$ & $A_{z}$ & $\not \rightarrow_{\text {Aut }}$ & $t_{z}$ & $A_{\text {Aut }}$ & $\not \rightarrow_{\text {Aut }}$ & $\not \rightarrow_{\text {Aut }}$ & $\not_{\text {Aut }}$ & $\not \rightarrow_{\text {Aut }}$ & $\not \rightarrow_{\text {Aut }}$ & $\not \rightarrow_{\text {Aut }}$ & $\not \rightarrow_{\text {Aut }}$ & $\stackrel{?}{\rightarrow}$ & $A_{\text {Aut }}$ \\
\hline$\partial_{56}$ & $\not_{\text {Aut }}$ & $\rightarrow_{\mathrm{DT}}$ & $A_{\text {Aut }}$ & $A_{z}$ & $\not \rightarrow_{\text {Aut }}$ & $A_{z}$ & $A_{z}$ & $\not \rightarrow_{\text {Aut }}$ & $A_{z}$ & $\not_{\text {Aut }}$ & $\not \rightarrow$ Aut & $A_{\text {Aut }}$ & $\not \rightarrow_{\text {Aut }}$ & $\not_{\text {Aut }}$ & $\not \rightarrow_{\text {Aut }}$ & $A_{\text {Aut }}$ & $\not_{\text {Aut }}$ & $\stackrel{?}{\rightarrow}$ & $\not \rightarrow_{\text {Aut }}$ \\
\hline$\partial_{57}$ & $\rightarrow$ & $\rightarrow$ & $\rightarrow_{\mathrm{DT}}$ & $t_{z}$ & $t_{z}$ & $t_{\mathrm{z}}$ & $t_{z}$ & $t_{\mathrm{z}}$ & $t_{z}$ & $t_{\text {Aut }}$ & $\not \rightarrow_{\text {Aut }}$ & $\not_{\text {Aut }}$ & $A_{\mathrm{Rad}}$ & $t_{\text {Aut }}$ & $\not \rightarrow_{\text {Aut }}$ & $A_{\text {Aut }}$ & $\stackrel{?}{\rightarrow}$ & $\stackrel{?}{\rightarrow}$ & $\stackrel{?}{\rightarrow}$ \\
\hline$J_{48}$ & $f_{\text {Aut }}$ & $f_{\text {Aut }}$ & $f_{\text {Aut }}$ & $\rightarrow_{\mathrm{DT}}$ & $f_{\text {Aut }}$ & $f_{\text {Aut }}$ & $f_{\text {Aut }}$ & $f_{\text {Aut }}$ & $f_{\text {Aut }}$ & $f_{\text {Aut }}$ & $f_{\text {Aut }}$ & $f_{\text {Aut }}$ & $f_{\text {Aut }}$ & $t_{\text {Aut }}$ & $A_{\text {Aut }}$ & $A_{\text {Aut }}$ & $A_{\text {Aut }}$ & $f_{\text {Aut }}$ & $A_{\text {Aut }}$ \\
\hline$J_{49}$ & $\nexists_{\text {Aut }}$ & $\not_{\text {Aut }}$ & $\not A_{\text {Aut }}$ & $\rightarrow$ & $\rightarrow \rightarrow_{\mathrm{DT}}$ & $\not_{\text {Ann }}$ & $\not_{\text {Ann }}$ & $\not_{\text {Aut }}$ & $\not_{\text {Ann }}$ & $\not \rightarrow_{\text {Aut }}$ & $\not A_{\text {Aut }}$ & $\not \Rightarrow_{\text {Aut }}$ & $\not_{\text {Aut }}$ & $t_{\text {Aut }}$ & $A_{\text {Aut }}$ & $\not_{\text {Aut }}$ & $\not A_{\text {Aut }}$ & $\stackrel{?}{\rightarrow}$ & $\not \rightarrow A_{\text {Aut }}$ \\
\hline$\partial_{50}$ & $A_{\text {Aut }}$ & $f_{\text {Aut }}$ & $f_{\text {Aut }}$ & $t_{\text {Aut }}$ & $f_{\text {Aut }}$ & $\rightarrow_{\mathrm{DT}}$ & $\not_{\text {Aut }}$ & $f_{\text {Aut }}$ & $\not_{\text {Aut }}$ & $\not_{\text {Aut }}$ & $\not_{\text {Aut }}$ & $f_{\text {Aut }}$ & $f_{\text {Aut }}$ & $\not A_{\text {Aut }}$ & $A_{\text {Aut }}$ & $\not A_{\text {Aut }}$ & $f_{\text {Aut }}$ & $f_{\text {Aut }}$ & $A_{\text {Aut }}$ \\
\hline$J_{58}$ & $f_{\text {Aut }}$ & $f_{\text {Aut }}$ & $A_{\text {Aut }}$ & $f_{\text {Aut }}$ & $f_{\text {Aut }}$ & $f_{\text {Aut }}$ & $\rightarrow_{\mathrm{DT}}$ & $f_{\text {Aut }}$ & $f_{\text {Aut }}$ & $f_{\text {Aut }}$ & $f_{\text {Aut }}$ & $f_{\text {Aut }}$ & $f_{\text {Aut }}$ & $t_{\text {Aut }}$ & $A_{\text {Aut }}$ & $f_{\text {Aut }}$ & $f_{\text {Aut }}$ & $f_{\text {Aut }}$ & $f_{\text {Aut }}$ \\
\hline$\partial_{59}$ & $\not_{\text {Aut }}$ & $\not_{\text {Aut }}$ & $\not_{\text {Aut }}$ & $t_{\text {npa }}$ & $\not_{\text {Aut }}$ & $t_{z}$ & $\rightarrow$ & $\rightarrow_{\mathrm{DT}}$ & $A_{z}$ & $\not_{\text {Aut }}$ & $\not \rightarrow_{\text {Aut }}$ & $\not_{\text {Aut }}$ & $\not_{\text {Aut }}$ & $t_{\text {Aut }}$ & $\not A_{\text {Aut }}$ & $\not A_{\text {Aut }}$ & $\not_{\text {Aut }}$ & $\stackrel{?}{\rightarrow}$ & $A_{\text {Aut }}$ \\
\hline$J_{60}$ & $f_{\text {Aut }}$ & $f_{\text {Aut }}$ & $A_{\text {Aut }}$ & $t_{\text {Aut }}$ & $\not A_{\text {Aut }}$ & $f_{\text {Aut }}$ & $\not_{\text {Aut }}$ & $\not \neg_{\text {Aut }}$ & $\rightarrow{ }_{\mathrm{DT}}$ & $t_{\text {Aut }}$ & $\nexists_{\text {Aut }}$ & $f_{\text {Aut }}$ & $f_{\text {Aut }}$ & $t_{A u t}$ & $A_{\text {Aut }}$ & $A_{\text {Aut }}$ & $A_{\text {Aut }}$ & $f_{\text {Aut }}$ & $\nexists_{\text {Aut }}$ \\
\hline$\partial_{6}$ & $\stackrel{?}{\rightarrow}$ & $\rightarrow_{\mathrm{T}}$ & $\stackrel{?}{\rightarrow}$ & $t_{\mathrm{A}}$ & $t_{\mathrm{z}}$ & $A_{z}$ & $A_{\mathrm{z}}$ & $t_{t} z$ & $A_{z}$ & $\rightarrow_{\mathrm{DT}}$ & $\not \rightarrow_{\text {Aut }}$ & $\not \rightarrow_{\text {Aut }}$ & $t_{z}$ & $t_{\text {Aut }}$ & $t_{\mathrm{Rad}}$ & $\stackrel{?}{\rightarrow}$ & $\stackrel{?}{\rightarrow}$ & $\rightarrow_{\mathrm{T}}$ & $\stackrel{?}{\rightarrow}$ \\
\hline$\partial_{7}$ & $t^{\prime} z^{2}$ & $f_{\text {npa }}$ & $t_{z^{2}}$ & $t_{z}$ & $t_{z} z$ & $A_{z}$ & $A_{z}$ & $t_{\mathrm{z}}$ & $t_{z}$ & $\not_{\text {Aut }}$ & $\rightarrow \rightarrow_{\mathrm{DT}}$ & $\not_{\text {Aut }}$ & $A_{z}$ & $\not_{\text {Aut }}$ & $f_{\mathrm{Rad}}$ & $\stackrel{?}{\rightarrow} \rightarrow$ & $\stackrel{?}{\rightarrow} \rightarrow$ & $\rightarrow_{\mathrm{T}}$ & $\stackrel{?}{\rightarrow} \rightarrow$ \\
\hline$\partial_{8}$ & $\not_{\text {Ann }}$ & $A_{\text {Ann }}$ & $A_{\text {Ann }}$ & $A_{z}$ & $A_{z}$ & $\not_{\text {Ann }}$ & $A_{\mathrm{Ann}}$ & $t_{\text {Ann }}$ & $A_{\text {Ann }}$ & $t_{\text {Aut }}$ & $\not \rightarrow$ Aut & $\rightarrow_{\mathrm{DT}}$ & $t_{\text {Ann }}$ & $t_{\text {Aut }}$ & $t_{\mathrm{Rad}}$ & $\stackrel{?}{\rightarrow}$ & $\stackrel{?}{\rightarrow}$ & $\stackrel{?}{\rightarrow-}$ & $\stackrel{?}{\rightarrow}$ \\
\hline$\partial_{9}$ & $t_{\text {Aut }}$ & $t_{\text {Aut }}$ & $t_{\text {Aut }}$ & $\rightarrow$ & $A_{\text {Aut }}$ & $t_{z}$ & $t_{z^{2}}$ & $t_{\text {Aut }}$ & $t_{z}$ & $t_{\text {Aut }}$ & $t_{\text {Aut }}$ & $t_{\text {Aut }}$ & $\rightarrow_{\mathrm{DT}}$ & $t_{\text {Aut }}$ & $t_{\text {Aut }}$ & $\rightarrow_{\text {Aut }}$ & $\not_{\text {Aut }}$ & $\stackrel{?}{\rightarrow-}$ & $\not \rightarrow_{\text {Aut }}$ \\
\hline $\mathcal{J}_{1}$ & $f_{z^{2}}$ & $f_{\text {npa }}$ & $f_{z^{2}}$ & $A \rightarrow z$ & $A_{z}$ & $t_{\mathrm{z} z}$ & $t_{z}$ & $A_{z}$ & $A_{\mathrm{z}}$ & $t_{z^{2}}$ & $\rightarrow_{\oplus}$ & $\rightarrow_{\oplus}$ & $t_{z}$ & $\rightarrow{ }_{\mathrm{DT}}$ & $t_{z}$ & $\rightarrow_{\mathrm{T}}$ & $\rightarrow_{\mathrm{T}}$ & $\rightarrow_{\mathrm{T}}$ & $\rightarrow_{\mathrm{T}}$ \\
\hline$\partial_{2}$ & $t_{\text {npa }}$ & $f_{\text {npa }}$ & $\nexists_{\text {Aut }}$ & $\rightarrow_{\mathrm{T}}$ & $\rightarrow$ & $A_{z}$ & $t_{z^{2}}$ & $t_{z^{2}}$ & $t_{z}$ & $t_{\text {Aut }}$ & $t_{\text {Aut }}$ & $\not A_{\text {Aut }}$ & $\rightarrow$ & $t_{\text {Aut }}$ & $\rightarrow_{\mathrm{DT}}$ & $\not A_{\text {Aut }}$ & $\stackrel{?}{\rightarrow}$ & $\stackrel{?}{\rightarrow} \rightarrow$ & $\stackrel{?}{\rightarrow} \rightarrow$ \\
\hline$\partial_{62}$ & $A_{\mathrm{Rad}}$ & $A_{\mathrm{Rad}}$ & $t_{\mathrm{Rad}}$ & $f_{\mathrm{Rad}}$ & $A_{\mathrm{Rad}}$ & $f_{\mathrm{Rad}}$ & $f_{\mathrm{Rad}}$ & $A_{\mathrm{Rad}}$ & $\not A_{\mathrm{Rad}}$ & $t_{\mathrm{Rad}}$ & $f_{\mathrm{Rad}}$ & $A_{\mathrm{Rad}}$ & $f_{\mathrm{Rad}}$ & $t_{\mathrm{Rad}}$ & $f_{\mathrm{Rad}}$ & $\rightarrow_{\mathrm{DT}}$ & $\rightarrow_{\text {Anc }}$ & $\rightarrow_{\mathrm{T}}$ & $\rightarrow_{\mathrm{Anc}}$ \\
\hline$J_{63}$ & $A_{\mathrm{Rad}}$ & $f_{\text {Rad }}$ & $A_{\mathrm{Rad}}$ & $f_{\mathrm{Rad}}$ & $f_{\text {Rad }}$ & $f_{\mathrm{Rad}}$ & $f_{\text {Rad }}$ & $f_{\text {Rad }}$ & $t_{\mathrm{Rad}}$ & $t_{\mathrm{Rad}}$ & $t_{\mathrm{Rad}}$ & $f_{\text {Rad }}$ & $f_{\mathrm{Rad}}$ & $t_{\mathrm{Rad}}$ & $t_{\mathrm{Rad}}$ & $t_{\text {Aut }}$ & $\rightarrow_{\mathrm{DT}}$ & $\rightarrow_{\text {Anc }}$ & $f_{\text {Aut }}$ \\
\hline$J_{64}$ & $A_{\mathrm{Rad}}$ & $f_{\mathrm{Rad}}$ & $A_{\mathrm{Rad}}$ & $f_{\mathrm{Rad}}$ & $t_{\mathrm{Rad}}$ & $f_{\mathrm{Rad}}$ & $f_{\mathrm{Rad}}$ & $t_{\mathrm{Rad}}$ & $f_{\mathrm{Rad}}$ & $f_{\mathrm{Rad}}$ & $t_{\mathrm{Rad}}$ & $f_{\mathrm{Rad}}$ & $t_{\mathrm{Rad}}$ & $t_{\mathrm{Rad}}$ & $f_{\mathrm{Rad}}$ & $f_{\text {Aut }}$ & $t_{\text {Aut }}$ & $\rightarrow_{\mathrm{DT}}$ & $\not A_{\text {Aut }}$ \\
\hline$\partial_{65}$ & $t_{\mathrm{Rad}}$ & $f_{\mathrm{Rad}}$ & $t_{\mathrm{Rad}}$ & $t_{\mathrm{Rad}}$ & $f_{\mathrm{Rad}}$ & $t_{\mathrm{Rad}}$ & $t_{\mathrm{Rad}}$ & $t_{\mathrm{Rad}}$ & $\not_{\mathrm{Rad}}$ & $t_{\mathrm{Rad}}$ & $t_{\mathrm{Rad}}$ & $t_{\text {Rad }}$ & $t_{\mathrm{Rad}}$ & $t_{\mathrm{Rad}}$ & $f_{\mathrm{Rad}}$ & $A_{\text {Aut }}$ & $\not A_{\text {Aut }}$ & $\stackrel{?}{\rightarrow}$ & $\rightarrow{ }_{\mathrm{DT}}$ \\
\hline
\end{tabular}




\subsection{Algumas PRopriedades DA VARIEDAde AlgÉbrica Jor 5}

Nesta pequena seção provaremos, essencialmente, que soma direta de álgebras (geometricamente) rígidas é rígida em Jor $r_{n}$ para $n \leqslant 5$ e daremos uma (boa) estimativa do número de componentes irredutíveis de $\mathrm{Jor}_{5}$.

Teorema 5.3. Soma direta de álgebras rígidas é de novo uma álgebra rígida em Jor $\mathrm{n}_{\mathrm{n}}$ para $\mathrm{n} \leqslant 5$.

Demonstração. Vimos nas Seções 5.1, 5.2 e 5.3 e no Teorema 5.2 que ke é a única álgebra rígida em Jor 1 e que a álgebra soma direta $\mathbf{k} e_{1} \oplus \mathbf{k} e_{2}$ é rígida em $\mathrm{Jor}_{2}, \mathcal{T}_{11}=\mathbf{k} \boldsymbol{e}_{1} \oplus$ $\mathbf{k}_{2} \oplus \mathbf{k} e_{3}$ é rígida em $\mathcal{J o r}_{3}$ e $\mathcal{J}_{3}=\mathbf{k} e_{1} \oplus \mathbf{k} e_{2} \oplus \mathbf{k} e_{3} \oplus \mathbf{k} e_{4}$ é rígida em $\mathrm{Jor}_{4}$. Além disso $\mathcal{B}_{2}$ é rígida em Jor 2 e as álgebras decomponíveis $\mathcal{T}_{19}=\mathcal{B}_{2} \oplus \mathbf{k} e_{2}$ é rígida em $\mathcal{J o r}_{3} \mathrm{e}$ $\mathcal{J}_{6}=\mathcal{B}_{2} \oplus \mathbf{k e}_{2} \oplus \mathbf{k} e_{3}$ e $\mathcal{J}_{13}=\mathcal{B}_{2} \oplus \mathcal{B}_{2}$ são rígidas em Jor 4 . Mais ainda em $\mathcal{J o r}_{3}$ temos as álgebras rígidas indecomponíveis $\mathcal{T}_{5}, \mathcal{T}_{7}$ e $\mathcal{T}_{9}$ das quais derivam as álgebras rígidas de $J_{0 r_{4}}: J_{1}=\mathcal{T}_{5} \oplus \mathbf{k} e_{4}, J_{12}=\mathcal{T}_{7} \oplus \mathbf{k} e_{2}$ e $\mathcal{J}_{24}=\mathcal{T}_{9} \oplus \mathbf{k} e_{2}$. Fazendo as somas diretas das álgebras rígidas de dimensões 1, 2, 3 e 4 com o fim de gerar uma álgebra de dimensão 5 obtemos as seguintes 13 álgebras decomponíveis de Jor $_{5}$ :

$$
\begin{aligned}
& \mathcal{C}_{1}=\mathbf{k} e_{1} \oplus \mathbf{k} e_{2} \oplus \mathbf{k} e_{3} \oplus \mathbf{k} e_{4} \oplus \mathbf{k} e_{5} ; \quad \mathcal{C}_{2}=\mathcal{B}_{2} \oplus \mathbf{k} e_{2} \oplus \mathbf{k} e_{3} \oplus \mathbf{k} e_{4} ; \\
& \mathcal{C}_{3}=\mathcal{B}_{2} \oplus \mathcal{B}_{2} \oplus \mathbf{k} e_{3} ; \quad \mathcal{C}_{4}=\mathcal{T}_{5} \oplus \mathbf{k} e_{4} \oplus \mathbf{k} e_{5} ; \quad \mathcal{C}_{5}=\mathcal{T}_{7} \oplus \mathbf{k} \mathbf{e}_{2} \oplus \mathbf{k} e_{3} ; \\
& \mathcal{C}_{6}=\mathcal{T}_{9} \oplus \mathbf{k} e_{2} \oplus \mathbf{k} e_{3} ; \quad \mathcal{C}_{7}=\mathcal{J}_{2} \oplus \mathbf{k} e_{5} ; \quad \mathcal{C}_{8}=\mathcal{J}_{16} \oplus \mathbf{k} e_{3} ; \\
& \mathcal{C}_{9}=\mathcal{J}_{33} \oplus \mathbf{k} e_{2} ; \quad \mathcal{C}_{10}=\mathcal{J}_{59} \oplus \mathbf{k} e_{2} ; \quad \mathcal{C}_{11}=\mathcal{B}_{2} \oplus \mathcal{T}_{5} ; \\
& \mathcal{C}_{12}=\mathcal{B}_{2} \oplus \mathcal{T}_{7} ; \quad \mathcal{C}_{13}=\mathcal{B}_{2} \oplus \mathcal{T}_{9} ;
\end{aligned}
$$

Verificamos utilizando um programa de computação simbólica elaborado pela autora (veja Apêndice $A$ ) que $\mathrm{H}^{2}\left(\mathfrak{C}_{i}, \mathcal{C}_{i}\right)=0$ para todo $1 \leqslant i \leqslant 13$ e portanto $\mathfrak{C}_{i}$ é rígida.

Teorema 5.4. O número de componentes irredutíveis em Jor 5 é maior ou igual a 26.

Demonstração. Sabemos da Proposição 4.36 que cada álgebra rígida de uma variedade gera uma componente irredutível. Vejamos que em Jor 5 temos no mínimo 26 álgebras rígidas.

Foi provado no Teorema 5.3 que as 13 álgebras de Jordan decomponíveis de dimensão $5, \mathcal{C}_{i}$ com $1 \leqslant i \leqslant 13$, são álgebras rígidas e claramente não isomorfas. Temos mais 3 álgebras rígidas não isomorfas provenientes dos Teoremas 4.65, 4.67 e 4.68: $A_{5}, B_{5}$ e $C_{5}$. Como elas são indecomponíveis, não são isomorfas às álgebras de Teorema 5·3.

As seguintes 10 álgebras de Jordan de dimensão 5 indecomponíveis têm segundo grupo de cohomologia nulo, isso foi calculado usando um programa de computação 
simbólica elaborado pela autora (veja Apêndice A) assim como também as dimensões dos respectivos grupos de automorfismos e aniquilador. O produto nas álgebras será dado em todos os casos na base $\left\{e_{1}, e_{2}, e_{3}, e_{4}, e_{5}\right\}$.

Tabela 5.7: Algumas álgebras de Jordan rígidas de dimensão 5

\begin{tabular}{|c|c|c|c|c|c|}
\hline e & Tabela de Multiplicação & $\begin{array}{c}\operatorname{dim} \\
\operatorname{Aut}(\mathcal{C})\end{array}$ & $\begin{array}{c}\operatorname{dim} \\
\operatorname{Ann}(\mathcal{C})\end{array}$ & $\begin{array}{c}\operatorname{dim} \\
\operatorname{Rad}(\mathcal{C})\end{array}$ & Obs. \\
\hline$e_{17}$ & $\begin{array}{c}e_{1}^{2}=e_{1} \quad e_{2}^{2}=e_{2} \quad e_{3}^{2}=e_{3} \\
e_{1} e_{5}=e_{2} e_{5}=\frac{1}{2} e_{5} \\
e_{2} e_{4}=e_{3} e_{4}=\frac{1}{2} e_{4}\end{array}$ & 4 & 0 & 2 & Unitária \\
\hline$e_{18}$ & 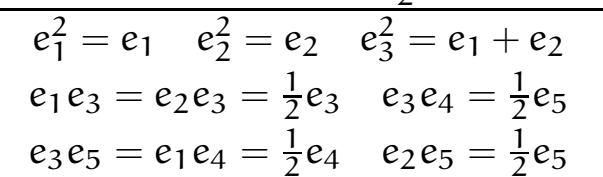 & 4 & 0 & 2 & \\
\hline$e_{19}$ & $\begin{array}{rrl}e_{1}^{2}=e_{1} & e_{2}^{2}=e_{2} & e_{3}^{2}=e_{5} \\
e_{4}^{2}=e_{5} & e_{1} e_{3}=\frac{1}{2} e_{3} & e_{2} e_{4}=\frac{1}{2} e_{4}\end{array}$ & 3 & 1 & 3 & \\
\hline$e_{20}$ & $\begin{array}{c}e_{1}^{2}=e_{1} \quad e_{2}^{2}=e_{2} \quad e_{3} e_{4}=e_{5} \\
e_{1} e_{3}=\frac{1}{2} e_{3} \quad e_{2} e_{4}=\frac{1}{2} e_{4} \\
e_{1} e_{5}=e_{2} e_{5}=\frac{1}{2} e_{5}\end{array}$ & 5 & 0 & 3 & \\
\hline$e_{21}$ & $\begin{array}{c}e_{1}^{2}=e_{1} \quad e_{2}^{2}=e_{2} \quad e_{3}^{2}=e_{4} \\
e_{5}^{2}=e_{4} \quad e_{1} e_{3}=\frac{1}{2} e_{3} \quad e_{1} e_{4}=e_{4} \\
e_{1} e_{5}=e_{2} e_{5}=\frac{1}{2} e_{5}\end{array}$ & 3 & 0 & 3 & \\
\hline$e_{22}$ & $\begin{array}{c}e_{1}^{2}=e_{1} \quad e_{2}^{2}=e_{2} \quad e_{4}^{2}=e_{5} \\
e_{1} e_{3}=\frac{1}{2} e_{3} \quad e_{2} e_{5}=e_{5} \\
e_{1} e_{4}=e_{2} e_{4}=\frac{1}{2} e_{4}\end{array}$ & 4 & 0 & 3 & \\
\hline $\mathrm{e}_{23}$ & $\begin{array}{c}e_{1}^{2}=e_{1} \quad e_{2}^{2}=e_{2} \quad e_{3}^{2}=e_{5} \\
e_{1} e_{3}=\frac{1}{2} e_{3} \quad e_{1} e_{4}=e_{2} e_{4}=\frac{1}{2} e_{4}\end{array}$ & 4 & 1 & 3 & \\
\hline$e_{24}$ & $\begin{array}{c}e_{1}^{2}=e_{1} \quad e_{1} e_{5}=\frac{1}{2} e_{5} \\
e_{1} e_{2}=e_{3}^{2}=e_{4} e_{5}=e_{2} \\
e_{1} e_{3}=\frac{1}{2} e_{3} \quad e_{1} e_{4}=\frac{1}{2} e_{4}\end{array}$ & 7 & 0 & 4 & \\
\hline$e_{25}$ & $\begin{array}{c}e_{1}^{2}=e_{1} \quad e_{1} e_{5}=\frac{1}{2} e_{5} \\
e_{1} e_{2}=e_{5}^{2}=e_{2} \quad e_{1} e_{4}=\frac{1}{2} e_{4} \\
e_{1} e_{3}=e_{4}^{2}=e_{4} e_{5}=e_{3}\end{array}$ & 4 & 0 & 4 & \\
\hline $\mathrm{e}_{26}$ & $\begin{array}{cl}e_{1}^{2}=e_{2}^{2} & =e_{3}^{2}=e_{4}^{2}=e_{5}^{2}=e_{1} \\
e_{1} e_{2} & =e_{2} \quad e_{1} e_{3}=e_{3} \\
e_{1} e_{4} & =e_{4} \quad e_{1} e_{5}=e_{5}\end{array}$ & 6 & 0 & 0 & $\begin{array}{l}\text { Unitária } \\
\text { Simples }\end{array}$ \\
\hline
\end{tabular}


Do Teorema 4.65 temos que $\operatorname{dim} \operatorname{Aut}\left(A_{5}\right)=20$ enquanto que

$$
\operatorname{dim} \operatorname{Aut}\left(B_{5}\right)=\operatorname{dim} \operatorname{Aut}\left(C_{5}\right)=8,
$$

logo fica claro pelas dimensões e pelo fato das álgebras serem decomponíveis ou indecomponíveis que todas as 26 álgebras são não isomorfas, logo em Jor 5 temos no mínimo 26 componentes irredutíveis. 



\section{6 \\ CLASSIFICAÇÃO GEOMÉTRICA DAS ÁLGEBRAS DE JORDAN DE DIMENSÃO MENOR OU IGUAL A 3 SO- BRE O CORPO DOS NÚMEROS REAIS}

Neste capítulo Jor $r_{n}^{\mathbb{R}}$ denotará a variedade algébrica das álgebra de Jordan de dimensão n sobre o corpo dos números reais $\mathbb{R}$.

É pouco o que se conhece em relação a deformações e álgebras rígidas sobre $\mathbb{R}$. Em 2007, Ancochea Bermúdez e outros classificaram algébrica e geometricamente as álgebras associativas reais de dimensão 2 no trabalho [5] e, posteriormente, as álgebras de Jordan reais de dimensão 2 em [3].

\subsection{A VARIEDADE ALGÉBRICA $\operatorname{jor}_{1}^{\mathbb{R}}$}

Analogamente ao caso sobre um corpo algebricamente fechado, em $\mathrm{Jor}_{1}^{\mathbb{R}}$ a única álgebra rígida é a álgebra simples $\mathbb{R e}$ e é claro que $\mathbb{R} e \rightarrow \mathbb{R} n$. Logo somente temos uma componente irredutível em Jor $_{1}^{\mathbb{R}}$ dada pelo fecho de Zariski da órbita da álgebra $\mathbb{R e}$, portanto Jor ${ }_{1}^{\mathbb{R}}$ é uma variedade afim conexa é irredutível de dimensão 1 com 2 órbitas sob a ação de GL(V).

\subsection{A VARIEDADE ALGÉBRICA $\operatorname{jor}_{2}^{\mathbb{R}}$}

No trabalho [3] os autores determinaram todas as contrações ${ }^{1}$ das álgebras de Jordan reais de dimensão 2 , isto nos permitiu determinar a existência de deformações entre tais álgebras e junto com os critérios de invariância através de deformações conseguimos dar uma descrição geométrica completa da variedade das álgebras de Jordan reais de dimensão 2.

Teorema 6.1. A variedade algébrica Jor $\mathbb{R}_{2}^{\mathbb{R}}$ é uma variedade afim conexa de dimensão 4 com 7 órbitas sob a ação de GL(V) e 3 componentes irredutíveis, duas de dimensão 4 dadas pelos fechos

1 Lembramos que se $\mathcal{J}$ é uma contração de $\mathcal{J}^{\prime}$ então $\mathcal{J}$ corresponde a um ponto no fecho da órbita de $\mathcal{J}^{\prime}, \log$ $\mathcal{J}^{\prime} \rightarrow \mathcal{J}$. 
de Zariski das órbitas das álgebras $\mathcal{B}_{4}^{\prime}$ e $\mathbb{R e}_{1} \oplus \mathbb{R e}_{2}$ e outra componente de dimensão 2 dada pelo fecho de Zariski da órbita da álgebra $\mathcal{B}_{2}^{\prime}$.

Demonstração. Segue do Lema 4.24 que Jor ${ }_{2}^{\mathbb{R}}$ é uma variedade afim e do Lema 4.40 sabemos que a variedade é conexa. Foi provado na Seção 3.1.2 que tem 7 órbitas sob a ação de $G L(V)$, elas são as órbitas representadas pelas álgebras $\mathcal{B}_{1}^{\prime}, \mathcal{B}_{2}^{\prime}, \mathcal{B}_{3}^{\prime}, \mathcal{B}_{4}^{\prime}, \mathbb{R e}_{1} \oplus \mathbb{R e}_{2}$, $\mathbb{R} e_{1} \oplus \mathbb{R} n_{1}$ e $\mathbb{R} n_{1} \oplus \mathbb{R} n_{2}$. Logo Jor $r_{2}^{\mathbb{R}}$ é uma união finita de órbitas que são conjuntos localmente fechados então a dimensão da variedade é o máximo das dimensões de suas órbitas

$$
\operatorname{dim} \operatorname{Dor}_{2}^{\mathbb{R}}=\operatorname{dim}\left(\mathcal{B}_{4}^{\prime}\right)^{\mathrm{G}}=2^{2}-\operatorname{dim} \operatorname{Aut}\left(\mathcal{B}_{4}^{\prime}\right)=4
$$

Em [3, Prop.6] foi provado que as órbitas das álgebras $\mathcal{B}_{2}^{\prime}, \mathcal{B}_{4}^{\prime}$ e $\mathbb{R} e_{1} \oplus \mathbb{R} e_{2}$ são abertas, logo seus fechos geram as componentes irredutíveis. Segue da Proposição 7 de [3] que $\mathcal{B}_{2}^{\prime}, \mathcal{B}_{4}^{\prime}$ e $\mathbb{R e}_{1} \oplus \mathbb{R} e_{2}$ são as únicas álgebras rígidas de $\mathcal{J o r}_{2}^{\mathbb{R}}$.

As deformações entre as álgebras de Jor ${ }_{2}^{\mathbb{R}}$ são descritas na Figura 6.1.

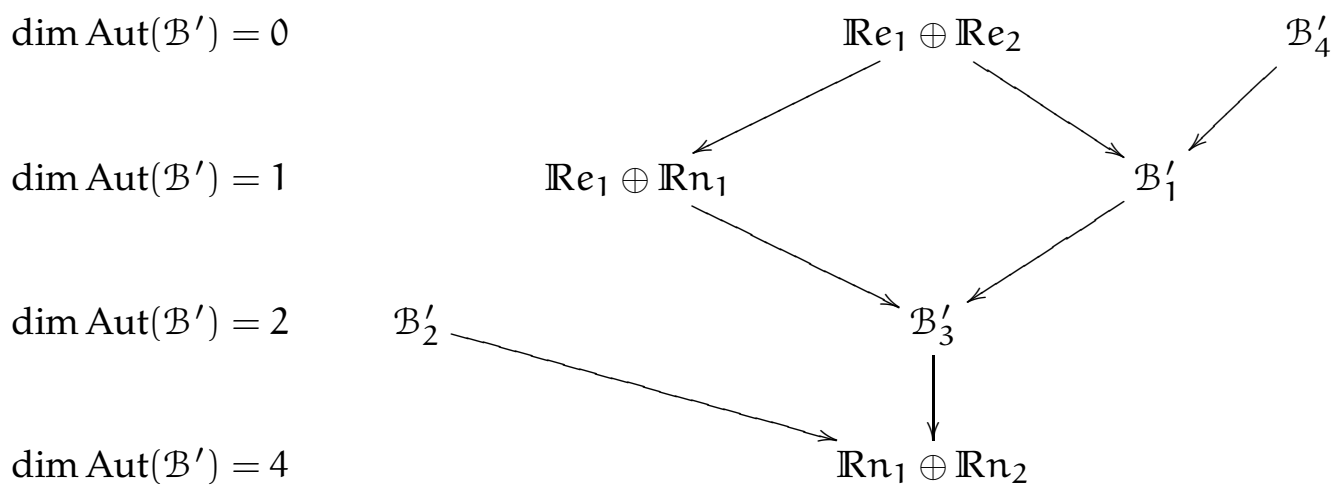

Fig. 6.1: Descrição completa das órbitas de Jorr

Um resumo da existência de deformações é dado na Tabela 6.1 e a simbologia respeita o padrão das tabelas do Capítulo 2 a menos de:

i. $\dashv_{R}$ : não existe deformação, pois foi provado em [3] que a álgebra da coluna é rígida;

ii. $\rightarrow$ : existe deformação e uma contração foi exibida em [3]. 
Tabela 6.1: Existência de deformações em Jor $_{2}^{\mathbb{R}}$

\begin{tabular}{|c|c|c|c|c|c|c|c|}
\hline & $\mathcal{B}_{1}^{\prime}$ & $\mathcal{B}_{2}^{\prime}$ & $\mathcal{B}_{3}^{\prime}$ & $\mathcal{B}_{4}^{\prime}$ & $\mathbb{R e}_{1} \oplus \mathbb{R} e_{2}$ & $\mathbb{R e}_{1} \oplus \mathbb{R} n_{1}$ & $\mathbb{R} n_{1} \oplus \mathbb{R} n_{2}$ \\
\hline $\mathcal{B}_{1}^{\prime}$ & $\rightarrow_{\mathrm{DT}}$ & $f_{R}$ & $\rightarrow$ & $A_{R}$ & $A_{R}$ & $\phi_{\text {Aut }}$ & $\rightarrow_{\mathrm{T}}$ \\
\hline $\mathcal{B}_{2}^{\prime}$ & $f_{\text {Aut }}$ & $\rightarrow_{\mathrm{DT}}$ & $t_{\text {Aut }}$ & $A_{R}$ & $\not A_{R}$ & $f_{\text {Aut }}$ & $\rightarrow$ \\
\hline $\mathcal{B}_{3}^{\prime}$ & $t_{A S}$ & $t_{R}$ & $\rightarrow_{\mathrm{DT}}$ & $t_{R}$ & $t_{R}$ & $A_{A S}$ & $\rightarrow$ \\
\hline $\mathcal{B}_{4}^{\prime}$ & $\rightarrow$ & $t_{R}$ & $\rightarrow_{\mathrm{T}}$ & $\rightarrow \rightarrow_{\mathrm{DT}}$ & $t_{R}$ & $t_{\text {npa }}$ & $\rightarrow_{\mathrm{T}}$ \\
\hline $\operatorname{Re}_{1} \oplus \mathbb{R} e_{2}$ & $\rightarrow$ & $t_{R}$ & $\rightarrow$ & $t_{R}$ & $\rightarrow_{\mathrm{DT}}$ & $\rightarrow$ & $\rightarrow \mathrm{T}$ \\
\hline $\mathbb{R} e_{1} \oplus \mathbb{R} n_{1}$ & $t_{\text {Aut }}$ & $t_{R}$ & $\rightarrow$ & $\not \rightarrow_{R}$ & $t_{R}$ & $\rightarrow$ DT & $\rightarrow_{\mathrm{T}}$ \\
\hline $\mathbb{R} n_{1} \oplus \mathbb{R} n_{2}$ & $t_{A S}$ & $A_{R}$ & $f_{A S}$ & $\not_{\mathrm{R}}$ & $f_{R}$ & $A_{A S}$ & $\rightarrow_{\mathrm{DT}}$ \\
\hline
\end{tabular}

\subsection{A VARIEDADE ALGÉBRICA $\operatorname{jor}_{3}^{\mathbb{R}}$}

Daremos a seguir uma descrição geométrica quase-completa das álgebras de Jordan reais de dimensão 3. Conseguimos determinar em um 97,6\% das vezes se uma álgebra de Jordan real de dimensão 3 pertence ou não ao fecho de Zariski da órbita de uma outra álgebra.

Teorema 6.2. A variedade algébrica Jor ${ }_{3}^{\mathbb{R}}$ é uma variedade afim conexa de dimensão 9 com 26 órbitas sob a ação de $\mathrm{GL}(\mathrm{V})$ e 8 componentes irredutíveis dadas pelos fechos de Zariski das órbitas das álgebras

$$
\Omega=\left\{\mathcal{T}_{1}^{\prime}, \mathcal{T}_{2}^{\prime}, \mathcal{T}_{3}^{\prime}, \mathcal{T}_{4}^{\prime}, \mathcal{T}_{5}^{\prime}, \mathcal{T}_{7}^{\prime}, \mathcal{T}_{12}^{\prime}, \mathcal{T}_{20}^{\prime}\right\},
$$

as quais determinam componentes de dimensões: 9, 9, 8, 8, 8, 7, 3 e 7, respetivamente.

Demonstração. Sabemos que $\operatorname{Jor}_{3}^{\mathbb{R}}$ é uma variedade afim pelo Lema 4.24, que é conexa segue do Lema 4.40 e que tem 26 órbitas sob a ação de $G$ foi provado na Seção 3.2. Assim temos que $\operatorname{Jor}_{3}^{\mathbb{R}}=\bigcup_{i=1}^{26}\left(\mathcal{T}_{i}^{\prime}\right)^{G}$ é uma união finita de órbitas que são conjuntos localmente fechados então a dimensão da variedade é o máximo das dimensões de suas órbitas

$$
\operatorname{dim} \operatorname{dor}_{3}^{\mathbb{R}}=\max _{1 \leqslant i \leqslant 26}\left\{\operatorname{dim} \mathcal{T}_{i}^{\prime G}\right\}=\operatorname{dim} \mathcal{T}_{1}^{\prime G}=3^{2}-\operatorname{dim} \operatorname{Aut}\left(\mathcal{T}_{1}^{\prime}\right)=9
$$

Analogamente à prova do Teorema 5.2, dividiremos o resto da prova em duas partes. Primeiramente, mostraremos que as álgebras em $\Omega$ são rígidas e depois provaremos que não existe outra álgebra rígida em $\mathrm{Jor}_{3}^{\mathbb{R}}$. 
As álgebras $\mathcal{T}_{1}^{\prime}$ e $\mathcal{T}_{2}^{\prime}$ têm $\operatorname{dim} \operatorname{Aut}\left(\mathcal{T}_{\mathfrak{i}}^{\prime}\right)=0$ logo, da Proposição 4.51, nenhuma outra álgebra pode ser uma deformação delas. Portanto $\mathcal{T}_{1}^{\prime}$ e $\mathcal{T}_{2}^{\prime}$ são álgebras rígidas. Pelo mesmo critério as únicas álgebras que podem ser deformação de $\mathcal{T}_{3}^{\prime}, \mathcal{T}_{4}^{\prime}$ e $\mathcal{T}_{5}^{\prime}$ são as álgebras $\mathcal{T}_{1}^{\prime}$ e $\mathcal{T}_{2}^{\prime}$ mas, da Proposição 4.56, uma álgebra associativa não pode ser uma deformação de uma álgebra não associativa. Logo $\mathcal{T}_{3}^{\prime}, \mathcal{T}_{4}^{\prime}$ e $\mathcal{T}_{5}^{\prime}$ também são as álgebras rígidas.

Nenhuma álgebra em $\mathcal{J o r}_{3}^{\mathbb{R}}$ domina $\mathcal{T}_{7}^{\prime}$ : pela Proposição 4.52 as únicas candidatas a serem deformação de $\mathcal{T}_{7}^{\prime}$ são as álgebras para as quais $\operatorname{dim} \operatorname{Rad}\left(\mathcal{T}_{\mathfrak{i}}^{\prime}\right) \leqslant 1$, i.e., as álgebras $\mathcal{T}_{1}^{\prime}$ a $\mathcal{T}_{11}^{\prime}$. Uma álgebra associativa não pode ser uma deformação de uma álgebra não associativa pela Proposição 4.56, logo podemos excluir da lista as álgebras $\mathcal{T}_{1}^{\prime}, \mathcal{T}_{2}^{\prime}, \mathcal{T}_{6}^{\prime}$, $\mathcal{T}_{9}^{\prime}$ e $\mathcal{T}_{10}^{\prime}$. Como a $\operatorname{dim} \operatorname{Aut}\left(\mathcal{T}_{i}^{\prime}\right)=2$, para $i=7,8,11$ nem $\mathcal{T}_{8}^{\prime}$ nem $\mathcal{T}_{11}^{\prime}$ podem ser deformações de $\mathcal{T}_{7}^{\prime}$ pois esta dimensão diminui através de deformações não triviais. A dimensão dos grupos de 2 -cociclos das três álgebras restantes, $\mathcal{T}_{i}^{\prime} \operatorname{com} i=3,4,5$, é 8 enquanto que $\operatorname{dim} Z^{2}\left(\mathcal{T}_{7}^{\prime}, \mathcal{T}_{7}^{\prime}\right)=7$, $\log$ o da Proposição 4.62 nenhuma delas domina $\mathcal{T}_{7}^{\prime}$. Concluímos que $\mathcal{T}_{7}^{\prime}$ somente tem as deformações triviais logo, da Proposição 4.39, $\mathcal{T}_{7}^{\prime}$ é rígida.

Observamos que a álgebra $\mathcal{T}_{12}^{\prime}$ é a álgebra $A_{3}$ do Teorema 4.65 logo é rígida.

Por último, vejamos que não existe uma álgebra em $\mathrm{Jor}_{3}^{\mathbb{R}}$ que deforme-se em $\mathcal{T}_{20}^{\prime}$ : Como $\operatorname{dim} \operatorname{Rad}\left(\mathcal{T}_{20}^{\prime}\right)=2$ nenhuma álgebra nilpotente, i.e., $\mathcal{T}_{\mathfrak{i}}^{\prime}$ para $22 \leqslant i \leqslant 26$, é deformação de $\mathcal{T}_{20}^{\prime}$. Pelo argumento da dimensão do grupo de automorfismos, nenhuma das álgebras $\mathcal{T}_{i}^{\prime}$ para $i=7,8,11, \cdots, 21$ domina $\mathcal{T}_{20}^{\prime}$. Também podemos excluir da lista as álgebras associativas $\mathcal{T}_{1}^{\prime}, \mathcal{T}_{2}^{\prime}, \mathcal{T}_{6}^{\prime}, \mathcal{T}_{9}^{\prime}$ e $\mathcal{T}_{10}^{\prime}$ pois $\mathcal{T}_{20}^{\prime}$ não é associativa. Por fim, $\mathcal{T}_{20}^{\prime}$ tem $\operatorname{dim} Z^{2}\left(\mathcal{T}_{20}^{\prime}, \mathcal{T}_{20}^{\prime}\right)=7$ o que implica que $\mathcal{T}_{i}^{\prime}$ para $i=3,4,5$ não é deformação de $\mathcal{T}_{20}^{\prime}$ portanto a álgebra $\mathcal{T}_{20}^{\prime}$ é rígida.

Vejamos agora que para cada álgebra $\mathcal{T}_{i}^{\prime}$ para $i=1, \cdots, 26$ da lista da Seção 3.2 existe uma álgebra $\mathcal{T}^{\prime} \in \Omega$ tal que $\mathcal{T}^{\prime}$ é uma deformação de $\mathcal{T}_{i}^{\prime}$. No que segue todas as deformações entre as álgebras serão dadas usando as bases do Capítulo 3, respeitando a ordem $\left\{e_{i}, n_{j}\right\}$ onde $1 \leqslant i, j \leqslant 3$.

Primeiramente, as órbitas das álgebras $\mathcal{T}_{6}^{\prime}, \mathcal{T}_{21}^{\prime}$ e $\mathcal{T}_{24}^{\prime}$, pertencem a $\overline{\mathcal{T}_{1}^{\prime \mathrm{G}}}$ : combinando as deformações para álgebras de Jordan reais de dimensão 2 obtidas na Seção 6.2 com a Proposição 4.41 temos: $\mathcal{T}_{1}^{\prime} \rightarrow \mathcal{T}_{6}^{\prime}$ e $\mathcal{T}_{6}^{\prime} \rightarrow \mathcal{T}_{21}^{\prime}$. Se consideramos a família de automorfismos de $\mathcal{T}_{21}^{\prime}$ dada por $A_{t}=\mathrm{tn}_{1}, B_{\mathrm{t}}=\mathrm{t}^{2} \mathrm{e}_{1}-\mathrm{n}_{2}$ e $C_{\mathrm{t}}=\mathrm{t}^{2} \mathrm{n}_{2}$ fazendo $\mathrm{t}$ tender a 0 obtemos a álgebra $\mathcal{T}_{24}^{\prime}$. Logo $\mathcal{T}_{21}^{\prime} \rightarrow \mathcal{T}_{24}^{\prime}$.

As órbitas das álgebras $\mathcal{T}_{9}^{\prime}, \mathcal{T}_{10}^{\prime}, \mathcal{T}_{13}^{\prime}, \mathcal{T}_{15}^{\prime}, \mathcal{T}_{18}^{\prime}, \mathcal{T}_{23}^{\prime}, \mathcal{T}_{25}^{\prime}$, e $\mathcal{T}_{26}^{\prime}$, pertencem a $\overline{\mathcal{T}_{2}^{\prime G}}$ : novamente, combinando as deformações para álgebras de Jordan reais de dimensão 2 obtidas na Seção 6.2 com a Proposição 4.41 temos: $\mathcal{T}_{2}^{\prime} \rightarrow \mathcal{T}_{9}^{\prime}, \mathcal{T}_{2}^{\prime} \rightarrow \mathcal{T}_{10}^{\prime}$ e $\mathcal{T}_{10}^{\prime} \rightarrow \mathcal{T}_{15}^{\prime}$. Observamos que as deformações entre álgebras de Jordan de dimensão 3 sobre um 
corpo algebricamente fechado obtidas na Seção $5 \cdot 3$ servem para as álgebras consideradas sobre $\mathbb{R}$ pois as famílias de automorfismos que dão tais deformações pertencem na verdade a $\mathrm{GL}\left(\mathbb{R}^{3}\right) \subseteq \mathrm{GL}\left(\mathbb{C}^{3}\right)$. Logo temos $\mathcal{T}_{9}^{\prime} \rightarrow \mathcal{T}_{18}^{\prime}, \mathcal{T}_{18}^{\prime} \rightarrow \mathcal{T}_{13}^{\prime}, \mathcal{T}_{15}^{\prime} \rightarrow \mathcal{T}_{23}^{\prime}, \mathcal{T}_{23}^{\prime} \rightarrow \mathcal{T}_{26}^{\prime}$ e $\mathcal{T}_{26}^{\prime} \rightarrow \mathcal{T}_{25}^{\prime}$.

A álgebra rígida $\mathcal{T}_{3}^{\prime}$ domina $\mathcal{T}_{8}^{\prime}$ e $\mathcal{T}_{14}^{\prime}$ : considere, para $\mathrm{t} \neq 0$, a família de automorfismos de $\mathcal{T}_{3}^{\prime}$ dada por $A_{t}=\frac{1}{2} e_{1}+\frac{1}{2} e_{2}, B_{t}=\frac{1}{2} e_{1}-\frac{1}{2} e_{2}$ e $C_{t}=t e_{3}$ quando fazemos $t$ tender a 0 obtemos a álgebra $\mathcal{T}_{8}^{\prime}, \operatorname{logo} \mathcal{T}_{3}^{\prime} \rightarrow \mathcal{T}_{8}^{\prime}$. Da Seção 5.3 obtemos $\mathcal{T}_{8}^{\prime} \rightarrow \mathcal{T}_{14}^{\prime}$.

A álgebra $\mathcal{T}_{11}^{\prime}$ pertence ao fecho da órbita de $\mathcal{T}_{4}^{\prime}$. Para ver isso considere a família de automorfismos de $\mathcal{T}_{4}$ dada por $A_{t}=e_{1}, B_{t}=e_{2}$ e $C_{t}=t e_{3}$ quando fazemos $t$ tender a 0 obtemos a álgebra $\mathcal{T}_{11}^{\prime}$. Da Seção 5.3 temos que a álgebra rígida $\mathcal{T}_{5}^{\prime}$ domina $\mathcal{T}_{19}^{\prime}$, analogamente $\mathcal{T}_{20}^{\prime}$ domina $\mathcal{T}_{16}^{\prime}$ e da Seção 6.2 segue que $\mathcal{T}_{7}^{\prime} \rightarrow \mathcal{T}_{17}^{\prime}$.

Por último, segue do Lema 4.40 que todo $\mathcal{T}^{\prime} \in \mathcal{J o r}_{3}^{\mathbb{R}}$ é uma deformação de $\mathcal{T}_{22}^{\prime}$. Isto completa a prova.

Para dar uma classificação geométrica o mais completa possível das álgebras de Jordan reais de dimensão 3 descreveremos a seguir todas as deformações que conseguimos achar em jor ${ }_{3}^{\mathbb{R}}$ :

Da combinação das deformações obtida na Seção 6.2 com a Proposição 4.41 temos as seguintes deformações dado que as álgebras envolvidas são soma direta de uma álgebra de Jordan real de dimensão 2 e $\mathbb{R e}$ ou $\mathbb{R} n$ :

$$
\begin{aligned}
& \mathcal{T}_{1}^{\prime} \rightarrow \mathcal{T}_{9}^{\prime} ; \quad \mathcal{T}_{6}^{\prime} \rightarrow \mathcal{T}_{15}^{\prime} ; \quad \mathcal{T}_{7}^{\prime} \rightarrow \mathcal{T}_{14}^{\prime} ; \quad \mathcal{T}_{21}^{\prime} \rightarrow \mathcal{T}_{17}^{\prime} \\
& \mathcal{T}_{9}^{\prime} \rightarrow \mathcal{T}_{15}^{\prime} ; \quad \mathcal{T}_{9}^{\prime} \rightarrow \mathcal{T}_{21}^{\prime} ; \quad \mathcal{T}_{17}^{\prime} \rightarrow \mathcal{T}_{25}^{\prime} .
\end{aligned}
$$

Da Seção $5 \cdot 3$ temos as seguintes deformações sobre $\mathbb{R}$ :

$$
\begin{aligned}
& \mathcal{T}_{5}^{\prime} \rightarrow \mathcal{T}_{8}^{\prime} ; \quad \mathcal{T}_{8}^{\prime} \rightarrow \mathcal{T}_{13}^{\prime} ; \quad \mathcal{T}_{19}^{\prime} \rightarrow \mathcal{T}_{14}^{\prime} ; \\
& \mathcal{T}_{18}^{\prime} \rightarrow \mathcal{T}_{23}^{\prime} ; \quad \mathcal{T}_{7}^{\prime} \rightarrow \mathcal{T}_{16}^{\prime} ; \quad \mathcal{T}_{21}^{\prime} \rightarrow \mathcal{T}_{23}^{\prime} ; \\
& \mathcal{T}_{14}^{\prime} \rightarrow \mathcal{T}_{26}^{\prime} ; \quad \mathcal{T}_{16}^{\prime} \rightarrow \mathcal{T}_{26}^{\prime} ; \quad \mathcal{T}_{13}^{\prime} \rightarrow \mathcal{T}_{25}^{\prime} .
\end{aligned}
$$

E das seguintes famílias de automorfismos obtemos as respectivas deformações:

i. Considere, para $t \neq 0$, a mudança de base $A_{t}=e_{1}, B_{t}=t e_{2}$ e $C_{t}=t n_{1}$ de $\mathcal{T}_{11}^{\prime}$. Fazendo $t$ tender a zero obtemos a estrutura da álgebra $\mathcal{T}_{13}^{\prime}$. Logo $\mathcal{T}_{11}^{\prime} \rightarrow \mathcal{T}_{13}^{\prime}$.

ii. Analogamente se consideramos a base $A_{t}=\frac{1}{2} e_{1}+\frac{1}{2} e_{2}, B_{t}=t e_{3}$ e $C_{t}=-\frac{t^{2}}{2} e_{1}+$ $\frac{t^{2}}{2} e_{2}$ de $\mathcal{T}_{3}^{\prime}$ obtemos a álgebra $\mathcal{T}_{19}^{\prime}$ quando $t$ tende a zero. Portanto, $\mathcal{T}_{3}^{\prime} \rightarrow \mathcal{T}_{19}^{\prime}$.

iii. A mudança de base $A_{t}=n_{1}, B_{t}=n_{3}$ e $C_{t}=\operatorname{tn} 2$ de $\mathcal{T}_{24}^{\prime}$, nos da $\mathcal{T}_{24}^{\prime} \rightarrow \mathcal{T}_{25}^{\prime}$. 
iv. A mudança de base $A_{t}=\frac{t}{2} e_{1}-\frac{t}{2} e_{2}, B_{t}=\frac{t}{2} e_{1}+\frac{t}{2} e_{2}+2 t^{2} n_{1}$ e $C_{t}=\frac{t^{2}}{2} e_{1}-\frac{t^{2}}{2} e_{2}+$ $t^{3} n_{1}$ de $\mathcal{T}_{11}^{\prime}$ tende a $\mathcal{T}_{24}^{\prime}$ quando $t \rightarrow 0$ o que implica que $\mathcal{T}_{11}^{\prime} \rightarrow \mathcal{T}_{24}^{\prime}$.

v. Para o caso da deformação $\mathcal{T}_{20}^{\prime} \rightarrow \mathcal{T}_{24}^{\prime}$ considere a família de automorfismo $A_{t}=$ $t e_{1}+t^{3} n_{2}, B_{t}=t^{2} n_{1}$ e $C_{t}=t^{2} e_{1}+2 t^{4} n_{2}$ de $\mathcal{T}_{20}^{\prime}$, passando o limite quando $\mathrm{t} \rightarrow 0$ obtemos a álgebra $\mathcal{T}_{24}^{\prime}$.

vi. A família de automorfismos $A_{t}=t e_{1}+-t_{2}, B_{t}=t n_{1}+n_{2}$ e $C_{t}=t^{2} e_{1}$ de $\mathcal{T}_{19}^{\prime}$ nos da $\mathcal{T}_{19}^{\prime} \rightarrow \mathcal{T}_{24}^{\prime}$.

vii. Se considerarmos a mudança de base $A_{t}=t e_{1}+t n_{2}, B_{t}=t n_{1}$ e $C_{t}=t^{2} e_{1}+$ $2 t^{2} n_{2}$ de $\mathcal{T}_{18}^{\prime}$, fazendo $t \rightarrow 0$ temos a álgebra $\mathcal{T}_{24}^{\prime}$ e portanto $\mathcal{T}_{18}^{\prime} \rightarrow \mathcal{T}_{24}^{\prime}$.

viii. Por último, a mudança de base $A_{t}=e_{1}, B_{t}=e_{2}+\sqrt{2} e_{3}$ e $C_{t}=t e_{2}+\frac{t}{\sqrt{2}} e_{3}$ de $\mathcal{T}_{3}^{\prime}$ nos da $\mathcal{T}_{3}^{\prime} \rightarrow \mathcal{T}_{11}^{\prime}$.

Mais uma vez resumimos a existência ou não existência de deformações em 3 tabelas. A simbologia utilizada respeita o padrão das tabelas dos capítulos e seções anteriores. 


\begin{tabular}{|c|c|c|c|c|c|c|c|c|c|c|c|c|c|}
\hline$\rightarrow$ & $\mathcal{T}_{1}^{\prime}$ & $\mathcal{T}_{2}^{\prime}$ & $\mathcal{T}_{3}^{\prime}$ & $\mathcal{T}_{4}^{\prime}$ & $\mathcal{T}_{5}^{\prime}$ & $\mathcal{T}_{6}^{\prime}$ & $\mathcal{T}_{7}^{\prime}$ & $\mathcal{T}_{8}^{\prime}$ & $\mathcal{T}_{9}^{\prime}$ & $\mathcal{T}_{10}^{\prime}$ & $\mathcal{T}_{11}^{\prime}$ & $\mathcal{T}_{12}^{\prime}$ & $\mathcal{T}_{13}^{\prime}$ \\
\hline $\mathcal{T}_{1}^{\prime}$ & $\rightarrow_{\mathrm{DT}}$ & $f_{\text {Aut }}$ & $A_{\text {Ass }}$ & $\not_{\text {Ass }}$ & $f_{\text {Ass }}$ & $\rightarrow$ & $f_{\text {Ass }}$ & $f_{\text {Ass }}$ & $\rightarrow$ & $A_{\text {npa }}$ & $A_{\mathrm{Ass}}$ & $f_{\text {Ass }}$ & $\rightarrow_{\mathrm{T}}$ \\
\hline $\mathcal{T}_{2}^{\prime}$ & $A_{\text {Aut }}$ & $\rightarrow{ }_{\mathrm{DT}}$ & $A_{\text {Ass }}$ & $\not \lambda_{\text {Ass }}$ & $\not \lambda_{\text {Ass }}$ & $A_{\text {npa }}$ & $A_{\text {Ass }}$ & $\not \lambda_{\text {Ass }}$ & $\rightarrow$ & $\rightarrow$ & $A_{\text {Ass }}$ & $\not \rightarrow_{\text {Ass }}$ & $\rightarrow_{\mathrm{T}}$ \\
\hline $\mathcal{T}_{3}^{\prime}$ & $A_{\text {Aut }}$ & $\lambda_{\text {Aut }}$ & $\rightarrow \mathrm{DT}$ & $\lambda_{\text {Aut }}$ & $f_{\text {Aut }}$ & $f_{\text {Aut }}$ & $f_{\text {npa }}$ & $\rightarrow$ & $A_{\text {Aut }}$ & $A_{\text {Aut }}$ & $\rightarrow$ & $A_{\text {npa }}$ & $\rightarrow_{\mathrm{T}}$ \\
\hline $\mathcal{T}_{4}^{\prime}$ & $f_{\text {Aut }}$ & $f_{\text {Aut }}$ & $\phi_{\text {Aut }}$ & $\rightarrow_{\mathrm{DT}}$ & $\phi_{\text {Aut }}$ & $\phi_{\text {Aut }}$ & $f_{\text {npa }}$ & $f_{\text {npa }}$ & $f_{\text {Aut }}$ & $A_{\text {Aut }}$ & $\rightarrow$ & $A_{\mathrm{npa}}$ & $\rightarrow_{\mathrm{T}}$ \\
\hline $\mathcal{T}_{5}^{\prime}$ & $t_{\text {Aut }}$ & $f_{\text {Aut }}$ & $A_{\text {Aut }}$ & $A_{\text {Aut }}$ & $\rightarrow \mathrm{DT}$ & $t_{\text {Aut }}$ & $A_{\text {npa }}$ & $\rightarrow$ & $t_{\text {Aut }}$ & $t_{\text {Aut }}$ & $f_{\text {npa }}$ & $A_{\text {npa }}$ & $\rightarrow_{\mathrm{T}}$ \\
\hline $\mathcal{T}_{6}^{\prime}$ & $\not_{\text {Aut }}$ & $A_{\text {Aut }}$ & $A_{\text {Aut }}$ & $A_{\text {Aut }}$ & $A_{\text {Aut }}$ & $\rightarrow \rightarrow_{\mathrm{DT}}$ & $A_{\text {Ass }}$ & $A_{\text {Ass }}$ & $\not_{\text {Ann }}$ & $A_{\text {npa }}$ & $\not_{\text {Ass }}$ & $\not_{\text {Ass }}$ & $A_{\text {Ann }}$ \\
\hline $\mathcal{T}_{7}^{\prime}$ & $\not_{\text {Aut }}$ & $A_{\text {Aut }}$ & $A_{\text {Aut }}$ & $A_{\text {Aut }}$ & $A_{\text {Aut }}$ & $A_{\text {Aut }}$ & $\rightarrow{ }_{\mathrm{DT}}$ & $\not_{\text {Aut }}$ & $A_{\text {Aut }}$ & $A_{\text {Aut }}$ & $A_{\text {Aut }}$ & $A_{\text {npa }}$ & $A_{\text {npa }}$ \\
\hline $\mathcal{T}_{8}^{\prime}$ & $A_{\text {Aut }}$ & $f_{\text {Aut }}$ & $A_{\text {Aut }}$ & $\lambda_{\text {Aut }}$ & $f_{\text {Aut }}$ & $A_{\text {Aut }}$ & $A_{\text {Aut }}$ & $\rightarrow{ }_{\mathrm{DT}}$ & $f_{\text {Aut }}$ & $A_{\text {Aut }}$ & $A_{\text {Aut }}$ & $A_{\text {npa }}$ & $\rightarrow$ \\
\hline $\mathcal{T}_{9}^{\prime}$ & $A_{\text {Aut }}$ & $\phi_{\text {Aut }}$ & $\phi_{\text {Aut }}$ & $\phi_{\text {Aut }}$ & $A_{\text {Aut }}$ & $A_{\text {npa }}$ & $\lambda_{\text {Ass }}$ & $f_{\text {Ass }}$ & $\rightarrow \mathrm{DT}$ & $A_{\text {npa }}$ & $A_{\text {Ass }}$ & $A_{\text {Ass }}$ & $\rightarrow \rightarrow_{\mathrm{T}}$ \\
\hline $\mathcal{T}_{10}^{\prime}$ & $\not \rightarrow{ }_{\text {Aut }}$ & $A_{\text {Aut }}$ & $\not \rightarrow$ Aut & $\not \rightarrow$ Aut & $f_{\text {Aut }}$ & $f_{\text {npa }}$ & $\not \rightarrow$ Ass & $\not \rightarrow$ Ass & $A_{\text {Ann }}$ & $\rightarrow \mathrm{DT}$ & $\not \rightarrow$ Ass & $\not \rightarrow$ Ass & $f_{\text {Ann }}$ \\
\hline $\mathcal{T}_{11}^{\prime}$ & $f_{\text {Aut }}$ & $f_{\text {Aut }}$ & $\nexists_{\text {Aut }}$ & $t_{\text {Aut }}$ & $f_{\text {Aut }}$ & $f_{\text {Aut }}$ & $f_{\text {Aut }}$ & $f_{\text {npa }}$ & $f_{\text {Aut }}$ & $f_{\text {Aut }}$ & $\rightarrow{ }_{\mathrm{DT}}$ & $f_{\text {npa }}$ & $\rightarrow$ \\
\hline$\frac{\mathcal{T}_{12}^{\prime}}{\tau^{\prime}}$ & $\not_{\text {Aut }}$ & $A_{\text {Aut }}$ & $\lambda_{\text {Aut }}$ & $A_{\text {Aut }}$ & $A_{\text {Aut }}$ & $A_{\text {Aut }}$ & $\not_{\text {Aut }}$ & $A_{\text {Aut }}$ & $A_{\text {Aut }}$ & $A_{\text {Aut }}$ & $\not_{\text {Aut }}$ & $\rightarrow \mathrm{DT}$ & $A_{\text {Aut }}$ \\
\hline $\mathcal{T}_{13}^{\prime}$ & $f_{\text {Aut }}$ & $\lambda_{\text {Aut }}$ & $\lambda_{\text {Aut }}$ & $\lambda_{\text {Aut }}$ & $A_{\text {Aut }}$ & $A_{\text {Aut }}$ & $A_{\text {Ass }}$ & $f_{\text {Ass }}$ & $f_{\text {Aut }}$ & $A_{\text {Aut }}$ & $\not \rightarrow_{\text {Ass }}$ & $f_{\text {Ass }}$ & $\rightarrow_{\mathrm{DT}}$ \\
\hline $\mathcal{T}_{14}^{\prime}$ & $\not \rightarrow_{\text {Aut }}$ & $A_{\text {Aut }}$ & $\not \rightarrow$ Aut & $\not \rightarrow_{\text {Aut }}$ & $f_{\text {Aut }}$ & $f_{\text {Aut }}$ & $\not \rightarrow_{\text {Aut }}$ & $t_{\text {Aut }}$ & $t_{\text {Aut }}$ & $f_{\text {Aut }}$ & $\not \rightarrow_{\text {Aut }}$ & $t_{A}$ Ann & $f_{\text {Ann }}$ \\
\hline $\mathcal{T}_{15}^{\prime}$ & $\nrightarrow_{\text {Aut }}$ & $\phi_{\text {Aut }}$ & $\lambda_{\text {Aut }}$ & $\nrightarrow_{\text {Aut }}$ & $\nexists_{\text {Aut }}$ & $\nexists_{\text {Aut }}$ & $t_{\text {Ass }}$ & $t_{\text {Ass }}$ & $A_{\text {Aut }}$ & $\nrightarrow_{\text {Aut }}$ & $\not_{\text {Ass }}$ & $t_{\text {Ass }}$ & $t_{\mathrm{Ann}}$ \\
\hline $\mathcal{T}_{16}^{\prime}$ & $\nrightarrow_{\text {Aut }}$ & $\lambda_{\text {Aut }}$ & $\lambda_{\text {Aut }}$ & $\lambda_{\text {Aut }}$ & $\nexists_{\text {Aut }}$ & $\nexists_{\text {Aut }}$ & $\Rightarrow_{\text {Aut }}$ & $f_{\text {Aut }}$ & $f_{\text {Aut }}$ & $\nexists_{\text {Aut }}$ & $\lambda_{\text {Aut }}$ & $f_{\text {npa }}$ & $t_{\text {npa }}$ \\
\hline $\mathcal{T}_{17}^{\prime}$ & $A_{\text {Aut }}$ & $A_{\text {Aut }}$ & $\not \rightarrow$ Aut & $\not \rightarrow{ }_{\text {Aut }}$ & $f_{\text {Aut }}$ & $f_{\text {Aut }}$ & $\not \rightarrow$ Ass & $\not \rightarrow$ Ass & $t_{\text {Aut }}$ & $f_{\text {Aut }}$ & $\not \rightarrow_{\text {Ass }}$ & $\not \rightarrow_{\text {Ass }}$ & $A_{\text {Aut }}$ \\
\hline $\mathcal{T}_{18}^{\prime}$ & $A_{\text {Aut }}$ & $\nexists_{\text {Aut }}$ & $\nexists_{\text {Aut }}$ & $\nrightarrow_{\text {Aut }}$ & $f_{\text {Aut }}$ & $f_{\text {Aut }}$ & $f_{\text {Ass }}$ & $\not \rightarrow$ Ass & $A_{\text {Aut }}$ & $f_{\text {Aut }}$ & $\not \rightarrow$ Ass & $t_{\text {Ass }}$ & $\rightarrow$ \\
\hline $\mathcal{T}_{19}^{\prime}$ & $\nrightarrow_{\text {Aut }}$ & $\nexists_{\text {Aut }}$ & $A_{\text {Aut }}$ & $\nexists_{\text {Aut }}$ & $f_{\text {Aut }}$ & $f_{\text {Aut }}$ & $\not \rightarrow$ Aut & $t_{\mathrm{Rad}}$ & $t_{\text {Aut }}$ & $\nexists_{\text {Aut }}$ & $\not>_{\mathrm{Rad}}$ & $\not_{\text {Ann }}$ & $f_{\text {Ann }}$ \\
\hline $\mathcal{T}_{20}^{\prime}$ & $t_{\text {Aut }}$ & $\lambda_{\text {Aut }}$ & $t_{\text {Aut }}$ & $\lambda_{\text {Aut }}$ & $\nexists_{\text {Aut }}$ & $\nexists_{\text {Aut }}$ & $\nexists_{\text {Aut }}$ & $\not t_{\mathrm{Rad}}$ & $f_{\text {Aut }}$ & $A_{\text {Aut }}$ & $t_{\mathrm{Rad}}$ & $\not_{\mathrm{npa}}$ & $\not A_{n p a}$ \\
\hline $\mathcal{T}_{21}^{\prime}$ & $A_{\text {Aut }}$ & $\not \rightarrow$ Aut & $A_{\text {Aut }}$ & $A_{\text {Aut }}$ & $f_{\text {Aut }}$ & $f_{\text {Aut }}$ & $\not \rightarrow$ Ass & $\not \rightarrow$ Ass & $t_{\text {Aut }}$ & $f_{\text {Aut }}$ & $\not A_{\text {Ass }}$ & $\not \rightarrow$ Ass & $A_{\text {Ann }}$ \\
\hline
\end{tabular}

Tabela 6.2: Existência de deformações em $\operatorname{Dor}_{3}^{\mathbb{R}}$ 


\begin{tabular}{|c|c|c|c|c|c|c|c|c|c|c|c|c|c|}
\hline$\rightarrow$ & $\mathcal{T}_{14}^{\prime}$ & $\mathcal{T}_{15}^{\prime}$ & $\mathcal{T}_{16}^{\prime}$ & $\mathcal{T}_{17}^{\prime}$ & $\mathcal{T}_{18}^{\prime}$ & $\mathcal{T}_{19}^{\prime}$ & $\mathcal{T}_{20}^{\prime}$ & $\mathcal{T}_{21}^{\prime}$ & $\mathcal{T}_{22}^{\prime}$ & $\mathcal{T}_{23}^{\prime}$ & $\mathcal{T}_{24}^{\prime}$ & $\mathcal{T}_{25}^{\prime}$ & $\mathcal{T}_{26}^{\prime}$ \\
\hline $\mathcal{T}_{1}^{\prime}$ & $\not \rightarrow$ Ass & $\rightarrow \mathrm{T}$ & $\not \rightarrow$ Ass & $\rightarrow_{\mathrm{T}}$ & $\rightarrow_{\mathrm{T}}$ & $f_{\text {Ass }}$ & $\not A_{\text {Ass }}$ & $\rightarrow_{\mathrm{T}}$ & $\rightarrow_{\mathrm{T}}$ & $\rightarrow_{\mathrm{T}}$ & $\rightarrow \mathrm{T}$ & $\rightarrow_{\mathrm{T}}$ & $\rightarrow \mathrm{T}$ \\
\hline $\mathcal{T}_{2}^{\prime}$ & $f_{\text {Ass }}$ & $\rightarrow_{\mathrm{T}}$ & $f_{\text {Ass }}$ & $\rightarrow_{\mathrm{T}}$ & $\rightarrow_{\mathrm{T}}$ & $f_{\text {Ass }}$ & $\not \rightarrow_{\text {Ass }}$ & $\rightarrow_{\mathrm{T}}$ & $\rightarrow_{\mathrm{T}}$ & $\rightarrow_{\mathrm{T}}$ & $\rightarrow_{\mathrm{T}}$ & $\rightarrow_{\mathrm{T}}$ & $\rightarrow_{\mathrm{T}}$ \\
\hline $\mathcal{T}_{3}^{\prime}$ & $\rightarrow_{\mathrm{T}}$ & $A_{\text {npa }}$ & $A_{\mathrm{npa}}$ & $A_{\text {npa }}$ & $\stackrel{?}{-\rightarrow}$ & $\rightarrow$ & $\not A_{\mathrm{npa}}$ & $A_{\text {npa }}$ & $\rightarrow \mathrm{T}$ & $\stackrel{?}{-\rightarrow}$ & $\rightarrow_{\mathrm{T}}$ & $\rightarrow_{\mathrm{T}}$ & $\rightarrow_{\mathrm{T}}$ \\
\hline $\mathcal{T}_{4}^{\prime}$ & $f_{\text {npa }}$ & $A_{\text {npa }}$ & $A_{\text {npa }}$ & $A_{\text {npa }}$ & $\begin{array}{l}? \\
-\stackrel{-\rightarrow}{*} \\
\end{array}$ & $A_{\text {npa }}$ & $A_{\text {npa }}$ & $A_{\text {npa }}$ & $\rightarrow_{\mathrm{T}}$ & $\begin{array}{l}? \\
-\rightarrow \\
\end{array}$ & $\rightarrow_{\mathrm{T}}$ & $\rightarrow_{\mathrm{T}}$ & $\begin{array}{l}? \\
-\stackrel{-\rightarrow}{*} \\
\end{array}$ \\
\hline $\mathcal{T}_{5}^{\prime}$ & $\rightarrow_{\mathrm{T}}$ & $A_{\text {npa }}$ & $A_{\text {npa }}$ & $A_{\text {npa }}$ & $\stackrel{?}{-\rightarrow}$ & $\rightarrow$ & $A_{\text {npa }}$ & $A_{\text {npa }}$ & $\rightarrow \mathrm{T}$ & $\stackrel{?}{-\rightarrow}$ & $\rightarrow_{\mathrm{T}}$ & $\rightarrow_{\mathrm{T}}$ & $\rightarrow_{\mathrm{T}}$ \\
\hline $\mathcal{T}_{6}^{\prime}$ & $\not A_{\text {Ass }}$ & $\rightarrow$ & $\not A_{\text {Ass }}$ & $\rightarrow_{\mathrm{T}}$ & $\not_{\text {Ann }}$ & $\not A_{\text {Ass }}$ & $\not \rightarrow$ Ass & $\rightarrow$ & $\rightarrow_{\mathrm{T}}$ & $\rightarrow_{\mathrm{T}}$ & $\rightarrow_{\mathrm{T}}$ & $\rightarrow_{\mathrm{T}}$ & $\rightarrow_{\mathrm{T}}$ \\
\hline $\mathcal{T}_{7}^{\prime}$ & $\rightarrow$ & $t_{\text {Aut }}$ & $\rightarrow$ & $\rightarrow$ & $t_{\text {Aut }}$ & $A_{\text {Aut }}$ & $t_{\text {Aut }}$ & $A_{\text {Aut }}$ & $\rightarrow_{\mathrm{T}}$ & $\stackrel{?}{--\rightarrow}$ & $\stackrel{?}{--\rightarrow}$ & $\rightarrow_{\mathrm{T}}$ & $\rightarrow_{\mathrm{T}}$ \\
\hline $\mathcal{T}_{8}^{\prime}$ & $\rightarrow$ & $A_{\text {Aut }}$ & $A_{\text {npa }}$ & $\not_{\text {npa }}$ & $A_{\text {Aut }}$ & $\nrightarrow_{\text {Aut }}$ & $\nrightarrow_{\text {Aut }}$ & $A_{\text {Aut }}$ & $\rightarrow_{\mathrm{T}}$ & $\stackrel{?}{-\rightarrow}$ & $\stackrel{?}{-\rightarrow}$ & $\rightarrow_{\mathrm{T}}$ & $\rightarrow_{\mathrm{T}}$ \\
\hline $\mathcal{T}_{9}^{\prime}$ & $\not t_{\text {Ass }}$ & $\rightarrow$ & $f_{\text {Ass }}$ & $\rightarrow_{\mathrm{T}}$ & $\rightarrow$ & $f_{\text {Ass }}$ & $f_{\text {Ass }}$ & $\rightarrow$ & $\rightarrow_{\mathrm{T}}$ & $\rightarrow_{\mathrm{T}}$ & $\rightarrow_{\mathrm{T}}$ & $\rightarrow_{\mathrm{T}}$ & $\rightarrow_{\mathrm{T}}$ \\
\hline $\mathcal{T}_{10}^{\prime}$ & $\not A_{\text {Ass }}$ & $\rightarrow$ & $\not A_{\text {Ass }}$ & $f_{\text {npa }}$ & $t_{\text {Ann }}$ & $f_{\text {Ass }}$ & $A_{\text {Ass }}$ & $f_{\text {npa }}$ & $\rightarrow_{\mathrm{T}}$ & $\rightarrow_{\mathrm{T}}$ & $\stackrel{?}{--\rightarrow}$ & $\rightarrow_{\mathrm{T}}$ & $\rightarrow_{\mathrm{T}}$ \\
\hline $\mathcal{T}_{11}^{\prime}$ & $t_{\text {npa }}$ & $A_{\text {npa }}$ & $f_{\text {npa }}$ & $f_{\text {npa }}$ & $\nrightarrow_{\text {Aut }}$ & $\not_{\text {npa }}$ & $A_{\text {Aut }}$ & $f_{\text {npa }}$ & $\rightarrow_{\mathrm{T}}$ & $\stackrel{?}{-\rightarrow}$ & $\rightarrow$ & $\rightarrow_{\mathrm{T}}$ & $\stackrel{?}{-\rightarrow}$ \\
\hline $\mathcal{T}_{12}^{\prime}$ & $A_{\text {Aut }}$ & $A_{\text {Aut }}$ & $A_{\text {Aut }}$ & $A_{\text {Aut }}$ & $\lambda_{\text {Aut }}$ & $A_{\text {Aut }}$ & $A_{\text {Aut }}$ & $A_{\text {Aut }}$ & $\rightarrow$ & $\lambda_{\text {Aut }}$ & $A_{\text {Aut }}$ & $f_{\text {Aut }}$ & $A_{\text {Aut }}$ \\
\hline $\mathcal{T}_{13}^{\prime}$ & $\not \rightarrow_{\text {Ass }}$ & $t_{\text {Aut }}$ & $A_{\text {Ass }}$ & $f_{\text {Aut }}$ & $f_{\text {Aut }}$ & $f_{\text {Ass }}$ & $f_{\text {Aut }}$ & $f_{\text {Aut }}$ & $\rightarrow$ & $A_{\text {Aut }}$ & $f_{\text {Aut }}$ & $\rightarrow$ & $f_{\text {Aut }}$ \\
\hline $\mathcal{T}_{14}^{\prime}$ & $\rightarrow \mathrm{DT}$ & $f_{\text {Aut }}$ & $f_{\text {Aut }}$ & $f_{\text {npa }}$ & $f_{\text {Aut }}$ & $f_{\text {Aut }}$ & $f_{\text {Aut }}$ & $f_{\text {Aut }}$ & $\rightarrow$ & $t_{\text {Aut }}$ & $\not \rightarrow_{M_{N u l}}$ & $\rightarrow_{\mathrm{T}}$ & $\rightarrow$ \\
\hline $\mathcal{T}_{15}^{\prime}$ & $\not \rightarrow_{\text {Ass }}$ & $\rightarrow \mathrm{DT}$ & $f_{\text {Ass }}$ & $f_{\text {npa }}$ & $t_{\text {Ann }}$ & $f_{\text {Ass }}$ & $f_{\text {Aut }}$ & $f_{\text {npa }}$ & $\rightarrow_{\mathrm{T}}$ & $\rightarrow$ & $\not \rightarrow_{M_{\mathrm{Nul}}}$ & $\rightarrow \mathrm{T}$ & $\rightarrow_{\mathrm{T}}$ \\
\hline $\mathcal{T}_{16}^{\prime}$ & $t_{\text {Aut }}$ & $t_{\text {Aut }}$ & $\rightarrow \mathrm{DT}$ & $f_{\text {npa }}$ & $f_{\text {Aut }}$ & $f_{\text {Aut }}$ & $f_{\text {Aut }}$ & $f_{\text {Aut }}$ & $\rightarrow$ & $A_{\text {Aut }}$ & $t_{M_{\mathrm{Nul}}}$ & $\rightarrow_{\mathrm{T}}$ & $\rightarrow$ \\
\hline $\mathcal{T}_{17}^{\prime}$ & $\not \rightarrow_{\text {Ass }}$ & $t_{\text {Aut }}$ & $f_{\text {Ass }}$ & $\rightarrow_{\mathrm{DT}}$ & $f_{\text {Aut }}$ & $f_{\text {Ass }}$ & $f_{\text {Aut }}$ & $f_{\text {Aut }}$ & $\rightarrow_{\mathrm{T}}$ & $A_{\text {Aut }}$ & $\not \rightarrow_{\text {Aut }}$ & $\rightarrow$ & $A_{\text {Aut }}$ \\
\hline $\mathcal{T}_{18}^{\prime}$ & $\not \rightarrow_{\text {Ass }}$ & $A_{\text {npa }}$ & $f_{\text {Ass }}$ & $A_{\text {npa }}$ & $\rightarrow \mathrm{DT}$ & $f_{\text {Ass }}$ & $A_{\text {Aut }}$ & $A_{\text {npa }}$ & $\rightarrow_{\mathrm{T}}$ & $\rightarrow$ & $\rightarrow$ & $\rightarrow_{\mathrm{T}}$ & $\rightarrow_{\mathrm{T}}$ \\
\hline $\mathcal{T}_{19}^{\prime}$ & $\rightarrow$ & $\not_{\text {npa }}$ & $\not_{\mathrm{Ann}}$ & $\not_{\text {npa }}$ & $\not \rightarrow$ Ann & $\rightarrow \mathrm{DT}$ & $\nrightarrow_{\text {Aut }}$ & $A_{\text {npa }}$ & $\rightarrow$ & $\stackrel{?}{-\rightarrow}$ & $\rightarrow$ & $\rightarrow_{\mathrm{T}}$ & $\rightarrow_{\mathrm{T}}$ \\
\hline $\mathcal{T}_{20}^{\prime}$ & $t_{\text {npa }}$ & $f_{\text {npa }}$ & $\rightarrow$ & $t_{\text {npa }}$ & $f_{\text {npa }}$ & $t_{\text {npa }}$ & $\rightarrow \mathrm{DT}$ & $A_{\text {npa }}$ & $\rightarrow_{\mathrm{T}}$ & $\stackrel{?}{-\rightarrow}$ & $\rightarrow$ & $\rightarrow_{\mathrm{T}}$ & $\rightarrow \mathrm{T}$ \\
\hline $\mathcal{T}_{21}^{\prime}$ & $\not A_{\text {Ass }}$ & $f_{\text {npa }}$ & $f_{\text {Ass }}$ & $\rightarrow$ & $\not A_{\text {Ann }}$ & $f_{\text {Ass }}$ & $f_{\text {Aut }}$ & $\rightarrow \mathrm{DT}$ & $\rightarrow_{\mathrm{T}}$ & $\rightarrow$ & $\rightarrow$ & $\rightarrow_{\mathrm{T}}$ & $\rightarrow_{\mathrm{T}}$ \\
\hline
\end{tabular}

Tabela 6.3: Existência de deformações em Jor ${ }_{3}^{\mathbb{R}}$ 
Na seguinte tabela colocaremos a relação de existência de deformações somente entre as álgebras nilpotentes, pois nas colunas que omitimos só teria o símbolo " $A_{\mathrm{Rad}}$ " $\mathrm{o}$ qual representa que "não existe deformação pela Proposição 4.52 que relaciona as dimensões dos radicais das álgebras envolvidas".

\begin{tabular}{|c|c|c|c|c|c|}
\hline & $\mathcal{T}_{22}^{\prime}$ & $\mathcal{T}_{23}^{\prime}$ & $\mathcal{T}_{24}^{\prime}$ & $\mathcal{T}_{25}^{\prime}$ & $\mathcal{T}_{26}^{\prime}$ \\
\hline$\overline{\mathcal{T}}_{22}^{\prime}$ & $\rightarrow \mathrm{DT}$ & $\nrightarrow_{\text {Aut }}$ & $\phi_{\text {Aut }}$ & $f_{\text {Aut }}$ & $f_{\text {Aut }}$ \\
\hline $\mathcal{T}_{23}^{\prime}$ & $\rightarrow_{\mathrm{T}}$ & $\rightarrow{ }_{\mathrm{DT}}$ & $f_{M_{N}}$ & $\rightarrow_{\mathrm{T}}$ & $\rightarrow$ \\
\hline $\mathcal{T}_{24}^{\prime}$ & $\rightarrow_{\mathrm{T}}$ & $f_{\text {Aut }}$ & $\rightarrow \mathrm{DT}$ & $\rightarrow$ & $f_{\text {Aut }}$ \\
\hline $\mathcal{T}_{25}^{\prime}$ & $\rightarrow$ & $f_{\text {Aut }}$ & $f_{\text {Aut }}$ & $\rightarrow \mathrm{DT}$ & $f_{\text {Aut }}$ \\
\hline $\mathcal{T}_{26}^{\prime}$ & $\rightarrow{ }_{\mathrm{T}}$ & $A_{\text {Aut }}$ & $A_{\text {Aut }}$ & $\rightarrow$ & $\rightarrow{ }_{\mathrm{DT}}$ \\
\hline
\end{tabular}

Tabela 6.4: Existência de deformações em $\operatorname{dor}_{3}^{\mathbb{R}}$

O diagrama quase-completo das álgebras de Jordan reais de dimensão 3 é dado na Figura 6.2.

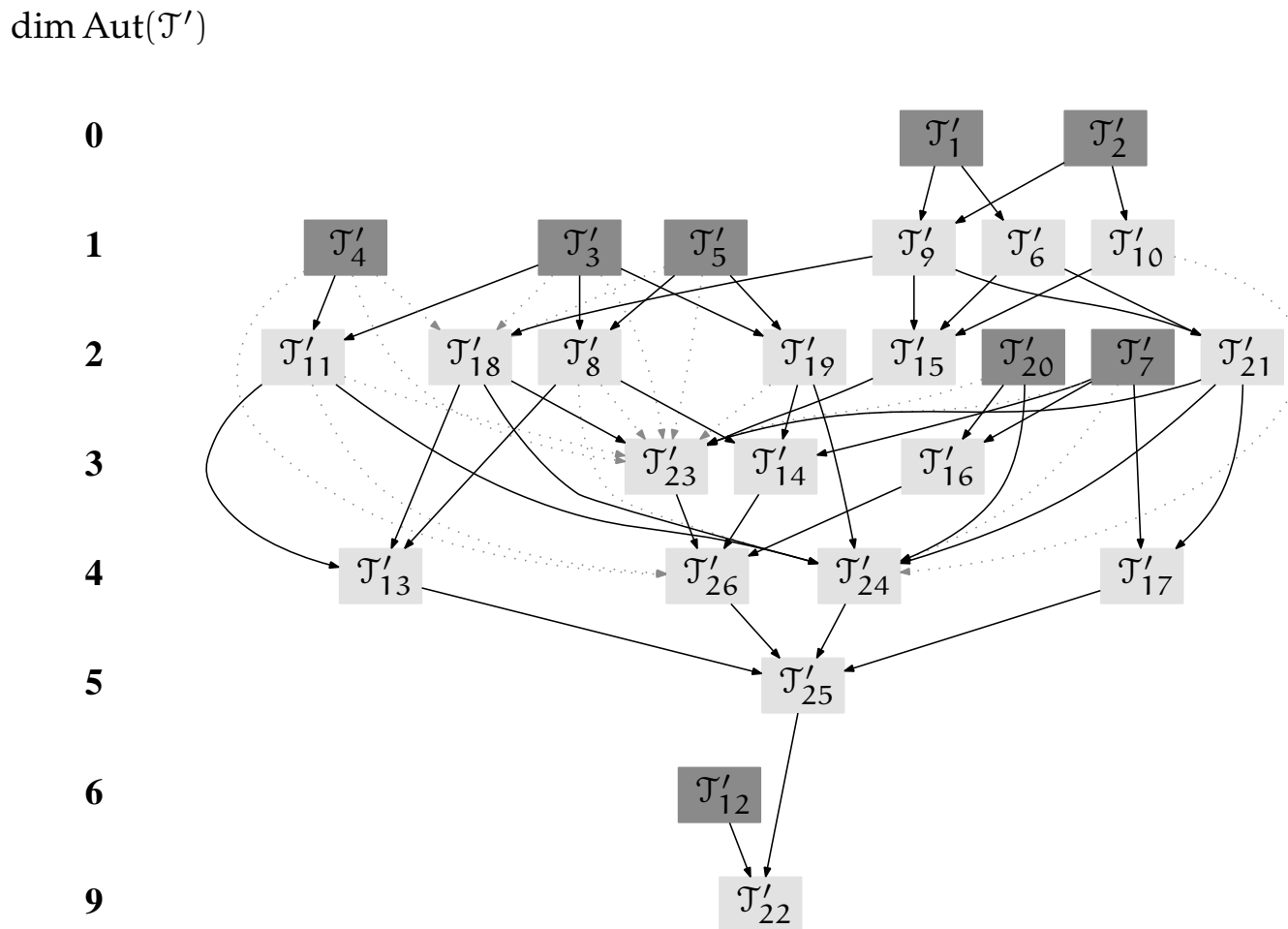

Fig. 6.2: Descrição quase-completa das órbitas de $\mathrm{Jor}_{3}^{\mathbb{R}}$ 


\subsection{COMPARAÇÃO DAS VARIEDAdES $\operatorname{jor}_{n}^{\mathbb{R}}$ E $\operatorname{jor}_{n}^{\mathbb{C}}$}

Nesta seção daremos uma rápida comparação das propriedades obtidas neste capítulo para a variedade das álgebras de Jordan reais com as propriedades obtidas no Capítulo 5 para álgebras de Jordan sobre um corpo algebricamente fechado, especificamente sobre o corpo $\mathbb{C}$ dos números complexos.

Tal como enuncia Gerstenhaber no seu trabalho [15] "uma k-álgebra $\mathcal{J}$ é rígida se e somente se $\mathcal{J} \otimes_{\mathbf{k}} \mathrm{L}$ é rígida sempre que $\mathrm{L}$ é uma extensão de $\mathbf{k}^{\prime \prime}$, as $8 \mathbb{R}$-álgebras de Jordan rígidas de dimensão 3: $\mathcal{T}_{1}^{\prime}, \mathcal{T}_{2}^{\prime}, \mathcal{T}_{3}^{\prime}, \mathcal{T}_{4}^{\prime}, \mathcal{T}_{5}^{\prime}, \mathcal{T}_{7}^{\prime}, \mathcal{T}_{12}^{\prime}$, e $\mathcal{T}_{20}^{\prime}$ são também rígidas quando consideradas como álgebras sobre $\mathbb{C}$, pois

$$
\begin{aligned}
& \mathcal{T}_{1}^{\prime} \otimes_{\mathbb{R}} \mathbb{C} \simeq_{\mathbb{C}} \mathcal{T}_{2}^{\prime} \otimes_{\mathbb{R}} \mathbb{C} \simeq_{\mathbb{C}} \mathcal{T}_{11} \\
& \mathcal{T}_{3}^{\prime} \otimes_{\mathbb{R}} \mathbb{C} \simeq_{\mathbb{C}} \mathcal{T}_{4}^{\prime} \otimes_{\mathbb{R}} \mathbb{C} \simeq_{\mathbb{C}} \mathcal{T}_{5}^{\prime} \otimes_{\mathbb{R}} \mathbb{C} \simeq_{\mathbb{C}} \mathcal{T}_{5} \\
& \mathcal{T}_{7}^{\prime} \otimes_{\mathbb{R}} \mathbb{C} \simeq_{\mathbb{C}} \mathcal{T}_{19} \quad \mathcal{T}_{12}^{\prime} \otimes_{\mathbb{R}} \mathbb{C} \simeq_{\mathbb{C}} \mathcal{T}_{7} \quad \mathcal{T}_{20}^{\prime} \otimes_{\mathbb{R}} \mathbb{C} \simeq_{\mathbb{C}} \mathcal{T}_{9} .
\end{aligned}
$$

Vimos nas Tabelas 6.2 a 6.4 da seção anterior que pelo fato da ação da parte semissimples não ser preservada (Proposição 4.59) não existem as seguintes deformações entre $\mathbb{R}$-álgebras:

$$
\begin{array}{lllll}
\mathcal{T}_{2}^{\prime} \not \rightarrow \mathcal{T}_{6}^{\prime} & \mathcal{T}_{1}^{\prime} \nrightarrow \mathcal{T}_{10}^{\prime} & \mathcal{T}_{4}^{\prime} \not \rightarrow \mathcal{T}_{8}^{\prime} & \mathcal{T}_{5}^{\prime} \not \rightarrow \mathcal{T}_{11}^{\prime} \\
\mathcal{T}_{4}^{\prime} \not \rightarrow \mathcal{T}_{19}^{\prime} & \mathcal{T}_{10}^{\prime} \not \rightarrow \mathcal{T}_{21}^{\prime} & \mathcal{T}_{10}^{\prime} \not \rightarrow & \mathcal{T}_{17}^{\prime} & \mathcal{T}_{11}^{\prime} \not \rightarrow \mathcal{T}_{14}^{\prime} .
\end{array}
$$

Mas da Seção 5.3 sabemos que sim existem as deformações das respectivas extensões das álgebras sobre $\mathbb{C}$ :

$$
\begin{aligned}
& \mathcal{T}_{11} \simeq_{\mathbb{C}} \mathcal{T}_{2}^{\prime} \otimes_{\mathbb{R}} \mathbb{C} \rightarrow \mathcal{T}_{6}^{\prime} \otimes_{\mathbb{R}} \mathbb{C} \simeq_{\mathbb{C}} \mathcal{T}_{13} \quad \mathcal{T}_{11} \simeq_{\mathbb{C}} \mathcal{T}_{1}^{\prime} \otimes_{\mathbb{R}} \mathbb{C} \rightarrow \mathcal{T}_{10}^{\prime} \otimes_{\mathbb{R}} \mathbb{C} \simeq_{\mathbb{C}} \mathcal{T}_{13} \\
& \mathcal{T}_{5} \simeq_{\mathbb{C}} \mathcal{T}_{4}^{\prime} \otimes_{\mathbb{R}} \mathbb{C} \rightarrow \mathcal{T}_{8}^{\prime} \otimes_{\mathbb{R}} \mathbb{C} \simeq_{\mathbb{C}} \mathcal{T}_{10} \quad \mathcal{T}_{5} \simeq_{\mathbb{C}} \mathcal{T}_{5}^{\prime} \otimes_{\mathbb{R}} \mathbb{C} \rightarrow \mathcal{T}_{11}^{\prime} \otimes_{\mathbb{R}} \mathbb{C} \simeq_{\mathbb{C}} \mathcal{T}_{10} \\
& \mathcal{T}_{5} \simeq_{\mathbb{C}} \mathcal{T}_{4}^{\prime} \otimes_{\mathbb{R}} \mathbb{C} \rightarrow \mathcal{T}_{19}^{\prime} \otimes_{\mathbb{R}} \mathbb{C} \simeq_{\mathbb{C}} \mathcal{T}_{8} \quad \mathcal{T}_{13} \simeq_{\mathbb{C}} \mathcal{T}_{10}^{\prime} \otimes_{\mathbb{R}} \mathbb{C} \rightarrow \mathcal{T}_{21}^{\prime} \otimes_{\mathbb{R}} \mathbb{C} \simeq_{\mathbb{C}} \mathcal{T}_{15} \\
& \mathcal{T}_{13} \simeq_{\mathbb{C}} \mathcal{T}_{10}^{\prime} \otimes_{\mathbb{R}} \mathbb{C} \rightarrow \mathcal{T}_{17}^{\prime} \otimes_{\mathbb{R}} \mathbb{C} \simeq_{\mathbb{C}} \mathcal{T}_{16} \quad \mathcal{T}_{10} \simeq_{\mathbb{C}} \mathcal{T}_{11}^{\prime} \otimes_{\mathbb{R}} \mathbb{C} \rightarrow \mathcal{T}_{14}^{\prime} \otimes_{\mathbb{R}} \mathbb{C} \simeq_{\mathbb{C}} \mathcal{T}_{20} .
\end{aligned}
$$

Isso nos diz que $\mathcal{J} \otimes_{\mathbb{R}} \mathbb{C}$ pode estar contido no fecho da órbita de $\mathcal{J}_{1} \otimes_{\mathbb{R}} \mathbb{C}$ sobre a ação de $\mathrm{GL}\left(\mathbb{C}^{\mathfrak{n}}\right)$ mesmo quando $\mathcal{J}^{\mathrm{GL}\left(\mathbb{R}^{\mathfrak{n}}\right)} \nsubseteq \overline{\mathcal{J}_{1}^{\mathrm{GL}\left(\mathbb{R}^{\mathfrak{n}}\right)}}$.

A seguinte tabela reúne e compara as informações obtidas nas seções anteriores sobre o número de órbitas e número de componentes irredutíveis das variedades Jor $_{\mathfrak{n}}$ e Jor $_{n}^{\mathbb{R}}$ em dimensões pequenas. 


\begin{tabular}{|c|c|c|c|c|}
\hline & \multicolumn{2}{|c|}{$\mathrm{Jor}_{\mathfrak{n}}^{\mathbb{R}}$} & \multicolumn{2}{c|}{ Jor $_{\mathfrak{n}}^{\mathrm{C}}$} \\
\hline $\mathrm{n}$ & $\mathrm{n}^{\text {o }}$ orbitas & $\mathrm{n}^{\text {o }}$ componentes & $\mathrm{n}^{\text {o }}$ órbitas & $\mathrm{n}^{\mathrm{o}}$ componentes \\
\hline 1 & 2 & 1 & 2 & 1 \\
\hline 2 & 7 & 3 & 6 & 2 \\
\hline 3 & 26 & 8 & 20 & 5 \\
\hline 4 & $>109$ & $\geqslant 18$ & 73 & 10 \\
\hline 5 & - & - & $\gg 223$ & $\geqslant 26$ \\
\hline
\end{tabular}

Tabela 6.5: Comparação das variedades $\operatorname{Jor}_{n}^{C}$ e Jor $_{n}^{\mathbb{R}}$ 



\section{ÁLGEBRAS ISOTÓPICAS}

Neste capítulo as álgebras de Jordan serão consideradas sobre um corpo $\mathbf{k}$ arbitrário de char $\mathbf{k} \neq 2$. Como referências para este capítulo citamos [21, Cap.I.12], [33, Cap.3.2, 4.6 e 7] e [28, Cap. IV.2].

Na Seção 7.1 trabalharemos majoritariamente com álgebras de Jordan unitárias e daremos as definições e resultados relativos a álgebras isotópicas. Omitiremos as provas dos resultados, mas elas podem ser facilmente encontradas na literatura.

Se duas álgebras são isomorfas então elas são isotópicas. Para álgebras associativas ou para álgebras sobre corpos algebricamente fechados a noção de isotopia não desempenha um papel importante pois os conceitos de álgebras isotópicas e isomorfas coincidem. Provaremos na Seção 7.2 que isso não acontece com as álgebras de Jordan, onde o conceito de isotopia fornece uma relação de equivalência mais ampla que o conceito de isomorfismo. Faremos isso classificando as álgebras de Jordan reais de dimensão 3 a menos de isotopia.

\subsection{INTRODUÇÃO Às ISOTOPIAS}

Uma álgebra homótopa de uma álgebra de Jordan é resumidamente uma nova álgebra de Jordan definida a partir de um elemento dado da álgebra original. A homótopa é unitária se e somente se a álgebra original é unitária e o elemento dado é invertível, em tal caso a álgebra homótopa é chamada de isótopa.

Definição 7.1. Seja $\mathcal{J}$ uma álgebra de Jordan com um elemento identidade 1. Então um elemento $a \in \mathcal{J}$ é chamado de invertível (ou Jordan invertível) se existe um elemento $\mathrm{b} \in \mathcal{J}$ tal que

$$
a b=1 \quad \text { e } \quad a^{2} b=a
$$

Se o elemento b existe ele é único e o chamaremos de elemento inverso de a e será denotado por $\mathrm{a}^{-1}$.

Uma álgebra de Jordan unitária na qual todo elemento não nulo é um elemento invertível é chamada de álgebra de divisão. 
Em $\mathcal{J}$ definimos o produto triplo de Jordan como sendo a identidade

$$
\{a, b, c\}=(a b) c+(c b) a-(a c) b .
$$

Denotaremos a aplicação linear que leva $x \mapsto\{a, x, b\}$ por $U_{a, b}$ e abreviaremos $U_{a, a}=$ $\mathrm{u}_{\mathrm{a}}$.

Definição 7.2. Um elemento $x$ de uma álgebra de Jordan $\mathcal{J}$ é chamado de trivial se $\mathrm{U}_{\mathrm{x}}(\mathcal{J})=0$ e $x^{2}=0$.

A identidade (7.1) nos permite definir, para um elemento qualquer $a \in \mathcal{J}$, o que chamaremos de a-multiplicação em $\mathcal{J}$

$$
x \cdot a y=\{x, a, y\}
$$

Esta multiplicação é claramente bilinear em x e em y, é comutativa e satisfaz a identidade de Jordan (1.2). Logo a álgebra $\mathcal{J}$ com produto $\cdot a$ é uma álgebra de Jordan que denotaremos por $(\mathcal{J}, a)$ e chamaremos de a-homótopa de $\mathcal{J}$.

Observamos que homotopia é reflexiva pois $(\mathcal{J}, 1)=\mathcal{J}$ e transitiva já que a b-homótopa da a-homótopa da álgebra de Jordan $\mathcal{J}$ é a $\{a, b, a\}$-homótopa de $\mathcal{J}$. Mas em geral não é simétrica, e portanto não é uma relação de equivalência. Então, se a não é invertível não podemos recuperar $\mathcal{J}$ de $(\mathcal{J}, a)$ e perdermos informação ao passar à homótopa.

Vamos a assumir agora que $\mathcal{J}$ tem um elemento identidade 1 e que $a \in \mathcal{J}$ é invertível. Então chamaremos $(\mathcal{J}, a)$ de a-isótopa ${ }^{1}$ de $\mathcal{J}$. A álgebra $(\mathcal{J}, a)$ é também uma álgebra unitária com elemento identidade $a^{-1}$ já que $\left\{a^{-1}, a, x\right\}=x$. É imediato ver que $1 \mathrm{e}$ $a^{-2}$ são inversos em $(\mathcal{J}, a)$ e também satisfazem $\left\{a, a^{-2}, a\right\}=1 \operatorname{logo} \mathcal{J}$ é a $a^{-2}$-isótopa de $(\mathcal{J}, a)$.

Definição 7·3. Sejam $\mathcal{J}$ e $\mathcal{J}^{\prime}$ duas álgebras de Jordan. Então uma aplicação $\alpha: \mathcal{J} \rightarrow \mathcal{J}^{\prime}$ é chamada de homotopia se $\alpha$ é linear e existe $a \in \mathcal{J}^{\prime}$ tal que $\alpha(x y)=\{\alpha(x), a, \alpha(y)\}$. Se $\mathcal{J}$ e $\mathcal{J}^{\prime}$ têm elementos identidades e $\alpha$ é um isomorfismo linear do espaço vetorial $\mathcal{J}$ sobre $\mathcal{J}^{\prime}$ e a é invertível, então chamaremos $\alpha$ de uma isotopia de $\mathcal{J}$ sobre $\mathcal{J}^{\prime}$ e diremos que $\mathcal{J}$ e $\mathcal{J}^{\prime}$ são isotópicas.

Esta definição de homotopia corresponde à definição de um homomorfismo de $\mathcal{J}$ na a-homótopa de $\mathcal{J}^{\prime}$ e a definição de isotopia corresponde à definição de um isomorfismo de $\mathcal{J}$ sobre a a-isótopa de $\mathcal{J}^{\prime}$.

Proposição 7.4. [33, Prop.7.2.1, p.223] Isotopia é uma relação de equivalência na classe das álgebras de Jordan unitárias.

1 A noção de isótopa é também chamada de mutação por alguns autores. 
Observamos que se $\mathcal{J}$ e $\mathcal{J}^{\prime}$ são isomorfas, então o isomorfismo entre elas é uma isotopia com $a=1, \log o \mathcal{J}$ e $\mathcal{J}^{\prime}$ são isotópicas.

A noção de isotopia não desempenha um papel importante na teoria de álgebras associativas pois álgebras associativas isotópicas são isomorfas. A saber, se $\mathcal{A}$ é uma álgebra associativa unitária e a um elemento invertível de $\mathcal{A}$, então definimos a a-multiplicação em $\mathcal{A}$ como sendo x · a $y=$ xay. Segue que a a-isótopa $(\mathcal{A}, \mathrm{a})$ de $\mathcal{A}$ é isomorfa a $\mathcal{A}$ já que a aplicação $\alpha: \mathcal{A} \rightarrow(\mathcal{A}, \mathrm{a})$ dada por $\chi \mapsto \mathrm{xa}^{-1}$ satisfaz:

$$
\alpha(x) \cdot a \alpha(y)=\left(x a^{-1}\right) \cdot a\left(y a^{-1}\right)=x a^{-1} a y a^{-1}=x y a^{-1}=\alpha(x y)
$$

é um isomorfismo. Quando considerarmos uma álgebra de Jordan associativa então a a-multiplicação é dada por

$$
x \cdot a y=\{x, a, y\}=(x a) y+x(a y)-a(x y)=x a y+x a y-a x y=x a y
$$

ou seja é a a-multiplicação definida para álgebras associativas, assim toda álgebra de Jordan associativa e sua a-isótopa são isomorfas.

No contexto de álgebras de Jordan não associativas as isotopias fornecem uma relação de equivalência mais ampla que isomorfismos. Veremos na Seção 7.2 que existem álgebras isotópicas que não são isomorfas.

A seguinte proposição fornece uma condição suficiente para álgebras isótopas serem isomorfas.

Proposição 7.5. [21, p.6o] Se a é o quadrado de um elemento invertível então J e sua a-isótopa $(\mathcal{J}$, a) são isomorfas.

Em particular, se o corpo for algebricamente fechado todo elemento invertível tem uma raiz quadrada:

Lema 7.6. [21, p.242] Se $\mathbf{k}$ é um corpo algebricamente fechado de char $\mathbf{k} \neq 2$ e $\mathbf{k}[\mathbf{a}]$ é a álgebra associativa e comutativa com unidade gerada por 1 e um elemento invertível a, então existe um elemento invertível $\mathrm{b}$ em $\mathbf{k}[\mathrm{a}]$ tal que $\mathrm{b}^{2}=\mathrm{a}$.

Combinando a Proposição 7.5 e o Lema 7.6 segue que se $\mathcal{J}$ é uma álgebra de Jordan de dimensão finita sobre um corpo algebricamente fechado, então toda isótopa de $\mathcal{J}$ é isomorfa a $\mathcal{J}$.

O seguinte teorema fornece uma caracterização de quando uma aplicação é uma isotopia.

Teorema 7.7. [21, p.59] Uma aplicação $\alpha: \mathcal{J} \rightarrow J^{\prime}$ é uma isotopia se e somente se $\alpha$ é bijetiva e linear e existe um $\beta \in \operatorname{Hom}_{\mathbf{k}}\left(\mathcal{J}, \mathcal{J}^{\prime}\right)$ tal que $\alpha \mathrm{U}_{\mathrm{c}}=\mathrm{U}_{\alpha(\mathrm{c})} \beta$ para $\mathrm{c} \in \mathcal{J}$. Em particular, $a$ 
restrição de $\alpha$ ao conjunto dos elementos invertíveis de J é uma bijeção linear deste conjunto sobre o conjunto de elementos invertíveis de $\mathrm{J}^{\prime}$.

Considere o conjunto $\Gamma(\mathcal{J})$ das isotopias de $\mathcal{J}$ sobre si mesma. Claramente $\Gamma(\mathcal{J})$ é um grupo de transformações lineares no espaço vetorial subjacente a $\mathcal{I}$ a qual chamaremos de grupo de estrutura de $\mathcal{J}$. Pode-se provar que $\Gamma(\mathcal{J})$ é na realidade um grupo algébrico, veja $[28$, p.79]

Teorema 7.8. [21, p.6o] J e qualquer isótopa (J, a) têm o mesmo grupo de estrutura.

Se $\alpha \in \Gamma(\mathcal{J})$ tal que $\alpha(1)=1$ então $\alpha$ é um isomorfismo de $\mathcal{J}$ sobre $(\mathcal{J}, 1)=\mathcal{J}$, logo $\alpha$ é um automorfismo de $\mathcal{J}$. Segue que o grupo de automorfismo Aut $(\mathcal{J})$ de $\mathcal{J}$ é o subgrupo do grupo de estrutura $\Gamma(\mathcal{J})$ que consiste de elementos que tem 1 como ponto fixo, i.e., $\operatorname{Aut}(\mathcal{J})=\operatorname{Stab}_{\Gamma(\mathfrak{g})}(1)$.

Proposição 7.9. [33, Prop.7.2.1, p.223] Uma isótopa tem exatamente os mesmos elementos triviais e invertíveis que a álgebra original:

$$
\begin{aligned}
& \text { xé trivial em }(\mathcal{J}, a) \Leftrightarrow x \text { é trivial em } \mathcal{J} ; \\
& \text { xé invertível em }(\mathcal{J}, a) \Leftrightarrow \text { x é invertível em } \mathcal{J}, \\
& \operatorname{com} x^{(-1, a)}=\mathrm{U}_{\mathrm{a}}^{-1} x^{-1} .
\end{aligned}
$$

Em particular, (J, a) é uma álgebra de divisão se e somente se J é uma álgebra de divisão.

\subsection{ClassificAÇÃo ALGÉbRICA A MENOS DE ISOTOPIA DAS ÁLGEBRAS DE JORDAN REAIS DE DIMENSÃO 3}

Nesta seção classificaremos as álgebras de Jordan reais unitárias de dimensão 3 a menos de isotopia. Veremos que o número de álgebras obtidas é 9 estritamente menor ao número de álgebras unitárias quando a classificação é feita a menos de isomorfismo. Isto prova que no cenário da álgebras de Jordan o conceito de isotopia fornece uma relação de equivalência mais ampla que o conceito de isomorfismo, a diferença do que acontece com as álgebras associativas onde ambos conceitos coincidem.

Se $\mathcal{J}$ e $\mathcal{J}^{\prime}$ são duas álgebras de Jordan unitárias e isomorfas, então o isomorfismo entre elas é uma isotopia com elemento invertível $a=1$, logo $\mathcal{J}$ e $\mathcal{J}^{\prime}$ são isotópicas. Então, a classificação algébrica feita no Capítulo 3 reduz o nosso problema a buscar isotopias entre as 10 álgebras de Jordan reais unitárias de dimensão 3. Assim, dada $\mathcal{T}^{\prime}$ uma álgebra de Jordan unitária real de dimensão 3 então $\mathcal{T}^{\prime}$ é isotópica a alguma (não necessariamente uma) das álgebras $\mathcal{T}_{i}^{\prime}$ para $i=1,2,3,4,5,8,9,11,13,18$. 
Agora, segue do fato que álgebras associativas isotópicas são isomorfas que as álgebras $\mathcal{T}_{i}^{\prime}$ para $i=1,2,9,13,18$ são duas a duas não isotópicas e também não são isotópicas a nenhuma das álgebras restantes.

Resta somente verificar se as álgebras $\left\{\mathcal{T}_{3}^{\prime}, \mathcal{T}_{4}^{\prime}, \mathcal{T}_{5}^{\prime}, \mathcal{T}_{8}^{\prime}, \mathcal{T}_{11}^{\prime}\right\}$ são duas a duas isotópicas.

Segue da Proposição 7.5 que se $\mathcal{J}$ é uma álgebra de Jordan de dimensão finita sobre um corpo algebricamente fechado, então toda isótopa de $\mathcal{J}$ é isomorfa a $\mathcal{J}$. Por outro lado, toda isotopia de $\mathbb{R}$-álgebras pode ser estendida a uma isotopia de $\mathbb{C}$-álgebras. Logo, se duas $\mathbb{R}$-álgebras de dimensão finita $\mathcal{T}$ e $\mathcal{T}^{\prime}$ são isotópicas então as $\mathbb{C}$-álgebras $\mathcal{T} \otimes_{\mathbb{R}} \mathbb{C}$ e $\mathcal{T}^{\prime} \otimes_{\mathbb{R}} \mathbb{C}$ são isomorfas. Isto implica que as $\mathbb{R}$-álgebras $\mathcal{T}_{i}^{\prime}$ e $\mathcal{T}_{j}^{\prime}$ para $i=3,4,5$ e $\mathfrak{j}=8,11$ não são isotópicas, restando somente comparar os seguintes pares de álgebras:

$$
\mathcal{T}_{3}^{\prime} \text { e } \mathcal{T}_{5}^{\prime} \quad \mathcal{T}_{3}^{\prime} \text { e } \mathcal{T}_{4}^{\prime} \quad \mathcal{T}_{4}^{\prime} \text { e } \mathcal{T}_{5}^{\prime} \quad \mathcal{T}_{8}^{\prime} \text { e } \mathcal{T}_{11}^{\prime}
$$

Em $\mathcal{T}_{5}^{\prime}$ considere o elemento invertível $a=e_{2}+e_{3}$ cujo inverso é $b=\frac{e_{2}}{2}+\frac{e_{3}}{2}$. Então a álgebra a-isótopa a $\mathcal{T}_{5}^{\prime}$ é dada pelo produto

\begin{tabular}{c|ccc}
$\left(\mathcal{T}_{5}^{\prime}, \mathrm{a}\right)$ & $e_{1}$ & $e_{2}$ & $e_{3}$ \\
\hline$e_{1}$ & $e_{2}+e_{3}$ & $e_{1}$ & $e_{1}$ \\
$e_{2}$ & $e_{1}$ & $e_{2}-e_{3}$ & $e_{2}+e_{3}$ \\
$e_{3}$ & $e_{1}$ & $e_{2}+e_{3}$ & $-e_{2}+e_{3}$
\end{tabular}

e é isomorfa a $\mathcal{T}_{3}^{\prime}$ com isomorfismo $\mathrm{T}: \mathcal{T}_{3}^{\prime} \rightarrow\left(\mathcal{T}_{5}^{\prime}, \mathrm{a}\right)$ dado por $\mathrm{T}\left(e_{1}\right)=\frac{1}{2} e_{2}+\frac{1}{2} e_{3}$, $\mathrm{T}\left(e_{2}\right)=-\frac{1}{\sqrt{2}} e_{1}$ e $\mathrm{T}\left(e_{3}\right)=-\frac{1}{2} e_{2}+\frac{1}{2} e_{3}$. Logo $\mathrm{T}$ e uma isotopia de $\mathcal{T}_{3}^{\prime}$ sobre $\mathcal{T}_{5}^{\prime}$ e portanto as álgebras $\mathcal{T}_{3}^{\prime}$ e $\mathcal{T}_{5}^{\prime}$ são isotópicas e não-isomorfas.

Vamos agora comparar $\mathcal{T}_{3}^{\prime}$ (ou, equivalentemente, $\mathcal{T}_{5}^{\prime}$ ) com $\mathcal{T}_{4}^{\prime}$. Observamos primeiramente que $\mathcal{T}_{4}^{\prime}$ é uma álgebra de divisão, i.e., é uma álgebra unitária cujos elementos não nulos são todos invertíveis. Seja, então, $a=\alpha e_{1}+\beta e_{2}+\gamma e_{3}$ um elemento não nulo de $\mathcal{T}_{4}^{\prime} \operatorname{com} \alpha, \beta, \gamma \in \mathbb{R}$ e pelo menos um deles é não nulo, então $a^{-1}=\frac{\alpha e_{1}}{\Delta}-\frac{\beta e_{2}}{\Delta}-\frac{\gamma e_{3}}{\Delta}$ onde $\Delta=\alpha^{2}+\beta^{2}+\gamma^{2} \neq 0$.

A álgebra a-isótopa a $\mathcal{T}_{4}^{\prime}$ é dada pelo produto

\begin{tabular}{c|ccc}
$\left(\mathcal{T}_{4}^{\prime}, \mathrm{a}\right)$ & $e_{1}$ & $e_{2}$ & $e_{3}$ \\
\hline$e_{1}$ & $\alpha e_{1}+\beta e_{2}+\gamma e_{3}$ & $-\beta e_{1}+\alpha e_{2}$ & $\alpha e_{3}-\gamma e_{1}$ \\
$e_{2}$ & $-\beta e_{1}+\alpha e_{2}$ & $-\alpha e_{1}-\beta e_{2}+\gamma e_{3}$ & $-\beta e_{3}-\gamma e_{2}$ \\
$e_{3}$ & $\alpha e_{3}-\gamma e_{1}$ & $-\beta e_{3}-\gamma e_{2}$ & $-\alpha e_{1}+\beta e_{2}-\gamma e_{3}$
\end{tabular}


e é isomorfa a $\mathcal{T}_{4}^{\prime}$ com isomorfismo $\mathrm{T}: \mathcal{T}_{4}^{\prime} \rightarrow\left(\mathcal{T}_{4}^{\prime}, \mathrm{a}\right)$ dado por

$$
\begin{aligned}
& \mathrm{T}\left(e_{1}\right)=\frac{\alpha}{\Delta} e_{1}-\frac{\beta}{\Delta} e_{2}-\frac{\gamma}{\Delta} e_{3}, \\
& \mathrm{~T}\left(e_{2}\right)=\frac{\gamma}{\sqrt{\left(\beta^{2}+\gamma^{2}\right) \Delta}} e_{2}-\frac{\beta}{\sqrt{\left(\beta^{2}+\gamma^{2}\right) \Delta}} e_{3}, \\
& \mathrm{~T}\left(e_{3}\right)=\frac{\sqrt{\beta^{2}+\gamma^{2}}}{\Delta} e_{1}+\frac{\alpha \beta}{\Delta \sqrt{\beta^{2}+\gamma^{2}}} e_{2}+\frac{\alpha \gamma}{\Delta \sqrt{\beta^{2}+\gamma^{2}}} e_{3},
\end{aligned}
$$

cujo determinante $\frac{1}{\sqrt{\Delta^{3}}}$ é bem definido e não nulo. Logo toda isótopa de $\mathcal{T}_{4}^{\prime}$ é isomorfa a $\mathcal{T}_{4}^{\prime}$. Portanto $\mathcal{T}_{3}^{\prime}$ e $\mathcal{T}_{5}^{\prime}$ não são isotópicas a $\mathcal{T}_{4}^{\prime}$.

Vejamos que também toda isótopa de $\mathcal{T}_{11}^{\prime}$ é isomorfa a $\mathcal{T}_{11}^{\prime}$. Seja $a=\alpha e_{1}+\beta e_{2}+\gamma e_{3}$ com $\alpha, \beta, \gamma \in \mathbb{R}$ um elemento invertível de $\mathcal{T}_{11}^{\prime}$ então $\alpha$ e $\beta$ não são ambos nulos. $\mathrm{O}$ inverso de a é $\mathrm{a}^{-1}=\frac{\alpha e_{1}}{\Delta}-\frac{\beta e_{2}}{\Delta}-\frac{\gamma e_{3}}{\Delta}$ onde $\Delta=\alpha^{2}+\beta^{2} \neq 0$.

A álgebra a-isótopa a $\mathcal{T}_{11}^{\prime}$ é dada pelo produto

\begin{tabular}{c|ccc}
$\left(\mathcal{T}_{11}^{\prime}, a\right)$ & $e_{1}$ & $e_{2}$ & $e_{3}$ \\
\hline$e_{1}$ & $\alpha e_{1}+\beta e_{2}+\gamma e_{3}$ & $-\beta e_{1}+\alpha e_{2}$ & $\alpha e_{3}$ \\
$e_{2}$ & $-\beta e_{1}+\alpha e_{2}$ & $-\alpha e_{1}-\beta e_{2}+\gamma e_{3}$ & $-\beta e_{3}$ \\
$e_{3}$ & $\alpha e_{3}$ & $-\beta e_{3}$ & 0
\end{tabular}

e é isomorfa a $\mathcal{T}_{11}^{\prime}$ com isomorfismo $\mathrm{T}: \mathcal{T}_{11}^{\prime} \rightarrow\left(\mathcal{T}_{11}^{\prime}, \mathrm{a}\right)$ dado por

$$
\begin{aligned}
\mathrm{T}\left(e_{1}\right) & =\frac{\alpha}{\Delta} e_{1}-\frac{\beta}{\Delta} e_{2}-\frac{\gamma}{\Delta} e_{3}, \\
\mathrm{~T}\left(e_{2}\right) & =\frac{\beta}{\Delta} e_{1}+\frac{\alpha}{\Delta} e_{2}, \\
\mathrm{~T}\left(e_{3}\right) & =e_{3},
\end{aligned}
$$

cujo determinante é $\frac{1}{\Delta}$ que é não nulo e está bem definido já que $\Delta \neq 0$. Isto implica que $\mathcal{T}_{8}^{\prime}$ não é isotópica a $\mathcal{T}_{11}^{\prime}$.

Assim, provamos o seguinte resultado:

Teorema 7.10. Seja $\mathcal{T}^{\prime}$ uma álgebra de Jordan unitária real de dimensão 3 então $\mathcal{T}^{\prime}$ é isotópica a uma e somente uma das álgebras $\mathcal{T}_{i}^{\prime}$ para $i=1,2,3,4,8,9,11,13,18$.

Na seguinte tabela reunimos e comparamos os resultados obtidos em relação à classificação algébrica das álgebras de Jordan unitárias de dimensão 3, seja a menos de isomorfismo ou a menos de isotopia, sobre $\mathbb{R}$ ou sobre $\mathbb{C}$. 


\begin{tabular}{|c|c|c|}
\hline $\mathrm{n}^{\text {o de álgebras a menos de: }}$ & sobre $\mathbb{R}$ & sobre $\mathbb{C}$ \\
\hline isomorfismo & 10 & 6 \\
\hline isotopia & 9 & 6 \\
\hline
\end{tabular}

Tabela 7.1: Comparação do número de álgebras de Jordan unitárias de dimensão 3 



\section{FUTURAS}

Durante a elaboração deste trabalho nos deparamos com novas questões ainda à serem respondidas. Neste capítulo indicaremos algumas possíveis direções para futuras pesquisas nesta área.

Primeiramente, acreditamos que seria interessante obter a classificação geométrica completa das variedades $\mathrm{Jor}_{3}, \mathrm{Jor}_{4}$ e $\mathrm{Jor}_{3}^{\mathbb{R}}$. Se bem neste trabalho foram determinadas todas as componentes irredutíveis de tais variedades, ainda resta determinar em média 2,5\% dos casos a existência ou não existência de deformação entre pares de álgebras. Estes casos são:

Existência de deformação desconhecida entre álgebras de Jordan de dimensão 3 sobre um corpo algebricamente fechado

$$
\begin{array}{ll} 
& \stackrel{?}{\mathcal{T}_{5}} \stackrel{?}{\rightarrow} \mathcal{T}_{1}, \quad \mathcal{T}_{5} \stackrel{?}{\rightarrow} \mathcal{T}_{3} \\
\mathcal{T}_{8} \stackrel{?}{\rightarrow} \mathcal{T}_{3} & \\
\mathcal{T}_{9} \stackrel{?}{\rightarrow} \mathcal{T}_{3} & \\
\mathcal{T}_{10} \stackrel{?}{\rightarrow} \mathcal{T}_{3} & \\
\mathcal{T}_{19} \stackrel{?}{\rightarrow} \mathcal{T}_{3} . &
\end{array}
$$

Existência de deformação desconhecida entre álgebras de Jordan de dimensão 4 sobre um corpo algebricamente fechado

$$
\begin{aligned}
& \partial_{42} \stackrel{?}{-} \rightarrow J_{70} \\
& \partial_{21} \stackrel{?}{\stackrel{?}{\rightarrow}} J_{66}, \quad J_{21} \stackrel{?}{\stackrel{?}{\rightarrow}} J_{70} \\
& \mathrm{~J}_{10} \stackrel{?}{\rightarrow} \mathcal{J}_{61}, \mathrm{~J}_{10} \stackrel{?}{\rightarrow} \mathcal{J}_{44}, \mathrm{~J}_{10} \stackrel{?}{\rightarrow} \mathrm{J}_{63}, \mathrm{~J}_{10} \stackrel{?}{\rightarrow} \rightarrow \mathrm{J}_{65}, \\
& J_{11} \stackrel{?}{\rightarrow} J_{61}, \quad J_{11} \stackrel{?}{\rightarrow} J_{44}, \quad J_{11} \stackrel{?}{\rightarrow} J_{63}, \quad J_{11} \stackrel{?}{\rightarrow} J_{64}, \quad J_{11} \stackrel{?}{\rightarrow} J_{65}, \\
& \partial_{12} \stackrel{?}{\rightarrow} \partial_{69}, \quad \partial_{12} \stackrel{?}{\rightarrow} \partial_{70}, \\
& \mathcal{J}_{13} \stackrel{?}{\stackrel{?}{\rightarrow}} \mathcal{J}_{66}, \quad J_{13} \stackrel{?}{\rightarrow} \mathcal{J}_{67}, \quad \mathcal{J}_{13} \stackrel{?}{\stackrel{?}{\rightarrow}} \mathcal{J}_{68}, \quad \mathcal{J}_{13} \stackrel{?}{\rightarrow} \mathcal{J}_{70}, \quad \mathcal{J}_{13} \stackrel{?}{\rightarrow} \mathcal{J}_{58}, \quad \mathcal{J}_{13} \stackrel{?}{\rightarrow} \mathcal{J}_{64}, \\
& \mathcal{J}_{14} \stackrel{?}{\rightarrow} \mathcal{J}_{61}, \quad \mathcal{J}_{14} \stackrel{?}{\rightarrow} \mathcal{J}_{63}, \mathcal{J}_{14} \stackrel{?}{\rightarrow} \mathcal{J}_{65} \\
& \mathcal{J}_{15} \stackrel{?}{\rightarrow} \mathcal{J}_{61}, \quad J_{15} \stackrel{?}{\rightarrow} \partial_{66}, \quad J_{15} \stackrel{?}{\rightarrow} J_{68}, \quad J_{15} \stackrel{?}{\rightarrow} J_{70}, \quad J_{15} \stackrel{?}{\rightarrow} J_{44}, \quad J_{15} \stackrel{?}{\rightarrow} J_{55}, \\
& \partial_{15} \stackrel{?}{\rightarrow} J_{63}, \quad J_{15} \stackrel{?}{\rightarrow} J_{64}, \quad \partial_{15} \stackrel{?}{\rightarrow} J_{65}, \\
& J_{16} \stackrel{?}{\rightarrow} \partial_{66}, \quad J_{16} \stackrel{?}{\rightarrow} J_{67}, \quad J_{16} \stackrel{?}{\rightarrow} J_{68}, \quad J_{16} \stackrel{?}{\rightarrow} J_{70}, \quad J_{16} \stackrel{?}{\rightarrow} J_{48}, \quad J_{16} \stackrel{?}{\rightarrow} J_{64},
\end{aligned}
$$




$$
\begin{aligned}
& \partial_{17} \stackrel{?}{\stackrel{?}{\rightarrow}} J_{61}, \quad J_{17} \stackrel{?}{\rightarrow} J_{44}, \quad J_{17} \stackrel{?}{\rightarrow} J_{63}, \quad J_{17} \stackrel{?}{\rightarrow} J_{65} \\
& \mathcal{J}_{18} \stackrel{?}{\rightarrow} \mathcal{J}_{69}, \partial_{18} \stackrel{?}{\rightarrow} \mathcal{J}_{70}
\end{aligned}
$$

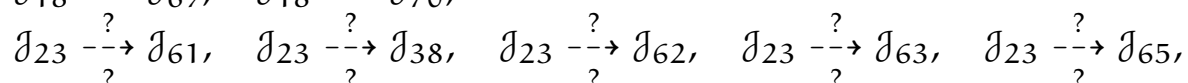

$$
\begin{aligned}
& J_{24} \stackrel{?}{\rightarrow} J_{61}, \quad J_{24} \stackrel{?}{\rightarrow} J_{38}, \quad J_{24} \stackrel{?}{\rightarrow} J_{52}, J_{24} \stackrel{?}{\rightarrow} J_{62}, \quad J_{24} \stackrel{?}{\rightarrow} J_{63}, J_{24} \stackrel{?}{\rightarrow} \rightarrow J_{65}, \\
& J_{25} \stackrel{?}{\rightarrow} J_{61}, \quad J_{25} \stackrel{?}{\rightarrow} J_{39}, \quad J_{25} \stackrel{?}{\rightarrow} J_{52} J_{52}, J_{25} \stackrel{?}{\rightarrow} J_{62}, \quad J_{25} \stackrel{?}{\rightarrow} J_{63}, \quad J_{25} \stackrel{?}{\rightarrow} J_{65}, \\
& J_{51} \stackrel{?}{\rightarrow} J_{61}, J_{51} \stackrel{?}{\rightarrow} J_{66}, J_{51} \stackrel{?}{\rightarrow} J_{63}, J_{51} \stackrel{?}{\rightarrow} J_{65}, \\
& J_{52} \stackrel{?}{\rightarrow} J_{61}, \quad J_{52} \stackrel{?}{\rightarrow} J_{66}, \quad J_{52} \stackrel{?}{\rightarrow} J_{68}, \quad J_{52} \stackrel{?}{\rightarrow} J_{63}, \quad J_{52} \stackrel{?}{\rightarrow} J_{65}, \\
& \partial_{53} \stackrel{?}{\rightarrow} J_{61}, \\
& \mathcal{J}_{44} \stackrel{?}{\stackrel{?}{\rightarrow}} \mathcal{J}_{67}, \quad \mathcal{J}_{44} \stackrel{?}{\stackrel{?}{\rightarrow}} \mathcal{J}_{68},
\end{aligned}
$$

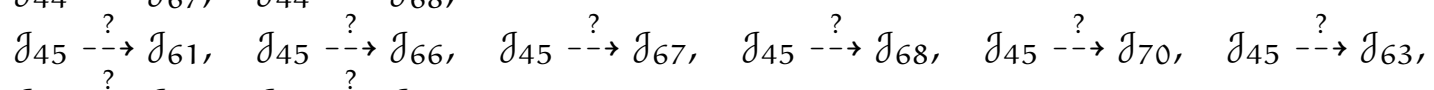

$$
\begin{aligned}
& \mathcal{J}_{45} \stackrel{?}{\rightarrow} \mathcal{J}_{64}, \quad \mathcal{J}_{45} \stackrel{?}{-} \rightarrow \mathcal{J}_{65},
\end{aligned}
$$

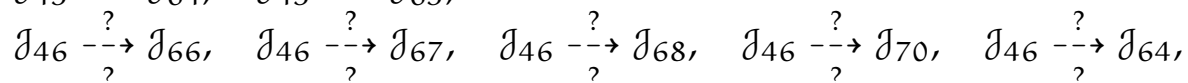

$$
\begin{aligned}
& J_{55} \stackrel{?}{\rightarrow} J_{66}, \quad J_{55} \stackrel{?}{\rightarrow} J_{67}, J_{55} \stackrel{?}{\rightarrow} J_{68}, J_{55} \stackrel{?}{\rightarrow} J_{70}, J_{55} \stackrel{?}{\rightarrow} J_{64},
\end{aligned}
$$

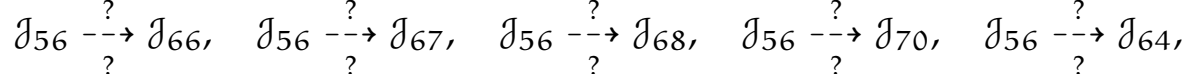

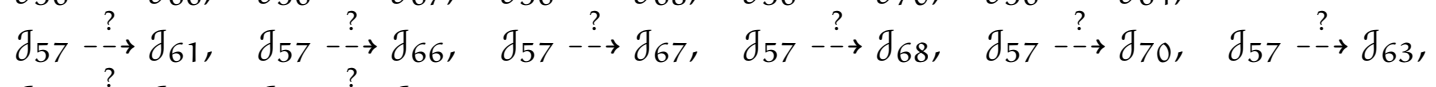

$$
\begin{aligned}
& \partial_{57} \stackrel{?}{\rightarrow} \partial_{64}, \quad \partial_{57} \stackrel{?}{\rightarrow} J_{65}, \\
& \mathrm{~J}_{48} \stackrel{?}{\stackrel{?}{\rightarrow}} \mathrm{J}_{67}, \mathrm{~J}_{48} \stackrel{?}{\stackrel{?}{\rightarrow}} \mathrm{J}_{68}, \mathrm{~J}_{48} \stackrel{?}{\stackrel{?}{\rightarrow}} \mathrm{J}_{69}, \mathrm{~J}_{48} \stackrel{?}{\stackrel{?}{\rightarrow}} \mathrm{J}_{70} \\
& J_{49} \stackrel{?}{\rightarrow} J_{66}, \quad J_{49} \stackrel{?}{\rightarrow} J_{67}, \quad J_{49} \stackrel{?}{\rightarrow} J_{68}, \quad J_{49} \stackrel{?}{\stackrel{?}{\rightarrow} J_{69},} J_{499} \stackrel{?}{\rightarrow} J_{70}, J_{49} \stackrel{?}{\rightarrow} J_{64},
\end{aligned}
$$

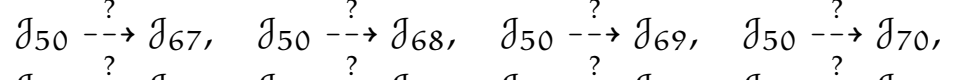

$$
\begin{aligned}
& \partial_{58} \stackrel{?}{\stackrel{?}{\rightarrow}} \partial_{67}, J_{58} \stackrel{?}{\rightarrow} J_{68}, J_{58} \stackrel{?}{\rightarrow} \partial_{69}, J_{58} \stackrel{?}{\rightarrow} J_{70}, \\
& J_{59} \stackrel{?}{\rightarrow} J_{66}, \quad J_{59} \stackrel{?}{\rightarrow} J_{67}, \quad J_{59} \stackrel{?}{\stackrel{?}{\rightarrow}} J_{68}, \quad J_{59} \stackrel{?}{\stackrel{?}{\rightarrow} J_{66},} J_{59} \stackrel{?}{\rightarrow} J_{70}, J_{59} \stackrel{?}{\rightarrow} J_{64}, \\
& \mathrm{~J}_{60} \stackrel{?}{?} \rightarrow \mathrm{J}_{67}, \mathrm{~J}_{60} \stackrel{?}{\rightarrow} \mathrm{J}_{68}, \mathrm{~J}_{60} \stackrel{?}{\rightarrow} \mathrm{J}_{69}, \mathrm{~J}_{60} \stackrel{?}{\rightarrow} \stackrel{?}{\rightarrow} \mathrm{J}_{70},
\end{aligned}
$$

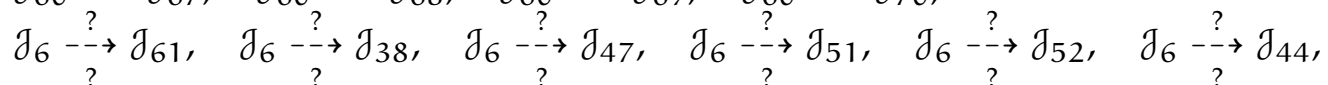

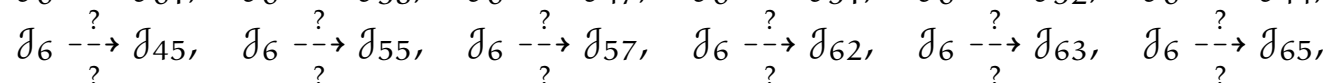

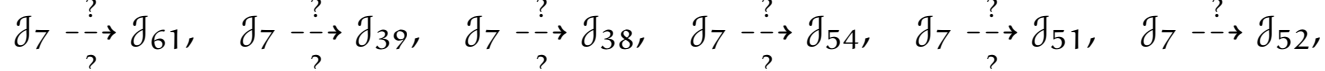

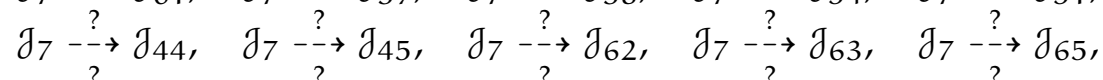

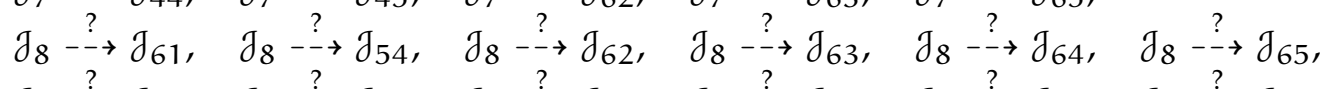

$$
\begin{aligned}
& \partial_{9} \stackrel{?}{\rightarrow} \partial_{66}, \quad J_{9} \stackrel{?}{\rightarrow} J_{67}, \quad \partial_{9} \stackrel{?}{\rightarrow} J_{68}, \quad \partial_{9} \stackrel{?}{\rightarrow} J_{69}, J_{9} \stackrel{?}{\rightarrow} J_{70}, J_{9} \stackrel{?}{\rightarrow} J_{42}, \\
& \partial_{9} \stackrel{?}{\rightarrow} \rightarrow \partial_{64}, \\
& \mathcal{J}_{1} \stackrel{?}{\stackrel{?}{\rightarrow}} \mathcal{J}_{61}, \quad \mathcal{J}_{1} \stackrel{?}{\stackrel{?}{\rightarrow}} \mathcal{J}_{39}, \quad \mathcal{J}_{1} \stackrel{?}{\stackrel{?}{\rightarrow}} \mathcal{J}_{38}, \quad \mathcal{J}_{1} \stackrel{?}{\stackrel{?}{\rightarrow}} \mathcal{J}_{54}, \quad \mathcal{J}_{1} \stackrel{?}{\stackrel{?}{\rightarrow}} \mathcal{J}_{27}, \\
& \mathcal{J}_{2} \stackrel{?}{\rightarrow} J_{61}, \quad J_{2} \stackrel{?}{\rightarrow} J_{66}, \quad J_{2} \stackrel{?}{\rightarrow} J_{67}, \quad J_{2} \stackrel{?}{\rightarrow} \rightarrow J_{68}, \quad J_{2} \stackrel{?}{\rightarrow} J_{69}, J_{2} \stackrel{?}{\rightarrow} J_{70}, \\
& \mathcal{J}_{2} \stackrel{?}{\rightarrow} \mathcal{J}_{43}, \quad J_{2} \stackrel{?}{\rightarrow} \mathcal{J}_{42}, \quad J_{2} \stackrel{?}{\rightarrow} \mathcal{J}_{63}, \quad J_{2} \stackrel{?}{\rightarrow} \mathcal{J}_{64}, \quad J_{2} \stackrel{?}{\rightarrow} \mathcal{J}_{65}, \\
& \partial_{65} \stackrel{?}{-\rightarrow} \partial_{64} \text {. }
\end{aligned}
$$

Existência de deformação desconhecida entre álgebras de Jordan de dimensão 3 sobre o corpo dos números reais

$$
\mathcal{T}_{3}^{\prime} \stackrel{?}{\rightarrow} \mathcal{T}_{18}^{\prime}, \quad \mathcal{T}_{3}^{\prime} \stackrel{?}{\rightarrow} \mathcal{T}_{23}^{\prime},
$$




$$
\begin{aligned}
& \mathcal{T}_{4}^{\prime} \stackrel{?}{-\rightarrow} \mathcal{T}_{18}^{\prime}, \quad \mathcal{T}_{4}^{\prime} \stackrel{?}{-\rightarrow} \mathcal{T}_{23}^{\prime}, \quad \mathcal{T}_{4}^{\prime} \stackrel{?}{-\rightarrow} \mathcal{T}_{26}^{\prime} \\
& \mathcal{T}_{5}^{\prime} \stackrel{?}{-\rightarrow} \mathcal{T}_{18}^{\prime}, \quad \mathcal{T}_{5}^{\prime} \stackrel{?}{-\rightarrow} \mathcal{T}_{23}^{\prime}, \\
& \mathcal{T}_{7}^{\prime} \stackrel{?}{\rightarrow} \rightarrow \mathcal{T}_{23}^{\prime}, \quad \mathcal{T}_{7}^{\prime} \stackrel{?}{\rightarrow} \rightarrow \mathcal{T}_{24}^{\prime}, \\
& \mathcal{T}_{8}^{\prime} \stackrel{?}{-\rightarrow} \mathcal{T}_{23}^{\prime}, \quad \mathcal{T}_{8}^{\prime} \stackrel{?}{-\rightarrow} \rightarrow \mathcal{T}_{24}^{\prime} \\
& \mathcal{T}_{10}^{\prime} \stackrel{?}{-\rightarrow} \rightarrow \mathcal{T}_{24}^{\prime} \\
& \mathcal{T}_{11}^{\prime} \stackrel{?}{\rightarrow} \mathcal{T}_{23}^{\prime}, \quad \mathcal{T}_{11}^{\prime} \stackrel{?}{\rightarrow} \mathcal{T}_{26}^{\prime} \\
& \mathcal{T}_{19}^{\prime} \stackrel{?}{?-\rightarrow} \mathcal{T}_{23}^{\prime}, \\
& \mathcal{T}_{20}^{\prime} \stackrel{?}{-\rightarrow} \rightarrow \mathcal{T}_{23}^{\prime}
\end{aligned}
$$

Outra questão que surge naturalmente ao tentar determinar a não existência de deformação está relacionada aos invariantes geométricos. Sabemos como se comportam a dimensão do grupo de automorfismos, do radical, aniquilador e das potências através de uma deformação. Isto nos levou a pesquisar o comportamento de outros invariantes tais como a dimensão do centro associador, do espaço dos 2-cociclos, dos 2-cobordos, da segunda cohomologia e da subálgebra maximal satisfazendo uma identidade. O que nos motiva a perguntar se seria possível determinar outros invariantes.

No final do Capítulo 5 obtivemos algumas propriedades da variedade algébrica Jor 5 . Conseguimos determinar que o número de componentes irredutíveis é maior ou igual a 26. Isto nos leva a questionarmos sobre a possibilidade de melhorar esta estimativa. Mais ainda, seria possível obter a classificação algébrica e geométrica das álgebras de Jordan de dimensão 5 sobre um corpo algebricamente fechado? Qual seria a dificuldade que isto pode apresentar em relação ao número de álgebras e ao tempo computacional dos programas desenvolvidos neste trabalho em dimensão 5? E também, será possível classificar algébrica e geometricamente as álgebras de Jordan de dimensão 4 sobre o corpo dos números reais? Uma estimativa do número de álgebras e do número de componentes irredutíveis em cada caso é dado na seguinte tabela.

\begin{tabular}{|c|c|c|c|c|}
\hline & \multicolumn{2}{|c|}{ Jor $_{n}^{\mathbb{R}}$} & \multicolumn{2}{c|}{ Jor $_{\mathrm{n}}$} \\
\hline $\mathrm{n}$ & $\mathrm{n}^{\mathrm{o}}$ órbitas & $\mathrm{n}^{\mathrm{o}}$ componentes & $\mathrm{n}^{\mathrm{o}}$ órbitas & $\mathrm{n}^{\mathrm{o}}$ componentes \\
\hline 1 & 2 & 1 & 2 & 1 \\
\hline 2 & 7 & 3 & 6 & 2 \\
\hline 3 & 26 & 8 & 20 & 5 \\
\hline 4 & $>109$ & $\geqslant 18$ & 73 & 10 \\
\hline 5 & - & - & $\gg 223$ & $\geqslant 26$ \\
\hline
\end{tabular}

Tabela 8.1: Número de órbitas e de componentes irredutíveis de $\operatorname{Jor}_{\mathfrak{n}}$ e $\operatorname{Jor}_{\mathfrak{n}}^{\mathbb{R}}$ 
Outra propriedade apresentada no Capítulo 5 é o fato da soma direta de álgebras rígidas ser rígida em Jor $\mathrm{n}$ para $\mathrm{n} \leqslant 5$. E ainda provamos que soma direta de álgebras infinitesimalmente rígidas é infinitesimalmente rígida, em qualquer dimensão. Isto nos motiva a perguntarmos se rigidez geométrica (respetivamente, analítica) é preservada por soma direta em todas as dimensões. Mais ainda, quais operações preservam rigidez?

Por outro lado, varias das proposições apresentadas no Capítulo 4 tem como hipóteses o fato da variedade ter um número finito de órbitas. De fato as variedades Jor $_{n}$ tem uma quantidade finita de órbitas para todo $n \leqslant 4$. Isto nos leva a questionarmos se a variedade das álgebras de Jordan sempre apresenta um número finito de órbitas ou se seria possível encontrar uma família infinita.

Por último, quando consideramos álgebras de dimensão finita:

$$
\text { rigidez infinitesimal } \Rightarrow \text { rigidez analítica } \Rightarrow \text { rigidez geométrica, }
$$

e ainda se o corpo tiver característica zero então rigidez geométrica $\Rightarrow$ rigidez analítica. $\mathrm{Na}$ categoria das álgebras associativas, para corpos de característica positiva, todas as implicações inversas foram provadas serem falsas mas, ainda permanece aberta a questão de se existe uma álgebra associativa, em char $=0$, que seja analiticamente rígida mas tenha segundo grupo de cohomologia não nulo. Enquanto que, esta última sentença, é verdadeira para álgebras de Lie. Mas, até o momento, nada se conhece em relação ás álgebras de Jordan. A pergunta que surge naturalmente é se seria possível provar ou achar contraexemplos para álgebras de Jordan de dimensão finita das seguintes implicações:

$\square$ para um corpo $\mathbf{k}$ com char $\mathbf{k}>0$, rigidez geométrica $\Rightarrow$ rigidez analítica?

$\square$ sobre quais hipóteses rigidez analítica $\Rightarrow$ rigidez infinitesimal?

$\square$ sobre quais hipóteses rigidez geométrica $\Rightarrow$ rigidez infinitesimal? 


\section{PROGRAMAS EM MATHEMATICA}

A seguir serão apresentados os algoritmos os quais são implementados utilizando o software Wolfram Mathematica versão 9.o.1.

\section{A.1 DEFINE O PRODUTO DE JORDAN NA BASE CANÔNICA}

Para o funcionamento dos seguintes programas devemos definir o produto de todas as álgebras de dimensão 4 , numa base fixa $\left\{e_{1}, e_{2}, e_{3}, e_{4}\right\}$, como feito no exemplo a seguir:

$$
\begin{aligned}
& \text { Álgebra J19 } \\
& g[e 1, e 1, J 19]=e 1 ; \\
& g[e 2, e 2, J 19]=e 2 ; \\
& g[e 3, e 3, J 19]=0 ; \\
& g[e 4, e 4, J 19]=0 ; \\
& g[e 1, e 2, J 19]=g[e 2, e 1, J 19]=0 ; \\
& g[e 1, e 3, J 19]=g[e 3, e 1, J 19]=0 ; \\
& g[e 1, e 4, J 19]=g[e 4, e 1, J 19]=0 ; \\
& g[e 2, e 3, J 19]=g[e 3, e 2, J 19]=0 ; \\
& g[e 2, e 4, J 19]=g[e 4, e 2, J 19]=0 ; \\
& g[e 3, e 4, J 19]=g[e 4, e 3, J 19]=0 ;
\end{aligned}
$$

Nomeamos as álgebras e as listamos de acordo com sua dimensão.

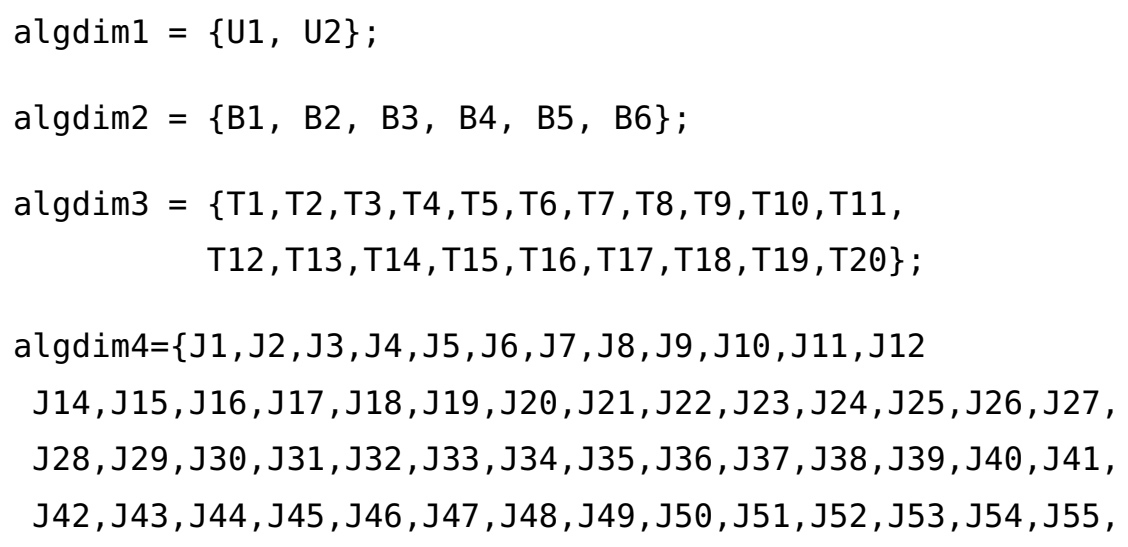


$\mathrm{J} 56$, J57 , J58, J59 , J60 , J61 , J62 , J63, J64 , J65 , J66 , J67 , J68 , J69,

$\mathrm{J} 70, \mathrm{~J} 71, \mathrm{~J} 72, \mathrm{~J} 73\}$;

A função dimensao[z] retorna a dimensão da álgebra z.

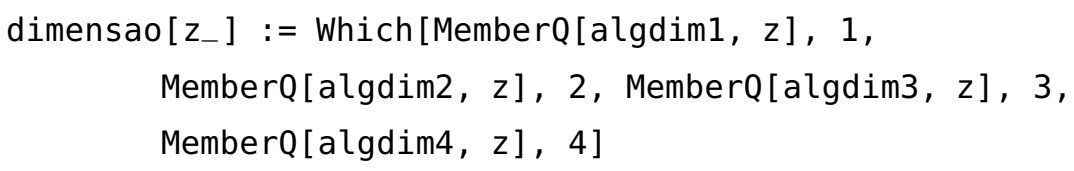

Definimos a base da álgebra.

$$
\text { base }=\{e 1, e 2, e 3, e 4\}
$$

Ingressando um elemento $z$ da forma $a e_{1}+b e_{2}+c e_{3}+d e_{4}$, a função paracan [z] retorna um vetor da forma $\{a, b, c, d\}$. As funções paracan3 e paracan2 fazem o procedimento análogo em dimensão 3 e 2 respetivamente.

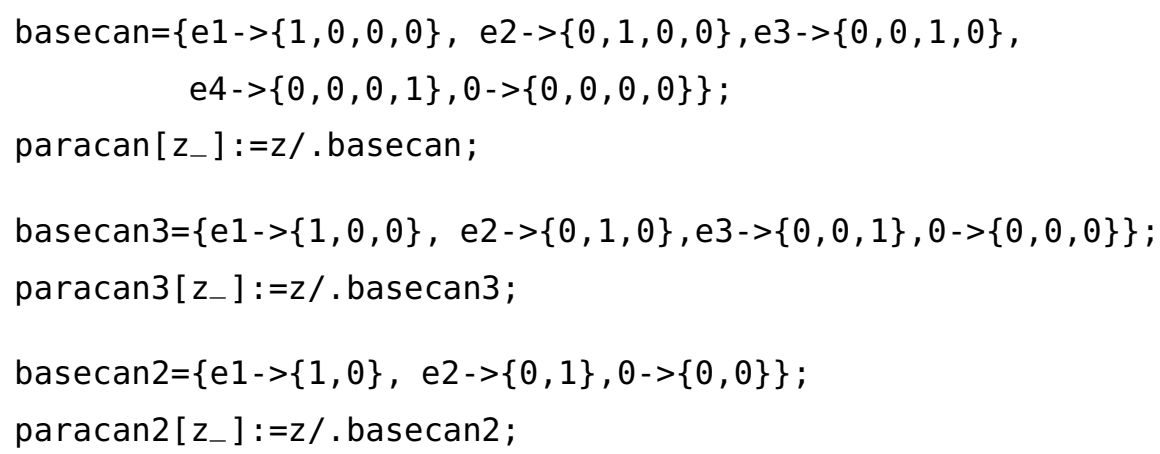

Reciprocamente, ingressando um vetor $z$ da forma $\{a, b, c, d\}$, a função decan $[z]$ retorna um elemento da forma $a e_{1}+b_{2}+c e_{3}+d e_{4}$. As funções decan3 e decan2 fazem $o$ procedimento análogo em dimensão 3 e 2 respetivamente.

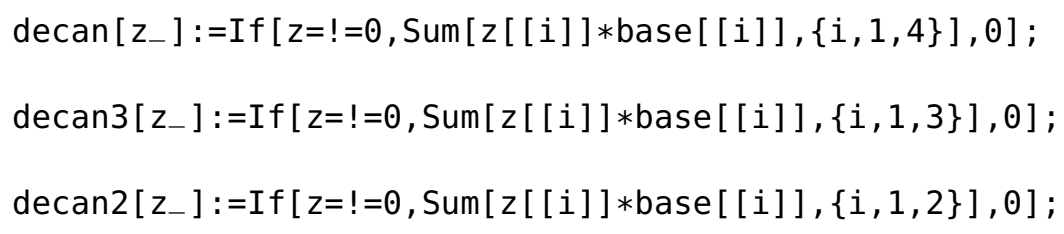

Estendendo o Produto da Álgebra por Linearidade.

Ingressando dois elementos $v$ e w da forma $a e_{1}+b e_{2}+c e_{3}+d e_{4}$, a função $g[v, w$, algebra $]$ retorna o produto deles na álgebra "algebra" na forma $\{a, b, c, d\}$. Analogamente g3 e g2 em dimensão 3 e 2 respetivamente. 


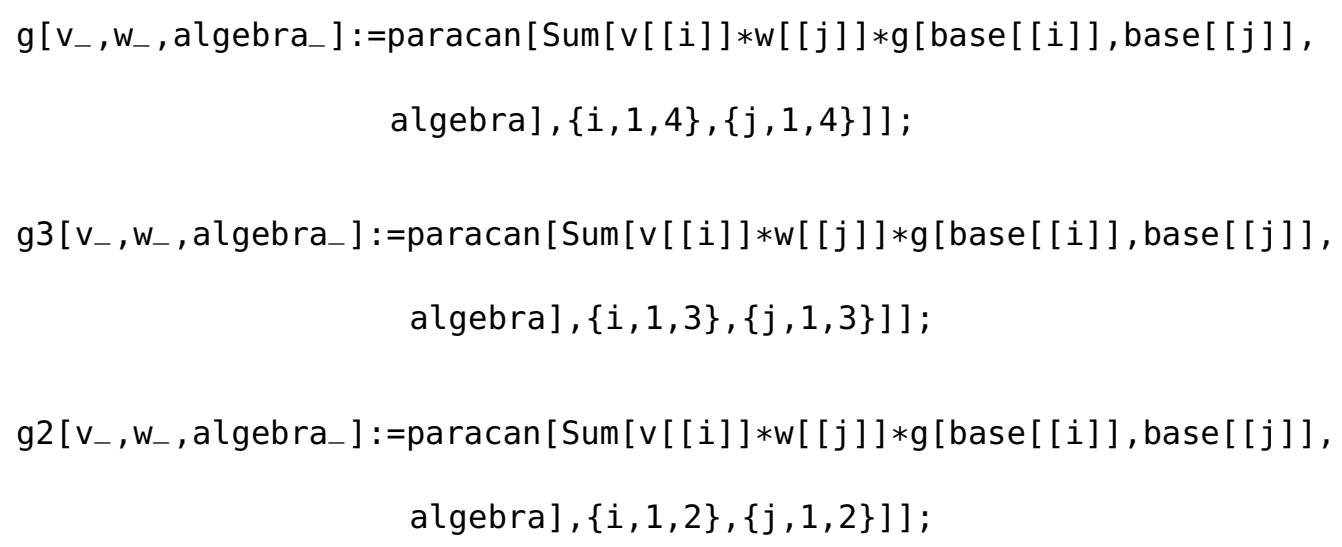

Ingressando dois elementos $v$ e $w$ da forma $a e_{1}+b e_{2}+c e_{3}+d e_{4}$, a função $G[v, w$, algebra] retorna o produto deles na álgebra "algebra" na forma $a e_{1}+b_{2}+c e_{3}+d e_{4}$. Analogamente G3 e G2 em dimensão 3 e 2 respetivamente.

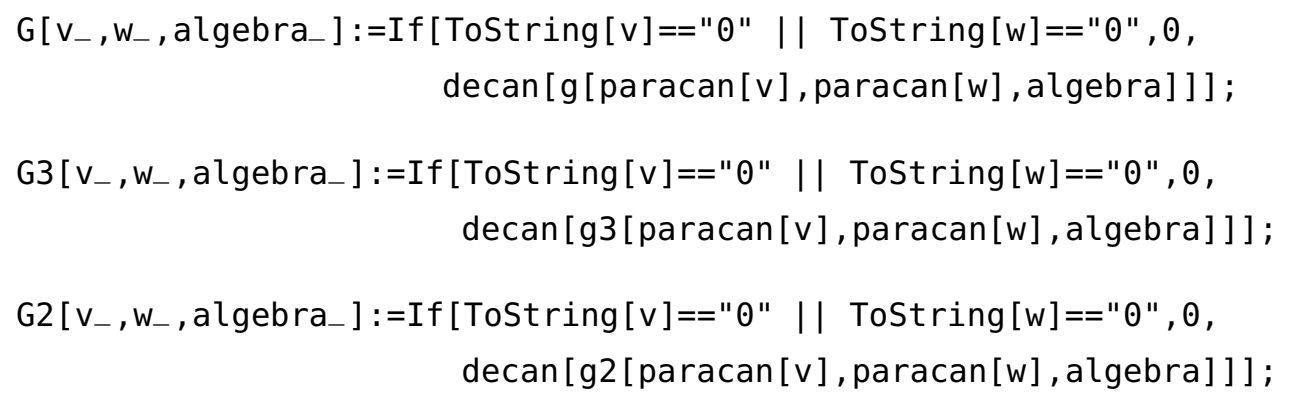

\section{A.2 VERIFICA SE DUAS ÁLGEBRAS SÃO ISOMORFAS}

Ingressando um elemento $z$ da forma $a e_{1}+b e_{2}+c e_{3}+d e_{4}$, a função TEF[z] troca a base $e_{i}$ por $f_{i}$.

$$
\operatorname{TEF}\left[z_{-}\right]:=z / .\{e 1->f 1, e 2->f 2, e 3->f 3, e 4->f 4\}
$$

Ingressando duas álgebras de dimensão 4 z e w, a função Relacoes [ $z, w]$ gera as condições nos elementos da base para duas álgebras serem isomorfas. As funções Relacoes 3 e Relacoes 2 realizam um procedimento análogo em dimensão 3 e 2 respetivamente.

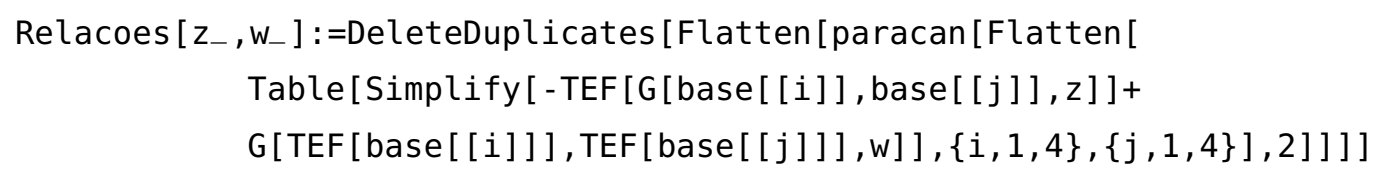




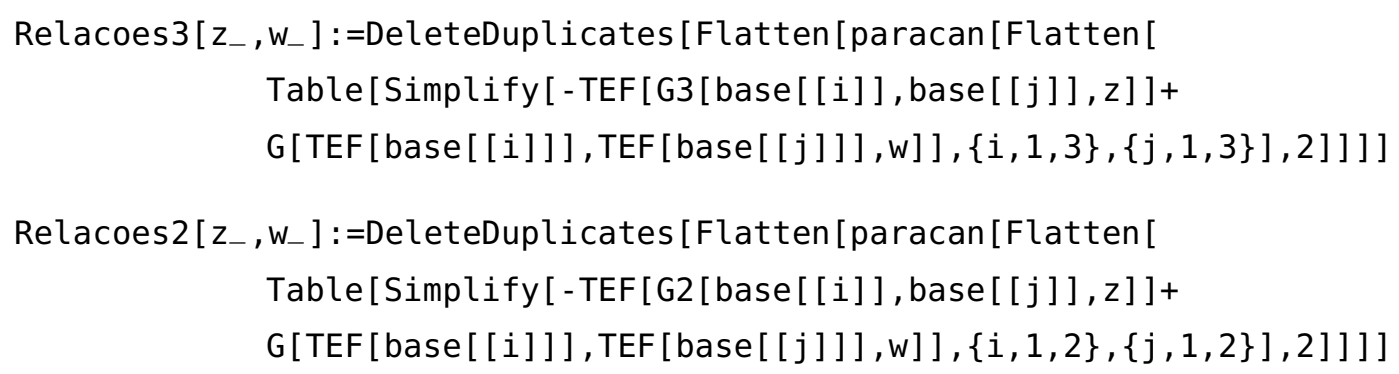

A função isomorfismo $[z, w]$ retorna um isomorfismo entre as álgebras $z$ e w, se existir.

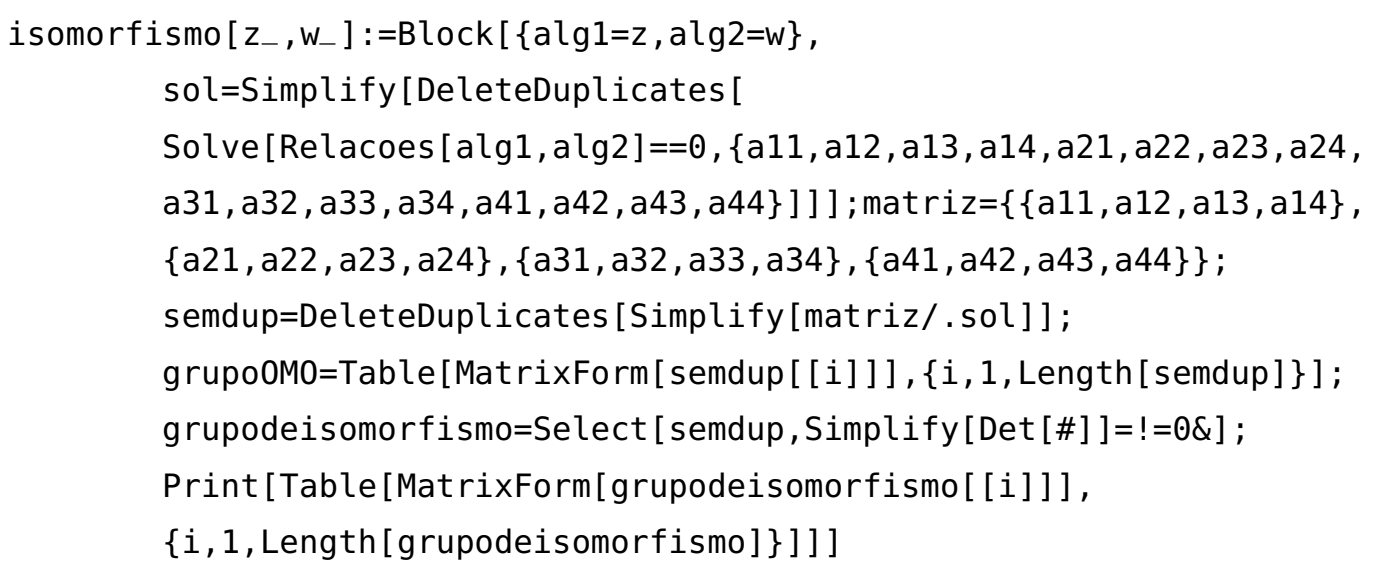

A função Qisomorfas $[z, w]$ retorna True ou False se duas álgebras z e w são ou não isomorfas.

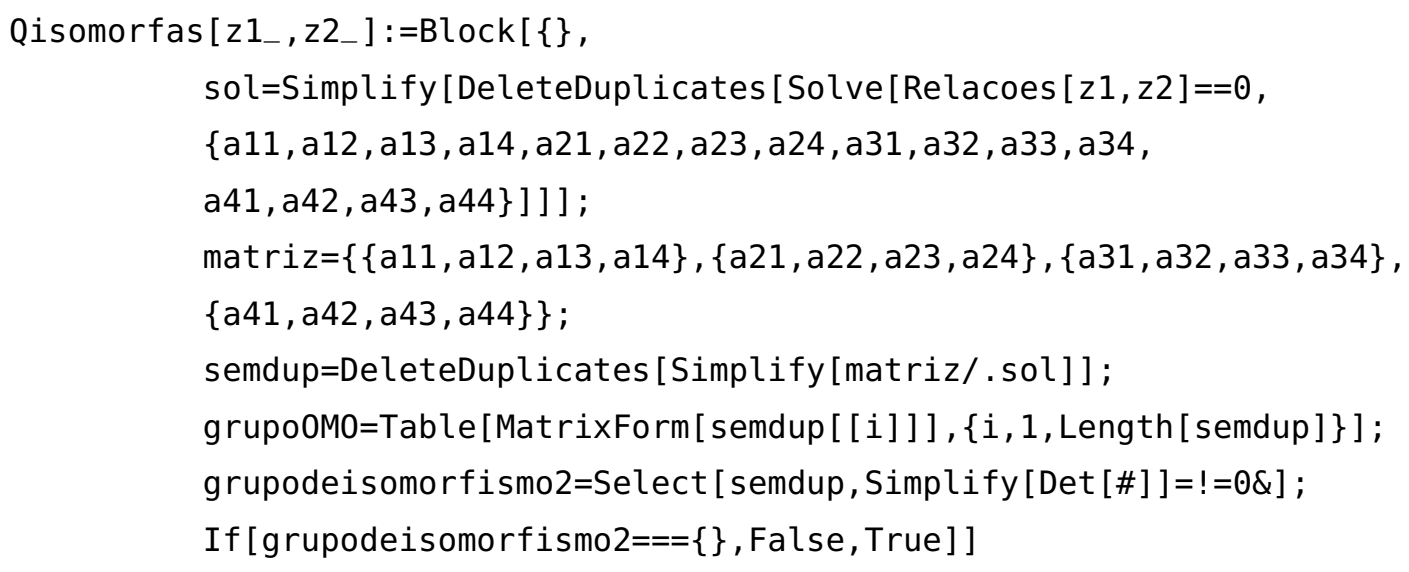

A.3 VERIficA SE UMA Álgebra É DE JORDAN, Flexível, ASSOCIATIVA, E/OU ALTERNATIVA.

Definimos quatro elementos arbitrários de uma álgebra. 


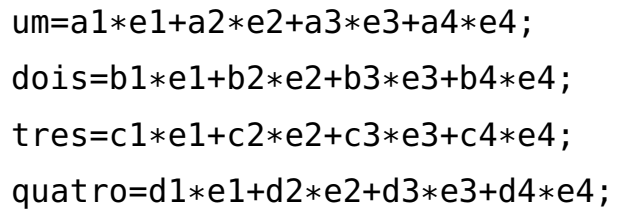

Definimos o associador dos elementos $\mathrm{x}, \mathrm{y}$ e $\mathrm{z}$ de uma álgebra alg.

$$
\operatorname{Ass}\left[x_{-}, y_{-}, z_{-}, a l g-\right]:=G[G[x, y, a l g], z, a l g]-G[x, G[y, z, a l g], a l g]
$$

A função Jor[alg] verifica nos elementos arbitrários e retorna se a álgebra alg é de Jordan.

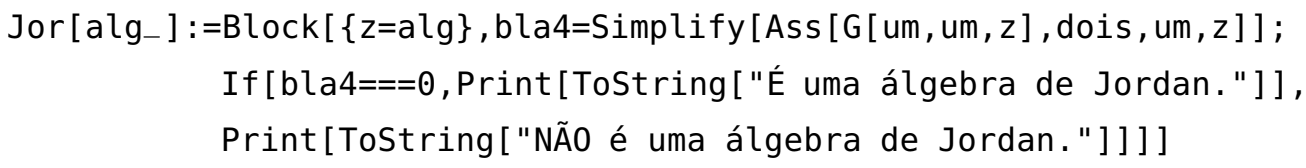

A função LinearJord[alg] verifica nos elementos arbitrários e retorna se a álgebra alg satisfaz a linearização da identidade de Jordan.

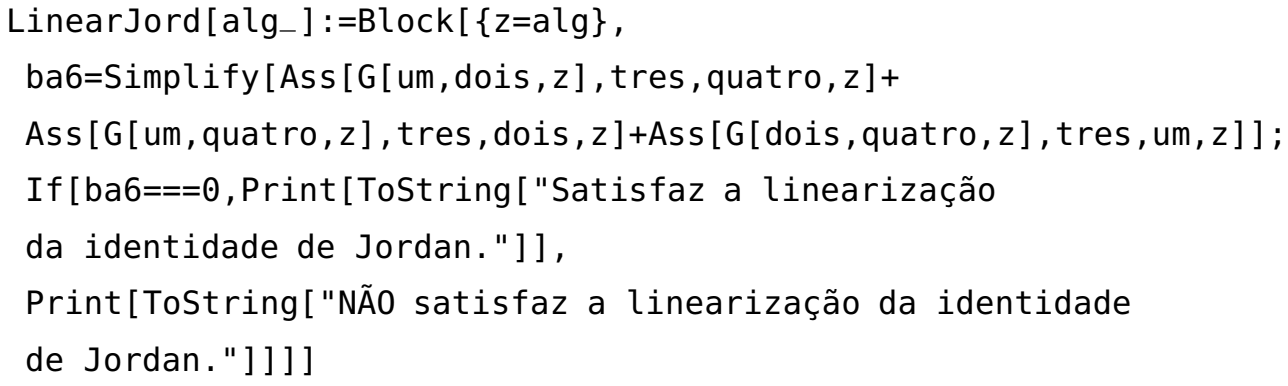

A função Asso [alg] verifica nos elementos arbitrários e retorna se a álgebra alg é associativa.

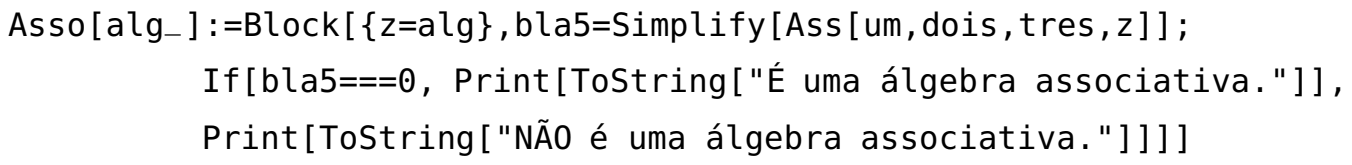

A função $A l t D[a l g]$ verifica nos elementos arbitrários e retorna se a álgebra alg é alternativa à direita.

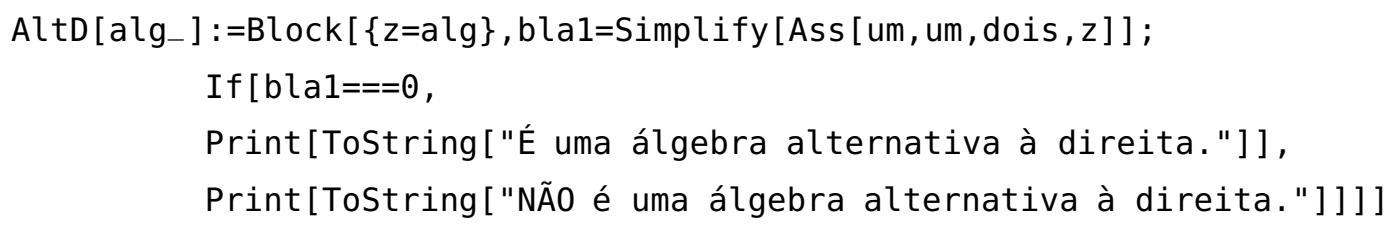


A função AltE[alg] verifica nos elementos arbitrários e retorna se a álgebra alg é alternativa à esquerda.

$$
\begin{aligned}
& \text { AltE[alg_ }:=B \operatorname{Block}[\{z=a l g\}, \text { bla2=Simplify[Ass[um,dois, dois, } z]] ; \\
& \text { If[bla2===0,Print[ToString["É uma álgebra alternativa à esquerda."]], } \\
& \text { Print[ToString["NÃO é uma álgebra alternativa à esquerda."]]]] }
\end{aligned}
$$

A função Flex[alg] verifica nos elementos arbitrários e retorna se a álgebra alg é flexível.

$$
\begin{aligned}
\text { Flex[alg_ }]:= & B l o c k[\{z=a l g\}, \text { blaß=Simplify[Ass[um,dois,um,z]]; } \\
& \text { If [bla3===0,Print[ToString["É uma álgebra flexível."]], } \\
& \text { Print[ToString["NÃO é uma álgebra flexível."]]]] }
\end{aligned}
$$

No caso que a álgebra z não seja de Jordan, a função Jor2 [z] mostra quais produtos $\left(x^{2}, y, x\right)$ são não nulos

$$
\begin{aligned}
& \operatorname{Jor} 2\left[z_{-}\right]:=\text {Table[Simplify[ Ass }[G[\text { base }[[i]], \text { base[[i]], } z] \\
& \text { base[[j]], }
\end{aligned}
$$

A função Jordan [z] resolve as condições (RelacoesJor[z]) para que a álgebra z satisfaça a linearização da identidade de Jordan

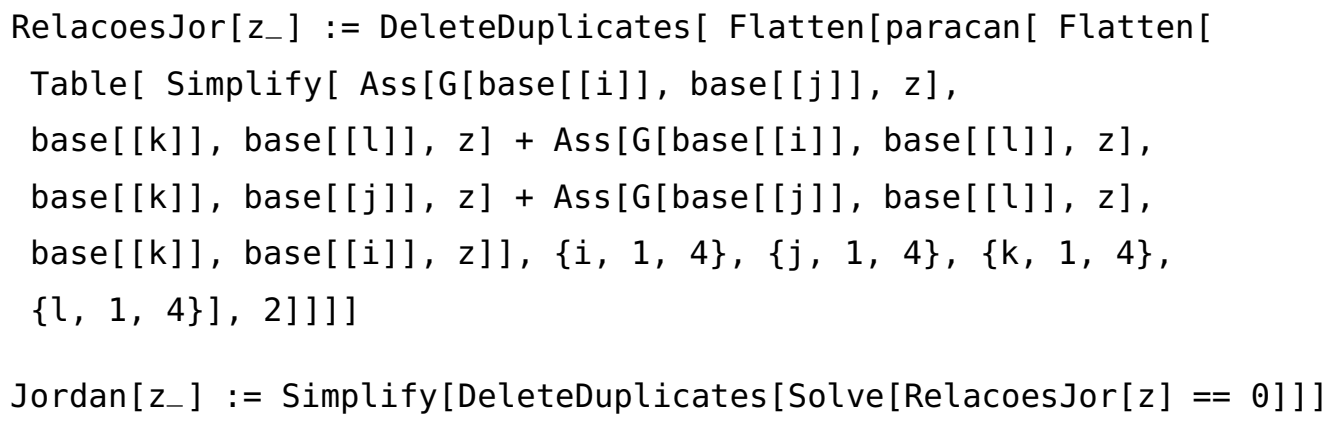

\section{A.4 VERIFICA SE UMA ÁLGEBRA É UNITÁRIA}

A função Unidade[alg] verifica se a álgebra alg possui o não uma unidade, no caso que possua retorna a unidade.

$$
\begin{aligned}
\text { Unidade }[\text { alg_ }]:= & \text { Block }[\{\operatorname{vet}=\{\mathrm{a} 1, \mathrm{a} 2, \mathrm{a} 3, \mathrm{a} 4\}, \\
& \text { unidade }:=\text { Solve }[\text { Table }[\text { paracan }[G[\text { decan [vet }],
\end{aligned}
$$




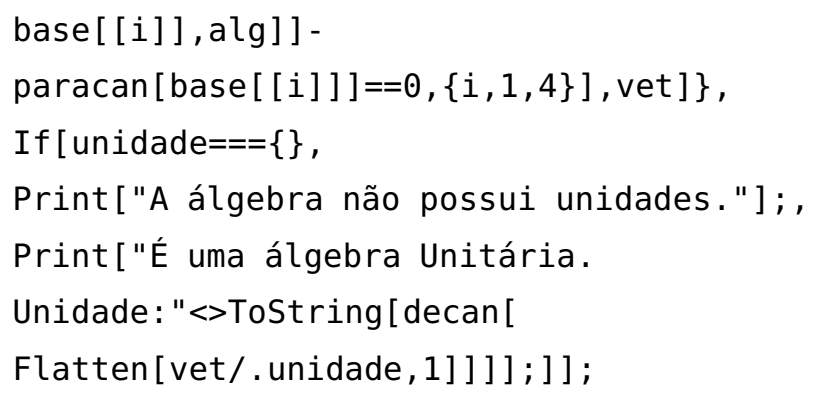

\section{A.5 VERIFICA SE É SUBÁlgeBra.}

A função subalgebradim34 [ $z, w]$ retorna se a álgebra $z$ de dimensão 3 é uma subálgebra da álgebra w de dimensão 4.

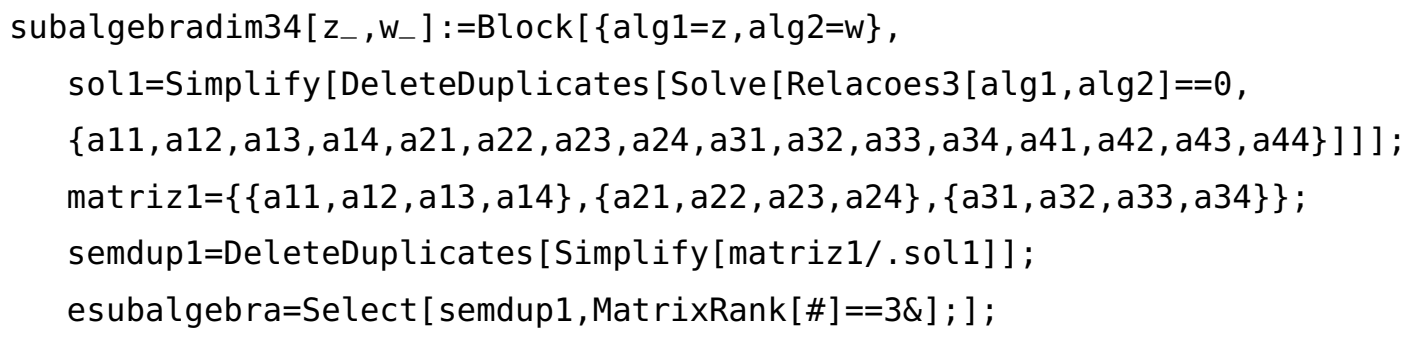

A função sub34[z] retorna a lista de todas as subálgebras de dimensão 3 da álgebra z de dimensão 4.

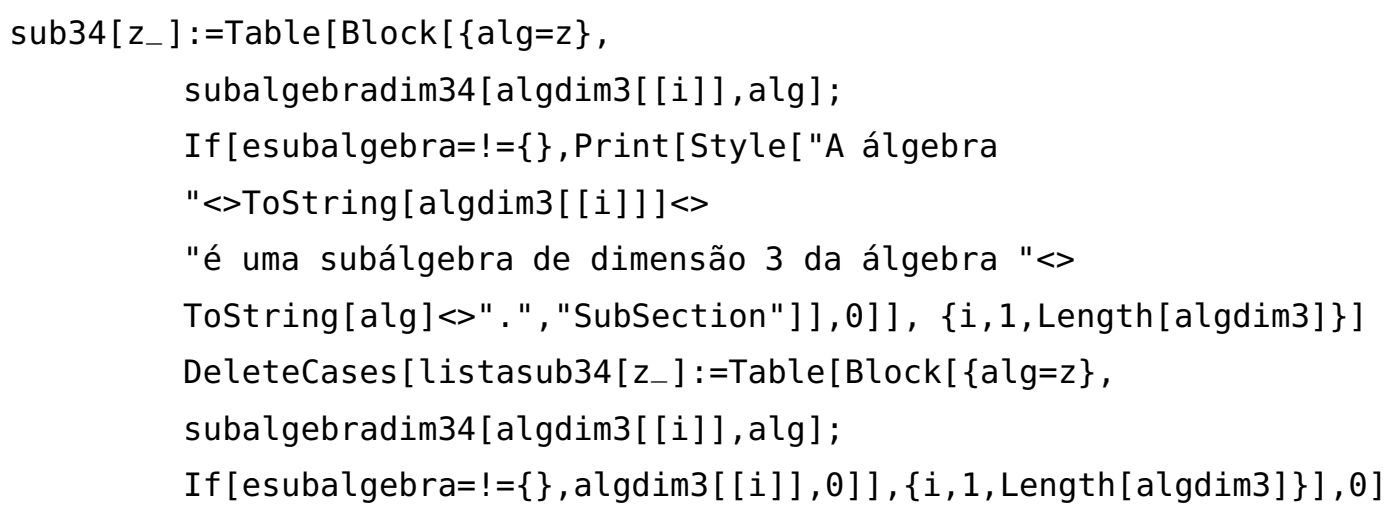

A função subalgebradim24 [ $z$, w ] retorna se a álgebra $z$ de dimensão 2 é uma subálgebra da álgebra w de dimensão 4.

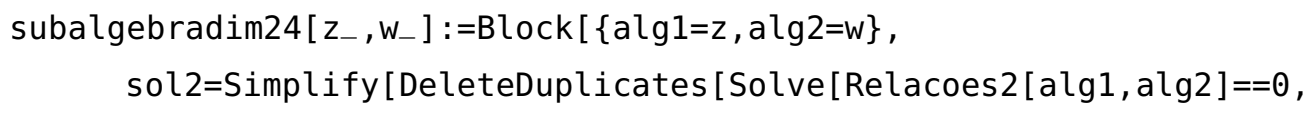




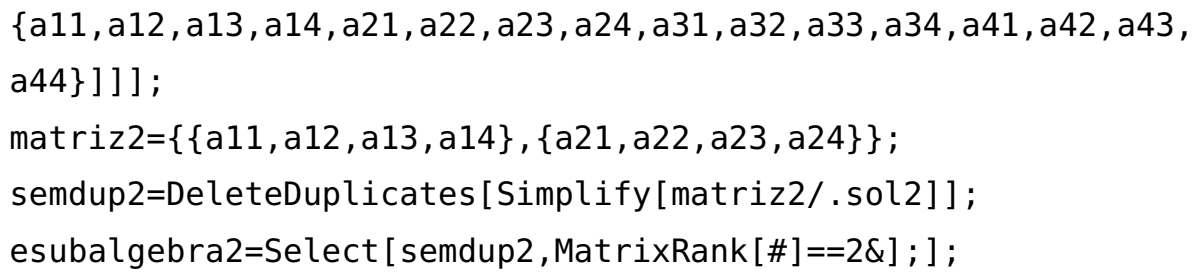

A função sub24[z] retorna a lista de todas as subálgebras de dimensão 2 da álgebra z de dimensão 4.

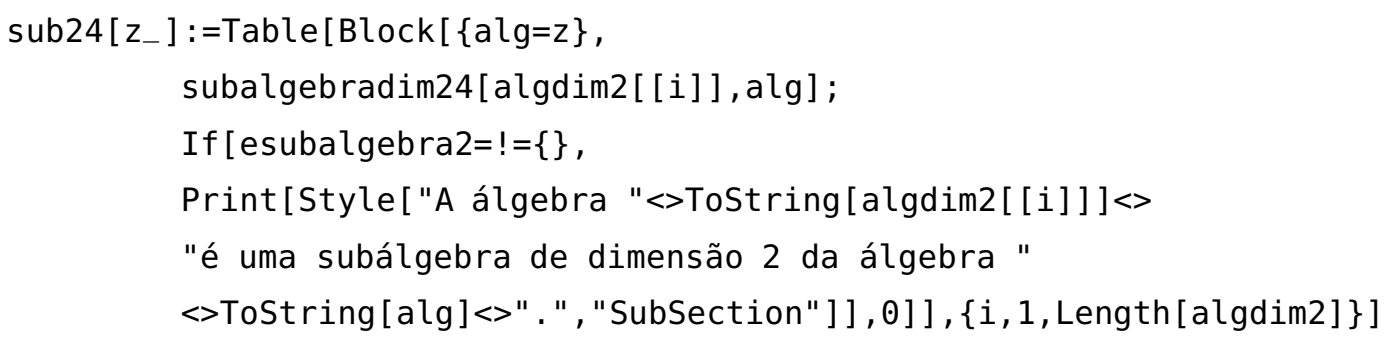

\section{A.6 CAlCula A Dimensẽo DO ANiquilador de UMA Ál- GEBRA}

A função ann [alg] retorna a dimensão do aniquilador da álgebra alg.

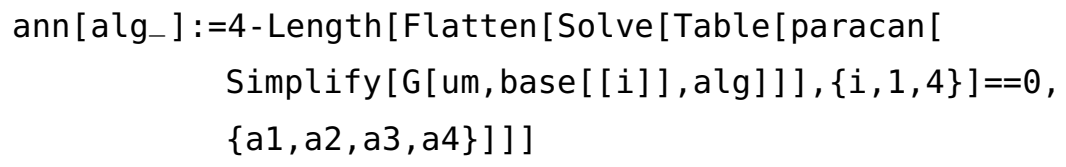

\section{A.7 CALCULA A DIMENSÃO DAS POTÊNCIAS}

A função produto2 $[u 1, u 2, w]$ retorna todas as palavras de comprimento 2 que podemse formar com os elementos u1 e u2 na álgebra w.

$$
\text { produto2 }\left[\mathrm{u} 1_{-}, \mathrm{u} 2_{-}, \mathrm{w}_{-}\right]:=\operatorname{paracan}[\mathrm{G}[\mathrm{u} 1, \mathrm{u} 2, \mathrm{w}]] \text {; }
$$

A função produto $3[u 1, u 2, u 3, w]$ retorna todas as palavras de comprimento 3 que podemse formar com os elementos u1, u2 e u3 na álgebra w.

$$
\begin{aligned}
\operatorname{produto}\left[\mathrm{u} 1_{-}, \mathrm{u} 2_{-}, \mathrm{u} 3_{-}, \mathrm{w}_{-}\right]:= & \{\operatorname{paracan}[\mathrm{G}[\mathrm{G}[\mathrm{u} 1, \mathrm{u} 2, \mathrm{w}], \mathrm{u} 3, \mathrm{w}]], \\
& \operatorname{paracan}[\mathrm{G}[\mathrm{u} 3, \mathrm{G}[\mathrm{u} 1, \mathrm{u} 2, \mathrm{w}], \mathrm{w}]]\} ;
\end{aligned}
$$


A função produto $4[u 1, u 2, u 3, u 4, w]$ retorna todas as palavras de comprimento 4 que podem-se formar com os elementos u1, u2, u3 e u4 na álgebra w.

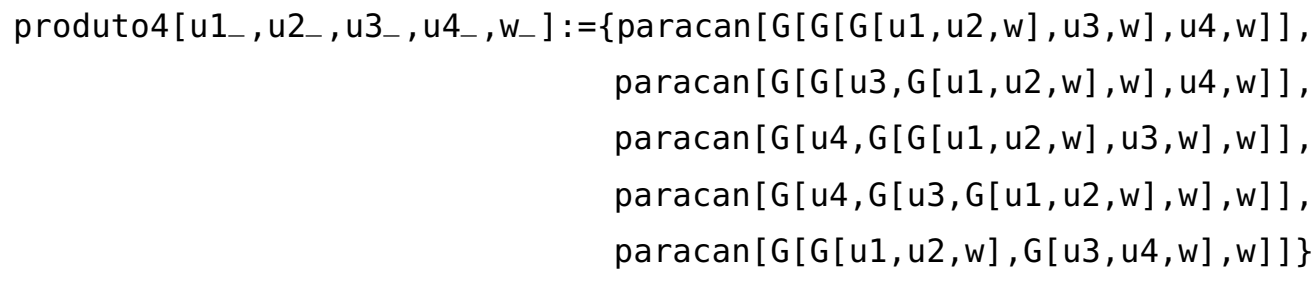

A função Potencia2 [w] retorna a dimensão da segunda potencia da álgebra w.

$$
\begin{aligned}
& \text { Potencia2 [w-] : =MatrixRank [Flatten [Table [Simplify [ } \\
& \text { produto2 [base [ [i]], base[[j]],w]], } \\
& \{i, 1,4\},\{j, 1,4\}], 1]] \text {; }
\end{aligned}
$$

A função Potencia3 [w] retorna a dimensão da terceira potencia da álgebra w.

$$
\begin{aligned}
\operatorname{Potencia} 3\left[\mathrm{w}_{-}\right]:= & \text {MatrixRank }[\text { Flatten }[\text { Table }[\text { Simplify }[ \\
& \operatorname{produto}[\text { base }[[i]], \text { base }[j]]], \text { base }[[k]], w]], \\
& \{i, 1,4\},\{j, 1,4\},\{k, 1,4\}], 3]] ;
\end{aligned}
$$

A função Potencia4[w] retorna a dimensão da quarta potencia da álgebra w.

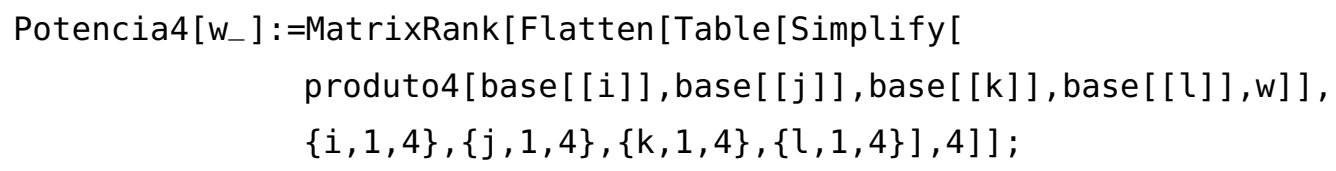

\section{A.8 CAlCula A Dimensão DO GRupo DE AUtomorfismo}

A função Relacoes7 [ $z$ ] gera as condições para uma aplicação ser um automorfismo da álgebra z.

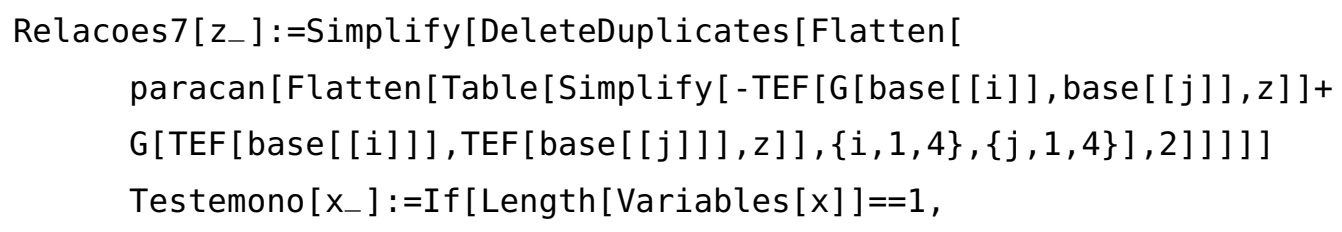




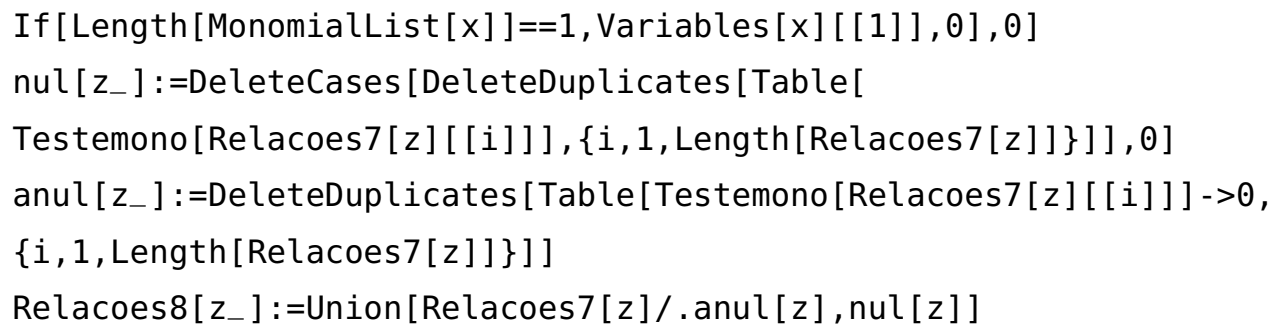

A função dim[z] retorna a dimensão do grupo de automorfismos da álgebra $z$.

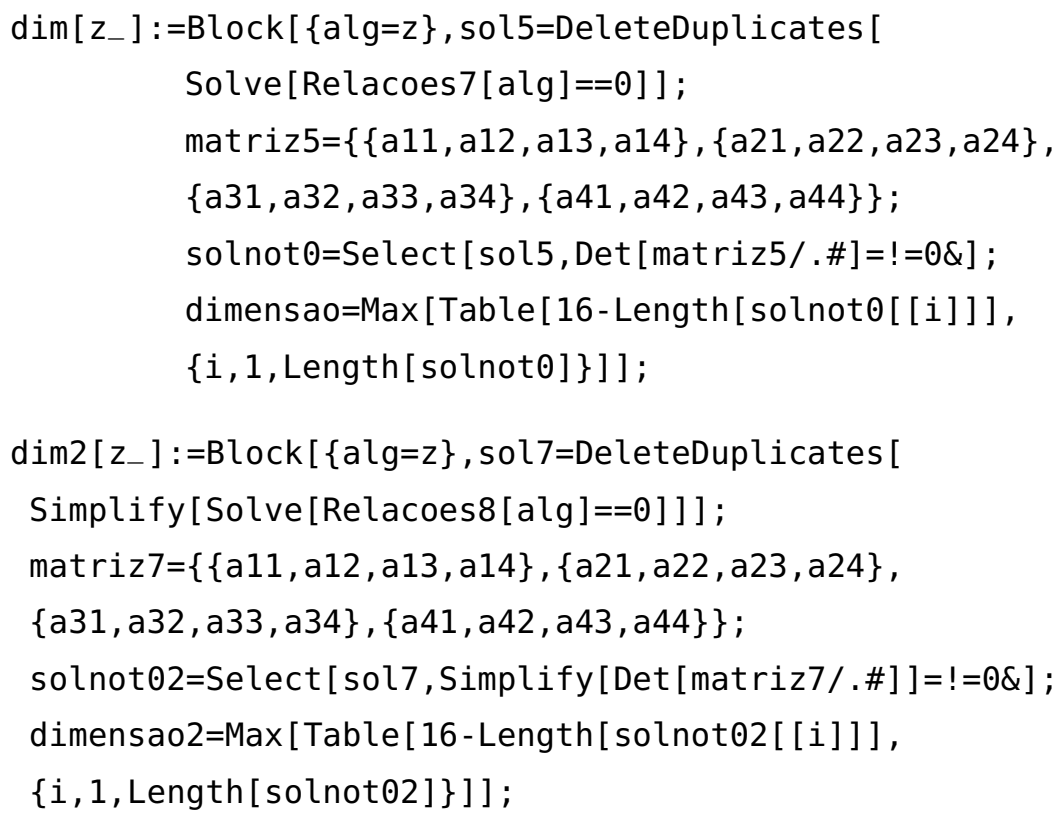

A função grupo [z] retorna o grupo de automorfismos da álgebra $z$.

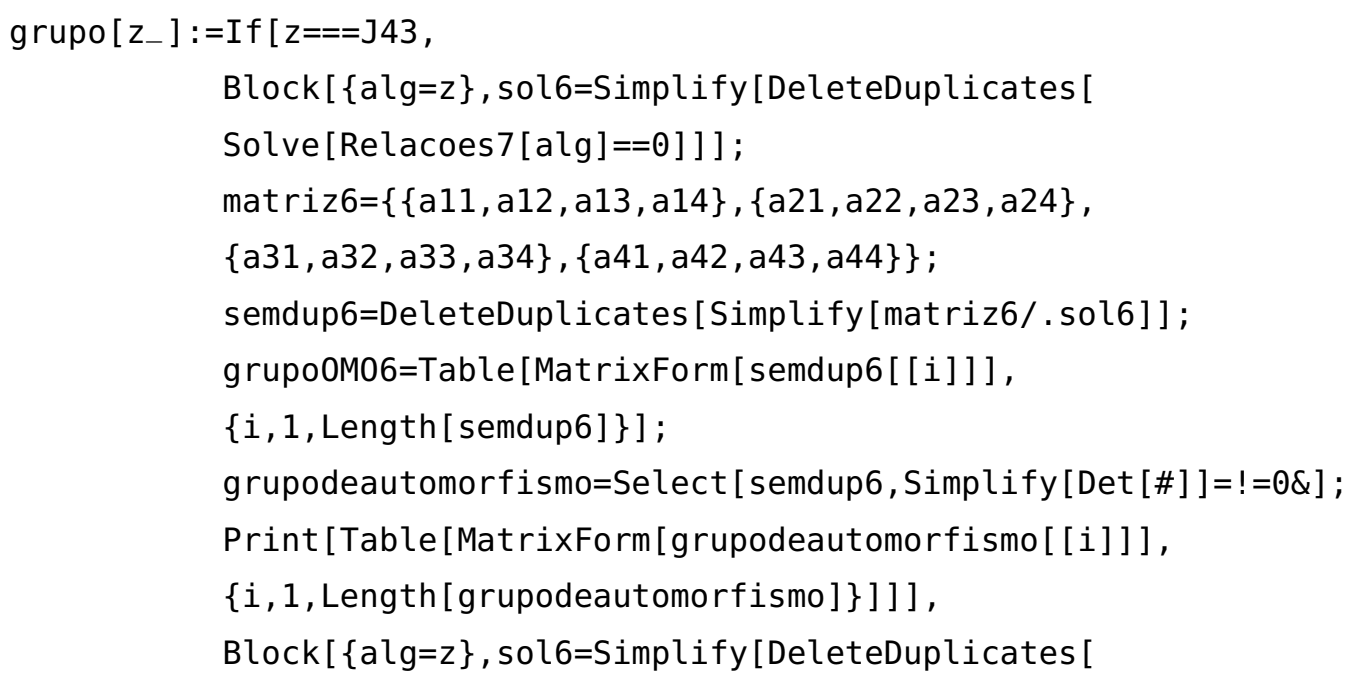




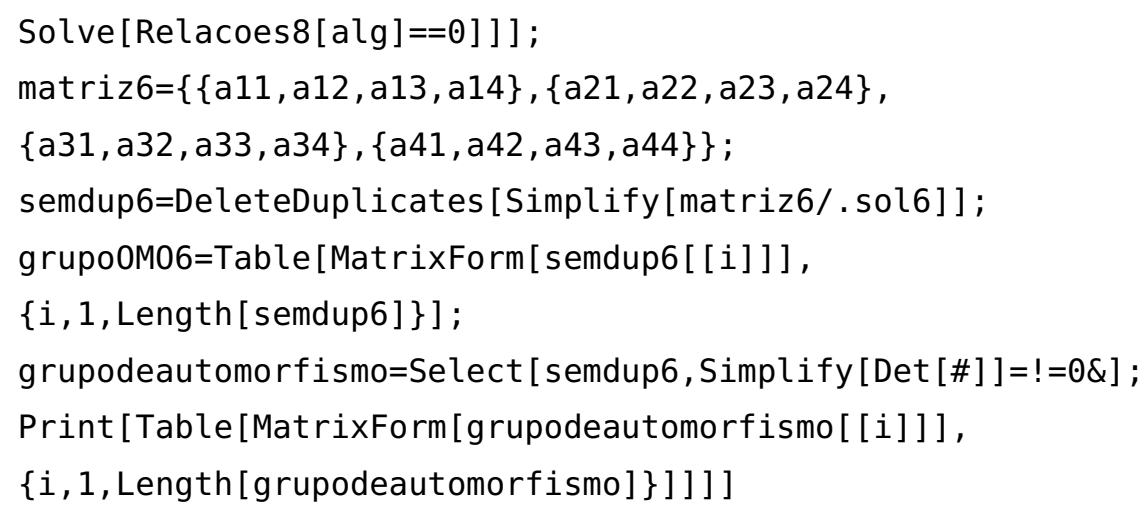

\section{A.9 CAlCula A Dimensão DE $h^{2}$.}

Definimos genericamente um 2-cociclo $\mathrm{h}: \mathcal{J} \times \mathcal{J} \rightarrow \mathcal{J}$ nos elementos da base.

$$
\begin{aligned}
& \mathrm{g}[\mathrm{e} 1, \mathrm{e} 1, \mathrm{H}]=\mathrm{a} 111 * \mathrm{e} 1+\mathrm{a} 112 * \mathrm{e} 2+\mathrm{a} 113 * \mathrm{e} 3+\mathrm{a} 114 * \mathrm{e} 4 ; \\
& \mathrm{g}[\mathrm{e} 2, \mathrm{e} 2, \mathrm{H}]=\mathrm{a} 221 * \mathrm{e} 1+\mathrm{a} 222 * \mathrm{e} 2+\mathrm{a} 223 * \mathrm{e} 3+\mathrm{a} 224 * \mathrm{e} 4 ; \\
& \mathrm{g}[\mathrm{e} 3, \mathrm{e} 3, \mathrm{H}]=\mathrm{a} 331 * \mathrm{e} 1+\mathrm{a} 332 * \mathrm{e} 2+\mathrm{a} 333 * \mathrm{e} 3+\mathrm{a} 334 * \mathrm{e} 4 ; \\
& \mathrm{g}[\mathrm{e} 4, \mathrm{e} 4, \mathrm{H}]=\mathrm{a} 441 * \mathrm{e} 1+\mathrm{a} 442 * \mathrm{e} 2+\mathrm{a} 443 * \mathrm{e} 3+\mathrm{a} 444 * \mathrm{e} 4 ; \\
& \mathrm{g}[\mathrm{e} 1, \mathrm{e} 2, \mathrm{H}]=\mathrm{g}[\mathrm{e} 2, \mathrm{e} 1, \mathrm{H}]=\mathrm{a} 121 * \mathrm{e} 1+\mathrm{a} 122 * \mathrm{e} 2+\mathrm{a} 123 * \mathrm{e} 3+\mathrm{a} 124 * \mathrm{e} 4 ; \\
& \mathrm{g}[\mathrm{e} 1, \mathrm{e} 3, \mathrm{H}]=\mathrm{g}[\mathrm{e} 3, \mathrm{e} 1, \mathrm{H}]=\mathrm{a} 131 * \mathrm{e} 1+\mathrm{a} 132 * \mathrm{e} 2+\mathrm{a} 133 * \mathrm{e} 3+\mathrm{a} 134 * \mathrm{e} 4 ; \\
& \mathrm{g}[\mathrm{e} 1, \mathrm{e} 4, \mathrm{H}]=\mathrm{g}[\mathrm{e} 4, \mathrm{e} 1, \mathrm{H}]=\mathrm{a} 141 * \mathrm{e} 1+\mathrm{a} 142 * \mathrm{e} 2+\mathrm{a} 143 * \mathrm{e} 3+\mathrm{a} 144 * \mathrm{e} 4 ; \\
& \mathrm{g}[\mathrm{e} 2, \mathrm{e} 3, \mathrm{H}]=\mathrm{g}[\mathrm{e} 3, \mathrm{e} 2, \mathrm{H}]=\mathrm{a} 231 * \mathrm{e} 1+\mathrm{a} 232 * \mathrm{e} 2+\mathrm{a} 233 * \mathrm{e} 3+\mathrm{a} 234 * \mathrm{e} 4 ; \\
& \mathrm{g}[\mathrm{e} 2, \mathrm{e} 4, \mathrm{H}]=\mathrm{g}[\mathrm{e} 4, \mathrm{e} 2, \mathrm{H}]=\mathrm{a} 241 * \mathrm{e} 1+\mathrm{a} 242 * \mathrm{e} 2+\mathrm{a} 243 * \mathrm{e} 3+\mathrm{a} 244 * \mathrm{e} 4 ; \\
& \mathrm{g}[\mathrm{e} 3, \mathrm{e} 4, \mathrm{H}]=\mathrm{g}[\mathrm{e} 4, \mathrm{e} 3, \mathrm{H}]=\mathrm{a} 341 * \mathrm{e} 1+\mathrm{a} 342 * \mathrm{e} 2+\mathrm{a} 343 * \mathrm{e} 3+\mathrm{a} 344 * \mathrm{e} 4 ;
\end{aligned}
$$

Definimos genericamente uma aplicação linear $\mathrm{U}: \mathcal{J} \rightarrow \mathcal{J}$ que implica que um 2-cociclo é equivalente a 0 , nos elementos da base.

$$
\begin{aligned}
& u[e 1, J]=u 11 * e 1+u 12 * e 2+u 13 * e 3+u 14 * e 4 ; \\
& u[e 2, J]=u 21 * e 1+u 22 * e 2+u 23 * e 3+u 24 * e 4 ; \\
& u[e 3, J]=u 31 * e 1+u 32 * e 2+u 33 * e 3+u 34 * e 4 ; \\
& u[e 4, J]=u 41 * e 1+u 42 * e 2+u 43 * e 3+u 44 * e 4 ;
\end{aligned}
$$

Estendemos U por linearidade.

$$
\begin{aligned}
& \mathrm{u}\left[\mathrm{v}_{-}, \text {algebra_] }:=\operatorname{paracan}[\text { Sum [v[[i]] } * u[\text { base [ [i] ], }\right. \\
& \text { algebra], }\{i, 1,4\}]] \text {; } \\
& \mathrm{U}\left[\mathrm{V}_{-}, \text {algebra_ }\right]:=\operatorname{If}[\text { ToString }[\mathrm{v}]==" \Theta ", \Theta, \operatorname{decan}[\mathrm{u}[\text { paracan [v], algebra] ] ] ; }
\end{aligned}
$$


As condições para uma aplicação bilinear h ser um 2-cociclo de Jordan são:

$$
\left\{\begin{array}{l}
h(a, b)=h(b, a) \\
(h(a, a) b) a+h\left(a^{2} b, a\right)+h\left(a^{2}, b\right) a=a^{2} h(b, a)+h(a, a)(b a)+h\left(a^{2}, b a\right)
\end{array}\right.
$$

Linearizando completamente estas condições temos:

$(h(x, y) b) z+(h(x, z) b) y+(h(y, z) b) x+h((x y) b, z)+h((x z) b, y)+h((y z) b, x)+h(x y, b) z+$ $h(x z, b) y+h(y z, b) x=(x y) h(b, z)+(x z) h(b, y)+(y z) h(b, x)+h(x, y)(b z)+h(x, z)(b y)+$ $h(y, z)(b x)+h(x y, b z)+h(x z, b y)+h(y z, b x)$

A função Relacoes5[z] gera as condições linearizadas (nos elementos da base) que deve satisfazer a aplicação bilinear $h$ para ser um 2-cociclo de Jordan da álgebra $z$.

$$
\begin{aligned}
& \text { Relacoes5[ } z_{-} \text {] : =Simplify [DeleteDuplicates [Flatten [ } \\
& \text { paracan [Flatten [Table [Simplify [G[G[G[base[[i]] ], base[[j]] ,H] , } \\
& \text { base [ [ l] ], z], base [ [m] ], z]+ } \\
& G[G[G[\text { base }[[i]] \text {, base }[[m]], H] \text {, base }[[l]], z] \text {, base }[[j]], z]+ \\
& \mathrm{G}[\mathrm{G}[\mathrm{G}[\text { base }[[j]] \text {, base }[\mathrm{m}]], \mathrm{H}] \text {, base [ [l]], z], base [[i]] ], z]+ } \\
& G[G[G[\text { base }[[i]] \text {, base }[[j]], z] \text {, base }[[\mathrm{l}]], z] \text {, base }[[m]], H]+ \\
& \mathrm{G}[\mathrm{G}[\mathrm{G}[\text { base }[[\mathrm{i}]] \text {, base }[\mathrm{m}]], \mathrm{z}] \text {, base }[\mathrm{l}]], \mathrm{z}] \text {, base }[\mathrm{j}]], \mathrm{H}]+ \\
& G[G[G[\text { base }[[j]] \text {, base }[[m]], z] \text {, base }[[\mathrm{l}]], z] \text {, base [ [i] ],H]+ } \\
& \mathrm{G}[\mathrm{G}[\mathrm{G}[\text { base }[[\mathrm{i}]] \text {, base }[[j]], z] \text {, base }[[\mathrm{l}]], \mathrm{H}] \text {, base }[\mathrm{m}]], \mathrm{z}]+ \\
& \mathrm{G}[\mathrm{G}[\mathrm{G}[\text { base }[[\mathrm{i}]] \text {, base }[\mathrm{m}]], \mathrm{z}] \text {, base }[\mathrm{l}]], \mathrm{H}] \text {, base }[\mathrm{j}]], \mathrm{z}]+ \\
& G[G[G[\text { base }[j]]] \text {, base }[[m]], z] \text {, base }[[l]], H] \text {, base [ [i]] , z] - } \\
& \mathrm{G}[\mathrm{G}[\text { base }[[\mathrm{i}]] \text {, base }[[j]], z], G[\text { base }[[\mathrm{l}]] \text {, base [ [m] ],H],z] - } \\
& G[G[\text { base }[[i]] \text {, base }[[m]], z], G[\text { base }[[\mathrm{l}]] \text {, base }[[j]], H], z] \text { - }
\end{aligned}
$$

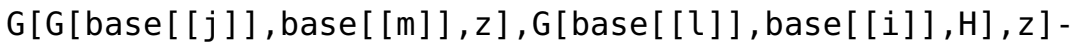

$$
\begin{aligned}
& \mathrm{G}[\mathrm{G}[\text { base }[[i]] \text {, base }[[j]], \mathrm{H}], \mathrm{G}[\text { base }[[\mathrm{l}]] \text {, base }[\mathrm{m}]], \mathrm{z}], \mathrm{z}] \text { - } \\
& \mathrm{G}[\mathrm{G}[\text { base }[\mathrm{i}]] \text {, base }[\mathrm{m}]], \mathrm{H}], \mathrm{G}[\text { base }[[\mathrm{l}]] \text {, base }[j]], \mathrm{z}], \mathrm{z}] \text { - }
\end{aligned}
$$

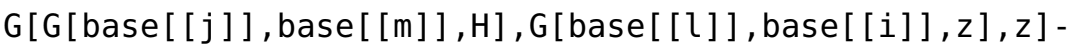

$$
\begin{aligned}
& \text { G[G[base [[i]] ], base[[j]],z], G[base[[l]] , base[[m] ],z],H] - }
\end{aligned}
$$

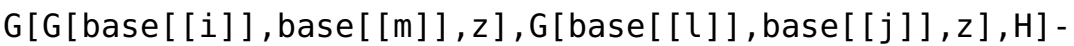

$$
\begin{aligned}
& \mathrm{G}[\mathrm{G}[\text { base }[[j]] \text {, base }[[\mathrm{m}]], z], G[\text { base [ [l]] , base[[i]] ,z],H] ], } \\
& \{i, 1,4\},\{j, 1,4\},\{m, 1,4\},\{l, 1,4\}], 2]]]]] \text {; }
\end{aligned}
$$

A função Relacoes71[z] gera as relações que deve satisfazer um 2-cociclo da álgebra z para ser equivalente a 0 , ou seja para ser um 2-cobordo.

$$
\begin{aligned}
& \text { Relacoes71[z-] := Simplify[DeleteDuplicates[ Flatten[paracan[ } \\
& \text { Flatten[Table[ Simplify[+U[G[base[[i]], base[[j]], z], J] - } \\
& \text { G[base[[i]], U[base[[j]], J], z] - G[U[base[[i]],J], base[[j]],z]], } \\
& \{i, 1,4\},\{j, 1,4\}], 2]]]]\} \text {; }
\end{aligned}
$$


Calcula a dimensão dos 2-cobordos

A função dimB2[z] retorna a dimensão do espaço dos 2-cobordos da álgebra z com coeficientes em $z$.

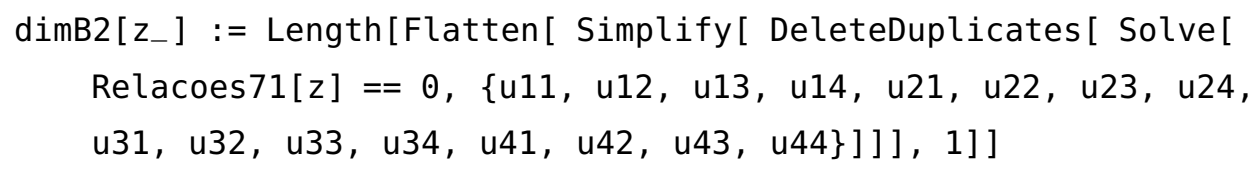

Calcula a dimensão dos 2-cociclos

A função dimZ2[z] retorna a dimensão do espaço dos 2-cociclos da álgebra z com coeficientes em $z$.

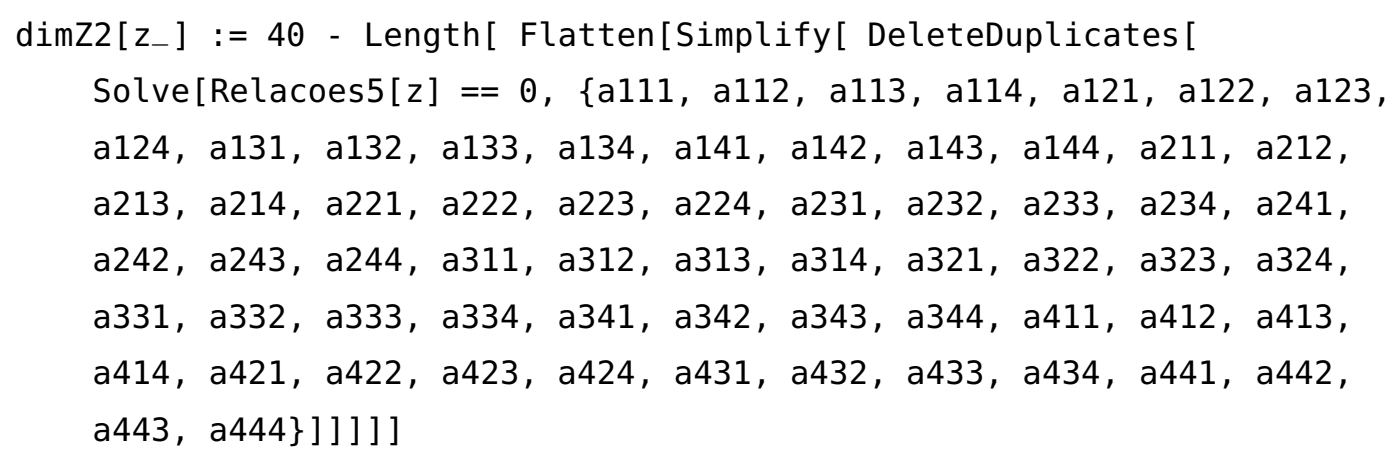

Calcula a dimensão de $\mathrm{H}^{2}$

A função dimH2 [z] retorna a dimensão do segundo grupo de cohomologia da álgebra $z$ com coeficientes em $z$.

$\operatorname{dimH} 2\left[z_{-}\right]:=\operatorname{dimZ2}[z]-\operatorname{dimB2}[z]$

\section{A.10 CAlCUla A Dimensão DO CEntro ASSOCiAdor}

A função ZAssociador [alg] retorna a dimensão do centro associador da álgebra alg.

$$
\begin{aligned}
\text { ZAssociador[alg_] } & :=4 \text { - Length [ Flatten[Solve[ Table[paracan[ } \\
& \text { Simplify }[\{\text { Ass }[\text { um, base }[i]], \text { base }[j]], \text { alg], } \\
& \text { Ass[base }[i]], \text { um, base[[j]], alg], }
\end{aligned}
$$


Ass[base[[i]], base[[j]], um, alg]\}]],

$\{i, 1,4\},\{j, 1,4\}]==0,\{a 1, a 2, a 3, a 4\}]\}]$

\section{A.11 REAlizA A SOMA DiRETA DE Álgebras DE JORDAN;}

A função Soma31 $[x, y]$ gera uma nova álgebra que é a soma direta da álgebra $x$ de dimensão 3 e a álgebra y de dimensão 1 .

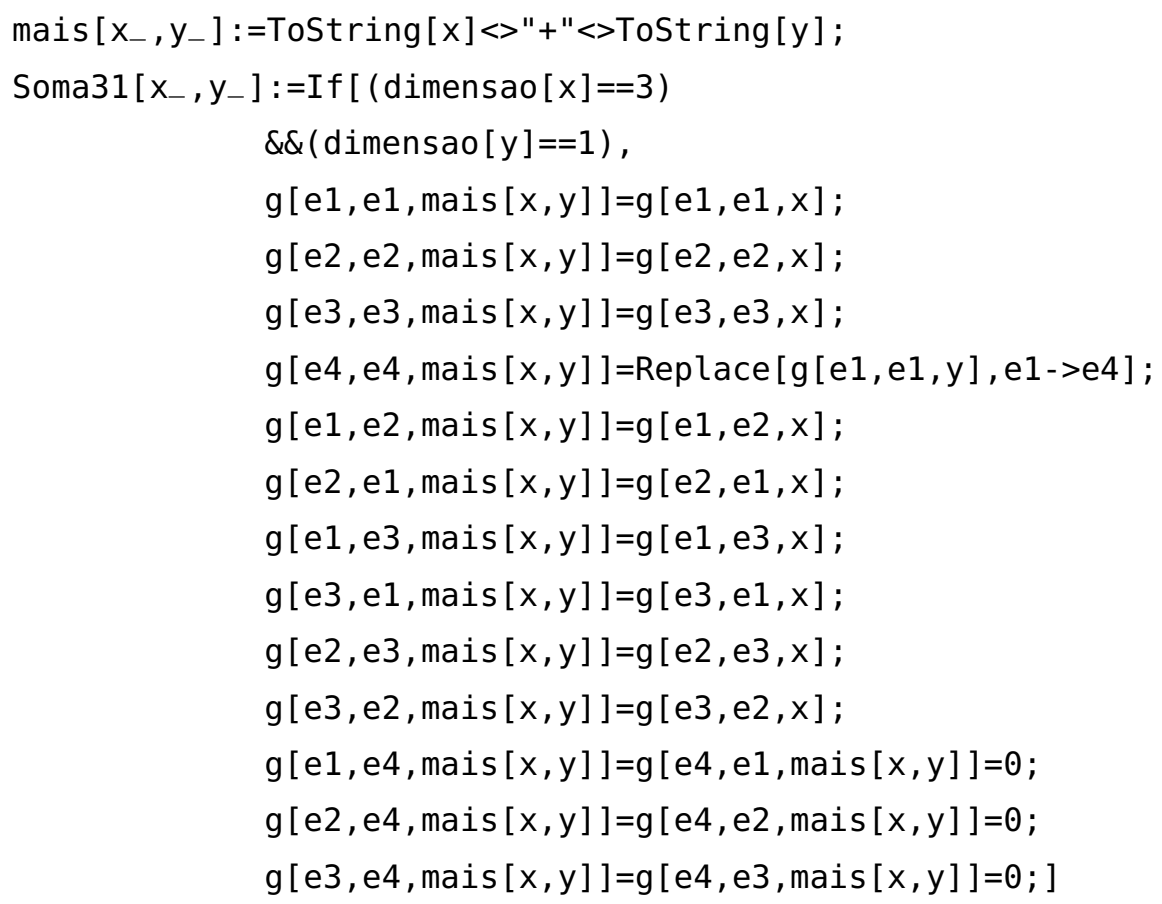

A função Soma22 $[x, y]$ gera uma nova álgebra que é a soma direta da álgebra $x$ de dimensão 2 e a álgebra y de dimensão 2 .

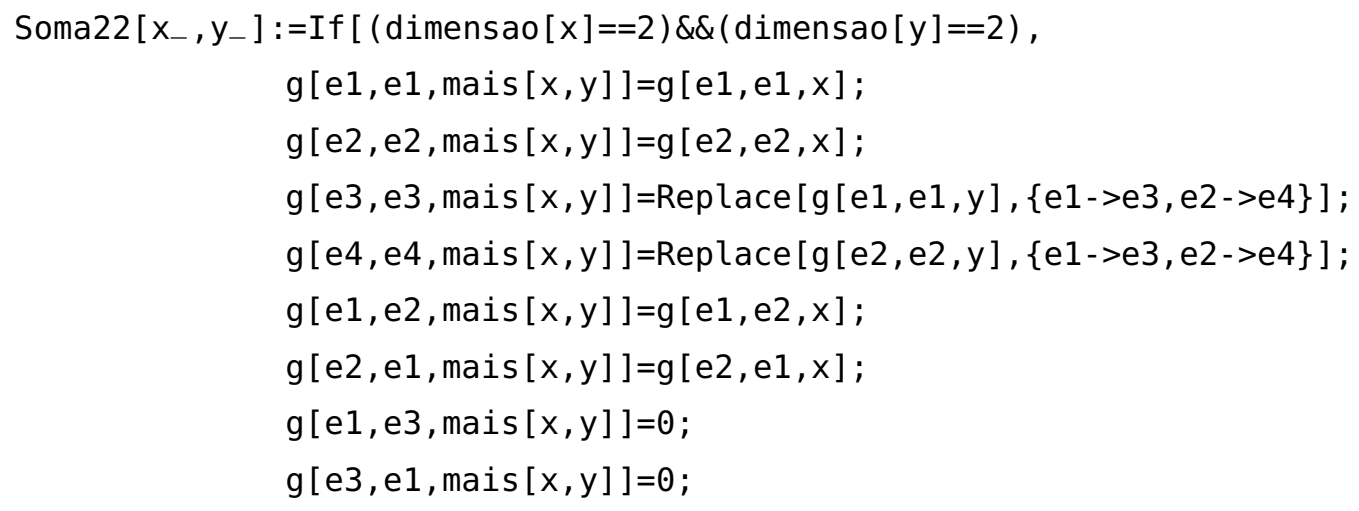




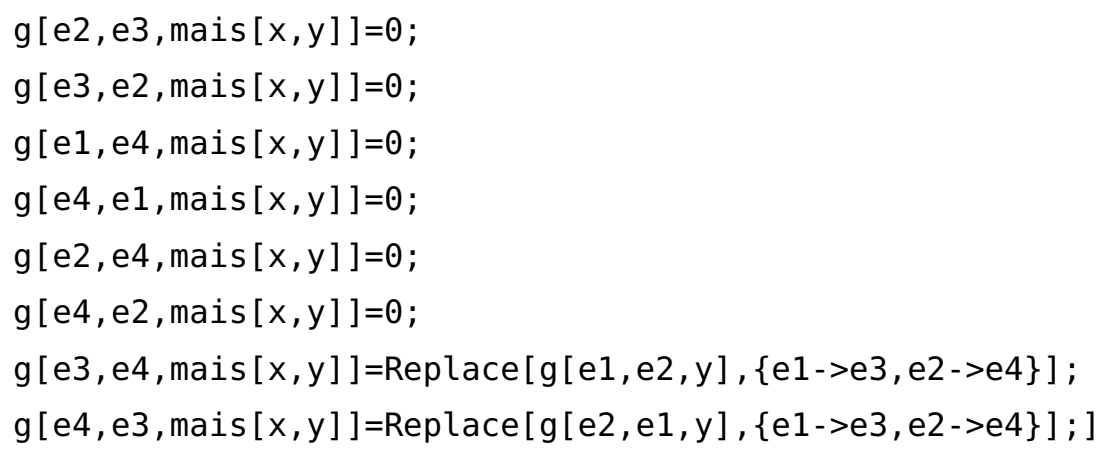

Geramos todas as álgebras que são soma direta de uma álgebra de dimensão $3 \mathrm{com}$ uma álgebra de dimensão 1.

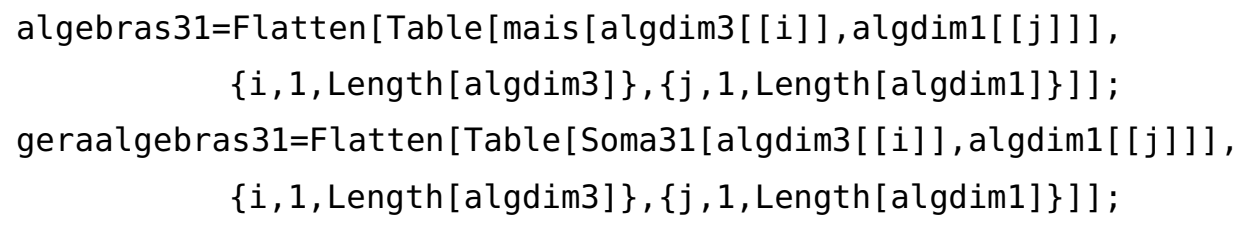

Geramos todas as álgebras que são soma direta de duas álgebras de dimensão 2 .

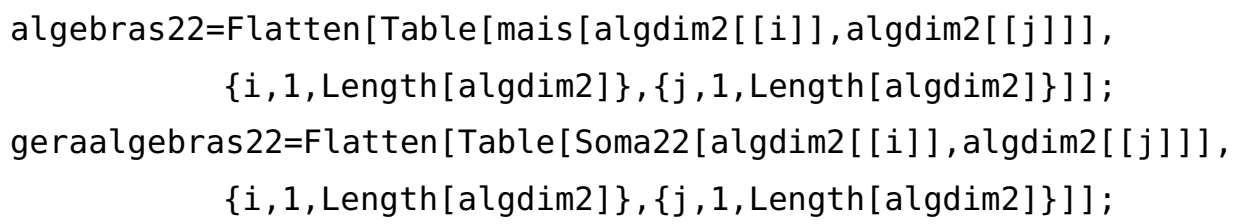

Geramos uma lista de todas as álgebras decomponíveis de dimensão 4.

algebradim4soma=Union [algebras31, algebras22] ;

\section{A.12 DECOMPÕE UMA ÁlgeBRA EM SOMA DiRETA}

A função Decomp2[z] compara a álgebra z com todas as álgebras decomponíveis de dimensão 4 procurando isomorfismos entre elas.

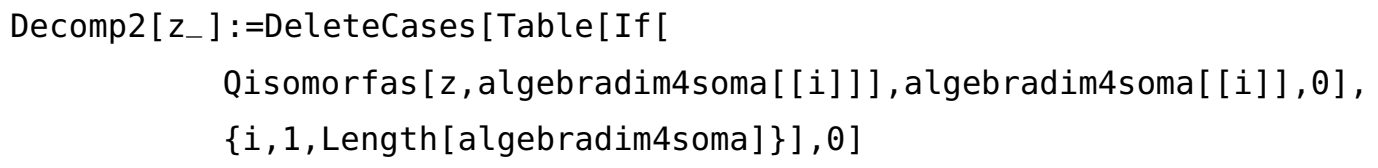

A função Decomp [ z ] retorna se a álgebra z é decomponível ou indecomponível. No caso que seja decomponível retorna sua decomposição. 


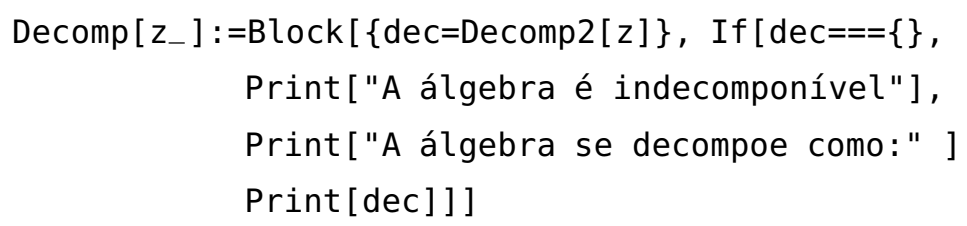

\section{A.13 EXIBE O PRODUTO DA ÁLGEBRA}

A função Exibir [z] exibe o produto da álgebra z na forma matricial.

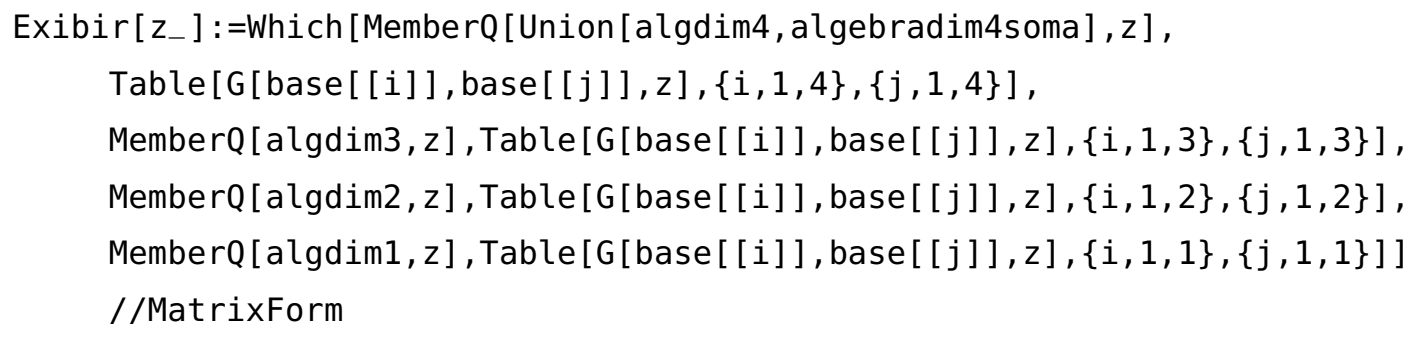

\section{A.14 EXIBE RESUMO DAS PROPRIEDAdes DA ÁlgeBrA}

A função Todo[z] exibe o resumo de todas as propriedades da álgebra $z$ :produto na forma matricial, se é de Jordan, associativa, não associativa, unitária, qual é a unidade, indecomponível, decomponível e qual é a sua decomposição, a dimensão do grupo de automorfismos, o grupo de automorfismos, a dimensão do aniquilador, subálgebras de dimensão 2 e 3 , dimensão do centro associador, dimensão de $\mathfrak{J}^{2}, \mathfrak{J}^{3}$ e $\mathfrak{f}^{4}$, dimensão do espaço de 2-cociclos, 2-cobordos e da segunda cohomologia.

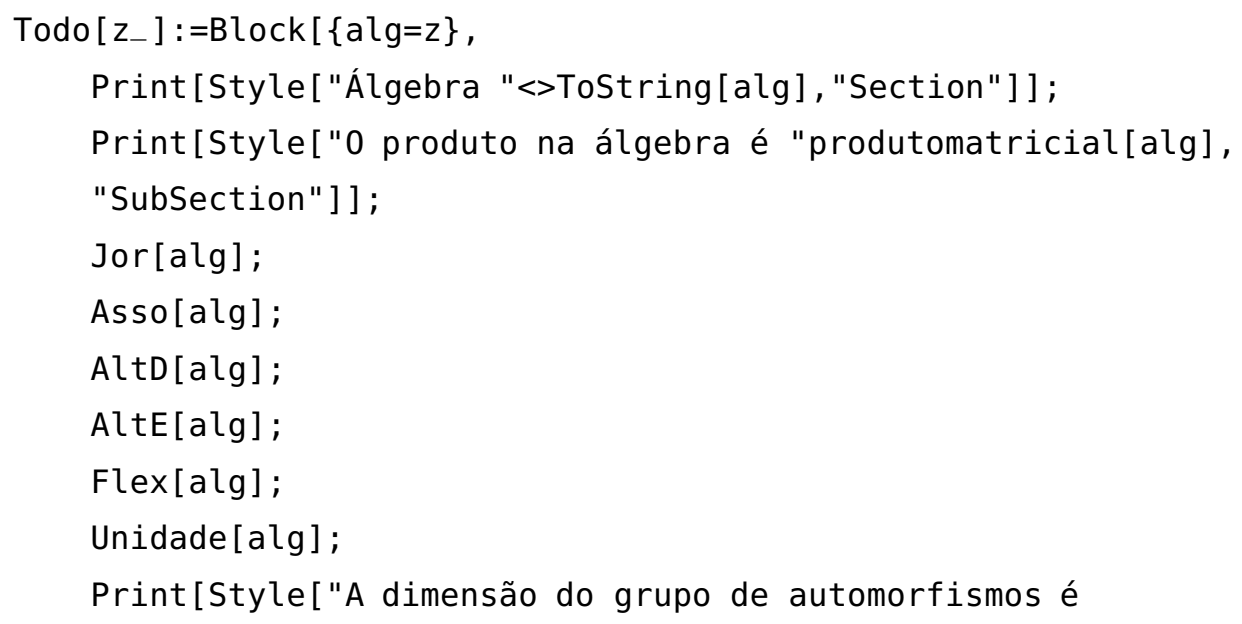


"<>ToString [dim2[alg] ]<>" . ", "SubSection"] ] ;

Print[Style["0 grupo de automorfismos é ","SubSection"]];grupo[alg]

Print[Style["A dimensão do aniquilador da álgebra é

"<>ToString [ann[alg] ]<>" . ", "SubSection"] ] ;

Print [Style["A dimensão de "<>ToString[alg]<>"^2 é

"<>ToString [Potencia2[alg] ] <>" . ", "SubSection"] ] ;

Print [Style["A dimensão de "<>ToString[alg]<>"^3 é

"<>ToString [Potencia3[alg] ] <>" . ", "SubSection"] ] ;

Print[Style["A dimensão de "<>ToString[alg]<>"^4 é

"<>ToString [Potencia4[alg] ]<>" . " , "SubSection"] ] ;

Decomp [alg] ;

sub34[alg];

sub24[alg];

Print[Style["A dimensão do centro associador é

"<>ToString [ZAssociador[alg] ]<>" . ", "SubSection"]] ;

Print [Style["A dimensão do grupo de 2-cociclos é

"<>ToString [dimZ2[alg] ]<>" . ", "SubSection"] ] ;

Print [Style["A dimensão do grupo de 2-cobordos é

"<>ToString [dimB2[alg] ]<>" . ", "SubSection"] ] ;

Print[Style["A dimensão do segundo grupo de Cohomologia é

"<>ToString [dimH2[alg] ]<>" . " , "SubSection"] ] ; ] 

DAN DE DIMENSÃO 4

Lembramos que as álgebras de Jordan de dimensão 4 sobre um corpo $\mathbf{k}$ algebricamente fechado, $\partial_{i}$, foram obtidas na Seção 2.2 e que as álgebras de Jordan indecomponíveis de dimensão 2 e 3 são:

Tabela B.1: k-álgebras de Jordan indecomponíveis de dimensão 2

\begin{tabular}{|c|ccc|}
\hline $\mathcal{B}$ & \multicolumn{4}{|c|}{ Tabela de Multiplicação } \\
\hline $\mathcal{B}_{1}$ & $e_{1}^{2}=e_{1}$ & $e_{1} n_{1}=n_{1}$ & $n_{1}^{2}=0$ \\
\hline $\mathcal{B}_{2}$ & $e_{1}^{2}=e_{1}$ & $e_{1} n_{1}=\frac{1}{2} n_{1}$ & $n_{1}^{2}=0$ \\
\hline $\mathcal{B}_{3}$ & $n_{1}^{2}=n_{2}$ & $n_{1} n_{2}=0$ & $n_{2}^{2}=0$ \\
\hline
\end{tabular}

Tabela B.2: k-álgebras de Jordan indecomponíveis de dimensão 3

\begin{tabular}{|c|c|}
\hline $\mathcal{T}$ & Tabela de Multiplicação \\
\hline $\mathcal{T}_{1}$ & $\begin{array}{ccc}e_{1}^{2}=e_{1} & n_{1}^{2}=n_{2} & n_{2}^{2}=0 \\
e_{1} n_{1}=n_{1} & e_{1} n_{2}=n_{2} & n_{1} n_{2}=0\end{array}$ \\
\hline $\mathcal{T}_{2}$ & $\begin{array}{c}e_{1}^{2}=e_{1} \quad n_{1}^{2}=0 \quad n_{2}^{2}=0 \\
e_{1} n_{1}=n_{1} \quad e_{1} n_{2}=n_{2} \quad n_{1} n_{2}=0\end{array}$ \\
\hline $\mathcal{T}_{3}$ & $\begin{array}{ccc}n_{1}^{2}=n_{2} & n_{2}^{2}=0 & n_{3}^{2}=0 \\
n_{1} n_{2}=n_{3} & n_{1} n_{3}=0 & n_{2} n_{3}=0\end{array}$ \\
\hline $\mathcal{T}_{4}$ & $\begin{array}{ccc}n_{1}^{2}=n_{2} & n_{2}^{2}=0 \quad n_{3}^{2}=0 \\
n_{1} n_{2}=0 \quad n_{1} n_{3}=n_{2} \quad n_{2} n_{3}=0\end{array}$ \\
\hline $\mathcal{T}_{5}$ & $\begin{aligned} e_{1}^{2} & =e_{1} \quad e_{2}^{2}=e_{2} \quad e_{3}^{2}=e_{1}+e_{2} \\
e_{1} e_{2} & =0 \quad e_{1} e_{3}=\frac{1}{2} e_{3} \quad e_{2} e_{3}=\frac{1}{2} e_{3}\end{aligned}$ \\
\hline $\mathcal{T}_{6}$ & $\begin{array}{c}e_{1}^{2}=e_{1} \quad n_{1}^{2}=0 \quad n_{2}^{2}=0 \\
e_{1} n_{1}=\frac{1}{2} n_{1} \quad e_{1} n_{2}=n_{2} \quad n_{1} n_{2}=0\end{array}$ \\
\hline $\mathcal{T}_{7}$ & $\begin{array}{c}e_{1}^{2}=e_{1} \quad n_{1}^{2}=0 \quad n_{2}^{2}=0 \\
e_{1} n_{1}=\frac{1}{2} n_{1} \quad e_{1} n_{2}=\frac{1}{2} n_{2} \quad n_{1} n_{2}=0\end{array}$ \\
\hline $\mathcal{T}_{8}$ & $\begin{array}{rll}e_{1}^{2}=e_{1} & n_{1}^{2}=n_{2} & n_{2}^{2}=0 \\
e_{1} n_{1}=\frac{1}{2} n_{1} & e_{1} n_{2}=0 & n_{1} n_{2}=0\end{array}$ \\
\hline
\end{tabular}




\begin{tabular}{|c|c|}
\hline $\mathcal{T}$ & Tabela de Multiplicação \\
\hline $\mathcal{T}_{9}$ & $\begin{array}{rll}e_{1}^{2}=e_{1} & n_{1}^{2}=n_{2} \quad n_{2}^{2}=0 \\
e_{1} n_{1}=\frac{1}{2} n_{1} & e_{1} n_{2}=n_{2} \quad n_{1} n_{2}=0\end{array}$ \\
\hline $\mathcal{T}_{10}$ & $\begin{array}{rrl}e_{1}^{2}=e_{1} \quad e_{2}^{2}=e_{2} & n_{1}^{2}=0 \\
e_{1} e_{2}=0 \quad e_{1} n_{1}=\frac{1}{2} n_{1} & e_{2} n_{1}=\frac{1}{2} n_{1}\end{array}$ \\
\hline
\end{tabular}

Todas as álgebras desta seção são apresentadas na base $\left\{e_{1}, e_{2}, e_{3}, e_{4}\right\}$.

ÁLGEBRA $\partial_{1}$

O produto na álgebra é:

\begin{tabular}{c|cccc}
$\jmath_{1}$ & $e_{1}$ & $e_{2}$ & $e_{3}$ & $e_{4}$ \\
\hline$e_{1}$ & $e_{1}$ & 0 & 0 & $\frac{e_{4}}{2}$ \\
$e_{2}$ & 0 & $e_{2}$ & 0 & $\frac{e_{4}}{2}$ \\
$e_{3}$ & 0 & 0 & $e_{3}$ & 0 \\
$e_{4}$ & $\frac{e_{4}}{2}$ & $\frac{e_{4}}{2}$ & 0 & $e_{1}+e_{2}$
\end{tabular}

É uma álgebra não associativa, unitária, com unidade: $e_{1}+e_{2}+e_{3}$. A dimensão do grupo de automorfismos é 1 . O grupo de automorfismos é:

$$
\begin{aligned}
& \left\{\left(\begin{array}{cccc}
\frac{1+a_{44}}{2} & \frac{1-a_{44}}{2} & 0 & \frac{\sqrt{1-a_{44}^{2}}}{2} \\
\frac{1-a_{44}}{2} & \frac{1+a_{44}}{2} & 0 & -\frac{1}{2} \sqrt{1-a_{44}^{2}} \\
0 & 0 & 1 & 0 \\
-\sqrt{1-a_{44}^{2}} & \sqrt{1-a_{44}^{2}} & 0 & a_{44}
\end{array}\right)\right. \\
& \left(\begin{array}{cccc}
\frac{1+a_{44}}{2} & \frac{1-a_{44}}{2} & 0 & -\frac{1}{2} \sqrt{1-a_{44}^{2}} \\
\frac{1-a_{44}}{2} & \frac{1+a_{44}}{2} & 0 & \frac{\sqrt{1-a_{44}^{2}}}{2} \\
0 & 0 & 1 & 0 \\
\sqrt{1-a_{44}^{2}} & -\sqrt{1-a_{44}^{2}} & 0 & a_{44}
\end{array}\right),\left(\begin{array}{cccc}
\frac{1-a_{44}}{2} & \frac{1+a_{44}}{2} & 0 & -\frac{1}{2} \sqrt{1-a_{44}^{2}} \\
\frac{1+a_{44}}{2} & \frac{1-a_{44}}{2} & 0 & \frac{\sqrt{1-a_{44}^{2}}}{2} \\
0 & 0 & 1 & 0 \\
-\sqrt{1-a_{44}^{2}} & \sqrt{1-a_{44}^{2}} & 0 & a_{44}
\end{array}\right) \text {, } \\
& \left(\begin{array}{cccc}
\frac{1-a_{44}}{2} & \frac{1+a_{44}}{2} & 0 & \frac{\sqrt{1-a_{44}^{2}}}{2} \\
\frac{1+a_{44}}{2} & \frac{1-a_{44}}{2} & 0 & -\frac{1}{2} \sqrt{1-a_{44}^{2}} \\
0 & 0 & 1 & 0 \\
\sqrt{1-a_{44}^{2}} & -\sqrt{1-a_{44}^{2}} & 0 & a_{44}
\end{array}\right) \\
& \left.\left(\begin{array}{cccc}
1 & 0 & 0 & 0 \\
0 & 1 & 0 & 0 \\
0 & 0 & 1 & 0 \\
0 & 0 & 0 & -1
\end{array}\right),\left(\begin{array}{llll}
1 & 0 & 0 & 0 \\
0 & 1 & 0 & 0 \\
0 & 0 & 1 & 0 \\
0 & 0 & 0 & 1
\end{array}\right)\left(\begin{array}{cccc}
0 & 1 & 0 & 0 \\
1 & 0 & 0 & 0 \\
0 & 0 & 1 & 0 \\
0 & 0 & 0 & -1
\end{array}\right),\left(\begin{array}{llll}
0 & 1 & 0 & 0 \\
1 & 0 & 0 & 0 \\
0 & 0 & 1 & 0 \\
0 & 0 & 0 & 1
\end{array}\right)\right\}
\end{aligned}
$$

A dimensão do aniquilador da álgebra é 0. É uma álgebra rígida. A álgebra se decompõe como: $\mathcal{T}_{5} \oplus \mathbf{k}$ e. Subálgebras de dimensão 2 e 3: $\mathbf{k} e_{1} \oplus \mathbf{k} e_{2} \oplus \mathbf{k} e_{3}, \mathcal{B}_{1} \oplus \mathbf{k} e_{2}, \mathcal{T}_{5}$, 
$\mathbf{k} e_{1} \oplus \mathbf{k} n_{1}, \mathcal{B}_{1}$ e $\mathbf{k} e_{1} \oplus \mathbf{k} e_{2}$. A dimensão do radical nilpotente é 0 . A dimensão da órbita é 15. A dimensão do centro associador é 2. A dimensão de $\mathfrak{\partial}^{2}$ é 4 . A dimensão de $\mathfrak{f}^{3}$ é 4 . A dimensão de $\mathfrak{J}^{4}$ é 4 . A dimensão do grupo de 2-cociclos é 15. A dimensão do grupo de 2-cobordos é 15. A dimensão do segundo grupo de Cohomologia é 0. A dimensão da subálgebra associativa maximal é 3. A dimensão da subálgebra nilpotente maximal é 0 . A dimensão da subálgebra nula maximal é 0 .

ÁLGEBRA $\partial_{2}$

O produto na álgebra é:

\begin{tabular}{c|cccc}
$\partial_{2}$ & $e_{1}$ & $e_{2}$ & $e_{3}$ & $e_{4}$ \\
\hline$e_{1}$ & $e_{1}$ & 0 & $\frac{e_{3}}{2}$ & $\frac{e_{4}}{2}$ \\
$e_{2}$ & 0 & $e_{2}$ & $\frac{e_{3}}{2}$ & $\frac{e_{4}}{2}$ \\
$e_{3}$ & $\frac{e_{3}}{2}$ & $\frac{e_{3}}{2}$ & 0 & $\frac{e_{1}+e_{2}}{2}$ \\
$e_{4}$ & $\frac{e_{4}}{2}$ & $\frac{e_{4}}{2}$ & $\frac{e_{1}+e_{2}}{2}$ & 0
\end{tabular}

É uma álgebra não associativa, unitária, com unidade: $e_{1}+e_{2}$. A dimensão do grupo de automorfismos é 3. A dimensão do aniquilador da álgebra é 0. É uma álgebra rígida. A álgebra é indecomponível. Subálgebras de dimensão 2 e 3: $\mathcal{T}_{5}, \mathcal{T}_{10}, \mathcal{B}_{1}, \mathcal{B}_{2}$ e $\mathbf{k}_{1} \oplus \mathbf{k} e_{2}$. A dimensão do radical nilpotente é 0 . A dimensão da órbita é 13. A dimensão do centro associador é 1 . A dimensão de $\mathfrak{J}^{2}$ é 4 . A dimensão de $\mathfrak{J}^{3}$ é 4 . A dimensão de $\mathfrak{J}^{4}$ é 4 . A dimensão do grupo de 2-cociclos é 13. A dimensão do grupo de 2-cobordos é 13. A dimensão do segundo grupo de Cohomologia é 0 . A dimensão da subálgebra associativa maximal é 2 . A dimensão da subálgebra nilpotente maximal é 0 . A dimensão da subálgebra nula maximal é 0 .

ÁLGEBRA $\partial_{3}$

O produto na álgebra é:

\begin{tabular}{c|cccc}
$\partial_{3}$ & $e_{1}$ & $e_{2}$ & $e_{3}$ & $e_{4}$ \\
\hline$e_{1}$ & $e_{1}$ & 0 & 0 & 0 \\
$e_{2}$ & 0 & $e_{2}$ & 0 & 0 \\
$e_{3}$ & 0 & 0 & $e_{3}$ & 0 \\
$e_{4}$ & 0 & 0 & 0 & $e_{4}$
\end{tabular}

É uma álgebra associativa, unitária, com unidade: $e_{1}+e_{2}+e_{3}+e_{4}$. A dimensão do grupo de automorfismos é 0 . A dimensão do aniquilador da álgebra é 0. É uma álgebra rígida. A álgebra se decompõe como: $\mathbf{k} e_{1} \oplus \mathbf{k} e_{2} \oplus \mathbf{k} e_{3} \oplus \mathbf{k} e_{4}$. Subálgebras de dimensão: 2 e $3: \mathbf{k} e_{1} \oplus \mathbf{k} e_{2} \oplus \mathbf{k} e_{3}$ e $\mathbf{k} e_{1} \oplus \mathbf{k} e_{2}$. A dimensão do radical nilpotente é 0 . A dimensão da órbita é 16. A dimensão do centro associador é 4. A dimensão de $\mathfrak{f}^{2}$ é 4 . A dimensão de $\mathfrak{J}^{3}$ é 4 . A dimensão de $\mathfrak{J}^{4}$ é 4 . A dimensão do grupo de 2-cociclos é 16 . A dimensão 
do grupo de 2-cobordos é 16. A dimensão do segundo grupo de Cohomologia é 0. A dimensão da subálgebra associativa maximal é 4. A dimensão da subálgebra nilpotente maximal é 0 . A dimensão da subálgebra nula maximal é 0 .

ÁLGEBRA $\partial_{4}$

\begin{tabular}{cc|cccc} 
& $\partial_{4}$ & $e_{1}$ & $e_{2}$ & $e_{3}$ & $e_{4}$ \\
\cline { 2 - 6 } O produto na álgebra é: & $e_{1}$ & $e_{1}$ & 0 & 0 & 0 \\
& $e_{2}$ & 0 & $e_{2}$ & 0 & 0 \\
& $e_{3}$ & 0 & 0 & $e_{3}$ & $e_{4}$ \\
& $e_{4}$ & 0 & 0 & $e_{4}$ & 0
\end{tabular}

É uma álgebra associativa, unitária, com unidade: $e_{1}+e_{2}+e_{3}$. A dimensão do grupo de automorfismos é 1. A dimensão do aniquilador da álgebra é 0 . A álgebra se decompõe como: $\mathcal{B}_{1} \oplus \mathbf{k} e_{2} \oplus \mathbf{k} e_{3}$. Subálgebras de dimensão 2 e 3: $\mathbf{k} e_{1} \oplus \mathbf{k} e_{2} \oplus \mathbf{k} e_{3}, \mathcal{B}_{1} \oplus \mathbf{k} e_{2}$, $\mathbf{k} e_{1} \oplus \mathbf{k} e_{2} \oplus \mathbf{k} n_{1}, \mathbf{k} e_{1} \oplus \mathbf{k} n_{1}, \mathcal{B}_{1}$ e $\mathbf{k} e_{1} \oplus \mathbf{k} e_{2}$. A dimensão do radical nilpotente é 1 . A dimensão da órbita é 15. A dimensão do centro associador é 4. A dimensão de $\mathfrak{J}^{2}$ é 4. A dimensão de $\mathfrak{J}^{3}$ é 4 . A dimensão de $\mathfrak{J}^{4}$ é 4 . A dimensão do grupo de 2-cociclos é 16. A dimensão do grupo de 2-cobordos é 15. A dimensão do segundo grupo de Cohomologia é 1. A dimensão da subálgebra associativa maximal é 4. A dimensão da subálgebra nilpotente maximal é 1. A dimensão da subálgebra nula maximal é 1 .

ÁLGEBRA $J_{5}$

O produto na álgebra é:

\begin{tabular}{c|cccc}
$\partial_{5}$ & $e_{1}$ & $e_{2}$ & $e_{3}$ & $e_{4}$ \\
\hline$e_{1}$ & $e_{1}$ & 0 & 0 & 0 \\
$e_{2}$ & 0 & $e_{2}$ & 0 & 0 \\
$e_{3}$ & 0 & 0 & $e_{3}$ & 0 \\
$e_{4}$ & 0 & 0 & 0 & 0
\end{tabular}

É uma álgebra associativa, não possui unidade. A dimensão do grupo de automorfismos é 1 .

O grupo de automorfismos é:

$$
\left\{\left(\begin{array}{cccc}
0 & 0 & 1 & 0 \\
0 & 1 & 0 & 0 \\
1 & 0 & 0 & 0 \\
0 & 0 & 0 & a_{44}
\end{array}\right),\left(\begin{array}{cccc}
0 & 0 & 1 & 0 \\
1 & 0 & 0 & 0 \\
0 & 1 & 0 & 0 \\
0 & 0 & 0 & a_{44}
\end{array}\right),\left(\begin{array}{cccc}
0 & 1 & 0 & 0 \\
0 & 0 & 1 & 0 \\
1 & 0 & 0 & 0 \\
0 & 0 & 0 & a_{44}
\end{array}\right),\right.
$$




$$
\left.\left(\begin{array}{cccc}
0 & 1 & 0 & 0 \\
1 & 0 & 0 & 0 \\
0 & 0 & 1 & 0 \\
0 & 0 & 0 & a_{44}
\end{array}\right),\left(\begin{array}{cccc}
1 & 0 & 0 & 0 \\
0 & 0 & 1 & 0 \\
0 & 1 & 0 & 0 \\
0 & 0 & 0 & a_{44}
\end{array}\right),\left(\begin{array}{cccc}
1 & 0 & 0 & 0 \\
0 & 1 & 0 & 0 \\
0 & 0 & 1 & 0 \\
0 & 0 & 0 & a_{44}
\end{array}\right)\right\}
$$

A dimensão do aniquilador da álgebra é 1. A álgebra se decompõe como: $\mathbf{k} e_{1} \oplus \mathbf{k} e_{2} \oplus$ $\mathbf{k} e_{3} \oplus \mathbf{k} n_{1}$. Subálgebras de dimensão 2 e 3: $\mathbf{k} e_{1} \oplus \mathbf{k} e_{2} \oplus \mathbf{k} e_{3}, \mathbf{k} e_{1} \oplus \mathbf{k} e_{2} \oplus \mathbf{k} n_{1}, \mathbf{k} e_{1} \oplus \mathbf{k} n_{1}$ e $\mathbf{k} e_{1} \oplus \mathbf{k} e_{2}$. A dimensão do radical nilpotente é 1. A dimensão da órbita é 15. A dimensão do centro associador é 4 . A dimensão de $\mathcal{J}^{2}$ é 3 . A dimensão de $\mathfrak{J}^{3}$ é 3 . A dimensão de $\mathfrak{J}^{4}$ é 3. A dimensão do grupo de 2-cociclos é 16. A dimensão do grupo de 2-cobordos é 15. A dimensão do segundo grupo de Cohomologia é 1. A dimensão da subálgebra associativa maximal é 4. A dimensão da subálgebra nilpotente maximal é 1. A dimensão da subálgebra nula maximal é 1.

ÁLGEBRA J 6

O produto na álgebra é:

\begin{tabular}{c|cccc}
$J_{6}$ & $e_{1}$ & $e_{2}$ & $e_{3}$ & $e_{4}$ \\
\hline$e_{1}$ & $e_{1}$ & 0 & 0 & $\frac{e_{4}}{2}$ \\
$e_{2}$ & 0 & $e_{2}$ & 0 & 0 \\
$e_{3}$ & 0 & 0 & $e_{3}$ & 0 \\
$e_{4}$ & $\frac{e_{4}}{2}$ & 0 & 0 & 0
\end{tabular}

É uma álgebra não associativa, não possui unidade. A dimensão do grupo de automorfismos é 2. A dimensão do aniquilador da álgebra é 0 . É uma álgebra rígida. A álgebra se decompõe como: $\mathcal{B}_{2} \oplus \mathbf{k} \mathbf{e}_{2} \oplus \mathbf{k} \boldsymbol{e}_{3}$. Subálgebras de dimensão 2 e $3: \mathbf{k} \boldsymbol{e}_{1} \oplus \mathbf{k} \mathbf{e}_{2} \oplus \mathbf{k} \mathbf{e}_{3}$, $\mathbf{k} e_{1} \oplus \mathbf{k} e_{2} \oplus \mathbf{k} n_{1}, \mathcal{B}_{2} \oplus \mathbf{k} e_{2}, \mathbf{k} e_{1} \oplus \mathbf{k} n_{1}, \mathcal{B}_{2}$ e $\mathbf{k} e_{1} \oplus \mathbf{k} e_{2}$. A dimensão do radical nilpotente é 1. A dimensão da órbita é 14. A dimensão do centro associador é 2. A dimensão de $\mathfrak{J}^{2}$ é 4 . A dimensão de $\mathfrak{J}^{3}$ é 4 . A dimensão de $\mathfrak{J}^{4}$ é 4 . A dimensão do grupo de 2 cociclos é 14. A dimensão do grupo de 2-cobordos é 14. A dimensão do segundo grupo de Cohomologia é 0. A dimensão da subálgebra associativa maximal é 3. A dimensão da subálgebra nilpotente maximal é 1. A dimensão da subálgebra nula maximal é 1.

\section{ÁLGEBRA $\partial_{7}$}

O produto na álgebra é:

\begin{tabular}{c|cccc}
$\partial_{7}$ & $e_{1}$ & $e_{2}$ & $e_{3}$ & $e_{4}$ \\
\hline$e_{1}$ & $e_{1}$ & 0 & 0 & $\frac{e_{4}}{2}$ \\
$e_{2}$ & 0 & $e_{2}$ & 0 & $\frac{e_{4}}{2}$ \\
$e_{3}$ & 0 & 0 & $e_{3}$ & 0 \\
$e_{4}$ & $\frac{e_{4}}{2}$ & $\frac{e_{4}}{2}$ & 0 & 0
\end{tabular}

É uma álgebra não associativa, unitária, com unidade: $e_{1}+e_{2}+e_{3}$. A dimensão do 
grupo de automorfismos é 2. A dimensão do aniquilador da álgebra é 0 . A álgebra se decompõe como: $\mathcal{T}_{10} \oplus \mathbf{k} \mathbf{e}_{3}$. Subálgebras de dimensão 2 e 3: $\mathbf{k} \boldsymbol{e}_{1} \oplus \mathbf{k} \mathbf{e}_{2} \oplus \mathbf{k} e_{3}, \mathcal{B}_{1} \oplus \mathbf{k} e_{2}$, $\mathcal{T}_{10}, \mathcal{B}_{2} \oplus \mathbf{k} e_{2}, \mathbf{k} e_{1} \oplus \mathbf{k} n_{1}, \mathcal{B}_{1}, \mathcal{B}_{2}$ e $\mathbf{k} e_{1} \oplus \mathbf{k} e_{2}$. A dimensão do radical nilpotente é 1. A dimensão da órbita é 14. A dimensão do centro associador é 2 . A dimensão de $\mathfrak{J}^{2}$ é 4. A dimensão de $\mathfrak{J}^{3}$ é 4. A dimensão de $\mathfrak{J}^{4}$ é 4 . A dimensão do grupo de 2-cociclos é 15. A dimensão do grupo de 2-cobordos é 14. A dimensão do segundo grupo de Cohomologia é 1. A dimensão da subálgebra associativa maximal é 3. A dimensão da subálgebra nilpotente maximal é 1. A dimensão da subálgebra nula maximal é 1 .

\section{ÁLGEBRA $\partial_{8}$}

O produto na álgebra é:

\begin{tabular}{c|cccc}
$\partial_{8}$ & $e_{1}$ & $e_{2}$ & $e_{3}$ & $e_{4}$ \\
\hline$e_{1}$ & $e_{1}$ & 0 & $\frac{e_{3}}{2}$ & 0 \\
$e_{2}$ & 0 & $e_{2}$ & $\frac{e_{3}}{2}$ & 0 \\
$e_{3}$ & $\frac{e_{3}}{2}$ & $\frac{e_{3}}{2}$ & $e_{1}+e_{2}$ & 0 \\
$e_{4}$ & 0 & 0 & 0 & 0
\end{tabular}

É uma álgebra não associativa, não possui unidade. A dimensão do grupo de automorfismos é 2 .

O grupo de automorfismos é:

$$
\begin{aligned}
& \left\{\begin{array}{cccc}
\frac{1+a_{33}}{2} & \frac{1-a_{33}}{2} & \frac{\sqrt{1-a_{33}^{2}}}{2} & 0 \\
\frac{1-a_{33}}{2} & \frac{1+a_{33}}{2} & -\frac{1}{2} \sqrt{1-a_{33}^{2}} & 0 \\
-\sqrt{1-a_{33}^{2}} & \sqrt{1-a_{33}^{2}} & a_{33} & 0 \\
0 & 0 & 0 & a_{44}
\end{array}\right) \\
& \left(\begin{array}{cccc}
\frac{1+a_{33}}{2} & \frac{1-a_{33}}{2} & -\frac{1}{2} \sqrt{1-a_{33}^{2}} & 0 \\
\frac{1-a_{33}}{2} & \frac{1+a_{33}}{2} & \frac{\sqrt{1-a_{33}^{2}}}{2} & 0 \\
\sqrt{1-a_{33}^{2}} & -\sqrt{1-a_{33}^{2}} & a_{33} & 0 \\
0 & 0 & 0 & a_{44}
\end{array}\right),\left(\begin{array}{cccc}
\frac{1-a_{33}}{2} & \frac{1+a_{33}}{2} & -\frac{1}{2} \sqrt{1-a_{33}^{2}} & 0 \\
\frac{1+a_{33}}{2} & \frac{1-a_{33}}{2} & \frac{\sqrt{1-a_{33}^{2}}}{2} & 0 \\
-\sqrt{1-a_{33}^{2}} & \sqrt{1-a_{33}^{2}} & a_{33} & 0 \\
0 & 0 & 0 & a_{44}
\end{array}\right), \\
& \left(\begin{array}{cccc}
\frac{1-a_{33}}{2} & \frac{1+a_{33}}{2} & \frac{\sqrt{1-a_{33}^{2}}}{2} & 0 \\
\frac{1+a_{33}}{2} & \frac{1-a_{33}}{2} & -\frac{1}{2} \sqrt{1-a_{33}^{2}} & 0 \\
\sqrt{1-a_{33}^{2}} & -\sqrt{1-a_{33}^{2}} & a_{33} & 0 \\
0 & 0 & 0 & a_{44}
\end{array}\right) \\
& \left(\begin{array}{cccc}
1 & 0 & 0 & 0 \\
0 & 1 & 0 & 0 \\
0 & 0 & -1 & 0 \\
0 & 0 & 0 & a_{44}
\end{array}\right),\left(\begin{array}{cccc}
1 & 0 & 0 & 0 \\
0 & 1 & 0 & 0 \\
0 & 0 & 1 & 0 \\
0 & 0 & 0 & a_{44}
\end{array}\right),
\end{aligned}
$$




$$
\left.\left(\begin{array}{cccc}
0 & 1 & 0 & 0 \\
1 & 0 & 0 & 0 \\
0 & 0 & -1 & 0 \\
0 & 0 & 0 & a_{44}
\end{array}\right),\left(\begin{array}{cccc}
0 & 1 & 0 & 0 \\
1 & 0 & 0 & 0 \\
0 & 0 & 1 & 0 \\
0 & 0 & 0 & a_{44}
\end{array}\right)\right\}
$$

A dimensão do aniquilador da álgebra é 1. A álgebra se decompõe como: $\mathcal{T}_{5} \oplus \mathbf{k} \mathbf{n}_{1}$. Subálgebras de dimensão 2 e $3: \mathbf{k} e_{1} \oplus \mathbf{k} e_{2} \oplus \mathbf{k} n_{1}, \mathcal{B}_{1} \oplus \mathbf{k} n_{2}, \mathcal{T}_{5}, \mathbf{k} e_{1} \oplus \mathbf{k} n_{1}, \mathcal{B}_{1}, \mathbf{k} e_{1} \oplus \mathbf{k} e_{2}$ e $\mathbf{k} n_{1} \oplus \mathbf{k} n_{2}$. A dimensão do radical nilpotente é 1. A dimensão da órbita é 14. A dimensão do centro associador é 2. A dimensão de $\mathfrak{d}^{2}$ é 3 . A dimensão de $\mathfrak{g}^{3}$ é 3 . A dimensão de $\mathcal{J}^{4}$ é 3. A dimensão do grupo de 2-cociclos é 15. A dimensão do grupo de 2-cobordos é 14. A dimensão do segundo grupo de Cohomologia é 1. A dimensão da subálgebra associativa maximal é 3. A dimensão da subálgebra nilpotente maximal é 2 . A dimensão da subálgebra nula maximal é 2 .

ÁLGEBRA Jq

O produto na álgebra é:

\begin{tabular}{c|cccc}
$\partial_{9}$ & $e_{1}$ & $e_{2}$ & $e_{3}$ & $e_{4}$ \\
\hline$e_{1}$ & $e_{1}$ & 0 & $\frac{e_{3}}{2}$ & $\frac{e_{4}}{2}$ \\
$e_{2}$ & 0 & $e_{2}$ & $\frac{e_{3}}{2}$ & $\frac{e_{4}}{2}$ \\
$e_{3}$ & $\frac{e_{3}}{2}$ & $\frac{e_{3}}{2}$ & $e_{1}+e_{2}$ & 0 \\
$e_{4}$ & $\frac{e_{4}}{2}$ & $\frac{e_{4}}{2}$ & 0 & 0
\end{tabular}

É uma álgebra não associativa, unitária, com unidade: $e_{1}+e_{2}$. A dimensão do grupo de automorfismos é 4 .

O grupo de automorfismos é:

$$
\begin{gathered}
\left\{\left(\begin{array}{cccc}
\frac{1-a_{33}}{2} & \frac{1+a_{33}}{2} & -\frac{1}{2} \sqrt{1-a_{33}^{2}} & -a_{24} \\
\frac{1+a_{33}}{2} & \frac{1-a_{33}}{2} & \frac{\sqrt{1-a_{33}^{2}}}{2} & a_{24} \\
-\sqrt{1-a_{33}^{2}} & \sqrt{1-a_{33}^{2}} & a_{33} & a_{34} \\
0 & 0 & 0 & a_{44}
\end{array}\right),\right. \\
\left(\begin{array}{cccc}
\frac{1-a_{33}}{2} & \frac{1+a_{33}}{2} & \frac{\sqrt{1-a_{33}^{2}}}{2} & -a_{24} \\
\frac{1+a_{33}}{2} & \frac{1-a_{33}}{2} & -\frac{1}{2} \sqrt{1-a_{33}^{2}} & a_{24} \\
\sqrt{1-a_{33}^{2}} & -\sqrt{1-a_{33}^{2}} & a_{33} & a_{34} \\
0 & 0 & 0 & a_{44}
\end{array}\right),\left(\begin{array}{cccc}
\frac{1+a_{33}}{2} & \frac{1-a_{33}}{2} & \frac{\sqrt{1-a_{33}^{2}}}{2} & -a_{24} \\
\frac{1-a_{33}}{2} & \frac{1+a_{33}}{2} & -\frac{1}{2} \sqrt{1-a_{33}^{2}} & a_{24} \\
-\sqrt{1-a_{33}^{2}} & \sqrt{1-a_{33}^{2}} & a_{33} & a_{34} \\
0 & 0 & 0 & a_{44}
\end{array}\right), \\
\left(\begin{array}{ccccc}
\frac{1+a_{33}}{2} & \frac{1-a_{33}}{2} & -\frac{1}{2} \sqrt{1-a_{33}^{2}} & -a_{24} \\
\frac{1-a_{33}}{2} & \frac{1+a_{33}}{2} & \frac{\sqrt{1-a_{33}^{2}}}{2} & a_{24} \\
\sqrt{1-a_{33}^{2}} & -\sqrt{1-a_{33}^{2}} & a_{33} & a_{34} \\
0 & 0 & 0 & a_{44}
\end{array}\right),\left(\begin{array}{cccc}
\frac{1-a_{33}}{2} & \frac{1+a_{33}}{2} & -\frac{1}{2} \sqrt{1-a_{33}^{2}} & 0 \\
\frac{1+a_{33}}{2} & \frac{1-a_{33}}{2} & \frac{\sqrt{1-a_{33}^{2}}}{2} & 0 \\
-\sqrt{1-a_{33}^{2}} & \sqrt{1-a_{33}^{2}} & a_{33} & a_{34} \\
0 & 0 & 0 & a_{44}
\end{array}\right),
\end{gathered}
$$




$$
\begin{aligned}
& \begin{array}{l}
\left(\begin{array}{cccc}
\frac{1-a_{33}}{2} & \frac{1+a_{33}}{2} & \frac{\sqrt{1-a_{33}^{2}}}{2} & 0 \\
\frac{1+a_{33}}{2} & \frac{1-a_{33}}{2} & -\frac{1}{2} \sqrt{1-a_{33}^{2}} & 0 \\
\sqrt{1-a_{33}^{2}} & -\sqrt{1-a_{33}^{2}} & a_{33} & a_{34} \\
0 & 0 & 0 & a_{44}
\end{array}\right) \\
\left(\begin{array}{cccc}
\frac{1+a_{33}}{2} & \frac{1-a_{33}}{2} & -\frac{1}{2} \sqrt{1-a_{33}^{2}} & 0 \\
\frac{1-a_{33}}{2} & \frac{1+a_{33}}{2} & \frac{\sqrt{1-a_{33}^{2}}}{2} & 0 \\
\sqrt{1-a_{33}^{2}} & -\sqrt{1-a_{33}^{2}} & a_{33} & a_{34} \\
0 & 0 & 0 & a_{44}
\end{array}\right)
\end{array} \\
& \left(\begin{array}{cccc}
0 & 1 & 0 & -a_{24} \\
1 & 0 & 0 & a_{24} \\
0 & 0 & -1 & a_{34} \\
0 & 0 & 0 & a_{44}
\end{array}\right),\left(\begin{array}{cccc}
0 & 1 & 0 & -a_{24} \\
1 & 0 & 0 & a_{24} \\
0 & 0 & 1 & a_{34} \\
0 & 0 & 0 & a_{44}
\end{array}\right),\left(\begin{array}{cccc}
1 & 0 & 0 & -a_{24} \\
0 & 1 & 0 & a_{24} \\
0 & 0 & -1 & a_{34} \\
0 & 0 & 0 & a_{44}
\end{array}\right), \\
& \left(\begin{array}{cccc}
1 & 0 & 0 & -a_{24} \\
0 & 1 & 0 & a_{24} \\
0 & 0 & 1 & a_{34} \\
0 & 0 & 0 & a_{44}
\end{array}\right),\left(\begin{array}{cccc}
0 & 1 & 0 & 0 \\
1 & 0 & 0 & 0 \\
0 & 0 & -1 & a_{34} \\
0 & 0 & 0 & a_{44}
\end{array}\right),\left(\begin{array}{cccc}
0 & 1 & 0 & 0 \\
1 & 0 & 0 & 0 \\
0 & 0 & 1 & a_{34} \\
0 & 0 & 0 & a_{44}
\end{array}\right), \\
& \left.\left(\begin{array}{cccc}
1 & 0 & 0 & 0 \\
0 & 1 & 0 & 0 \\
0 & 0 & -1 & a_{34} \\
0 & 0 & 0 & a_{44}
\end{array}\right),\left(\begin{array}{cccc}
1 & 0 & 0 & 0 \\
0 & 1 & 0 & 0 \\
0 & 0 & 1 & a_{34} \\
0 & 0 & 0 & a_{44}
\end{array}\right)\right\}
\end{aligned}
$$

A dimensão do aniquilador da álgebra é 0. A álgebra é indecomponível. Subálgebras de dimensão 2 e 3: $\mathcal{T}_{2}, \mathcal{T}_{5}, \mathcal{T}_{10}, \mathcal{B}_{1}, \mathcal{B}_{2}, \mathbf{k}_{1} \oplus \mathbf{k} e_{2}$ e $\mathbf{k n}_{1} \oplus \mathbf{k} n_{2}$. A dimensão do radical nilpotente é 1. A dimensão da órbita é 12. A dimensão do centro associador é 1. A dimensão de $\mathfrak{J}^{2}$ é 4 . A dimensão de $\mathfrak{J}^{3}$ é 4 . A dimensão de $\mathfrak{J}^{4}$ é 4 . A dimensão do grupo de 2-cociclos é 13. A dimensão do grupo de 2-cobordos é 12. A dimensão do segundo grupo de Cohomologia é 1. A dimensão da subálgebra associativa maximal é 3. A dimensão da subálgebra nilpotente maximal é 2. A dimensão da subálgebra nula maximal é 2.

ÁLGEBRA $\jmath_{10}$

O produto na álgebra é:

\begin{tabular}{c|cccc}
$\partial_{10}$ & $e_{1}$ & $e_{2}$ & $e_{3}$ & $e_{4}$ \\
\hline$e_{1}$ & $e_{1}$ & 0 & $\frac{e_{3}}{2}$ & 0 \\
$e_{2}$ & 0 & $e_{2}$ & 0 & 0 \\
$e_{3}$ & $\frac{e_{3}}{2}$ & 0 & 0 & 0 \\
$e_{4}$ & 0 & 0 & 0 & 0
\end{tabular}

É uma álgebra não associativa, não possui unidade. A dimensão do grupo de automorfismos é 3. 
O grupo de automorfismos é:

$$
\left\{\left(\begin{array}{cccc}
1 & 0 & a_{13} & 0 \\
0 & 1 & 0 & 0 \\
0 & 0 & a_{33} & 0 \\
0 & 0 & 0 & a_{44}
\end{array}\right)\right\}
$$

A dimensão do aniquilador da álgebra é 1. A álgebra se decompõe como: $\mathcal{B}_{2} \oplus \mathbf{k} e_{2} \oplus$ $\mathbf{k} n_{2}$. Subálgebras de dimensão 2 e 3: $\mathbf{k} e_{1} \oplus \mathbf{k} e_{2} \oplus \mathbf{k} n_{1}, \mathbf{k} e_{1} \oplus \mathbf{k} n_{1} \oplus \mathbf{k} n_{2}, \mathcal{B}_{2} \oplus \mathbf{k} e_{2}$, $\mathcal{B}_{2} \oplus \mathbf{k} n_{2}, \mathbf{k} e_{1} \oplus \mathbf{k} n_{1}, \mathcal{B}_{2}, \mathbf{k} e_{1} \oplus \mathbf{k} e_{2}$ e $\mathbf{k} n_{1} \oplus \mathbf{k} n_{2}$. A dimensão do radical nilpotente é 2. A dimensão da órbita é 13. A dimensão do centro associador é 2 . A dimensão de $\mathfrak{J}^{2}$ é 3. A dimensão de $\mathfrak{g}^{3}$ é 3. A dimensão de $\mathfrak{g}^{4}$ é 3. A dimensão do grupo de 2-cociclos é 16. A dimensão do grupo de 2-cobordos é 13. A dimensão do segundo grupo de Cohomologia é 3. A dimensão da subálgebra associativa maximal é 3. A dimensão da subálgebra nilpotente maximal é 2 . A dimensão da subálgebra nula maximal é 2 .

ÁLGEBRA $\mathcal{J}_{11}$

O produto na álgebra é:

\begin{tabular}{c|cccc}
$\partial_{11}$ & $e_{1}$ & $e_{2}$ & $e_{3}$ & $e_{4}$ \\
\hline$e_{1}$ & $e_{1}$ & 0 & $\frac{e_{3}}{2}$ & 0 \\
$e_{2}$ & 0 & $e_{2}$ & $\frac{e_{3}}{2}$ & 0 \\
$e_{3}$ & $\frac{e_{3}}{2}$ & $\frac{e_{3}}{2}$ & 0 & 0 \\
$e_{4}$ & 0 & 0 & 0 & 0
\end{tabular}

É uma álgebra não associativa, não possui unidade. A dimensão do grupo de automorfismos é 3.

O grupo de automorfismos é:

$$
\begin{aligned}
& \left\{\left(\begin{array}{cccc}
0 & 1 & -a_{23} & 0 \\
1 & 0 & a_{23} & 0 \\
0 & 0 & a_{33} & 0 \\
0 & 0 & 0 & a_{44}
\end{array}\right),\left(\begin{array}{cccc}
1 & 0 & -a_{23} & 0 \\
0 & 1 & a_{23} & 0 \\
0 & 0 & a_{33} & 0 \\
0 & 0 & 0 & a_{44}
\end{array}\right),\right. \\
& \left.\left(\begin{array}{cccc}
0 & 1 & 0 & 0 \\
1 & 0 & 0 & 0 \\
0 & 0 & a_{33} & 0 \\
0 & 0 & 0 & a_{44}
\end{array}\right),\left(\begin{array}{cccc}
1 & 0 & 0 & 0 \\
0 & 1 & 0 & 0 \\
0 & 0 & a_{33} & 0 \\
0 & 0 & 0 & a_{44}
\end{array}\right)\right\}
\end{aligned}
$$

A dimensão do aniquilador da álgebra é 1. A álgebra se decompõe como: $\mathcal{T}_{10} \oplus \mathbf{k} \mathbf{n}_{2}$. Subálgebras de dimensão 2 e 3: $\mathbf{k} e_{1} \oplus \mathbf{k} e_{2} \oplus \mathbf{k} n_{1}, \mathcal{B}_{1} \oplus \mathbf{k} n_{2}, \mathcal{T}_{10}, \mathcal{B}_{2} \oplus \mathbf{k} n_{2}, \mathbf{k} e_{1} \oplus \mathbf{k} n_{1}$, $\mathcal{B}_{1}, \mathcal{B}_{2}, \mathbf{k} e_{1} \oplus \mathbf{k} e_{2}$ e $\mathbf{k} n_{1} \oplus \mathbf{k} n_{2}$. A dimensão do radical nilpotente é 2 . A dimensão da órbita é 13. A dimensão do centro associador é 2. A dimensão de $\mathfrak{J}^{2}$ é 3 . A dimensão de $\mathfrak{J}^{3}$ é 3. A dimensão de $\mathfrak{J}^{4}$ é 3 . A dimensão do grupo de 2-cociclos é 15 . A dimensão do grupo de 2-cobordos é 13. A dimensão do segundo grupo de Cohomologia é 2. A 
dimensão da subálgebra associativa maximal é 3. A dimensão da subálgebra nilpotente maximal é 2. A dimensão da subálgebra nula maximal é 2 .

ÁLGEBRA $\jmath_{12}$

O produto na álgebra é:

\begin{tabular}{c|cccc}
$J_{12}$ & $e_{1}$ & $e_{2}$ & $e_{3}$ & $e_{4}$ \\
\hline$e_{1}$ & $e_{1}$ & 0 & $\frac{e_{3}}{2}$ & $\frac{e_{4}}{2}$ \\
$e_{2}$ & 0 & $e_{2}$ & 0 & 0 \\
$e_{3}$ & $\frac{e_{3}}{2}$ & 0 & 0 & 0 \\
$e_{4}$ & $\frac{e_{4}}{2}$ & 0 & 0 & 0
\end{tabular}

É uma álgebra não associativa, não possui unidade. A dimensão do grupo de automorfismos é 6.

O grupo de automorfismos é:

$$
\left\{\left(\begin{array}{cccc}
1 & 0 & a_{13} & a_{14} \\
0 & 1 & 0 & 0 \\
0 & 0 & a_{33} & a_{34} \\
0 & 0 & a_{43} & a_{44}
\end{array}\right)\right\}
$$

A dimensão do aniquilador da álgebra é 0. É uma álgebra rígida. A álgebra se decompõe como: $\mathcal{T}_{7} \oplus \mathbf{k} e_{2}$. Subálgebras de dimensão 2 e 3: $\mathbf{k} e_{1} \oplus \mathbf{k} n_{1} \oplus \mathbf{k} n_{2}, \mathcal{T}_{7}, \mathcal{B}_{2} \oplus \mathbf{k} e_{2}$, $\mathbf{k} e_{1} \oplus \mathbf{k} n_{1}, \mathcal{B}_{2}, \mathbf{k} e_{1} \oplus \mathbf{k} e_{2}$ e $\mathbf{k} n_{1} \oplus \mathbf{k} n_{2}$. A dimensão do radical nilpotente é 2 . A dimensão da órbita é 10. A dimensão do centro associador é 1. A dimensão de $\mathfrak{J}^{2}$ é 4 . A dimensão de $\mathfrak{J}^{3}$ é 4 . A dimensão de $\mathfrak{J}^{4}$ é 4 . A dimensão do grupo de 2-cociclos é 10. A dimensão do grupo de 2-cobordos é 10. A dimensão do segundo grupo de Cohomologia é 0 . A dimensão da subálgebra associativa maximal é 3. A dimensão da subálgebra nilpotente maximal é 2 . A dimensão da subálgebra nula maximal é 2.

ÁLGEBRA $\partial_{13}$

O produto na álgebra é:

\begin{tabular}{c|cccc}
$\partial_{13}$ & $e_{1}$ & $e_{2}$ & $e_{3}$ & $e_{4}$ \\
\hline$e_{1}$ & $e_{1}$ & 0 & 0 & $\frac{e_{4}}{2}$ \\
$e_{2}$ & 0 & $e_{2}$ & $\frac{e_{3}}{2}$ & 0 \\
$e_{3}$ & 0 & $\frac{e_{3}}{2}$ & 0 & 0 \\
$e_{4}$ & $\frac{e_{4}}{2}$ & 0 & 0 & 0
\end{tabular}

É uma álgebra não associativa, não possui unidade. A dimensão do grupo de automorfismos é 4. 
O grupo de automorfismos é:

$$
\left\{\left(\begin{array}{cccc}
0 & 1 & a_{13} & 0 \\
1 & 0 & 0 & a_{24} \\
0 & 0 & 0 & a_{34} \\
0 & 0 & a_{43} & 0
\end{array}\right),\left(\begin{array}{cccc}
1 & 0 & 0 & a_{14} \\
0 & 1 & a_{23} & 0 \\
0 & 0 & a_{33} & 0 \\
0 & 0 & 0 & a_{44}
\end{array}\right)\right\}
$$

A dimensão do aniquilador da álgebra é 0. É uma álgebra rígida. A álgebra se decompõe como: $\mathcal{B}_{2} \oplus \mathcal{B}_{2}$. Subálgebras de dimensão: 2 e 3: $\mathcal{T}_{7}, \mathcal{B}_{2} \oplus \mathbf{k} e_{2}, \mathcal{B}_{2} \oplus \mathbf{k} n_{2}, \mathbf{k} e_{1} \oplus \mathbf{k n} n_{1}$, $\mathcal{B}_{2}, \mathbf{k} e_{1} \oplus \mathbf{k} e_{2}$ e $\mathbf{k} n_{1} \oplus \mathbf{k} n_{2}$. A dimensão do radical nilpotente é 2 . A dimensão da órbita é 12. A dimensão do centro associador é 0 . A dimensão de $\mathfrak{J}^{2}$ é 4 . A dimensão de $\mathfrak{J}^{3}$ é 4 . A dimensão de $\mathfrak{J}^{4}$ é 4 . A dimensão do grupo de 2-cociclos é 12. A dimensão do grupo de 2-cobordos é 12. A dimensão do segundo grupo de Cohomologia é 0. A dimensão da subálgebra associativa maximal é 2. A dimensão da subálgebra nilpotente maximal é 2. A dimensão da subálgebra nula maximal é 2 .

ÁLGEBRA J J14

O produto na álgebra é:

\begin{tabular}{c|cccc}
$\partial_{14}$ & $e_{1}$ & $e_{2}$ & $e_{3}$ & $e_{4}$ \\
\hline$e_{1}$ & $e_{1}$ & 0 & $e_{3}$ & $\frac{e_{4}}{2}$ \\
$e_{2}$ & 0 & $e_{2}$ & 0 & 0 \\
$e_{3}$ & $e_{3}$ & 0 & 0 & 0 \\
$e_{4}$ & $\frac{e_{4}}{2}$ & 0 & 0 & 0
\end{tabular}

É uma álgebra não associativa, não possui unidade. A dimensão do grupo de automorfismos é 3 .

O grupo de automorfismos é:

$$
\left\{\left(\begin{array}{cccc}
1 & 0 & 0 & a_{14} \\
0 & 1 & 0 & 0 \\
0 & 0 & a_{33} & 0 \\
0 & 0 & 0 & a_{44}
\end{array}\right)\right\}
$$

A dimensão do aniquilador da álgebra é 0. A álgebra se decompõe como: $\mathcal{T}_{6} \oplus \mathbf{k} \mathbf{e}_{2}$. Subálgebras de dimensão 2 e 3: $\mathcal{B}_{1} \oplus \mathbf{k} e_{2}, \mathbf{k} e_{1} \oplus \mathbf{k} n_{1} \oplus \mathbf{k} n_{2}, \mathcal{T}_{6}, \mathcal{B}_{2} \oplus \mathbf{k} e_{2}, \mathbf{k} e_{1} \oplus \mathbf{k} n_{1}$, $\mathcal{B}_{1}, \mathcal{B}_{2}, \mathbf{k} e_{1} \oplus \mathbf{k} e_{2}$ e $\mathbf{k} n_{1} \oplus \mathbf{k} n_{2}$. A dimensão do radical nilpotente é 2. A dimensão da órbita é 13. A dimensão do centro associador é 2. A dimensão de $\mathfrak{J}^{2}$ é 4 . A dimensão de $\mathfrak{J}^{3}$ é 4 . A dimensão de $\mathfrak{J}^{4}$ é 4 . A dimensão do grupo de 2-cociclos é 15. A dimensão do grupo de 2-cobordos é 13. A dimensão do segundo grupo de Cohomologia é 2. A dimensão da subálgebra associativa maximal é 3. A dimensão da subálgebra nilpotente maximal é 2. A dimensão da subálgebra nula maximal é 2 . 
O produto na álgebra é:

\begin{tabular}{c|cccc}
$\partial_{15}$ & $e_{1}$ & $e_{2}$ & $e_{3}$ & $e_{4}$ \\
\hline$e_{1}$ & $e_{1}$ & 0 & 0 & $\frac{e_{4}}{2}$ \\
$e_{2}$ & 0 & $e_{2}$ & $e_{3}$ & 0 \\
$e_{3}$ & 0 & $e_{3}$ & 0 & 0 \\
$e_{4}$ & $\frac{e_{4}}{2}$ & 0 & 0 & 0
\end{tabular}

É uma álgebra não associativa, não possui unidade. A dimensão do grupo de automorfismos é 3.

O grupo de automorfismos é:

$$
\left\{\left(\begin{array}{cccc}
1 & 0 & 0 & a_{14} \\
0 & 1 & 0 & 0 \\
0 & 0 & a_{33} & 0 \\
0 & 0 & 0 & a_{44}
\end{array}\right)\right\}
$$

A dimensão do aniquilador da álgebra é 0. A álgebra se decompõe como: $\mathcal{B}_{2} \oplus \mathcal{B}_{1}$. Subálgebras de dimensão 2 e 3: $\mathcal{B}_{1} \oplus \mathbf{k} e_{2}, \mathcal{B}_{1} \oplus \mathbf{k} n_{2}, \mathcal{T}_{6}, \mathcal{B}_{2} \oplus \mathbf{k} e_{2}, \mathcal{B}_{2} \oplus \mathbf{k} n_{2}, \mathbf{k} e_{1} \oplus \mathbf{k} n_{1}$, $\mathcal{B}_{1}, \mathcal{B}_{2}, \mathbf{k} e_{1} \oplus \mathbf{k} e_{2}$ e $\mathbf{k} n_{1} \oplus \mathbf{k} n_{2}$. A dimensão do radical nilpotente é 2. A dimensão da órbita é 13. A dimensão do centro associador é 2. A dimensão de $f^{2}$ é 4 . A dimensão de $\mathfrak{J}^{3}$ é 4 . A dimensão de $\mathfrak{J}^{4}$ é 4 . A dimensão do grupo de 2-cociclos é 14 . A dimensão do grupo de 2-cobordos é 13. A dimensão do segundo grupo de Cohomologia é 1. A dimensão da subálgebra associativa maximal é 3. A dimensão da subálgebra nilpotente maximal é 2. A dimensão da subálgebra nula maximal é 2 .

ÁLGEBRA $J_{16}$

O produto na álgebra é:

\begin{tabular}{c|cccc}
$\partial_{16}$ & $e_{1}$ & $e_{2}$ & $e_{3}$ & $e_{4}$ \\
\hline$e_{1}$ & $e_{1}$ & 0 & $\frac{e_{3}}{2}$ & $\frac{e_{4}}{2}$ \\
$e_{2}$ & 0 & $e_{2}$ & $\frac{e_{3}}{2}$ & 0 \\
$e_{3}$ & $\frac{e_{3}}{2}$ & $\frac{e_{3}}{2}$ & 0 & 0 \\
$e_{4}$ & $\frac{e_{4}}{2}$ & 0 & 0 & 0
\end{tabular}

É uma álgebra não associativa, não possui unidade. A dimensão do grupo de automorfismos é 4.

O grupo de automorfismos é:

$$
\left\{\left(\begin{array}{cccc}
1 & 0 & -a_{23} & a_{14} \\
0 & 1 & a_{23} & 0 \\
0 & 0 & a_{33} & 0 \\
0 & 0 & 0 & a_{44}
\end{array}\right),\left(\begin{array}{cccc}
1 & 0 & 0 & a_{14} \\
0 & 1 & 0 & 0 \\
0 & 0 & a_{33} & 0 \\
0 & 0 & 0 & a_{44}
\end{array}\right)\right\}
$$


A dimensão do aniquilador da álgebra é 0. É uma álgebra rígida. A álgebra é indecomponível. Subálgebras de dimensão 2 e 3: $\mathcal{T}_{6}, \mathcal{T}_{7}, \mathcal{T}_{10}, \mathcal{B}_{2} \oplus \mathbf{k} e_{2}, \mathcal{B}_{2} \oplus \mathbf{k} n_{2}, \mathbf{k} e_{1} \oplus \mathbf{k} n_{1}$, $\mathcal{B}_{1}, \mathcal{B}_{2}, \mathbf{k} e_{1} \oplus \mathbf{k} e_{2}$ e $\mathbf{k} n_{1} \oplus \mathbf{k} n_{2}$. A dimensão do radical nilpotente é 2. A dimensão da órbita é 12. A dimensão do centro associador é 0 . A dimensão de $\mathfrak{g}^{2}$ é 4 . A dimensão de $\mathfrak{J}^{3}$ é 4 . A dimensão de $\mathfrak{J}^{4}$ é 4 . A dimensão do grupo de 2-cociclos é 12. A dimensão do grupo de 2-cobordos é 12. A dimensão do segundo grupo de Cohomologia é 0. A dimensão da subálgebra associativa maximal é 2 . A dimensão da subálgebra nilpotente maximal é 2. A dimensão da subálgebra nula maximal é 2 .

ÁLGEBRA $\jmath_{17}$

O produto na álgebra é:

\begin{tabular}{c|cccc}
$\partial_{17}$ & $e_{1}$ & $e_{2}$ & $e_{3}$ & $e_{4}$ \\
\hline$e_{1}$ & $e_{1}$ & 0 & $\frac{e_{3}}{2}$ & $e_{4}$ \\
$e_{2}$ & 0 & $e_{2}$ & $\frac{e_{3}}{2}$ & 0 \\
$e_{3}$ & $\frac{e_{3}}{2}$ & $\frac{e_{3}}{2}$ & 0 & 0 \\
$e_{4}$ & $e_{4}$ & 0 & 0 & 0
\end{tabular}

É uma álgebra não associativa, unitária, com unidade: $e_{1}+e_{2}$. A dimensão do grupo de automorfismos é 3 .

O grupo de automorfismos é:

$$
\left\{\left(\begin{array}{cccc}
1 & 0 & -a_{23} & 0 \\
0 & 1 & a_{23} & 0 \\
0 & 0 & a_{33} & 0 \\
0 & 0 & 0 & a_{44}
\end{array}\right),\left(\begin{array}{cccc}
1 & 0 & 0 & 0 \\
0 & 1 & 0 & 0 \\
0 & 0 & a_{33} & 0 \\
0 & 0 & 0 & a_{44}
\end{array}\right)\right\}
$$

A dimensão do aniquilador da álgebra é 0. A álgebra é indecomponível. Subálgebras de dimensão 2 e 3: $\mathcal{B}_{1} \oplus \mathbf{k} e_{2}, \mathcal{T}_{2}, \mathcal{T}_{6}, \mathcal{T}_{10}, \mathcal{B}_{2} \oplus \mathbf{k} n_{2}, \mathbf{k} e_{1} \oplus \mathbf{k} n_{1}, \mathcal{B}_{1}, \mathcal{B}_{2}, \mathbf{k} e_{1} \oplus \mathbf{k} e_{2}$ e $\mathbf{k} n_{1} \oplus \mathbf{k} n_{2}$. A dimensão do radical nilpotente é 2. A dimensão da órbita é 13. A dimensão do centro associador é 2. A dimensão de $\mathfrak{g}^{2}$ é 4 . A dimensão de $\mathfrak{J}^{3}$ é 4 . A dimensão de $\mathfrak{J}^{4}$ é 4 . A dimensão do grupo de 2-cociclos é 15. A dimensão do grupo de 2-cobordos é 13. A dimensão do segundo grupo de Cohomologia é 2. A dimensão da subálgebra associativa maximal é 3. A dimensão da subálgebra nilpotente maximal é 2 . A dimensão da subálgebra nula maximal é 2 . 
O produto na álgebra é:

\begin{tabular}{c|cccc}
$\partial_{18}$ & $e_{1}$ & $e_{2}$ & $e_{3}$ & $e_{4}$ \\
\hline$e_{1}$ & $e_{1}$ & 0 & $\frac{e_{3}}{2}$ & $\frac{e_{4}}{2}$ \\
$e_{2}$ & 0 & $e_{2}$ & $\frac{e_{3}}{2}$ & $\frac{e_{4}}{2}$ \\
$e_{3}$ & $\frac{e_{3}}{2}$ & $\frac{e_{3}}{2}$ & 0 & 0 \\
$e_{4}$ & $\frac{e_{4}}{2}$ & $\frac{e_{4}}{2}$ & 0 & 0
\end{tabular}

É uma álgebra não associativa, unitária, com unidade: $e_{1}+e_{2}$. A dimensão do grupo de automorfismos é 6 . A dimensão do grupo de automorfismos é 6 .

O grupo de automorfismos é:

$$
\begin{aligned}
& \left\{\left(\begin{array}{cccc}
0 & 1 & -a_{23} & -a_{24} \\
1 & 0 & a_{23} & a_{24} \\
0 & 0 & a_{33} & a_{34} \\
0 & 0 & a_{43} & a_{44}
\end{array}\right),\left(\begin{array}{cccc}
1 & 0 & -a_{23} & -a_{24} \\
0 & 1 & a_{23} & a_{24} \\
0 & 0 & a_{33} & a_{34} \\
0 & 0 & a_{43} & a_{44}
\end{array}\right),\left(\begin{array}{cccc}
0 & 1 & 0 & -a_{24} \\
1 & 0 & 0 & a_{24} \\
0 & 0 & a_{33} & a_{34} \\
0 & 0 & a_{43} & a_{44}
\end{array}\right),\right. \\
& \left(\begin{array}{cccc}
1 & 0 & 0 & -a_{24} \\
0 & 1 & 0 & a_{24} \\
0 & 0 & a_{33} & a_{34} \\
0 & 0 & a_{43} & a_{44}
\end{array}\right),\left(\begin{array}{ccccc}
0 & 1 & -a_{23} & 0 \\
1 & 0 & a_{23} & 0 \\
0 & 0 & a_{33} & a_{34} \\
0 & 0 & a_{43} & a_{44}
\end{array}\right),\left(\begin{array}{cccc}
1 & 0 & -a_{23} & 0 \\
0 & 1 & a_{23} & 0 \\
0 & 0 & a_{33} & a_{34} \\
0 & 0 & a_{43} & a_{44}
\end{array}\right) \\
& \left.\left(\begin{array}{cccc}
0 & 1 & 0 & 0 \\
1 & 0 & 0 & 0 \\
0 & 0 & a_{33} & a_{34} \\
0 & 0 & a_{43} & a_{44}
\end{array}\right),\left(\begin{array}{cccc}
1 & 0 & 0 & 0 \\
0 & 1 & 0 & 0 \\
0 & 0 & a_{33} & a_{34} \\
0 & 0 & a_{43} & a_{44}
\end{array}\right)\right\}
\end{aligned}
$$

A dimensão do aniquilador da álgebra é 0. A álgebra é indecomponível. Subálgebras de dimensão 2 e 3: $\mathcal{T}_{2}, \mathcal{T}_{7}, \mathcal{T}_{10}, \mathcal{B}_{1}, \mathcal{B}_{2}, \mathbf{k e}_{1} \oplus \mathbf{k} e_{2}$ e $\mathbf{k} n_{1} \oplus \mathbf{k} n_{2}$. A dimensão do radical nilpotente é 2. A dimensão da órbita é 10. A dimensão do centro associador é 1. A dimensão de $\mathfrak{J}^{2}$ é 4 . A dimensão de $\mathfrak{J}^{3}$ é 4 . A dimensão de $\mathfrak{J}^{4}$ é 4 . A dimensão do grupo de 2-cociclos é 13. A dimensão do grupo de 2-cobordos é 10. A dimensão do segundo grupo de Cohomologia é 3. A dimensão da subálgebra associativa maximal é 3. A dimensão da subálgebra nilpotente maximal é 2. A dimensão da subálgebra nula maximal é 2.

ÁLGEBRA $J_{19}$

O produto na álgebra é:

\begin{tabular}{c|cccc}
$J_{19}$ & $e_{1}$ & $e_{2}$ & $e_{3}$ & $e_{4}$ \\
\hline$e_{1}$ & $e_{1}$ & 0 & 0 & 0 \\
$e_{2}$ & 0 & $e_{2}$ & 0 & 0 \\
$e_{3}$ & 0 & 0 & 0 & 0 \\
$e_{4}$ & 0 & 0 & 0 & 0
\end{tabular}


É uma álgebra associativa, não possui unidade. A dimensão do grupo de automorfismos é 4 .

O grupo de automorfismos é:

$$
\left\{\left(\begin{array}{cccc}
0 & 1 & 0 & 0 \\
1 & 0 & 0 & 0 \\
0 & 0 & a_{33} & a_{34} \\
0 & 0 & a_{43} & a_{44}
\end{array}\right),\left(\begin{array}{cccc}
1 & 0 & 0 & 0 \\
0 & 1 & 0 & 0 \\
0 & 0 & a_{33} & a_{34} \\
0 & 0 & a_{43} & a_{44}
\end{array}\right)\right\}
$$

A dimensão do aniquilador da álgebra é 2. A álgebra se decompõe como: $\mathbf{k} e_{1} \oplus \mathbf{k} \mathbf{e}_{2} \oplus$ $\mathbf{k} n_{1} \oplus \mathbf{k} n_{2}$. Subálgebras de dimensão 2 e 3: $\mathbf{k} e_{1} \oplus \mathbf{k} e_{2} \oplus \mathbf{k} n_{1}, \mathbf{k} e_{1} \oplus \mathbf{k} n_{1} \oplus \mathbf{k} n_{2}, \mathbf{k} e_{1} \oplus$ $\mathbf{k} n_{1}, \mathbf{k} e_{1} \oplus \mathbf{k} e_{2}$ e $\mathbf{k} n_{1} \oplus \mathbf{k} n_{2}$. A dimensão do radical nilpotente é 2 . A dimensão da órbita é 12. A dimensão do centro associador é 4. A dimensão de $\mathfrak{f}^{2}$ é 2 . A dimensão de $\mathfrak{J}^{3}$ é 2 . A dimensão de $\mathfrak{J}^{4}$ é 2 . A dimensão do grupo de 2-cociclos é 18 . A dimensão do grupo de 2-cobordos é 12. A dimensão do segundo grupo de Cohomologia é 6. A dimensão da subálgebra associativa maximal é 4. A dimensão da subálgebra nilpotente maximal é 2. A dimensão da subálgebra nula maximal é 2 .

ÁLGEBRA $\partial_{20}$

O produto na álgebra é:

\begin{tabular}{c|cccc}
$\partial_{20}$ & $e_{1}$ & $e_{2}$ & $e_{3}$ & $e_{4}$ \\
\hline$e_{1}$ & $e_{1}$ & 0 & 0 & $e_{4}$ \\
$e_{2}$ & 0 & $e_{2}$ & 0 & 0 \\
$e_{3}$ & 0 & 0 & 0 & 0 \\
$e_{4}$ & $e_{4}$ & 0 & 0 & 0
\end{tabular}

É uma álgebra associativa, não possui unidade. A dimensão do grupo de automorfismos é 2 .

O grupo de automorfismos é:

$$
\left\{\left(\begin{array}{cccc}
1 & 0 & 0 & 0 \\
0 & 1 & 0 & 0 \\
0 & 0 & a_{33} & 0 \\
0 & 0 & 0 & a_{44}
\end{array}\right)\right\}
$$

A dimensão do aniquilador da álgebra é 1. A álgebra se decompõe como: $\mathcal{B}_{1} \oplus \mathbf{k} e_{2} \oplus$ $\mathbf{k n}_{2}$. Subálgebras de dimensão 2 e 3: $\mathcal{B}_{1} \oplus \mathbf{k} \mathbf{e}_{2}, \mathbf{k} e_{1} \oplus \mathbf{k} \mathbf{e}_{2} \oplus \mathbf{k} n_{1}, \mathcal{B}_{1} \oplus \mathbf{k} n_{2}, \mathbf{k} e_{1} \oplus \mathbf{k} n_{1} \oplus$ $\mathbf{k} n_{2}, \mathbf{k} e_{1} \oplus \mathbf{k} n_{1}, \mathcal{B}_{1}, \mathbf{k} e_{1} \oplus \mathbf{k} e_{2}$ e $\mathbf{k} n_{1} \oplus \mathbf{k} n_{2}$. A dimensão do radical nilpotente é 2. A dimensão da órbita é 14. A dimensão do centro associador é 4. A dimensão de $g^{2}$ é 3. A dimensão de $\mathfrak{J}^{3}$ é 3. A dimensão de $\mathfrak{J}^{4}$ é 3. A dimensão do grupo de 2-cociclos é 16. A dimensão do grupo de 2-cobordos é 14. A dimensão do segundo grupo de 
Cohomologia é 2. A dimensão da subálgebra associativa maximal é 4. A dimensão da subálgebra nilpotente maximal é 2 . A dimensão da subálgebra nula maximal é 2 .

ÁLGEBRA $\partial_{21}$

O produto na álgebra é:

\begin{tabular}{c|cccc}
$\partial_{21}$ & $e_{1}$ & $e_{2}$ & $e_{3}$ & $e_{4}$ \\
\hline$e_{1}$ & $e_{1}$ & 0 & $e_{3}$ & $e_{4}$ \\
$e_{2}$ & 0 & $e_{2}$ & 0 & 0 \\
$e_{3}$ & $e_{3}$ & 0 & 0 & 0 \\
$e_{4}$ & $e_{4}$ & 0 & 0 & 0
\end{tabular}

É uma álgebra associativa, unitária, com unidade: $e_{1}+e_{2}$. A dimensão do grupo de automorfismos é 4 .

O grupo de automorfismos é:

$$
\left\{\left(\begin{array}{cccc}
1 & 0 & 0 & 0 \\
0 & 1 & 0 & 0 \\
0 & 0 & a_{33} & a_{34} \\
0 & 0 & a_{43} & a_{44}
\end{array}\right)\right\}
$$

A dimensão do aniquilador da álgebra é 0. A álgebra se decompõe como: $\mathcal{T}_{2} \oplus \mathbf{k} e_{2}$. Subálgebras de dimensão 2 e 3: $\mathcal{B}_{1} \oplus \mathbf{k} e_{2}, \mathcal{T}_{2}, \mathbf{k} e_{1} \oplus \mathbf{k} n_{1} \oplus \mathbf{k} n_{2}, \mathbf{k} e_{1} \oplus \mathbf{k} n_{1}, \mathcal{B}_{1}, \mathbf{k} e_{1} \oplus \mathbf{k} e_{2}$ e $\mathbf{k} n_{1} \oplus \mathbf{k} n_{2}$. A dimensão do radical nilpotente é 2. A dimensão da órbita é 12. A dimensão do centro associador é 4 . A dimensão de $\mathcal{J}^{2}$ é 4 . A dimensão de $\mathcal{J}^{3}$ é 4 . A dimensão de $\mathfrak{J}^{4}$ é 4 . A dimensão do grupo de 2-cociclos é 19. A dimensão do grupo de 2-cobordos é 12. A dimensão do segundo grupo de Cohomologia é 7. A dimensão da subálgebra associativa maximal é 4. A dimensão da subálgebra nilpotente maximal é 2 . A dimensão da subálgebra nula maximal é 2 .

ÁLGEBRA $\partial_{22}$

\begin{tabular}{cc|cccc} 
& $J_{22}$ & $e_{1}$ & $e_{2}$ & $e_{3}$ & $e_{4}$ \\
\cline { 2 - 6 } O produto na álgebra é: & $e_{1}$ & $e_{1}$ & 0 & $e_{3}$ & 0 \\
& $e_{2}$ & 0 & $e_{2}$ & 0 & $e_{4}$ \\
& $e_{3}$ & $e_{3}$ & 0 & 0 & 0 \\
& $e_{4}$ & 0 & $e_{4}$ & 0 & 0
\end{tabular}

É uma álgebra associativa, unitária, com unidade: $e_{1}+e_{2}$. A dimensão do grupo de automorfismos é 2 . 
O grupo de automorfismos é:

$$
\left\{\left(\begin{array}{cccc}
0 & 1 & 0 & 0 \\
1 & 0 & 0 & 0 \\
0 & 0 & 0 & a_{34} \\
0 & 0 & a_{43} & 0
\end{array}\right),\left(\begin{array}{cccc}
1 & 0 & 0 & 0 \\
0 & 1 & 0 & 0 \\
0 & 0 & a_{33} & 0 \\
0 & 0 & 0 & a_{44}
\end{array}\right)\right\}
$$

A dimensão do aniquilador da álgebra é 0. A álgebra se decompõe como: $\mathcal{B}_{1} \oplus \mathcal{B}_{1}$. Subálgebras de dimensão 2 e 3: $\mathcal{B}_{1} \oplus \mathbf{k} e_{2}, \mathcal{T}_{2}, \mathcal{B}_{1} \oplus \mathbf{k} n_{2}, \mathbf{k} e_{1} \oplus \mathbf{k} n_{1}, \mathcal{B}_{1}, \mathbf{k} e_{1} \oplus \mathbf{k} e_{2}$ e $\mathbf{k} n_{1} \oplus \mathbf{k} n_{2}$. A dimensão do radical nilpotente é 2. A dimensão da órbita é 14. A dimensão do centro associador é 4 . A dimensão de $\mathfrak{J}^{2}$ é 4 . A dimensão de $\mathfrak{J}^{3}$ é 4 . A dimensão de $\mathfrak{J}^{4}$ é 4 . A dimensão do grupo de 2-cociclos é 16. A dimensão do grupo de 2-cobordos é 14. A dimensão do segundo grupo de Cohomologia é 2. A dimensão da subálgebra associativa maximal é 4. A dimensão da subálgebra nilpotente maximal é 2 . A dimensão da subálgebra nula maximal é 2 .

ÁLGEBRA $\partial_{23}$

O produto na álgebra é:

\begin{tabular}{c|cccc}
$\partial_{23}$ & $e_{1}$ & $e_{2}$ & $e_{3}$ & $e_{4}$ \\
\hline$e_{1}$ & $e_{1}$ & 0 & 0 & $\frac{e_{4}}{2}$ \\
$e_{2}$ & 0 & $e_{2}$ & 0 & 0 \\
$e_{3}$ & 0 & 0 & 0 & 0 \\
$e_{4}$ & $\frac{e_{4}}{2}$ & 0 & 0 & $e_{3}$
\end{tabular}

É uma álgebra não associativa, não possui unidade. A dimensão do grupo de automorfismos é 2.

O grupo de automorfismos é:

$$
\left\{\left(\begin{array}{cccc}
1 & 0 & a_{14}^{2} & a_{14} \\
0 & 1 & 0 & 0 \\
0 & 0 & a_{44}^{2} & 0 \\
0 & 0 & 2 a_{14} a_{44} & a_{44}
\end{array}\right),\left(\begin{array}{cccc}
1 & 0 & 0 & 0 \\
0 & 1 & 0 & 0 \\
0 & 0 & a_{44}^{2} & 0 \\
0 & 0 & 0 & a_{44}
\end{array}\right)\right\}
$$

A dimensão do aniquilador da álgebra é 1. A álgebra se decompõe como: $\mathcal{T}_{8} \oplus \mathbf{k} \boldsymbol{e}_{2}$. Subálgebras de dimensão 2 e 3: $\mathbf{k} e_{1} \oplus \mathbf{k} e_{2} \oplus \mathbf{k} n_{1}, \mathcal{B}_{3} \oplus \mathbf{k} e_{1}, \mathcal{T}_{8}, \mathbf{k} e_{1} \oplus \mathbf{k} n_{1}, \mathbf{k} e_{1} \oplus \mathbf{k} e_{2}$ e $\mathcal{B}_{3}$. A dimensão do radical nilpotente é 2. A dimensão da órbita é 14. A dimensão do centro associador é 2. A dimensão de $\partial^{2}$ é 4. A dimensão de $\mathfrak{g}^{3}$ é 4 . A dimensão de $J^{4}$ é 4. A dimensão do grupo de 2-cociclos é 15. A dimensão do grupo de 2cobordos é 14. A dimensão do segundo grupo de Cohomologia é 1. A dimensão da subálgebra associativa maximal é 3. A dimensão da subálgebra nilpotente maximal é 2 . A dimensão da subálgebra nula maximal é 1. 
O produto na álgebra é:

\begin{tabular}{c|cccc}
$J_{24}$ & $e_{1}$ & $e_{2}$ & $e_{3}$ & $e_{4}$ \\
\hline$e_{1}$ & $e_{1}$ & 0 & $e_{3}$ & $\frac{e_{4}}{2}$ \\
$e_{2}$ & 0 & $e_{2}$ & 0 & 0 \\
$e_{3}$ & $e_{3}$ & 0 & 0 & 0 \\
$e_{4}$ & $\frac{e_{4}}{2}$ & 0 & 0 & $e_{3}$
\end{tabular}

É uma álgebra não associativa, não possui unidade. A dimensão do grupo de automorfismos é 2 .

O grupo de automorfismos é:

$$
\left\{\left(\begin{array}{cccc}
1 & 0 & -a_{14}^{2} & a_{14} \\
0 & 1 & 0 & 0 \\
0 & 0 & a_{44}^{2} & 0 \\
0 & 0 & -2 a_{14} a_{44} & a_{44}
\end{array}\right),\left(\begin{array}{cccc}
1 & 0 & 0 & 0 \\
0 & 1 & 0 & 0 \\
0 & 0 & a_{44}^{2} & 0 \\
0 & 0 & 0 & a_{44}
\end{array}\right)\right\}
$$

A dimensão do aniquilador da álgebra é 0. É uma álgebra rígida. A álgebra se decompõe como: $\mathcal{T}_{9} \oplus \mathbf{k} e_{2}$. Subálgebras de dimensão 2 e 3: $\mathcal{B}_{1} \oplus \mathbf{k} e_{2}, \mathcal{B}_{3} \oplus \mathbf{k} e_{1}, \mathcal{T}_{9}, \mathbf{k}_{1} \oplus \mathbf{k} n_{1}$, $\mathcal{B}_{1}, \mathbf{k} e_{1} \oplus \mathbf{k} e_{2}$ e $\mathcal{B}_{3}$. A dimensão do radical nilpotente é 2 . A dimensão da órbita é 14. A dimensão do centro associador é 2 . A dimensão de $\mathfrak{f}^{2}$ é 4 . A dimensão de $\mathfrak{J}^{3}$ é 4 . A dimensão de $\mathfrak{J}^{4}$ é 4 . A dimensão do grupo de 2-cociclos é 14. A dimensão do grupo de 2-cobordos é 14. A dimensão do segundo grupo de Cohomologia é 0. A dimensão da subálgebra associativa maximal é 3. A dimensão da subálgebra nilpotente maximal é 2 . A dimensão da subálgebra nula maximal é 1.

ÁLGEBRA $J_{25}$

O produto na álgebra é:

\begin{tabular}{c|cccc}
$\partial_{25}$ & $e_{1}$ & $e_{2}$ & $e_{3}$ & $e_{4}$ \\
\hline$e_{1}$ & $e_{1}$ & 0 & $e_{3}$ & $\frac{e_{4}}{2}$ \\
$e_{2}$ & 0 & $e_{2}$ & 0 & $\frac{e_{4}}{2}$ \\
$e_{3}$ & $e_{3}$ & 0 & 0 & 0 \\
$e_{4}$ & $\frac{e_{4}}{2}$ & $\frac{e_{4}}{2}$ & 0 & $e_{3}$
\end{tabular}

É uma álgebra não associativa, unitária, com unidade: $e_{1}+e_{2}$. A dimensão do grupo de automorfismos é 2 .

O grupo de automorfismos é:

$$
\left\{\left(\begin{array}{cccc}
1 & 0 & -a_{24}^{2} & -a_{24} \\
0 & 1 & a_{24}^{2} & a_{24} \\
0 & 0 & a_{44}^{2} & 0 \\
0 & 0 & 2 a_{24} a_{44} & a_{44}
\end{array}\right),\left(\begin{array}{cccc}
1 & 0 & 0 & 0 \\
0 & 1 & 0 & 0 \\
0 & 0 & a_{44}^{2} & 0 \\
0 & 0 & 0 & a_{44}
\end{array}\right)\right\}
$$


A dimensão do aniquilador da álgebra é 0. A álgebra é indecomponível. Subálgebras de dimensão 2 e 3: $\mathcal{B}_{1} \oplus \mathbf{k} e_{2}, \mathcal{T}_{1}, \mathcal{T}_{8}, \mathcal{T}_{9}, \mathbf{k} e_{1} \oplus \mathbf{k} n_{1}, \mathcal{B}_{1}, \mathbf{k} e_{1} \oplus \mathbf{k} e_{2}$ e $\mathcal{B}_{3}$. A dimensão do radical nilpotente é 2 . A dimensão da órbita é 14. A dimensão do centro associador é 2. A dimensão de $\mathfrak{g}^{2}$ é 4 . A dimensão de $\mathfrak{g}^{3}$ é 4 . A dimensão de $\mathfrak{g}^{4}$ é 4 . A dimensão do grupo de 2-cociclos é 15. A dimensão do grupo de 2-cobordos é 14. A dimensão do segundo grupo de Cohomologia é 1. A dimensão da subálgebra associativa maximal é 3. A dimensão da subálgebra nilpotente maximal é 2. A dimensão da subálgebra nula maximal é 1.

ÁLGEBRA $\jmath_{26}$

\begin{tabular}{cc|cccc} 
& $J_{26}$ & $e_{1}$ & $e_{2}$ & $e_{3}$ & $e_{4}$ \\
\hline \multirow{4}{*}{ O produto na álgebra é: } & $e_{1}$ & $e_{1}$ & 0 & 0 & 0 \\
& $e_{2}$ & 0 & $e_{2}$ & 0 & 0 \\
& $e_{3}$ & 0 & 0 & $e_{4}$ & 0 \\
& $e_{4}$ & 0 & 0 & 0 & 0
\end{tabular}

É uma álgebra associativa, não possui unidade. A dimensão do grupo de automorfismos é 2 .

O grupo de automorfismos é:

$$
\left\{\left(\begin{array}{cccc}
0 & 1 & 0 & 0 \\
1 & 0 & 0 & 0 \\
0 & 0 & a_{33} & a_{34} \\
0 & 0 & 0 & a_{33}^{2}
\end{array}\right),\left(\begin{array}{cccc}
1 & 0 & 0 & 0 \\
0 & 1 & 0 & 0 \\
0 & 0 & a_{33} & a_{34} \\
0 & 0 & 0 & a_{33}^{2}
\end{array}\right)\right\}
$$

A dimensão do aniquilador da álgebra é 1. A álgebra se decompõe como: $\mathcal{B}_{3} \oplus \mathbf{k} e_{1} \oplus$ $\mathbf{k}_{2}$. Subálgebras de dimensão 2 e $3: \mathbf{k} e_{1} \oplus \mathbf{k} e_{2} \oplus \mathbf{k} n_{1}, \mathcal{B}_{3} \oplus \mathbf{k} e_{1}, \mathbf{k} e_{1} \oplus \mathbf{k} n_{1}, \mathbf{k} e_{1} \oplus \mathbf{k} e_{2}$ e $\mathcal{B}_{3}$. A dimensão do radical nilpotente é 2. A dimensão da órbita é 14. A dimensão do centro associador é 4. A dimensão de $\partial^{2}$ é 3. A dimensão de $\mathfrak{g}^{3}$ é 2. A dimensão de $J^{4}$ é 2. A dimensão do grupo de 2-cociclos é 16. A dimensão do grupo de 2cobordos é 14. A dimensão do segundo grupo de Cohomologia é 2. A dimensão da subálgebra associativa maximal é 4. A dimensão da subálgebra nilpotente maximal é 2 . A dimensão da subálgebra nula maximal é 1. 
O produto na álgebra é:

\begin{tabular}{c|cccc}
$J_{27}$ & $e_{1}$ & $e_{2}$ & $e_{3}$ & $e_{4}$ \\
\hline$e_{1}$ & $e_{1}$ & 0 & $e_{3}$ & $e_{4}$ \\
$e_{2}$ & 0 & $e_{2}$ & 0 & 0 \\
$e_{3}$ & $e_{3}$ & 0 & $e_{4}$ & 0 \\
$e_{4}$ & $e_{4}$ & 0 & 0 & 0
\end{tabular}

É uma álgebra associativa, unitária, com unidade: $e_{1}+e_{2}$. A dimensão do grupo de automorfismos é 2 .

O grupo de automorfismos é:

$$
\left\{\left(\begin{array}{cccc}
1 & 0 & 0 & 0 \\
0 & 1 & 0 & 0 \\
0 & 0 & a_{33} & a_{34} \\
0 & 0 & 0 & a_{33}^{2}
\end{array}\right)\right\}
$$

A dimensão do aniquilador da álgebra é 0. A álgebra se decompõe como: $\mathcal{T}_{1} \oplus \mathbf{k} \mathbf{e}_{2}$. Subálgebras de dimensão 2 e 3: $\mathcal{B}_{1} \oplus \mathbf{k} e_{2}, \mathcal{T}_{1}, \mathcal{B}_{3} \oplus \mathbf{k} e_{1}, \mathbf{k} e_{1} \oplus \mathbf{k} n_{1}, \mathcal{B}_{1}, \mathbf{k} e_{1} \oplus \mathbf{k} e_{2}$ e $\mathcal{B}_{3}$. A dimensão do radical nilpotente é 2. A dimensão da órbita é 14. A dimensão do centro associador é 4 . A dimensão de $\mathfrak{J}^{2}$ é 4 . A dimensão de $\mathfrak{J}^{3}$ é 4 . A dimensão de $J^{4}$ é 4 . A dimensão do grupo de 2-cociclos é 16. A dimensão do grupo de 2cobordos é 14. A dimensão do segundo grupo de Cohomologia é 2. A dimensão da subálgebra associativa maximal é 4. A dimensão da subálgebra nilpotente maximal é 2 . A dimensão da subálgebra nula maximal é 1.

ÁLGEBRA $\partial_{28}$

O produto na álgebra é:

\begin{tabular}{c|cccc}
$J_{28}$ & $e_{1}$ & $e_{2}$ & $e_{3}$ & $e_{4}$ \\
\hline$e_{1}$ & $e_{1}$ & 0 & 0 & $\frac{e_{4}}{2}$ \\
$e_{2}$ & 0 & 0 & 0 & 0 \\
$e_{3}$ & 0 & 0 & 0 & 0 \\
$e_{4}$ & $\frac{e_{4}}{2}$ & 0 & 0 & 0
\end{tabular}

É uma álgebra não associativa, não possui unidade. A dimensão do grupo de automorfismos é 6 .

O grupo de automorfismos é:

$$
\left\{\left(\begin{array}{cccc}
1 & 0 & 0 & a_{14} \\
0 & a_{22} & a_{23} & 0 \\
0 & a_{32} & a_{33} & 0 \\
0 & 0 & 0 & a_{44}
\end{array}\right)\right\}
$$


A dimensão do aniquilador da álgebra é 2. A álgebra se decompõe como: $\mathcal{B}_{2} \oplus \mathbf{k} n_{2} \oplus$ $\mathbf{k n}_{3}$. Subálgebras de dimensão 2 e 3: $\mathbf{k} e_{1} \oplus \mathbf{k} n_{1} \oplus \mathbf{k} n_{2}, \mathbf{k} n_{1} \oplus \mathbf{k} n_{2} \oplus \mathbf{k} n_{3}, \mathcal{B}_{2} \oplus \mathbf{k} n_{2}$, $\mathbf{k} e_{1} \oplus \mathbf{k} n_{1}, \mathcal{B}_{2}$ e $\mathbf{k} n_{1} \oplus \mathbf{k} n_{2}$. A dimensão do radical nilpotente é 3. A dimensão da órbita é 10. A dimensão do centro associador é 2. A dimensão de $\partial^{2}$ é 2 . A dimensão de $\mathfrak{J}^{3}$ é 2 . A dimensão de $\mathfrak{J}^{4}$ é 2 . A dimensão do grupo de 2-cociclos é 20. A dimensão do grupo de 2-cobordos é 10. A dimensão do segundo grupo de Cohomologia é 10. A dimensão da subálgebra associativa maximal é 3. A dimensão da subálgebra nilpotente maximal é 3. A dimensão da subálgebra nula maximal é 3.

ÁLGEBRA J29

O produto na álgebra é:

\begin{tabular}{c|cccc}
$J_{29}$ & $e_{1}$ & $e_{2}$ & $e_{3}$ & $e_{4}$ \\
\hline$e_{1}$ & $e_{1}$ & $e_{2}$ & 0 & $\frac{e_{4}}{2}$ \\
$e_{2}$ & $e_{2}$ & 0 & 0 & 0 \\
$e_{3}$ & 0 & 0 & 0 & 0 \\
$e_{4}$ & $\frac{e_{4}}{2}$ & 0 & 0 & 0
\end{tabular}

É uma álgebra não associativa, não possui unidade. A dimensão do grupo de automorfismos é 4.

O grupo de automorfismos é:

$$
\left\{\left(\begin{array}{cccc}
1 & 0 & 0 & a_{14} \\
0 & a_{22} & 0 & 0 \\
0 & 0 & a_{33} & 0 \\
0 & 0 & 0 & a_{44}
\end{array}\right)\right\}
$$

A dimensão do aniquilador da álgebra é 1. A álgebra se decompõe como: $\mathcal{T}_{6} \oplus \mathbf{k} n_{3}$. Subálgebras de dimensão 2 e 3: $\mathcal{B}_{1} \oplus \mathbf{k} \mathbf{n}_{2}, \mathbf{k} \mathbf{n}_{1} \oplus \mathbf{k} n_{2} \oplus \mathbf{k} n_{3}, \mathcal{T}_{6}, \mathcal{B}_{2} \oplus \mathbf{k} n_{2}, \mathbf{k} e_{1} \oplus \mathbf{k} n_{1}$, $\mathcal{B}_{1}, \mathcal{B}_{2}$ e $\mathbf{k} n_{1} \oplus \mathbf{k} n_{2}$. A dimensão do radical nilpotente é 3. A dimensão da órbita é 12. A dimensão do centro associador é 2. A dimensão de $\mathfrak{g}^{2}$ é 3. A dimensão de $\mathfrak{d}^{3}$ é 3. A dimensão de $\mathfrak{J}^{4}$ é 3. A dimensão do grupo de 2-cociclos é 17. A dimensão do grupo de 2-cobordos é 12. A dimensão do segundo grupo de Cohomologia é 5. A dimensão da subálgebra associativa maximal é 3. A dimensão da subálgebra nilpotente maximal é 3 . A dimensão da subálgebra nula maximal é 3. 
O produto na álgebra é:

\begin{tabular}{c|cccc}
$\partial_{30}$ & $e_{1}$ & $e_{2}$ & $e_{3}$ & $e_{4}$ \\
\hline$e_{1}$ & $e_{1}$ & $\frac{e_{2}}{2}$ & 0 & $\frac{e_{4}}{2}$ \\
$e_{2}$ & $\frac{e_{2}}{2}$ & 0 & 0 & 0 \\
$e_{3}$ & 0 & 0 & 0 & 0 \\
$e_{4}$ & $\frac{e_{4}}{2}$ & 0 & 0 & 0
\end{tabular}

É uma álgebra não associativa, não possui unidade. A dimensão do grupo de automorfismos é 7.

O grupo de automorfismos é:

$$
\left\{\left(\begin{array}{cccc}
1 & a_{12} & 0 & a_{14} \\
0 & a_{22} & 0 & a_{24} \\
0 & 0 & a_{33} & 0 \\
0 & a_{42} & 0 & a_{44}
\end{array}\right)\right\}
$$

A dimensão do aniquilador da álgebra é 1. A álgebra se decompõe como: $\mathcal{T}_{7} \oplus \mathbf{k} \mathbf{n}_{3}$. Subálgebras de dimensão 2 e 3: $\mathbf{k n}_{1} \oplus \mathbf{k} n_{2} \oplus \mathbf{k} n_{3}, \mathcal{T}_{7}, \mathcal{B}_{2} \oplus \mathbf{k} n_{2}, \mathbf{k} e_{1} \oplus \mathbf{k} n_{1}, \mathcal{B}_{2}$ e $\mathbf{k} n_{1} \oplus \mathbf{k} n_{2}$. A dimensão do radical nilpotente é 3. A dimensão da órbita é 9 . A dimensão do centro associador é 1 . A dimensão de $\mathfrak{J}^{2}$ é 3 . A dimensão de $\mathfrak{J}^{3}$ é 3 . A dimensão de $J^{4}$ é 3. A dimensão do grupo de 2-cociclos é 17. A dimensão do grupo de 2cobordos é 9. A dimensão do segundo grupo de Cohomologia é 8. A dimensão da subálgebra associativa maximal é 3. A dimensão da subálgebra nilpotente maximal é 3 . A dimensão da subálgebra nula maximal é 3.

ÁLGEBRA $\partial_{31}$

O produto na álgebra é:

\begin{tabular}{c|cccc}
$J_{31}$ & $e_{1}$ & $e_{2}$ & $e_{3}$ & $e_{4}$ \\
\hline$e_{1}$ & $e_{1}$ & $e_{2}$ & $e_{3}$ & $\frac{e_{4}}{2}$ \\
$e_{2}$ & $e_{2}$ & 0 & 0 & 0 \\
$e_{3}$ & $e_{3}$ & 0 & 0 & 0 \\
$e_{4}$ & $\frac{e_{4}}{2}$ & 0 & 0 & 0
\end{tabular}

É uma álgebra não associativa, não possui unidade. A dimensão do grupo de automorfismos é 6 .

O grupo de automorfismos é:

$$
\left\{\left(\begin{array}{cccc}
1 & 0 & 0 & a_{14} \\
0 & a_{22} & a_{23} & 0 \\
0 & a_{32} & a_{33} & 0 \\
0 & 0 & 0 & a_{44}
\end{array}\right)\right\}
$$


A dimensão do aniquilador da álgebra é 0. A álgebra é indecomponível. Subálgebras de dimensão 2 e 3: $\mathcal{T}_{2}, \mathbf{k} n_{1} \oplus \mathbf{k} n_{2} \oplus \mathbf{k} n_{3}, \mathcal{T}_{6}, \mathcal{B}_{1}, \mathcal{B}_{2}$ e $\mathbf{k n}_{1} \oplus \mathbf{k} n_{2}$. A dimensão do radical nilpotente é 3. A dimensão da órbita é 10. A dimensão do centro associador é 2. A dimensão de $\mathfrak{J}^{2}$ é 4 . A dimensão de $\mathfrak{J}^{3}$ é 4 . A dimensão de $\mathfrak{J}^{4}$ é 4 . A dimensão do grupo de 2-cociclos é 18. A dimensão do grupo de 2-cobordos é 10. A dimensão do segundo grupo de Cohomologia é 8. A dimensão da subálgebra associativa maximal é 3. A dimensão da subálgebra nilpotente maximal é 3. A dimensão da subálgebra nula maximal é 3.

ÁLGEBRA $J_{32}$

O produto na álgebra é:

\begin{tabular}{c|cccc}
$J_{32}$ & $e_{1}$ & $e_{2}$ & $e_{3}$ & $e_{4}$ \\
\hline$e_{1}$ & $e_{1}$ & $\frac{e_{2}}{2}$ & $e_{3}$ & $\frac{e_{4}}{2}$ \\
$e_{2}$ & $\frac{e_{2}}{2}$ & 0 & 0 & 0 \\
$e_{3}$ & $e_{3}$ & 0 & 0 & 0 \\
$e_{4}$ & $\frac{e_{4}}{2}$ & 0 & 0 & 0
\end{tabular}

É uma álgebra não associativa, não possui unidade. A dimensão do grupo de automorfismos é 7.

O grupo de automorfismos é:

$$
\left\{\left(\begin{array}{cccc}
1 & a_{12} & 0 & a_{14} \\
0 & a_{22} & 0 & a_{24} \\
0 & 0 & a_{33} & 0 \\
0 & a_{42} & 0 & a_{44}
\end{array}\right)\right\}
$$

A dimensão do aniquilador da álgebra é 0. A álgebra é indecomponível. Subálgebras de dimensão 2 e 3: $\mathbf{k n}_{1} \oplus \mathbf{k} n_{2} \oplus \mathbf{k} n_{3}, \mathcal{T}_{6}, \mathcal{T}_{7}, \mathcal{B}_{1}, \mathcal{B}_{2}$ e $\mathbf{k n}_{1} \oplus \mathbf{k} n_{2}$. A dimensão do radical nilpotente é 3. A dimensão da órbita é 9. A dimensão do centro associador é 1. A dimensão de $\mathfrak{g}^{2}$ é 4 . A dimensão de $\mathfrak{g}^{3}$ é 4 . A dimensão de $\mathfrak{g}^{4}$ é 4 . A dimensão do grupo de 2-cociclos é 16. A dimensão do grupo de 2-cobordos é 9. A dimensão do segundo grupo de Cohomologia é 7. A dimensão da subálgebra associativa maximal é 3. A dimensão da subálgebra nilpotente maximal é 3. A dimensão da subálgebra nula maximal é 3. 
O produto na álgebra é:

\begin{tabular}{c|cccc}
$J_{33}$ & $e_{1}$ & $e_{2}$ & $e_{3}$ & $e_{4}$ \\
\hline$e_{1}$ & $e_{1}$ & $\frac{e_{2}}{2}$ & $\frac{e_{3}}{2}$ & $\frac{e_{4}}{2}$ \\
$e_{2}$ & $\frac{e_{2}}{2}$ & 0 & 0 & 0 \\
$e_{3}$ & $\frac{e_{3}}{2}$ & 0 & 0 & 0 \\
$e_{4}$ & $\frac{e_{4}}{2}$ & 0 & 0 & 0
\end{tabular}

É uma álgebra não associativa, não possui unidade. A dimensão do grupo de automorfismos é 12 .

O grupo de automorfismos é:

$$
\left\{\left(\begin{array}{llll}
1 & a_{12} & a_{13} & a_{14} \\
0 & a_{22} & a_{23} & a_{24} \\
0 & a_{32} & a_{33} & a_{34} \\
0 & a_{42} & a_{43} & a_{44}
\end{array}\right)\right\}
$$

A dimensão do aniquilador da álgebra é 0. É uma álgebra rígida. A álgebra é indecomponível. Subálgebras de dimensão 2 e 3: $\mathbf{k} n_{1} \oplus \mathbf{k} n_{2} \oplus \mathbf{k} n_{3}, \mathcal{T}_{7}, \mathcal{B}_{2}$ e $\mathbf{k} n_{1} \oplus \mathbf{k} n_{2}$. A dimensão do radical nilpotente é 3. A dimensão da órbita é 4. A dimensão do centro associador é 0 . A dimensão de $\mathfrak{f}^{2}$ é 4 . A dimensão de $\mathfrak{J}^{3}$ é 4 . A dimensão de $\mathfrak{J}^{4}$ é 4. A dimensão do grupo de 2-cociclos é 4. A dimensão do grupo de 2-cobordos é 4. A dimensão do segundo grupo de Cohomologia é 0. A dimensão da subálgebra associativa maximal é 3. A dimensão da subálgebra nilpotente maximal é 3. A dimensão da subálgebra nula maximal é 3 .

ÁLGEBRA $J_{34}$

O produto na álgebra é:

\begin{tabular}{c|cccc}
$J_{34}$ & $e_{1}$ & $e_{2}$ & $e_{3}$ & $e_{4}$ \\
\hline$e_{1}$ & $e_{1}$ & 0 & 0 & 0 \\
$e_{2}$ & 0 & 0 & 0 & 0 \\
$e_{3}$ & 0 & 0 & 0 & 0 \\
$e_{4}$ & 0 & 0 & 0 & 0
\end{tabular}

É uma álgebra associativa, não possui unidade. A dimensão do grupo de automorfismos é 9 .

O grupo de automorfismos é:

$$
\left\{\left(\begin{array}{cccc}
1 & 0 & 0 & 0 \\
0 & a_{22} & a_{23} & a_{24} \\
0 & a_{32} & a_{33} & a_{34} \\
0 & a_{42} & a_{43} & a_{44}
\end{array}\right)\right\}
$$


A dimensão do aniquilador da álgebra é 3. A álgebra se decompõe como: $\mathbf{k e}_{1} \oplus \mathbf{k} \mathbf{n}_{1} \oplus$ $\mathbf{k} n_{2} \oplus \mathbf{k} n_{3}$. Subálgebras de dimensão 2 e 3: $\mathbf{k} e_{1} \oplus \mathbf{k} n_{1} \oplus \mathbf{k} n_{2}, \mathbf{k} n_{1} \oplus \mathbf{k} n_{2} \oplus \mathbf{k} n_{3}, \mathbf{k} e_{1} \oplus$ $\mathbf{k} n_{1}$ e $\mathbf{k} n_{1} \oplus \mathbf{k} n_{2}$. A dimensão do radical nilpotente é 3. A dimensão da órbita é 7. A dimensão do centro associador é 4 . A dimensão de $\mathcal{J}^{2}$ é 1 . A dimensão de $\mathfrak{J}^{3}$ é 1 . A dimensão de $\mathfrak{J}^{4}$ é 1 . A dimensão do grupo de 2-cociclos é 25. A dimensão do grupo de 2-cobordos é 7. A dimensão do segundo grupo de Cohomologia é 18. A dimensão da subálgebra associativa maximal é 4 . A dimensão da subálgebra nilpotente maximal é 3. A dimensão da subálgebra nula maximal é 3.

ÁLGEBRA $\mathcal{J}_{35}$

\begin{tabular}{ll|cccc} 
O produto na álgebra é: & $e_{2}$ & 0 & 0 & 0 & 0 \\
& $e_{3}$ & $e_{3}$ & 0 & 0 & 0 \\
& $e_{4}$ & 0 & 0 & 0 & 0
\end{tabular}

É uma álgebra associativa, não possui unidade. A dimensão do grupo de automorfismos é 5 .

O grupo de automorfismos é:

$$
\left\{\left(\begin{array}{cccc}
1 & 0 & 0 & 0 \\
0 & a_{22} & 0 & a_{24} \\
0 & 0 & a_{33} & 0 \\
0 & a_{42} & 0 & a_{44}
\end{array}\right)\right\}
$$

A dimensão do aniquilador da álgebra é 2. A álgebra se decompõe como: $\mathcal{B}_{1} \oplus \mathbf{k} n_{2} \oplus$ $\mathbf{k n}_{3}$. Subálgebras de dimensão 2 e $3: \mathcal{B}_{1} \oplus \mathbf{k} n_{2}, \mathbf{k} e_{1} \oplus \mathbf{k} n_{1} \oplus \mathbf{k} n_{2}, \mathbf{k} n_{1} \oplus \mathbf{k} n_{2} \oplus \mathbf{k} n_{3}$, $\mathbf{k} e_{1} \oplus \mathbf{k} n_{1}, \mathcal{B}_{1}$ e $\mathbf{k} n_{1} \oplus \mathbf{k} n_{2}$. A dimensão do radical nilpotente é 3. A dimensão da órbita é 11. A dimensão do centro associador é 4. A dimensão de $\partial^{2}$ é 2. A dimensão de $\mathfrak{J}^{3}$ é 2 . A dimensão de $\mathfrak{J}^{4}$ é 2 . A dimensão do grupo de 2-cociclos é 18 . A dimensão do grupo de 2-cobordos é 11. A dimensão do segundo grupo de Cohomologia é 7. A dimensão da subálgebra associativa maximal é 4. A dimensão da subálgebra nilpotente maximal é 3. A dimensão da subálgebra nula maximal é 3. 
O produto na álgebra é:

\begin{tabular}{c|cccc}
$J_{36}$ & $e_{1}$ & $e_{2}$ & $e_{3}$ & $e_{4}$ \\
\hline$e_{1}$ & $e_{1}$ & $e_{2}$ & $e_{3}$ & $e_{4}$ \\
$e_{2}$ & $e_{2}$ & 0 & 0 & 0 \\
$e_{3}$ & $e_{3}$ & 0 & 0 & 0 \\
$e_{4}$ & $e_{4}$ & 0 & 0 & 0
\end{tabular}

É uma álgebra associativa, unitária, com unidade: $e_{1}$. A dimensão do grupo de automorfismos é 9.

O grupo de automorfismos é:

$$
\left\{\left(\begin{array}{cccc}
1 & 0 & 0 & 0 \\
0 & a_{22} & a_{23} & a_{24} \\
0 & a_{32} & a_{33} & a_{34} \\
0 & a_{42} & a_{43} & a_{44}
\end{array}\right)\right\}
$$

A dimensão do aniquilador da álgebra é 0. A álgebra é indecomponível. Subálgebras de dimensão 2 e 3: $\mathcal{T}_{2}, \mathbf{k} n_{1} \oplus \mathbf{k} n_{2} \oplus \mathbf{k} n_{3}, \mathcal{B}_{1}$ e $\mathbf{k} n_{1} \oplus \mathbf{k} n_{2}$. A dimensão do radical nilpotente é 3. A dimensão da órbita é 7. A dimensão do centro associador é 4. A dimensão de $\mathfrak{g}^{2}$ é 4. A dimensão de $\mathfrak{g}^{3}$ é 4. A dimensão de $\mathfrak{g}^{4}$ é 4 . A dimensão do grupo de 2-cociclos é 28. A dimensão do grupo de 2-cobordos é 7. A dimensão do segundo grupo de Cohomologia é 21. A dimensão da subálgebra associativa maximal é 4. A dimensão da subálgebra nilpotente maximal é 3. A dimensão da subálgebra nula maximal é 3 .

ÁLGEBRA $\jmath_{37}$

O produto na álgebra é:

\begin{tabular}{c|cccc}
$\partial_{37}$ & $e_{1}$ & $e_{2}$ & $e_{3}$ & $e_{4}$ \\
\hline$e_{1}$ & $e_{1}$ & $e_{2}$ & $e_{3}$ & 0 \\
$e_{2}$ & $e_{2}$ & 0 & 0 & 0 \\
$e_{3}$ & $e_{3}$ & 0 & 0 & 0 \\
$e_{4}$ & 0 & 0 & 0 & 0
\end{tabular}

É uma álgebra associativa, não possui unidade. A dimensão do grupo de automorfismos é 5 .

O grupo de automorfismos é:

$$
\left\{\left(\begin{array}{cccc}
1 & 0 & 0 & 0 \\
0 & a_{22} & a_{23} & 0 \\
0 & a_{32} & a_{33} & 0 \\
0 & 0 & 0 & a_{44}
\end{array}\right)\right\}
$$


A dimensão do aniquilador da álgebra é 1. A álgebra se decompõe como: $\mathcal{T}_{2} \oplus \mathbf{k} n_{3}$. Subálgebras de dimensão 2 e 3: $\mathcal{T}_{2}, \mathcal{B}_{1} \oplus \mathbf{k} \mathbf{n}_{2}, \mathbf{k} n_{1} \oplus \mathbf{k} n_{2} \oplus \mathbf{k} n_{3}, \mathbf{k} e_{1} \oplus \mathbf{k} n_{1}, \mathcal{B}_{1}$ e $\mathbf{k} n_{1} \oplus \mathbf{k} n_{2}$. A dimensão do radical nilpotente é 3. A dimensão da órbita é 11. A dimensão do centro associador é 4. A dimensão de $\partial^{2}$ é 3. A dimensão de $\mathfrak{J}^{3}$ é 3 . A dimensão de $\mathfrak{J}^{4}$ é 3. A dimensão do grupo de 2-cociclos é 19. A dimensão do grupo de 2-cobordos é 11. A dimensão do segundo grupo de Cohomologia é 8. A dimensão da subálgebra associativa maximal é 4 . A dimensão da subálgebra nilpotente maximal é 3. A dimensão da subálgebra nula maximal é 3.

ÁLGEBRA $\mathcal{J}_{38}$

\begin{tabular}{ll|cccc} 
O produto na álgebra é: & $e_{2}$ & 0 & 0 & 0 & $e_{3}$ \\
& $e_{3}$ & 0 & 0 & 0 & 0 \\
& $e_{4}$ & 0 & $e_{3}$ & 0 & $e_{2}$
\end{tabular}

É uma álgebra associativa, não possui unidade. A dimensão do grupo de automorfismos é 3 .

O grupo de automorfismos é:

$$
\left\{\left(\begin{array}{cccc}
1 & 0 & 0 & 0 \\
0 & a_{44}^{2} & 2 a_{42} a_{44} & 0 \\
0 & 0 & a_{44}^{3} & 0 \\
0 & a_{42} & a_{43} & a_{44}
\end{array}\right)\right\}
$$

A dimensão do aniquilador da álgebra é 1. A álgebra se decompõe como: $\mathcal{T}_{3} \oplus \mathbf{k} e_{1}$. Subálgebras de dimensão 2 e 3: $\mathcal{T}_{3}, \mathbf{k} e_{1} \oplus \mathbf{k} n_{1} \oplus \mathbf{k} n_{2}, \mathbf{k} e_{1} \oplus \mathbf{k} n_{1}$ e $\mathbf{k} n_{1} \oplus \mathbf{k} n_{2}$. A dimensão do radical nilpotente é 3. A dimensão da órbita é 13. A dimensão do centro associador é 4. A dimensão de $\mathfrak{\partial}^{2}$ é 3. A dimensão de $\mathfrak{g}^{3}$ é 2. A dimensão de $\mathfrak{d}^{4}$ é 1 . A dimensão do grupo de 2-cociclos é 16. A dimensão do grupo de 2-cobordos é 13. A dimensão do segundo grupo de Cohomologia é 3. A dimensão da subálgebra associativa maximal é 4. A dimensão da subálgebra nilpotente maximal é 3. A dimensão da subálgebra nula maximal é 2 . 
O produto na álgebra é:

\begin{tabular}{c|cccc}
$J_{39}$ & $e_{1}$ & $e_{2}$ & $e_{3}$ & $e_{4}$ \\
\hline$e_{1}$ & $e_{1}$ & $e_{2}$ & $e_{3}$ & $e_{4}$ \\
$e_{2}$ & $e_{2}$ & 0 & 0 & $e_{3}$ \\
$e_{3}$ & $e_{3}$ & 0 & 0 & 0 \\
$e_{4}$ & $e_{4}$ & $e_{3}$ & 0 & $e_{2}$
\end{tabular}

É uma álgebra associativa, unitária, com unidade: $e_{1}$. A dimensão do grupo de automorfismos é 3 .

O grupo de automorfismos é:

$$
\left\{\left(\begin{array}{cccc}
1 & 0 & 0 & 0 \\
0 & a_{44}^{2} & 2 a_{42} a_{44} & 0 \\
0 & 0 & a_{44}^{3} & 0 \\
0 & a_{42} & a_{43} & a_{44}
\end{array}\right)\right\}
$$

A dimensão do aniquilador da álgebra é 0. A álgebra é indecomponível. Subálgebras de dimensão 2 e 3: $\mathcal{T}_{2}, \mathcal{T}_{3}, \mathcal{B}_{1}$ e $\mathbf{k} n_{1} \oplus \mathbf{k} n_{2}$. A dimensão do radical nilpotente é 3. A dimensão da órbita é 13. A dimensão do centro associador é 4. A dimensão de $\mathfrak{J}^{2}$ é 4. A dimensão de $\mathfrak{g}^{3}$ é 4. A dimensão de $\mathfrak{g}^{4}$ é 4 . A dimensão do grupo de 2-cociclos é 16. A dimensão do grupo de 2-cobordos é 13. A dimensão do segundo grupo de Cohomologia é 3. A dimensão da subálgebra associativa maximal é 4. A dimensão da subálgebra nilpotente maximal é 3. A dimensão da subálgebra nula maximal é 3 .

ÁLGEBRA $\partial_{40}$

O produto na álgebra é:

\begin{tabular}{c|cccc}
$J_{40}$ & $e_{1}$ & $e_{2}$ & $e_{3}$ & $e_{4}$ \\
\hline$e_{1}$ & $e_{1}$ & 0 & 0 & 0 \\
$e_{2}$ & 0 & 0 & 0 & 0 \\
$e_{3}$ & 0 & 0 & 0 & 0 \\
$e_{4}$ & 0 & 0 & 0 & $e_{2}$
\end{tabular}

É uma álgebra associativa, não possui unidade. A dimensão do grupo de automorfismos é 5 .

O grupo de automorfismos é:

$$
\left\{\left(\begin{array}{cccc}
1 & 0 & 0 & 0 \\
0 & a_{44}^{2} & 0 & 0 \\
0 & a_{32} & a_{33} & 0 \\
0 & a_{42} & a_{43} & a_{44}
\end{array}\right)\right\}
$$

A dimensão do aniquilador da álgebra é 2. A álgebra se decompõe como: $\mathcal{B}_{3} \oplus \mathbf{k} e_{1} \oplus$ $\mathbf{k n}_{3}$. Subálgebras de dimensão 2 e 3: $\mathcal{B}_{3} \oplus \mathbf{k} e_{1}, \mathbf{k} e_{1} \oplus \mathbf{k} n_{1} \oplus \mathbf{k} n_{2}, \mathcal{B}_{3} \oplus \mathbf{k} n_{3}, \mathbf{k} e_{1} \oplus \mathbf{k} n_{1}$, 
$\mathbf{k} n_{1} \oplus \mathbf{k} n_{2}$ e $\mathcal{B}_{3}$. A dimensão do radical nilpotente é 3. A dimensão da órbita é 11. A dimensão do centro associador é 4 . A dimensão de $\mathcal{J}^{2}$ é 2 . A dimensão de $\mathfrak{J}^{3}$ é 1 . A dimensão de $\mathfrak{g}^{4}$ é 1 . A dimensão do grupo de 2-cociclos é 21. A dimensão do grupo de 2-cobordos é 11. A dimensão do segundo grupo de Cohomologia é 10. A dimensão da subálgebra associativa maximal é 4 . A dimensão da subálgebra nilpotente maximal é 3 . A dimensão da subálgebra nula maximal é 2.

ÁLGEBRA $\partial_{41}$

\begin{tabular}{cc|cccc} 
& & & & \\
O produto na álgebra é: & $e_{1}$ & $e_{1}$ & 0 & 0 & 0 \\
& $e_{2}$ & 0 & 0 & 0 & 0 \\
& $e_{3}$ & 0 & 0 & $e_{2}$ & 0 \\
& $e_{4}$ & 0 & 0 & 0 & $e_{2}$
\end{tabular}

É uma álgebra associativa, não possui unidade. A dimensão do grupo de automorfismos é 4 .

O grupo de automorfismos é:

$$
\begin{aligned}
& \left\{\left(\begin{array}{cccc}
1 & 0 & 0 & 0 \\
0 & a_{43}^{2}+a_{44}^{2} & 0 & 0 \\
0 & a_{32} & -a_{44} & a_{43} \\
0 & a_{42} & a_{43} & a_{44}
\end{array}\right),\left(\begin{array}{ccccc}
1 & 0 & 0 & 0 \\
0 & a_{43}^{2}+a_{44}^{2} & 0 & 0 \\
0 & a_{32} & a_{44} & -a_{43} \\
0 & a_{42} & a_{43} & a_{44}
\end{array}\right),\right. \\
& \left.\left(\begin{array}{cccc}
1 & 0 & 0 & 0 \\
0 & a_{44}^{2} & 0 & 0 \\
0 & a_{32} & -a_{44} & 0 \\
0 & a_{42} & 0 & a_{44}
\end{array}\right),\left(\begin{array}{cccc}
1 & 0 & 0 & 0 \\
0 & a_{44}^{2} & 0 & 0 \\
0 & a_{32} & a_{44} & 0 \\
0 & a_{42} & 0 & a_{44}
\end{array}\right)\right\}
\end{aligned}
$$

A dimensão do aniquilador da álgebra é 2. A álgebra se decompõe como: $\mathcal{T}_{4} \oplus \mathbf{k} e_{1}$. Subálgebras de dimensão 2 e 3: $\mathcal{B}_{3} \oplus \mathbf{k} e_{1}, \mathbf{k} e_{1} \oplus \mathbf{k} n_{1} \oplus \mathbf{k} n_{2}, \mathcal{T}_{4}, \mathbf{k} e_{1} \oplus \mathbf{k} n_{1}, \mathbf{k} n_{1} \oplus \mathbf{k} n_{2}$ e $\mathcal{B}_{3}$. A dimensão do radical nilpotente é 3. A dimensão da órbita é 12. A dimensão do centro associador é 4. A dimensão de $\mathcal{J}^{2}$ é 2. A dimensão de $\mathfrak{J}^{3}$ é 1 . A dimensão de $J^{4}$ é 1 . A dimensão do grupo de 2-cociclos é 19. A dimensão do grupo de 2cobordos é 12. A dimensão do segundo grupo de Cohomologia é 7. A dimensão da subálgebra associativa maximal é 4 . A dimensão da subálgebra nilpotente maximal é 3. A dimensão da subálgebra nula maximal é 2 . 
O produto na álgebra é:

\begin{tabular}{c|cccc}
$\partial_{42}$ & $e_{1}$ & $e_{2}$ & $e_{3}$ & $e_{4}$ \\
\hline$e_{1}$ & $e_{1}$ & $e_{2}$ & $e_{3}$ & $e_{4}$ \\
$e_{2}$ & $e_{2}$ & 0 & 0 & 0 \\
$e_{3}$ & $e_{3}$ & 0 & 0 & 0 \\
$e_{4}$ & $e_{4}$ & 0 & 0 & $e_{2}$
\end{tabular}

É uma álgebra associativa, unitária, com unidade: $e_{1}$. A dimensão do grupo de automorfismos é 5 .

O grupo de automorfismos é:

$$
\left\{\left(\begin{array}{cccc}
1 & 0 & 0 & 0 \\
0 & a_{44}^{2} & 0 & 0 \\
0 & a_{32} & a_{33} & 0 \\
0 & a_{42} & a_{43} & a_{44}
\end{array}\right)\right\}
$$

A dimensão do aniquilador da álgebra é 0. A álgebra é indecomponível. Subálgebras de dimensão 2 e 3: $\mathcal{T}_{1}, \mathcal{T}_{2}, \mathcal{B}_{3} \oplus \mathbf{k} n_{3}, \mathcal{B}_{1}, \mathbf{k} n_{1} \oplus \mathbf{k} n_{2}$ e $\mathcal{B}_{3}$. A dimensão do radical nilpotente é 3. A dimensão da órbita é 11. A dimensão do centro associador é 4. A dimensão de $\mathfrak{g}^{2}$ é 4. A dimensão de $\mathfrak{g}^{3}$ é 4. A dimensão de $\mathfrak{J}^{4}$ é 4 . A dimensão do grupo de 2-cociclos é 19. A dimensão do grupo de 2-cobordos é 11. A dimensão do segundo grupo de Cohomologia é 8. A dimensão da subálgebra associativa maximal é 4. A dimensão da subálgebra nilpotente maximal é 3. A dimensão da subálgebra nula maximal é 2 .

ÁLGEBRA $\partial_{43}$

O produto na álgebra é:

\begin{tabular}{c|cccc}
$J_{43}$ & $e_{1}$ & $e_{2}$ & $e_{3}$ & $e_{4}$ \\
\hline$e_{1}$ & $e_{1}$ & $e_{2}$ & $e_{3}$ & $e_{4}$ \\
$e_{2}$ & $e_{2}$ & 0 & 0 & 0 \\
$e_{3}$ & $e_{3}$ & 0 & $e_{2}$ & 0 \\
$e_{4}$ & $e_{4}$ & 0 & 0 & $e_{2}$
\end{tabular}

É uma álgebra associativa, unitária, com unidade: $e_{1}$. A dimensão do grupo de automorfismos é 4.

O grupo de automorfismos é:

$$
\left\{\left(\begin{array}{cccc}
1 & 0 & 0 & 0 \\
0 & a_{33}^{2}+a_{34}^{2} & 0 & 0 \\
0 & a_{32} & a_{33} & a_{34} \\
0 & a_{42} & a_{34} & -a_{33}
\end{array}\right),\left(\begin{array}{cccc}
1 & 0 & 0 & 0 \\
0 & a_{33}^{2}+a_{34}^{2} & 0 & 0 \\
0 & a_{32} & a_{33} & a_{34} \\
0 & a_{42} & -a_{34} & a_{33}
\end{array}\right),\right.
$$




$$
\begin{gathered}
\left(\begin{array}{cccc}
1 & 0 & 0 & 0 \\
0 & a_{33}^{2}+a_{43}^{2} & 0 & 0 \\
0 & a_{32} & a_{33} & -a_{43} \\
0 & a_{42} & a_{43} & a_{33}
\end{array}\right),\left(\begin{array}{cccc}
1 & 0 & 0 & 0 \\
0 & a_{33}^{2}+a_{43}^{2} & 0 & 0 \\
0 & a_{32} & a_{33} & a_{43} \\
0 & a_{42} & a_{43} & -a_{33}
\end{array}\right) \\
\left(\begin{array}{ccccc}
1 & 0 & 0 & 0 \\
0 & a_{33}^{2} & 0 & 0 \\
0 & a_{32} & a_{33} & 0 \\
0 & a_{42} & 0 & -a_{33}
\end{array}\right),\left(\begin{array}{cccc}
1 & 0 & 0 & 0 \\
0 & a_{33}^{2} & 0 & 0 \\
0 & a_{32} & a_{33} & 0 \\
0 & a_{42} & 0 & a_{33}
\end{array}\right), \\
\left.\left(\begin{array}{cccc}
1 & 0 & 0 & 0 \\
0 & a_{43}^{2} & 0 & 0 \\
0 & a_{32} & 0 & -a_{43} \\
0 & a_{42} & a_{43} & 0
\end{array}\right),\left(\begin{array}{cccc}
1 & 0 & 0 & 0 \\
0 & a_{43}^{2} & 0 & 0 \\
0 & a_{32} & 0 & a_{43} \\
0 & a_{42} & a_{43} & 0
\end{array}\right)\right\}
\end{gathered}
$$

A dimensão do aniquilador da álgebra é 0 . A álgebra é indecomponível. Subálgebras de dimensão 2 e 3: $\mathcal{T}_{1}, \mathcal{T}_{2}, \mathcal{T}_{4}, \mathcal{B}_{1}, \mathbf{k n}_{1} \oplus \mathbf{k n}_{2}$ e $\mathcal{B}_{3}$. A dimensão do radical nilpotente é 3. A dimensão da órbita é 12. A dimensão do centro associador é 4 . A dimensão de $\mathfrak{f}^{2}$ é 4. A dimensão de $\mathfrak{J}^{3}$ é 4 . A dimensão de $\mathfrak{J}^{4}$ é 4 . A dimensão do grupo de 2-cociclos é 19. A dimensão do grupo de 2-cobordos é 12. A dimensão do segundo grupo de Cohomologia é 7. A dimensão da subálgebra associativa maximal é 4. A dimensão da subálgebra nilpotente maximal é 3. A dimensão da subálgebra nula maximal é 2 .

\section{ÁLGEBRA $J_{44}$}

\section{O produto na álgebra é:}

\begin{tabular}{c|cccc}
$\partial_{44}$ & $e_{1}$ & $e_{2}$ & $e_{3}$ & $e_{4}$ \\
\hline$e_{1}$ & $e_{1}$ & 0 & 0 & $\frac{e_{4}}{2}$ \\
$e_{2}$ & 0 & 0 & 0 & 0 \\
$e_{3}$ & 0 & 0 & 0 & 0 \\
$e_{4}$ & $\frac{e_{4}}{2}$ & 0 & 0 & $e_{3}$
\end{tabular}

É uma álgebra não associativa, não possui unidade. A dimensão do grupo de automorfismos é 4.

O grupo de automorfismos é:

$$
\left\{\left(\begin{array}{cccc}
1 & 0 & a_{14}^{2} & a_{14} \\
0 & a_{22} & a_{23} & 0 \\
0 & 0 & a_{44}^{2} & 0 \\
0 & 0 & 2 a_{14} a_{44} & a_{44}
\end{array}\right),\left(\begin{array}{cccc}
1 & 0 & 0 & 0 \\
0 & a_{22} & a_{23} & 0 \\
0 & 0 & a_{44}^{2} & 0 \\
0 & 0 & 0 & a_{44}
\end{array}\right)\right\}
$$

A dimensão do aniquilador da álgebra é 2. A álgebra se decompõe como: $\mathcal{T}_{8} \oplus \mathbf{k} \mathbf{n}_{3}$. Subálgebras de dimensão 2 e $3: \mathbf{k} e_{1} \oplus \mathbf{k} n_{1} \oplus \mathbf{k} n_{2}, \mathcal{B}_{3} \oplus \mathbf{k} n_{3}, \mathcal{T}_{8}, \mathbf{k} e_{1} \oplus \mathbf{k} n_{1}, \mathbf{k} n_{1} \oplus \mathbf{k} \mathbf{n}_{2}$ e $\mathcal{B}_{3}$. A dimensão do radical nilpotente é 3. A dimensão da órbita é 12. A dimensão do centro associador é 2. A dimensão de $\mathfrak{f}^{2}$ é 3 . A dimensão de $\mathfrak{J}^{3}$ é 3 . A dimensão de $J^{4}$ é 3. A dimensão do grupo de 2-cociclos é 16. A dimensão do grupo de 2- 
cobordos é 12. A dimensão do segundo grupo de Cohomologia é 4. A dimensão da subálgebra associativa maximal é 3. A dimensão da subálgebra nilpotente maximal é 3 . A dimensão da subálgebra nula maximal é 2 .

ÁLGEBRA $\partial_{45}$

O produto na álgebra é:

\begin{tabular}{c|cccc}
$J_{45}$ & $e_{1}$ & $e_{2}$ & $e_{3}$ & $e_{4}$ \\
\hline$e_{1}$ & $e_{1}$ & 0 & 0 & $\frac{e_{4}}{2}$ \\
$e_{2}$ & 0 & $e_{3}$ & 0 & 0 \\
$e_{3}$ & 0 & 0 & 0 & 0 \\
$e_{4}$ & $\frac{e_{4}}{2}$ & 0 & 0 & $e_{3}$
\end{tabular}

É uma álgebra não associativa, não possui unidade. A dimensão do grupo de automorfismos é 3.

O grupo de automorfismos é:

$$
\begin{aligned}
& \left\{\left(\begin{array}{cccc}
1 & 0 & a_{14}^{2} & a_{14} \\
0 & -a_{44} & a_{23} & 0 \\
0 & 0 & a_{44}^{2} & 0 \\
0 & 0 & 2 a_{14} a_{44} & a_{44}
\end{array}\right),\left(\begin{array}{cccc}
1 & 0 & a_{14}^{2} & a_{14} \\
0 & a_{44} & a_{23} & 0 \\
0 & 0 & a_{44}^{2} & 0 \\
0 & 0 & 2 a_{14} a_{44} & a_{44}
\end{array}\right),\right. \\
& \left.\left(\begin{array}{cccc}
1 & 0 & 0 & 0 \\
0 & -a_{44} & a_{23} & 0 \\
0 & 0 & a_{44}^{2} & 0 \\
0 & 0 & 0 & a_{44}
\end{array}\right),\left(\begin{array}{cccc}
1 & 0 & 0 & 0 \\
0 & a_{44} & a_{23} & 0 \\
0 & 0 & a_{44}^{2} & 0 \\
0 & 0 & 0 & a_{44}
\end{array}\right)\right\}
\end{aligned}
$$

A dimensão do aniquilador da álgebra é 1. A álgebra é indecomponível. Subálgebras de dimensão 2 e 3: $\mathcal{B}_{3} \oplus \mathbf{k} e_{1}, \mathcal{T}_{4}, \mathcal{T}_{8}, \mathbf{k} e_{1} \oplus \mathbf{k} n_{1}, \mathbf{k} n_{1} \oplus \mathbf{k} n_{2}$ e $\mathcal{B}_{3}$. A dimensão do radical nilpotente é 3. A dimensão da órbita é 13. A dimensão do centro associador é 2. A dimensão de $\mathfrak{J}^{2}$ é 3. A dimensão de $\mathfrak{J}^{3}$ é 3. A dimensão de $\mathfrak{J}^{4}$ é 3 . A dimensão do grupo de 2-cociclos é 15. A dimensão do grupo de 2-cobordos é 13. A dimensão do segundo grupo de Cohomologia é 2. A dimensão da subálgebra associativa maximal é 3. A dimensão da subálgebra nilpotente maximal é 3. A dimensão da subálgebra nula maximal é 3.

ÁLGEBRA $J_{46}$

O produto na álgebra é:

\begin{tabular}{c|cccc}
$J_{46}$ & $e_{1}$ & $e_{2}$ & $e_{3}$ & $e_{4}$ \\
\hline$e_{1}$ & $e_{1}$ & 0 & 0 & $\frac{e_{4}}{2}$ \\
$e_{2}$ & 0 & $e_{3}$ & 0 & 0 \\
$e_{3}$ & 0 & 0 & 0 & 0 \\
$e_{4}$ & $\frac{e_{4}}{2}$ & 0 & 0 & 0
\end{tabular}


É uma álgebra não associativa, não possui unidade. A dimensão do grupo de automorfismos é 4.

O grupo de automorfismos é:

$$
\left\{\left(\begin{array}{cccc}
1 & 0 & 0 & a_{14} \\
0 & a_{22} & a_{23} & 0 \\
0 & 0 & a_{22}^{2} & 0 \\
0 & 0 & 0 & a_{44}
\end{array}\right)\right\}
$$

A dimensão do aniquilador da álgebra é 1. A álgebra se decompõe como: $\mathcal{B}_{2} \oplus \mathcal{B}_{3}$. Subálgebras de dimensão 2 e 3: $\mathcal{B}_{3} \oplus \mathbf{k} e_{1}, \mathcal{B}_{3} \oplus \mathbf{k} n_{3}, \mathcal{B}_{2} \oplus \mathbf{k} n_{2}, \mathbf{k} e_{1} \oplus \mathbf{k} n_{1}, \mathcal{B}_{2}, \mathbf{k} n_{1} \oplus$ $\mathbf{k n}_{2}$ e $\mathcal{B}_{3}$. A dimensão do radical nilpotente é 3. A dimensão da órbita é 12. A dimensão do centro associador é 2 . A dimensão de $\mathfrak{J}^{2}$ é 3 . A dimensão de $\mathfrak{J}^{3}$ é 2 . A dimensão de $\mathfrak{J}^{4}$ é 2. A dimensão do grupo de 2-cociclos é 16. A dimensão do grupo de 2-cobordos é 12. A dimensão do segundo grupo de Cohomologia é 4. A dimensão da subálgebra associativa maximal é 3. A dimensão da subálgebra nilpotente maximal é 3 . A dimensão da subálgebra nula maximal é 2 .

ÁLGEBRA J47

O produto na álgebra é:

\begin{tabular}{c|cccc}
$J_{47}$ & $e_{1}$ & $e_{2}$ & $e_{3}$ & $e_{4}$ \\
\hline$e_{1}$ & $e_{1}$ & 0 & $e_{3}$ & 0 \\
$e_{2}$ & 0 & $e_{4}$ & 0 & 0 \\
$e_{3}$ & $e_{3}$ & 0 & 0 & 0 \\
$e_{4}$ & 0 & 0 & 0 & 0
\end{tabular}

É uma álgebra associativa, não possui unidade. A dimensão do grupo de automorfismos é 3 .

O grupo de automorfismos é:

$$
\left\{\left(\begin{array}{cccc}
1 & 0 & 0 & 0 \\
0 & a_{22} & 0 & a_{24} \\
0 & 0 & a_{33} & 0 \\
0 & 0 & 0 & a_{22}^{2}
\end{array}\right)\right\}
$$

A dimensão do aniquilador da álgebra é 1. A álgebra se decompõe como: $\mathcal{B}_{1} \oplus \mathcal{B}_{3}$. Subálgebras de dimensão 2 e 3: $\mathcal{B}_{1} \oplus \mathbf{k} \mathbf{n}_{2}, \mathcal{B}_{3} \oplus \mathbf{k} e_{1}, \mathcal{B}_{3} \oplus \mathbf{k} n_{3}, \mathbf{k} e_{1} \oplus \mathbf{k} n_{1}, \mathcal{B}_{1}, \mathbf{k} n_{1} \oplus$ $\mathbf{k n}_{2}$ e $\mathcal{B}_{3}$. A dimensão do radical nilpotente é 3. A dimensão da órbita é 13. A dimensão do centro associador é 4. A dimensão de $\mathfrak{g}^{2}$ é 3 . A dimensão de $\mathfrak{J}^{3}$ é 2 . A dimensão de $\mathfrak{J}^{4}$ é 2. A dimensão do grupo de 2-cociclos é 16. A dimensão do grupo de 2-cobordos é 13. A dimensão do segundo grupo de Cohomologia é 3. A dimensão da 
subálgebra associativa maximal é 4. A dimensão da subálgebra nilpotente maximal é 3. A dimensão da subálgebra nula maximal é 2.

ÁLGEBRA $\partial_{48}$

O produto na álgebra é:

\begin{tabular}{c|cccc}
$\mathcal{J}_{48}$ & $e_{1}$ & $e_{2}$ & $e_{3}$ & $e_{4}$ \\
\hline$e_{1}$ & $e_{1}$ & $\frac{e_{2}}{2}$ & 0 & $\frac{e_{4}}{2}$ \\
$e_{2}$ & $\frac{e_{2}}{2}$ & 0 & 0 & 0 \\
$e_{3}$ & 0 & 0 & 0 & 0 \\
$e_{4}$ & $\frac{e_{4}}{2}$ & 0 & 0 & $e_{3}$
\end{tabular}

É uma álgebra não associativa, não possui unidade. A dimensão do grupo de automorfismos é 5 .

O grupo de automorfismos é:

$$
\left\{\left(\begin{array}{cccc}
1 & a_{12} & a_{14}^{2} & a_{14} \\
0 & a_{22} & 0 & 0 \\
0 & 0 & a_{44}^{2} & 0 \\
0 & a_{42} & 2 a_{14} a_{44} & a_{44}
\end{array}\right),\left(\begin{array}{cccc}
1 & a_{12} & 0 & 0 \\
0 & a_{22} & 0 & 0 \\
0 & 0 & a_{44}^{2} & 0 \\
0 & a_{42} & 0 & a_{44}
\end{array}\right)\right\}
$$

A dimensão do aniquilador da álgebra é 1. A álgebra é indecomponível. Subálgebras de dimensão 2 e 3: $\mathcal{B}_{3} \oplus \mathbf{k} n_{3}, \mathcal{T}_{8}, \mathcal{B}_{2} \oplus \mathbf{k} n_{2}, \mathbf{k} e_{1} \oplus \mathbf{k} n_{1}, \mathcal{B}_{2}, \mathbf{k} n_{1} \oplus \mathbf{k} n_{2}$ e $\mathcal{B}_{3}$. A dimensão do radical nilpotente é 3. A dimensão da órbita é 11. A dimensão do centro associador é 1. A dimensão de $\mathfrak{J}^{2}$ é 4 . A dimensão de $\mathfrak{J}^{3}$ é 4 . A dimensão de $\mathfrak{J}^{4}$ é 4 . A dimensão do grupo de 2-cociclos é 13. A dimensão do grupo de 2-cobordos é 11. A dimensão do segundo grupo de Cohomologia é 2. A dimensão da subálgebra associativa maximal é 3. A dimensão da subálgebra nilpotente maximal é 3. A dimensão da subálgebra nula maximal é 2 .

ÁLGEBRA $\partial_{49}$

O produto na álgebra é:

\begin{tabular}{c|cccc}
$\partial_{49}$ & $e_{1}$ & $e_{2}$ & $e_{3}$ & $e_{4}$ \\
\hline$e_{1}$ & $e_{1}$ & $\frac{e_{2}}{2}$ & 0 & $\frac{e_{4}}{2}$ \\
$e_{2}$ & $\frac{e_{2}}{2}$ & 0 & 0 & $e_{3}$ \\
$e_{3}$ & 0 & 0 & 0 & 0 \\
$e_{4}$ & $\frac{e_{4}}{2}$ & $e_{3}$ & 0 & $e_{3}$
\end{tabular}

É uma álgebra não associativa, não possui unidade. A dimensão do grupo de automorfismos é 4. 
O grupo de automorfismos é:

$$
\begin{gathered}
\left\{\left(\begin{array}{cccc}
1 & a_{12} & a_{14}\left(2 a_{12}+a_{14}\right) & a_{14} \\
0 & 2 a_{42}+a_{44} & 2 a_{14}\left(2 a_{42}+a_{44}\right) & 0 \\
0 & 0 & a_{44}\left(2 a_{42}+a_{44}\right) & 0 \\
0 & a_{42} & 2\left(a_{12} a_{44}+a_{14}\left(a_{42}+a_{44}\right)\right) & a_{44}
\end{array}\right),\right. \\
\left(\begin{array}{cccc}
1 & a_{12} & a_{14}\left(2 a_{12}+a_{14}\right) & a_{14} \\
0 & -a_{44} & 2\left(2 a_{12}+a_{14}\right) a_{44} & 2 a_{44} \\
0 & 0 & a_{44}\left(2 a_{42}+a_{44}\right) & 0 \\
0 & a_{42} & 2\left(a_{12} a_{44}+a_{14}\left(a_{42}+a_{44}\right)\right) & a_{44}
\end{array}\right),\left(\begin{array}{ccccc}
1 & a_{12} & 0 & 0 \\
0 & 2 a_{42}+a_{44} & 0 & 0 \\
0 & 0 & a_{44}\left(2 a_{42}+a_{44}\right) & 0 \\
0 & a_{42} & 2 a_{12} a_{44} & a_{44}
\end{array}\right), \\
\left(\begin{array}{cccc}
1 & a_{12} & 0 & 0 \\
0 & -a_{44} & 4 a_{12} a_{44} & 2 a_{44} \\
0 & 0 & a_{44}\left(2 a_{42}+a_{44}\right) & 0 \\
0 & a_{42} & 2 a_{12} a_{44} & a_{44}
\end{array}\right),\left(\begin{array}{ccccc}
1 & 0 & a_{14}^{2} & a_{14} \\
0 & -a_{44} & 2 a_{14} a_{44} & 2 a_{44} \\
0 & 0 & a_{44}\left(2 a_{42}+a_{44}\right) & 0 \\
0 & a_{42} & 2 a_{14}\left(a_{42}+a_{44}\right) & a_{44}
\end{array}\right) \\
\left.\begin{array}{ccccccc}
1 & 0 & a_{14}^{2} & a_{14} \\
0 & 2 a_{42}+a_{44} & 2 a_{14}\left(2 a_{42}+a_{44}\right) & 0 \\
0 & 0 & a_{44}\left(2 a_{42}+a_{44}\right) & 0 \\
0 & a_{42} & 2 a_{14}\left(a_{42}+a_{44}\right) & a_{44}
\end{array}\right),\left(\begin{array}{cccc}
1 & 0 & 0 & 0 \\
0 & -a_{44} & 0 & 2 \\
0 & 0 & a_{44}\left(2 a_{42}+a_{44}\right) & 0 \\
0 & a_{42} & 0 & a_{44}
\end{array}\right), \\
0 \\
0
\end{gathered}
$$

A dimensão do aniquilador da álgebra é 1. A álgebra é indecomponível. Subálgebras de dimensão 2 e 3: $\mathcal{T}_{4}, \mathcal{T}_{8}, \mathcal{B}_{2} \oplus \mathbf{k} n_{2}, \mathbf{k} e_{1} \oplus \mathbf{k} n_{1}, \mathcal{B}_{2}, \mathbf{k} n_{1} \oplus \mathbf{k} n_{2}$ e $\mathcal{B}_{3}$. A dimensão do radical nilpotente é 3. A dimensão da órbita é 12. A dimensão do centro associador é 1. A dimensão de $\mathfrak{J}^{2}$ é 4 . A dimensão de $\mathfrak{J}^{3}$ é 4 . A dimensão de $\mathfrak{J}^{4}$ é 4 . A dimensão do grupo de 2-cociclos é 13. A dimensão do grupo de 2-cobordos é 12. A dimensão do segundo grupo de Cohomologia é 1. A dimensão da subálgebra associativa maximal é 3. A dimensão da subálgebra nilpotente maximal é 3. A dimensão da subálgebra nula maximal é 2 .

ÁLGEBRA $J_{50}$

O produto na álgebra é:

\begin{tabular}{c|cccc}
$J_{50}$ & $e_{1}$ & $e_{2}$ & $e_{3}$ & $e_{4}$ \\
\hline$e_{1}$ & $e_{1}$ & $\frac{e_{2}}{2}$ & 0 & $\frac{e_{4}}{2}$ \\
$e_{2}$ & $\frac{e_{2}}{2}$ & 0 & 0 & 0 \\
$e_{3}$ & 0 & 0 & 0 & $e_{2}$ \\
$e_{4}$ & $\frac{e_{4}}{2}$ & 0 & $e_{2}$ & 0
\end{tabular}

É uma álgebra não associativa, não possui unidade. A dimensão do grupo de automorfismos é 5 . 
O grupo de automorfismos é:

$$
\left\{\left(\begin{array}{cccc}
1 & a_{12} & 0 & a_{14} \\
0 & a_{33} a_{44} & 0 & 0 \\
0 & -2 a_{14} a_{33} & a_{33} & 0 \\
0 & a_{42} & 0 & a_{44}
\end{array}\right),\left(\begin{array}{cccc}
1 & a_{12} & 0 & 0 \\
0 & a_{33} a_{44} & 0 & 0 \\
0 & 0 & a_{33} & 0 \\
0 & a_{42} & 0 & a_{44}
\end{array}\right)\right\}
$$

A dimensão do aniquilador da álgebra é 1. A álgebra é indecomponível. Subálgebras de dimensão 2 e 3: $\mathcal{T}_{4}, \mathcal{T}_{7}, \mathcal{B}_{2} \oplus \mathbf{k} n_{2}, \mathbf{k} e_{1} \oplus \mathbf{k} n_{1}, \mathcal{B}_{2}, \mathbf{k} n_{1} \oplus \mathbf{k} n_{2}$ e $\mathcal{B}_{3}$. A dimensão do radical nilpotente é 3. A dimensão da órbita é 11. A dimensão do centro associador é 0. A dimensão de $\mathfrak{J}^{2}$ é 3 . A dimensão de $\mathfrak{J}^{3}$ é 3. A dimensão de $\mathfrak{J}^{4}$ é 3 . A dimensão do grupo de 2-cociclos é 12. A dimensão do grupo de 2-cobordos é 11. A dimensão do segundo grupo de Cohomologia é 1. A dimensão da subálgebra associativa maximal é 3. A dimensão da subálgebra nilpotente maximal é 3. A dimensão da subálgebra nula maximal é 2.

ÁLGEBRA $J_{51}$

O produto na álgebra é:

\begin{tabular}{c|cccc}
$\partial_{51}$ & $e_{1}$ & $e_{2}$ & $e_{3}$ & $e_{4}$ \\
\hline$e_{1}$ & $e_{1}$ & $e_{2}$ & 0 & $\frac{e_{4}}{2}$ \\
$e_{2}$ & $e_{2}$ & 0 & 0 & 0 \\
$e_{3}$ & 0 & 0 & 0 & 0 \\
$e_{4}$ & $\frac{e_{4}}{2}$ & 0 & 0 & $e_{2}$
\end{tabular}

É uma álgebra não associativa, não possui unidade. A dimensão do grupo de automorfismos é 3.

O grupo de automorfismos é:

$$
\left\{\left(\begin{array}{cccc}
1 & -a_{14}^{2} & 0 & a_{14} \\
0 & a_{44}^{2} & 0 & 0 \\
0 & 0 & a_{33} & 0 \\
0 & -2 a_{14} a_{44} & 0 & a_{44}
\end{array}\right),\left(\begin{array}{cccc}
1 & 0 & 0 & 0 \\
0 & a_{44}^{2} & 0 & 0 \\
0 & 0 & a_{33} & 0 \\
0 & 0 & 0 & a_{44}
\end{array}\right)\right\}
$$

A dimensão do aniquilador da álgebra é 1. A álgebra se decompõe como: $\mathcal{T}_{9} \oplus \mathbf{k} \mathbf{n}_{3}$. Subálgebras de dimensão 2 e 3: $\mathcal{B}_{1} \oplus \mathbf{k} n_{2}, \mathcal{B}_{3} \oplus \mathbf{k} n_{3}, \mathcal{T}_{9}, \mathbf{k} e_{1} \oplus \mathbf{k} n_{1}, \mathcal{B}_{1}, \mathbf{k} n_{1} \oplus \mathbf{k} n_{2}$ e $\mathcal{B}_{3}$. A dimensão do radical nilpotente é 3. A dimensão da órbita é 13. A dimensão do centro associador é 2. A dimensão de $\mathfrak{g}^{2}$ é 3. A dimensão de $\mathfrak{g}^{3}$ é 3. A dimensão de $J^{4}$ é 3. A dimensão do grupo de 2-cociclos é 16. A dimensão do grupo de 2cobordos é 13. A dimensão do segundo grupo de Cohomologia é 3. A dimensão da subálgebra associativa maximal é 3. A dimensão da subálgebra nilpotente maximal é 3 . A dimensão da subálgebra nula maximal é 2 . 
O produto na álgebra é:

\begin{tabular}{c|cccc}
$J_{52}$ & $e_{1}$ & $e_{2}$ & $e_{3}$ & $e_{4}$ \\
\hline$e_{1}$ & $e_{1}$ & $e_{2}$ & 0 & $\frac{e_{4}}{2}$ \\
$e_{2}$ & $e_{2}$ & 0 & 0 & 0 \\
$e_{3}$ & 0 & 0 & 0 & 0 \\
$e_{4}$ & $\frac{e_{4}}{2}$ & 0 & 0 & $e_{3}$
\end{tabular}

É uma álgebra não associativa, não possui unidade. A dimensão do grupo de automorfismos é 3 .

O grupo de automorfismos é:

$$
\left\{\left(\begin{array}{cccc}
1 & 0 & a_{14}^{2} & a_{14} \\
0 & a_{22} & 0 & 0 \\
0 & 0 & a_{44}^{2} & 0 \\
0 & 0 & 2 a_{14} a_{44} & a_{44}
\end{array}\right),\left(\begin{array}{cccc}
1 & 0 & 0 & 0 \\
0 & a_{22} & 0 & 0 \\
0 & 0 & a_{44}^{2} & 0 \\
0 & 0 & 0 & a_{44}
\end{array}\right)\right\}
$$

A dimensão do aniquilador da álgebra é 1. A álgebra é indecomponível. Subálgebras de dimensão 2 e 3: $\mathcal{B}_{1} \oplus \mathbf{k} n_{2}, \mathcal{B}_{3} \oplus \mathbf{k} n_{3}, \mathcal{T}_{8}, \mathbf{k} e_{1} \oplus \mathbf{k} n_{1}, \mathcal{B}_{1}, \mathbf{k} n_{1} \oplus \mathbf{k} n_{2}$ e $\mathcal{B}_{3}$. A dimensão do radical nilpotente é 3. A dimensão da órbita é 13. A dimensão do centro associador é 2. A dimensão de $\mathfrak{g}^{2}$ é 4. A dimensão de $\mathfrak{g}^{3}$ é 4. A dimensão de $\mathfrak{g}^{4}$ é 4 . A dimensão do grupo de 2-cociclos é 15. A dimensão do grupo de 2-cobordos é 13. A dimensão do segundo grupo de Cohomologia é 2. A dimensão da subálgebra associativa maximal é 3. A dimensão da subálgebra nilpotente maximal é 3. A dimensão da subálgebra nula maximal é 2 .

ÁLGEBRA $\jmath_{53}$

O produto na álgebra é:

\begin{tabular}{c|cccc}
$\partial_{53}$ & $e_{1}$ & $e_{2}$ & $e_{3}$ & $e_{4}$ \\
\hline$e_{1}$ & $e_{1}$ & $e_{2}$ & 0 & $\frac{e_{4}}{2}$ \\
$e_{2}$ & $e_{2}$ & 0 & 0 & 0 \\
$e_{3}$ & 0 & 0 & 0 & 0 \\
$e_{4}$ & $\frac{e_{4}}{2}$ & 0 & 0 & $e_{2}+e_{3}$
\end{tabular}

É uma álgebra não associativa, não possui unidade. A dimensão do grupo de automorfismos é 2 .

O grupo de automorfismos é:

$$
\left\{\left(\begin{array}{cccc}
1 & -a_{14}^{2} & a_{14}^{2} & a_{14} \\
0 & a_{44}^{2} & 0 & 0 \\
0 & 0 & a_{44}^{2} & 0 \\
0 & -2 a_{14} a_{44} & 2 a_{14} a_{44} & a_{44}
\end{array}\right),\left(\begin{array}{cccc}
1 & 0 & 0 & 0 \\
0 & a_{44}^{2} & 0 & 0 \\
0 & 0 & a_{44}^{2} & 0 \\
0 & 0 & 0 & a_{44}
\end{array}\right)\right\}
$$


A dimensão do aniquilador da álgebra é 1. A álgebra é indecomponível. Subálgebras de dimensão 2 e 3: $\mathcal{B}_{1} \oplus \mathbf{k} n_{2}, \mathcal{B}_{3} \oplus \mathbf{k} n_{3}, \mathbf{k} e_{1} \oplus \mathbf{k} n_{1}, \mathcal{B}_{1}, \mathbf{k} n_{1} \oplus \mathbf{k} n_{2}$ e $\mathcal{B}_{3}$. A dimensão do radical nilpotente é 3. A dimensão da órbita é 14. A dimensão do centro associador é 2. A dimensão de $\mathfrak{g}^{2}$ é 4 . A dimensão de $\mathfrak{g}^{3}$ é 4 . A dimensão de $\mathfrak{J}^{4}$ é 4 . A dimensão do grupo de 2-cociclos é 15. A dimensão do grupo de 2-cobordos é 14. A dimensão do segundo grupo de Cohomologia é 1. A dimensão da subálgebra associativa maximal é 3. A dimensão da subálgebra nilpotente maximal é 3. A dimensão da subálgebra nula maximal é 2.

ÁLGEBRA $\mathcal{J}_{54}$

O produto na álgebra é:

\begin{tabular}{c|cccc}
$J_{54}$ & $e_{1}$ & $e_{2}$ & $e_{3}$ & $e_{4}$ \\
\hline$e_{1}$ & $e_{1}$ & $e_{2}$ & $e_{3}$ & 0 \\
$e_{2}$ & $e_{2}$ & 0 & 0 & 0 \\
$e_{3}$ & $e_{3}$ & 0 & $e_{2}$ & 0 \\
$e_{4}$ & 0 & 0 & 0 & 0
\end{tabular}

É uma álgebra associativa, não possui unidade. A dimensão do grupo de automorfismos é 3 .

O grupo de automorfismos é:

$$
\left\{\left(\begin{array}{cccc}
1 & 0 & 0 & 0 \\
0 & a_{33}^{2} & 0 & 0 \\
0 & a_{32} & a_{33} & 0 \\
0 & 0 & 0 & a_{44}
\end{array}\right)\right\}
$$

A dimensão do aniquilador da álgebra é 1. A álgebra se decompõe como: $\mathcal{T}_{1} \oplus \mathbf{k} n_{3}$. Subálgebras de dimensão 2 e 3: $\mathcal{T}_{1}, \mathcal{B}_{1} \oplus \mathbf{k} \mathbf{n}_{2}, \mathcal{B}_{3} \oplus \mathbf{k} \mathbf{n}_{3}, \mathbf{k} e_{1} \oplus \mathbf{k} n_{1}, \mathcal{B}_{1}, \mathbf{k} n_{1} \oplus \mathbf{k} n_{2}$ e $\mathcal{B}_{3}$. A dimensão do radical nilpotente é 3. A dimensão da órbita é 13. A dimensão do centro associador é 4. A dimensão de $\mathfrak{d}^{2}$ é 3. A dimensão de $\mathfrak{\partial}^{3}$ é 3. A dimensão de $\mathfrak{J}^{4}$ é 3. A dimensão do grupo de 2-cociclos é 16. A dimensão do grupo de 2cobordos é 13. A dimensão do segundo grupo de Cohomologia é 3. A dimensão da subálgebra associativa maximal é 4. A dimensão da subálgebra nilpotente maximal é 3. A dimensão da subálgebra nula maximal é 2 . 
O produto na álgebra é:

\begin{tabular}{c|cccc}
$J_{55}$ & $e_{1}$ & $e_{2}$ & $e_{3}$ & $e_{4}$ \\
\hline$e_{1}$ & $e_{1}$ & $e_{2}$ & $e_{3}$ & $\frac{e_{4}}{2}$ \\
$e_{2}$ & $e_{2}$ & 0 & 0 & 0 \\
$e_{3}$ & $e_{3}$ & 0 & 0 & 0 \\
$e_{4}$ & $\frac{e_{4}}{2}$ & 0 & 0 & $e_{3}$
\end{tabular}

É uma álgebra não associativa, não possui unidade. A dimensão do grupo de automorfismos é 4.

O grupo de automorfismos é:

$$
\left\{\left(\begin{array}{cccc}
1 & 0 & -a_{14}^{2} & a_{14} \\
0 & a_{22} & a_{23} & 0 \\
0 & 0 & a_{44}^{2} & 0 \\
0 & 0 & -2 a_{14} a_{44} & a_{44}
\end{array}\right),\left(\begin{array}{cccc}
1 & 0 & 0 & 0 \\
0 & a_{22} & a_{23} & 0 \\
0 & 0 & a_{44}^{2} & 0 \\
0 & 0 & 0 & a_{44}
\end{array}\right)\right\}
$$

A dimensão do aniquilador da álgebra é 0. A álgebra é indecomponível. Subálgebras de dimensão 2 e 3: $\mathcal{T}_{2}, \mathcal{B}_{3} \oplus \mathbf{k} n_{3}, \mathcal{T}_{9}, \mathcal{B}_{1}, \mathbf{k} n_{1} \oplus \mathbf{k} n_{2}$ e $\mathcal{B}_{3}$. A dimensão do radical nilpotente é 3. A dimensão da órbita é 12. A dimensão do centro associador é 2. A dimensão de $\mathfrak{g}^{2}$ é 4. A dimensão de $\mathfrak{g}^{3}$ é 4. A dimensão de $\mathfrak{g}^{4}$ é 4 . A dimensão do grupo de 2-cociclos é 14. A dimensão do grupo de 2-cobordos é 12. A dimensão do segundo grupo de Cohomologia é 2. A dimensão da subálgebra associativa maximal é 3. A dimensão da subálgebra nilpotente maximal é 3. A dimensão da subálgebra nula maximal é 2 .

ÁLGEBRA J56

O produto na álgebra é:

\begin{tabular}{c|cccc}
$\partial_{56}$ & $e_{1}$ & $e_{2}$ & $e_{3}$ & $e_{4}$ \\
\hline$e_{1}$ & $e_{1}$ & $e_{2}$ & $e_{3}$ & $\frac{e_{4}}{2}$ \\
$e_{2}$ & $e_{2}$ & $e_{3}$ & 0 & 0 \\
$e_{3}$ & $e_{3}$ & 0 & 0 & 0 \\
$e_{4}$ & $\frac{e_{4}}{2}$ & 0 & 0 & 0
\end{tabular}

É uma álgebra não associativa, não possui unidade. A dimensão do grupo de automorfismos é 4.

O grupo de automorfismos é:

$$
\left\{\left(\begin{array}{cccc}
1 & 0 & 0 & a_{14} \\
0 & a_{22} & a_{23} & 0 \\
0 & 0 & a_{22}^{2} & 0 \\
0 & 0 & 0 & a_{44}
\end{array}\right)\right\}
$$


A dimensão do aniquilador da álgebra é 0. A álgebra é indecomponível. Subálgebras de dimensão 2 e 3: $\mathcal{T}_{1}, \mathcal{B}_{3} \oplus \mathbf{k} n_{3}, \mathcal{T}_{6}, \mathcal{B}_{1}, \mathcal{B}_{2}, \mathbf{k} n_{1} \oplus \mathbf{k} n_{2}$ e $\mathcal{B}_{3}$. A dimensão do radical nilpotente é 3. A dimensão da órbita é 12. A dimensão do centro associador é 2. A dimensão de $\mathfrak{J}^{2}$ é 4. A dimensão de $\mathfrak{g}^{3}$ é 4. A dimensão de $\mathfrak{J}^{4}$ é 4 . A dimensão do grupo de 2-cociclos é 15. A dimensão do grupo de 2-cobordos é 12. A dimensão do segundo grupo de Cohomologia é 3. A dimensão da subálgebra associativa maximal é 3. A dimensão da subálgebra nilpotente maximal é 3. A dimensão da subálgebra nula maximal é 2.

ÁLGEBRA $J_{57}$

O produto na álgebra é:

\begin{tabular}{c|cccc}
$\partial_{57}$ & $e_{1}$ & $e_{2}$ & $e_{3}$ & $e_{4}$ \\
\hline$e_{1}$ & $e_{1}$ & $e_{2}$ & $e_{3}$ & $\frac{e_{4}}{2}$ \\
$e_{2}$ & $e_{2}$ & $e_{3}$ & 0 & 0 \\
$e_{3}$ & $e_{3}$ & 0 & 0 & 0 \\
$e_{4}$ & $\frac{e_{4}}{2}$ & 0 & 0 & $e_{3}$
\end{tabular}

É uma álgebra não associativa, não possui unidade. A dimensão do grupo de automorfismos é 3 .

O grupo de automorfismos é:

$$
\begin{aligned}
& \left\{\left(\begin{array}{cccc}
1 & 0 & -a_{14}^{2} & a_{14} \\
0 & a_{22} & a_{23} & 0 \\
0 & 0 & a_{22}^{2} & 0 \\
0 & 0 & -2 a_{14} a_{22} & a_{22}
\end{array}\right),\left(\begin{array}{cccc}
1 & 0 & -a_{14}^{2} & a_{14} \\
0 & a_{22} & a_{23} & 0 \\
0 & 0 & a_{22}^{2} & 0 \\
0 & 0 & 2 a_{14} a_{22} & -a_{22}
\end{array}\right),\right. \\
& \left.\left(\begin{array}{cccc}
1 & 0 & 0 & 0 \\
0 & a_{22} & a_{23} & 0 \\
0 & 0 & a_{22}^{2} & 0 \\
0 & 0 & 0 & -a_{22}
\end{array}\right),\left(\begin{array}{cccc}
1 & 0 & 0 & 0 \\
0 & a_{22} & a_{23} & 0 \\
0 & 0 & a_{22}^{2} & 0 \\
0 & 0 & 0 & a_{22}
\end{array}\right)\right\}
\end{aligned}
$$

A dimensão do aniquilador da álgebra é 0. A álgebra é indecomponível. Subálgebras de dimensão 2 e 3: $\mathcal{T}_{1}, \mathcal{T}_{4}, \mathcal{T}_{9}, \mathcal{B}_{1}, \mathbf{k} n_{1} \oplus \mathbf{k} n_{2}$ e $\mathcal{B}_{3}$. A dimensão do radical nilpotente é 3. A dimensão da órbita é 13. A dimensão do centro associador é 2 . A dimensão de $\mathfrak{J}^{2}$ é 4. A dimensão de $\mathfrak{J}^{3}$ é 4 . A dimensão de $\mathfrak{J}^{4}$ é 4 . A dimensão do grupo de 2-cociclos é 14. A dimensão do grupo de 2-cobordos é 13. A dimensão do segundo grupo de Cohomologia é 1. A dimensão da subálgebra associativa maximal é 3. A dimensão da subálgebra nilpotente maximal é 3. A dimensão da subálgebra nula maximal é 2 . 
O produto na álgebra é:

\begin{tabular}{c|cccc}
$\partial_{58}$ & $e_{1}$ & $e_{2}$ & $e_{3}$ & $e_{4}$ \\
\hline$e_{1}$ & $e_{1}$ & $\frac{e_{2}}{2}$ & $e_{3}$ & $\frac{e_{4}}{2}$ \\
$e_{2}$ & $\frac{e_{2}}{2}$ & 0 & 0 & 0 \\
$e_{3}$ & $e_{3}$ & 0 & 0 & 0 \\
$e_{4}$ & $\frac{e_{4}}{2}$ & 0 & 0 & $e_{3}$
\end{tabular}

É uma álgebra não associativa, não possui unidade. A dimensão do grupo de automorfismos é 5 .

O grupo de automorfismos é:

$$
\left\{\left(\begin{array}{cccc}
1 & a_{12} & -a_{14}^{2} & a_{14} \\
0 & a_{22} & 0 & 0 \\
0 & 0 & a_{44}^{2} & 0 \\
0 & a_{42} & -2 a_{14} a_{44} & a_{44}
\end{array}\right),\left(\begin{array}{cccc}
1 & a_{12} & 0 & 0 \\
0 & a_{22} & 0 & 0 \\
0 & 0 & a_{44}^{2} & 0 \\
0 & a_{42} & 0 & a_{44}
\end{array}\right)\right\}
$$

A dimensão do aniquilador da álgebra é 0. A álgebra é indecomponível. Subálgebras de dimensão 2 e 3: $\mathcal{B}_{3} \oplus \mathbf{k} n_{3}, \mathcal{T}_{6}, \mathcal{T}_{9}, \mathcal{B}_{1}, \mathcal{B}_{2}, \mathbf{k} n_{1} \oplus \mathbf{k} n_{2}$ e $\mathcal{B}_{3}$. A dimensão do radical nilpotente é 3. A dimensão da órbita é 11. A dimensão do centro associador é 1. A dimensão de $\mathfrak{g}^{2}$ é 4. A dimensão de $\mathfrak{g}^{3}$ é 4. A dimensão de $\mathfrak{g}^{4}$ é 4 . A dimensão do grupo de 2-cociclos é 12. A dimensão do grupo de 2-cobordos é 11. A dimensão do segundo grupo de Cohomologia é 1. A dimensão da subálgebra associativa maximal é 3. A dimensão da subálgebra nilpotente maximal é 3. A dimensão da subálgebra nula maximal é 2.

ÁLGEBRA J59

O produto na álgebra é:

\begin{tabular}{c|cccc}
$J_{59}$ & $e_{1}$ & $e_{2}$ & $e_{3}$ & $e_{4}$ \\
\hline$e_{1}$ & $e_{1}$ & $\frac{e_{2}}{2}$ & $e_{3}$ & $\frac{e_{4}}{2}$ \\
$e_{2}$ & $\frac{e_{2}}{2}$ & 0 & 0 & $e_{3}$ \\
$e_{3}$ & $e_{3}$ & 0 & 0 & 0 \\
$e_{4}$ & $\frac{e_{4}}{2}$ & $e_{3}$ & 0 & $e_{3}$
\end{tabular}

É uma álgebra não associativa, não possui unidade. A dimensão do grupo de automorfismos é 4.

O grupo de automorfismos é:

$$
\left\{\left(\begin{array}{cccc}
1 & a_{12} & -a_{14}\left(2 a_{12}+a_{14}\right) & a_{14} \\
0 & 2 a_{42}+a_{44} & -2 a_{14}\left(2 a_{42}+a_{44}\right) & 0 \\
0 & 0 & a_{44}\left(2 a_{42}+a_{44}\right) & 0 \\
0 & a_{42} & -2\left(a_{12} a_{44}+a_{14}\left(a_{42}+a_{44}\right)\right) & a_{44}
\end{array}\right),\right.
$$




$$
\begin{aligned}
& \left(\begin{array}{cccc}
1 & a_{12} & -a_{14}\left(2 a_{12}+a_{14}\right) & a_{14} \\
0 & -a_{44} & -2\left(2 a_{12}+a_{14}\right) a_{44} & 2 a_{44} \\
0 & 0 & a_{44}\left(2 a_{42}+a_{44}\right) & 0 \\
0 & a_{42} & -2\left(a_{12} a_{44}+a_{14}\left(a_{42}+a_{44}\right)\right) & a_{44}
\end{array}\right),\left(\begin{array}{cccc}
1 & a_{12} & 0 & 0 \\
0 & 2 a_{42}+a_{44} & 0 & 0 \\
0 & 0 & a_{44}\left(2 a_{42}+a_{44}\right) & 0 \\
0 & a_{42} & -2 a_{12} a_{44} & a_{44}
\end{array}\right), \\
& \left(\begin{array}{cccc}
1 & a_{12} & 0 & 0 \\
0 & -a_{44} & -4 a_{12} a_{44} & 2 a_{44} \\
0 & 0 & a_{44}\left(2 a_{42}+a_{44}\right) & 0 \\
0 & a_{42} & -2 a_{12} a_{44} & a_{44}
\end{array}\right),\left(\begin{array}{cccc}
1 & 0 & -a_{14}^{2} & a_{14} \\
0 & -a_{44} & -2 a_{14} a_{44} & 2 a_{44} \\
0 & 0 & a_{44}\left(2 a_{42}+a_{44}\right) & 0 \\
0 & a_{42} & -2 a_{14}\left(a_{42}+a_{44}\right) & a_{44}
\end{array}\right), \\
& \left(\begin{array}{cccc}
1 & 0 & -a_{14}^{2} & a_{14} \\
0 & 2 a_{42}+a_{44} & -2 a_{14}\left(2 a_{42}+a_{44}\right) & 0 \\
0 & 0 & a_{44}\left(2 a_{42}+a_{44}\right) & 0 \\
0 & a_{42} & -2 a_{14}\left(a_{42}+a_{44}\right) & a_{44}
\end{array}\right),\left(\begin{array}{cccc}
1 & 0 & 0 & 0 \\
0 & -a_{44} & 0 & 2 a_{44} \\
0 & 0 & a_{44}\left(2 a_{42}+a_{44}\right) & 0 \\
0 & a_{42} & 0 & a_{44}
\end{array}\right), \\
& \left.\left(\begin{array}{cccc}
1 & 0 & 0 & 0 \\
0 & 2 a_{42}+a_{44} & 0 & 0 \\
0 & 0 & a_{44}\left(2 a_{42}+a_{44}\right) & 0 \\
0 & a_{42} & 0 & a_{44}
\end{array}\right)\right\}
\end{aligned}
$$

A dimensão do aniquilador da álgebra é 0. É uma álgebra rígida. A álgebra é indecomponível. Subálgebras de dimensão 2 e 3: $\mathcal{T}_{4}, \mathcal{T}_{6}, \mathcal{T}_{9}, \mathcal{B}_{1}, \mathcal{B}_{2}, \mathbf{k n}_{1} \oplus \mathbf{k} n_{2}$ e $\mathcal{B}_{3}$. A dimensão do radical nilpotente é 3. A dimensão da órbita é 12. A dimensão do centro associador é 1. A dimensão de $\mathfrak{J}^{2}$ é 4 . A dimensão de $\mathfrak{J}^{3}$ é 4 . A dimensão de $\mathfrak{J}^{4}$ é 4 . A dimensão do grupo de 2-cociclos é 12. A dimensão do grupo de 2-cobordos é 12. A dimensão do segundo grupo de Cohomologia é 0. A dimensão da subálgebra associativa maximal é 3. A dimensão da subálgebra nilpotente maximal é 3. A dimensão da subálgebra nula maximal é 2 .

\section{ÁLGEBRA $\partial_{60}$}

O produto na álgebra é:

\begin{tabular}{c|cccc}
$\partial_{60}$ & $e_{1}$ & $e_{2}$ & $e_{3}$ & $e_{4}$ \\
\hline$e_{1}$ & $e_{1}$ & $\frac{e_{2}}{2}$ & $e_{3}$ & $\frac{e_{4}}{2}$ \\
$e_{2}$ & $\frac{e_{2}}{2}$ & 0 & 0 & 0 \\
$e_{3}$ & $e_{3}$ & 0 & 0 & $e_{2}$ \\
$e_{4}$ & $\frac{e_{4}}{2}$ & 0 & $e_{2}$ & 0
\end{tabular}

É uma álgebra não associativa, não possui unidade. A dimensão do grupo de automorfismos é 5 .

O grupo de automorfismos é:

$$
\left\{\left(\begin{array}{cccc}
1 & a_{12} & 0 & a_{14} \\
0 & a_{33} a_{44} & 0 & 0 \\
0 & 2 a_{14} a_{33} & a_{33} & 0 \\
0 & a_{42} & 0 & a_{44}
\end{array}\right),\left(\begin{array}{cccc}
1 & a_{12} & 0 & 0 \\
0 & a_{33} a_{44} & 0 & 0 \\
0 & 0 & a_{33} & 0 \\
0 & a_{42} & 0 & a_{44}
\end{array}\right)\right\}
$$


A dimensão do aniquilador da álgebra é 0 . A álgebra é indecomponível. Subálgebras de dimensão 2 e 3: $\mathcal{T}_{4}, \mathcal{T}_{6}, \mathcal{T}_{7}, \mathcal{B}_{1}, \mathcal{B}_{2}, \mathbf{k} n_{1} \oplus \mathbf{k} n_{2}$ e $\mathcal{B}_{3}$. A dimensão do radical nilpotente é 3. A dimensão da órbita é 11. A dimensão do centro associador é 0 . A dimensão de $\mathfrak{J}^{2}$ é 4. A dimensão de $\mathfrak{g}^{3}$ é 4 . A dimensão de $\mathfrak{J}^{4}$ é 4 . A dimensão do grupo de 2-cociclos é 12. A dimensão do grupo de 2-cobordos é 11. A dimensão do segundo grupo de Cohomologia é 1. A dimensão da subálgebra associativa maximal é 3. A dimensão da subálgebra nilpotente maximal é 3. A dimensão da subálgebra nula maximal é 2 .

ÁLGEBRA $J_{61}$

\begin{tabular}{cc|cccc} 
& $J_{61}$ & $e_{1}$ & $e_{2}$ & $e_{3}$ & $e_{4}$ \\
\cline { 2 - 6 } O produto na álgebra é: & $e_{1}$ & $e_{2}$ & $e_{3}$ & $e_{4}$ & 0 \\
& $e_{2}$ & $e_{3}$ & $e_{4}$ & 0 & 0 \\
& $e_{3}$ & $e_{4}$ & 0 & 0 & 0 \\
& $e_{4}$ & 0 & 0 & 0 & 0
\end{tabular}

É uma álgebra associativa, não possui unidade. A dimensão do grupo de automorfismos é 4.

O grupo de automorfismos é:

$$
\left\{\left(\begin{array}{cccc}
a_{11} & a_{12} & a_{13} & a_{14} \\
0 & a_{11}^{2} & 2 a_{11} a_{12} & a_{12}^{2}+2 a_{11} a_{13} \\
0 & 0 & a_{11}^{3} & 3 a_{11}^{2} a_{12} \\
0 & 0 & 0 & a_{11}^{4}
\end{array}\right)\right\}
$$

A dimensão do aniquilador da álgebra é 1. A álgebra é indecomponível. Subálgebras de dimensão 2 e 3: $\mathcal{B}_{3} \oplus \mathbf{k} n_{3}, \mathbf{k} n_{1} \oplus \mathbf{k} n_{2}$ e $\mathcal{B}_{3}$. A dimensão do radical nilpotente é 4 . A dimensão da órbita é 12. A dimensão do centro associador é 4. A dimensão de $\mathfrak{J}^{2}$ é 3. A dimensão de $\mathfrak{J}^{3}$ é 2 . A dimensão de $\mathcal{J}^{4}$ é 1 . A dimensão do grupo de 2-cociclos é 16. A dimensão do grupo de 2-cobordos é 12. A dimensão do segundo grupo de Cohomologia é 4. A dimensão da subálgebra associativa maximal é 4. A dimensão da subálgebra nilpotente maximal é 4. A dimensão da subálgebra nula maximal é 2 .

ÁLGEBRA $J_{62}$

O produto na álgebra é:

\begin{tabular}{c|cccc}
$J_{62}$ & $e_{1}$ & $e_{2}$ & $e_{3}$ & $e_{4}$ \\
\hline$e_{1}$ & $e_{2}$ & $e_{3}$ & 0 & 0 \\
$e_{2}$ & $e_{3}$ & 0 & 0 & 0 \\
$e_{3}$ & 0 & 0 & 0 & 0 \\
$e_{4}$ & 0 & 0 & 0 & $e_{2}$
\end{tabular}


É uma álgebra não associativa, não possui unidade. A dimensão do grupo de automorfismos é 3.

O grupo de automorfismos é:

$$
\left\{\left(\begin{array}{cccc}
a_{11} & 0 & a_{13} & 0 \\
0 & a_{11}^{2} & 0 & 0 \\
0 & 0 & a_{11}^{3} & 0 \\
0 & 0 & a_{43} & -a_{11}
\end{array}\right),\left(\begin{array}{cccc}
a_{11} & 0 & a_{13} & 0 \\
0 & a_{11}^{2} & 0 & 0 \\
0 & 0 & a_{11}^{3} & 0 \\
0 & 0 & a_{43} & a_{11}
\end{array}\right)\right\}
$$

A dimensão do aniquilador da álgebra é 2. A álgebra é indecomponível. Subálgebras de dimensão 2 e 3: $\mathcal{T}_{3}, \mathcal{T}_{4}, \mathcal{B}_{3} \oplus \mathbf{k} n_{3}, \mathbf{k} n_{1} \oplus \mathbf{k} n_{2}$ e $\mathcal{B}_{3}$. A dimensão do radical nilpotente é 4. A dimensão da órbita é 13. A dimensão do centro associador é 2. A dimensão de $\mathfrak{J}^{2}$ é 2. A dimensão de $\mathfrak{J}^{3}$ é 1 . A dimensão de $\mathfrak{J}^{4}$ é 0 . A dimensão do grupo de 2-cociclos é 16. A dimensão do grupo de 2-cobordos é 13. A dimensão do segundo grupo de Cohomologia é 3. A dimensão da subálgebra associativa maximal é 3. A dimensão da subálgebra nilpotente maximal é 4 . A dimensão da subálgebra nula maximal é 2 .

ÁLGEBRA $J_{63}$

O produto na álgebra é:

\begin{tabular}{c|cccc}
$J_{63}$ & $e_{1}$ & $e_{2}$ & $e_{3}$ & $e_{4}$ \\
\hline$e_{1}$ & $e_{2}$ & $e_{3}$ & 0 & 0 \\
$e_{2}$ & $e_{3}$ & 0 & 0 & $e_{3}$ \\
$e_{3}$ & 0 & 0 & 0 & 0 \\
$e_{4}$ & 0 & $e_{3}$ & 0 & $-e_{2}-e_{3}$
\end{tabular}

É uma álgebra não associativa, não possui unidade. A dimensão do grupo de automorfismos é 4. A dimensão do aniquilador da álgebra é 2. A álgebra é indecomponível. Subálgebras de dimensão 2 e 3: $\mathcal{T}_{3}, \mathcal{T}_{4}, \mathcal{B}_{3} \oplus \mathbf{k} n_{3}, \mathbf{k} n_{1} \oplus \mathbf{k} n_{2}$ e $\mathcal{B}_{3}$. A dimensão do radical nilpotente é 4 . A dimensão da órbita é 12. A dimensão do centro associador é 2. A dimensão de $\mathfrak{J}^{2}$ é 2 . A dimensão de $\mathfrak{J}^{3}$ é 1 . A dimensão de $\mathfrak{J}^{4}$ é 0 . A dimensão do grupo de 2-cociclos é 16. A dimensão do grupo de 2-cobordos é 12. A dimensão do segundo grupo de Cohomologia é 4. A dimensão da subálgebra associativa maximal é 3. A dimensão da subálgebra nilpotente maximal é 4. A dimensão da subálgebra nula maximal é 2 . 
O produto na álgebra é:

\begin{tabular}{c|cccc}
$J_{64}$ & $e_{1}$ & $e_{2}$ & $e_{3}$ & $e_{4}$ \\
\hline$e_{1}$ & $e_{2}$ & $e_{3}$ & 0 & 0 \\
$e_{2}$ & $e_{3}$ & 0 & 0 & $e_{3}$ \\
$e_{3}$ & 0 & 0 & 0 & 0 \\
$e_{4}$ & 0 & $e_{3}$ & 0 & $-e_{2}$
\end{tabular}

É uma álgebra não associativa, não possui unidade. A dimensão do grupo de automorfismos é 5 .

O grupo de automorfismos é:

$$
\begin{aligned}
& \left\{\left(\begin{array}{cccc}
a_{44} & -a_{42} & a_{13} & a_{41} \\
0 & -a_{41}^{2}+a_{44}^{2} & -2 a_{42}\left(a_{41}+a_{44}\right) & 0 \\
0 & 0 & -\left(a_{41}-a_{44}\right)\left(a_{41}+a_{44}\right)^{2} & 0 \\
a_{41} & a_{42} & a_{43}
\end{array}\right),\right. \\
& \left.\left\{\begin{array}{cccc}
a_{44} & -a_{42} & a_{13} & 0 \\
0 & a_{44}^{2} & -2 a_{42} a_{44} & 0 \\
0 & 0 & a_{44}^{3} & 0 \\
0 & a_{42} & a_{43} & a_{44}
\end{array}\right)\right\}
\end{aligned}
$$

A dimensão do aniquilador da álgebra é 2. A álgebra é indecomponível. Subálgebras de dimensão 2 e 3: $\mathcal{T}_{3}, \mathcal{T}_{4}, \mathbf{k} \mathfrak{n}_{1} \oplus \mathbf{k} n_{2} \oplus \mathbf{k} n_{3}, \mathbf{k} n_{1} \oplus \mathbf{k} n_{2}$ e $\mathcal{B}_{3}$. A dimensão do radical nilpotente é 4. A dimensão da órbita é 11. A dimensão do centro associador é 2. A dimensão de $\mathfrak{J}^{2}$ é 2 . A dimensão de $\mathfrak{\partial}^{3}$ é 1 . A dimensão de $\mathfrak{J}^{4}$ é 0 . A dimensão do grupo de 2-cociclos é 17. A dimensão do grupo de 2-cobordos é 11. A dimensão do segundo grupo de Cohomologia é 6 . A dimensão da subálgebra associativa maximal é 3. A dimensão da subálgebra nilpotente maximal é 4. A dimensão da subálgebra nula maximal é 3 .

ÁLGEBRA $J_{65}$

\begin{tabular}{cc|cccc} 
& $J_{65}$ & $e_{1}$ & $e_{2}$ & $e_{3}$ & $e_{4}$ \\
\hline \multirow{4}{*}{ O produto na álgebra é: } & $e_{1}$ & $e_{2}$ & $e_{3}$ & 0 & 0 \\
& $e_{2}$ & $e_{3}$ & 0 & 0 & $e_{3}$ \\
& $e_{3}$ & 0 & 0 & 0 & 0 \\
& $e_{4}$ & 0 & $e_{3}$ & 0 & 0
\end{tabular}

É uma álgebra não associativa, não possui unidade. A dimensão do grupo de automorfismos é 4 . 
O grupo de automorfismos é:

$$
\left\{\left(\begin{array}{cccc}
a_{11} & 0 & a_{13} & a_{14} \\
0 & a_{11}^{2} & 0 & 0 \\
0 & 0 & a_{11}^{2}\left(a_{11}+a_{14}\right) & 0 \\
0 & 0 & a_{43} & a_{11}+a_{14}
\end{array}\right)\right\}
$$

A dimensão do aniquilador da álgebra é 2. A álgebra é indecomponível. Subálgebras de dimensão 2 e 3: $\mathcal{T}_{3}, \mathcal{T}_{4}, \mathcal{B}_{3} \oplus \mathbf{k} n_{3}, \mathbf{k} n_{1} \oplus \mathbf{k} n_{2}$ e $\mathcal{B}_{3}$. A dimensão do radical nilpotente é 4. A dimensão da órbita é 12. A dimensão do centro associador é 2. A dimensão de $\mathfrak{J}^{2}$ é 2. A dimensão de $\mathfrak{J}^{3}$ é 1 . A dimensão de $\mathfrak{f}^{4}$ é 0 . A dimensão do grupo de 2-cociclos é 16. A dimensão do grupo de 2-cobordos é 12. A dimensão do segundo grupo de Cohomologia é 4. A dimensão da subálgebra associativa maximal é 3. A dimensão da subálgebra nilpotente maximal é 4 . A dimensão da subálgebra nula maximal é 2 .

ÁLGEBRA $J_{66}$

\begin{tabular}{cc|cccc} 
& $J_{66}$ & $e_{1}$ & $e_{2}$ & $e_{3}$ & $e_{4}$ \\
\hline \multirow{4}{*}{ O produto na álgebra é: } & $e_{1}$ & $e_{2}$ & $e_{3}$ & 0 & 0 \\
& $e_{2}$ & $e_{3}$ & 0 & 0 & 0 \\
& $e_{3}$ & 0 & 0 & 0 & 0 \\
& $e_{4}$ & 0 & 0 & 0 & $e_{3}$
\end{tabular}

É uma álgebra associativa, não possui unidade. A dimensão do grupo de automorfismos é 5 .

O grupo de automorfismos é:

$$
\begin{aligned}
& \left\{\left(\begin{array}{cccc}
a_{11} & a_{12} & a_{13} & a_{14} \\
0 & a_{11}^{2} & 2 a_{11} a_{12}+a_{14}^{2} & 0 \\
0 & 0 & a_{11}^{3} & 0 \\
0 & -\sqrt{a_{11}} a_{14} & a_{43} & a_{11}^{3 / 2}
\end{array}\right),\right. \\
& \left.\left\{\begin{array}{cccc}
a_{11} & a_{12} & a_{13} & a_{14} \\
0 & a_{11}^{2} & 2 a_{11} a_{12}+a_{14}^{2} & 0 \\
0 & 0 & a_{11}^{3} & 0 \\
0 & \sqrt{a_{11}} a_{14} & a_{43} & -a_{11}^{3 / 2}
\end{array}\right)\right\}
\end{aligned}
$$

A dimensão do aniquilador da álgebra é 1. A álgebra é indecomponível. Subálgebras de dimensão 2 e 3: $\mathcal{T}_{3}, \mathcal{B}_{3} \oplus \mathbf{k} n_{3}, \mathbf{k} n_{1} \oplus \mathbf{k} n_{2}$ e $\mathcal{B}_{3}$. A dimensão do radical nilpotente é 4. A dimensão da órbita é 11. A dimensão do centro associador é 4 . A dimensão de $\mathfrak{J}^{2}$ é 2. A dimensão de $\mathfrak{g}^{3}$ é 1 . A dimensão de $\mathfrak{g}^{4}$ é 0 . A dimensão do grupo de 2 -cociclos é 19. A dimensão do grupo de 2-cobordos é 11. A dimensão do segundo grupo de Cohomologia é 8. A dimensão da subálgebra associativa maximal é 4. A dimensão da subálgebra nilpotente maximal é 4 . A dimensão da subálgebra nula maximal é 2 . 
O produto na álgebra é:

\begin{tabular}{c|cccc}
$J_{67}$ & $e_{1}$ & $e_{2}$ & $e_{3}$ & $e_{4}$ \\
\hline$e_{1}$ & $e_{2}$ & $e_{3}$ & 0 & 0 \\
$e_{2}$ & $e_{3}$ & 0 & 0 & 0 \\
$e_{3}$ & 0 & 0 & 0 & 0 \\
$e_{4}$ & 0 & 0 & 0 & $e_{3}$
\end{tabular}

É uma álgebra associativa, não possui unidade. A dimensão do grupo de automorfismos é 6 .

O grupo de automorfismos é:

$$
\left\{\left(\begin{array}{cccc}
a_{11} & a_{12} & a_{13} & a_{14} \\
0 & a_{11}^{2} & 2 a_{11} a_{12} & 0 \\
0 & 0 & a_{11}^{3} & 0 \\
0 & 0 & a_{43} & a_{44}
\end{array}\right)\right\}
$$

A dimensão do aniquilador da álgebra é 2. A álgebra se decompõe como: $\mathcal{T}_{3} \oplus \mathbf{k} n_{4}$. Subálgebras de dimensão 2 e 3: $\mathcal{T}_{3}, \mathbf{k n}_{1} \oplus \mathbf{k} n_{2} \oplus \mathbf{k} n_{3}$ e $\mathbf{k} n_{1} \oplus \mathbf{k} n_{2}$. A dimensão do radical nilpotente é 4 . A dimensão da órbita é 10. A dimensão do centro associador é 4. A dimensão de $\mathfrak{g}^{2}$ é 2. A dimensão de $\mathfrak{J}^{3}$ é 1 . A dimensão de $\mathcal{J}^{4}$ é 0 . A dimensão do grupo de 2-cociclos é 21. A dimensão do grupo de 2-cobordos é 10. A dimensão do segundo grupo de Cohomologia é 11. A dimensão da subálgebra associativa maximal é 4. A dimensão da subálgebra nilpotente maximal é 4. A dimensão da subálgebra nula maximal é 3.

ÁLGEBRA $J_{68}$

O produto na álgebra é:

\begin{tabular}{c|cccc}
$J_{68}$ & $e_{1}$ & $e_{2}$ & $e_{3}$ & $e_{4}$ \\
\hline$e_{1}$ & $e_{2}$ & $e_{3}$ & 0 & 0 \\
$e_{2}$ & $e_{3}$ & 0 & 0 & 0 \\
$e_{3}$ & 0 & 0 & 0 & 0 \\
$e_{4}$ & 0 & 0 & 0 & $e_{3}$
\end{tabular}

É uma álgebra associativa, não possui unidade. A dimensão do grupo de automorfismos é 6 .

O grupo de automorfismos é:

$$
\left\{\left(\begin{array}{cccc}
0 & a_{12} & a_{13} & a_{14} \\
0 & 0 & 0 & a_{13}^{2} \\
a_{31} & a_{32} & 0 & a_{34} \\
0 & a_{31}^{2} & 0 & 0
\end{array}\right),\left(\begin{array}{cccc}
a_{11} & a_{12} & 0 & a_{14} \\
0 & a_{11}^{2} & 0 & 0 \\
0 & a_{32} & a_{33} & a_{34} \\
0 & 0 & 0 & a_{33}^{2}
\end{array}\right)\right\}
$$


A dimensão do aniquilador da álgebra é 2. A álgebra se decompõe como: $\mathcal{B}_{3} \oplus \mathcal{B}_{3}$. Subálgebras de dimensão 2 e 3: $\mathcal{B}_{3} \oplus \mathbf{k} n_{3}, \mathbf{k} n_{1} \oplus \mathbf{k} n_{2}$ e $\mathcal{B}_{3}$. A dimensão do radical nilpotente é 4. A dimensão da órbita é 10. A dimensão do centro associador é 4. A dimensão de $\mathfrak{\partial}^{2}$ é 2. A dimensão de $\mathfrak{\partial}^{3}$ é 0 . A dimensão de $\partial^{4}$ é 0 . A dimensão do grupo de 2-cociclos é 22. A dimensão do grupo de 2-cobordos é 10. A dimensão do segundo grupo de Cohomologia é 12. A dimensão da subálgebra associativa maximal é 4. A dimensão da subálgebra nilpotente maximal é 4. A dimensão da subálgebra nula maximal é 2 .

ÁLGEBRA $\mathcal{J}_{69}$

O produto na álgebra é:

\begin{tabular}{c|cccc}
$\partial_{69}$ & $e_{1}$ & $e_{2}$ & $e_{3}$ & $e_{4}$ \\
\hline$e_{1}$ & $e_{2}$ & 0 & $e_{4}$ & 0 \\
$e_{2}$ & 0 & 0 & 0 & 0 \\
$e_{3}$ & $e_{4}$ & 0 & 0 & 0 \\
$e_{4}$ & 0 & 0 & 0 & 0
\end{tabular}

É uma álgebra associativa, não possui unidade. A dimensão do grupo de automorfismos é 7 .

O grupo de automorfismos é:

$$
\left\{\left(\begin{array}{cccc}
a_{11} & a_{12} & a_{13} & a_{14} \\
0 & a_{11}^{2} & 0 & 2 a_{11} a_{13} \\
0 & a_{32} & a_{33} & a_{34} \\
0 & 0 & 0 & a_{11} a_{33}
\end{array}\right)\right\}
$$

A dimensão do aniquilador da álgebra é 2. A álgebra é indecomponível. Subálgebras de dimensão 2 e 3: $\mathcal{B}_{3} \oplus \mathbf{k} n_{3}, \mathbf{k} n_{1} \oplus \mathbf{k} n_{2} \oplus \mathbf{k} n_{3}, \mathbf{k} n_{1} \oplus \mathbf{k} n_{2}$ e $\mathcal{B}_{3}$. A dimensão do radical nilpotente é 4. A dimensão da órbita é 9. A dimensão do centro associador é 4. A dimensão de $\partial^{2}$ é 2. A dimensão de $\mathfrak{J}^{3}$ é 0 . A dimensão de $\partial^{4}$ é 0 . A dimensão do grupo de 2-cociclos é 24. A dimensão do grupo de 2-cobordos é 9. A dimensão do segundo grupo de Cohomologia é 15. A dimensão da subálgebra associativa maximal é 4. A dimensão da subálgebra nilpotente maximal é 4. A dimensão da subálgebra nula maximal é 3 . 
O produto na álgebra é:

\begin{tabular}{c|cccc}
$\partial_{70}$ & $e_{1}$ & $e_{2}$ & $e_{3}$ & $e_{4}$ \\
\hline$e_{1}$ & $e_{2}$ & 0 & 0 & 0 \\
$e_{2}$ & 0 & 0 & 0 & 0 \\
$e_{3}$ & 0 & 0 & 0 & $e_{2}$ \\
$e_{4}$ & 0 & 0 & $e_{2}$ & 0
\end{tabular}

É uma álgebra associativa, não possui unidade. A dimensão do grupo de automorfismos é 7. A dimensão do aniquilador da álgebra é 3. A álgebra é indecomponível. Subálgebras de dimensão 2 e 3: $\mathcal{T}_{4}, \mathcal{B}_{3} \oplus \mathbf{k} n_{3}, \mathbf{k} n_{1} \oplus \mathbf{k} n_{2}$ e $\mathcal{B}_{3}$. A dimensão do radical nilpotente é 4. A dimensão da órbita é 9. A dimensão do centro associador é 4. A dimensão de $\mathfrak{J}^{2}$ é 1 . A dimensão de $\mathfrak{J}^{3}$ é 0 . A dimensão de $\mathfrak{J}^{4}$ é 0 . A dimensão do grupo de 2-cociclos é 28. A dimensão do grupo de 2-cobordos é 9. A dimensão do segundo grupo de Cohomologia é 19. A dimensão da subálgebra associativa maximal é 4. A dimensão da subálgebra nilpotente maximal é 4. A dimensão da subálgebra nula maximal é 2.

\section{ÁLGEBRA $J_{71}$}

O produto na álgebra é:

\begin{tabular}{c|cccc}
$\partial_{71}$ & $e_{1}$ & $e_{2}$ & $e_{3}$ & $e_{4}$ \\
\hline$e_{1}$ & $e_{2}$ & 0 & 0 & 0 \\
$e_{2}$ & 0 & 0 & 0 & 0 \\
$e_{3}$ & 0 & 0 & $e_{2}$ & 0 \\
$e_{4}$ & 0 & 0 & 0 & 0
\end{tabular}

É uma álgebra associativa, não possui unidade. A dimensão do grupo de automorfismos é 8 .

O grupo de automorfismos é:

$$
\begin{aligned}
& \left\{\left(\begin{array}{cccc}
-a_{33} & a_{12} & a_{31} & a_{14} \\
0 & a_{31}^{2}+a_{33}^{2} & 0 & 0 \\
a_{31} & a_{32} & a_{33} & a_{34} \\
0 & a_{42} & 0 & a_{44}
\end{array}\right),\left(\begin{array}{cccc}
a_{33} & a_{12} & -a_{31} & a_{14} \\
0 & a_{31}^{2}+a_{33}^{2} & 0 & 0 \\
a_{31} & a_{32} & a_{33} & a_{34} \\
0 & a_{42} & 0 & a_{44}
\end{array}\right),\right. \\
& \left.\left(\begin{array}{cccc}
-a_{33} & a_{12} & 0 & a_{14} \\
0 & a_{33}^{2} & 0 & 0 \\
0 & a_{32} & a_{33} & a_{34} \\
0 & a_{42} & 0 & a_{44}
\end{array}\right),\left(\begin{array}{cccc}
a_{33} & a_{12} & 0 & a_{14} \\
0 & a_{33}^{2} & 0 & 0 \\
0 & a_{32} & a_{33} & a_{34} \\
0 & a_{42} & 0 & a_{44}
\end{array}\right)\right\}
\end{aligned}
$$

A dimensão do aniquilador da álgebra é 3. A álgebra se decompõe como: $\mathcal{T}_{4} \oplus \mathbf{k} \mathfrak{n}_{4}$ . Subálgebras de dimensão 2 e 3: $\mathcal{T}_{4}, \mathcal{B}_{3} \oplus \mathbf{k} n_{3}, \mathbf{k n}_{1} \oplus \mathbf{k} n_{2} \oplus \mathbf{k} n_{3}, \mathbf{k n}_{1} \oplus \mathbf{k} n_{2}$ e $\mathcal{B}_{3}$. A dimensão do radical nilpotente é 4. A dimensão da órbita é 8. A dimensão do centro associador é 4. A dimensão de $\mathfrak{J}^{2}$ é 1 . A dimensão de $\mathfrak{J}^{3}$ é 0 . A dimensão 
de $\mathfrak{J}^{4}$ é 0 . A dimensão do grupo de 2-cociclos é 31. A dimensão do grupo de 2cobordos é 8. A dimensão do segundo grupo de Cohomologia é 23. A dimensão da subálgebra associativa maximal é 4 . A dimensão da subálgebra nilpotente maximal é 4. A dimensão da subálgebra nula maximal é 3.

ÁLGEBRA $\partial_{72}$

O produto na álgebra é:

\begin{tabular}{c|cccc}
$\partial_{72}$ & $e_{1}$ & $e_{2}$ & $e_{3}$ & $e_{4}$ \\
\hline$e_{1}$ & $e_{2}$ & 0 & 0 & 0 \\
$e_{2}$ & 0 & 0 & 0 & 0 \\
$e_{3}$ & 0 & 0 & 0 & 0 \\
$e_{4}$ & 0 & 0 & 0 & 0
\end{tabular}

É uma álgebra associativa, não possui unidade. A dimensão do grupo de automorfismos é 10.

O grupo de automorfismos é:

$$
\left\{\left(\begin{array}{cccc}
a_{11} & a_{12} & a_{13} & a_{14} \\
0 & a_{11}^{2} & 0 & 0 \\
0 & a_{32} & a_{33} & a_{34} \\
0 & a_{42} & a_{43} & a_{44}
\end{array}\right)\right\}
$$

A dimensão do aniquilador da álgebra é 3. A álgebra se decompõe como: $\mathcal{B}_{3} \oplus \mathbf{k} n_{3} \oplus$ $\mathbf{k n}_{4}$. Subálgebras de dimensão 2 e 3: $\mathcal{B}_{3} \oplus \mathbf{k} n_{3}, \mathbf{k n}_{1} \oplus \mathbf{k} n_{2} \oplus \mathbf{k} n_{3}, \mathbf{k} n_{1} \oplus \mathbf{k} n_{2}$ e $\mathcal{B}_{3}$. A dimensão do radical nilpotente é 4. A dimensão da órbita é 6. A dimensão do centro associador é 4. A dimensão de $\mathfrak{J}^{2}$ é 1 . A dimensão de $\mathfrak{J}^{3}$ é 0 . A dimensão de $J^{4}$ é 0 . A dimensão do grupo de 2-cociclos é 34. A dimensão do grupo de 2cobordos é 6. A dimensão do segundo grupo de Cohomologia é 28. A dimensão da subálgebra associativa maximal é 4 . A dimensão da subálgebra nilpotente maximal é 4. A dimensão da subálgebra nula maximal é 3.

ÁLGEBRA $J_{73}$

O produto na álgebra é:

\begin{tabular}{c|cccc}
$\partial_{73}$ & $e_{1}$ & $e_{2}$ & $e_{3}$ & $e_{4}$ \\
\hline$e_{1}$ & 0 & 0 & 0 & 0 \\
$e_{2}$ & 0 & 0 & 0 & 0 \\
$e_{3}$ & 0 & 0 & 0 & 0 \\
$e_{4}$ & 0 & 0 & 0 & 0
\end{tabular}

É uma álgebra associativa, não possui unidade. A dimensão do grupo de automorfismos é 16 . 
O grupo de automorfismos é:

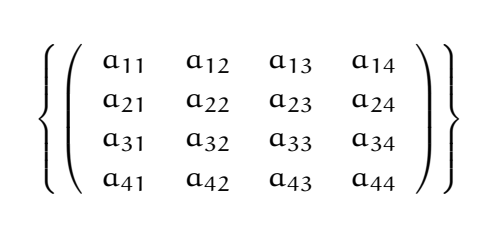

A dimensão do aniquilador da álgebra é 4. A álgebra se decompõe como: $\mathbf{k} n_{1} \oplus \mathbf{k} \mathbf{n}_{2} \oplus$ $\mathbf{k n}_{3} \oplus \mathbf{k} n_{4}$. Subálgebras de dimensão 2 e 3: $\mathbf{k n}_{1} \oplus \mathbf{k} n_{2} \oplus \mathbf{k} n_{3}$ e $\mathbf{k} n_{1} \oplus \mathbf{k} n_{2}$. A dimensão do radical nilpotente é 4. A dimensão da órbita é 0 . A dimensão do centro associador é 4. A dimensão de $\mathfrak{J}^{2}$ é 0 . A dimensão de $\mathfrak{J}^{3}$ é 0 . A dimensão de $\mathcal{J}^{4}$ é 0 . A dimensão do grupo de 2-cociclos é 40. A dimensão do grupo de 2-cobordos é 0 . A dimensão do segundo grupo de Cohomologia é 40. A dimensão da subálgebra associativa maximal é 4. A dimensão da subálgebra nilpotente maximal é 4. A dimensão da subálgebra nula maximal é 4 . 



\section{C INFORMAÇÕES SOBRE AS ÁLGEBRAS DE JOR- DAN REAIS DE DIMENSÃO 3}

Lembramos que as álgebras de Jordan reais de dimensão $3, \mathcal{T}_{i}^{\prime}$, foram obtidas na Seção 3.2 e que as álgebras de Jordan reais de dimensão 2 indecomponíveis são:

Tabela C.1: $\mathbb{R}$-álgebras de Jordan indecomponíveis de dimensão 2

\begin{tabular}{|c|ccc|}
\hline $\mathcal{B}^{\prime}$ & \multicolumn{3}{|c|}{ Tabela de Multiplicação } \\
\hline $\mathcal{B}_{1}^{\prime}$ & $e_{1}^{2}=e_{1}$ & $e_{1} n_{1}=n_{1}$ & $n_{1}^{2}=0$ \\
\hline $\mathcal{B}_{2}^{\prime}$ & $e_{1}^{2}=e_{1}$ & $e_{1} n_{1}=\frac{1}{2} n_{1}$ & $n_{1}^{2}=0$ \\
\hline $\mathcal{B}_{3}^{\prime}$ & $n_{1}^{2}=n_{2}$ & $n_{1} n_{2}=0$ & $n_{2}^{2}=0$ \\
\hline $\mathcal{B}_{4}^{\prime}$ & $e_{1}^{2}=e_{1}$ & $e_{1} e_{2}=e_{2}$ & $e_{2}^{2}=-e_{1}$ \\
\hline
\end{tabular}

\section{ÁLGEBRA $\mathcal{T}_{1}^{\prime}$}

A dimensão da órbita é 9. A dimensão do grupo de automorfismos é 0. A dimensão do aniquilador é 0 . A dimensão do centro associador é 3. A dimensão de $\mathcal{T}^{2}$ é 3. A dimensão de $\mathcal{T}^{3}$ é 3. A dimensão de $\mathcal{T}^{4}$ é 3. A dimensão do grupo de 2-cociclos é 9. A dimensão do grupo de 2-cobordos é 9 . A dimensão do segundo grupo de Cohomologia é 0. A dimensão do radical nilpotente é 0 . A dimensão da subálgebra associativa maximal é 3. A dimensão da subálgebra nilpotente maximal é 0 . A dimensão da subálgebra nula maximal é 0 . É uma álgebra associativa, unitária, semisimples. A álgebra se decompõe como: $\mathbb{R e}_{1} \oplus \mathbb{R} e_{2} \oplus \mathbb{R} e_{3}$. Subálgebras de dimensão 2 : $\mathbb{R e}_{1} \oplus \mathbb{R e}_{2}$.

\section{ÁLGEBRA $\mathcal{T}_{2}^{\prime}$}

A dimensão da órbita é 9. A dimensão do grupo de automorfismos é 0. A dimensão do aniquilador é 0. A dimensão do centro associador é 3. A dimensão de $\mathcal{T}^{2}$ é 3. A dimensão de $\mathcal{T}^{3}$ é 3. A dimensão de $\mathcal{T}^{4}$ é 3. A dimensão do grupo de 2-cociclos é 9 . A dimensão do grupo de 2-cobordos é 9 . A dimensão do segundo grupo de Cohomologia 
é 0. A dimensão do radical nilpotente é 0. A dimensão da subálgebra associativa maximal é 3. A dimensão da subálgebra nilpotente maximal é 0 . A dimensão da subálgebra nula maximal é 0 . É uma álgebra associativa, unitária, semisimples. A álgebra se decompõe como: $\mathcal{B}_{4}^{\prime} \oplus \mathbb{R e}_{3}$. Subálgebras de dimensão 2: $\mathbb{R e}_{1} \oplus \mathbb{R e}_{2}$ e $\mathcal{B}_{4}^{\prime}$.

\section{ÁLGEBRA $\mathcal{T}_{3}^{\prime}$}

A dimensão da órbita é 8. A dimensão do grupo de automorfismos é 1. A dimensão do aniquilador é 0 . A dimensão do centro associador é 1. A dimensão de $\mathcal{T}^{2}$ é 3. A dimensão de $\mathcal{T}^{3}$ é 3. A dimensão de $\mathcal{T}^{4}$ é 3. A dimensão do grupo de 2-cociclos é 8. A dimensão do grupo de 2-cobordos é 8. A dimensão do segundo grupo de Cohomologia é 0 . A dimensão do radical nilpotente é 0 . A dimensão da subálgebra associativa maximal é 2. A dimensão da subálgebra nilpotente maximal é 0 . A dimensão da subálgebra nula maximal é 0. É uma álgebra unitária, semisimples, indecomponível. Subálgebras de dimensão 2: $\mathbb{R} e_{1} \oplus \mathbb{R} e_{2}, \mathcal{B}_{4}^{\prime}$ e $\mathcal{B}_{1}^{\prime}$.

ÁLGEBRA $\mathcal{T}_{4}^{\prime}$

A dimensão da órbita é 8. A dimensão do grupo de automorfismos é 1. A dimensão do aniquilador é 0 . A dimensão do centro associador é 1. A dimensão de $\mathcal{T}^{2}$ é 3 . A dimensão de $\mathcal{T}^{3}$ é 3 . A dimensão de $\mathcal{T}^{4}$ é 3. A dimensão do grupo de 2-cociclos é 8 . A dimensão do grupo de 2-cobordos é 8. A dimensão do segundo grupo de Cohomologia é 0. A dimensão do radical nilpotente é 0. A dimensão da subálgebra associativa maximal é 2. A dimensão da subálgebra nilpotente maximal é 0 . A dimensão da subálgebra nula maximal é 0 . É uma álgebra unitária, semisimples, indecomponível. Subálgebras de dimensão 2: $\mathcal{B}_{4}^{\prime}$.

\section{ÁLGEBRA $\mathcal{T}_{5}^{\prime}$}

A dimensão da órbita é 8. A dimensão do grupo de automorfismos é 1. A dimensão do aniquilador é 0 . A dimensão do centro associador é 1. A dimensão de $\mathcal{T}^{2}$ é 3 . A dimensão de $\mathcal{T}^{3}$ é 3 . A dimensão de $\mathcal{T}^{4}$ é 3. A dimensão do grupo de 2-cociclos é 8 . A dimensão do grupo de 2-cobordos é 8. A dimensão do segundo grupo de Cohomologia é 0 . A dimensão do radical nilpotente é 0 . A dimensão da subálgebra associativa 
maximal é 2. A dimensão da subálgebra nilpotente maximal é 0. A dimensão da subálgebra nula maximal é 0 . É uma álgebra unitária, semisimples, indecomponível. Subálgebras de dimensão 2: $\mathbb{R e}_{1} \oplus \mathbb{R} e_{2}$.

\section{ÁLGEBRA $\mathcal{T}_{6}^{\prime}$}

A dimensão da órbita é 8. A dimensão do grupo de automorfismos é 1. A dimensão do aniquilador é 1. A dimensão do centro associador é 3. A dimensão de $\mathcal{T}^{2}$ é 2 . A dimensão de $\mathcal{T}^{3}$ é 2 . A dimensão de $\mathcal{T}^{4}$ é 2. A dimensão do grupo de 2-cociclos é 9. A dimensão do grupo de 2-cobordos é 8 . A dimensão do segundo grupo de Cohomologia é 1. A dimensão do radical nilpotente é 1. A dimensão da subálgebra associativa maximal é 3. A dimensão da subálgebra nilpotente maximal é 1. A dimensão da subálgebra nula maximal é 1. É uma álgebra associativa. A álgebra se decompõe como: $\mathbb{R} e_{1} \oplus \mathbb{R} e_{2} \oplus \mathbb{R} n_{1}$. Subálgebras de dimensão 2: $\mathbb{R e}_{1} \oplus \mathbb{R} e_{2}$ e $\mathbb{R} e_{1} \oplus \mathbb{R} n_{1}$.

\section{ÁLGEBRA $\mathcal{T}_{7}^{\prime}$}

A dimensão da órbita é 7. A dimensão do grupo de automorfismos é 2. A dimensão do aniquilador é 0 . A dimensão do centro associador é 1 . A dimensão de $\mathcal{T}^{2}$ é 3. A dimensão de $\mathcal{T}^{3}$ é 3. A dimensão de $\mathcal{T}^{4}$ é 3. A dimensão do grupo de 2-cociclos é 7. A dimensão do grupo de 2-cobordos é 7. A dimensão do segundo grupo de Cohomologia é 0. A dimensão do radical nilpotente é 1. A dimensão da subálgebra associativa maximal é 2. A dimensão da subálgebra nilpotente maximal é 1. A dimensão da subálgebra nula maximal é 1 . A álgebra se decompõe como: $\mathcal{B}_{2}^{\prime} \oplus \mathbb{R e}_{2}$. Subálgebras de dimensão 2: $\mathbb{R e}_{1} \oplus \mathbb{R} e_{2}, \mathbb{R e}_{1} \oplus \mathbb{R} n_{1}$ e $\mathcal{B}_{2}^{\prime}$.

\section{ÁLGEBRA $\mathcal{T}_{8}^{\prime}$}

A dimensão da órbita é 7. A dimensão do grupo de automorfismos é 2. A dimensão do aniquilador é 0 . A dimensão do centro associador é 1 . A dimensão de $\mathcal{T}^{2}$ é 3. A dimensão de $\mathcal{T}^{3}$ é 3. A dimensão de $\mathcal{T}^{4}$ é 3. A dimensão do grupo de 2-cociclos é 8 . A dimensão do grupo de 2-cobordos é 7. A dimensão do segundo grupo de Cohomologia é 1. A dimensão do radical nilpotente é 1. A dimensão da subálgebra associativa maximal é 2. A dimensão da subálgebra nilpotente maximal é 1. A dimensão da 
subálgebra nula maximal é 1. É uma álgebra unitária, indecomponível. Subálgebras de dimensão 2: $\mathbb{R e}_{1} \oplus \mathbb{R e}_{2}, \mathcal{B}_{1}^{\prime}$ e $\mathcal{B}_{2}^{\prime}$.

ÁLGEBRA $\mathcal{T}_{9}^{\prime}$

A dimensão da órbita é 8. A dimensão do grupo de automorfismos é 1. A dimensão do aniquilador é 0 . A dimensão do centro associador é 3. A dimensão de $\mathcal{T}^{2}$ é 3 . A dimensão de $\mathcal{T}^{3}$ é 3. A dimensão de $\mathcal{T}^{4}$ é 3. A dimensão do grupo de 2-cociclos é 9. A dimensão do grupo de 2-cobordos é 8. A dimensão do segundo grupo de Cohomologia é 1. A dimensão do radical nilpotente é 1. A dimensão da subálgebra associativa maximal é 3. A dimensão da subálgebra nilpotente maximal é 1. A dimensão da subálgebra nula maximal é 1. É uma álgebra associativa, unitária. A álgebra se decompõe como: $\mathcal{B}_{1}^{\prime} \oplus \mathbb{R e}_{2}$. Subálgebras de dimensão 2: $\mathbb{R} e_{1} \oplus \mathbb{R} e_{2}, \mathcal{B}_{1}^{\prime}$ e $\mathbb{R} e_{1} \oplus \mathbb{R} n_{1}$.

\section{ÁLGEBRA $\mathcal{T}_{10}^{\prime}$}

A dimensão da órbita é 8. A dimensão do grupo de automorfismos é 1. A dimensão do aniquilador é 1. A dimensão do centro associador é 3. A dimensão de $\mathcal{T}^{2}$ é 2 . A dimensão de $\mathcal{T}^{3}$ é 2 . A dimensão de $\mathcal{T}^{4}$ é 2. A dimensão do grupo de 2-cociclos é 9 . A dimensão do grupo de 2-cobordos é 8. A dimensão do segundo grupo de Cohomologia é 1. A dimensão do radical nilpotente é 1. A dimensão da subálgebra associativa maximal é 3. A dimensão da subálgebra nilpotente maximal é 1. A dimensão da subálgebra nula maximal é 1. É uma álgebra associativa. A álgebra se decompõe como: $\mathcal{B}_{4}^{\prime} \oplus \mathbb{R} n_{1}$. Subálgebras de dimensão $2: \mathbb{R} e_{1} \oplus \mathbb{R} n_{1}$ e $\mathcal{B}_{4}^{\prime}$.

ÁLGEBRA $\mathcal{T}_{11}^{\prime}$

A dimensão da órbita é 7. A dimensão do grupo de automorfismos é 2. A dimensão do aniquilador é 0 . A dimensão do centro associador é 1. A dimensão de $\mathcal{T}^{2}$ é 3 . A dimensão de $\mathcal{T}^{3}$ é 3 . A dimensão de $\mathcal{T}^{4}$ é 3. A dimensão do grupo de 2-cociclos é 8 . A dimensão do grupo de 2-cobordos é 7. A dimensão do segundo grupo de Cohomologia é 1. A dimensão do radical nilpotente é 1. A dimensão da subálgebra associativa maximal é 2. A dimensão da subálgebra nilpotente maximal é 1. A dimensão da 
subálgebra nula maximal é 1. É uma álgebra unitária, indecomponível. Subálgebras de dimensão 2: $\mathcal{B}_{1}^{\prime}$ e $\mathcal{B}_{4}^{\prime}$.

ÁLGEBRA $\mathcal{T}_{12}^{\prime}$

A dimensão da órbita é 3. A dimensão do grupo de automorfismos é 6. A dimensão do aniquilador é 0 . A dimensão do centro associador é 0 . A dimensão de $\mathcal{T}^{2}$ é 3. A dimensão de $\mathcal{T}^{3}$ é 3. A dimensão de $\mathcal{T}^{4}$ é 3. A dimensão do grupo de 2-cociclos é 3. A dimensão do grupo de 2-cobordos é 3. A dimensão do segundo grupo de Cohomologia é 0. A dimensão do radical nilpotente é 2. A dimensão da subálgebra associativa maximal é 2. A dimensão da subálgebra nilpotente maximal é 2. A dimensão da subálgebra nula maximal é 2. É uma álgebra indecomponível. Subálgebras de dimensão 2: $\mathbb{R} n_{1} \oplus \mathbb{R} n_{2}$ e $\mathcal{B}_{2}^{\prime}$.

ÁLGEBRA $\mathcal{T}_{13}^{\prime}$

A dimensão da órbita é 5. A dimensão do grupo de automorfismos é 4. A dimensão do aniquilador é 0 . A dimensão do centro associador é 3. A dimensão de $\mathcal{T}^{2}$ é 3. A dimensão de $\mathcal{T}^{3}$ é 3. A dimensão de $\mathcal{T}^{4}$ é 3. A dimensão do grupo de 2-cociclos é 12. A dimensão do grupo de 2-cobordos é 5. A dimensão do segundo grupo de Cohomologia é 7. A dimensão do radical nilpotente é 2. A dimensão da subálgebra associativa maximal é 3. A dimensão da subálgebra nilpotente maximal é 2. A dimensão da subálgebra nula maximal é 2. É uma álgebra associativa, unitária, indecomponível. Subálgebras de dimensão 2: $\mathcal{B}_{1}^{\prime}$ e $\mathbb{R} n_{1} \oplus \mathbb{R} n_{2}$.

ÁLGEBRA $\mathcal{T}_{14}^{\prime}$

A dimensão da órbita é 6. A dimensão do grupo de automorfismos é 3. A dimensão do aniquilador é 1. A dimensão do centro associador é 1. A dimensão de $\mathcal{T}^{2}$ é 2. A dimensão de $\mathcal{T}^{3}$ é 2 . A dimensão de $\mathcal{T}^{4}$ é 2. A dimensão do grupo de 2-cociclos é 9 . A dimensão do grupo de 2-cobordos é 6 . A dimensão do segundo grupo de Cohomologia é 3. A dimensão do radical nilpotente é 2. A dimensão da subálgebra associativa maximal é 2. A dimensão da subálgebra nilpotente maximal é 2. A dimensão da 
subálgebra nula maximal é 2 . A álgebra se decompõe como: $\mathcal{B}_{2}^{\prime} \oplus \mathbb{R} n_{2}$. Subálgebras de dimensão 2: $\mathbb{R} e_{1} \oplus \mathbb{R} n_{1}, \mathbb{R} n_{1} \oplus \mathbb{R} n_{2}$ e $\mathcal{B}_{2}^{\prime}$.

ÁLGEBRA $\mathcal{T}_{15}^{\prime}$

A dimensão da órbita é 7. A dimensão do grupo de automorfismos é 2. A dimensão do aniquilador é 1. A dimensão do centro associador é 3. A dimensão de $\mathcal{T}^{2}$ é 2 . A dimensão de $\mathcal{T}^{3}$ é 2 . A dimensão de $\mathcal{T}^{4}$ é 2 . A dimensão do grupo de 2-cociclos é 9 . A dimensão do grupo de 2-cobordos é 7. A dimensão do segundo grupo de Cohomologia é 2. A dimensão do radical nilpotente é 2. A dimensão da subálgebra associativa maximal é 3. A dimensão da subálgebra nilpotente maximal é 2. A dimensão da subálgebra nula maximal é 2. É uma álgebra associativa. A álgebra se decompõe como: $\mathcal{B}_{1}^{\prime} \oplus \mathbb{R} n_{2}$. Subálgebras de dimensão $2: \mathcal{B}_{1}^{\prime}, \mathbb{R e}_{1} \oplus \mathbb{R} n_{1}$ e $\mathbb{R} n_{1} \oplus \mathbb{R} n_{2}$.

\section{ÁLGEBRA $\mathcal{T}_{16}^{\prime}$}

A dimensão da órbita é 6. A dimensão do grupo de automorfismos é 3. A dimensão do aniquilador é 0 . A dimensão do centro associador é 1. A dimensão de $\mathcal{T}^{2}$ é 3 . A dimensão de $\mathcal{T}^{3}$ é 3. A dimensão de $\mathcal{T}^{4}$ é 3. A dimensão do grupo de 2-cociclos é 8 . A dimensão do grupo de 2-cobordos é 6 . A dimensão do segundo grupo de Cohomologia é 2. A dimensão do radical nilpotente é 2. A dimensão da subálgebra associativa maximal é 2. A dimensão da subálgebra nilpotente maximal é 2. A dimensão da subálgebra nula maximal é 2. É uma álgebra indecomponível. Subálgebras de dimensão 2: $\mathcal{B}_{1}^{\prime}, \mathbb{R} n_{1} \oplus \mathbb{R} n_{2}$ e $\mathcal{B}_{2}^{\prime}$.

\section{ÁLGEBRA $\mathcal{T}_{17}^{\prime}$}

A dimensão da órbita é 5. A dimensão do grupo de automorfismos é 4. A dimensão do aniquilador é 2. A dimensão do centro associador é 3. A dimensão de $\mathcal{T}^{2}$ é 1. A dimensão de $\mathcal{T}^{3}$ é 1 . A dimensão de $\mathcal{T}^{4}$ é 1 . A dimensão do grupo de 2-cociclos é 11. A dimensão do grupo de 2-cobordos é 5. A dimensão do segundo grupo de Cohomologia é 6. A dimensão do radical nilpotente é 2. A dimensão da subálgebra associativa maximal é 3. A dimensão da subálgebra nilpotente maximal é 2. A dimensão da 
subálgebra nula maximal é 2. É uma álgebra associativa. A álgebra se decompõe como: $\mathbb{R e}_{1} \oplus \mathbb{R} n_{1} \oplus \mathbb{R} n_{2}$. Subálgebras de dimensão 2: $\mathbb{R} e_{1} \oplus \mathbb{R} n_{1}$ e $\mathbb{R} n_{1} \oplus \mathbb{R} n_{2}$.

\section{ÁLGEBRA $\mathcal{T}_{18}^{\prime}$}

A dimensão da órbita é 7. A dimensão do grupo de automorfismos é 2. A dimensão do aniquilador é 0 . A dimensão do centro associador é 3. A dimensão de $\mathcal{T}^{2}$ é 3. A dimensão de $\mathcal{T}^{3}$ é 3. A dimensão de $\mathcal{T}^{4}$ é 3. A dimensão do grupo de 2-cociclos é 9 . A dimensão do grupo de 2-cobordos é 7. A dimensão do segundo grupo de Cohomologia é 2. A dimensão do radical nilpotente é 2. A dimensão da subálgebra associativa maximal é 3. A dimensão da subálgebra nilpotente maximal é 2. A dimensão da subálgebra nula maximal é 1. É uma álgebra associativa, unitária, indecomponível. Subálgebras de dimensão 2: $\mathcal{B}_{1}^{\prime}$ e $\mathcal{B}_{3}^{\prime}$.

\section{ÁLGEBRA $\mathcal{T}_{19}^{\prime}$}

A dimensão da órbita é 7. A dimensão do grupo de automorfismos é 2. A dimensão do aniquilador é 1. A dimensão do centro associador é 1. A dimensão de $\mathcal{T}^{2}$ é 3. A dimensão de $\mathcal{T}^{3}$ é 3. A dimensão de $\mathcal{T}^{4}$ é 3. A dimensão do grupo de 2-cociclos é 8 . A dimensão do grupo de 2-cobordos é 7. A dimensão do segundo grupo de Cohomologia é 1. A dimensão do radical nilpotente é 2. A dimensão da subálgebra associativa maximal é 2. A dimensão da subálgebra nilpotente maximal é 2. A dimensão da subálgebra nula maximal é 1. É uma álgebra indecomponível. Subálgebras de dimensão 2: $\mathbb{R} e_{1} \oplus \mathbb{R} n_{1}$ e $\mathcal{B}_{3}^{\prime}$.

\section{ÁLGEBRA $\mathcal{T}_{20}^{\prime}$}

A dimensão da órbita é 7. A dimensão do grupo de automorfismos é 2. A dimensão do aniquilador é 0 . A dimensão do centro associador é 1. A dimensão de $\mathcal{T}^{2}$ é 3. A dimensão de $\mathcal{T}^{3}$ é 3. A dimensão de $\mathcal{T}^{4}$ é 3. A dimensão do grupo de 2-cociclos é 7. A dimensão do grupo de 2-cobordos é 7. A dimensão do segundo grupo de Cohomologia é 0. A dimensão do radical nilpotente é 2. A dimensão da subálgebra associativa maximal é 2. A dimensão da subálgebra nilpotente maximal é 2. A dimensão da 
subálgebra nula maximal é 1. É uma álgebra indecomponível. Subálgebras de dimensão 2: $\mathcal{B}_{1}^{\prime}$ e $\mathcal{B}_{3}^{\prime}$.

ÁLGEBRA $\mathcal{T}_{21}^{\prime}$

A dimensão da órbita é 7. A dimensão do grupo de automorfismos é 2. A dimensão do aniquilador é 1. A dimensão do centro associador é 3. A dimensão de $\mathcal{T}^{2}$ é 2 . A dimensão de $\mathcal{T}^{3}$ é 1 . A dimensão de $\mathcal{T}^{4}$ é 1 . A dimensão do grupo de 2-cociclos é 9 . A dimensão do grupo de 2-cobordos é 7. A dimensão do segundo grupo de Cohomologia é 2. A dimensão do radical nilpotente é 2. A dimensão da subálgebra associativa maximal é 3. A dimensão da subálgebra nilpotente maximal é 2. A dimensão da subálgebra nula maximal é 1. É uma álgebra associativa. A álgebra se decompõe como: $\mathcal{B}_{3}^{\prime} \oplus \mathbb{R} e_{1}$. Subálgebras de dimensão 2: $\mathbb{R} e_{1} \oplus \mathbb{R} n_{1}$ e $\mathcal{B}_{3}^{\prime}$.

\section{ÁLGEBRA $\mathcal{T}_{22}^{\prime}$}

A dimensão da órbita é 0 . A dimensão do grupo de automorfismos é 9. A dimensão do aniquilador é 3. A dimensão do centro associador é 3. A dimensão de $\mathcal{T}^{2}$ é 0 . A dimensão de $\mathcal{T}^{3}$ é 0 . A dimensão de $\mathcal{T}^{4}$ é 0 . A dimensão do grupo de 2-cociclos é 18. A dimensão do grupo de 2-cobordos é 0 . A dimensão do segundo grupo de Cohomologia é 18. A dimensão do radical nilpotente é 3. A dimensão da subálgebra associativa maximal é 3. A dimensão da subálgebra nilpotente maximal é 3. A dimensão da subálgebra nula maximal é 3. É uma álgebra associativa, nilpotente. A álgebra se decompõe como: $\mathbb{R} n_{1} \oplus \mathbb{R} n_{2} \oplus \mathbb{R} n_{3}$. Subálgebras de dimensão 2: $\mathbb{R} n_{1} \oplus \mathbb{R} n_{2}$.

ÁLGEBRA $\mathcal{T}_{23}^{\prime}$

A dimensão da órbita é 6. A dimensão do grupo de automorfismos é 3. A dimensão do aniquilador é 1. A dimensão do centro associador é 3. A dimensão de $\mathcal{T}^{2}$ é 2 . A dimensão de $\mathcal{T}^{3}$ é 1 . A dimensão de $\mathcal{T}^{4}$ é 0 . A dimensão do grupo de 2-cociclos é 9 . A dimensão do grupo de 2-cobordos é 6 . A dimensão do segundo grupo de Cohomologia é 3. A dimensão do radical nilpotente é 3. A dimensão da subálgebra associativa maximal é 3. A dimensão da subálgebra nilpotente maximal é 3. A dimensão da 
subálgebra nula maximal é 2. É uma álgebra associativa, nilpotente, indecomponível. Subálgebras de dimensão $2: \mathbb{R} n_{1} \oplus \mathbb{R} n_{2}$.

\section{ÁLGEBRA $\mathcal{T}_{24}^{\prime}$}

A dimensão da órbita é 5. A dimensão do grupo de automorfismos é 4. A dimensão do aniquilador é 1. A dimensão do centro associador é 3. A dimensão de $\mathcal{T}^{2}$ é 1. A dimensão de $\mathcal{T}^{3}$ é 0 . A dimensão de $\mathcal{T}^{4}$ é 0 . A dimensão do grupo de 2-cociclos é 12. A dimensão do grupo de 2-cobordos é 5. A dimensão do segundo grupo de Cohomologia é 7. A dimensão do radical nilpotente é 3. A dimensão da subálgebra associativa maximal é 3. A dimensão da subálgebra nilpotente maximal é 3. A dimensão da subálgebra nula maximal é 1. É uma álgebra associativa, nilpotente, indecomponível. Subálgebras de dimensão 2: $\mathcal{B}_{3}^{\prime}$.

\section{ÁLGEBRA $\mathcal{T}_{25}^{\prime}$}

A dimensão da órbita é 4. A dimensão do grupo de automorfismos é 5. A dimensão do aniquilador é 2. A dimensão do centro associador é 3. A dimensão de $\mathcal{T}^{2}$ é 1 . A dimensão de $\mathcal{T}^{3}$ é 0 . A dimensão de $\mathcal{T}^{4}$ é 0 . A dimensão do grupo de 2-cociclos é 14. A dimensão do grupo de 2-cobordos é 4. A dimensão do segundo grupo de Cohomologia é 10. A dimensão do radical nilpotente é 3. A dimensão da subálgebra associativa maximal é 3. A dimensão da subálgebra nilpotente maximal é 3. A dimensão da subálgebra nula maximal é 2. É uma álgebra associativa, nilpotente. A álgebra se decompõe como: $\mathcal{B}_{3}^{\prime} \oplus \mathbb{R} n_{3}$. Subálgebras de dimensão 2 : $\mathbb{R} n_{1} \oplus \mathbb{R} n_{2}$ e $\mathcal{B}_{3}^{\prime}$.

\section{ÁLGEBRA $\mathcal{T}_{26}^{\prime}$}

A dimensão da órbita é 5. A dimensão do grupo de automorfismos é 4. A dimensão do aniquilador é 1. A dimensão do centro associador é 3. A dimensão de $\mathcal{T}^{2}$ é 1. A dimensão de $\mathcal{T}^{3}$ é 0 . A dimensão de $\mathcal{T}^{4}$ é 0 . A dimensão do grupo de 2-cociclos é 12. A dimensão do grupo de 2-cobordos é 5. A dimensão do segundo grupo de Cohomologia é 7. A dimensão do radical nilpotente é 3. A dimensão da subálgebra associativa maximal é 3. A dimensão da subálgebra nilpotente maximal é 3. A dimensão da 
subálgebra nula maximal é 2. É uma álgebra associativa, nilpotente, indecomponível. Subálgebras de dimensão 2: $\mathbb{R} n_{1} \oplus \mathbb{R} n_{2}$ e $\mathcal{B}_{3}^{\prime}$. 


\section{REFERÊNCIAS BIBLIOGRÁFICAS}

[1] A. A. Albert, On a Certain Algebra of Quantum Mechanics, The Annals of Mathematics, 35 (1934), pp. 65-73.

[2] — A Structure Theory for Jordan Algebras, The Annals of Mathematics, 48 (1947), pp. 546-567.

[3] J. M. Ancochea Bermúdez, R. Campoamor-Stursberg, L. García Vergnolle, e J. SÁnchez Hernández, Contractions d'Algèbres de Jordan en Dimension 2, Journal of Algebra, 319 (2008), pp. 2395-2409.

[4] J. M. Ancochea Bermúdez, J. Fresán, e J. M. Bentabol, Contractions of LowDimensional Nilpotent Jordan Algebras, Communications in Algebra, 39 (2011), pp. 1139-1151.

[5] J. M. Ancochea Bermudez, J. Fresán, e J. Sánchez Hernández, On the Variety of two Dimensional Real Associative Algebras, Int. J. Contemp. Math. Sciences, 2 (2007), pp. 1293-1305.

[6] J. Bochnak, M. Coste, E M. F. Roy, Real Algebraic Geometry, Ergebnisse der Mathematik und ihrer Grenzgebiete, Springer, Berlin, 1998.

[7] A. Borel, Linear Algebraic Groups, Springer, New York, 1991.

[8] W. Crawley-Boevey, Geometry of Representations of Algebras, Lecture Notes, Oxford University, 1993.

[9] M. Demazure e P. Gabriel, Introduction to Algebraic Geometry and Algebraic Groups, North-Holland Mathematics Studies, Elsevier Science, Amsterdam, 1980.

[10] W. Ferrer Santos E A. Rittatore, Actions and Invariants of Algebraic Groups, vol. 269 of Pure and Applied Mathematics, CRC Press, Boca Raton, 2005.

[11] F. Flanigan, Algebraic Geography: Varieties of Structure Constants, Pacific Journal of Mathematics, 27 (1968), pp. 71-79.

[12] — Straightening-out and Semirigidity in Associative Algebras, Transactions of the American Mathematical Society, 138 (1969), pp. 415-425. 
[13] P. Gabriel, Finite Representation Type is Open, Lectures Notes in Mathematics, 488 (1975), pp. 132-155.

[14] M. Gerstenhaber, On the Deformation of Rings and Algebras, The Annals of Mathematics, 79 (1964), pp. 59-103.

[15] — Relative Hochschild Cohomology, Rigid Algebras, and the Bockstein, Journal of Pure and Applied Algebra, 43 (1986), pp. 53-74.

[16] R. Hartshorne, Algebraic Geometry, Graduate Texts in Mathematics, Springer, New York, 1977.

[17] A. Hegazi e H. Авdelwahab, Construction of Nilpotent Jordan Algebras Over any Arbitrary Fields, eprint arXiv:1301.6349, (2013), pp. 1-41.

[18] J. Humphreys, Linear Algebraic Groups, Springer-Verlag, New York, 1995.

[19] N. Jacobson, A Theorem on the Structure of Jordan Algebras, Proceedings of the National Academy of Sciences, 42 (1956), pp. 140-147.

[20] —_ Exceptional Lie Algebras, Lecture Notes in Pure and Applied Mathematics, Mimeographed Notes, Yale University, New Haven, 1958.

[21] — Structure and Representations of Jordan Algebras, vol. 39 of American Mathematical Society Colloquium Publications, American Mathematical Society, Providence, 1968.

[22] I. Kashuba, Variedades de Álgebras de Jordan/Tipos de Representações de Álgebras de Jordan, tese de doutorado, Universidade de São Paulo, 2004.

[23] _ - Variety of Jordan Algebras in Small Dimensions, Algebra and Discrete Mathematics, 2 (2006), pp. 62-76.

[24] I. Kashuba e M. E. Martin, Deformations of Jordan Algebras of Dimension Four, Aceito para publicação no Journal of Algebra, (2013).

[25] I. Kashuba e I. Shestakov, Jordan Algebras of Dimension Three: Geometric Classification and Representation Type, Actas del XVI Coloquio Latinoamericano de Álgebra (Colonia del Sacramento, Uruguay). Bibl. Rev. Iber. Mat, I (2005), pp. 295-315.

[26] A. A. Kirillov E Y. A. Neretin, The Variety $A_{n}$ of n-dimensional Lie Algebra Structures, American Mathematical Society, 137 (1987), pp. 21-30. 
[27] M. Koecher, An Elementary Approach to Bounded Symmetric Domains, Rice University, Houston, 1969.

[28] M. Koecher, The Minnesota Notes on Jordan Algebras and Their Applications, Springer, Berlin, 1999.

[29] E. Kunz, Introduction to Commutative Algebra and Algebraic Geometry, Modern Birkhauser Classics, Birkhauser, Boston, 1985.

[30] M. E. Martin, Four Dimensional Jordan Algebras, International Journal of Mathematics, Game Theory and Algebra, 20 (2013), pp. 41-59.

[31] G. Mazzola, The Algebraic and Geometric Classification of Associative Algebras of Dimension Five, Manuscripta Math., 27 (1979), pp. 81-101.

[32] _ Generic Finite Schemes and Hochschild Cocycles, Commentarii Mathematici Helvetici, 55 (2011), pp. 267-293.

[33] K. McCrimmon, A Taste of Jordan Algebras, Springer, New York, 2004.

[34] M. Nathanson, Elementary Methods in Number Theory, Springer, New York, 2000.

[35] Y. A. Neretin, An Estimate of the Number of Parameters Defining an n-Dimensional Algebra, Math. USSR Izvestiya, 20 (1988), pp. 283-294.

[36] A. Nijenhuis e R. W. Richardson, Commutative Algebra Cohomology and Deformations of Lie and Associative Algebras, Journal of Algebra, 9 (1968), pp. 42-53.

[37] S. S. PAGE, A Characterization of Rigid Algebras, Journal of the London Mathematical Society, 2 (1970), pp. 237-240.

[38] A. J. Penico, The Wedderburn Principal Theorem for Jordan Algebras, Transactions of the American Mathematical Society, 70 (1951), pp. 404-420.

[39] R. Richardson, On the Rigidity of Semi-direct Products of Lie Algebras, Pacific Journal of Mathematics, 22 (1967), pp. 339-344.

[40] S. Roman, Advanced Linear Algebra, Springer, New York, 2008.

[41] R. Schafer, The Exceptional Simple Jordan Algebras, American Journal of Mathematics, 70 (1948), pp. 82-94.

[42] R. D. Schafer, Structure and Representation of Nonassociative Algebras, vol. 61 of Department of Mathematics at Oklahoma State University, Oklahoma, 1955. 
[43] H. J. Sherlukov, Jordan Algebras in Small Dimensions, dissertação de mestrado, University of Novosibirsk, 1989.

[44] А. M. SLIN'ко, Special Varieties of Jordan Algebras, Matematieheskie Zametki, 26 (1979), pp. 661-665.

[45] V. E. Study, Über Systeme Complexer Zahlen und ihre Anwendung in der Theorie der Transformationsgruppen., Monatsh. Math. Phys, 1 (1890), pp. 283-254.

[46] E. TAFT, Invariant Wedderburn Factors, Illinois Journal of Mathematics, 1 (1957), pp. 565-573.

[47] C. A. WeIbel, An Introduction to Homological Algebra, Cambridge Studies in Advanced Mathematics, Cambridge University Press, Cambridge, 1995.

[48] H. Wesseler, Der Klassification der Jordan-Algebren niedrigen Dimensionen., tese de doutorado, Staatexamensarbeit für der Lehramt am Gymnasium Münster, 1978.

[49] E. Wigner, P. Jordan, E J. v. Neumann, On an Algebraic Generalization of the Quantum Mechanical Formalism, Annals of Mathematics, 35 (1934), pp. 29-64.

[50] K. Zhevlakov, A. Slin'Ko, I. Shestakov, e A. Shirshov, Rings that are Nearly Associative, Academic Press, New York, 1982. 


\section{ÍNDICE REMISSIVO}

2-cobordos, 18

2-cociclo, 18

equivalentes, 18

a-homótopa, 134

a-isótopa, 134

a-multiplicação, 134

n-espaço afim, 59

álgebra, 8

analiticamente rígida, 66

associativa, 9

nilpotente, 11

de composição, 10

de divisão, 134

de Jordan, 8

de uma forma bilinear simétrica,

9

especial, 9

excepcional, 9

ideal, 8

subálgebra, 8

de multiplicação, 14

geometricamente rígida, 71

infinitesimalmente rígida, 66

nilpotente, 11

rígida, 71

semissimples, 12

separável, 12

simples, 12

central, 12

unitária, 10

órbita, 62 índice

de nilpotência, 11

adjunção formal, 10

analiticamente rígida, 66

aniquilador, 23

anulador, 58

associador, 8

bimódulo de Jordan, 18

centro associativo, 82

centroide, 14

cobordos, 18

cociclo, 18

componentes

de Peirce, 16

conjunto algébrico, 58

constantes

estruturais, 68

decomposição

de Peirce, 16

deformação, 70

a um parâmetro, 63

trivial, 64

trivial, 70

deformações

equivalentes, 65

diferencial, 64

dimensão, 60

elemento

idempotente, 15 
inverso, 133

invertível, 133

trivial, 134

espaço afim, 59

estabilizador, 62

excepcional, 9

extensão

cinde, 19

de álgebras, 19

nula, 19

extensão escalar, 11

extensões

equivalentes, 19

função polinomial, 58

função regular, 59

geometricamente rígida, 71

grupo

de estrutura, 135

grupo algébrico, 61

ação, 62

morfismo, 61

homótopa, 134

homotopia, 134

ideal, 8

idempotente

próprio, 15

idempotentes ortogonais, 15

identidade, 10

identidade de Jordan, 8

infinitesimalmente rígida, 66

integrável, 64

involução, 9

padrão, 10

irredutível, 59

isótopa, 134 isotópicas, 134

isotopia, 134

localmente fechado, 59

morfismo, 60

nil-índice, 11

nilpotente, 11

operador de multiplicação, 14

parte semissimples, 12

ponto genérico, 60

potência, 11

produto

de Kronecker, 11

produto triplo de Jordan, 134

rígida

analiticamente, 66

geometricamente, 71

infinitesimalmente, 66

radical, 12

nilpotente, 12

série central inferior, 11

segundo grupo de cohomologia, 18

subálgebra, 8

subvariedade, 59

tipo de nilpotência, 12

topologia de Zariski, 59

transporte de estrutura, 69

unidade, 10

unitária, 10

variedade, 59

afim, 60

algébrica, 59

das álgebras de Jordan, 69 